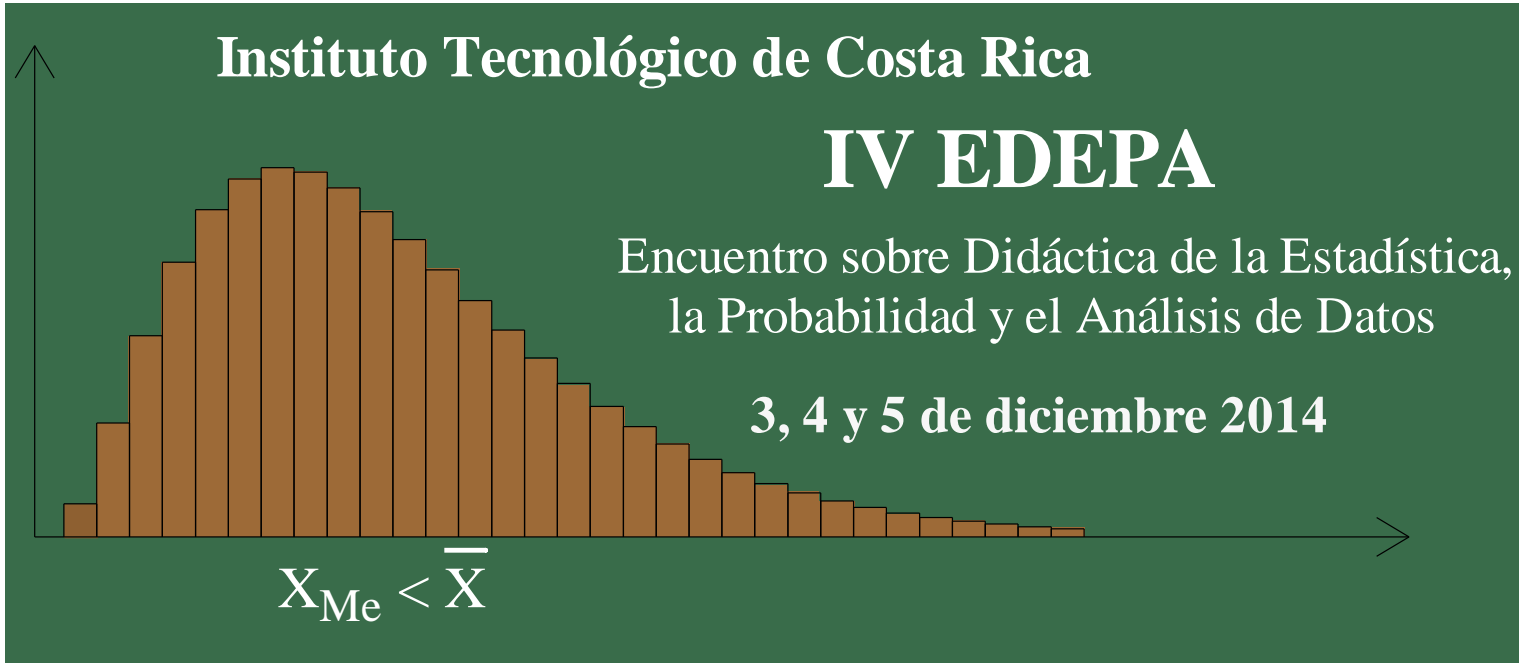

\title{
Memorias
}

\section{Encuentro sobre Didáctica de la Estadística, la Probabilidad y el Análisis de Datos}

Costa Rica, 3,4 y 5 de diciembre de 2014

ISBN: 978-9968-641-17-3 


\section{Presentación}

Recientemente en nuestro país se aprobaron nuevos programas de estudio en el área de matemática, que abarca la enseñanza primaria y la secundaria. Dichos programas contemplan, entre otros cambios, la inserción de una serie de contenidos y modificaciones en el abordaje de otros ya existentes que tienen que ver con probabilidad y estadística.

Pese a que hay consenso tanto en el plano internacional como en el nacional de que estos temas constituyen una parte fundamental deseable en la educación general de los futuros ciudadanos adultos, para llevar al aula estos temas se requiere de capacitaciones y una formación continua de los docentes de matemática en servicio.

Como Institución comprometida con el desarrollo de la educación de nuestro país, la Escuela de Matemática del Tecnológico de Costa Rica (TEC) ha venido desarrollando actividades tendientes a colaborar con ese requerimiento. En el año 2009 y en 2011 se organizaron en la sede central del TEC, el I EDEPA y el II EDEPA respectivamente, en los que a través de charlas, ponencias, talleres y conferencias, los participantes se nutrieron, tanto de propuestas didácticas como de aplicaciones, que tenían que ver con la Estadística y la Probabilidad.

Por otro lado, en 2012 se organizó la I Escuela de Verano, en la que se desarrollaron semi-cursos, talleres y charlas en los que se abordaron temas de probabilidad y estadística y la computadora, como recurso didáctico, permeó todas las actividades realizadas, brindando así aportes metodológicos significativos a los docentes participantes para desarrollar en el aula.

Más recientemente, en 2013, los días 27, 28 y 29 de noviembre, se organizó el III EDEPA, con líneas temáticas que tomaron en cuenta las propuestas del Ministerio de Educación Pública en la reforma de los programas de matemática.

Se contó en todas las ediciones con la participación de excelentes conferencistas nacionales e internacionales quienes compartieron los resultados de sus experiencias e investigaciones. Las actividades promovieron la participación activa de los docentes en el sentido de fomentar y potenciar el uso de la computadora como recurso de apoyo en los procesos de enseñanzaaprendizaje de los saberes que desean establecer.

Conscientes de la importancia del Encuentro, El Tecnológico de Costa Rica y su Escuela de Matemática tienen el propósito de que el EDEPA constituya un punto de encuentro y espacio académico entre profesores, investigadores y administradores educativos de Iberoamérica. El Comité Organizador del Encuentro tiene la convicción de continuar avanzando en la construcción de un espacio de crítica, debate y comunicación sobre el estado actual y el desarrollo reciente de la investigación en la didáctica de la estadística, la probabilidad y el análisis de datos a nivel internacional.

Es por ello que, en los días 3, 4 y 5 de diciembre 2014, la Escuela de Matemática del Tecnológico de Costa Rica organiza la cuarta edición del EDEPA (IV EDEPA) cuyas líneas temáticas contempladas, intentan responder a las necesidades de la educación estocástica, no solo en Costa Rica, sino también en otras latitudes:

- Datos y contexto en educación estadística: hacia una sociedad basada en la evidencia.

- La educación estadística en el ámbito escolar.

- Formación del profesorado en estadística 
- Educación estadística en educación superior.

- Evaluación en educación estadística.

- Educación estadística en el ámbito laboral.

- Investigación en educación estadística.

- Tecnología computacional en educación estadística.

- La educación estadística en la perspectiva internacional.

Para tal fin, se consideró pertinente integrar un Comité Científico con académicos líderes en el campo de la educación estadística, tanto a nivel nacional como internacional, el cual realizó trabajo preciso y detallado en la revisión de las diferentes propuestas presentadas por distinguidos académicos de Iberoamérica.

Entregamos con mucha satisfacción a la comunidad académica esta valiosa colección de extensos y resúmenes de los trabajos presentados en el IV EDEPA que esperamos sea de gran utilidad.

Con nuestra estima,

M.Sc. Giovanni Sanabria Brenes.

M.Sc. Félix Núñez Vanegas.

Coordinadores del IV EDEPA

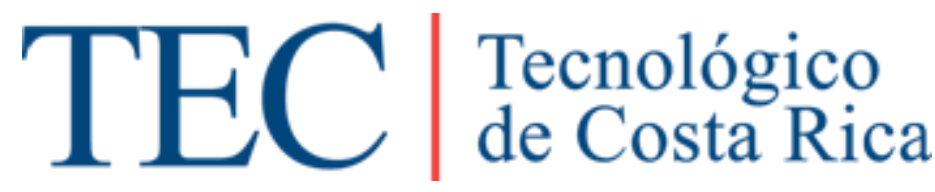




\section{Comité Organizador del IV EDEPA}

M.Sc. Giovanni Sanabria Brenes, Instituto Tecnológico de Costa Rica (co-coordinador).

M.Sc. Félix Núñez Vanegas, Instituto Tecnológico de Costa Rica (co-coordinador).

M.Sc. Greivin Ramírez Arce, Instituto Tecnológico de Costa Rica.

Dr. Jorge Monge Fallas, Instituto Tecnológico de Costa Rica.

Dr. Jesús Humberto Cuevas Acosta, Instituto Tecnológico de Chihuahua II, México.

\section{Comité Científico del IV EDEPA}

\section{Comité científico internacional}

Dra. Carmen Batanero Bernabeu, Universidad de Granada, Coordinadora del Grupo de Investigación sobre Educación Estadística, España

Dr. José Miguel Contreras García, Universidad de Granada, España

Dr. Jesús Humberto Cuevas Acosta, Instituto Tecnológico de Chihuahua II, México.

Dr. Mario Olguin Scherffig, Director del área de Estadística de la Universidad de Las Américas, Chile.

Dr. Sergio Hernández González. Universidad Veracruzana, presidente de la Asociación Mexicana de Estadística

M.Sc. Pedro Ramos, Universidad de El Salvador.

\section{Comité científico local}

Dr. Javier Trejos Zelaya, Universidad de Costa Rica.

Dr. Pedro Méndez Hernández, Universidad de Costa Rica.

Dra. Tania Elena Moreira Mora, Instituto Tecnológico de Costa Rica.

M.Sc. Giovanni Sanabria Brenes, Instituto Tecnológico de Costa Rica.

M.Sc. Félix Núñez Vanegas, Instituto Tecnológico de Costa Rica.

MSc. Greivin Ramírez Arce, Instituto Tecnológico de Costa Rica.

MSc. Luis Acuña Prado, Instituto Tecnológico de Costa Rica.

Lic. Luis Rojas Torres, Universidad de Costa Rica. 


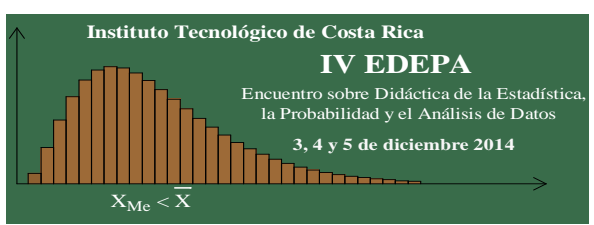

\section{Listado de presentaciones del IV EDEPA CONFERENCIAS}

\begin{tabular}{|l|l|l|l|}
\hline \multicolumn{2}{|c|}{ Ponentes } & \multicolumn{1}{|c|}{ Lugar } & \multicolumn{1}{c|}{ Título } \\
\hline C01 & $\begin{array}{l}\text { Roberto Behar Gutiérrez, } \\
\text { Pere Grima }\end{array}$ & $\begin{array}{l}\text { Universidad del Valle, Cali, } \\
\text { Colombia. Departamento de } \\
\text { Estadistica e l/O, UPC, } \\
\text { España }\end{array}$ & $\begin{array}{l}\text { Restricciones y obstáculos para la implantación de una propuesta de Reforma del } \\
\text { proceso de enseñanza y aprendizaje de la Estadística }\end{array}$ \\
\hline C02 & Assumpta Estrada Roca & $\begin{array}{l}\text { Universidad de Lleida, } \\
\text { España }\end{array}$ & Los Proyectos en la Formación Estadística \\
\hline
\end{tabular}

\section{EDEPA}

\begin{tabular}{|c|c|c|c|}
\hline $\mathrm{CO3}$ & Roberto Behar Gutiérrez & $\begin{array}{l}\text { Universidad del Valle, Cali, } \\
\text { Colombia }\end{array}$ & Estadística: Aprendizaje a Largo Plazo. Factores que inciden y estrategias plausibles \\
\hline C04 & Assumpta Estrada Roca & $\begin{array}{l}\text { Universidad de Lleida, } \\
\text { España }\end{array}$ & Las Actitudes hacia la Estadística y la Formación del Profesorado \\
\hline C05 & Eiliana Montero Rojas & $\begin{array}{l}\text { Proyecto Estado de la } \\
\text { Educación y Universidad de } \\
\text { Costa Rica }\end{array}$ & $\begin{array}{l}\text { Variables del estudiante y de la institución que predicen los puntajes de Alfabetización } \\
\text { Matemática en la muestra costarricense de PISA } 2009 \text { y 2012: análisis de regresión } \\
\text { multinivel e implicaciones educativas }\end{array}$ \\
\hline C06 & Edwin Chaves Esquivel & $\begin{array}{l}\text { Universidad Nacional, Costa } \\
\text { Rica }\end{array}$ & ¿Miente la información generada por medio de las Estadísticas y las Probabilidades? \\
\hline $\mathrm{CO}$ & $\begin{array}{l}\text { Jesús Humberto Cuevas } \\
\text { Acosta }\end{array}$ & $\begin{array}{l}\text { Instituto Tecnológico de } \\
\text { Chihuahua II México }\end{array}$ & Investigación Reproducible en la Educación Estadística \\
\hline
\end{tabular}

\section{MESA REDONDA}

\begin{tabular}{|l|l|l|l|}
\hline MR & $\begin{array}{l}\text { Assumpta Estrada Roca, } \\
\text { Roberto Behar Gutiérrez, } \\
\text { Giovanni Sanabria Brenes, } \\
\text { Jesús Humberto Cuevas } \\
\text { Acosta (moderador) }\end{array}$ & $\begin{array}{l}\text { España, Colombia, Costa Rica } \\
\text { y México }\end{array}$ & El papel de la tecnología computacional en la educación estadística \\
\hline
\end{tabular}

\section{PONENCIAS}

\begin{tabular}{|l|l|l|l|}
\hline$\#$ & \multicolumn{1}{|c|}{ Ponentes } & \multicolumn{1}{c|}{ Lugar } & \multicolumn{1}{c|}{ Título } \\
\hline P01 & $\begin{array}{l}\text { Assumpta Estrada } \\
\text { Roca }\end{array}$ & $\begin{array}{l}\text { Universidad de Lleida, } \\
\text { España }\end{array}$ & La Web Social Para Enseñar Estadística: Una Experiencia Innovadora \\
\hline P02 & $\begin{array}{l}\text { Roberto Behar } \\
\text { Gutiérrez }\end{array}$ & $\begin{array}{l}\text { Universidad del Valle, } \\
\text { Cali, Colombia }\end{array}$ & $\begin{array}{l}\text { La Desviación Estándar. La reina de la estadística. Pero, ¿Cómo } \\
\text { explicarla? }\end{array}$ \\
\hline P08 & $\begin{array}{l}\text { Armel Brizuela } \\
\text { Rodríguez, Guaner } \\
\text { Rojas Rojas }\end{array}$ & $\begin{array}{l}\text { Universidad de Costa } \\
\text { Rica }\end{array}$ & $\begin{array}{l}\text { Aplicación del modelo mixto de Rasch para la identificación de } \\
\text { subpoblaciones no observadas de examinados }\end{array}$ \\
\hline
\end{tabular}




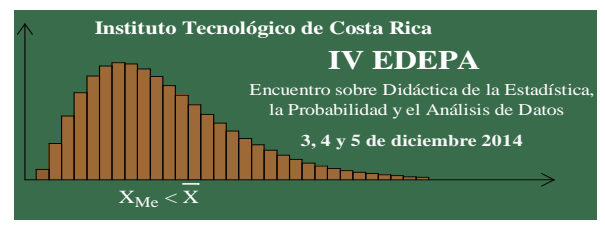

\section{PONENCIAS}

\begin{tabular}{|c|c|c|c|}
\hline P09 & $\begin{array}{l}\text { Sara } \\
\text { RodríguezRodríguez, } \\
\text { Hortensia Reyes } \\
\text { Cervantes ,Gabriel } \\
\text { Huerta , Fernando } \\
\text { Velasco, Hugo Cruz } \\
\text { Suárez }\end{array}$ & $\begin{array}{l}\text { Facultad de Ciencias } \\
\text { Físico Matemáticas- } \\
\text { Benemérita } \\
\text { Universidad Autónoma } \\
\text { de Puebla, México. } \\
\text { Department of } \\
\text { Mathematics and } \\
\text { StatisticsTheUniversity } \\
\text { of New México- } \\
\text { Albuquerque. }\end{array}$ & La modelación estadística en fenómenos ambientales un reto actual \\
\hline P10 & $\begin{array}{l}\text { Ana Luisa Nieto } \\
\text { Méndez, Hortensia } \\
\text { Reyes Cervantes, } \\
\text { Flaviano Godínez } \\
\text { Jaimes, Francisco } \\
\text { Tajonar Sanabria, } \\
\text { Víctor Hugo Guevara }\end{array}$ & $\begin{array}{l}\text { Benemérita } \\
\text { Universidad Autónoma } \\
\text { de Puebla, Universidad } \\
\text { Autónomo de } \\
\text { Guerrero. México }\end{array}$ & $\begin{array}{l}\text { Un análisis de las generaciones 2000-2008 de la Facultad de Físico- } \\
\text { Matemáticas de la Benemérita Universidad Autónoma de Puebla }\end{array}$ \\
\hline P12 & $\begin{array}{l}\text { Lorena Salazar } \\
\text { Solorzano }\end{array}$ & $\begin{array}{l}\text { Universidad Nacional. } \\
\text { Universidad de Costa } \\
\text { Rica. }\end{array}$ & $\begin{array}{l}\text { Induciendo a la creación de problemas de probabilidad: una } \\
\text { experiencia con docentes de matemática de secundaria }\end{array}$ \\
\hline P13 & $\begin{array}{l}\text { Ailton Paulo de } \\
\text { Oliveira Júnior, } \\
\text { Márcia Lopes Vieira }\end{array}$ & $\begin{array}{l}\text { Universidade Federal } \\
\text { do Triângulo Mineiro, } \\
\text { Instituto Federal do } \\
\text { Triângulo Mineiro, } \\
\text { Brasil } \\
\end{array}$ & $\begin{array}{l}\text { Concepciones de Maestros de Escuelas Públicas en los Primeros } \\
\text { Años de Escuela Primaria en Uberlândia en Relación con las } \\
\text { Estadísticas }\end{array}$ \\
\hline P14 & $\begin{array}{l}\text { Ailton Paulo de } \\
\text { Oliveira Júnior, Joana } \\
\text { dos Santos Silva, } \\
\text { Roberta Cristina de } \\
\text { Faria Moreira, Valéria } \\
\text { Ciabotti }\end{array}$ & $\begin{array}{l}\text { Universidade Federal } \\
\text { do Triângulo Mineiro. } \\
\text { Escola Municipal } \\
\text { Urbana Frei Eugênio. } \\
\text { Brasil. }\end{array}$ & $\begin{array}{l}\text { Juego Pedagógico y Resolución de Problemas en el Aprendizaje de } \\
\text { Estadística en la Escuela Primaria }\end{array}$ \\
\hline P15 & $\begin{array}{l}\text { Ailton Paulo de } \\
\text { Oliveira Júnior, } \\
\text { Beatriz Cristina da } \\
\text { Silva Delalíbera, } \\
\text { Oraide Rodrigues } \\
\text { Martins, Vanderleia } \\
\text { Conceição Ribeiro }\end{array}$ & $\begin{array}{l}\text { Universidade Federal } \\
\text { do Triângulo Mineiro . } \\
\text { Bolsista do Programa } \\
\text { Institucional de Bolsas } \\
\text { de Iniciação à } \\
\text { Docência. Brasil }\end{array}$ & $\begin{array}{l}\text { La Historia de la Estadística en el caso de la Enseñanza y el } \\
\text { Aprendizaje de la Estadística en la Escuela Secundaria }\end{array}$ \\
\hline
\end{tabular}




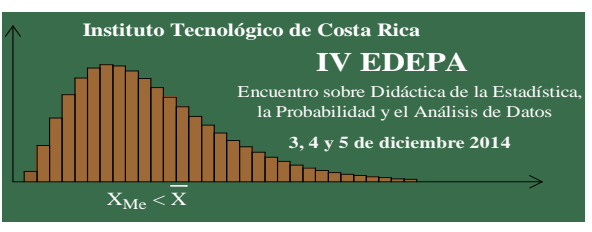

\section{PONENCIAS}

\begin{tabular}{|c|c|c|c|}
\hline P16 & $\begin{array}{l}\text { José Leonardo } \\
\text { Fernández Castro }\end{array}$ & $\begin{array}{l}\text { Universidad Estatal a } \\
\text { Distancia, Costa Rica }\end{array}$ & $\begin{array}{l}\text { La estadística vista desde un enfoque popular. Una estrategia de } \\
\text { clase útil y amena en la educación secundaria de Costa Rica. }\end{array}$ \\
\hline P18 & $\begin{array}{l}\text { Augusta Osorio } \\
\text { Gonzales, Elizabeth } \\
\text { Advíncula Clemente }\end{array}$ & $\begin{array}{l}\text { Pontificia Universidad } \\
\text { Católica del Perú }\end{array}$ & $\begin{array}{l}\text { Medición de Logros en Estudiantes de Educación Básica mediante un } \\
\text { Mapa de Progreso en Estadística y Probabilidad }\end{array}$ \\
\hline P19 & $\begin{array}{l}\text { Augusta Osorio } \\
\text { Gonzales, Elizabeth } \\
\text { Advíncula Clemente } \\
\end{array}$ & $\begin{array}{l}\text { Pontificia Universidad } \\
\text { Católica del Perú }\end{array}$ & $\begin{array}{l}\text { Desarrollo del Pensamiento Estadístico en Docentes de Educación } \\
\text { Básica Regular } \\
\text { mediante el Mapa de Progreso en Estadística y Probabilidad }\end{array}$ \\
\hline P20 & $\begin{array}{l}\text { MariCarmen González- } \\
\text { Videgaray, Rubén } \\
\text { Romero-Ruiz, Nora } \\
\text { del Consuelo Goris- } \\
\text { Mayans }\end{array}$ & $\begin{array}{l}\text { Universidad Nacional } \\
\text { Autónoma de México }\end{array}$ & $\begin{array}{l}\text { Uso de R para Mejorar el Aprendizaje de la Estadística en Alumnos } \\
\text { Universitarios de Ciencias Sociales }\end{array}$ \\
\hline P21 & Liliana Tauber & $\begin{array}{l}\text { Universidad Nacional } \\
\text { del Litoral - Santa Fe - } \\
\text { Argentina }\end{array}$ & $\begin{array}{l}\text { Argumentos utilizados por profesores de matemática para explicar } \\
\text { conceptos asociados a la idea de aleatoriedad }\end{array}$ \\
\hline P22 & $\begin{array}{l}\text { Víctor Hugo Vázquez } \\
\text { Guevara, Hugo Adán } \\
\text { Cruz Suárez, } \\
\text { Francisco Solano } \\
\text { Tajonar Sanabria, } \\
\text { Fernando Velasco } \\
\text { Luna y Hortensia } \\
\text { Reyes Cervantes }\end{array}$ & $\begin{array}{l}\text { Benemérita } \\
\text { Universidad Autónoma } \\
\text { de Puebla }\end{array}$ & Combinatoria: ¿por qué es tan difícil para algunos? \\
\hline P23 & $\begin{array}{l}\text { Francisco Solano } \\
\text { Tajonar Sanabria, } \\
\text { Yoanna Arenas } \\
\text { Martínez, Hugo Cruz } \\
\text { Suárez, Víctor Hugo } \\
\text { Vázquez Guevara, y } \\
\text { Hortensia Reyes } \\
\text { Cervantes }\end{array}$ & $\begin{array}{l}\text { Benemérita } \\
\text { Universidad Autónoma } \\
\text { de Puebla }\end{array}$ & Modelos Paramétricos en el Análisis de Supervivencia \\
\hline P24 & $\begin{array}{l}\text { Kenner Ordóñez } \\
\text { Lacayo, Guaner Rojas } \\
\text { Rojas, Karol Jiménez } \\
\text { Alfaro }\end{array}$ & $\begin{array}{l}\text { Universidad de Costa } \\
\text { Rica }\end{array}$ & Evaluación del ajuste de ítems en la teoría de respuesta al ítem \\
\hline
\end{tabular}




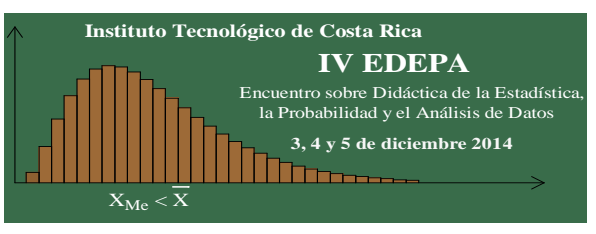

\section{PONENCIAS}

\begin{tabular}{|c|c|c|c|}
\hline P25 & Guaner Rojas Rojas & $\begin{array}{l}\text { Universidad de Costa } \\
\text { Rica }\end{array}$ & Una alternativa para la clasificación diagnóstica \\
\hline P26 & $\begin{array}{l}\text { María Inés Rodríguez, } \\
\text { Héctor Agnelli }\end{array}$ & $\begin{array}{l}\text { Universidad Nacional } \\
\text { de Río Cuarto, } \\
\text { Argentina }\end{array}$ & $\begin{array}{l}\text { El Bootstrap como Herramienta para la Enseñanza de la Distribución } \\
\text { Muestral }\end{array}$ \\
\hline P29 & $\begin{array}{l}\text { Alejandra Alfaro } \\
\text { Barquero, Greivin } \\
\text { Ramírez Arce } \\
\end{array}$ & $\begin{array}{l}\text { Instituto Tecnológico } \\
\text { de Costa Rica }\end{array}$ & $\begin{array}{l}\text { Nivel de razonamiento en la solución de problemas matemáticos y } \\
\text { su relación con variables cognitivas, metacognitivas y la dificultad de } \\
\text { la tarea }\end{array}$ \\
\hline P30 & $\begin{array}{l}\text { Giovanni Sanabria } \\
\text { Brenes }\end{array}$ & $\begin{array}{l}\text { Instituto Tecnológico } \\
\text { de Costa Rica - } \\
\text { Universidad de Costa } \\
\text { Rica, Costa Rica. }\end{array}$ & Mundial de Futbol Brasil 2014 y la probabilidad \\
\hline P31 & Rocío Mora Fallas & $\begin{array}{l}\text { Universidad de Costa } \\
\text { Rica }\end{array}$ & $\begin{array}{l}\text { Uso Del Software Power Point a Través De La Metodología de } \\
\text { Proyectos: Una Aplicación Para La Enseñanza De La Estadística En } \\
\text { Secundaria }\end{array}$ \\
\hline P32 & $\begin{array}{l}\text { Pedro Armando } \\
\text { Ramos Alberto, Javier } \\
\text { Eliseo Chávez Mancía }\end{array}$ & $\begin{array}{l}\text { Universidad de El } \\
\text { Salvador, El Salvador }\end{array}$ & $\begin{array}{l}\text { Análisis Estadístico sobre la aplicación de la Responsabilidad Social } \\
\text { de la Empresa (RSE) en seis industrias textiles en El Salvador, } 2014\end{array}$ \\
\hline P33 & $\begin{array}{l}\text { Andrea Ordóñez } \\
\text { Delgado }\end{array}$ & $\begin{array}{l}\text { Escuela Colombiana de } \\
\text { Ingeniería Julio Gravito, } \\
\text { Colombia. }\end{array}$ & Didáctica del Análisis de Datos para Estudiantes de Ingeniería \\
\hline P34 & $\begin{array}{l}\text { Dicleny Castro } \\
\text { Carvajal, John Jairo } \\
\text { Zabala Corrales }\end{array}$ & $\begin{array}{l}\text { Universidad del Tolima. } \\
\text { Ibagué Colombia }\end{array}$ & $\begin{array}{l}\text { Evaluando Actitudes de los Estudiantes de la Universidad del Tolima } \\
\text { Hacia la Estadística }\end{array}$ \\
\hline P35 & $\begin{array}{l}\text { Grettel Gutiérrez Ruíz, } \\
\text { Lourdes Quesada } \\
\text { Villalobos }\end{array}$ & $\begin{array}{l}\text { Instituto Tecnológico } \\
\text { de Costa Rica }\end{array}$ & $\begin{array}{l}\text { Un ejemplo metodológico de cómo aplicar la técnica ABP ( } \\
\text { Aprendizaje Basado en Problemas) en Estadística }\end{array}$ \\
\hline P36 & Hugo Cruz-Suárez & $\begin{array}{l}\text { Facultad de Ciencias } \\
\text { Físico-Matemáticas, } \\
\text { Benemérita } \\
\text { Universidad Autónoma } \\
\text { de Puebla, México }\end{array}$ & $\begin{array}{l}\text { Enseñanza de sistemas estocásticos de control en las áreas de } \\
\text { inventarios y finanzas }\end{array}$ \\
\hline P37 & $\begin{array}{l}\text { Luis Gerardo Meza } \\
\text { Cascante, Evelyn } \\
\text { Agüero Calvo, Zuleyka } \\
\text { Suárez Valdés-Ayala }\end{array}$ & $\begin{array}{l}\text { Instituto Tecnológico } \\
\text { de Costa Rica }\end{array}$ & $\begin{array}{l}\text { Elementos de análisis factorial aplicados al estudio de cualidades } \\
\text { psicométricas de la escala de "ansiedad matemática" de Fennema- } \\
\text { Sherman }\end{array}$ \\
\hline P38 & $\begin{array}{l}\text { Deyanira Meza } \\
\text { Cascante }\end{array}$ & $\begin{array}{l}\text { Instituto Tecnológico } \\
\text { de Costa Rica }\end{array}$ & $\begin{array}{l}\text { Aplicación del método de ecuaciones estructurales en el estudio del } \\
\text { estilo de liderazgo de los directores de escuela o departamento } \\
\text { académico en universidades costarricenses }\end{array}$ \\
\hline
\end{tabular}




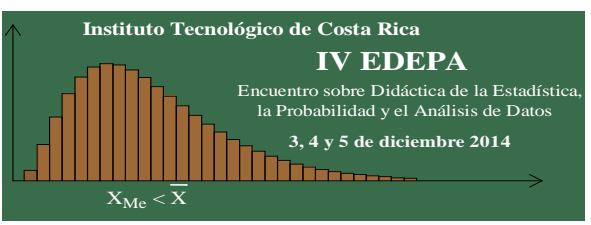

TALLERES

\begin{tabular}{|c|c|c|c|}
\hline \# & Ponentes & Lugar & Título \\
\hline T01 & Augusta Osorio Gonzales & $\begin{array}{l}\text { Pontificia Universidad } \\
\text { Católica del Perú } \\
\end{array}$ & El uso de las Situaciones Aleatorias en la Enseñanza de la Probabilidad \\
\hline T02 & $\begin{array}{l}\text { Estibaliz Rojas Quesada, } \\
\text { Jeffry Barrantes Gutierrez }\end{array}$ & $\begin{array}{l}\text { Universidad Estatal a } \\
\text { Distancia }\end{array}$ & $\begin{array}{l}\text { Propuesta didáctica para la enseñanza de la Estadística y la Probabilidad en } \\
\text { Secundaria }\end{array}$ \\
\hline T03 & $\begin{array}{l}\text { Liliana Tauber, María Jesús } \\
\text { Bianchi, Mariela Cravero }\end{array}$ & $\begin{array}{l}\text { Universidad Nacional del } \\
\text { Litoral - Santa Fe - Argentina }\end{array}$ & $\begin{array}{l}\text { Utilización de Applets para favorecer la construcción de ideas fundamentales de la } \\
\text { inferencia estadística informal }\end{array}$ \\
\hline T04 & $\begin{array}{l}\text { José Juan Castro Alva, Félix } \\
\text { Almendra Arao, Hortensia } \\
\text { Reyes Cervantes, Francisco } \\
\text { Tajonar Sanabria, Hugo } \\
\text { Cruz Juárez }\end{array}$ & $\begin{array}{l}\text { Benemérita Universidad } \\
\text { Autónoma de Puebla, } \\
\text { México. Instituto Politécnico } \\
\text { Nacional, México, D.F. }\end{array}$ & Un acercamiento a las pruebas de Hipótesis \\
\hline T05 & Welman Rosa Alvarado & EI Salvador, FEDECREDITO & $\begin{array}{l}\text { Aplicación de la Teoría de Modelos Multinivel Lineal y No-Lineal utilizando el software } \\
\text { especializado HLM7 }\end{array}$ \\
\hline T06 & $\begin{array}{l}\text { Kendall Eduardo Rodríguez } \\
\text { Bustos }\end{array}$ & $\begin{array}{l}\text { Instituto Tecnológico de } \\
\text { Costa Rica }\end{array}$ & Simulación de eventos probabilísticos con Excel \\
\hline T07 & $\begin{array}{l}\text { Gerardo Josué Cruz } \\
\text { Márquez }\end{array}$ & $\begin{array}{l}\text { Universidad Pedagógica } \\
\text { Nacional Francisco Morazán, } \\
\text { Honduras }\end{array}$ & Introducción a la Construcción de Tablas y Gráficas \\
\hline T08 & Luis Rojas Torres & Universidad de Costa Rica & Introducción al uso del software R \\
\hline T09 & $\begin{array}{l}\text { Noé Alonso Navarro } \\
\text { Martínez, Kendall Eduardo } \\
\text { Rodríguez Bustos }\end{array}$ & $\begin{array}{l}\text { Instituto Tecnológico de } \\
\text { Costa Rica, Costa Rica. }\end{array}$ & Geogebra como herramienta de simulación en eventos de probabilidad geométrica \\
\hline T10 & $\begin{array}{l}\text { Carlos Monge Madriz, } \\
\text { Steven Gabriel Sánchez } \\
\text { Ramírez }\end{array}$ & $\begin{array}{l}\text { Instituto Tecnológico de } \\
\text { Costa Rica }\end{array}$ & Exelearning como recurso didáctico para la enseñanza de la estadística en secundaria \\
\hline T12 & $\begin{array}{l}\text { Franklin Hernández Clavera, } \\
\text { Rebeca Arce Núñez }\end{array}$ & $\begin{array}{l}\text { IPEC Arabela Jiménez, Costa } \\
\text { Rica. Liceo Nocturno, Costa } \\
\text { Rica }\end{array}$ & Resolviendo problemas de probabilidad binomial usando Geogebra \\
\hline T13 & \begin{tabular}{|l|} 
Edwin Chaves Esquivel, \\
Ricardo Poveda Vásquez, \\
Jonathan Espinoza \\
González, Claudia Martínez \\
Pacheco y Miguel González \\
Ortega
\end{tabular} & $\begin{array}{l}\text { Universidad Nacional, Costa } \\
\text { Rica }\end{array}$ & $\begin{array}{l}\text { La integración de habilidades en la resolución de problemas de Probabilidad y } \\
\text { Estadística }\end{array}$ \\
\hline T14 & Tania Elena Moreira Mora & $\begin{array}{l}\text { Instituto Tecnológico de } \\
\text { Costa Rica }\end{array}$ & Análisis descriptivos con EXCEL de ítems de selección única en pruebas de aula \\
\hline T15 & Marilyn Calderón Mora & Colegio Saint Paul, Costa Rica & $\begin{array}{l}\text { La enseñanza de la probabilidad geométrica en primaria y secundaria con ayuda de } \\
\text { GEOGEBRA }\end{array}$ \\
\hline T16 & $\begin{array}{l}\text { Jesús Humberto Cuevas } \\
\text { Acosta }\end{array}$ & $\begin{array}{l}\text { Instituto Tecnológico de } \\
\text { Chihuahua II México }\end{array}$ & Investigación Reproducible con Markdown-Knitr-R \\
\hline
\end{tabular}


Conferencias 


\title{
Restricciones y Obstáculos para la implantación de una Propuesta de Reforma del Proceso de Enseñanza-Aprendizaje de la Estadística
}

\author{
Roberto Behar ${ }^{1} \&$ Pere Grima ${ }^{2}$
}

\begin{abstract}
Resumen
Se plantea el Proceso de Enseñanza-Aprendizaje como un sistema, en el cual se pone en evidencia como las componentes del curso (Objetivos, metodología, evaluación, profesor y su perfil) interactúan con las características del estudiante y estas relaciones están mediadas por el ambiente institucional, sus normas, sus políticas, sus mecanismos de control, sus recursos, para potencializar o para limitar cambios en el proceso, conducentes a un mejor logro de los Objetivos (Cambio de actitud del estudiante, su aprendizaje y su persistencia).

Palabras clave: Proceso de Enseñanza Aprendizaje, Objetivos, Reforma del Proceso, Restricciones y Obstáculos.
\end{abstract}

${ }^{1}$ Escuela de Estadística, Universidad del Valle, Colombia. roberto.behar@correounivalle.edu.co

${ }^{2}$ Departamento de Estadistica e I/O, UPC, España. pere.grima@ upc.es 


\title{
Los Proyectos en la Formación Estadística
}

\author{
Assumpta Estrada Roca ${ }^{1}$
}

\begin{abstract}
Resumen
Las recomendaciones recientes sobre metodología de enseñanza de la estadística presentan el trabajo con proyectos como método alternativo a la enseñanza tradicional al introducir en la clase por un lado una filosofía exploratoria y participativa y por otro la integración de la estadística y el contexto pues los datos tienen un significado y tienen que ser interpretados.

En este taller/presentación se analiza la metodología del trabajo con proyectos (interés, objetivos de enseñanza, recursos informáticos y evaluación del aprendizaje) así como su papel en la formación estadística de los futuros profesores de Educación Primaria.

Palabras clave: enseñanza de la estadística, proyectos, formación de profesores, didáctica, objetivos de aprendizaje.
\end{abstract}

${ }^{1}$ Universidad de Lleida, España. aestrada@matematica.udl.cat 


\title{
Estadística: Aprendizaje a largo plazo. Factores que inciden y estrategias plausibles
}

\author{
Roberto Behar ${ }^{1}$
}

\begin{abstract}
Resumen
En el marco de un primer (o único) curso de Estadística, se pretende discutir sobre el impacto de algunos factores en el aprendizaje a largo plazo. Los objetivos que planteamos en nuestro curso hoy, son los mismos que los que platearíamos en el largo plazo? ¿Nuestra manera de conducir el proceso de enseñanza-aprendizaje hoy, es compatible con los objetivos que queremos lograr en el largo plazo? ¿Qué factores limitan el logro de los objetivos a largo plazo? Si nos referimos a un primer curso de estadística, de 50 horas de duración, ¿Cuáles serían unos objetivos plausibles? ¿Y qué sobre la metodología?
\end{abstract}

Palabras clave: Primer curso, Proceso de Enseñanza Aprendizaje, Objetivos a largo Plazo, limitantes del aprendizaje.

${ }^{1}$ Escuela de Estadística, Universidad del Valle, Colombia. roberto.behar@correounivalle.edu.co 


\title{
Las Actitudes hacia la Estadística y la Formación del Profesorado
}

\author{
Assumpta Estrada Roca ${ }^{1}$
}

\begin{abstract}
Resumen
El profesorado vive en la práctica mucho más alejado del dominio afectivo en la enseñanza de la Estadística que de la comprensión de conceptos y procesos y del desarrollo de destrezas en el dominio cognoscitivo. Pero olvidar las propias actitudes preconcebidas del profesorado ante la materia lleva a menudo al fracaso de la educación. El objetivo de esta conferencia es presentar las actitudes hacia la Estadística analizando sus componentes, las variables que las afectan, así como los diferentes instrumentos de evaluación. Describimos también resumidamente los resultados de las principales investigaciones sobre actitudes de los profesores de educación primaria.

Palabras clave: Actitudes hacia la Estadística, Educación Estadística, Formación de profesores, Escalas de Actitudes.
\end{abstract}

\footnotetext{
${ }^{1}$ Universidad de Lleida, España. aestrada@matematica.udl.cat
} 


\title{
Variables del estudiante y de la institución que predicen los puntajes de Alfabetización Matemática en la muestra costarricense de PISA 2009 y 2012: análisis de regresión multinivel e implicaciones educativas
}

Eiliana Montero Rojas 1

\begin{abstract}
Resumen
Las pruebas PISA (Programme for International Student Assesment) son exámenes estandarizados internacionales en tres áreas: Competencia Lectora, Alfabetización Matemática y Alfabetización Científica. Se intenta medir el grado en que los jóvenes de 15 años logran aplicar conocimientos en esas áreas para resolver problemas en situaciones de la vida real. Costa Rica participa desde el año 2009 en este programa. En este estudio específico se llevaron a cabo análisis descriptivos y multivariados con los datos costarricenses de las aplicaciones 2009 y 2012 en Alfabetización Matemática. A nivel descriptivo se destacan los niveles bajos de desempeño, de hecho casi un $25 \%$ de la muestra no puede ser siquiera ubicada en la escala de 6 niveles de PISA en Alfabetización Matemática. Se trata entonces de estudiantes que no muestran evidencia de logro en las tareas más básicas. Aunado a esto, el nivel de cobertura del marco muestral solo incluye alrededor del $50 \%$ de la población de 15 años (los que son estudiantes de la llamada educación regular), y se infiere que posiblemente si se incluyera en la muestra a la población no representada los desempeños estimados serían aún más bajos. Con análisis de regresión corregidos por la estructura multinivel de los datos se logró identificar un conjunto de variables del estudiante y del centro educativo que predicen los desempeños en este examen, varias de ellas pueden ser objeto de intervenciones educativas. Las diferencias sustanciales que presentan en sus puntajes promedio estudiantes de colegios públicos y privados, se disminuyen considerablemente cuando se controlan variables del estudiante. Los resultados también refuerzan la hipótesis en torno al papel fundamental que juegan las dimensiones de lectura como factor predictivo del rendimiento en Alfabetización Matemática.
\end{abstract}

Palabras clave: Pruebas PISA, Costa Rica, Alfabetización Matemática, regresión multinivel, factores asociados.

${ }^{1}$ Proyecto Estado de la Educación - Universidad de Costa Rica, Costa Rica. eilianamontero@gmail.com 


\title{
¿Miente la información generada por medio de las Estadísticas y las Probabilidades?
}

Edwin Chaves Esquivel ${ }^{1}$

\begin{abstract}
Resumen
Regularmente se ha escuchado hablar que la información estocástica no siempre es confiable, al respecto se han planteado ejemplos por medio de los cuales dicha información tiende a confundir al lector, tal como se ha evidenciado en diversas encuestas políticas. Mucho se ha escrito sobre este tema, y tiene como antecedente la famosa frase "Las Mentiras, las Malditas Mentiras y las Estadísticas" que se atribuye al Primer Ministro del Reino Unido, Benjamín Disraeli (1804-1881).
\end{abstract}

El propósito de la conferencia consiste en analizar estas creencias desde el origen de los datos y el mensaje que comunican. Se muestran diferentes ejemplos que evidencia que las mal llamadas mentiras estadísticas obedecen a un uso inadecuado de las herramientas estadísticas y probabilísticas, o una mala interpretación del mensaje que los datos comunican.

La discusión de este tema resulta de vital importancia dentro del campo educativo, debido a que la generación de una cultura estadística en los jóvenes pretende romper con las ideas equívocas que podrían estarse presentando en la población adulta. En este sentido, es necesario que los educadores matemáticos lleven a cabo un análisis del tema y planteen problemas a los estudiantes que potencien esta discusión.

Palabras clave: ¿mienten las estadísticas?, mentiras estadísticas, las estadísticas de lo seguro.

${ }^{1}$ Escuela de Matemática-UNA, Escuela de Estadística-UCR. echavese@gmail.com 


\title{
Investigación Reproducible en la Educación Estadística
}

\author{
Jesús Humberto Cuevas Acosta ${ }^{1}$
}

\begin{abstract}
Resumen
Se describen las características elementales que debe reunir una investigación reproducible a través del uso de tecnología computacional. Especialmente se hace énfasis en la importancia de diseñar, implantar y promover protocolos de reproducibilidad en la realización de estudios y experimentos científico-académicos. También se describe un método para incorporar la realización de estudios reproducibles en la educación estadística, y se hace una demostración breve.
\end{abstract}

Palabras clave: investigación reproducible, educación estadística, tecnología computacional.

${ }^{1}$ Instituto Tecnológico de Chihuahua II, México. jesus.humberto.cuevas@ outlook.com 
Mesa Redonda 


\title{
El papel de la tecnología computacional en la educación estadística
}

\author{
Roberto Bejar Gutiérrez ${ }^{1}$ \\ Assumpta Estrada Roca ${ }^{2}$ \\ Geovanni Sanabria Brennes ${ }^{3}$ \\ Jesús Humberto Cuevas Acosta ${ }^{4}$ (Moderador)
}

\begin{abstract}
Resumen
Esta mesa de trabajo tuvo como propósito conocer las opiniones de tres profesores e investigadores de prestigio internacional, en relación al papel que actualmente guarda la tecnología computacional en la educación estadística, especialmente en la educación secundaria. Las preguntas planteadas a cada uno de los participantes fueron (1) ¿En qué medida consideran importante enseñar programación de computadoras a la par que se enseña estadística? (2) ¿Consideran ustedes que el perfil del profesorado actual permite incorporar y articular de forma efectiva la tecnología computacional en la enseñanza de la matemática y estadística? y (3) En su opinión, ¿Qué pueden hacer las Universidades para apoyar este proceso de incorporación y articulación tecnológica?
\end{abstract}

Palabras clave: tecnología computacional, educación estadística, educación secundaria

\footnotetext{
${ }^{1}$ Escuela de Estadística, Universidad del Valle, Colombia. roberto.behar@correounivalle.edu.co

${ }^{2}$ Universidad de Lleida. aestrada@matematica.udl.cat

${ }^{3}$ Instituto Tecnológico de Costa Rica. gsanabriab@yahoo.com

${ }^{4}$ Instituto Tecnológico de Chihuahua II, México. jesus.humberto.cuevas@outlook.com
} 


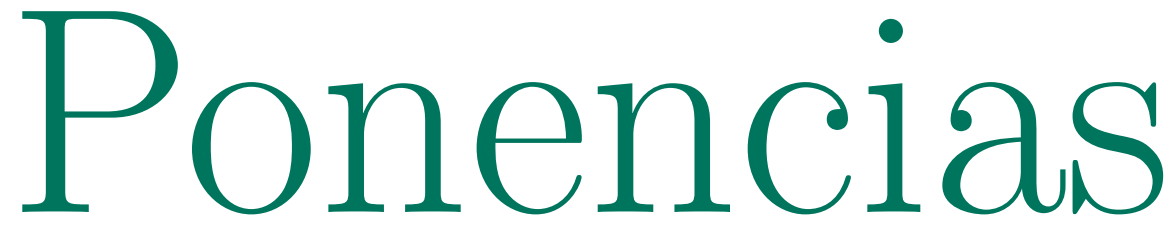




\title{
La Web Social Para Enseñar Estadística: Una Experiencia Innovadora
}

\author{
Assumpta Estrada Roca ${ }^{1}$
}

\begin{abstract}
Resumen
Se presenta una experiencia pedagógica innovadora realizada en el marco de los estudios de Educación Social, de la asignatura obligatoria "Informática y estadística" de 6 ECTS. Se utiliza una metodología que culmina con la realización (3 o 4 alumnos) de un proyecto de investigación en el que se requiere la utilización de los conocimientos teóricos y prácticos, tanto informáticos como estadísticos, adquiridos en clase.

Este proyecto consiste en: diseñar, construir y dinamizar un espacio de información y difusión temático a través de Internet, editado a través de una aplicación online (Blogger), sobre algún tema o aspecto concreto de los diferentes ámbitos de intervención en educación no formal.
\end{abstract}

Palabras clave: educación estadística, tecnología educativa, didáctica.

\footnotetext{
${ }^{1}$ Universidad de Lleida, España. aestrada@matematica.udl.cat
} 


\title{
La Desviación Estándar. La Reina de la Estadística, pero ¿Cómo Explicarla?
}

\author{
Roberto Behar ${ }^{1}$
}

\begin{abstract}
Resumen
Hay cierto consenso sobre que la Desviación Estándar es la medida estadística por excelencia, sin embargo, cuando la intentamos explicarla, en el marco de la estadística descriptiva de un curso introductorio, nos cuesta dificultad justificar esta categoría y además nos cuesta hacer útil interpretación de un valor particular en un problema específico. En esta ponencia se comparte una experiencia que pretende aportar un granito de arena en esta dirección, usando el principio de Chebyshev y la Distribución Normal.
\end{abstract}

Palabras clave:. Desviación Estándar, interpretación útil, inclusión de la variabilidad. Principio de Chebyshev, Distribución Normal.

${ }^{1}$ Escuela de Estadística, Universidad del Valle, Colombia. roberto.behar@correounivalle.edu.co 


\title{
Aplicación del modelo mixto de Rasch para la identificación de subpoblaciones no observadas de examinados
}

\author{
Armel Brizuela Rodríguez y Guaner Rojas Rojas ${ }^{2}$
}

\begin{abstract}
Resumen
Se expone la aplicación de un modelo estadístico para identificar subagrupaciones en una muestra de aspirantes a ingreso a dos universidades públicas costarricenses. La detección de subagrupaciones permitiría la diferenciación de los aspirantes en niveles de desempeño en cada subgrupo. En primer lugar presentan las principales características del modelo mixto de Rasch, mediante el cual se estima la probabilidad de pertenencia de los examinados a cada subagrupación o clase latente. Posteriormente, se discuten las implicaciones prácticas del modelo en el contexto de la docencia. La implementación del modelo se llevó a cabo mediante el lenguaje de programación R.
\end{abstract}

Palabras clave: Análisis de datos, modelo mixto de Rasch, clases latentes, docencia.

\begin{abstract}
In this paper the application of a statistical model to identify sub-groups in a sample of applicants entering two Costa Rican public universities is presented. The detection of subgroups allows the differentiation in performance levels for each subgroup of applicants. First, the main characteristics of the mixture Rasch model are presented; in this model the probability of examinees belonging to each subgroup or latent class is estimated. Subsequently, the practical implications of the model in the context of teaching are discussed. The model was implemented using the programming language $\mathrm{R}$.
\end{abstract}

Keywords: Data analysis, mixture Rasch model, latent classes, teaching.

${ }^{1}$ Universidad de Costa Rica, Costa Rica. armel9@gmail.com

${ }^{2}$ Universidad de Costa Rica, Costa Rica. guanerdavid@yahoo.com 


\title{
La modelación estadística en fenómenos ambientales un reto actual
}

\author{
Sara Rodríguez Rodríguez ${ }^{1}$, Hortensia Reyes Cervantes ${ }^{2}$, Gabriel Huerta ${ }^{3}$, Fernando \\ Velasco $^{4} \&$ Hugo Cruz Suárez 5
}

\begin{abstract}
Resumen
El trabajo está dividido en dos partes: la primera se analiza la dirección del viento para cada estación que monitorea concentraciones de ozono, apoyándose de estadística circular con el enfoque clásico y como segunda parte se realiza una estimación bayesiana de los parámetros de la distribución de Valores Extremos Generalizada incluyendo la covariable tiempo en el parámetro de escala, con el propósito de encontrar la tendencia de las concentraciones de ozono para cada estación de Monitoreo. Se observa que la mayoría de los vientos vienen en dirección Noreste y la tendencia de concentraciones máximas de ozono para 7 estaciones en la Ciudad de México es decreciente.
\end{abstract}

Palabras clave: Distribución de Valores Extremos Generalizada, Estadística Circular, Estadística Bayesiana, Ozono.

\begin{abstract}
The work is divided into two parts, the first the wind direction is analyzed for each station monitors ozone concentrations, based on circular statistics to the classical approach and as a second part a Bayesian estimation of the parameters of the distribution of values is performed Generalized extreme weather including the covariate scale parameter, in order to find the trend of ozone concentrations for each monitoring station. It is observed that most of the winds are going northeast and the trend of maximum ozone concentrations for 7 stations in Mexico City is decreasing.
\end{abstract}

Keywords: Generalized Extreme Values, Circular Statistic, Bayesian Statistic, Ozone. Modalidad: Ponencia

\footnotetext{
${ }^{1}$ Facultad de Ciencias Físico Matemáticas-Benemérita Universidad Autónoma de Puebla, México. rguez.sara@gmail.com

${ }^{2}$ FCFM-Benemérita Universidad Autónoma de Puebla, México. hreyes@fcfm.buap.mx

${ }^{3}$ Department of Mathematics and Statistics The University of New México- Estados Unidos ghuerta@stat.unm.edu

${ }^{4}$ FCFM-Benemérita Universidad Autónoma de Puebla, México fvelasco@ @fcfm.buap.mx

${ }^{5}$ FCFM-Benemérita Universidad Autónoma de Puebla, México. hadan4@ yahoo.com
} 


\section{Introducción}

El comportamiento del ozono se ha estado monitoreando en todo el mundo especialmente en las grandes ciudades por sus efectos nocivos a la vida. Diversas metodologías se han realizado por los investigadores de diferentes campos del conocimiento, en particular en el campo de la estadística, modelos: multivariados, regresiones no lineales, series de tiempo, redes neuronales, cópulas y otros, se han usado tratando de entender y modelar su conducta para predecir estados de emergencia en los valores del ozono, Porter S., Rao T., Zurbenko G., Dunker M. y Wolff T.G. [8], Thompson M. L., Joel R., Lawrencw H.C., Peter G. y Paul D.S., 2001 [13], Wolff T. et al., 2001 [14], entre otros. Desgraciadamente es un problema bastante complicado para resolver ya que cada país tiene sus características geográficas (altitud, longitud), políticas y economías diferentes. Aún más, cada sitio tiene variabilidad en los precursores que forman al ozono (algunos de ellos son: bióxido de carbono, monóxido de carbono, dióxido de nitrógeno, dióxido de azufre, metano y otros. Aparte en el sitio hay variables ambientales, como: temperatura, velocidad de viento, humedad relativa, dirección del viento, radiación ultravioleta y otras). En las normas de salud (se refleja la conciencia de los ciudadanos para emitir decisiones y cuidar a la población en riesgo: viejos y niños), los países tienen un uso del petróleo y sus derivados (restos fósiles) como única fuente de energía para mover una ciudad, y además no hay conciencia en el crecimiento de la población. Ya que cada ciudadano requerirá en un tiempo dado un espacio para vivir, servicios transporte, agua, luz, muebles, etc., esto generará más contaminación ambiental y se verá reflejado también en la contaminación del agua y la pérdida de los espacios verdes por contaminación de suelos, entre otras cosas.

El Programa de Contingencias Ambientales Atmosféricas (PCAA); en México; reúne un conjunto de medidas que se aplican cuando se presenta un episodio de contaminación severa, durante el cual, las concentraciones de ozono o de partículas menores a 10 micrómetros alcanzan niveles que ponen en riesgo la salud de la población en general y producen efectos adversos en los grupos sensibles como niños, adultos mayores y personas con enfermedades respiratorias o cardiovasculares [10]. Menciona Bravo H. [1] que los mecanismos de formación de ozono son aún más complicados de lo que se suponía, encontrándose una gran evidencia de acumulación nocturna de contaminantes atmosféricos precursores de ozono por arriba de la capa de inversión de temperatura típica del Valle de México.

Estas lecturas de las variables ambientales son consideradas por investigadores para estimar parámetros, suponer ciertas distribuciones de las concentraciones de ozono, realizar simulaciones y de esta manera ver la tendencia, concentración, efectos de la dirección del viento o de otras covariables del contaminante en estudio. En la Zona Metropolitana del Valle de México un régimen de viento importante que se forma es el de brisa de valle y de montaña. Los vientos más fuertes son los producidos por factores relacionados con la dinámica de la atmósfera. La velocidad del viento influye sobre los contaminantes debido a que su estancamiento depende de las bajas magnitudes de este parámetro. Sin embargo, las altas concentraciones de cualquier contaminante en las capas más bajas de la tropósfera no dependen enteramente de la componente horizontal del viento, sino que existe una gran dependencia de la componente vertical, ya que esta juega un papel importante en la dispersión.

Huerta et al.[3] muestra un trabajo donde consideran lecturas por hora de concentraciones de ozono en la Ciudad de México y proponen un modelo espacial así como de interpolación temporal y predicción, incluyendo una covariable (temperatura), los resultados se observan consistentes con el comportamiento cíclico de los datos y sobre la dispersión del ozono en la ciudad de México, la contaminación producida alrededor de las 12 p.m. y los picos entre las 2 y 4 p.m., al estimar el coeficiente de altitud en el modelo para la temperatura revela que hay un significante efecto de tal 
variable, concluyen que las teorías sobre la dispersión del ozono obtenidos con el modelo parecen razonables para pequeños términos de interpolación espacial.

En el trabajo de Rodríguez et al. [11], se muestra que se pueden ingresar diferentes covariables para verificar si influyen en la tendencia del ozono, sin embargo los conjuntos que explican de manera significativa las concentraciones de ozono en muchos casos son los que contienen las variables tiempo, monóxido de carbono y temperatura. En este trabajo al intentar de ingresar la dirección de viento como covariable en una regresión lineal se observa que no es adecuada, ya que es una variable circular (se puede ver como puntos situado en el círculo unitario centrado en el origen), de allí el interés de tratar de modelar la iteración de las concentraciones de ozono con esta variable usando estadística circular propuesta en la primera parte de este trabajo.

Hay varias razones para realizar un análisis bayesiano de valores extremos, una es debida a la escasez de datos, la facilidad de incluir otras fuentes de información a través de una distribución a priori; segunda es que la salida de un análisis bayesiano (distribución a posteriori) da una inferencia completa que corresponde a un análisis de máximo verosimilitud; una tercera razón, es que esta no depende de la suposición requerida por la teoría asintótica de máxima verosimilitud, para mayor información ver Coles [2]. Procederemos a ver la forma de aplicar un análisis bayesiano de Valores Extremos en concentraciones máximas de ozono en la ciudad de México.

\section{Marco Teórico}

Presentamos una aplicación en estadística clásica, particularmente, el uso de estadística circular en el modelamiento de dirección de viento de las concentraciones de ozono. Debido a que analizamos máximos de las concentraciones de ozono con un enfoque bayesiano, se da una introducción sobre la estadística Bayesiana, además se supone que cada observación máxima de ozono sigue una distribución de Valores Extremos Generalizada, donde en el parámetro de localización se ingresa una regresión que incorpora un parámetro que representa la tendencia de las concentraciones máximas de ozono.

\section{II.1 Estadística Circular}

Como se sabe, el modelado de análisis estadístico y la inferencia constituyen la base para generalizaciones objetivas a partir de datos observados. Los datos de dirección se presentan en muchos estudios científicos, por ejemplo en la biología puede ser medida la dirección de vuelo de un pájaro o de orientación de un animal, mientras que en geología se puede interesar en el polo magnético de la tierra. De aquí las llamadas para el uso de herramientas y técnicas estadísticas especializadas, que no son todavía ampliamente conocidas y apreciadas.

Dado que las direcciones no tienen magnitud, estas pueden ser representadas convenientemente como puntos sobre una circunferencia de un círculo unitario centrado en el origen, o como vectores unitarios conectados al origen. Rao J. [4] señala que los datos circulares pueden ser representados como ángulos o como puntos en una circunferencia, para este propósito, se usa un sistema coordenado rectangular con origen en 0 y dos ejes $X, Y$ perpendiculares, cualquier punto $P$ sobre el plano puede ser representado como $(x, y)$, en término de sus coordenadas rectangulares, $\mathrm{o}$ como $(r, \propto)$ en término de sus coordenadas polares, donde $r$ es la distancia al origen y $\propto$ su dirección. Así tenemos $x=r \cos \propto, y=$ 
$r \operatorname{sen} \propto$. Como la dirección no tiene magnitud tenemos que $r=1$, luego la conversión de coordenadas polares a rectangulares es:

$$
x=\cos \propto, y=\operatorname{sen} \propto
$$

Una medida de la media direccional para un conjunto de direcciones es obtenido por el tratamiento de datos como vectores unitarios y usando la dirección de su vectores resultantes. Sean $\propto_{1}, \propto_{2}, \ldots, \propto_{n}$ un conjunto de observaciones circulares dado en términos de ángulos, y consideremos la transformación polar a rectangular para cada observación i.e. $\left(\cos \propto_{i}\right.$, sen $\left.\propto_{i}\right), i=1, \ldots, n$. Se obtiene el vector:

$$
\overrightarrow{\mathrm{R}}=\left(\sum_{i=1}^{n} \cos \propto_{i}, \sum_{i=1}^{n} \operatorname{sen} \propto_{i}\right)=(C, S)
$$

Así $\mathbf{R}=|| \overrightarrow{\mathrm{R}} \|=\sqrt{C^{2}+S^{2}}$ representa la longitud del vector resultante $\overrightarrow{\mathrm{R}}$, y se propone como la media circular a $\overline{\alpha_{0}}$ definida como en (3)

$$
\overline{\alpha_{0}}=\left\{\begin{array}{c}
\arctan \left(\frac{S}{C}\right), \quad \text { si } C>0, S \geq 0, \\
\frac{\pi}{2}, \quad \text { si } C=0, S>0 \\
\arctan \left(\frac{S}{C}\right)+\pi, \text { si } C<0, \\
\arctan \left(\frac{s}{C}\right)+2 \pi, \text { si } C \geq 0, S<0 \\
\text { indefinido, si } C=0, S=0
\end{array}\right.
$$

Además de que la dirección $\overline{\alpha_{0}}$ del vector resultante $\overrightarrow{\mathrm{R}}$ proporciona una media direccional, $R=\|\vec{R}\|$ es usada como medida de datos unimodales y del grado de concentración de los datos.

Una razonable medida de la distancia circular entre dos puntos $A$ y $B$, es tomada como el más pequeño de los arcos formados entre los puntos a lo largo de la circunferencia, i.e., para cualesquiera dos ángulos $\alpha$ y $\beta$ la distancia circular está definida como en (4)

$$
d_{0}(\alpha, \beta)=(1-\cos (\alpha-\beta))
$$

En los trabajos Rao J. se justifica a $(n-R)$ como una medida de dispersión, con motivos similares a $\boldsymbol{s}^{\mathbf{2}}$ la varianza muestral del caso lineal. Un valor de $\boldsymbol{R}$ cerca de 0 significa que la dispersión es grande mientras que los valores de $\boldsymbol{R}$ cerca de $\boldsymbol{n}$ implica que el conjunto de observaciones tiene pequeña dispersión o más concentración hacia el centro. Sin embargo si se da una pequeña media direccional poblacional $\mu_{0}$, (también llamada dirección polar), entonces denotamos al vector polar por $P=\left(\cos \mu_{0}, \operatorname{sen} \mu_{0}\right)$, así es natural medir la dispersión alrededor de esta media direccional conocida, por lo que Rao J. propone $D_{P}=n-$ $\sum_{i=1}^{n} \cos \left(\alpha_{i}-\mu_{0}\right)=n-V_{0}$ donde $V_{0}=R \cos \left(\alpha_{0}-\mu_{0}\right)$. A partir del resultado anterior $y$ posteriormente haciendo transformaciones se define a una varianza circular como $2(1-\bar{R})$

$$
2(1-\bar{R})=\frac{2}{n} \sum_{i=1}^{n}\left(1-\cos \left(\alpha_{i}-\overline{\alpha_{0}}\right)\right) \cong \frac{1}{n} \sum_{i=1}^{n}\left(\alpha_{i}-\overline{\alpha_{0}}\right)^{2}=s^{2}
$$




\section{Esta es una medida preferible en lugar de tratar de encontrar una aproximación lineal o equivalente.}

\section{II.2 Distribución de Valores Extremos}

El efecto de datos extremos en un análisis estadístico es de extraordinaria importancia, ya que exhibir el escenario de variables aleatorias en diferentes enfoques como inundaciones, huracanes, altas concentraciones de contaminación, climas extremos, aseguradoras, crisis de precios, etc., ayudaría a evitar grandes catástrofes naturales o financieras.

La distribución de valores extremos surge del teorema del límite de Fisher-Tippet mostrado en Pickands J. [7] sobre valores extremos o máximos en muestras de datos. El modelo se enfoca sobre el comportamiento estadístico de $\mathrm{Z}_{\mathrm{n}}=\operatorname{máx}\left\{\mathrm{X}_{1}, \ldots, \mathrm{X}_{\mathrm{n}}\right\}$ donde $\mathrm{X}_{1}, \ldots, \mathrm{X}_{\mathrm{n}}$ es una sucesión de variables aleatorias independientes idénticamente distribuidas, con función de distribución $\mathrm{F}$.

El teorema del límite demuestra que $\mathrm{Z}_{\mathrm{n}}$ tiene por función de distribución $\mathrm{G}$ que es no degenerada y pertenece a la familia de Valores Extremos (VE) tales como la Gumbel, Fréchet o Weibull.

La distribución de Valores Extremos Generalizada (VEG) unifica la representación paramétrica de las tres diferentes familias de distribuciones de VE, Coles [2], Reiss [9]. La expresión de la distribución VEG estandarizada, está dada por:

$$
\begin{array}{cc}
G_{\theta}(\mathrm{z})=\mathrm{G}_{\mu, \sigma, \varepsilon}(\mathrm{z})=\exp \left\{-\left[1+\varepsilon \frac{\mathrm{z}-\mu}{\sigma}\right]^{-\frac{1}{\varepsilon}}\right\} & \text { si } 1+\varepsilon \frac{\mathrm{z}-\mu}{\sigma}>0 \\
\mathrm{G}_{\mu, \sigma, 0}(\mathrm{z})=\exp \left\{-\exp ^{-\frac{\mathrm{z}-\mu}{\sigma}}\right\} & \text { si } \quad \varepsilon=0
\end{array}
$$

con $\theta=(\mu, \sigma, \varepsilon) \in\left(\mathbf{R} \times \mathbf{R}^{+} \times \mathbf{R}\right)$. Los parámetros $\mu, \sigma>0$ y $\varepsilon$ son los parámetros de localización, de escala y forma, respectivamente.

Bajo la suposición de que $\mathrm{Z}_{1, \ldots, \mathrm{Z}_{\mathrm{n}}}$ son variables dependientes con distribución VEG, la función de verosimilitud cuando $\varepsilon \neq 0$ es:

$$
L(\mu, \sigma, \varepsilon)=-m \log \sigma-\left(1+\frac{1}{\varepsilon}\right) \sum_{i=1}^{n} \log \left[1+\varepsilon\left(\frac{z_{i}-\mu}{\sigma}\right)\right]-\sum_{i=1}^{n}\left[1+\varepsilon\left(\frac{z_{i}-\mu}{\sigma}\right)\right]^{-1 / \varepsilon}
$$

Siempre que $1+\varepsilon\left(\frac{z_{i}-\mu}{\sigma}\right)>0$, para $i=1, \ldots, m$. Cuando $\varepsilon>-0.5$, los estimadores de máxima verosimilitud son regulares, en el sentido de tener las propiedades asintóticas usuales, si $-1<\varepsilon<$ -0.5 los estimadores se pueden obtener pero no con las propiedades asintóticas y finalmente si $\varepsilon<$ -1 los estimadores son improbables de obtener.

\section{II.3 Estadística Bayesiana}

La estadística Bayesiana a diferencia de la teoría de la estadística clásica, considera a los parámetros desconocidos como variables aleatorias. Ntzoufras I. [6] menciona que la inferencia bayesiana está basada en el razonamiento, donde los datos observados $\left(y_{1}, y_{2}, \ldots, y_{n}\right), \boldsymbol{\theta}$ que denota las posibles causas que provocan las $y_{i}, \mathrm{i}=1, \ldots, \mathrm{n}, \mathrm{y} P\left(y_{i} \mid \boldsymbol{\theta}\right)$ como la probabilidad de que suceda $y_{i}$ cuando la causa $\boldsymbol{\theta}$ este presente, se quiere encontrar las probabilidad de que $\boldsymbol{\theta}$ sea la respuesta para la ocurrencia de las $y_{i}$, denotado como $P\left(\boldsymbol{\theta} \mid y_{i}\right)$, esto se puede escribir de la siguiente manera:

$$
P\left(\boldsymbol{\theta} \mid y_{i}\right)=P\left(y_{i} \mid \boldsymbol{\theta}\right) P(\boldsymbol{\theta})
$$


entonces se define la distribución a priori como $f(\theta)$ que expresa la información posible para

los resultados antes de cualquier dato, la función de verosimilitud como $f(y \mid \theta)$, luego el interés radica en calcular la distribución posterior $f(\theta \mid y)$ del parámetro $\theta$ dados los datos observados $y$, así usando la ecuación (8) tenemos:

$$
f(\theta \mid y)=f(y \mid \theta) f(\theta) .
$$

Sin embargo en la vida real la dificultad radica en encontrar la distribución posterior, así que se recurre al uso de técnicas numéricas. Lee P. M. [5] menciona que los métodos usados son los relacionados con métodos de Monte Carlo debido a su flexibilidad aunada a un desarrollo masivo de facilidades computacionales.

\section{Metodología y Resultados}

Se realiza un análisis con datos de concentraciones de Ozono para 16 estaciones pertenecientes al Sistema de Monitoreo Atmosférico de la Ciudad de México (SIMAT), se extraen los máximo de concentraciones de ozono de cada 72 horas, a partir del año 2007 al 2013, sólo para los meses de Abril, Mayo y Junio. En la Figura 2 a) mostramos el mapa de la ciudad de México con la ubicación geográfica de las estaciones y en la Figura 2 b) se muestran los nombres de cada estación y el área a la que pertenecen.

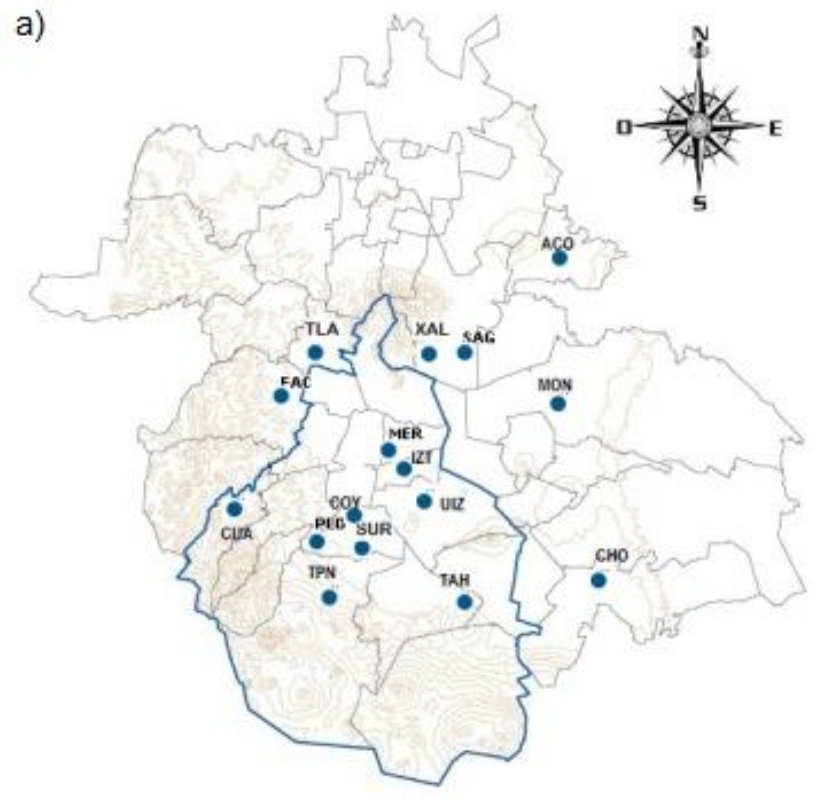

b)

\begin{tabular}{lrl} 
Estación & Abreviación & Zona \\
\hline Fes Acatlán & FAC & Noroeste \\
Tlalnepantla & TLA & Noroeste \\
Acolman & ACO & Noroeste \\
Montecillo & MON & Noreste \\
San Agustín & SAG & Noreste \\
Xalostoc & XAL & Noreste \\
Iztacalco & IZT & Centro \\
Merced & MER & Centro \\
Coyoacán & COY & Suroeste \\
Cuajimalpa & CUA & Suroeste \\
Pedregal & PED & Suroeste \\
Santa Ursula & SUR & Suroeste \\
Tlalpan & TPN & Suroeste \\
Chalco & CHO & Sureste \\
Tláhuac & TAH & Sureste \\
UAM Iztapalapa & UIZ & Sureste \\
\hline
\end{tabular}

Figura 2. En a) Ubicación de las estaciones de monitoreo atmosférico en la Ciudad de México y en b) Nombres y área a la que pertenece cada estación.

\section{III.1 Modelación de Dirección de viento}


En trabajos anteriores nos dimos a la tarea de investigar si influían diferentes covariables químicas ambientales en el comportamiento del ozono, allí notamos el hecho que la covariable dirección del viento no se debía interpretar de la misma manera que una variable que toma valores lineales, por lo que ahora se da otra manera de tomar los valores alrededor del círculo. Para esta parte del trabajo solo once estaciones pertenecientes al SIMAT monitorean ozono y direcciones de viento, con las cuales se ha trabajado, estas estaciones son FAC, TLA, MON, SAG, XAL, MER, CUA, PED, SUR, TPN, TAH.

La dirección del viento se especifica en relación con el norte verdadero en el lugar de observación y hace referencia a la dirección desde la cual el viento está soplando, reportándose en unidades de grados (en el sentido de las manecillas del reloj).

Apoyándose del paquete Circular del software $R$-project [10] convertimos la información de la dirección del viento a datos circulares y obtenemos lo que se muestra en la Figura 3, los diagramas de rosa en color azul, se leen como un histograma que representa la frecuencia de las velocidades del viento, presentado en la circunferencia de un círculo. Para cada barra de frecuencia se tiene un número que representa la concentración de ozono que llega en dicha dirección de viento. Se observa
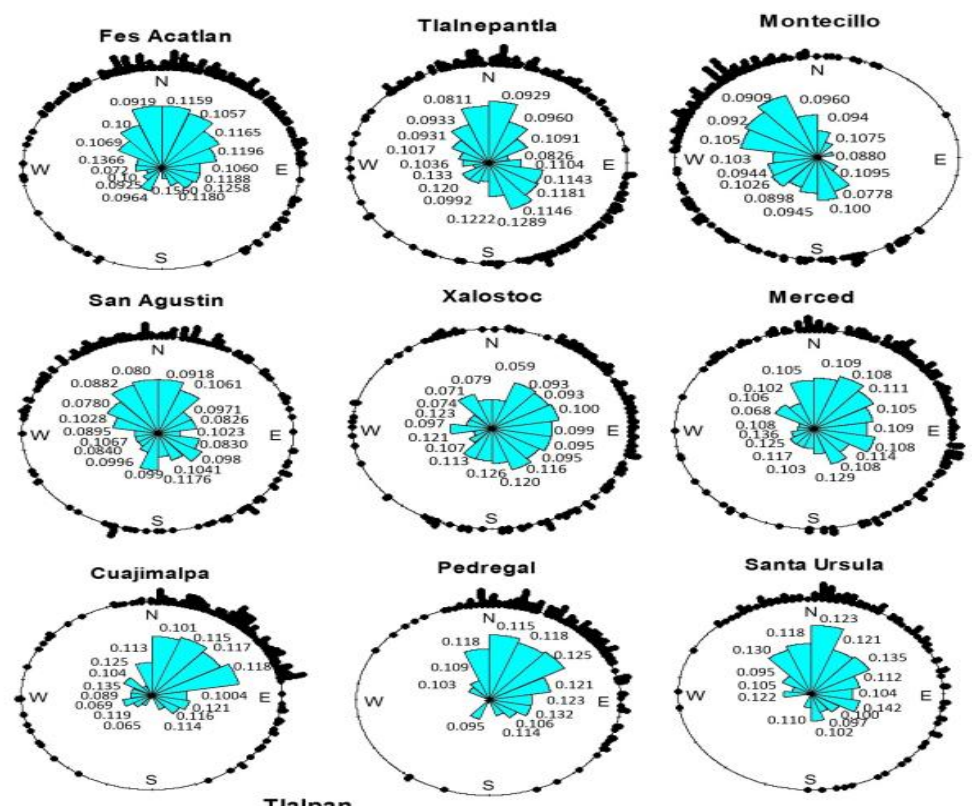

\begin{tabular}{lc} 
Estación & Media de WDR(var.) \\
\hline Fes Acatlan & $16.4460572(0.3882088)$ \\
Tlalnepantla & $33.8123483(0.6932154)$ \\
Montecillo & $-71.3461440(0.5241615)$ \\
San Agustin & $0.2748525(0.6354878)$ \\
Xalostoc & $84.9503619(0.6414759)$ \\
Merced & $49.1032122(0.5166122)$ \\
Cuajimalpa & $41.3853073(0.2967726)$ \\
Pedregal & $36.5050577(0.2528227)$ \\
Santa Ursula & $26.1326147(0.3852788)$ \\
Tlalpan & $35.4749474(0.2427923)$ \\
Tlahuac & $-17.8915236(0.5791574)$ \\
\hline
\end{tabular}
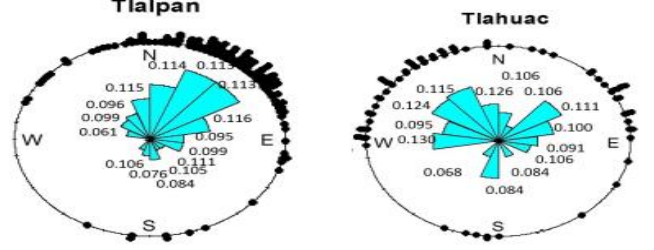

Figura 3. Diagrama de Rosas de vientos con la concentración de ozono y en la Tabla se muestra la Media y la varianza circular para cada una de las estaciones de monitoreo atmosférico. 
que los vientos predominantes llegan de la dirección Noreste en siete de las once estaciones, de la tabla de la Figura 3 notemos que la media de los vientos se concentra en el primer cuadrante (noreste) lo que corresponde a lo dicho anteriormente. Sin embargo la magnitud de las concentraciones de ozono que llegan de esta dirección son menores en comparación con las que llegan en dirección suroeste.

\section{III.1 Modelación de Bayesiana}

Para este análisis se usaron las 16 estaciones que monitorean concentraciones de ozono en la Ciudad de México, los máximos de ozono se denotan por $z_{1}, \ldots, z_{n} \quad(\mathrm{n}=212)$ realizaciones independientes de una variable aleatoria $Z$ que tiene densidad de probabilidad $V E G(z ; \mu, \sigma, \varepsilon)$, donde $\mu=\beta_{0}+\beta_{1} t$ con $\beta_{0}$ representa el intercepto, $\beta_{1}$ el parámetro representa la tendencia del ozono y $t=(1,2, \ldots, 212)$ el tiempo.

Para cada una de las estaciones se desea obtener las distribuciones a posteriori de cada parámetro de la distribución de Valores Extremos Generalizada, para ellos se eligen las siguientes distribuciones a priori: $\quad \beta_{0} \sim$ Uniforme $(-10000,10000), \quad \beta_{1} \sim$ Uniforme $(-10000,10000)$, $\sigma \sim \operatorname{Gamma}^{-1}(-10000,10000)$ y $\varepsilon \sim$ Uniforme $(-0.5,0.5)$. Apoyados del paquete Brugs del software R-projec generamos las distribuciones posteriores de los parámetros de la distribución de Valores Extremos, se realizaron 3000 iteraciones de calentamiento y posteriormente 50000 más, en la Figura 4 sólo mostramos las densidades de la tendencia, y en la Tabla 1 se muestra la información posterior del parámetro de tendencia.

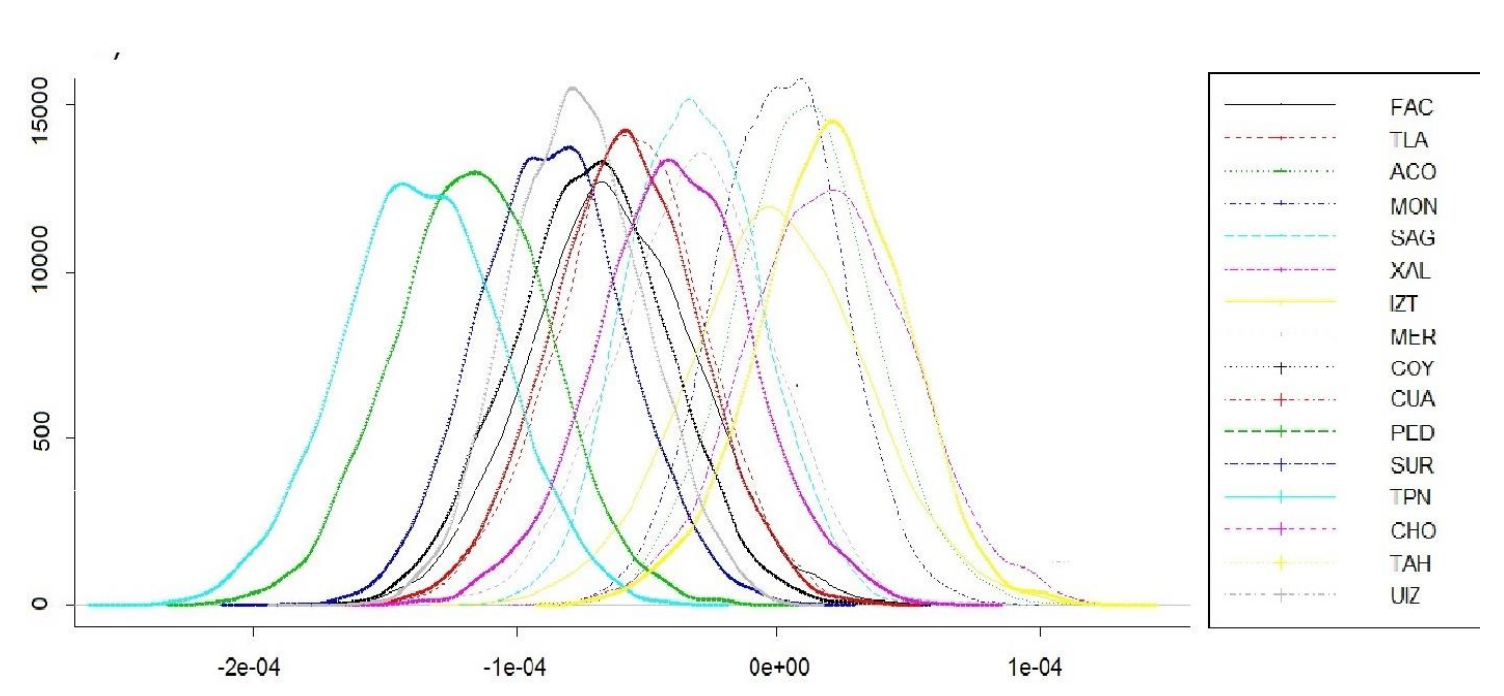

Figura 4. Densidad posterior del parámetro que representa la tendencia $\beta_{1}$, para todas las estaciones del SIMAT. 


\begin{tabular}{llcl} 
Station & Media & de. & IC. 97.5\% \\
\hline FAC & $-6.26 \mathrm{E}-05$ & $3.22 \mathrm{E}-05$ & $(-0.0001245,1.11 \mathrm{E}-06)$ \\
TLA & $-5.62 \mathrm{E}-05$ & $2.82 \mathrm{E}-05$ & $(-0.0001125,-1.01 \mathrm{E}-06)$ \\
ACO & $9.86 \mathrm{E}-06$ & $2.71 \mathrm{E}-05$ & $(-4.30 \mathrm{E}-05,6.44 \mathrm{E}-05)$ \\
MON & $3.76 \mathrm{E}-07$ & $2.43 \mathrm{E}-05$ & $(-4.76 \mathrm{E}-05,4.70 \mathrm{E}-05)$ \\
SAG & $-3.16 \mathrm{E}-05$ & 0.000025 & $(-7.82 \mathrm{E}-05,1.85 \mathrm{E}-05)$ \\
XAL & 0.0000201 & $3.14 \mathrm{E}-05$ & $(-4.19 \mathrm{E}-05,8.34 \mathrm{E}-05)$ \\
IZT & $-1.35 \mathrm{E}-07$ & $3.42 \mathrm{E}-05$ & $(-6.81 \mathrm{E}-05,6.77 \mathrm{E}-05)$ \\
MER & $-3.20 \mathrm{E}-05$ & $3.04 \mathrm{E}-05$ & $(-9.25 \mathrm{E}-05,2.51 \mathrm{E}-05)$ \\
COY & $-7.14 \mathrm{E}-05$ & $3.02 \mathrm{E}-05$ & $(-0.0001299,-1.20 \mathrm{E}-05)$ \\
CUA & $-5.85 \mathrm{E}-05$ & $2.86 \mathrm{E}-05$ & $(-0.0001149,-2.72 \mathrm{E}-06)$ \\
PED & -0.0001169 & 0.00003 & $(-0.0001749,-5.84 \mathrm{E}-05)$ \\
SUR & $-8.61 \mathrm{E}-05$ & $2.83 \mathrm{E}-05$ & $(-0.0001404,-2.95 \mathrm{E}-05)$ \\
TPN & -0.0001362 & $3.04 \mathrm{E}-05$ & $(-0.0001972,-7.69 \mathrm{E}-05)$ \\
CHO & $-3.93 \mathrm{E}-05$ & 0.0000301 & $(-0.0001,0.0000214)$ \\
TAH & $2.06 \mathrm{E}-05$ & $2.86 \mathrm{E}-05$ & $(-3.88 \mathrm{E}-05,7.51 \mathrm{E}-05)$ \\
UIZ & $-7.64 \mathrm{E}-05$ & $2.56 \mathrm{E}-05$ & $(-0.0001248,-2.48 \mathrm{E}-05)$ \\
\hline
\end{tabular}

Tabla 1. Información posterior del parámetro $\beta_{1}$, se muestra la media, desviación estándar (de), y el intervalo de credibilidad al 97.5\%, para cada una de las estaciones del SIMAT.

De los resultados de la distribución a posteriori para el parámetro $\beta_{1}$ que representa la tendencia de las concentraciones máximas de ozono, se toma atención al intervalo de credibilidad al $97.5 \%$, de tal manera que si incluye al cero, lo que indica que no hay evidencia para dar alguna tendencia (creciente o decreciente), si el intervalo tiene valores negativos hay evidencia para decir que la tendencia de máximos ozono del año 2007 al 2013 ha decrecido.

\section{Siete estaciones presentan una tendencia decreciente para los máximos de concentraciones de ozono, estas son Tlalnepantla, UAM Iztapalapa, Coyoacán, Cuajimalpa, Pedregal, Santa Úrsula y Tlalpan, se observa que todas las estaciones que pertenecen al área suroeste presentan una tendencia a la baja.}

\section{Conclusiones}

Podemos concluir que existe una relación entre las concentraciones de Ozono y con el comportamiento de la dirección del viento, ya que los resultados permiten ver que los vientos arrastran los contaminantes a la parte suroeste de la cuenca de México donde se encuentran las estaciones CUA, PED, SUR y TPN, además que debido a las condiciones geográficas hace difícil la circulación de dichos contaminantes, lo cual provoca que la mayor concentración de ozono se ubique en esta área.

Para las estaciones MON y SAG que se encuentran en el primer cuadrante de la ciudad de México, las concentraciones de ozono que llegan del noreste y del suroeste son parecidas oscilando entre $0.090 \mathrm{ppm}$ y $0.101 \mathrm{ppm}$. La estación que representa el centro de la ciudad de México es Merced a la cual le llega en su mayoría viento del noreste percibe la misma concentración de ozono que en su mayoría es mayor a $0.11 \mathrm{ppm}$. 
Del análisis bayesiano se obtuvo que las concentraciones máximas de ozono tienen una tendencia a la baja para el $43.75 \%$ de las estaciones y es de señalar que estas son las que presentan los más altos niveles de contaminación, además que el resto de las estaciones de monitoreo aún no están por debajo de los .11 ppm, lo que es preocupante y es una exhortación para que se sigan tomando leyes ambientales que prevengan la emisión masiva de contaminantes en la Ciudad de México.

Como trabajos posteriores es importante retomar la iteración de la dirección del viento junto con otras variables ambientales y químicas, modelar el comportamiento espacial de las concentraciones de ozono, ya sea con un enfoque clásico o bayesiano.

\section{Bibliografía}

[1] Bravo H., (1992) La contaminación atmosférica por ozono en la zona metropolitana de la Ciudad de México: evolución histórica y perspectivas", IX Comisión Nacional de los Derechos Humanos.

[2] Coles A., "An introduction to statistical modeling of extreme values", tercera edición, editorial Springer Verlag, Great Britain, 2004.

[3] Huerta G., Sansó B., Stroud J. “A spatiotemporal model for Mexico City ozone levels”, Journal of the Royal Statistical Society (Applied Statistics), Vol. 53, No. 2, 2004

[4] Jammalamadaka, Rao S. \& SenGupta, A. Topics in circular statistics, primers edn, World Scientific, USA-INDIA. 2001

[5] Lee P.M. "Bayesian Statics An Introduction", segunda edición, editorial Arnold, Great Britain, ISBN 047119481, 1997.

[6 ] Ntzoufras, I. Bayesian Modeling Using WinBUGS. John Wiley \& Sons, 2009.

[7] Pickands J. III, "Statistical inference using extreme order statistics", The Annals of Statistics, Vol. 3, No. 1, pp. 119-131, 1975.

[8]Porter S., Rao T., Zurbenko G., Dunker M. y Wolff T.G., Ozone Air Quality over North America: Part II - An Analysis of Trend Detection and Attribution Techniques, Air and Waste Management

Association, vol. 51, pp. 283-306, 2001.

[9] Reiss R. y Thomas M." Statistical analysis of extreme values", segunda edición, editorial B9rkhauser Verlag, Germany, 2001.

[10] R Development Core Team (2007), R: A Language and Environment for Statistical Computing, R Foundation for Statistical Computing, Vienna, Austria. ISBN 3-900051-07-0.

*http://www.R-project.org

[11] Rodríguez, S, Reyes, H, Pérez, P. y Vaquera H." Selection of a subset of meteorological variables for ozone analysis: study case of Pedregal Station in Mexico city". Journal of Environmental Science and Engineering, pp: 11-20, Vol.1, 2012.

[12] SIMAT, "Sistema de Monitoreo Atmosférico de la Ciudad de México". Agricultura No.

21, Primer Piso, Col. Escandón, Del. Miguel Hidalgo, C.P. 11800, 2010, http://www.sma.df.gob.mx/simat2

[13]Thompson M.L., Joel R., Lawrencw H.C., Peter G. y Paul D.S., A review of statistical methods for the meteorological adjustment of tropospheric ozone, Atmospheric environment, vol. 35, pp. 617630, 2001.

[14]Wolff T., Dunker M., Rao T., Porter S. y Zurbenko G.I., Ozone Air Quality over North America: Part I - A Review of Reported Trends, Air and Waste Management Association, vol. 51, pp. 273-282, 2001. 


\section{Un análisis de las generaciones 2000-2008 de la Facultad de Físico- Matemáticas de la Benemérita Universidad Autónoma de Puebla.}

Ana Luisa Nieto Méndez ${ }^{1}$, Hortensia Reyes Cervantes² ${ }^{2}$ Flaviano Godínez Jaimes ${ }^{3}$, Francisco Tajonar Sanabria ${ }^{4}$ y Víctor Hugo Vázquez Guevara ${ }^{5}$.

Resumen: En este trabajo se analizaron las generaciones de estudiantes de la Facultad de Físico-Matemáticas; de las carreras de Matemáticas y Matemáticas Aplicadas, de 2000-2008. Hay una deserción del 80\%, sólo se revisaron algunas materias básicas de los primeros dos años ya que se consideran estas materias como representativas de las carreras debido a su grado de dificultad y significancia a lo largo de la carrera. Los alumnos se clasificaron en dos tipos: egresados y no egresados. Se observó que el promedio final, y las calificaciones de matemáticas elementales y cálculo integral en varias variables son las variables que explican si un alumno egresa de la carrera.

Palabras clave: materias básicas, dificultades en el estudio, regresión logística.

Abstract: In this work the generations of students of the Faculty of Physics and Mathematics were analyzed; degree Mathematics and Applied Mathematics, 2000-2008. There is $80 \%$ dropout, just some basic materials of the first two years were reviewed and considered these matters as representative of degree due to its difficulty and significance throughout the degree. Students were classified into two types: graduates and non-graduates. It was found that the variables that explain if a student is graduated are final grades average, grades in elementary mathematics and integral calculus in several variables.

Keywords: basic materials, difficulty in the study, logistic regression.

\footnotetext{
${ }^{1}$ FCFM-BUAP, Puebla-México, ana_mate89@hotmail.com

${ }^{2}$ FCFM-BUAP, Puebla-México, hreyes@fcfm.buap.mx

${ }^{3}$ UAGro, Guerrero-México, fgodinezj@gmail.com

${ }^{4}$ FCFM-BUAP, Puebla-México, ftajonar@fcfm.buap.mx

${ }^{5}$ FCFM-BUAP, Puebla-México, vvazquez@fcfm.buap.mx
} 


\section{Introducción}

Reconocer al alumno como el eje de la atención de los programas académicos de las Instituciones de Educación Superiores (IES) mexicanas, implica considerar su existencia y su transformación a lo largo de un espacio temporal que comprende tres momentos claramente identificables: la etapa anterior a su ingreso y hasta su integración a la institución educativa (primer momento), el transcurso de los estudios regulares durante su permanencia en la institución (segundo), y el lapso que se observa entre el momento de egreso y la obtención de un título que acredita sus aprendizajes y su calidad como profecionista en alguna o algunas áreas del conocimiento (tercero). En ese extenso recorrido, las instituciones educativas prevén o debieran prever un conjunto de actividades dirigidas a los alumnos, con la aspiración de atenderlos de manera integral, es decir, ocuparse de los estudiantes desde antes de su ingreso, con programas de información y orientación efectivos y mecanismos de selección justos; y hasta después de su egreso, con mecanismos de apoyo para la inserción laboral [4].

Los modelos de regresión logística son una herramienta que permite explicar el comportamiento de una variable con respuesta binaria mediante una o varias variables independientes explicativas de naturaleza cuantitativa y/o cualitativa. Los modelos de respuesta discreta son un caso particular de los modelos lineales generalizados formulados por Nelder y Wedderburn en 1972, al igual que los modelos de regresión lineal o el análisis de varianza [6].

\section{Objetivo}

Análizar los factores académicos que se relacionan con la eficiencia terminal, para así identificar los factores que la propician o no, esto usando la técnica estadística regresión logística. Así probar o descartar afirmaciones acerca de, que tanto la formación académica de un alumno de ciencias influye para que egrese o no de su respectivo plan de estudios, además de tratar de caracterizar a la población de alumnos de matemáticas y matemáticas aplicadas para futuras comparaciones con los alumnos de física y física aplicada.

\subsection{Justificación}

Se ha encontrado que la eficiencia terminal es un indicador importante en las metas y objetivos que tienen las universidades hoy en día, aunque se ha visto que con esto no se puede juzgar la calidad de las instituciones y el aprendizaje de los estudiantes. 
En las instituciones de educación media y superior, se mantienen vivos problemas que a todos preocupa o debiera preocupar, estos son, los asuntos de reprobación, rezago y deserción entre los estudiantes del área de ciencias [1].

En ciencias los alumnos se enfrentan a un ambiente escolar diferente al que estaban acostumbrados. Ahora, los alumnos deben ser responsables por sí mismos, de sus logros académicos pues se esmeran en sus tareas, estudios y exámenes de las materias que cursan. También los alumnos empiezan a conocer las matemáticas desde sus propiedades elementales, axiomas y se aprende a manejar la lógica para demostrar sus resultados.

\section{Marco Teórico}

Los estudios de egresados constituyen una de las estrategias apropiadas para retroalimentar los programas de formación de profesionales e investigadores en las instituciones de educación superior. Estos también son considerados como mecanismos poderosos de diagnóstico de la realidad, con el potencial de inducir en las instituciones la reflexión a fondo sobre sus fines y sus valores. Los resultados de estos estudios pueden asimismo, aportar elementos para redefinir el proyecto de desarrollo de aquellas instituciones que se mantienen alerta ante las nuevas necesidades sociales, permitiéndoles reconocer y asumir las nuevas formas de práctica profesional que se requieren para sustentar un proceso social menos inequitativo y dependiente. Los estudios de egresados pueden ser una herramienta básica para la definición de políticas en el nivel regional, estatal e incluso nacional y para el diseño de estrategias tendientes a propiciar el desarrollo y el fortalecimiento de todas las instituciones educativas del país [17].

En la Facultad de Ciencias Físico Matemáticas (FCFM) de la Benemérita Universidad Autónoma de Puebla (BUAP) existen algunos estudios acerca de los factores que influyen en la aprobación de la materia de Matemáticas Básicas y/o Matemáticas Elementales, tales como los siguientes:

- Una aplicación de regresión lineal en el aprovechamiento de los alumnos de nuevo ingreso en el área de matemáticas de la FCFM, en el que se encontró que los alumnos no tiene buenos hábitos de estudio, pues la mayoría estudia 
muy pocas horas diariamente y esto influye en sus calificaciones que los alumnos obtienen [2]. Algunas variables se encontraron importantes, fueron:

a) Si la carrera fue su primera opción de ingreso y

b) La confianza que tienen con el profesor para preguntarle dudas.

- Un estudio de uso de la regresión logística para estudiar la aprobación de la materia de Matemáticas Básicas de la FCFM en las generaciones 2010 y 2011. Se encontró que los alumnos tienen malos hábitos de estudio. Las dos generaciones se comportan de manera diferente: la mejor en aprobación es la 2011 y se debe a que estos alumnos van estudiando diariamente los conceptos vistos durante la clase, mientras que los alumnos de la generación 2010 deciden estudiar hasta la fecha del examen [9].

- Un análisis estadístico de algunos factores que afectan el proceso de enseñanza aprendizaje en la FCFM, usando técnicas estadísticas multivariadas, donde encontró que uno de los principales factores fue el hecho de que la Licenciatura en Matemáticas no fuera su primera opción, ya que esto afecta en el desempeño académico, otro factor que influye es la atención que tiene el maestro en sus cursos, así como los factores económico, social y cultural, entre otros [7].

- En un estudio acerca del proceso de enseñanza aprendizaje en la FCFM-BUAP se encontró que el lugar de Procedencia es un indicador, ya que los alumnos que provienen de otros estados son los que más acreditan la materia de Matemáticas Básicas [8].

- Un estudio de identificación de factores que intervienen en la reprobación del curso de Matemáticas Básicas de la FCFM de la BUAP. El análisis se realizó del período Primavera 2007 a Otoño 2010, y encontró que los principales factores son:

1. El profesor;

2. La falta de asistencia a asesorías que compete tanto a estudiantes como a profesores;

3. La literatura empleada en el curso. 
Se mencionan estos trabajos,ya que sus resultados son importantes y proporcionan los antecedentes para investigar a la población de egresados y no egresados [13].

Es conocido por los profesores que dan los cursos Matemáticas Elementales (ME), Cáculo Diferencial (CD), Cálculo Integral (CI), Cálculo Diferencial en Varias Variables CDV) y Cálculo Integral en Varias Variables (CIV) que los primeros dos años, los alumnos desertan más que en cualquier otro período. Los alumnos egresados tienen mejores promedios que los alumnos no egresados (pero que ya han estado en la carrera el tiempo suficiente para haberse titulado), y que los alumnos que desertaron. El promedio que los alumnos tienen en la preparatoria no es un indicador de que los alumnos serán capaces de terminar la carrera [18].

\subsection{Eficiencia Terminal}

La eficiencia terminal es determinante para conocer la eficacia de un programa de estudio; aunado a esto se encuentra la deserción en los programas lo que ocaciona un problema serio en el Sistema Educativo Mexicano por su incidencia negativa sobre los procesos políticos, económicos, sociales y culturales del desarrollo nacional [14].

El término eficiencia es polisémico por las aplicaciones que se hacen de él. En educación se denomina eficiencia terminal porque el proceso educativo se desarrolla durante un número de ciclos preestablecidos hasta la conclusión de un plan de estudios [15]. La eficiencia terminal del sistema educativo, como manifestación del rendimiento escolar, permite una serie de posibilidades de análisis descriptivo en la evaluación del rendimiento. Constituye asimismo, un referente cuantitativo en el análisis del recorrido escolar de los alumnos matrículados en un ciclo o nivel educativo. Estas dos condiciones de la eficiencia terminal, descripción y cuantificación, señalan de entrada las limitaciones que ella tiene para la explicación de procesos escolares. Sin embargo, sus potencialidades analíticas pueden constituir un punto de partida para el desarrollo de estudios más complejos y profundos sobre la institución escolar, y puede ser también un elemento de apoyo tanto para la elaboración conceptual, como para el conocimiento empírico de los comportamientos escolares de los alumnos [3]. 


\section{Metodología}

Este es un análisis de tipo inferencial, previamente se ha hecho un análisis descriptivo de la información y se observó que un $20 \%$ es egresado, así en este estudio nos enfocaremos a determinar los factores que determinan la eficiencia terminal por medio de la regresión logística.

\subsection{Regresión Logística}

El Análisis de Regresión Logística (Berenson y Levine, 1996; Hosmer y Lemeshow, 1989; Mather, 1976; Soares y Bartman, 1983), es una técnica estadística que permite, a través del planteamiento de un modelo matemático, establecer una relación funcional entre la variable dependiente binaria y las variables independientes o regresoras para la estimación de los parámetros del modelo matemático.

El modelo de regresión logística para una variable se deriva de la función matemática:

$$
\begin{gathered}
\pi(x)=P(Y=1 \mid x)=\frac{e^{g(x)}}{1+e^{g(x)}} \quad \text { con }-\infty<g(x)<\infty \\
g(x)=\beta_{0}+\beta_{1} x
\end{gathered}
$$

y para el caso de $\mathrm{k}$ variables: $g\left(x_{1}, x_{2}, \cdots, x_{k}\right)=\beta_{0}+\beta_{1} x_{1}+\cdots+\beta_{k} x_{k}$

La función $\pi(x)$ toma valores desde cero hasta uno de una forma creciente y tiene forma sigmoidea. La variable dependiente o de respuesta es una variable dicotómica que toma valores de 0 ó 1 , con $k$ variables regresoras evaluadas en un tiempo inicial [16]

\subsection{Descripción de la información}

El Universo consta de 1047 alumnos inscritos de dos de la licenciaturas Matemáticas y Matemáticas Aplicadas que se ofrecen en la FCFM de la BUAP, la información se obtuvo de la Dirección de Administración Escolar por medio de sus departamentos 
de Cómputo y de Titulación y de la Dirección General de Profeciones (SEP). El cohorte generacional fue hecho al periodo verano 2013; la información se analizó en SPSS 19 [19].

De la información obtenida de todos los alumnos de las dos licenciaturas, estos se clasificaron como alumnos que desertaron (alumnos que no se inscribieron en dos semestres consecutivos [20]), alumnos no egresados (alumnos que estan inscritos actualmente, pero que de acuerdo a los planes de estudio de la facultad podrían haberse titulado pues por el cohorte generacional considerado tienen al menos 5 años tomando cursos), y alumnos egresados (alumnos que han alcanzado el 100 por ciento de créditos y estan titulados). Así los datos con los que se trabajaron fueron con los alumnos no egresados y egresados; el total de estos alumnos son 308.

Se usó la siguiente codificación. La variable respuesta fue egreso que tuvo dos valores: egresado $(Y=1)$ y no egresados $(Y=0)$. Las variables independientes consideradas fueron Carrera (Licenciaturas de matemáticas (MAT) y matemáticas aplicadas (LMA)); las calificaciones en las materias ME, CD, CI, CDV, CIV; y otras variables como Años Cursados (AÑOS), Promedio Primer año (PROM1), Promedio Segundo año (PROM2), Promedio Tercer Año (PROM3), Promedio final (PROMAC), Promedio Preparatoria o Bachiller (PROMPREP), Puntaje exámen de admisión (PUNTAJE) y sexo (SEXO).

\section{Análisis de Datos}

Realizando la comparación de medias para la variable respuesta Y (categórica) y las variables explicativas (cuantitativas), obtuvimos que para la variable $C D$, no se rechaza la hipótesis nula de igualdad de medias y por tanto ésta variable ya no se considerará como variable explicativa.

Se realizó selección de variables hacia adelante y hacia atrás considerando las siguientes: Carrera, ME, CI, CDV, CIV, AÑOS, PROM1, PROM2, PROM3, PROMAC, PROMPREP, PUNTAJE y SEXO en SPSS. Las variables que resultaron en el modelo final fueron ME, CIV y PROMAC que como puede observarse tienen alta significancia (Tabla 1).

\subsection{Interpretación de los coeficientes}

La interpretación de los coeficientes en un modelo de regresión logística comúnmente se realiza mediante cambios en la escala logit, a través de razones de odds 
Tabla 1: Variables en la ecuación; salida de SPSS

\begin{tabular}{crrrrrr} 
Variables en la ecuación & B & ET & Wald & gl & Sig & $\operatorname{Exp}(\mathrm{B})$ \\
\hline ME & -.396 & .179 & 4.872 & 1 & .029 & 0.673 \\
CIV & .344 & .144 & 5.682 & 1 & .017 & 1.410 \\
PROMAC & 2.695 & .587 & 21.060 & 1 & .000 & 14.800 \\
Constante & -21.074 & 4.386 & 23.085 & 1 & .000 & 0.000 \\
\hline
\end{tabular}

condicionales. Sin embargo dichos coeficientes no tiene una interpretación directa en términos de probabilidad, que podría considerarse como la mas frecuente para la mayoría de los usuarios [12].

Otra forma de presentar los resultados del modelo de regresión logística es a través de una estimación ajustada de las probabilidades asociadas a cada covariable. El calculo de probabilidades ajustadas puede ser mas conveniente para una persona no experta en las nociones de estimación de parámetros de regresión y probabilidad asociada [11].

El signo de los coeficientes estimados del modelo de regresión logística en la Tabla 1 da una explicación de las variables predicadoras usadas, luego los signos de CIV y PROMAC son positivos y por consiguiente producen un crecimiento en la probabilidad para egresar. En cambio el signo de la variable ME es negativo con lo cual produce un decrecimiento en la misma probabilidad.

La razón de momios (RM) se obtuvo exponenciando la estimación del parámetro y corresponde a la columna $\operatorname{Exp}(\mathrm{B})$ en la Tabla 1 por lo tanto:

- La RM par ME es 0.673, lo que indica que un incremento de la calificación de ME disminuye la probabilidad de que un alumno egrese de alguna de las licenciaturas (MAT y LMA).

- La RM para CIV es 1.416, esto indica que un incremento de la calificación de CIV incrementa la probabilidad de que un alumno egrese de alguna de las lilcenciaturas (LMA y MAT).

- La RM para PROMAC es 14.8, lo que indica que un incremento en el PROMAC incrementa la probabilidad de que un alumno egrese de alguna de las licenciaturas (LMA y MAT).

\subsection{Bondad de Ajuste}

Del modelo obtenido se debe verificar que efectivamente este modelo se ajusta correctamente a los datos, para esto en SPSS se tiene la opción que al momento de 
realizar el análisis de regresión logística se obtenga en la misma salida el resultado de la Prueba de Hosmer y Lemeshow. En esta prueba la hipótesis nula se define por: no hay diferencia entre los valores observados y los valores pronosticados. En la Tabla 2, el valor calculado del estadístico de Hosmer y Lemeshow de bondad de ajuste para el modelo fue $\mathrm{c}=8.22$, con un $\mathrm{p}$ valor de 0.394 , y por tanto podemos concluir que no se rechaza la hipótesis nula.

\begin{tabular}{cccc}
\multicolumn{4}{c}{ Tabla 2: Prueba de Hosmer y Lemeshow } \\
\hline Paso & Chi cuadrado & gl & Significancia \\
3 & 8.420 & 8 & .394 \\
\hline
\end{tabular}

En la Tabla 3 observamos la tabla de Clasificación tenemos que: $40 \%$ de los alumnos no egresados se pronosticaron correctamente y 52 se clasificaron incorrectamente. El $96 \%$ de los alumnos egresados se pronosticaron correctamente, y por tanto esto nos da un 85 de porcentaje global pronosticado correctamente. Con lo cual el modelo se puede considerar bueno.

Tabla 3: Tabla de clasificación de valores observados y pronosticados

\begin{tabular}{cccc}
\hline Observado & Pronosticado & & \\
\hline & 0 & 1 & Porcentaje Correcto \\
0 & 20 & 30 & $40.0 \%$ \\
1 & 8 & 202 & $96.2 \%$ \\
& Porcentaje global & & $85.4 \%$ \\
\hline
\end{tabular}

El valor de corte es .500

Una forma de valorar el ajuste del modelo de regresión logística es midiendo su capacidad discriminadora. La Curva Característica de funcionamiento del receptor (COR) es una gráfica de la probabilidad de detectar una señal verdadera(sensibilidad) y la señal falsa (1-sensibilidad) para todo un rango de posibles puntos de corte que van de 0 a 1 . Una medida de la precisión de la clasificación está dada por el área bajo la curva (A) COR. Según Hosmer y Lemeshow (2013)(Véase [10]):

1) $\mathrm{Si} \mathrm{A}=0.5$, entonces el modelo no permite predecir el éxito o el fracaso y por tanto el modelo no es útil. 
Tabla 4: Área bajo la curva COR

Variables resultado de contraste: Probabilidad pronosticada

\begin{tabular}{ccccc}
\hline Área & Error & & & \\
& & & Límite inferior & Límite superior \\
\hline .776 & .032 & 0.000 & .713 & .839 \\
\hline
\end{tabular}

a. Bajo el supuesto no parámetrico

b. Ho: área verdadera $=0.5$

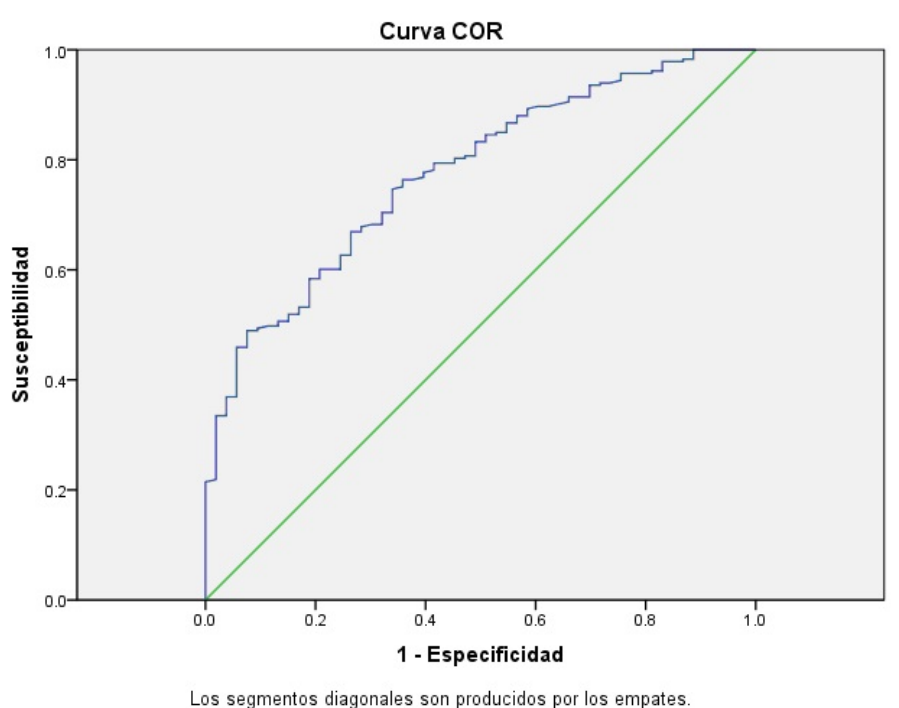

Figura 1: Área bajo la curva COR

2) Si $0.7<a<0.8$ hay una discriminación aceptable.

3) Si $0.8<a<0.9$ hay una discriminación excelente.

SPSS permite probar la hipótesis de que $A=0.5$ contra $A \neq 0.5$, esto es de que el modelo no tiene capacidad discriminante contra de que si la tiene. En la tabla $4 \mathrm{~A}=0.776$ con intervalo de confianza del $95 \%$ de 0.713 a 0.839 y un valor $\mathrm{p}=0.000$ por tanto el modelo tiene capacidad discriminante, es decir clasificará de manera correcta a los alumnos. Aún más, el modelo tiene una capacidad discriminante aceptable. 


\section{Conclusiones}

En este análisis, se estudiaron los factores para que un alumno egrese o no de alguna de las dos licenciaturas Matemáticas o Matemáticas Aplicadas, usando el modelo de regresión logística. Los factores usados fueron Matemáticas Elementales, Cálculo Diferencial, Cálculo Integral, Cálculo Diferencial en Varias, Cálculo Integral en varias variables, Años cursados, Promedio primer año, Promedio segundo año, Promedio tercer año, Promedio final, Promedio Preparatoria o Bachiller, Puntaje examen de admisión, Carrera y Sexo.

El resultado de la selección de variables fue la calificación de las materias Matemáticas Elementales y Cálculo Integral en varias variables, éstas influyen negativa y positivamente respectivamente en la probabilidad de que un alumno egrese o no; además la variable Promedio final aumenta de manera muy significativa esta probabilidad.

En cuanto al test de Hosmer y Lemeshow, dada la hipótesis nula de que el modelo no tiene capacidad discriminante; se obtuvó no rechazar la hipotesis nula y por lo tanto el modelo tiene un buen ajuste, ésto se confirmó con la curva COR al rechazar la hipótesis nula: área bajo la curva igual a 0.5. En la tabla de clasificación se pudo observar que un $85 \%$ de la población total fue pronosticada correctamente.

\section{Bibliografía}

[1] Aparicio L. E., Un estudio sobre factores que obstaculizan la permanencia, logro educativo y eficiencia terminal en las áreas de matemáticas del nivel superior: el caso de la Facultad de Matemáticas de la Universidad Autónoma de Yucatán.

[2] Arenas Y., Una Aplicación de Regresión Lineal en el Aprovechamiento de los alumnos de Nuevo Ingreso en el Área de Matemáticas de la FCFM. Tesis de licenciatura, 2011.

[3] Camarena R., Chavez A., Gómez J., Reflexiones en torno al rendimiento escolar, y a la eficiencia terminal.

[4] Díaz J., Análisis Descriptivo de los Egresados y Titulados de las Licenciaturas de Matemáticas y Matemáticas Aplicadas de las Generaciones 2000 a 2004, Tesis de licenciatura en matemáticas FCFM-BUAP, 2013

[5] Romo A., Fresan M., Los factores curriculares y académicos relacionados con el abandono y el rezago.

[6] De la Fuente S., Regresión Logística, Facultad de Ciencias Económicas y Empresariales, UAM, Madrid, 2011. 
[7] Hernández S., Reyes H., Linares G., Análisis Estadístico de algunos factores que afectan el proceso de enseñanza aprendizaje en la FCFM-BUAP, usando técnicas estadísticas multivariadas, VI Encuentro Participación de la Mujer en la Ciencia, CIO, 2009.

[8] Hernández S., Reyes H., Ibarra M., Linares G., Proceso de enseñanza aprendizaje, VII encuentro Participación de la mujer en la ciencia, CIO, 2010.

[9] Hernández, S. Uso del modelo de Regresión Logística para estudiar la aprobación de la materia de Matemáticas Básicas de la FCFM en las generaciones 2010 y 2011, tesis de licenciatura, México, 2013.

[10] Hosmer D., Lemeshow S., Applied Logistic Regression, Wiley, 2013.

[11] Iglesias Cabo T., Métodos de Bondad de ajuste en regresión logística, Trabajo fin de Máster, 2013.

[12] Lopéz J., García J., Eventos por variable en Regresión Logística y Redes Bayesianas para Predecir Actitudes Emprendedoras, Universidad de Almeria, 2011.

[13] Maldonado A., Identificación de los Factores que intervienen en la reprobación del curso de Matemáticas Básicas de la FCFM de la BUAP, Tesis de Licenciatura, México, 2012.

[14] Montoya S., Análisis de la eficiencia terminal de la generación 1999-2003 de la maestría en ciencias son especialidad en administración pública, campus virtual politécnico de la escuela superior de comercio y administración, unidad Santo Tomás, IPN, Tesis de Maestría, junio 2006.

[15] Morales E., Nolasco S., Arizaldo E., Vida académica y eficiencia terminal del nivel superior de la Universidad Autónoma de Nayarit 1995-2001: una mirada retrospectiva en el marco del modelo educativo tradicional, Universidad Autónoma de Nayarit, Artículo

[16] Muller M., Generalized Linear Models, Gemany, 2004.

[17] Ontiveros I., Seguimiento de Egresados de la Licenciatura en Artes Visuales de la Excuela de Pintura, Escultura y Artesanías de la UJED, Tesis de Maestría, 2006.

[18] Reyes J., Canizo J., Meza E., Herrera A., Cruz H., Nieto A., Godínez J.; Descripción de las generaciones 2000-2008 para los alumnos que desertan en dos licenciaturas de la FCFM-BUAP, Encuentro Participación de la Mujer en la ciencia, CIO 2014. 
IV Encuentro sobre Didáctica de la Estadística, la Probabilidad y el Análisis de Datos 13

[19] SPSS(2010), IBM SPSS Statistics 19 para Windows.

[20] Tinto V., Una consideración de las teorías de la deserción estudiantil en la trayectoria escolar en la educación superior, México: ANUIES 1987. 


\title{
Induciendo a la creación de problemas de probabilidad: una experiencia con docentes de matemática de secundaria
}

\author{
Autor: Lorena Salazar Solórzano ${ }^{1}$
}

\section{Resumen.}

Un famoso provervio chino dice: "Regala un pescado a un hombre y le darás alimento para un día, enseñale a pescar y lo alimentarás para el resto de su vida". Se exponen aquí los resultados de una actividad implementada con profesores de secundaria en ejercicio, en la que se les indujo a realizar creaciones de problemas de probabilidad, contextualizados y que respondieran a un objetivo específico. Para ello se les proporcionaron datos reales nacionales, tomados del Compendio de Estadísticas del Informe del Estado de la Nación (Costa Rica, 2013) de modo que les sirva de insumo para la creación de sus problemas. La aprobación en el 2012 de nuevos programas de matemática en Costa Rica, plantean una demanda nacional que exige que los docentes tengan, no solo un dominio sólido en conceptos de combinatoria y probabilidad, sino que además desarrollen competencias para elegir y crear su propio material y no se limiten a seguir libros de texto y materiales, escritos en la mayoría de los casos, para otros contextos que resultan ajenos a la realidad nacional. Se lograron evidencias que indican que esta estrategia de creación de problemas, no solo logra la afirmación de los conceptos de probabilidad, sino también que les da más seguridad a los profesores para preparar sus clases en secundaria.

\begin{abstract}
. the teachers, self-confidence to prepare their lessons. educación matemática. cation.

Modalidad: Ponencia

\footnotetext{
${ }^{1}$ Universidad Nacional. Universidad de Costa Rica. lorena.salazarsolorzano@ucr.ac.cr
}

A famous Chinese proverb says, " if you give a man a fish, then you feed him for a day, if you teach him to fish, then you will feed him for the rest of his life". This paper presents the results of an activity implemented with high school teachers, which was intended to induce them to make creations of probability problems, contextualized and that they respond to a specific objective. They were provided with real data taken from the National Statistical Abstract of the of Costa Rica, 2013, so that they coul use for the creation of their problems. The adoption in 2012 of new math programs in Costa Rica, demandes that teachers have to have, not only solid concepts of combinatorics and probability, but also develop skills to choose and create their own material and not just follow textbooks and materials written in most cases, for other contexts that are outside the national reality. We got some evidences, that indicates that this strategy of creating problems, not only does affirm the concepts of probability, but also gives to

Palabras clave: probabilidad, didáctica de la probabilidad, creación de problemas,

Key Words: probability, didactic of probability, creating problems, mathematics edu- 


\section{Introducción:}

En el 2012 el Consejo Superior de Educación de Costa Rica, aprobó los nuevos programas de matemática que involucran desde primaria hasta secundaria. En el 2013 se inició un plan de transición para la aplicación de los mismos, y se espera que para el 2016, ya esten siendo aplicados en todos los niveles. Estos nuevos programas exigen un profesor de matemática altamente competente en diversas aristas, una de ellas es la resolución de problemas, que los nuevos programas han declarado como su enfoque principal. Por primera vez se incluye el tema de probabilidad y estadística en todos los niveles, y esto hace que el profesor en ejercicio requiera competencias que le permitan elegir y crear material para la enseñanza de este tema.

\section{Nociones Teóricas:}

En la última década, se ha dado un auge en investigaciones que centran su atención no solo a la resolución de problemas, sino también al planteamiento de problemas. Algunas de las investigaciones sobre creación de problemas que se han centrado en los profesores, consideran que, en la práctica docente, los profesores deben mostrar competencia en la creación de problemas. Sin embargo, hay investigaciones como la de Singer y Voica (2013), que muestran que los problemas que crean los profesores tienen serias limitaciones que son relevantes para el aprendizaje de sus alumnos. Estas investigaciones son el origen de otras que investigan sobre propuestas de cómo el planteamiento de problemas, puede ser una parte integral de los programas de formación de profesores (Ellerton, 2013). En Salazar (2014) se muestran algunas experiencias de aula en las áreas de análisis y álgebra en cursos de matemática formal para profesores de matemática, en donde la creación de problemas resultaron ser una actividad muy productiva para la comprensión y aprendizaje de algunos temas de estas áreas. Malaspina (2013), también reporta resultados positivos al aplicar esta estrategia con profesores de matemática, en la que ellos crean problemas pre y problemas pos, que luego se resuelven y comentan grupalmente. Según algunas de sus conclusiones, la capacidad de crear y resolver problemas lleva a reflexiones didácticas y matemáticas que muestran la importancia de una redacción adecuada del enunciado, elemento importantísimo en problemas de probabilidad. Por otro lado, en Espinoza, Lupiañez y Segovia (2014) se hace un estudio de los propósitos de la invención de problemas en la disciplina de matemáticas. Para estos autores, la invención de problemas es una forma de desarrollar la actividad creativa y su responsabilidad en el aprendizaje, además de que mejora su disposición y actitudes hacia las matemáticas. En esta experiencia, se pretende que el docente realice inicialmente, algunas variaciones sencillas de problemas dados, para luego adaptarlos y contextualizarlos al entorno estudiantil.

\section{Metodología}

El taller se aplicó a un grupo de 30 profesores de matemática en ejercicio en el IV Encuentro Provincial de Educación Matemática. Primeramente se hizo una introducción al tema, mostrando cómo se podían variar problemas en primera instancia, hasta crear problemas propios. Se les proporcionó una guía de trabajo con algunos ejemplos de variaciones y creaciones de problemas. La modalidad de trabajo fue la de trabajo colaborativo en grupos de 2 a 3 personas en una sesión de 3 horas. Para ello se les proporcionaron datos reales nacionales, tomados del Compendio de Estadísticas del In- 
forme del Estado de la Nación (Costa Rica, 2013) de modo que les sirviera de insumo para la creación de sus problemas. Cada grupo tuvo un problema diferente con temáticas relacionadas con su entorno laboral con datos reales del sistema educativo en Costa Rica desde 2000 al 2012. Explícitamente, los temas tratados fueron sobre matrícula en el sistema educativo,adecuaciones, repitencia, deserción, resultados de exámenes de bachillerato e ingreso a las universidades. También se les dio una lectura de un artículo del periódico La Nación, alusivo a cada tema, con el fin de motivarlos a la reflexión y a obtener provecho a los datos proporcionados, de modo que sus creaciones buscaran obtener respuestas a sus inquietudes. Se les dió un tiempo de 1 hora para la creación de sus problemas y luego se les solicitó que cada grupo expusiera su trabajo al resto del grupo.

Para la recolección de datos, se usó una bitácora para captar los acontecimientos que se fueron dando en el aula, donde se fueron anotando comentarios y reflexiones dadas por los profesores. Se recolectaron evidencias escritas del trabajo de ellos, así como de algunas grabaciones de la voz de algunos de los grupos de trabajo, para obtener información sobre el proceso desarrollado, previo concentimientos de los docentes.

\section{Variaciones de problemas}

La creación de problemas, al igual que la resolución de problemas, es una estrategia que debe desarrollarse, entrenarse y ejercitarse, iniciando con niveles básicos hasta llegar a niveles superiores. De acuerdo a otras experiencias realizadas por la autora de este trabajo, es recomendable iniciar esta estrategia de creación de problemas, con simples modificaciones a problemas tomados de libros de texto. Cambiando primero los objetos matemáticos, se puede lograr otro problema contextualizado a la realidad de los estudiantes.

Como un ejemplo se presenta el siguiente problema típico que involucra el concepto laplaciano de probabilidad, tomado del libro Mendenhall(2002), para ilustrar como se le pueden hacer adaptaciones o variaciones a este problema para obtener otro más cercano a los intereses estudiantiles.

\section{Problema Modelo 1:}

En una comarca hay dos periódicos, el Nacional (N) y el Liberal (L). Se sabe que el $55 \%$ lee el (N), mientras que un $40 \%$, lee (L) y $25 \%$ no lee ninguno de los dos. Si se escoge una persona al azar, estime la probabilidad de que una persona tomada al azar lea alguno de los dos, los dos, solo uno de ellos, que no lea ni uno ni el otro, es decir halle

$$
P(N \cup L), P(N \cap L), P(N-L) \cup P(L-N), P(-(N \cup L)) .
$$

En un problema como este, se espera que lo primero que hagan es contextualizarlo al entorno nuestro. En este caso específico, se pueden cambiar los nombres de los periódicos a los nacionales y buscando datos reales en la web, se puede variar el problema, fortalecerlo y sacarle más provecho que el original, como se presenta a continuación. 
Variación del Problema Modelo 1:

En la página http://www.mediatico.com/es/periodicos/america-latina/costarica/, se presentan las visitas en internet a 20 periódicos nacionales. A continuación se presentan los datos de los más populares, a saber: La Extra (E), La Nación (N), Al Día (D), La Gaceta (G), La República (R), La Prensa Libre (PL), El Tico Times (T), El Financiero (F) y el Semanario Universidad (S).

\begin{tabular}{|l|c|c|c|c|c|c|c|c|c|c|}
\hline $\begin{array}{l}\text { Nombre } \\
\text { del } \\
\text { Periódico }\end{array}$ & $\begin{array}{c}\text { Diario La } \\
\text { Extra }\end{array}$ & $\begin{array}{c}\text { Nación } \\
\text { Digital }\end{array}$ & $\begin{array}{c}\text { Periódico } \\
\text { al Día }\end{array}$ & $\begin{array}{c}\text { La } \\
\text { Gaceta }\end{array}$ & $\begin{array}{c}\text { La } \\
\text { República }\end{array}$ & $\begin{array}{c}\text { Prensa } \\
\text { Libre }\end{array}$ & $\begin{array}{c}\text { Tico } \\
\text { Times }\end{array}$ & $\begin{array}{c}\text { Financier } \\
0\end{array}$ & $\begin{array}{c}\text { Semanario } \\
\text { Universidad }\end{array}$ & $\begin{array}{c}\text { Total de } \\
\text { visitas }\end{array}$ \\
\hline $\begin{array}{l}\text { \# de } \\
\text { Visitas }\end{array}$ & 140971 & 59344 & 48749 & 33786 & 33056 & 22833 & 21658 & 16489 & 12111 & 317415 \\
\hline
\end{tabular}

Usando los datos anteriores, estime la probabilidad de que al tomar una persona al azar, este visite los periódicos indicados, $P(E), P(N), P(R), P(P L), P(N \cup R), P(-D \cap$ $S), P(F \cup P L), P(-(P L \cap T))$

Cuando se hace una variación a un problema, es común que se les ocurra hacer variaciones a la variación ya hecha, pues de una cosa se sigue la otra. Como los adolescentes usualmente no les gusta leer periódicos, quizás convenga hacer una variación que les sea más atractiva. A continuación se presenta a modo de ejemplo, una variación a la variación del problema original, en la que se cambian los objetos involucrados (los periódicos) por series de películas que se ofrecen en línea en la internet, muy común en los jóvenes y por lo tanto posiblemente más aceptado por ellos.

Variación de la variación del Problema Modelo 1:

A continuación se presentan datos, tomados de sobre la puntuación que la aundiencia da a diferentes series de películas que se descargan de internet: Walking Dead (WD), Los Simpsons (S), Games or Thrones (GT), The Big Bang Theory (BT), House (H), Dexter (D), Breaking Bad (BB), How I met your mother (HM) y The vampires diaries (VD).

\begin{tabular}{|l|l|l|c|c|c|c|c|c|c|}
\hline $\begin{array}{l}\text { Nombre de } \\
\text { la Serie }\end{array}$ & $\begin{array}{l}\text { Walking } \\
\text { Dead }\end{array}$ & $\begin{array}{l}\text { Los } \\
\text { Simpsons }\end{array}$ & $\begin{array}{c}\text { Game or } \\
\text { Thrones }\end{array}$ & $\begin{array}{c}\text { The Big } \\
\text { Bang } \\
\text { Theory }\end{array}$ & House & Dexter & $\begin{array}{c}\text { Breaking } \\
\text { Bad }\end{array}$ & $\begin{array}{c}\text { How I } \\
\text { met your } \\
\text { mother }\end{array}$ & $\begin{array}{c}\text { The } \\
\text { vam pire } \\
\text { diaries }\end{array}$ \\
\hline Puntuación & 8341 & 6129 & 6087 & 5328 & 4665 & 3349 & 4295 & 3153 & 2556 \\
\hline
\end{tabular}

Usando los datos dados, estime la probabilidad de que al tomar una persona al azar, este siga la serie indicada. $P(W D), P(S), P(G T), P(B T), P(H \cup D), P(-B B \cap$ $V D), P(H M \cup W D), P(-G T \cap . V D)$

\section{Creación de problemas}

A continuación se presentan los resultados de la actividad, donde los docentes debían crear sus problemas en los temas asignados a cada grupo. 


\section{Tema 1: Matrícula en el sistema educativo}

\begin{tabular}{|c|c|c|c|c|c|c|c|c|c|c|c|c|c|c|}
\hline & 2000 & 2001 & 2002 & 2003 & 2004 & 2005 & 2006 & 2007 & 2008 & 2009 & 2010 & 2011 & 2012 & Total \\
\hline Total & 283.989 & 298.889 & 327.042 & 346.870 & 368.126 & 375.481 & 385.302 & 387.493 & 391.330 & 418.185 & 426.735 & 433.077 & 441.296 & 4.883 .815 \\
\hline Tradicional & 252.828 & 266.058 & 284.841 & 301.300 & 317.539 & 330.562 & 338.508 & 338.748 & 337.445 & 349.595 & 350.791 & 354.413 & 359.138 & 4.181 .766 \\
\hline Pública & 219.019 & 231.346 & 248.861 & 264.173 & 279.989 & 292.611 & 301.189 & 300.197 & 297.519 & 309.251 & 310.442 & 313.823 & 317.950 & 3.686 .370 \\
\hline Privada & 21.265 & 22.049 & 23.337 & 24.475 & 24.870 & 25.190 & 24.754 & 25.748 & 27.406 & 27.705 & 27.445 & 27.983 & 28.589 & 330.816 \\
\hline $\begin{array}{c}\text { Privada } \\
\text { subvencionada }\end{array}$ & 12.544 & 12.663 & 12.643 & 12.652 & 12.680 & 12.761 & 12.565 & 12.803 & 12.520 & 12.639 & 12.904 & 12.607 & 12.599 & 164.580 \\
\hline $\begin{array}{c}\text { Académica } \\
\text { diurna }\end{array}$ & 181.089 & 192.465 & 204.250 & 214.090 & 224.522 & 234.118 & 238.434 & 237.237 & 236.812 & 244.121 & 244.997 & 246.875 & 244.670 & 2.943 .680 \\
\hline Técnica diurna & 48.360 & 49.960 & 52.943 & 55.913 & 57.414 & 58.592 & 60.386 & 62.370 & 64.109 & 66.927 & 67.092 & 68.492 & 73.408 & 785.966 \\
\hline $\begin{array}{c}\text { Académica } \\
\text { nocturna }\end{array}$ & 22.847 & 23.059 & 26.782 & 30.281 & 34.534 & 36.565 & 37.981 & 37.442 & 35.026 & 36.742 & 36.371 & 36.007 & 35.749 & 429.386 \\
\hline $\begin{array}{c}\text { Técnica } \\
\text { nocturna }\end{array}$ & 532 & 574 & 866 & 1.016 & 1.069 & 1.287 & 1.707 & 1.699 & 1.498 & 1.805 & 2.331 & 3.039 & 5.311 & 22.734 \\
\hline No tradicional & 31.161 & 32.831 & 42.201 & 45.570 & 50.587 & 44.919 & 46.794 & 48.745 & 53.885 & 68.590 & 75.944 & 78.664 & 82.158 & 702.049 \\
\hline $\begin{array}{c}\text { suficiencia } \\
\text { (MEP) }\end{array}$ & 9.144 & 8.698 & 12.189 & 12.305 & 12.655 & 8.986 & 8.894 & 8.568 & 8.718 & 12.209 & 13.842 & 14.989 & 20.197 & 151.394 \\
\hline $\begin{array}{l}\text { distancia } \\
\text { (Coned) }\end{array}$ & & & & & & & 2.463 & 2.760 & 3.476 & 4.794 & 5.107 & 5.436 & 5.037 & 29.073 \\
\hline $\begin{array}{c}\text { Nuevas } \\
\text { Oportunidades }\end{array}$ & 10.414 & 10.457 & 14.377 & 15.853 & 16.152 & 10.791 & 10.593 & 12.588 & 14.648 & 20.012 & 23.271 & 19.727 & 16.382 & 195.265 \\
\hline $\begin{array}{c}\text { IPEC (Plan } \\
125)\end{array}$ & 396 & 803 & 511 & 547 & 694 & 455 & 246 & 654 & 515 & 209 & 0 & 0 & 0 & 5.030 \\
\hline $\begin{array}{c}\text { Cindea (II-III } \\
\text { nivel) }\end{array}$ & 8.392 & 9.780 & 11.000 & 12.225 & 15.903 & 19.251 & 18.237 & 17.701 & 20.395 & 24.627 & 26.436 & 30.619 & 32.627 & 247.193 \\
\hline $\begin{array}{c}\text { Educación } \\
\text { especial }\end{array}$ & 2.815 & 3.093 & 4.124 & 4.640 & 5.183 & 5.436 & 6.361 & 6.474 & 6.133 & 6.739 & 7.288 & 7.893 & 7.915 & 74.094 \\
\hline
\end{tabular}

Algunos de los problemas creados conjuntamente entre la instructora y los docentes, se dan a continuación.

1. ¿Cuál es la probabilidad de que un estudiante, tomado al azar entre los años 2000 y 2012, del III ciclo o ciclo diversificado tradicional, esté en una institución privada? ¿En una institución pública? ¿En una subvencionada?

Las probabilidades solicitadas son

$$
\begin{aligned}
& P(\text { Priv })=\frac{330816}{4181766}=0,07910 \approx 0,08 \\
& P(\text { Públ })=\frac{3686370}{4181766}=0,8815 \approx 0,88 \\
& P(\text { Subv })=\frac{164580}{4181766}=0,0393 \approx 0,04
\end{aligned}
$$

2. Estime la probabilidad de que un estudiante de secundaria costarricense, tomado al azar, esté estudiando en una institución técnica?

Dado que se esta tomando un estudiante de secundaria costarricense en general, es decir no se limita a los años del 2000 al 2012, se trata más bien de una probabilidad frecuencial, por lo que es importante el uso de la palabra "estimar" e lugar de "hallar" una probabilidad. Por otro lado, como no se especifica si la institución debe ser Técnica diurna (D) o Técnica nocturna(N), se debe estimar la probabilidad de que este estudiando en alguna de ellas. Como los eventos son independientes, pues se supone que un estudiante no estará matriculado en los dos tipos de instituciones, la probabilidad solicitada es: 
IV Encuentro sobre Didáctica de la Estadística, la Probabilidad y el Análisis de Datos

$$
P(D \cup N)=\frac{785966}{4883815}+\frac{22734}{4883815} \approx 0,16
$$

\section{Tema 2: Adecuaciones curriculares}

Adecuaciones curriculares en Costa Rica del 2000 al 2011

\begin{tabular}{|c|c|c|c|c|c|c|c|c|c|c|c|c|c|}
\hline & 2000 & 2001 & 2002 & 2003 & 2004 & 2005 & 2006 & 2007 & 2008 & 2009 & 2010 & 2011 & Total \\
\hline De acceso & 8.994 & 10.169 & 10.767 & 10.47 & 10.747 & 10.72 & 11.679 & 10.766 & 10.759 & 11.178 & 11.397 & 10.513 & 128.168 \\
\hline No significativa & 59.548 & 68.824 & 78.610 & 89.42 & 95.996 & $102.26 \mathrm{z}$ & 113.666 & 112.142 & 109.098 & 113.027 & 113.592 & 114.228 & 1.170 .506 \\
\hline Significativa & 2.778 & 3.846 & 4.891 & 6.43 & 7.530 & 9.03 & 11.095 & 11.025 & 11.650 & 12.237 & 13.087 & 13.482 & 107.089 \\
\hline Total & & & & & & & & & & & & & 1.405 .763 \\
\hline & & & & & & & & & & & & & \\
\hline Preescolar & & & & & & & & & & & & & \\
\hline De acceso & 816 & 705 & 767 & 89 & 995 & 958 & 1.201 & 729 & 789 & 1.012 & \begin{tabular}{r|r|}
902 \\
\end{tabular} & 889 & 10.657 \\
\hline No significativa & 2.668 & 2.487 & 2.156 & 2.47 & 2.514 & 2.268 & 2.931 & 1.831 & 1.638 & 1.865 & 1.559 & 1.584 & 25.978 \\
\hline Total & & & & & & & & & & & & & 36.635 \\
\hline & & & & & & & & & & & & & \\
\hline | y || ciclos & & & & & & & & & & & & & 0 \\
\hline De acceso & 6.965 & 7.826 & 8.003 & 7.538 & 7.764 & 7.195 & 7.884 & 8.028 & 8.116 & 8.158 & 8.009 & 7.215 & 92.705 \\
\hline No significativa & 45.979 & 51.927 & 57.920 & 63.142 & 66.043 & 69.32 & 74.225 & 72.835 & \begin{tabular}{ll|}
72.027 & \\
\end{tabular} & 73.272 & 73.313 & 73.416 & 793.427 \\
\hline Significativa & 2.597 & 3.585 & 4.510 & 5.80 & 6.786 & 7.85 & 9.172 & 9.271 & 9.486 & 9.756 & 10.496 & \begin{tabular}{|l|l}
10.707 \\
\end{tabular} & 90.027 \\
\hline Total & & & & & & & & & & & & & 976.159 \\
\hline & & & & & & & & & & & & & \\
\hline Escuelas nocturnas & & & & & & & & & & & & & 0 \\
\hline De acceso & 7 & 143 & 9 & (c) & 6 & 66 & 10 & 3 & 11 & 0 & 33 & 1 & 377 \\
\hline No significativa & 125 & 35 & 18 & 9 & 125 & 75 & 161 & 17 & 59 & 137 & 50 & 38 & 931 \\
\hline Significativa & 0 & 4 & 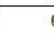 & 15 & 10 & 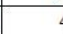 & 5 & 0 & 6 & & 1 & 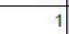 & 53 \\
\hline Total & & & & & & & & & & & & & 1.361 \\
\hline & & & & & & & & & & & & & \\
\hline III ciclo y educación diversificada & & & & & & & & & & & & & 0 \\
\hline De acceso & 1.206 & 1.495 & 1.90 & 2.038 & 1.982 & 2.50 & 2.584 & 2.006 & 1.843 & 2.008 & 2.453 & 2.408 & 24.429 \\
\hline No significativa & 10.776 & 14.375 & 18.51 & 23.715 & 27.314 & 30.59 & 36.349 & 37.459 & 35.374 & 37.753 & 38.720 & 39.228 & 350.170 \\
\hline Significativa & 181 & 257 & 37 & 612 & 734 & 1.17 & 1.918 & 1.754 & 2.158 & 2.480 & 2.590 & 2.774 & 17.009 \\
\hline Total & & & & & & & & & & & & & 391.608 \\
\hline & & & & & & & & & & & & & \\
\hline
\end{tabular}

Con los datos anteriores, es mejor hacer un cuadro con los totales de los datos para facilitar la creación de problemas de probabilidad.

Total de adecuaciones curriculares en Costa Rica entre el 2000 y 2011

\begin{tabular}{|c|c|c|c|c|}
\hline & $\begin{array}{c}\text { Adecuación } \\
\text { de Acceso }\end{array}$ & $\begin{array}{c}\text { Adecuación no } \\
\text { Significativa }\end{array}$ & $\begin{array}{c}\text { Adecuación } \\
\text { Significativa }\end{array}$ & Total \\
\hline Preescolar (K) & 10.657 & 25.978 & 0 & 36.635 \\
\hline I y II ciclo (E) & 92.705 & 793.427 & 90.027 & 976.159 \\
\hline $\begin{array}{c}\text { Escuela Nocturna } \\
\text { (N) }\end{array}$ & 377 & 931 & 53 & 1.361 \\
\hline $\begin{array}{c}\text { III ciclo y } \\
\text { Diversificado (C) }\end{array}$ & 24.429 & 350.170 & 17.009 & 391.608 \\
\hline & 128168 & 1170506 & 107089 & 1.405 .763 \\
\hline
\end{tabular}

Algunos de los problemas creados en conjunto con la instructora, de modo que se repasaran los conceptos de probabilidad condicional fueron los siguientes. 


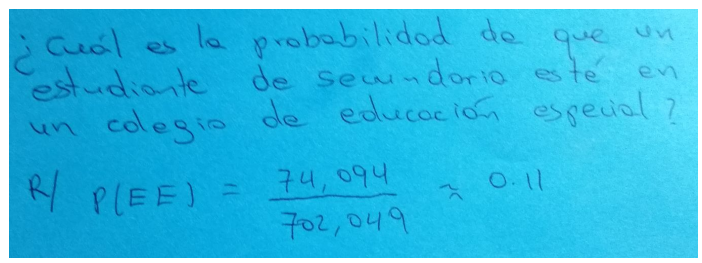

Figura 1: Un problema con solución errónea

1. ¿Cuál es la probabilidad de que un niño de prescolar con alguna adecuación curricular en el 2000 tenga adecuación de acceso?

2. ¿Cuál es la probabilidad de que un estudiante con alguna adecuación curricular entre el 2000 y el 2012 tenga una adecuación significativa, dado que está en secundaria?

3. Estime la probabilidad de que un estudiante costarricense con alguna adecuación curricular, tenga adecuación significativa.

Se muestran a continuación varios problemas creados por los docentes, en trabajo colaborativo en grupos. Cada uno de ellos fueron seleccionados para ilustrar algún elemento a destacar. Por razones de espacio muchos problemas no se incluyeron en este documento.

El problema de la figura 1, presenta un error en la solución. Se les instó a que interpretaran el resultado, a lo que concluyeron que no podía ser cierto que de cada 100 estudiantes de secundaria, 11 estuvieran en una institución de educación especial. ¿Dónde podría estar el error? Después de varias discusiones, se llegó a la conclusión de que el denominador tomado estaba incorrecto, dado que se debió tomar el total de matriculados en secundaria entre el 2000 y el 2012 y no solamente los del sistema tradicional. Esto porque la pregunta solo dice "un estudiante de secundaria", y esto se interpreta como cualquier estudiante de secundaria tomado al azar. De este modo se concluyó que la solución correcta era:

$$
P(E E)=\frac{74094}{4883815} \approx 0,015
$$

Este resultado indica que aproximadamente 1.5 estudiante de cada 100, estará en una institución de educación especial, lo cual es más creíble. Este error ayudó a la comprensión del tema y se reflexionó sobre la importancia de la redacción al crear un problema en general y que en probabilidad es aún más delicado por lo que se debe poner mucha atención a este aspecto. Luego se les planteó la pregunta: ¿Cómo se debería redactarse la pregunta de modo que la respuesta sea la probabilidad antes planteada $P(E E)=\frac{74094}{702049}$ ? A lo cual respondieron acertadamente.

El problema de la figura 2 hace un encabezado formal al problema, lo cual es importante a la hora de crear problemas, e indicar la fuente de donde fueron tomados los datos.

Otro de los problemas creados, vea figura 3 , ilustra un interesante caso donde se le pide interpretar una razón de números. Este podría catalogarse de un nivel superior a los anteriores, donde se se desarrolla el proceso de analisis. 


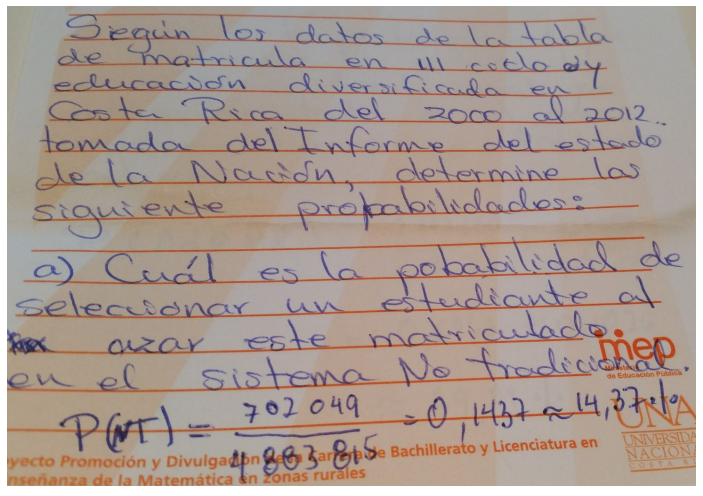

Figura 2: Problema que incluye un encabezado más completo

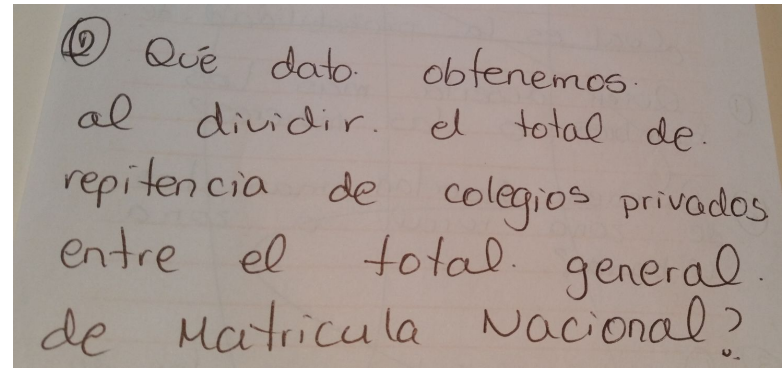

Figura 3: Problema para desarrollar la habilidad de interpretación

Otro de los temas tratados para crear problemas, fue el de deserción de los estudiantes del sistema educativo. Cabe mencionar que los docentes tuvieron problemas para interpretar los datos, dado que no eran números absolutos ni porcentuales, sino más bien estaban en forma decimal. Se hizo una reflexión de todas las formas de expresar las probabilidades.

\section{Tema 3: Deserción}

\begin{tabular}{|c|c|c|c|c|c|c|c|c|c|c|c|c|c|c|}
\hline & \multicolumn{12}{|c|}{ Deserción intra-anual en III ciclo y educación diversificada diurna } & \multirow[b]{2}{*}{2012} & \multirow[b]{2}{*}{ Total } \\
\hline & 2000 & 2001 & 2002 & 2003 & 2004 & 2005 & 2006 & 2007 & 2008 & 2009 & 2010 & 2011 & & \\
\hline Total & 10.2 & 11.3 & 10,8 & 9.4 & 10,3 & $\pi, 0$ & 11.5 & n.0 & 10.5 & 9,5 & 8.5 & 9.4 & 8.7 & 10.2 \\
\hline III ciclo & 119 & 12.9 & 12.5 & 0.7 & 11.7 & 12.7 & 13.2 & 12.7 & 12.1 & 11.2 & 10,0 & 11.1 & 10.2 & 11.8 \\
\hline$\pi$ & 18.6 & 19.5 & 19.1 & 6,6 & 18,3 & 19,2 & 20,1 & 19,5 & 18.6 & 16,1 & 14,4 & 16,0 & 14.7 & 17.8 \\
\hline gr & 79 & 9,1 & 7.8 & 6.8 & 8.5 & 9.4 & 9,3 & 9.1 & 8.3 & 88 & 8.2 & 8.7 & 7.9 & 8.5 \\
\hline$g r$ & 4.4 & 4.7 & 4.7 & 4.1 & 4.0 & 5.0 & 5,3 & 4.9 & 5.1 & 5,5 & 4.6 & 5,4 & 4.9 & 4.8 \\
\hline Educación diversificada & 5,7 & 7.3 & 6.6 & 6.2 & 6.6 & 6.9 & 8.0 & 7.1 & 7.2 & 58 & 5,3 & 5,5 & 5.1 & 6.4 \\
\hline tor & 80 & 10.4 & 9.7 & 8.7 & 9.4 & 10.2 & 12.0 & 100 & 10.5 & 89 & 7.6 & 8.2 & 7.4 & 9.4 \\
\hline$\pi r$ & 2.7 & 3.1 & 2.6 & 3.0 & 2,9 & 2.9 & 3.3 & 26 & 3.4 & 2.6 & 3,1 & 3.1 & 3.0 & 2.9 \\
\hline$z^{2}$ & 4.3 & 3.8 & 3.9 & 5.3 & 4.6 & 1.8 & 1.1 & 1.2 & 1.9 & 0.1 & 0.7 & -0.5 & 0.3 & 2.2 \\
\hline \multicolumn{15}{|l|}{ Por dependencia } \\
\hline Públicas & 11.6 & 12.8 & 12.2 & 0,5 & 11.5 & 12,4 & 13,0 & 12,3 & 11.9 & 10.6 & 9.5 & 10.5 & 9,6 & 11,4 \\
\hline Privads & 1.4 & 1.7 & 1.6 & 3,3 & 1.6 & 1.1 & 1.5 & 0.7 & 1.0 & 1.1 & 1.2 & 0.9 & 1,5 & 1.4 \\
\hline \begin{tabular}{|l|l|} 
Privada \\
subvencionadat
\end{tabular} & 20 & 2,1 & 1.4 & 1.2 & 1.7 & 1.7 & 1.8 & 24 & 2.8 & 1.7 & 2.9 & 2.5 & 2.2 & 2.0 \\
\hline \multicolumn{15}{|l|}{ Por zona } \\
\hline Lrbena & 9,5 & 10.5 & 10,2 & 8.4 & 9.6 & 10.2 & 10.4 & 9.9 & 9.6 & 8.6 & 7,3 & 8.5 & 7.9 & 9.3 \\
\hline Burser & 11,5 & 12.7 & 11.7 & 12,1 & 11,8 & 13,0 & 14,2 & 19,3 & 12,4 & 11.2 & 10.7 & 11,0 & 10,0 & 12,0 \\
\hline \multicolumn{15}{|l|}{ Por sexo } \\
\hline Hombres & $\pi 14$ & 12.7 & 12.2 & 0.9 & 11.8 & 12.8 & 13,4 & 12.7 & 12,2 & 10.7 & 9.6 & 10.5 & 9.9 & 11.6 \\
\hline Mujeres & 90 & 9.9 & 9,4 & 8.0 & 8.8 & 9.3 & 9,9 & 9.3 & 8.9 & 8.4 & 7,5 & 8.3 & 7,4 & 8.8 \\
\hline
\end{tabular}




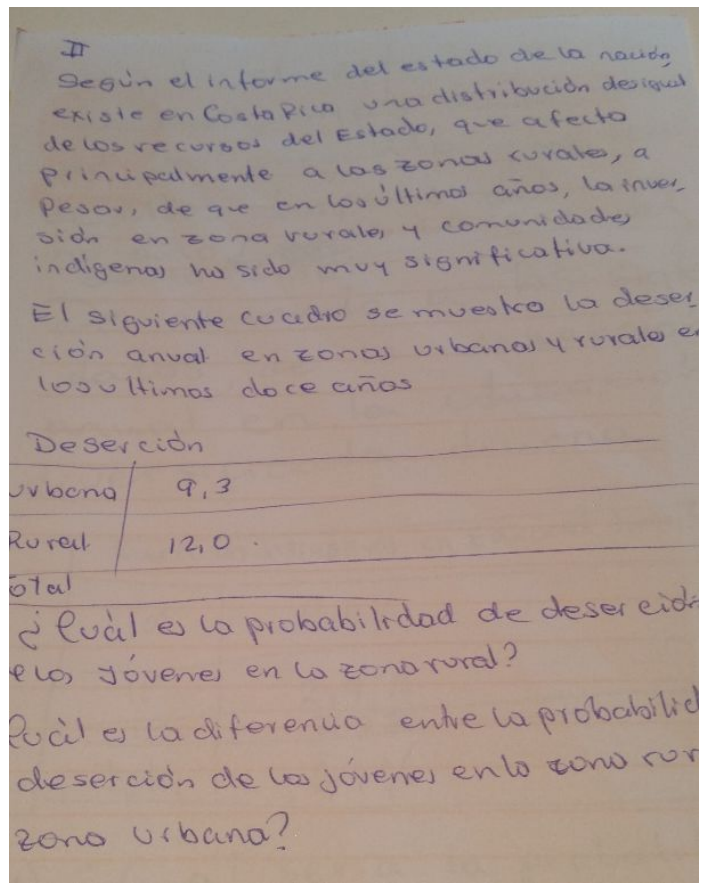

Figura 4: Un problema con una redacción motivadora

Aunque el problema creado por uno de los grupos en la figura 5, es errónea al aplicar mal la probabilidad condicional, si se percataron que la población total a tomar era 100, cosa que les costó a muchos.

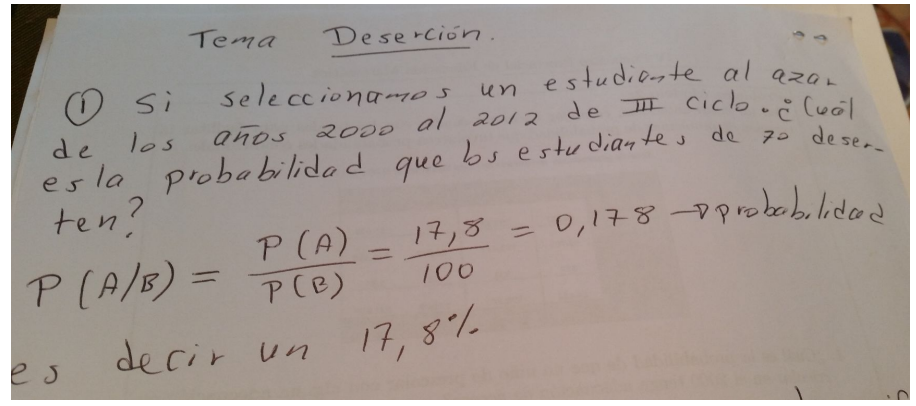

Figura 5: Otro problema con un error

A continuación se presenta un ejemplo de una de las lecturas asignadas, en el que se señala las dificultades de los jóvenes para graduarse de secundaria. Esto con el fin de que motivara a los profesores a la reflexión y a obtener provecho a los datos proporcionados, de modo que se obtuvieran respuestas a sus inquietudes. 
Lectura introductoria de motivación:

Discuta con su grupo de trabajo el siguiente artículo de La Nación.

LA nịclōn

NOTICIA ESTADO DE EDUCACIÓN: SOLO 46\% DE MUCHACHOS DE 17 A 21 AÑOS LOGRA TÍTULO

\section{Menos de la mitad de jóvenes ticos logra graduarse del cole}

AMY ROSS A. - 11 de septiembre de 2013 a las 12:00 a.m.

- Probabilidades de éxito descienden a $28 \%$ si papás no superaron escuela

- $52 \%$ de mujeres jóvenes obtienen diploma frente a un $40 \%$ de varones

Si los colegiales constituyeran ese "ejército de estudiantes" del cual se jacta Costa Rica, la tropa estaría perdiendo a más de la mitad de sus "soldados".

Aunque el pais ya ganó la batalla para universalizar la escuela primaria, en la contienda por el título de colegio sigue en desventaja: solo 46 de cada 100 estudiantes de 17 a 21 años gana el diploma.

Este es uno de los hallazgos del IV Informe Estado de la Educación (http://www.estadonacion.or.cr/estadoeducacion/educacion-informe-ultimo), elaborado por el Programa Estado de la Nación, con base en datos del 2011.

Además, el campo de batalla está más embarrialado e inclinado en perjuicio de algunos: cuando hay una desigualdad de por medio, las probabilidades de graduarse descienden a un $35 \%$.

Un joven cuyos padres no superaron la escuela, por ejemplo, tiene la balanza inclinada drásticamente en su contra: de 100 estudiantes en esta situación, solo 28 logran concluir la secundaria.

Si el mismo alumno hubiera nacido en un hogar con papás que asistieron a la universidad, sus probabilidades se triplicarian.

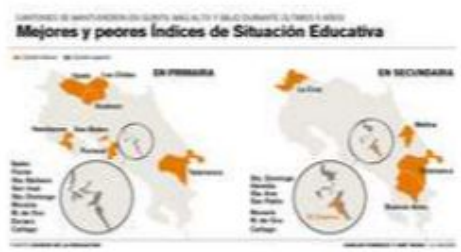

Mejores y peores índices de Situación Educativa (CARLOS FONSECA Y AMY ROSS / LANACIÓN)

número baja a un $36,8 \%$, en tanto que para un hijo único es del $54,5 \%$.

Estas brechas las evidencia el indice de oportunidades educativas, el cual permite ver qué circunstancias generan más desigualdad al completar la secundaria.

Mejor pero insuficiente. Aunque la conclusión de la secundaria ha crecido un $80 \%$ en los últimos 25 años (en 1987 era del $25,8 \%$ y en el 211 subió a $46,3 \%$ ), Costa Rica aún se sitúa entre los países latinoamericanos con niveles mediocres del indicador de logro.

El ministro de Educación Pública, Leonardo Garnier, puntualizó que los datos entre países no siempre son comparables. Recalcó que el aumento en asistencia escolar ha venido de la mano con una reducción en las brechas entre los más ricos y los más pobres, pero también entre zonas urbanas y rurales.

"La asistencia (a clases) de jóvenes de 13 a 17 años provenientes de hogares con climas educativos bajos, aumentó de $58,2 \%$ en el 2003 a $73,2 \%$ en el 2012 . La brecha se redujo de un $68 \%$ a un $29 \%$ ", enfatizó. 
IV Encuentro sobre Didáctica de la Estadística, la Probabilidad y el Análisis de Datos

\section{Tema 4: Repitencia}

Repitencia en III ciclo y educación diversificada diurna del 2000 al 2012

\begin{tabular}{|c|c|c|c|c|c|c|c|c|c|c|c|c|c|c|}
\hline & $\mathbf{2 0 0 0}$ & $\mathbf{2 0 0 1}$ & $\mathbf{2 0 0 2}$ & $\mathbf{2 0 0 3}$ & $\mathbf{2 0 0 4}$ & $\mathbf{2 0 0 5}$ & $\mathbf{2 0 0 6}$ & $\mathbf{2 0 0 7}$ & $\mathbf{2 0 0 8}$ & $\mathbf{2 0 0 9}$ & $\mathbf{2 0 1 0}$ & $\mathbf{2 0 1 1}$ & $\mathbf{2 0 1 2}$ & Total \\
\hline Total & 8,8 & 9,2 & 10,2 & 10,4 & 10,2 & 11,4 & 11,4 & 12,2 & 11,6 & 10,0 & 11,6 & 12,8 & 11,9 & 10,9 \\
\hline III ciclo & 10,3 & 10,9 & 11,4 & 11,7 & 11,7 & 12,9 & 12,9 & 13,9 & 13,2 & 11,2 & 13,3 & 14,4 & 13,5 & 12,4 \\
\hline $7^{\circ}$ & 14,5 & 14,2 & 14,5 & 15,2 & 15,4 & 15,4 & 15,7 & 16,8 & 16,2 & 13,4 & 14,3 & 15,3 & 14,5 & 15,0 \\
\hline $8^{\circ}$ & 8,7 & 12,1 & 11,5 & 11,1 & 10,6 & 12,3 & 13,1 & 14,0 & 14,0 & 11,8 & 14,5 & 15,3 & 14,6 & 12,6 \\
\hline $9^{\circ}$ & 4,5 & 2,7 & 4,7 & 5,4 & 6,2 & 9,1 & 7,4 & 8,5 & 6,8 & 6,6 & 9,8 & 11,3 & 10,2 & 7,2 \\
\hline $\begin{array}{c}\text { cuucuacurvI } \\
\text { diversificada }\end{array}$ & 4,9 & 4,9 & 7,4 & 7,3 & 6,5 & 7,6 & 8,1 & 8,2 & 7,9 & 7,2 & 8,0 & 9,4 & 8,3 & 7,4 \\
\hline $10^{\circ}$ & 8,2 & 7,8 & 12,1 & 12,1 & 10,4 & 11,7 & 12,2 & 13,2 & 12,2 & 11,4 & 11,6 & 13,3 & 11,8 & 11,4 \\
\hline $11^{\circ}$ & 0,9 & 1,2 & 1,7 & 2,0 & 1,8 & 2,8 & 2,8 & 2,4 & 2,9 & 2,5 & 4,2 & 5,4 & 4,5 & 2,7 \\
\hline $12^{\circ}$ & 0,8 & 0,7 & 0,5 & 0,9 & 1,0 & 1,9 & 2,4 & 1,6 & 1,4 & 1,6 & 2,8 & 3,6 & 4,6 & 1,8 \\
\hline Por dependencia & & & & & & & & & & & & & & \\
\hline Püblica & 9,8 & 10,2 & 11,4 & 11,6 & 11,3 & 12,6 & 12,6 & 13,4 & 12,8 & 11,0 & 12,9 & 14,3 & 13,3 & 12,1 \\
\hline Privada & 2,7 & 2,9 & 2,6 & 2,9 & 2,6 & 3,4 & 3,4 & 3,5 & 3,4 & 2,3 & 2,3 & 2,5 & 2,0 & 2,8 \\
\hline rinvurac & 3,4 & 2,7 & 3,8 & 3,0 & 2,7 & 2,9 & 3,1 & 3,7 & 3,6 & 3,5 & 3,3 & 3,7 & 2,8 & 3,2 \\
\hline Por zona & & & & & & & & & & & & & & \\
\hline Urbana & 9,1 & 9,6 & 10,5 & 10,8 & 10,5 & 12,1 & 12,0 & 12,5 & 11,7 & 10,6 & 11,7 & 12,9 & 12,6 & 11,3 \\
\hline Rural & 8,3 & 8,3 & 9,8 & 9,4 & 9,4 & 9,7 & 10,1 & 11,4 & 11,3 & 8,8 & 11,4 & 12,8 & 10,7 & 10,1 \\
\hline Por sexo & & & & & & & & & & & & & & \\
\hline Hombres & 10,3 & 10,8 & 11,8 & 12,1 & 12,0 & 13,1 & 13,2 & 14,0 & 13,4 & 11,2 & 13,2 & 14,5 & 13,7 & 12,6 \\
\hline Mujeres & 7,5 & 7,6 & 8,7 & 8,8 & 8,4 & 9,7 & 9,7 & 10,4 & 9,8 & 8,7 & 10,1 & 11,2 & 10,1 & 9,3 \\
\hline
\end{tabular}

\begin{tabular}{|c|c|c|c|c|}
\hline \multicolumn{5}{|c|}{ Repitencia en III ciclo y educación diversificada en } \\
\hline & $\begin{array}{c}\text { Costa Rica del } 2000 \text { al } 2012 \\
\text { Colegios } \\
\text { Públicos }\end{array}$ & $\begin{array}{c}\text { Colegios } \\
\text { Privados }\end{array}$ & $\begin{array}{c}\text { Privados } \\
\text { subvenciona } \\
\text { dos }\end{array}$ & Total \\
\hline Repiten & 442.364 & 9.263 & 5.267 & 456.894 \\
\hline Aprueban & 3.244 .006 & 321.553 & 159.313 & 3.724 .872 \\
\hline & 3686370 & 330816 & 164580 & 4.181 .766 \\
\hline
\end{tabular}

En la figura 6, se muestra una creación muy sencilla de un problema. Observe que, aunque se les proporcionó una tabla con la intención de que hicieran probabilidades condicionales, continuaron planteando problemas con la regla laplaciana.

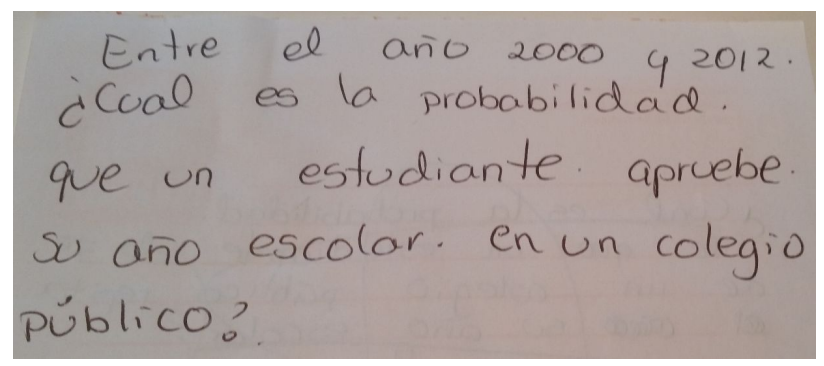

Figura 6: Problema sobre repitencia 
IV Encuentro sobre Didáctica de la Estadística, la Probabilidad y el Análisis de Datos

Tema 5: Examen de Bachillerato

Pruebas Nacionales de Bachillerato en Costa Rica 2000 a 2012

\begin{tabular}{|c|c|c|c|c|c|c|c|c|c|c|c|c|c|}
\hline & 2000 & 2001 & 2002 & 2003 & 2004 & 2005 & 2006 & 2007 & 2008 & 2009 & 2010 & 2011 & Total \\
\hline Total & 25.159 & 24.007 & 26.291 & 28.878 & 27.479 & 29.790 & 30.503 & 32.424 & 32.551 & 34.076 & 35.294 & 35.467 & 361.919 \\
\hline Aprobados & 15.482 & 15.954 & 17.527 & 18.584 & 17.062 & 18.794 & 19.123 & 21.788 & 21.025 & 23.303 & 23.995 & 23.739 & 236.376 \\
\hline Aplazados & 9.677 & 8.053 & 8.764 & 10.294 & 10.417 & 10.996 & 11.380 & 10.636 & 11.526 & 10.773 & 11.299 & 11.728 & 125.543 \\
\hline Promedio nota de examen & 76,9 & 78,0 & 78,2 & 78,0 & 75,8 & 76,3 & 72,6 & 72,6 & 70,7 & 70,8 & 69,9 & 69,3 & 74 \\
\hline Promedio nota de bachillerato & 78,3 & 79,4 & 79,9 & 79,8 & 78,7 & 78,8 & 79,6 & 79,3 & 78,7 & 79.9 & 80,3 & 81,0 & 79 \\
\hline Porcentaje de promoción & 61,5 & 66,5 & 66,7 & 64,4 & 62,1 & 63,1 & 62.7 & 67,2 & 64,6 & 68,4 & 68,0 & 66,9 & 65 \\
\hline Promoción por materia & & & & & & & & & & & & & \\
\hline Españof & 91,3 & 91,4 & 93,6 & 92,9 & 91,9 & 90,2 & 96,0 & 96,3 & 93.7 & 90,4 & 90,9 & 92,1 & 93 \\
\hline Esfudios Sociales & 92,0 & 96,2 & 97,0 & 96,6 & 92,0 & 95,4 & 94,4 & 92,4 & 89,4 & 91,4 & 92,9 & 88,0 & 93 \\
\hline Matemática & 66,5 & 72,5 & 75,1 & 72,2 & 72,5 & 78,9 & 72,2 & 80,6 & 79,4 & 80,1 & 77,0 & 71,0 & 75 \\
\hline Bjiologia & 87.9 & 88,7 & 88,0 & 89,3 & 85,2 & 82,5 & 87,2 & 86,2 & 86,0 & 90,3 & 87,6 & 86,4 & 87 \\
\hline Fisica & 81,3 & 86,5 & 87,0 & 86,5 & 86,7 & 86,2 & 86,4 & 77.5 & 76,6 & 84,9 & 87,2 & 89.7 & 85 \\
\hline Quimica & 86,2 & 92,1 & 94,2 & 92,5 & 88.7 & 80,1 & 86,1 & 82,3 & 86,2 & 83.7 & 83.7 & 86,7 & 87 \\
\hline Francés & 96,9 & 98,4 & 97,2 & 92,4 & 87,6 & 98,3 & 96,4 & 93,4 & 92,7 & 92,2 & 90,3 & 89,3 & 94 \\
\hline Inglés & 95,1 & 97,9 & 88.5 & 91,9 & 91,1 & 81,2 & 86,0 & 87,7 & 88,3 & 85.7 & 85,3 & 92,1 & 89 \\
\hline Educación Civica & & & 98,4 & 97,1 & 96,0 & 93.7 & 95,7 & 94,1 & 92,5 & 93,7 & 92,3 & 97,2 & 95 \\
\hline
\end{tabular}

Otro de los problemas creados se muestra en la figura 7, con respecto a los resultados del exámen de bachillerato en nuestro país. Note que no se percatan de la diferencia entre probabilidad laplaciana y probabilidad frecuencial.

(1) ¿Cuál es la probabilidad de que un estudiante que haya aplicado la prueba de bachillerato, en el 2011 , se encuentre aplazado dado que aprobó las asignaturas de Español y Matemática?

(2) ¿ cuál es la probabilidad de que un estudiante que haya aplicado prueba de bachillerato en el año 2008, eligió Química como ciencia?

(3) ¿Cuál es la probabilidad de que un estudiante que aplicó prueba de bachillerato haya aprobado, dado que escogió Francés como idioma?

¿Cuál es la probabilidad que un estudiante que aplicó pruebade bachillerato, obtuviera en el examen, en promedio, una nota inferior a 70 ?

Figura 7: Problema sobre resultados del exámen de bachillerato 


\section{Tema 6: Sistema universitario}

Por último, otro de los grupos trabajó en el tema de el acceso a las universidades estatales privadas. Se les proporcionó el siguiente cuadro de datos.

\section{Diplomas otorgados por la educación superior}

\begin{tabular}{|c|c|c|c|c|c|c|c|c|c|c|c|c|c|}
\hline & 2000 & 2001 & 2002 & 2003 & 2004 & 2005 & 2006 & 2007 & 2008 & 2009 & 2010 & 2011 & Total \\
\hline Total & 26.469 & 23.302 & 26.051 & 25.739 & 26.472 & 26.800 & 28.956 & 30.754 & 31.847 & 33.796 & 38.163 & 40.310 & 358.659 \\
\hline Universidad de Costa Rica & 3.955 & 4.131 & 4.118 & 4.378 & 4.112 & 4.234 & 4.258 & 4.421 & 4.330 & 4.684 & 5.054 & 5.206 & 52.881 \\
\hline Universidad Nacional & 2.586 & 2.389 & 2.830 & 2.849 & 3.152 & 3.264 & 2.868 & 2.495 & 2.476 & 2.720 & 3.127 & 2.784 & 33.540 \\
\hline Instituto Tecnológico & 876 & 834 & 1.084 & 1.104 & 996 & 1.287 & 1.369 & 1.243 & 1.326 & 1.181 & 1.448 & 1.277 & 14.025 \\
\hline Universidad Estatal a Distancia & 2.173 & 1.659 & 2.214 & 2.176 & 2.114 & 2.372 & 2.353 & 2.323 & 2.197 & 2.293 & 2.306 & 2.270 & 26.450 \\
\hline Universidades privadas & 16.879 & 14.289 & 15.805 & 15.232 & 16.098 & 15.643 & 18.108 & 20.272 & 21.518 & 22.918 & 26.228 & 28.115 & 231.105 \\
\hline \multicolumn{14}{|l|}{ Por grado académico } \\
\hline Universidades estatales & 9.590 & 9.013 & 10.246 & 10.507 & 10.374 & 11.157 & 10.848 & 10.482 & 10.329 & 10.878 & 11.935 & 12.195 & 127.554 \\
\hline Diplomado & 1.566 & 1.048 & 1.449 & 1.491 & 1.456 & 1.486 & 1.373 & 1.219 & 1.027 & 1.108 & 1.343 & 1.868 & 16.434 \\
\hline Frofesorado & 284 & 332 & 301 & 277 & 325 & 257 & 266 & 243 & 157 & 200 & 188 & 172 & 3.002 \\
\hline Bachillerato & 4.390 & 4.348 & 4.717 & 4.633 & 4.845 & 5.473 & 5.276 & 4.826 & 4.920 & 5.286 & 5.536 & 5.647 & 59.897 \\
\hline Licenciatura & 2.515 & 2.267 & 2.429 & 2.895 & 2.652 & 2.681 & 2.733 & 2.949 & 2.940 & 3.024 & 3.279 & 3.234 & 33.598 \\
\hline Especialidad profesional & 214 & 190 & 188 & 169 & 188 & 168 & 140 & 174 & 186 & 202 & 194 & 246 & 2.259 \\
\hline Maestria & 614 & 820 & 1.146 & 1.030 & 889 & 1.072 & 1.030 & 1.047 & 1.064 & 1.024 & 1.363 & 987 & 12.086 \\
\hline Dochorado & 7 & 8 & 16 & 12 & 19 & 20 & 30 & 24 & 35 & 34 & 32 & 41 & 278 \\
\hline Universidades privadas & 16.879 & 14.289 & 15.805 & 15.232 & 16.098 & 15.643 & 17.933 & 20.272 & 21.518 & 22.918 & 26.228 & 28.115 & 230.930 \\
\hline Bachillerato & 8.567 & 7.643 & 8.802 & 7.535 & 8.236 & 7.588 & 8.735 & 10.152 & 10.308 & 10.968 & 12.497 & 13.513 & 114.544 \\
\hline Licenciatura & 6.577 & 5.306 & 5.663 & 6.382 & 6.011 & 6.412 & 6.991 & 7.811 & 8.670 & 9.295 & 10.760 & 11.502 & 91.380 \\
\hline Mosestria & 11 & 8 & 4 & 4 & 4 & 12 & 142 & 207 & 290 & 267 & 315 & 2.755 & 4.019 \\
\hline Doctorado & 1.707 & 1.308 & 1.315 & 1.297 & 1.836 & 1.622 & 2.000 & 2.025 & 2.206 & 2.321 & 2.571 & 71 & 20.279 \\
\hline Especialidad profesional & 17 & 24 & 21 & 14 & 11 & 9 & 65 & 77 & 44 & 67 & 85 & 274 & 708 \\
\hline
\end{tabular}

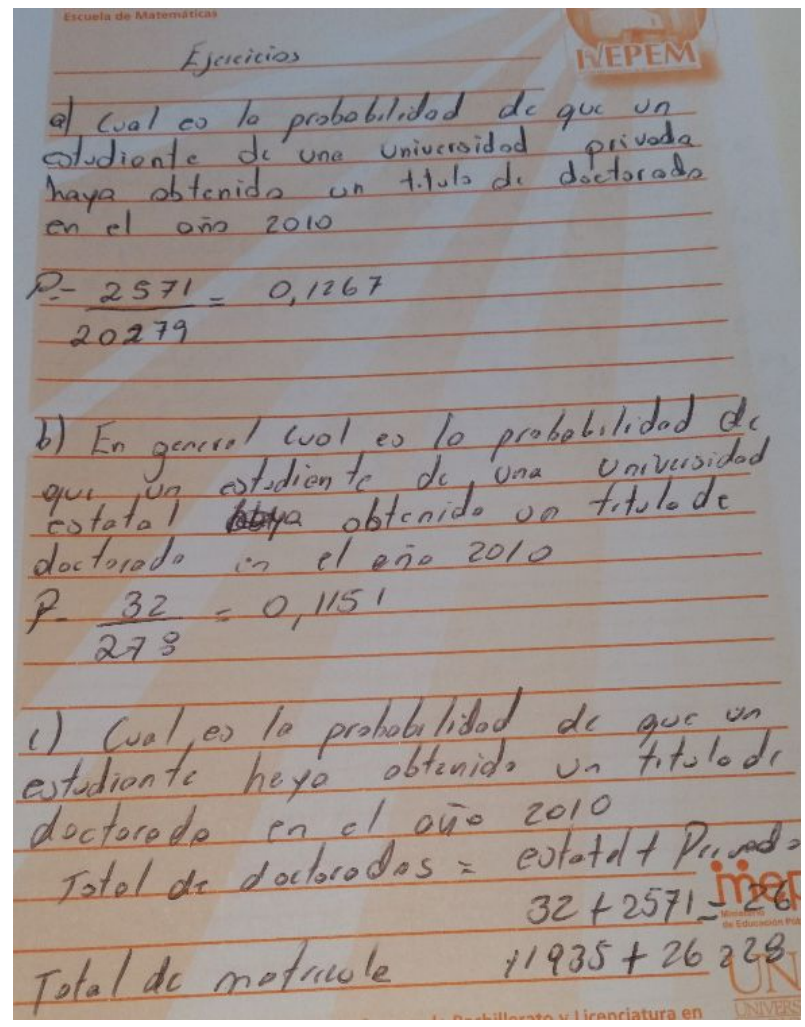

Figura 8: Comparación entre universidades públicas y privadas 


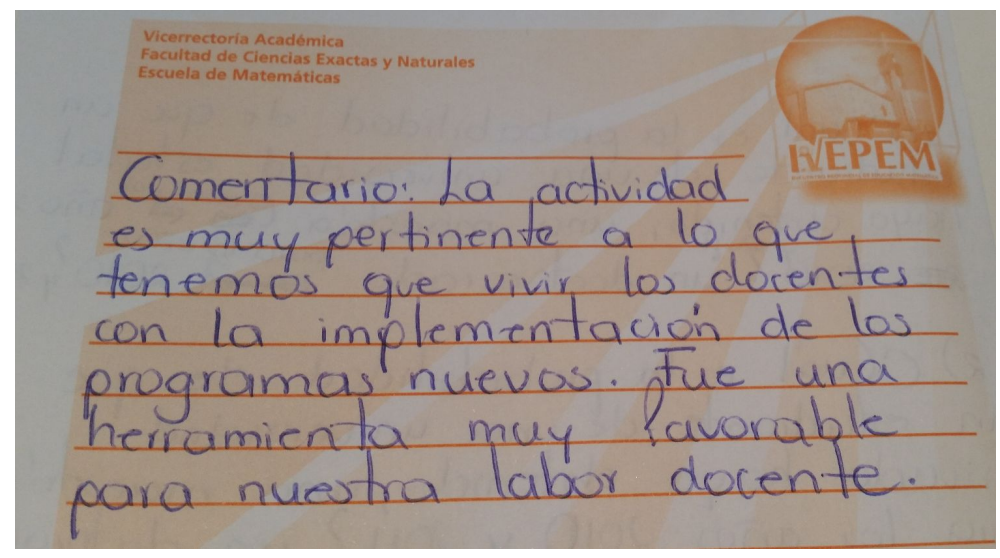

Figura 9: Comentario sobre la actividad

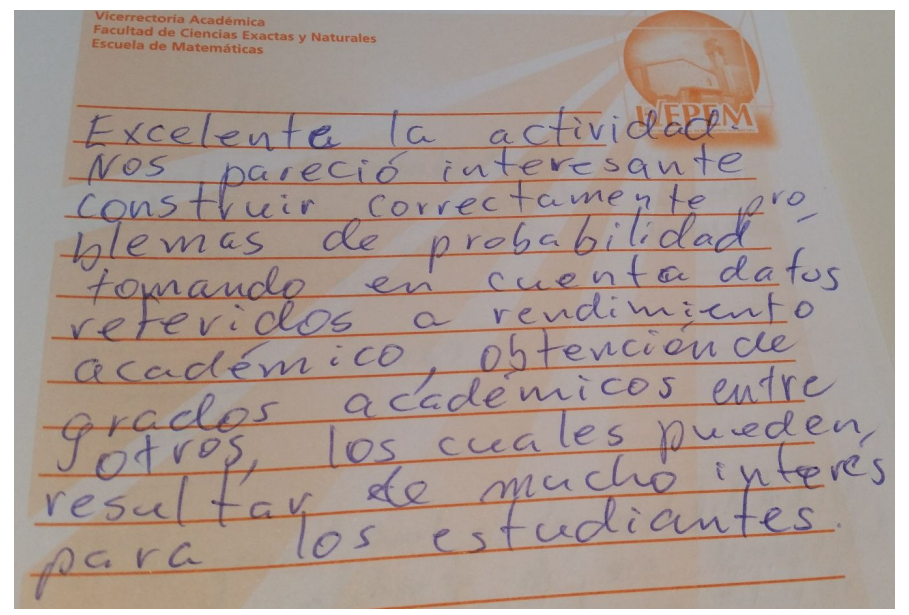

Figura 10: Comentario sobre la actividad

\section{Resultados y conclusiones}

La actividad resultó positiva y aunque en su gran mayoría, solo se basaron en la regla laplaciana clásica y a algunos problemas que involucraron probabilidad frecuencial, aunque no siempre lograron visualizar la diferencia, podría decirse que realizaron importantes creaciones. Cabe recalcar que en realidad para ser una primera experiencia, se logró al menos consolidar algunos conceptos matemáticos y que crearan problemas muy sencillos, pero propios. Al inicio, les tomó un tiempo considerable en comprender la matriz de datos, y había una a actitud de poco interés en realizar las actividades solicitadas. Sin embargo, ha medida que iban obteniendo resultados, se fue dando un cambio en su actitud, más confianza en sí mismos al notar la posibilidad de inventar sus propios problemas, y eso los entusiasmó, de modo que las creaciones se realizaron cada vez más rápido. Los temas escogidos para la creación de los porblemas, resultaron muy bien aceptados y por los comentarios obtenidos, se puede inferir que esto los motivó a buscar respuestas a preguntas, que de forma natural se fueron planteando. Es decir los temas lograron el propósito buscado, que fue el de lograr motivar a la invención de problemas. Algunos comentarios señalaban que les parecían mejor sus problemas 
creados, en comparación con los clásicos problemas de cartas, dador y juegos de azar, dado que estos podían relacionarlos con problemas del contexto educativo familiar a los estudiantes. Sin embargo, los problemas de dados y cartas son muy útiles, y no se deben dejar de lado, pues en ellos se tiene control del espacio muestral.

El tiempo de una hora resultó ser muy poco, por lo que se recomienda que en actividades similares, se dedique mucho más tiempo. Sin embargo, el taller si permitió que los docentes se abrieran, y se convencieran a sí mismos, de la posibilidad de crear material propio y no depender de terceros. Varios de ellos preguntaron donde se podían sacar datos, a lo que se les dio la dirección en la web de estadísticas y censos del país, además de que solicitaron el material del taller. Algunos de los comentarios sobre la actividad se presentan en las figuras 9 y 10 .

Es importante repetir estas tareas con los docentes, y enseñarles a "pescar", en lugar de darles simplente material hecho.

\section{Referencias bibliográficas}

[1] Espinoza, J, Lupiañez, J y Segovia, I (2014). La invención de problemas y sus ámbitos de investigación en educación matemática. Revista digital Matemática, Educación e Internet. Vol 14, Marzo 2014.

[2] Informe del Estado de la Nación. Compendio de Estadísticas. San José, Costa Rica. 2013.

[3] Malaspina, U. (2013). Nuevos horizontes matemáticos mediante variaciones de un problema. Unión, 35, 135-143.

[4] Mendenhall,W, Beaber, R y Beaber,B. Introducción a la probabilidad y estadística. Editorial Thomson Learning. 2002.

[5] Ministerio de Educación Pública. Documento de Apoyo curricular III Ciclo y Ciclo Diversificado. San José, Costa Rica. 2011.

[6] Polya, G. Cómo plantear y resolver problemas. México D.F. Editorial Trillas. 1965.

[7] Salazar, L (2014). Diseño de tareas a partir de la modificación de problemas planteados en libros de texto y su implementación con futuros profesores de matemática. Revista Paradigma vol XXXV (1) junio 2014 V-2.

[8] Salazar, L (2014). Creación de problemas: un método alternativo para introducir y rea?rmar el concepto de grupo. Revista Digital Matemática, Educación e Internet. Agosto 2014.

[9] Singer. F. M. y Voica, C. (2013). A problem-solving conceptual framework and its implications in designing problem-posing tasks. Educational Studies in Mathematics, 83(1), 9-26. 


\title{
Concepciones de Maestros de Escuelas Públicas en los Primeros Años de Escuela Primaria en Uberlândia en Relación con la Estadística
}

\author{
Ailton Paulo de Oliveira Júnior ${ }^{1}$, Márcia Lopes Vieira ${ }^{2}$
}

\begin{abstract}
Resumen
En este trabajo se presenta la concepción hacia la estadística de 26 profesores de matemáticas en los primeros años de la escuela primaria (de $1^{\circ}$ a $5^{\circ}$ años) de cuatro escuelas públicas en Uberlândia, Brasil. Se observó que el 30,77\% de los profesores conceptualizaron la estadística como un conjunto de datos que deben acopiarse, organizarse y analizarse, utilizando elementos matemáticos para su desarrollo; 38,46\% cree que el conocimiento estadístico se adquiere mediante el estudio y la vinculación a situaciones prácticas; $46.15 \%$ incorpora situaciones cotidianas en sus clases de estadística a través de situaciones problemáticas. Las respuestas ofrecidas por los profesores en los primeros años de la escuela primaria nos permiten indicar cómo se relacionan con la enseñanza de la Estadística.
\end{abstract}

Palabras clave: enseñanza de la estadística, concepción profesores, educación primaria

\begin{abstract}
This paper presents the conception towards Statistics of 26 mathematics teachers in the early years of elementary school (1 to 5 year) of four public schools in Uberlândia, Brazil. It was observed that $30.77 \%$ of teachers conceptualized Statistics as being a set of data that must be collected, organized and analyzed, which uses mathematical elements for their development; $38.46 \%$ believe that statistical knowledge is acquired by studying and linking to practical situations; $46.15 \%$ incorporate everyday situations in their classes statistical through problem situations. The answers offered by teachers in the early years of elementary school allow us to indicate how they relate to the teaching of Statistics.
\end{abstract}

Keywords: teaching statistics, conception’s teacher, primary education

\footnotetext{
${ }^{1}$ Universidade Federal do Triângulo Mineiro - Brasil - drapoj@uol.com.br.

${ }^{2}$ Instituto Federal do Triângulo Mineiro - Uberlândia -Brasil - $\underline{\text { marcialopes @iftm.edu.br. }}$
} 


\section{Introducción}

Según Prestes (2004) el curso de Pedagogía es responsable por gran parte de los profesores que enseñan en los primeros años de la Escuela Primaria (seis a 10 años de edad). Como el ejercicio magisterio en los primeros años se caracteriza por la unidocencia, el pedagogo necesita de un vasto marco teórico para que pueda comprender la complejidad del proceso educativo y de una amplia visión de las diferentes áreas del conocimiento.

De esta forma, en los años iniciales, además del valor inmediato para la vida del educando, se forman las bases para las series siguientes, principalmente de los conceptos y relaciones en Matemáticas. Aunque las Directrices Curriculares Nacionales - DCN para el Curso de Pedagogía (Brasil, 2006) establezcan el perfil de la formación inicial de los profesores polivalentes, en muchas ocasiones ese curso no los prepara adecuadamente para enseñar los conceptos matemáticos.

De acuerdo con los Parámetros Curriculares Nacionales (PCN) del Brasil (1998), para la asignatura de matemática en los grados iniciales de la Escuela Primaria, el conocimiento de los conceptos matemáticos debe acontecer en la formación de profesores de tal manera que muestran los alumnos que la Matemática es una ciencia dinámica y abierta a la incorporación de nuevos conocimientos. En este sentido, el profesor necesita identificar las características de esa ciencia, de tener métodos y precisión de sus propias concepciones sobre la Matemática, una vez que la práctica áulica y la metodología pedagógica están profundamente mezcladas. Segundo Tardif (2000) los saberes que sirven como bases para la enseñanza, son caracterizadas por el sincretismo, es decir, las diferentes concepciones que los profesores tienen con su práctica, además de su visión de realidad.

Curi e Pires (2004) destacan que éstos conciben la Matemática a partir de las experiencias que tuvieron como alumnos y profesores, del conocimiento que construyeron, de las opiniones de sus maestros, es decir, de las influencias socioculturales que atravesaron de sus vidas.

Además, fue a partir de los PCN que la Estadística pasó a ser el objetivo de muchos educadores y libros didácticos en los años iniciales de la Escuela Primaria (seis a diez años de edad), pues uno de sus principios rectores reconoce la importancia de las diferentes formas de representar datos y su relación significativa con la realidad del educando. Enseñar Estadística para niños se tornó una necesidad social con el objetivo de devolver al alumno la habilidad de colectar, organizar, interpretar y tomar decisiones efectivas.

Los PCN resaltan que la Estadística posibilita el desarrollo de formas específicas de pensamiento y raciocinio, dejando muy claro que no se pretende únicamente el desarrollo de un trabajo basado en la definición de términos o de fórmulas (Brasil, 1998). De acuerdo con los PCN (Brasil, 1998), la enseñanza de la estadística debe ser conducida para contribuir con el desarrollo de una visión crítica de los acontecimientos 
en los individuos, ayudándoles a hacer previsiones y tomar decisiones que impacten su vida personal y colectiva.

Según Lemos y Gitirana (2004) la mayoría de los profesores de la Primaria tienen dificultades en entender representaciones gráficas, y reconocen no estar preparados para trabajar esos tópicos con sus alumnos. Los estudios relacionados a los años iniciales de la Estadística representan una gran contribución para el área de la Educación Matemática, por tanto la necesidad de indagar y conocer más sobre los profesores que actúan en este nivel de enseñanza.

Para Ponte (1992), las concepciones tienen naturaleza esencialmente cognitiva, actúan como una especie de filtro, dando sentido a las cosas o actuando como bloqueador para nuevas situaciones, limitando la posibilidad de actuación y comprensión.

Lopes (1999) dice que en los elementos constitutivos de las concepciones de Estadística y Probabilidad se destaca la permisividad de ejercitar un modo de pensar que posibilita lidiar con situaciones no deterministas en que la incertidumbre se encuentra presente.

Colodel y Brandalise (2010) hicieron una investigación de naturaleza cualitativa y carácter interpretativo, buscando diagnosticar las percepciones de los profesores en los años iniciales de la Primaria $\left(1^{\circ}\right.$ al $\left.5^{\circ}\right)$ de una red municipal de enseñanza sobre las concepciones de enseñanza y aprendizaje sobre el conocimiento estadístico que desarrollan en sus prácticas pedagógicas, específicamente considerando el bloque "Tratamiento de la Información" propuesto en los PCN de Matemática. El análisis de documentos y el cuestionario fueron los procedimientos utilizados para la colecta de datos. Para el análisis de los 67 docentes participante se usó una metodología del discurso del sujeto colectivo - DSC, la cual reveló que los contenidos matemáticos referentes a este bloque están presentes en las prácticas pedagógicas de la mayoría de los profesores. Los autores del estudio también señalan la necesidad de asesoramiento cuanto a las metodologías de enseñanza a ser desarrolladas, afín de contribuir para el desarrollo de habilidades y competencias matemáticas en los alumnos.

Investigadores como Kataoka, Oliveira, Souza, Rodrigues y Oliveira (2011) y Guimarães, Gitirana, Marques y Cavalcanti (2009) afirman que una de las mayores dificultades para el desarrollo de la Educación Estadística en la primaria se refiere al hecho de que los profesores no tuvieron una formación sistematizada en relación a la Educación Estadística. En esa misma perspectiva, Lopes (2010) afirma que los contenidos de Estadística y Probabilidad aún no son prioridad en la escuela, tampoco en los programas de formación inicial del profesorado de matemática. En esta misma dirección, Borba, Monteiro, Guimarães, Coutinho y Kataoka (2011) resaltan que la implementación de la Educación Estadística en la educación básica es aún incipiente, comparada al Álgebra, a Geometría o Aritmética.

Para Moron y Brito (2001), la última concepción es una creencia, una vez que las concepciones son relativas al dominio cognitivo, mientras las creencias son 
influenciadas por la cultura y se refieren a la aceptación de una idea sin el debido soporte teórico.

Entrelazadas a las concepciones están las actitudes, las expectativas y el entendimiento que cada profesor tiene de su papel en una situación dada. La actitud es una condición psicológica necesaria para que el individuo realice una tarea, y posee un carácter cognitivo y afectivo con tendencia para la acción. Moron y Brito (2001) optan por la definición de actitud como una disposición personal, idiosincrática, presente en todos los individuos, dirigida a objetos, eventos o personas, que asume dirección e intensidad de acuerdo con las experiencias de individuo.

Si se considera que en la enseñanza de la Estadística es fundamental evaluar la práctica pedagógica del profesor, examinando su formación profesional y su praxis, en consecuencia surge las siguiente interrogante ¿Cuáles las concepciones de profesores que enseñan Matemáticas en los años iniciales de la escuela primaria con relación a la Estadística?

Debido a las características del tema y las características de la interrogante planteada anteriormente, el objetivo de este estudio fue investigar las concepciones de profesores de los años iniciales de la escuela primaria en relación a la enseñanza a la Estadística en cuatro escuelas de la ciudad de Uberlândia - Triángulo Minero.

\section{Procedimientos Metodológicos}

El estudio se efectuó con 26 profesores de Matemáticas adscritos a Escuelas Primarias Públicas ubicadas en Uberlândia, Minas Gerais, 13 provenientes de dos escuelas provinciales y 13 profesores de escuelas municipales. Las escuelas participantes fueron seleccionadas de acuerdo con su Índice de Desarrollo de Educación Básica - IDEB, una por debajo y otra por encima de la media nacional.

A través de un cuestionario con cuatro cuestiones abiertas se verificó la concepción de este grupo de profesores con relación a la enseñanza de Estadística, a saber, (1) ¿Cómo conceptualizan la Estadística los profesores de los primeros años de la escuela primaria? (2) ¿Cómo conciben los profesores de escuela primaria que los estudiantes de educación básica adquiere el conocimiento estadístico? (3) ¿Cómo enseñan los contenidos estadísticos en sus clases en los grados iniciales de la Educación Primaria? (4) ¿Cómo las situaciones cotidianas son incorporadas a las clases de estadística en los años iniciales de la Enseñanza Primaria?

Para Bardin (2009), el análisis de contenido se torna un conjunto de técnicas de análisis de comunicación que utiliza procedimientos sistemáticos y objetivos de descripción de contenidos de los mensajes y los organiza en tres fases: (1) Pre Análisis - fase en que el material es organizado a fin de sistematizar ideas iniciales; (2) Exploración del material 
- la definición de categorías e identificación de las unidades de contexto expresado en documentos; (3) Tratamiento de los resultados inferencia e interpretación - que resume las principales informaciones para análisis, culminando en interpretaciones inferenciales, este es el momento para la intuición, el análisis reflexivo y la crítica.

Así, las respuestas de los profesores fueron trascritas e identificadas de acuerdo con cada tema generado, con el propósito de comprender el mensaje contenido en el texto. Cada respuesta fue examinada individualmente y se asoció en función de la semejanza entre sus contenidos y categorías previamente definidas.

\section{III.Resultados}

Las respuestas obtenidas en esta encuesta fueron organizadas en varias categorías. Así, se observó que para el 30,77\% de los profesores que participaron de esta encuesta, la Estadística es un conjunto de datos que deben ser colectados, organizados, analizados y que utiliza elementos matemáticos para su conocimiento. A continuación se muestran segmentos de diálogos estabelecidos con los professores participantes en el estudio:

"Estadística es una rama de la matemática que tiene como objetivo obtener, organizar y analizar datos.” Profesor 19

"Estadística es para hacer la investigación, colecta de datos para determinada cuestión y utilizará la matemática para los resultados finales." Profesor 24

Sobresale el hecho que un grupo de profesores $(26,92 \%)$ consideraran la Estadística como importante instrumento para un mejor conocimiento diario:

"Ciencia, área de conocimiento que permite al individuo comprender mejor el mundo a su alrededor." Profesor 7

"Estadística es una asignatura fundamental para diversos asuntos, sea en el mundo profesional o hasta en el día a día.” Profesor 21

A partir de los testimonios de los profesores, se consideró que estos se aproximan a lo que fue puesto en el sitio web de la Escuela Nacional de Ciencias Estadísticas - ENCE ${ }^{3}$, donde se define Estadística como un conjunto de técnicas y métodos de investigación que, entre otros tópicos, involucra la planeación de un experimento a ser realizado, la colecta calificada de los datos, la inferencia, el procesamiento, el análisis y la diseminación de los resultados. En este trabajo se considera que esta definición tiene atributos importantes sobre la conceptualización de la Estadística, mostrando que más allá de ser un conjunto de técnicas y métodos, involucra aspectos como la colecta, tratamiento, presentación y análisis de datos que son importantes para auxiliar en la toma de decisiones en diversas áreas del conocimiento.

\footnotetext{
${ }^{3}$ http://www.ence.ibge.gov.br/index.php/sobre-estatistica/o-que-e-estatistica
} 
Un aspecto importante en este trabajo es detectar como consideran los participantes que una persona adquiere conocimientos en Estadística. Se detectó que el 38,46\% creen que se adquiere estudiando, asociando las situaciones prácticas:

"Estudiando, claro, comprendiendo los caminos de raciocinio de las mismas y principalmente el hecho de saber usarlas en su cotidiano, entendiendo como la Estadística es importante en otros aspectos de vivencia diaria."

\section{Professor 7}

"Adquiere conocimientos a través de estudios, concentración y clases prácticas." Profesor 21

Destacan también aquellos profesores $(23,08 \%)$ que suponen la adquisición de conocimiento estadístico a partir de situaciones cotidianas:

"Haciendo ejercicios e investigación, procurando traer esos conocimientos a la vida real, mostrando como la Estadística está presente en nuestras vidas." Profesor 24

"Yo creo que para adquirir conocimiento en Estadística es preciso que el alumno viva algo concreto, o sea, pruebe ese conocimiento para que él vea algo real." Profesor 25

Según Gauthier et al. (2006), el saber empírico lleva el profesor a creer y a elaborar casi un ritual de procedimientos para que el aprendizaje de los alumnos sea efectivo.

Otro aspecto destacado es la forma en que este grupo de profesores trabaja los contenidos estadísticos en sus clases. Por tanto, el análisis de su discurso se agrupó en tres categorías. Así, el 19,23\% de los profesores declaran que trabajan la Estadística a partir de representaciones gráficas relacionadas a la realidad de sus alumnos:

"Como doy clases para la Primaria $\left(1^{\circ}\right.$ al $5^{\circ}$ año) trabajo con gráficos provenientes de investigaciones relacionadas a la realidad del alumno."

\section{Profesor 15}

"Gráficos hechos con números reales que el alumno hace. Ej.: ¿Cuántos alumnos faltaron el mes anterior?" Profesor 20

De igual forma, 26,92\% de los profesores, dicen trabajar la Estadística a partir de situaciones que suceden a diario:

"Trabajo relacionado con la realidad de los alumnos, utilizando datos traídos por ellos.” Profesor 16

"Dando ejemplos del cotidiano de los niños, haciendo la relación entre los contenidos y sus vivencias con ejercicios y trabajos de investigación, usando teoría y prácticas de manera paralela." Profesor 24 
También se encontró que 34,62\% de los profesores trabajan con material concreto, pero no especificaron como lo hacen, pudiendo indicar que piensan solamente en el contenido matemático y no en el estadístico.

Según López (2008), es necesario el desarrollo de prácticas pedagógicas que involucren situaciones en que los estudiantes realicen actividades considerando sus contextos cotidianos, y que estos puedan observar y construir todos eventos posibles por medio de experimentación y organización de datos.

La Estadística debe ser aplicada a diario, ya que es una ciencia que al servicio de las otras. Considerando estos aspectos, algunos profesores de este grupo esta consciente de la importancia de esta disciplina en el tratamiento de los datos.

Con relación a la manera como profesores incorporan situaciones en sus clases de Estadística, 46,15\% de estos lo hacen en sus clases a través de situaciones problema:

"Reflexionando en las más diversas situaciones en que usamos la Estadística, conduciendo a los alumnos en el entendimiento de su utilidad como auxiliar en las más diversas situaciones, mostrando que lo que parece complicado, muchas veces es mucho más sencillo." Profesor 7

"Situaciones problemas usando hasta el nombre de los alumnos." Profesor 10

Onuchic y Allevato (2009) defienden que el problema es punto de partida para alcanzar el conocimiento y posicionan el profesor como guía y el alumno como cooperador en los procesos de enseñanza aprendizaje. En este aspecto, 30,77\% de los profesores destacan situaciones prácticas y cotidianas:

"A partir de gráficos realizados de acuerdo con el planeamiento curricular y por medio de cuestionarios llevados para casa, o de forma oral. Preguntas realizadas con los propios alumnos en clase.” Profesor 15

"Haciendo investigación, por ejemplo, películas preferidas de los alumnos; boletos, supermercado y otros.” Profesor 24

"Utilizando datos rutinarios, por ejemplo: ¿Cuántos somos? ¿Cuántos chicos y cuántas chicas? Son más los chicos o las chicas? Profesor 24

Gal (2002) apunta los estudios estadísticos como herramientas importantes para la formación de un ciudadano capacitado a resolver situaciones problema que están presentes en su cotidiano con mejor desempeño. 


\section{IV.Consideraciones /Recomendaciones}

Es preciso indagar, difundir y profundizar más en los conocimientos estadísticos durante los encuentros de formación de profesores y en los contextos de trabajo de la escuela, destacando los tratamientos teórico-metodológicos que pueden ser utilizados en los años iniciales, especialmente cuando se examinan los contenidos estadísticos para el tratamiento de la información. También deben incentivarse los estudios que puedan contribuir para el desarrollo de la Educación Estadística.

Es necesario hacer énfasis en la necesidad de una capacitación didáctica para el profesorado de Matemáticas, especialmente en lo relacionado con la enseñanza de la estadística. Como resaltan Batanero, Ottaviani y Truran (2000), el conocimiento didáctico que el profesor de Matemáticas debe tener para enseñar Estadística es:

- Reflexión epistemológica de los significados de los conceptos sobre estadística en el campo histórico, filosófico y los conceptos sobre estadística como campo histórico, filosófico y cultural.

- Experiencia en la adaptación de este conocimiento a diferentes niveles de enseñanza a través de metodologías variadas;

- Capacidad crítica para el análisis de libros y materiales didácticos sobre Estadística;

- Predicción de las dificultades de entendimiento - comprensión que los alumnos puedan presentar en la solución de problemas que implican el uso de métodos estadísticos;

- Experiencia con buenos ejemplos de situaciones de enseñanza de Estadística añadida a buenas herramientas didácticas y materiales que auxilien en la preparación de las clases.

Si los profesores realmente desarrollan esas competencias y modo de pensar la Estadística y su enseñanza tiene todo para adquirir una situación favorable a la educación en nuestro País.

\section{Bibliografia}

[1] Batanero, C., Ottaviani, G. \& Truran, J. (2000). Investigación en educación estadística: Algunas cuestones prioritárias. Statistical Education Research Newsletter, 1 (2).

[2] Borba, R. E. S.; Monteiro, C. E.; Guimarães, G. L.; Coutinho, C.; Kataoka, V. I. (2011). Educação Estatística no Ensino Básico: Currículo, pesquisa e prática em sala de aula. EM TEIA: Revista de Educação Matemática e Tecnológica Iberoamericana, 2, 1-18.

[3] Brasil. Índice de desenvolvimento da educação básica. Em http://portal.mec.gov.br. 
[4] Brasil. (1998). Ministério da Educação. Secretaria de Ensino Fundamental. Parâmetros curriculares nacionais: ensino fundamental: matemática. Brasília: MEC/SEF.

[5] Brasil. (2006). Diretrizes Curriculares para Graduação em Pedagogia. Brasília: MEC/CNE. Em http://www.mec.gov.br/sesu/ftp/curdiretriz/estatistica/esdire.rtf.

[6] Colodel, D. L.; Brandalise, A. T. (2010). Tratamento da Informação nos Anos Iniciais do Ensino Fundamental: entre concepções e práticas. In II Simpósio Nacional de Ensino de Ciência e Tecnologia, 07 a 09 de outubro.

[7] Curi, E.; Pires, C. M. C. (2004). A formação matemática de professores dos anos iniciais do ensino fundamental face às novas demandas nacionais. In VIII Encontro Nacional de Educação Matemática. Recife, Brasil. Em www.sbem.com.br/files/viii/pdf/13/MR20.pdf.

[8] Gal, I. (2002). Adult's statistical literacy: meanings, components, responsibilities appeares. Internacional Statistical Review, Espanha, 70 (1), 1-33

[9] Gauthier, C. et al. (2006). Por uma teoria da pedagogia: pesquisas contemporâneas sobre os saberes docentes. Ijuí, RS: Ed. Unijuí.

[10] Guimarães, G.; Gitirana, V.; Marques, M.; Cavalcanti, M. (2009); Educação estatística na educação infantil e nos anos iniciais. Zetetiké. Cempem-FE. Unicamp, 17 (32), jul/dez.

[11] Kataoka, V. Y.; Oliveira, A. C. S.; Souza, A. A.; Rodrigues, A; Oliveira, M. S. (2011). A educação estatística no ensino fundamental II em Lavras, Minas Gerais, Brasil: avaliação e intervenção. Revista Latinoamericana de Investigación em Matemática Educativa, 14 (2), 233-263.

[12] Lemos, M.P.F. e Gitirana, V. A. (2004). A formação de professores através de analises a priori de atividades em interpretação de gráficos de barras. In: Anais do VIII ENEM (Encontro Nacional de educação Básica).

[13] Lopes, C. A. E. (1999). A Probabilidade e a Estatística no currículo de Matemática do ensino fundamental brasileiro. In: Anais da Conferência Internacional: Experiências e Perspectivas do Ensino da Estatística - Desafios para o século XXI. (167-174) Florianópolis.

[14] Lopes, C. A. E. (2008). O ensino de estatística e da probabilidade na Educação Básica e a formação de professores. Caderno Cedes, 28 (74), 57-73, jan/abr.

[15] Moron, C. F.; Brito, M. R. F. (2001). Atitudes e concepções dos professores da educação infantil em relação à Matemática, In BRITO (org). Psicologia da Educação Matemática. Teoria e Pesquisa. Florianópolis: Editora Insular, 263-277.

[16] Onuchic, L. R.; Allevato, N. S. G. (2009). Ensinando Matemática na sala de aula através da Resolução de Problemas. Boletim GEPEM, Rio de Janeiro, 55 (19).

[17] Ponte, J. P. (1992). Concepções de professores de Matemática e processos de formação. In Ponte, J. P. Educação Matemática: Temas de investigação. Lisboa: Instituto de Inovação Educacional, 185-239.

[18] Prestes, L. M. A formação matemática docente para os anos iniciais do ensino fundamental - desafios e perspectivas. Em http://revistas.fw.uri.br/index.php/revistadech/article/view/246.

[19] Tardif, M. (2002). Saberes docentes e formação profissional. Petrópolis: Vozes. 


\title{
Juego Pedagógico y Resolución de Problemas en el Aprendizaje de Estadística en la Escuela Primaria
}

\author{
Ailton Paulo de Oliveira Júnior ${ }^{1}$, José António Fernandes ${ }^{2}$, Joana dos Santos Silva ${ }^{3}$, \\ Roberta Cristina de Faria Moreira ${ }^{4}$, Valéria Ciabotti ${ }^{5}$
}

\begin{abstract}
Resumen
Este trabajo tiene como objetivo presentar la importancia del juego pedagógico en el proceso de enseñanza y aprendizaje de conceptos básicos de Estadística y Probabilidad utilizando la metodología de la Resolución de Problemas. Para ello fue desarrollado el juego "Jugando con la Estadística y la Probabilidad" para ser aplicado a alumnos del $9^{\circ}$ año de la Primaria con la intención de facilitar la comprensión de tales contenidos. Tras la aplicación del juego la mayoría de los alumnos declaró haber gustado de la actividad y que habían aprendido con la misma. Con la aplicación del juego, se puede percibir que el juego confeccionado sirvió de apoyo metodológico para la clase de Estadística y Probabilidad, dejándola más estimulante y atractiva para los alumnos.
\end{abstract}

Palabras clave: juegos educativos, la enseñanza de la estadística y la probabilidad, educación primaria.

\begin{abstract}
This paper aims to present the importance of teaching the game in the teaching and learning of basic concepts of Statistics and Probability using the methodology of Problem Solving. Therefore, we developed the game "Playing with Statistics and Probability" to 9th grade students of elementary school to be applied with the intention of facilitating comprehension of such content. After application of the game most of the students said they enjoyed the activity and what they learned with the same. With the game application, it can be noticed that the game made worked as a methodological support to class Statistics and Probability, making it more exciting and attractive to students.
\end{abstract}

Keywords: educational games, teaching statistics and probability, elementary education

\footnotetext{
${ }^{1}$ Universidade Federal do Triângulo Mineiro - Brasil. drapoj@uol.com.br.

${ }^{2}$ Universidade do Minho - Braga - Portugal - jfernandes@ie.uminho.pt.

${ }^{3}$ Universidade Federal do Triângulo Mineiro - Brasil. Bolsista do Programa Institucional de Bolsas de Iniciação à Docência.jo.uftm@ hotmail.com.

${ }^{4}$ Universidade Federal do Triângulo Mineiro - Brasil. Bolsista do Programa Institucional de Bolsas de Iniciação à Docência. betinha20cris@ hotmail.com.

${ }^{5}$ Escola Municipal Urbana Frei Eugênio - Uberaba - Brasil - valeria_ciabotti@ hotmail.com.
} 


\section{Introducción}

La estadística es una ciencia destinada a la colecta, análisis e interpretación de datos, que envuelve un conjunto de métodos para la obtención de informaciones, organizaciones y presentaciones de las mismas. Tiene como objetivo la comprensión de una realidad específica, auxiliando en un mejor entendimiento de las situaciones de nuestro cotidiano.

La formación adecuada, no sólo de los técnicos que producen estadísticas, sino de los profesionales y ciudadanos que deben interpretarlas y tomar a su vez decisiones basadas en esta información, así como de los que deben colaborar en la obtención de los datos requeridos es, por tanto, un motor del desarrollo.

El interés por la enseñanza de la estadística dentro de la Educación Matemática, viene ligado al rápido desarrollo de la estadística como ciencia y como útil en la investigación, la técnica y la vida profesional, impulsado notablemente por la difusión de los ordenadores y el crecimiento espectacular de la potencia y rapidez de cálculo de los mismos, así como por las posibilidades de comunicación.

Los nuevos currículos de educación primaria y secundaria incluyen en forma generalizada recomendaciones sobre la enseñanza de la estadística. Sin embargo, en la práctica son todavía pocos los profesores que enseñan este tema y en otros casos se trata muy brevemente, o en forma excesivamente formalizada.

Estamos rodeados por la Estadística, esta aparece en periódicos, en revistas, en las radios, en la televisión y hasta mismo en Internet. Según Francisco de Paulo Buscácio ${ }^{6}$, la Estadística puede ser aplicada en diversas áreas, como por ejemplo, "en la demografía, en la industria, en el área de los recursos humanos, en la salud, en las pesquisas de mercado y de opinión, etc".

En relación a la Probabilidad, se considera que esta puede promover la comprensión de gran parte de los acontecimientos del cotidiano que son de naturaleza aleatoria, posibilitando la identificación de resultados posibles de esos acontecimientos.

Se puede también verificar la Probabilidad a diario, como, por ejemplo, en la Biología, cuando se procura obtener previsiones de carácter genético; en la política es muy utilizado en las previsiones electorales, y hasta mismo en un simple juego de cara o corona. Entonces se percibe que la Probabilidad también desempeña un papel fundamental en nuestras vidas.

Frecuentemente se presencia la falta de preparación de profesores en relación a contenidos estadísticos, siendo que profesores de Matemáticas, aún los recién formados tienen pocos conocimientos sobre estadística en su preparación profesional y que segundo Bratton (2000) acaba dificultando su enseñanza.

6 Ex-Presidente do Conselho Federal de Estatística em comentário no site. Disponível em: <http://www.ibge.gov.br/ibgeteen/datas /estatístico/ palavra estatistico.html>. Acesso em: 24 ago. 2011. 
Los Parámetros Curriculares Nacionales - PCN (1998) recomiendan usar la Estadística con la finalidad de que el estudiante construya procedimientos para colectar, organizar, comunicar e interpretar datos, utilizando tablas, gráficos y representaciones, y que sea capaz de escribir e interpretar su realidad, usando conocimientos matemáticos, como por ejemplo, estudios sobre Salud, Medio Ambiente, Trabajo y Consumo etc.

En relación a la Probabilidad, los PCN consideran que auxilia en la comprensión de los acontecimientos que son de naturaleza aleatoria, permitiendo la identificación de resultados posibles. Destaca la incertidumbre que se manifiesta intuitivamente, por tanto la escuela debe proponer situaciones en que los niños puedan realizar experimentos y hacer observaciones de los eventos.

Lopes (2008) apunta que el estudio de la Estadística y de la Probabilidad en la Educación Básica es indispensable para el ciudadano en la actualidad y en su futuro, imponiendo a la Matemáticas el compromiso de no sólo enseñar el dominio de los números como la organización de datos, lectura de gráficos y análisis estadísticos. Para este mismo autor, la enseñanza y el aprendizaje de Estadística y de Probabilidad deben ser basados en investigaciones y en resoluciones de problemas, de modo a permitir que el conocimiento matemático y estadístico posibilite al estudiante adquirir habilidades para comprender y manejar adecuadamente a su realidad.

Los PCN (1998) sugieren el recurso a los juegos como uno de los caminos para "hacer Matemáticas" en la clase, en contextos donde se presentan problemas específicos, o sirviendo como instrumento para la construcción de estrategias de resolución de problemas. En ellos se observa que:

Los juegos constituyen una forma interesante de proponer problemas, pues permiten que estos sean presentados de modo atractivo y favorecen la creatividad en la elaboración de estrategias de resolución y busca soluciones. Propicia la simulación de situaciones problemas que exigen soluciones vivas e inmediatas, lo que estimula el planeamiento de acciones, posibilitando la construcción de una actitud positiva frente a los errores, una vez que las situaciones suceden rápidamente y pueden ser corregidas de forma natural, en el decorrer de la acción, sin dejar huellas negativas.

Para Polya (1978), resolver problemas es la realización específica de la inteligencia, y si la educación no contribuye para el desarrollo de la inteligencia, obviamente está incompleta.

La resolución de problemas es destacada como uno de los patrones de proceso para la enseñanza de Matemáticas (Onuchic e Allevato, 2005). En el caso de D’Ambrósio y Ohio (2008), fue a partir de los años 90 que la resolución de problemas se tornó una parte integrante de la clase de Matemáticas y surgieron propuestas curriculares que situaban la enseñanza de la Matemática vía resolución de problemas. 
Por otra parte, se observa en los PCN un vínculo entre Estadística, Resolución de Problemas y la realidad de los alumnos, como defiende Dewey (1933) y D‘Ambrósio y Ohio (2008, p. 1) al proponer que los proyectos curriculares sean basados en las experiencias de los alumnos, y que todo lo que se exponga al alumno sin una conexión con su experiencia se tornaría "inútil, como basura, criando barreras y obstaculizando la posibilidad de pensar sobre los problemas afrentados”.

Así, el tipo de problema a ser escogido es un punto crítico. Según Dante (2005) las características de un buen problema son: (1) que constituya un reto para los alumnos; (2) sea real; (3) sea interesante; (4) sea el elemento desconocido de un problema original; (5) no consistir en la aplicación evidente y directa de una o más operaciones aritméticas; (6) tener un nivel adecuado de dificultad.

Las actividades con juegos representan un importante recurso metodológico en clase, pues es una forma interesante de proponer problemas debido a que es atractivo para el alumno y por favorecer la creatividad en la elaboración de estrategias.

Normalmente pensamos en los juegos como una distracción placentera, simplemente algo que hacemos por diversión. Sin embargo, una evidencia cada vez mayor sugiere que los juegos dan más que entretenimiento, especialmente cuando se trata de aprendizaje en un salón de clases.

Puesto que la estadística es un tema que no resulta fácil para la mayoría de las personas y suele no ser vista como algo divertido, el uso de juegos adecuadamente diseñados para enseñarla es una herramienta muy útil para despertar interés y ayudar a explicar conceptos difíciles.

Almeida (1998), afirma que los juegos constituyeron siempre una forma de actividades humanas. Entre los primitivos, la actividad de la danza, caza, pesca, luchas, eran tenidas como sobrevivencia, ultrapasando muchas veces el carácter restricto de divertimiento y placer natural.

Souza (2002, p. 132), expresa la importancia de trabajar con el juego en clase diciendo que:

La propuesta de trabajar con juegos en el proceso enseñanza aprendizaje de la Matemática es una opción didáctico metodológica por parte del profesor, vinculada a sus concepciones de educación, de Matemática, de mundo, pues es a partir de tales concepciones que se definen normas, maneras y objetivos a ser trabajados, coherentes con metodología de enseñanza adoptada por el profesor.

El juego puede ser considerado como medio por el cual el educando expresa sus cualidades espontáneas y que permite al educador comprender mejor sus alumnos. En las palabras de Santos (2001, p. 90): 
Juego es una palabra, una manera de expresar el mundo y, por lo tanto, de interpretarlo. Precisamos pues reconocer que estamos tratando de una concepción compleja en la medida en que, en vuelta de un nudo de significaciones, giran valores muy diferentes: la noción abierta a interpretaciones y a nuevas posibilidades de análisis. Se puede descubrir un paradigma dominante en vuelta de la oposición al trabajo, mas también potencialidades diversas conforme se favorezca esa o aquella dirección de su desarrollo.

Así, los juegos pueden estimular y ser usados para estimular y desarrollar la habilidad de pensar de forma independiente, contribuyendo para su proceso de construcción de conocimiento lógico matemático (Kamii e Joseph, 1992).

Los juegos pueden ser utilizados para introducir, fijar o concluir un contenido, es decir, preparar el alumno para profundizar los ítems trabajados antes. Así, uno de los motivos para la introducción de juegos en las clases de matemáticas es la posibilidad de disminuir obstáculos por temor a la matemática (Souza, 2006).

Además del juego ser un agente facilitador para la asimilación de los contenidos matemáticos, posibilita una interacción social entre los alumnos, estimula un pensamiento crítico reflexivo, ayuda en el desarrollo del raciocinio lógico y de la capacidad de resolver situaciones problemas.

Por tanto, es interesante desarrollarlo conforme lo propuesto por Kamii (1991) y Krulik (1993) apud Smole, Diniz y Candido (2007), quienes señalan que el juego debe ser implementado para dos o más jugadores, teniendo un objetivo a ser alcanzado; las decisiones deben ser discutidas con todo el grupo. El juego no debe ser mecánico y sin significados para los jugadores, en cambio debe permitir la posibilidad de usar estrategias, establecer planos, ejecutar jugadas e evaluar la eficacia de estos elementos en los resultados obtenidos.

Con este recurso didáctico el profesor consigue verificar las dificultades que sus alumnos presentan. Incluso, hasta los alumnos más tímidos que muchas veces no participan de las clases por miedo de equivocarse, se vuelven más activos tornándose más autónomos, permitiendo al profesor esclarecer dudas que aquéllos no se arriesgarían en exponerlas en el transcurso de la clase expositiva. Según Borin (1996),

Otro motivo para la introducción de juegos en las clases de matemáticas es la posibilidad de disminuir obstáculos presentados por muchos de nuestros estudiantes que temen a las Matemáticas y se sienten incapaces de aprenderla. Dentro de la situación de juego, donde es imposible una actitud pasiva y la motivación es grande, percibimos que, al mismo tiempo en que estos alumnos hablan Matemáticas, presentan también un mejor desarrollo y actitudes más positivas a sus procesos de aprendizaje (Borin, 1996, p.9). 
Según Grando (2000) la resolución de problemas son las actividades de juegos relacionadas cuando, al observar el comportamiento de un niño en situaciones de bromas y/o juego, se percibe lo cuanto ella desarrolla su capacidad de hacer preguntas, buscar diferentes soluciones, repensar situaciones, evaluar actitudes, encontrar y reestructurar nuevas relaciones, es decir, resolver problemas.

Lopes (2008) dice que la utilización de la Resolución de Problemas permite al alumno la construcción de noción y conceptos matemáticos como herramientas para resolver problemas. Considera que no hace sentido trabajar con actividades estadísticas desvinculadas a una problemática. Es necesario despertar en el alumno la criticidad, la idea del aleatorio, de las probabilidades y las análisis. Esas actividades no son conceptos estadísticos, tampoco matemáticos, era un listado de ejercicios a seguir y resolver por la aplicación de conceptos desarrollados significativamente por los alumnos en el proceso de resolución de problemas.

De acuerdo con los Parámetros Curriculares Nacionales:

La resolución de problemas no es una actividad para ser desarrollada en paralelo o como aplicación del aprendizaje, más una orientación para el aprendizaje, pues proporciona el contexto en que se pueden aprender conceptos, procedimientos y actitudes matemáticas. (Brasil, 2000, p. 43-44).

El juego como una actividad de resolución de problemas desencadena la construcción de nuevos conceptos o ideas de forma motivadora, placentera y desafiante para el alumno. No obstante, es preciso saber todo el juego antes de presentarlo al alumno, debe ser minuciosamente estudiado, pues a él le tocó el deber de contribuir para la enseñanza aprendizaje de forma a dar resultados positivos.

Además de ello, debe acordarse que el ambiente educativo debe ser entendido como un sitio de fascinación donde se desarrollan habilidades en los alumnos, principalmente, el raciocinio, la creatividad y la autonomía.

Grando (2004) destaca que la inserción de los juegos en las clases puede darse en todos los niveles de la enseñanza, cuidando que los objetivos sean claros, la metodología a ser utilizada adecuada al nivel de enseñanza y que la actividad sea retadora para el alumno.

De esta forma, es trabajado conceptos de Estadística y Probabilidad referentes al $9^{\circ}$ año de la Primaria, por medio de la utilización de un juego, con la intención de facilitar el proceso de enseñanza aprendizaje tanto para el profesor como para el alumno, posibilitando además de la asimilación del contenido, una mayor interacción entre alumno/alumno y alumno/profesor.

Se considera este trabajo relevante una vez que presenta la necesidad, no sólo de una buena formación profesional del profesor en relación a los contenidos en cuestiones, pero también la concientización de la importancia de tales contenidos para los años finales de la Enseñanza Primaria, así como la elaboración de recursos didácticos, como 
juegos, por decir, que visan contribuir en el proceso enseñanza aprendizaje de Estadística y Probabilidad.

De esta forma, esta pesquisa tiene como objetivo presentar la contribución de los juegos pedagógicos en el proceso enseñanza aprendizaje de la Estadística y de la Probabilidad, utilizando la metodología de la Resolución de Problemas.

\section{Metodologia}

Este trabajo fue desarrollado con un grupo del $9^{\circ}$ año de la Escuela Primaria Provincial Corina de Oliveira, Uberaba, Minas Gerais, Brasil. Escuela participante Programa Institucional de Bolsa de Iniciación a la Docencia - PIBID.

El programa tiene como objetivo anticipar el vínculo entre los futuros profesores y las clases y con esa iniciativa, hace una articulación entre la Educación Superior (por medio de los cursos de Formación de Profesores), la escuela de Educación Básica y los sistemas provinciales y municipales de Educación.

Se concede becas a alumnos de cursos de Formación de Profesores en las diversas áreas del conocimiento que participan en proyectos de iniciación a la docencia desarrollada por Instituciones de Educación Superior (IES) en asociación con escuelas de Educación Básica de la rede pública de enseñanza. Los proyectos deben promover la inserción de los estudiantes en el contexto de las escuelas públicas desde el inicio de su formación académica para que desenvuelvan actividades didácticas pedagógicas con la orientación de docentes que imparte clases en curso de formación de profesores de instituciones de Enseñanza Superior y de un profesor de Educación Básica.

De esta forma fue desarrollado un juego sobre Estadística y Probabilidad, considerando propuestas de los Parámetros Curriculares Nacionales - PCN, de forma a posibilitar a los alumnos la lectura, interpretación y organización de datos; construcción de tablas y gráficos; concepción y comprensión de: espacio muestral, media, moda y mediana; cálculo de la probabilidad de un evento por medio de una razón; frecuencia absoluta y relativa.

El juego pedagógico presentado en la Figura 1 fue confeccionado con la intención de utilizarlo para la fijación de los contenidos de Estadística y Probabilidad en el $9^{\circ}$ Año de la Enseñanza Secundaria, a través de Resolución de Problemas. 


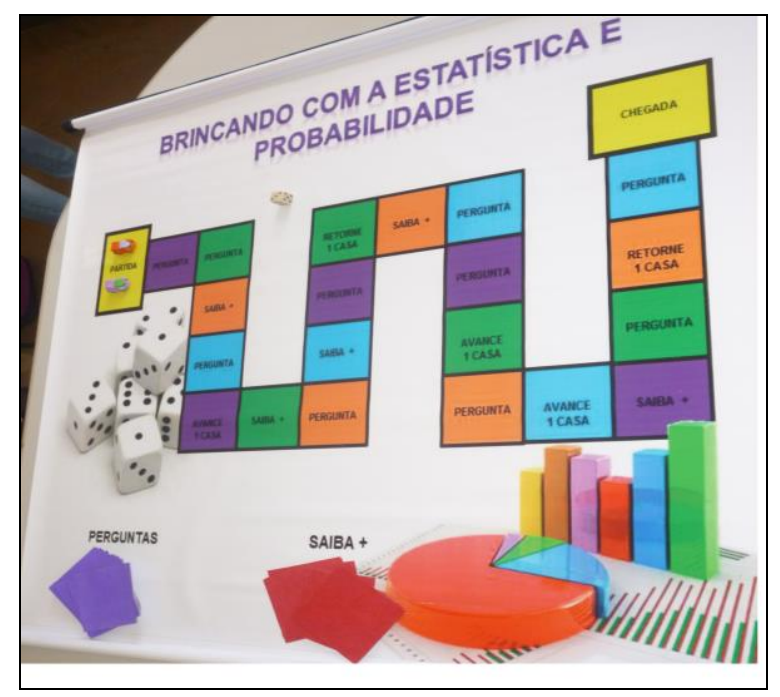

Figura 1 - Tablero del Juego "Jugando con Estadística y Probabilidad”.

En la aplicación inicial del juego para evaluación del mismo, se utilizó un diario de campo en que fueron registradas las reacciones de los alumnos, dudas y comentarios durante el proceso del juego.

Durante la realización del juego, fue entregada una hoja de registro a los alumnos, para que estos hiciesen los apuntes de los cálculos realizados durante la actividad, la cual se recogió para evaluación.

Se sugiere usar este juego en $9^{\circ}$ de la Enseñanza Primaria ya que auxilia en la fijación de contenidos estadísticos y probabilísticos. Presenta también situaciones problemas para que el alumno construya su pensamiento estadístico y probabilístico. Además, el juego auxilia el profesor a identificar posibles dificultades con relación a contenidos específicos. El juego está compuesto por casas de Preguntas y de Sepa Más, hay también casas de Adelanta y Retrocede.

Deben estructurarse grupos de entre dos a ocho integrantes. Los recursos necesarios para efectuar la actividad son: un tablero conforme modelo de la Figura 1, piezas coloridas (1 de cada color) para la representación de cada uno de los grupos, y un dado común.

El juego tiene las siguientes reglas:

1. En el inicio del juego, los grupos deben poner sus piezas en la casilla "Partida" y en seguida, se lanza el dado para indicar cual grupo iniciará el juego, o sea, quien saca el número mayor del dado comienza la partida. El grupo que obtuvo el mayor número en el lanzamiento del dado, lo lanza nuevamente y posiciona su pieza en la casilla correspondiente al valor del dado y así sucesivamente los otros grupos.

2. Si la pieza que representa el grupo caer en la casa de las preguntas, uno de los jugadores de este tendrá que sacar una pregunta del mazo de "Preguntas", leer para 
todos los otros miembros del grupo, y posteriormente todos responderán a la pregunta en una hoja de papel. Al contestar acertadamente la pregunta, deberá moverse en el tablero la cantidad de casillas indicadas en la ficha de la pregunta que se sacó, en caso que no la hayan contestado bien, no se moverá ni retrocederá ninguna casilla, más pero el grupo que no esté participando del partido, tendrá derecho de responderla, pudiendo mover el total de casas correspondientes a la cuestión salvo la acerte. Si los dos grupos erraren en la respuesta, el profesor podrá interferir en el juego, indicando la respuesta correcta y comentando los erros cometidos por los grupos.

3. Si la pieza representante del grupo caer en la casa "Sepa +", deberá leer la curiosidad en alta voz para todos los jugadores del grupo y después deberá mover el tablero la cantidad de casillas correspondientes en la ficha.

4. Si la pieza representante del grupo se posiciona en la casilla "Adelante Casillas", deberá avanzar la cantidad correspondiente. Si la pieza cae en la casilla "Retroceda Casillas", deberá retornar la cantidad correspondiente.

5. Gana el partido el grupo que completar una vuelta completa en el tablero.

\section{Exponiendo el Juego Pedagógico}

La aplicación del juego fue realizada para alumnos del $9^{\circ}$ de la Escuela Provincial Corina de Oliveira, en Uberaba, Minas Gerais, Brasil, y se tuvieron clases para la práctica. Inicialmente se informó a los alumnos sobre la actividad, Figura 2, y en seguida les explicaron las reglas del juego.

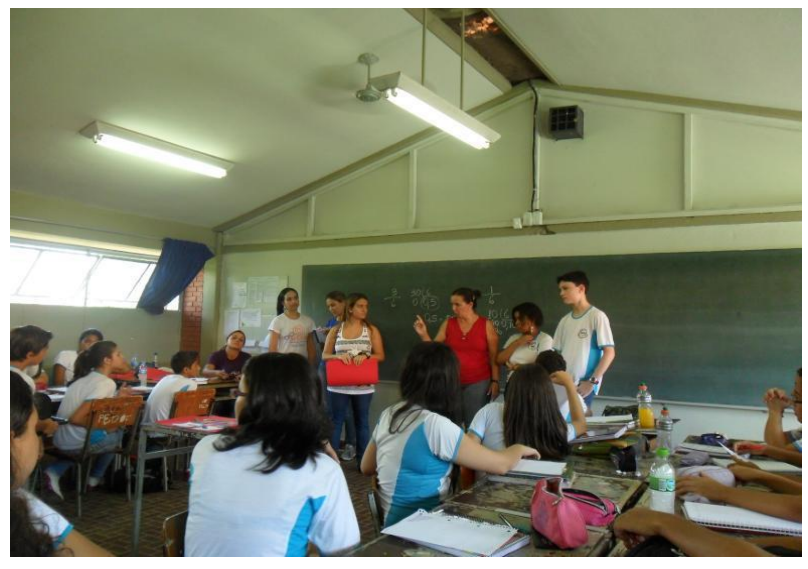

Figura 2 - Presentación de la actividad en la escuela.

Como se trataba de un test para verificación de las cuestiones elaboradas y las reglas del juego fueron presentados dos tableros para 32 alumnos. Para que pudiese ser empezado el juego, se pidieron a los alumnos que se dividiesen en dos grupos, cada uno con 16 integrantes. 
Debido al número de integrantes en cada grupo se solicitó que eligieran un representante para hacer las acciones del juego en el tablero, ejemplo, mover la pieza en el tablero y leer las fichas para el grupo.

Así, un tablero fue dado a cada grupo y en seguida se indicó que tendrían 5 minutos para responderla. Inmediatamente después se inició el juego. Se estuvo al pendiente de dar asistencia a los alumnos en el transcurso de la partida.

Al final del juego se recogieron las hojas que los alumnos utilizaron para dar respuesta a las preguntas y se les pidió que escribiesen sus opiniones sobre la actividad realizada. La mayoría de los alumnos dijo que les había gustado la actividad y que aprendieron de una forma divertida. Abajo se encuentra algunos fragmentos de estas opiniones:

"La clase de hoy fue bien diferente y divertida. Aprendemos bastante y reforzamos aquello que ya sabíamos. Fue estupendo, pues salimos de la rutina y nos entretuvimos." - Alumno 1.

"Me gustó mucho el juego, me ayudó mucho, creo. Reforzó bastante lo que el profesor había enseñado. Creo que si otra asignatura tuviera un juego así, mucho nos ayudaría." - Alumno 2.

Durante la aplicación del juego al Grupo 1, Figura 3, se observó que los alumnos mostraron entusiasmo. Expusieron que las clases de Matemáticas quedarían mejores si hubiese un juego como éste y que era bueno para entender los contenidos.

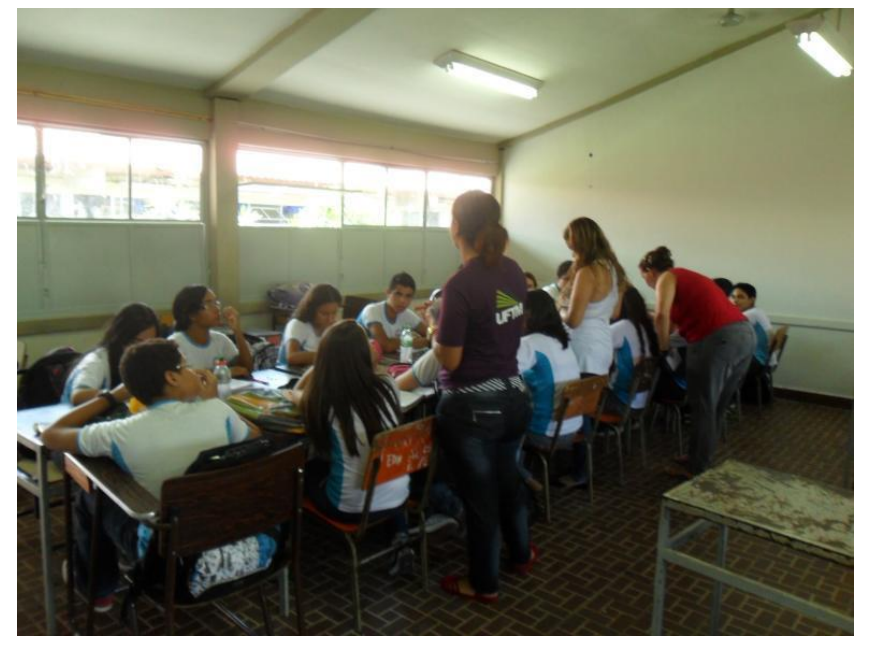

Figura 3 - Grupo 1 durante la aplicación del juego.

Se describe también una de las etapas del juego en que los alumnos tuvieron dificultades. Específicamente fue una cuestión en que se presentaba muchos valores como, por ejemplo: "Los siguientes datos representan diferentes precios (en RealesMoneda Nacional Brasilera-R \$) de un determinado producto pesquisado en 20 tiendas: $50,50,51,51,51,51,51,52,52,52,52,52,52,53,53,53,53,53,53,54$. 
Considerando los datos referidos anteriormente, ¿cuál es el percentual de ocurrencia de los diferentes precios? Comenta los resultados".

Cuestiones similares a esta también provocaron dificultades durante el juego, debido a la dificultad de memorización de tantos valores por los alumnos, una vez que había apenas una ficha para los 16 integrantes. En este caso, fue necesario que se redactaran los números en la pizarra para que todos los jugadores tuviesen acceso a los valores de la pregunta, aunque esto generó monotonía.

A pesar de que la mayoría se interesó en el juego, se observó que algunos aprovecharon la situación para salir del aula. Se cree que los alumnos presentaron deficiencias o evitaron la pregunta por falta de atención en la realización de operaciones básicas de matemáticas como: adición; porcentaje; regla de tres; y división.

Gran parte de los alumnos mostró dominio con relación a los contenidos estadísticos y probabilísticos y solo en determinadas situaciones consultaron el cuaderno de apuntes y el libro didáctico. Algunas veces se intervino en el proceso auxiliando a los alumnos para el desarrollo de operaciones matemáticas.

Respecto de la aplicación del juego en el Grupo 2, Figura 4, se observó que la mayoría de los alumnos participó del juego, aunque por la cantidad de ellos, los alumnos que estaban alejados del tablero no participaron activamente de la actividad y tomaron las respuestas ya calculadas y discutidas por algunos.

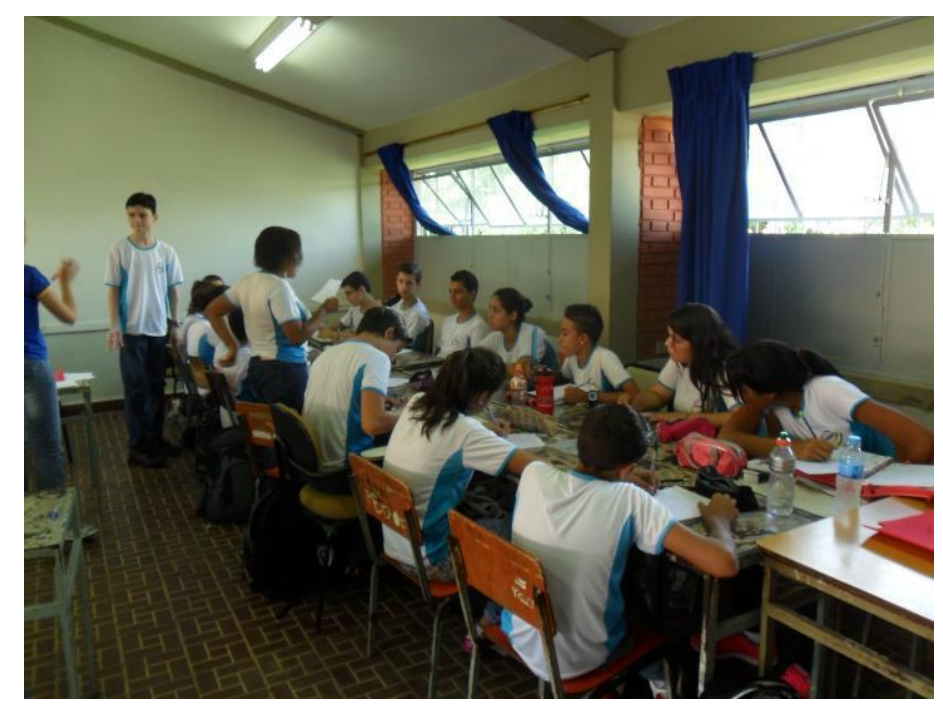

Figura 4 - Grupo 2 durante la aplicación del juego.

Además de esta observación, se notó que la mayoría de los alumnos acertaron las preguntas, independientemente de quien la había planteado. Los alumnos mostraron el contenido, principalmente aquellos relacionados a la Probabilidad. 
A continuación se señalan algunas preguntas sorteadas durante el juego: ¿Cuál es la probabilidad de salir el número siete en el lanzamiento de un dado? A partir de esa pregunta los alumnos se miraron medio confusos, y entonces, uno de los alumnos comentó:

"Ello es trampa, no es posible salir un siete en el dado".

A partir del planteamiento de esta pregunta y la respuesta emitida por el estudiante, se explicó que cuando se trata de un acontecimiento imposible, la probabilidad de ocurrencia es igual a 0 (cero).

Es importante explicar que sucede si la pieza representativa del grupo cae en la casa "Sepa +" donde el representante debía que leer la información para todos los otros jugadores y después avanzar el número de casillas correspondientes al que indicaba la ficha. Se percibió que durante la lectura de la tarjeta "Sepa +" muchos alumnos se dispersaron, charlando.

También destacó una pregunta que contemplaba la lectura de un gráfico e indicar el valor de la "Moda", valor más frecuente de los datos suministrados, obsérvese la Figura 5.

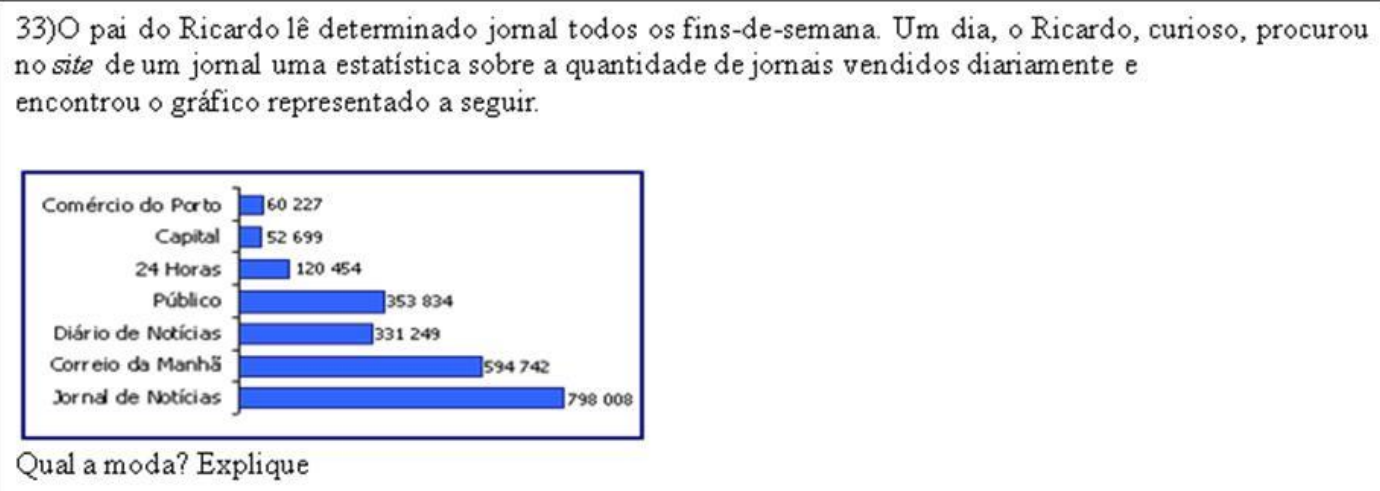

Figura 5 - Cuestión 33 del test de evaluación.

Se observó que los alumnos no conseguían resolverlo, pues intentaban efectuar operaciones, siendo que esta pregunta implicaba únicamente la lectura del gráfico para la obtención de la respuesta.

A partir de algunos comentarios surgidos a partir de las dudas de los alumnos. Cuando se percibió que los alumnos intentaban hacer operaciones y que estaban alejándose del objetivo de la cuestión, se cuestionó a los alumnos de la siguiente forma:

“¿Ustedes tienen certeza que en esta actividad precisa hacer cuenta?”

"Den una mirada en el anunciado de la actividad." 
En ese momento los alumnos leyeron nuevamente la pregunta, y entonces se les preguntó:

“QQué es moda?”.

Ellos respondieron:

"Es lo que más acontece".

Entonces se preguntó:

"Si la moda es el evento que ocurre con más frecuencia y es lo que más acontece, cuál es la moda en esta pregunta?"

Entonces comenzaron darse cuenta que no se requería hacer operaciones matemáticas, pero sí analizar los gráficos con atención. Se indicó:

“Entonces la moda es el Periódico de Noticias”.

Se cuestionó entonces a los alumnos por qué el Periódico de Noticias ser la moda, y ellos contestaron:

"Porque es lo que más se vende".

Después de confirmar que la respuesta estaba correcta se formalizó el concepto de moda.

La aplicación del juego permitió darse cuenta que a la mayoría de los alumnos les gustó la actividad, debido a que fue divertida y les permitió que salieran de la rutina. La mayoría de los alumnos participó activamente del juego, aunque algunos ni intentaron responder las preguntas ya que tomaron sus respuestas listas de otros estudiantes. Lo anterior pudo haber ocurrido debido la falta de interés del alumno en jugar, o tal vez debido al hecho de tener apenas un tablero para 16 de ellos, provocando el desaliento de los que se ubicaban a mayor distancia del tablero.

Se considera que el juego debe ser jugado por un máximo 8 integrantes (cuatro contra cuatro) para posibilitar que todos participen de la solución de las cuestiones y que estas no deben tener muchos valores pues también desalienta el juego se torna monótono.

A pesar del desinterés de algunos alumnos, la actividad fue gratificante debido a que la mayoría de los alumnos demostró interés intentando resolver las cuestiones, cuestionándonos sobre el contenido y sobre la forma de resolver las cuestiones.

Y en sus relatorios la mayoría de los alumnos dijo haberle gustado la actividad y que habían aprendido bastante con la misma. Uno de los relatos se muestra a continuación:

"El juego elaborado por las chicas del Pibid fue muy creativo, en él pudimos aprender más de una manera más divertida”. - Alumno del $9^{\circ}$ Año. 


\section{Consideraciones Finales}

Gran parte de los alumnos mostró dominio con relación a los contenidos estadísticos y probabilísticos, únicamente en determinadas situaciones, se percibió que consultaban el cuaderno de apuntes de clases y el libro didáctico. Algunas veces intervino en el proceso auxiliando los alumnos a que desarrollen operaciones matemáticas.

Se verificó con la aplicación del juego que la mayoría de los alumnos les gustó la actividad, pues esta ocurrió de forma divertida y les permitió que salieran de la rutina. La mayoría de los alumnos participaron activamente. También se percibió que el juego confeccionado sirvió como apoyo metodológico para la clase de Estadística y Probabilidad. El juego posibilitó la participación en la construcción del conocimiento, dejando de ser pasivo y tornándose agente de su aprendizaje. En la situación del juego el alumno se tornó más confiado, más abierto.

El juego favorece el desarrollo de la creatividad, su habilidad crítica, su capacidad de participación en el diálogo, en la convivencia sana, observación, uso del lenguaje, y sobre todo, favoreció el interés en aprender (Grando, 2004).

Se recomienda tomar en cuenta que durante la aplicación del juego es fundamental que el profesor realice buenas intervenciones pedagógicas para que los alumnos puedan percibir y participar de la construcción de los conceptos matemáticos. También es necesario que el profesor planee con cuidado la actividad, eligiendo juegos que proporcionen desafíos a los alumnos, teniendo presente los objetivos y el contenido a ser tratado, es decir, evitar generar una concepción de "jugar por jugar". Al hacer lo anterior, se podrá auxiliar a los alumnos en el proceso de enseñanza aprendizaje en Matemáticas, principalmente con relación a la Estadística y la Probabilidad.

\section{Bibliografia}

[1] Almeida, P. N. (1998). Educação Lúdica; prazer de estudar - Técnicas e jogos pedagógicos. Rio de Janeiro: Loyola.

[2] Borin, J. (1996). Jogos e resolução de problemas: uma estratégia para as aulas de matemática. São Paulo: IME-USP.

[3] Brasil. (1998). Secretaria de Educação Fundamental. Parâmetros curriculares nacionais: Matemática/Secretaria de Educação Fundamental. MEC / SEF, Brasília.

[4] Brasil. (2000). Ministério da Educação. Secretaria de Educação Fundamental.

Parâmetros Curriculares Nacionais. V.3. Rio de Janeiro: DP\&A.

[5] Bratton, G. (2004). The Role of Technology in Introductory Statistics Classes. Statistical Education Research Newsletter, 1(1).

[6] D’Ambrósio, B. S.; Ohio, M. U. (2008). A Evolução da Resolução de Problemas no Currículo Matemático. In Seminário de Resolução de Problemas, 1., 2008, Rio Claro. Anais eletrônicos... Rio Claro: GTERP.

[7] Dante, L. R. (2005). Didática da resolução de problemas. São Paulo: Ática.

[8] Grando, R. C. (2000). O conhecimento matemático e o uso de jogos na sala de aula. Campinas, SP. Tese de Doutorado. Faculdade de Educação, UNICAMP, 2000. 
[9] Grando, R. C. (2004). O jogo e a matemática no contexto da sala de aula. São Paulo: Paulus.

[10] Lopes, C. A. E. (2008). O ensino da estatística e da probabilidade na educação básica e a formação dos professores. Caderno Cedes, Campinas, 28 (74), 57-73.

[11] Kamii, C. (1991). Piaget para a educação pré-escolar. Trad. Maria Alice Bad Denise. Porto Alegre: Artes Médicas.

[12] Kamii, C.; Joseph, L.L. (1992). Aritmética: Novas Perspectivas - implicações da teoria de Piaget. Tradução de Marcelo Cestari T. Lellis, Marta Rabioglio e Jorge José de Oliveira. Campinas: Papirus.

[13] Onuchic, L. R.; Allevato, N. S. G. (2005). Novas reflexões sobre o ensinoaprendizagem de matemática através da resolução de problemas. In Bicudo, M. A. V.; Borba, M. C. (Orgs). Educação Matemática - pesquisa em movimento. São Paulo: Cortez.

[14] Polya, G. (1978). A arte de resolver problemas. Rio de Janeiro: Interciência. [15] SANTOS, S. M. P. (2001). A ludicidade como ciência. Petrópolis, RJ. Vozes

[16] Smole, K.S.; Diniz, M.; Cândido, P. (2007). Jogos de Matemática de $1^{\circ}$ a $6^{\circ}$ ano. Porto Alegre: Artmed.

[17] Souza, M. F. G. (2002). Fundamentos da Educação Básica para Crianças. Volume 3, In Módulo 2. Curso PIE - Pedagogia para Professores em Exercício no Início de Escolarização. Brasília, UnB. 


\title{
La Historia de la Estadística en el caso de la Enseñanza y el Aprendizaje de la Estadística en la Escuela Secundaria
}

\author{
Ailton Paulo de Oliveira Júnior ${ }^{1}$, Beatriz Cristina da Silva Delalíbera ${ }^{2}$, \\ Oraide Rodrigues Martinss ${ }^{3}$, Vanderleia Conceição Ribeiro ${ }^{4}$
}

\begin{abstract}
Resumen
En este trabajo se presenta la secuencia de instrucción que trabaja usando la Historia de la Estadística como un recurso para su enseñanza. Su desarrollo se basa en una revisión de la literatura sobre la Historia de Estadística, que se inicia con los informes obtenidos en el uso de las estadísticas de los tiempos antiguos, en los que los gobiernos utilizan para recopilar datos sobre la población y la recaudación de impuestos, entre otras cosas, llegando a la contemporaneidad, donde se observa problemas de análisis de la sociedad, entre otros. De esta manera se pretendía mostrar que el conocimiento se desarrolla a partir de las necesidades, la asistencia en la comprensión de la importancia de los contenidos de aprendizaje como experiencia y desempeño en la sociedad.
\end{abstract}

Palabras clave: historia de las estadísticas, la enseñanza de la estadística, secuencia instrucciones.

\begin{abstract}
In this paper, we report instructional sequence that works using the History of Statistics as a resource for your education. Its development is based on a literature review on the History of Statistics, which begins with reports obtained on the use of statistics from ancient times, where governments used to collect data on population and tax collection among other things, coming to contemporaneity, where it notes that remain about the same, ie, tax collection, analysis problems of society, among others. In this way it was intended to show that knowledge is developed from needs, assisting in understanding the importance of learning content such experience and performance in society.
\end{abstract}

Keywords: history of statistics, teaching statistics, instructional sequence

\footnotetext{
${ }^{1}$ Universidade Federal do Triângulo Mineiro - Brasil. drapoj@uol.com.br.

${ }^{2}$ Universidade Federal do Triângulo Mineiro - Brasil. Bolsista do Programa Institucional de Bolsas de Iniciação à Docência. beatriz_delalibera@yahoo.com.br.

${ }^{3}$ Universidade Federal do Triângulo Mineiro - Brasil. Bolsista do Programa Institucional de Bolsas de Iniciação à Docência. martins5286@ hotmail.com.

${ }^{4}$ Universidade Federal do Triângulo Mineiro - Brasil. Bolsista do Programa Institucional de Bolsas de Iniciação à Docência. vanderleia_cr@hotmail.com.
} 


\section{Introducción}

En la escuela es frecuente oír alumnos cuestionarse la utilidad del estudio de ciertos contenidos. Preguntan a cada rato el motivo el porqué de tales asignaturas, en aquel momento y de aquella manera, preguntan si estos contenidos realmente son importantes, mientras podrían estar aprendiendo a hacer otras cosas más relevantes.

Se cree que a partir del instante en que el alumno pueda conocer mejor el origen, su utilidad y necesidad, este aprendizaje se tornará más interesante y agradable. Entender cómo hacer, por qué hacer, dónde hacer, y cuándo, puede ser mucho más atractivo, tanto para los alumnos como para profesores, que simplemente aprender a hacer.

Se pretende entonces, que un abordaje histórico pueda llevarlos a comprender la necesidad y el surgimiento de tales contenidos.

Nobre (1996) sugiere partir del desarrollo histórico de los conceptos matemáticos envés de enseñar la practicidad de los contenidos escolares e invertir en la fundamentación de ellos. Buscar el real motivo de por qué se enseña las cosas.

Se considera que la Educación Matemática necesita de nuevos instrumentos metodológicos, que busquen sanar los problemas y las dificultades encontradas en el actual ambiente escolar. En ese sentido, Baroni y Nobre (1999) destacan que el movimiento de la Educación Matemática incorpora, de tiempos en tiempos, componentes que visan fornecer instrumentos que pueden ser utilizados por el profesor de Matemáticas. Entre estos la Resolución de Problemas, la Configuración Matemática, la Etnomatemática y la Informática. Mas esta pesquisa incluye entre ellos la Historia de las Matemáticas, que en los últimos tiempos, viene logrando espacio.

Por un lado, Baroni y Nobre (1999) afirman que la Historia de las Matemáticas (así como el análisis, el Álgebra, la Estadística, la Geometría, etc.) constituye un área del conocimiento matemático, un campo de investigación. Por lo tanto, es una ingenuidad considerarla tan sólo un instrumento metodológico.

Lo que se tiene, entonces, no es la Historia de las Matemáticas como un simple instrumento de enseñanza, mas sí, un amplio campo, que conciliado con una didáctica de cualidad, puede auxiliar de manera considerable en el proceso de enseñanza aprendizaje, y en la relación de alumnos y profesores con los contenidos con los cuales trabajan.

Garcia (2005) en su pesquisa dice que los profesores, aun creyendo importante el uso de la Historia de las Matemáticas en clase, dicen no saber cómo utilizarla, pues tienen poco conocimiento del tema, aunque les gustaría hacerlo.

Las pesquisas y el desarrollo de nuevos recursos metodológicos de nada sirven si no son aplicados y utilizados, y para ello es necesaria la divulgación y la capacitación de 
profesores, para que tales recursos puedan interferir de forma efectiva en el proceso de enseñanza aprendizaje. Es importante que los estudios se tornen herramientas palpables y en el ambiente escolar práctico.

El uso de la Historia de las Matemáticas, además de propiciar una buena oportunidad de auxilio en el aprendizaje y comprensión de los contenidos de forma significativa, comprobada en estudios, también ya se hace presente en los propios instrumentos norteadores de la educación nacional, y en las competencias necesarias para la formación escolar.

Cuanto a las habilidades y competencias de la contextualización socio cultural, los Parámetros Curriculares Nacionales - PCN (Brasil, 1997) establecen que el alumno deba:

- Reconocer el sentido histórico de la ciencia y de la tecnología, percibiendo su papel en la vida humana en diferentes épocas y en la capacidad humana de transformar el medio;

- Comprender las ciencias como construcciones humanas, entendiendo como ellas se desarrollan por acumulación, continuidad o ruptura de paradigmas, relacionando el desarrollo científico con la transformación de la sociedad;

- Relacionar etapas de la historia de las Matemáticas con la evolución de la humanidad.

Según el Programa Nacional del Libro Didáctico para la Enseñanza Secundaria PNLEM (Brasil, 2004), la Historia de las Matemáticas ofrece un ámbito de contextualización importante del conocimiento matemático. Un libro didáctico debe hacer referencias a los procesos históricos de producción del conocimiento matemático y utilizar esos procesos como instrumento para auxiliar el aprendizaje de las matemáticas.

La Historia de las Matemáticas utilizada como recurso pedagógico en clase es una fuerte aliada para que sea posible enseñar y aprender los orígenes de las ideas y el desarrollo de las mismas. Ella es un importante instrumento para el aprendizaje de la Estadística y su conexión con otras áreas del conocimiento.

Generalmente, en las licenciaturas, los profesores en formación no tienen muchas informaciones acerca de la historia y evolución de los conceptos de Estadística o de la evolución curricular de esa asignatura, lo que podría contribuir para la comprensión adecuada de sus ideas. Por esa razón, muchas veces, en sus clases, dejan de utilizar una posibilidad rica e importante: la propia Historia de la Estadística. Los estudios históricos son muy importantes, en cualquier área del conocimiento, como lo señala Machado (2000): 
(...) la construcción del conocimiento jamás es definitiva. Jamás se puede fundamentar en definiciones cerradas. La red se encuentra en permanente estado de actualización. Para aprender el sentido de las transformaciones, el camino es uno sólo: es preciso estudiar Historia. Nadie puede enseñar cualquier contenido, de las ciencias a las lenguas, pasando por las matemáticas, sin una visión histórica de su desarrollo. Es en la historia que se puede percibir las razones que llevaron tal o cual relación, este o aquel concepto, a ser constituidos, reforzados o desechados. (p.103)

Muchos otros autores muestran la Historia de las Matemáticas como un importante auxilio para el aprendizaje de los conceptos matemáticos, de forma especial, Miguel (1997), al analizar las razones referidas por varios autores para utilizarse o no, la Historia de las Matemáticas en la enseñanza, listó doce argumentos que refuerzan las potencialidades pedagógicas de la Historia de las Matemáticas y cuatro argumentos cuestionadores. El autor se contrapuso de forma convincente a cada uno de los argumentos cuestionadores mostrándonos las grandes posibilidades pedagógicas que la Historia ofrece.

Así, a través de la realización de una pesquisa bibliográfica sobre la Historia de la Estadística, desarrollando una secuencia didáctica trabajando los conceptos estadísticos, utilizando como recurso didáctico la Historia de las Matemáticas.

\section{Metodología}

Inicialmente se hizo una pesquisa bibliográfica analizando los documentos encontrados en el área de la Historia de las Matemáticas sobre el desarrollo de la Estadística. En seguida fue realizado un censo de informaciones encontradas, las cuales fueron reportadas en forma de texto, para que a partir de este texto fuesen desarrolladas actividades componentes de una secuencia didáctica que busca reproducir hechos constituyentes del desarrollo de contenidos estadísticos tales como elaboración y análisis de gráficos y tablas, bien como conceptos básicos que fundamentan el área del conocimiento, para que a partir de estas actividades sea posible por parte de los alumnos la absorción de los contenidos estadísticos estudiados.

\section{Resultados}

Popularmente el significado de Estadística está asociado a colectas de datos numéricos presentados en forma de gráficos o cuadros que contengan informaciones demográficas o económicas, generalmente de interés de los gobiernos, para que puedan ejecutar plan a través del perfil de la población, además de ello, la etimología de la palabra status (estado) proviene del latín y era utilizada para designar la colecta y la presentación de datos cuantitativos del intereses del Estado (Memória, 2004). 
En los días de hoy la Estadística es una herramienta indispensable para el ejercicio de la ciudadanía. Ella permite analizar informaciones y subsidiar la tomada de decisiones sea en la vida personal o laboral. Su presencia es tan fuerte que se puede hasta pensar que sus métodos y técnicas son frutos exclusivos del mundo contemporáneo, sin embargo, la historia nos informa que la estadística ya era utilizada como pilar para la tomada de decisiones en el mundo antiguo. Para mejor explicitación de la relevancia y presencia del raciocinio estadístico en el desarrollo socio histórico de la relevancia y presencia del raciocinio estadístico en el desarrollo socio histórico, vamos destacar los principales momentos de la historia de la Estadística. Además de ello, con el objetivo de establecer el entendimiento del concepto a lo largo de los tiempos observamos el análisis etimológico del vocablo hecha por Lopes y Meirelles (2005).

Al verificar la etimología de la palabra estadística, nos chocamos con el registro de la forma italiana stadística, desde 1633, con el sentido de "ciencia del estado". La palabra Statistik venida del alemán, se originó la palabra francesa Statistique en 1771; la española Stadística en 1776; la inglesa statistics en 1787; y, finalmente, la portuguesa Estadística al principio del siglo XIX.

La palabra Estadística se originó de "Status" (Estado) y significaba un conjunto de descripciones y datos relativos al Estado, representando así, una poderosa herramienta para los administradores.

Otro raciocinio con relación al término "Estadística" lo relaciona al término "Censo", palabra que viene del latín census, y quiere decir "conjunto de los datos estadísticos de los habitantes de una ciudad, provincia, estado nación, etc." (IBGE, 2013). En el Diccionario Aurelio, la palabra "Censo" se refiere a un conjunto de datos estadísticos de los habitantes de una ciudad, provincia, nación, etc., con todas sus características; censo demográfico; empadronamiento.

Así siendo, pasamos a considerar el empleo del término "estadística" adoptando una perspectiva histórica. Con la invención de los sistemas de numeración y el surgimiento de las ciudades, el hombre no solamente pasó a cuantificar el mundo de la producción y del consumo como también a contar y recontar, con el objetivo de mejor planear sus acciones administrativas. O sea, el hombre entendió el uso de los números a la producción (tornarse productivo) de datos que favorecía el planeamiento de acciones de la administración pública o bélicas. Conforme destaca Memória (2004),

Hasta hoy, en el concepto popular, la palabra estadística evoca datos numéricos presentados en cuadros o gráficos, publicados por agencias gubernamentales, referentes a hechos demográficos o económicos. La etimología de la palabra, del latín status (estado), usada aquí para designar la colecta y la presentación de datos cuantitativos de interés el Estado, ello refleja bien esa idea. Entretanto, la mera colecta de datos así presentados está lejos de ser lo que entendemos, hoy, por Estadística. La verdad, su deseo esencial es de tornarse un conjunto de métodos (métodos estadísticos), especialmente apropiado, (...) el tratamiento de datos 
numéricos afectados por una multiplicidad de causas. Esos métodos hacen uso de las Matemáticas, particularmente del cálculo de probabilidades, en la colecta, presentación, análisis e interpretación de datos cuantitativos (p.10).

El interés político, militar y económico sobre la expansión territorial y las cuestiones tributarias, históricamente, fueron decisivos para la realización de los censos (Wada, 1996).

En otra perspectiva, Martin, (2001, p. 3) afirma que las primeras tentativas de enumeración de individuos o de bienes comienzan con grandes imperios de la Antigüedad y, cuyas estructuras administrativas eran fuertes:

Preocupados en manejar y administrar su imperio del mejor modo, los poderes centrales procuraron conocer mejor su extensión territorial y el número de sus súbditos. Fue así que las civilizaciones egipcia, mesopotámica y china, como anteriormente la civilización de los sumerios (5000 a 2000 A.C.), realizaban pesquisas censitarias de las cuales algunos llegaron hasta nosotros. El objetivo de esos censos era, ante todo, responder a la necesidad de la administración del imperio: responder a las necesidades de mano de obra en vista de la construcción de las grandes pirámides; responder a las preocupaciones fiscales.

La utilización de la Historia de la Estadística no debe ser apenas un abordaje de su origen, como también de su evolución y desarrollo de acuerdo con las necesidades que surgieron, de esta manera, los alumnos podrán percibir que la Estadística no es algo que pronto se encontró y está lejos de ser un asunto tenido por consumado.

Así, la secuencia didáctica construida tuvo como objetivo llevar al conocimiento de los alumnos como fueron realizados los avances en la Estadística a lo largo de la historia de la humanidad, de esa manera, se espera que los alumnos consigan percibir a través de textos y actividades propuestas, el progreso del área, tan real a diario.

El inicio de las pesquisas bibliográficas apuntan el recontar como siendo una preocupación en todas las culturas y antigua civilizaciones. Las civilizaciones siempre necesitan conocer la población en nivel económico y social; los emperadores ordenaban el recontar con los objetivos de cobrar impuestos y para el alistamiento militar (Ferreira y Tavares, 2002).

El registro de informaciones se pierde en el tiempo. En China Confucio hizo un levantamiento hace más de 2000 años antes de la era común. En el antiguo Egipto, los faraones hicieron uso sistemático de informaciones de carácter estadístico, conforme evidenciaron pesquisas arqueológicas. De esos registros también se utilizaron las civilizaciones pre colombianas de loa mayas, aztecas e incas. Es conocido de todos los cristianos el censo de los judíos, ordenado por el Imperador César Augusto, en la época del nacimiento de Jesús (Memória, 2004). 
Vale destacar que para hacer esos alistamientos eran apenas para los individuos relevantes, eran enumerados, tales como, propietarios, jefes de familia u hombres sujetos al reclutamiento militar.

En esta secuencia didáctica proponemos a los alumnos la lectura de los parágrafos siguientes y enseguida las actividades.

1. ¿Usted sabe qué significa el término "reclutamiento" en Estadística en los días de hoy? ¿Para qué es utilizado? ¿Es diferente de su uso en la antigüedad? Vamos a pensarlo y discutirlo.

2. Así como las civilizaciones realizaban censos de datos en el desarrollo de la Estadística, también iremos hacer uno. Pesquise y apunte cuántos alumnos hay en su clase, cuántos son del sexo femenino, cuántos del masculino y cuáles son sus edades.

En aquella época, el objetivo más importante del censo era saber el número de personas disponibles para hacer la guerra, cobrar impuestos. La punición para quienes no respondían al censo, generalmente era la muerte.

La Biblia (1995), en Lucas 2: 1-5, relata la historia que San José y la virgen María salieron de la ciudad de Nazaré, de la Galilea, para Belén, en la Judea, para responder al censo ordenado por César Augusto (las personas tenían que ser empadronadas en su ciudad de origen). Esto se dio en Belén, ciudad que Jesús nació.

Se presenta a seguir otra actividad que se fundamenta en una historia conocida por gran parte de la población que es la vida de Jesucristo. Figura 1, a través de un fragmento bíblico, para que los alumnos puedan percibir la importancia y la influencia de la Estadística en la vida de las personas:

"Aconteció en aquellos días que se promulgó un edicto de parte de Augusto César, que el mundo fuera empadronado. Este primer censo se hizo siendo Cirenio gobernador de Siria. E iban todos para ser empadronados, casa uno a su ciudad. También José subió de Galilea, de la ciudad de Nazaret, a Judea, a la ciudad de David, que se llama Belén, para ser empadronado con María, desposada con él, la cual estaba encinta. Lucas 2: 1-5 (Bíblia, Reina Valera, 1995). 


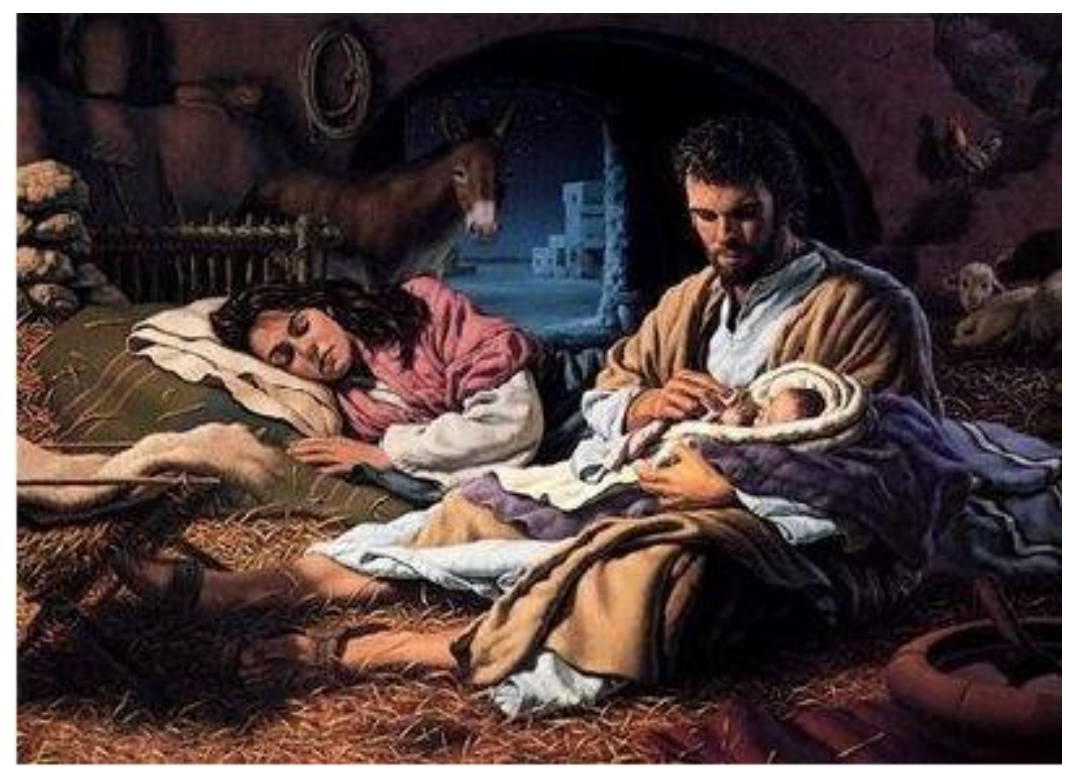

Figura 1 - Imagen retratando el nacimiento del niño Jesús.

Las actividades siguen en paralelo con el acontecimiento de los hechos, así los alumnos pueden reproducir lo que fue realizado en otros periodos de la historia de la humanidad y que hacen parte del desarrollo de muchas ciencias y conceptos utilizados por la actual sociedad. Observando lo que fue dicho por Memória (2004) proponemos la siguiente actividad:

3- Vamos a hacer un levanto en la clase y verificar cuántos alumnos nacieron en Uberaba y cuántos nacieron en otras ciudades.

Consecuentemente, se debe narrar a los alumnos que con el Renacimiento se despertó el interese por los datos estadísticos y su aplicación en la administración pública, se debe mencionar el reconocimiento de la Iglesia Católica Romana sobre la importancia de los registros de bautismos, casamientos y óbitos (Memória, 2004).

Además, el desarrollo de la Estadística está entrelazado a sus aplicaciones. John Graunt (1620 - 1674) fue el primer quien colectó datos en las parroquias de Londres para posteriores conclusiones y comparaciones sobre mortandad y sexo (Memória, 2004). 


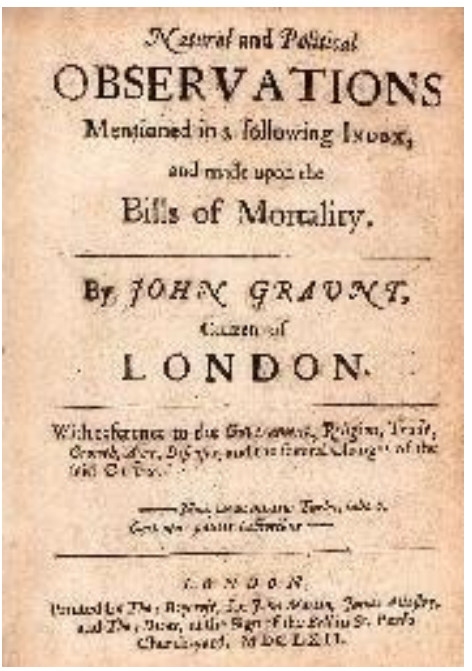

Figura 2 - Capa del libro de John Graunt publicado en 1662.

La tentativa referida arriba hecha por John Graunt, Figura 3, un próspero negociante londrino de telas que en 1662, publicó un pequeño libro intitulado Natural and Political Observations Mentioned in a Following Index and Made upon the Bills of Mortality. Su análisis fue basado sobre razones y proporciones de hechos vitales, en los cuales él observó una regularidad estadística en un gran número de datos (Memória, 2004).

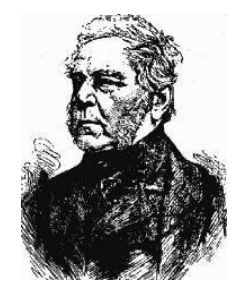

Figura 3 - Foto de John Graunt.

Para facilitar la análisis, interpretación y llegar a las conclusiones, Graunt (1662) organizó los datos colectados en una tabla en la Figura 4.

\begin{tabular}{|c|c|c|c|c|}
\hline \multicolumn{5}{|c|}{ Now to know in which of these 4. was the greatest Mortality at large, we reason thus, } \\
\hline $\begin{array}{l}\text { Anno } \\
1592\end{array}$ & $\begin{array}{l}\text { Buried } \\
\text { Christned }\end{array}$ & $\left.\begin{array}{c}26490 \\
4277\end{array}\right\}$ & oras & $\left\{\begin{array}{l}6 \\
1\end{array}\right.$ \\
\hline $\begin{array}{l}\text { Anno } \\
1603 .\end{array}$ & $\begin{array}{l}\text { There died in the whole Year of all or as } \\
\text { Christned }\end{array}$ & $\left.\begin{array}{c}38244 \\
4784\end{array}\right\}$ & & $\left\{\begin{array}{l}8 \\
1\end{array}\right.$ \\
\hline $\begin{array}{l}\text { 1. to 8. or } \\
11 / 4 \text {. to } \\
10 .\end{array}$ & $\begin{array}{l}\text { Anno Died in the whole Year } \\
\text { 1625. Christned. }\end{array}$ & $\left.\begin{array}{c}54265 \\
6983\end{array}\right\}$ & oras & $\left\{\begin{array}{l}8 \\
1\end{array}\right.$ \\
\hline $\begin{array}{l}\text { Anno } \\
1636 .\end{array}$ & $\begin{array}{l}\text { There died, ut supra } \\
\text { Christned }\end{array}$ & $\left.\begin{array}{c}23359 \\
9522\end{array}\right\}$ & oras & $\left\{\begin{array}{l}5 \\
2\end{array}\right.$ \\
\hline
\end{tabular}

Figura 4 - Formato de la tabla de John Graunt. 
Traduciendo la tabla de Graunt (1662), y adecuándola a las tablas utilizadas en los días actuales, se tiene:

Tabla 1 - Traducción propia de la tabla John Graunt.

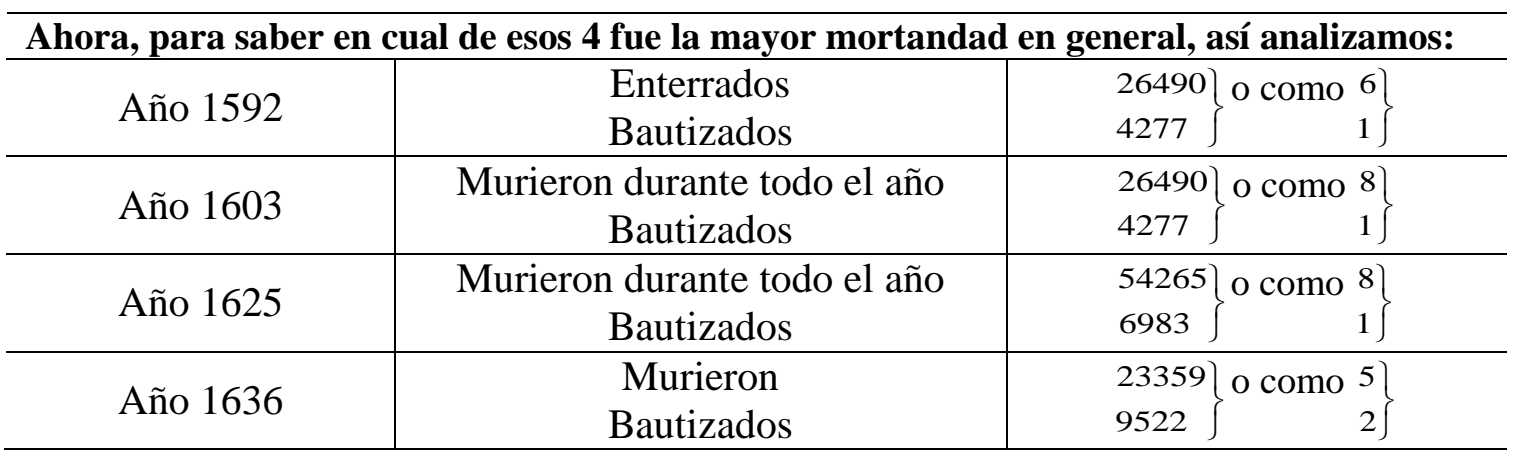

A través de la experiencia de Graunt será propuesto a los alumnos que organicen los datos colectados durante las actividades. La realización de un proceso semejante al que fue realizado por Graunt permitirá que el alumno perciba que esa organización facilita la comprensión y análisis de los datos, tales como:

1. Así como John Graunt, vamos a organizar los datos recogidos en las tablas.

2. Analizando los datos que colectamos en clase, ¿es posible obtener alguna conclusión de ellos?

3. ¿Usted sabe qué significan los términos "amuestra" y "población" en Estadística?

Con estos cuestionamientos se espera que los alumnos consigan hacer entrelazar los textos y a las actividades, de manera que puedan construir su propio conocimiento basado en reflexiones hechas a través de pesquisa realizada sobre la Historia de la Estadística.

En la secuencia será enseñado a los alumnos que la Estadística fue desarrollada a través de las necesidades que fueron surgiendo a diario, y para entenderlo, se puede observar que después de John Graunt, otros estudiosos trabajan en esa cuestión de comprensión de datos, uno de ellos fue Edmond Halley (1656-1742) que contribuyó para el desarrollo de la Estadística a través de la construcción de la primera regla de sobrevivencia que contenía registros vitales, elemento básico para calcular seguros de vida, se debe destacar también Richard Price (1723-1791) que fundó, en la Inglaterra, la actuaria, el área del conocimiento que analiza los riesgos y expectativas financieros y económicos, principalmente en la administración de seguros y pensiones.

Paralelamente, la Astronomía y la Geodésia desarrollaban métodos útiles al tratamiento de los datos de observación, surgiendo la Teoría de los Yerros, incorporadas a la Teoría Estadística (Memória, 2004), sin embargo, fue con el auxilio de los estudios de la Probabilidad que los conocimientos estadísticos vinieron a dar una nueva dimensión, 
considerando una nueva etapa que comienza a hacer Inferencia Estadística, una vez conocidas ciertas propiedades conseguidas a través de una análisis descriptiva de la amuestra, que vengan a considerar proposiciones generales que traduzcan la existencia de leyes en la población (Ferreira y Tavares, 2002).

Adolphe Quételet colaboró con el desarrollo de la Estadística cuando notó que la misma debería ser basada en los conocimientos sobe Probabilidad que a misma debería ser basada nos conocimientos sobre Probabilidad, su contribución fue marcante por considerar las dispersiones de las medidas, descubriendo que la curva normal se podía ajustarse a las medidas corporales de reclutas franceses, además de ello, era un eficiente organizador, colectó datos sobre criminalidad y delincuencia, asoció las características de predisposición al crimen haciendo agrupamientos conforme sexo, edad, escolaridad y tipo de delito (Memória, 2004).

El término curva de errores recibió el nombre de curva normal a través de Galton y Pearson, Galton sugirió que la distribución normal es determinada por la mediana y el desvío semi cuartílico, en un estudio donde hacía comparaciones de estaturas de padres e hijos usó el término regresión tras analizar la población observada. Es considerado segundo Stigler apud Memória, "un personaje romántico en la Historia de la Estadística” (Memória, 2004).

En fin, será desarrollada la siguiente actividad:

Considerando quién primero abogó el uso de muestra en levantos (sample surveys) fue Kiaer, con su método de representatividad, en la reunión del Instituto Internacional de Estadística (inventado 10 años antes, en Londres) en 1895, en Berna, Suiza. Andrés Nicolás Kiaer (1838 - 1919) era entonces Director del Bureau Central de Estadística en Cristiana, como era llamada la capital de Noruega, que en 1925 pasó a llamarse Oslo. A idea de Kiaer sobre amuestra representativa era la de ser una miniatura aproximada de la población. El método que él propuso correspondía al que, actualmente, sería un bien trabajado método de estratificación, llevándose en contra factores geográficos, sociales y económicos. Además, él introdujo una selección proporcional en cada estrato, basada en detalles del previo censo (Memória, 2004, p. 69).

De esta forma, se proponen las siguientes actividades:

1. ¿Las conclusiones extraídas sobre los datos cogidos en nuestra clase, podrían ser generalizadas para todas las aulas del $2^{\circ}$ año de la Secundaria de nuestra escuela?

2. ¿Ustedes saben qué es Media Aritmética?

Gauss llegó a la curva de los errores con espíritu empírico, adoptando como axioma el principio de que el valor más probable de una cantidad desconocida, observada con igual precisión varias veces sobre las mismas circunstancias, es la media aritmética de las observaciones (Memória, 2004, p.18-19). 
2.1. Calcule la media de las edades de los alumnos de la clase.

3. ¿Ustedes saben que es Mediana?

3.1. ¿Cuál la Mediana de las edades?

4. Y qué es Moda, ¿ustedes saben?

4.1. Existe Moda en las edades de los alumnos de las clases? Si existe, ¿cuál es?

5. Llevando en consideración las tres medidas de posición nombradas aquí (Media Aritmética, Mediana y Moda), ¿cuál representaría mejor la edad de los alumnos de la clase?

En la actualidad, es muy importante como ciudadanos, tener algunos conocimientos estadísticos para que posamos ser críticos en relación a las informaciones disponibles en la sociedad, haciéndose entender, comunicándose y tomar decisiones basadas en esas afirmaciones. Es por ello que la estadística está inclusa en los contenidos a ser estudiados en las escuelas.

6. ¿Ustedes pueden dar ejemplos de uso de la estadística en la sociedad actual, de la cual están inseridos?

Con el pasar del tiempo la Estadística se tornó herramienta indispensable para cualquier profesión que esté asociada en analizar informaciones y con la llegada de los ordenadores y tecnología avanzada, estando cada vez más accesible a las personas, pues los datos estadísticos son encontrados fácilmente en los medios de comunicación, lo que permite que cualquier persona aunque no tenga el menor esclarecimiento sobre el asunto, pueda estar en contacto asiduo con gráficos, tablas e informaciones estadísticas, habiendo así una gran necesidad de abordar el tema en las escuelas.

\section{Consideraciones Finales}

Para mostrar como la Estadística puede influenciar en la formación de opinión de los ciudadanos es fundamental que el alumno construya su saber a través de las informaciones disponibilidades a él. Al hacerlo analizar que la Estadística fue construida a través de las necesidades y situaciones que fueron surgiendo a lo largo de la historia, el alumno percibirá que este conocimiento no fue algo a ser pensado y solucionado instantáneamente.

Las informaciones estadísticas son disponibilidades a todo el momento para la sociedad a través de los medios de comunicación. Partiendo de este presupuesto, consideramos que los alumnos ya poseen cierto saber sobre el asunto, y de esa forma, debemos los auxiliar en la construcción de un nuevo conocimiento crítico y autónomo a través de la disponibilidad de nuevos saberes. Creemos que fundamentados en la Historia de las 
Matemáticas, los alumnos tendrán recursos y conocimientos necesarios para evaluar las informaciones estadísticas ofrecidas por la midia, percibiendo cuando hay manipulación de datos para obtención de resultados forzados por pretensiones políticas y/o capitalistas.

Con el auxilio de la Historia de la Estadística en el proceso de enseñanza aprendizaje, se cree entonces, que además de aprender nuevos contenidos, los alumnos estén también aptos a analizar y criticar los resultados obtenidos y las informaciones disponibilidades en su cotidiano y en sus vidas prácticas, para que el que aprende en la escuela no sea un fin en sí mismo, mas que colabore para la construcción de la ciudadanía y de la participación activa en la sociedad.

Después de la pesquisa bibliográfica y la creación de una secuencia didáctica, la pretensión es aplicarla en dos aulas de la Secundaria y dos escuelas provinciales de Uberaba, la Escuela Provincial Profesora Corina de Oliveira y Escuela Provincial Santa Terezinha, para entones, ser realizada una análisis para percibir si el uso de este recurso influenció de forma efectiva, y como, en el aprendizaje de los alumnos.

\section{Bibliografia}

[1] Baroni, R. L. S.; Nobre, S. (1999). A Pesquisa em História da Matemática e Suas Relações com a Educação Matemática. In: BICUDO, M. A.(org.). Pesquisa em Educação Matemática: concepções e perspectivas. São Paulo: UNESP, 129- 136.

[2] Bíblia. (1997). Português. Antigo e Novo Testamento. Traduzida em português por João Ferreira de Almeida. 2. ed. rev. e corrigida no Brasil. Rio de Janeiro: Liga Bíblica Brasileira.

[3] Brasil. (2004). Programa Nacional do Livro Didático para o Ensino Médio PNLEM. Brasília: Ministério de Educação e Cultura.

[4] Brasil. (1997). Secretaria da Educação Fundamental. Parâmetros Curriculares Nacionais. Brasília: MEC/SEF.

[5] Ferreira, M. J.; Tavares, I. VI Notas sobre a História da Estatística. Em http://www.alea.pt/Html/statofic/html/dossier/doc/dossier6.pdf.

[6] Garcia, F. T. (2005). A participação da História da Matemática no Ensino da Matemática: A visão dos professores das séries finais do Ensino Fundamental de Itabirito. Monografia (Especialização) Curso de Especialização em Educação Matemática, UFOP, Ouro Preto.

[7] Graunt, J. (1662). Natural and Political Observations Mentioned in a following Index, and made upon the Bills of Mortality. Em <http://www.neonatology.org/pdf/graunt.pdf> .

[8] IBGE - Instituto Brasileiro de Geografia e Estatística. Censo Demográfico 2010:

Resultados Gerais da Amostra por áreas de ponderação. Rio de Janeiro, 2013.

[9] Lopes, C. A. E.; Meirelles, E. (2005). O Desenvolvimento da Probabilidade e da Estatística, In XVIII Encontro Regional de Professores de Matemática LEM/IMECC/UNICAMP.

[10] Machado, N. J. (2000). Educação: Projetos e Valores. (Coleção Ensaios Transversais). São Paulo: Escrituras Editora. 
[11] Martin, O. (2001). Da estatística política à sociologia estatística.

Desenvolvimento e transformações da análise estatística da sociedade (séculos XVIIXIX). In Rev. bras. Hist., São Paulo, 21 (41).

[12] Memória, J. M. P. (2004). Breve História da Estatística. Brasília: Embrapa Informação Tecnológica.

[13] Miguel, A. (1997). As potencialidades pedagógicas da história da matemática em questão: argumentos reforçadores e questionadores. Zetetiké, FE/UNICAMP, 5 (8), 73-129.

[14] Nobre, S. (1996). Alguns "porquês" na História da Matemática e suas contribuições para a Educação Matemática. Cadernos CEDES 40. História e Educação Matemática. Campinas, SP: Papirus.

[15] Wada, R. S. (1996). Estatística e Ensino: um estudo sobre representações de professores do $3^{\circ}$ grau, Doutorado, UNICAMP - EDUCAÇÃO. 


\title{
La estadística vista desde un enfoque popular: Una estrategia de clase útil y amena en la educación secundaria de Costa Rica
}

\author{
José Leonardo Fernández Castro ${ }^{1}$
}

\begin{abstract}
Resumen
El presente trabajo presenta describe la experiencia desarrollada para enseñar estadística en secundaria más cercana a el espacio próximo de los estudiantes y haciendo del trabajo en clase un pequeño laboratorio de conocimientos aplicados a su vida próxima. La experiencia se desarrolló en el año 2005, en el pueblo de Cascajal de Coronado, como parte de la práctica profesional en un grupo de dicentes de octavo año. El trabajo que realizaron por los dicentes se basó en realizar un estudio estadístico que permitiera entrelazar ecología con estadísticas, tomando datos de sus alrededores y guiados por el profesional a cargo.
\end{abstract}

Palabras clave: enseñanza de la estadística, ecología, contextualización.

\section{Introducción}

La estadística es una herramienta que permite acercar al estudiante a su entorno próximo, tomando en cuenta los intereses de los estudiantes con el fin de provocar un aprendizaje significativo.

Según Rojano M. (2014) “el método docente conocido también como programa educativo o currículo a nivel aula, y que ahora manejamos como plan de estudios flexible, se define como: un plan de estudios que sobre la base de unos fundamentos racionales, organiza el contenido o material en forma secuencial y coordinada para facilitar la elaboración de actividades de enseñanza aprendizaje y lograr una meta u objetivos"

Todo plan de estudio debe de tener flexibilidad y a la vez relacionarse con el contexto en el que se desenvuelve el educando, es solo así como un aprendizaje puede calar en su vida y marcarlo positivamente para su futuro.

Así mismo, referente a los ejes transversales Segura M. (2006) opina "La política educativa vigente y la ley fundamental de la Educación (1957), toman en cuenta los valores fundamentales del humanismo, estos valores se relacionan con la solidaridad individual, social y con la naturaleza, dignifican la esencia de los seres humanos"

Los ejes transversales son parte importante en la formación integral del educando, pues permiten un afrontamiento de los contenidos desde un punto global e inclusivo.

La ecología es un eje trasversal que debe de estar implícito en la vida cotidiana, pues el manejo de los desechos es un problema próximo a ser una dificultad mundial que amenaza con contaminarnos.

${ }^{1}$ Universidad Estatal a Distancia, Costa Rica. leofe910@gmail.com 
Con el fin de acercar a la problemática social a los dicentes, se diseñó un trabajo extra clase donde el educando durante un mes, una vez por semana, recogiera la basura en la ruta de la carretera que va desde Cascajal de Coronado hasta las Nubes de Coronado, ruta que asiduamente caminaban para asistir a la Institución donde recibían lecciones.

Se procede con bolsas plásticas a recoger la basura que los choferes de los autos que transitan por el sector botan por la ventana, sin ningún tipo de cultura ecológica.

Luego de recolectar la basura se selecciona y se cuenta, con el fin de realizar una base de datos, necesaria para realizar los gráficos pertinentes.

Los estudiantes toman la información, realizan rótulos con diferentes ilustraciones donde desarrollan gráficos de barras con frecuencia absoluta, identifican la moda, la mediana y la media aritmética.

Finalmente en un proceso de exposición los dicentes proceden a compartir con sus iguales los hallazgos encontrados en una pequeña investigación.

La necesidad de una cultura donde la proximidad sea parte indispensable del currículo de los educando, permitiendo accesar de una manera grata a un aprendizaje de los contenidos.

\section{Bibliografía}

Rojano M. El aprendizaje significativo de la estadística; Un Ensayo cuasi experimental. 2014. En http://dcb.fi-c.unam.mx/Eventos/Foro3/Memorias/Ponencia_93.pdf.

Segura M. Los valores en el planeamiento didáctico. Eje transversal del currículo costarricense. 2006. En http://www.educadem.oas.org/documentos/boletin3 /valoresyplaneamiento $\% 20$ costa\%20rica.pdf 
IV Encuentro sobre Didáctica de la Estadística, la Probabilidad y el Análisis de Datos

\title{
Medición de Logros en Estudiantes de Educación Básica mediante un Mapa de Progreso en Estadística y Probabilidad
}

Augusta Osorio Gonzales - Elizabeth Advíncula Clemente

\begin{abstract}
Resumen:
En nuestro país, se viene elaborando los Estándares de Aprendizaje Nacionales descritos como Mapas de Progreso del Aprendizaje. Nuestra investigación, de carácter descriptivo, los utilizó para conocer los logros de aprendizaje, en temas de Estadística y Probabilidad, en de una muestra dirigida de estudiantes del nivel educativo básico. Logramos la identificación de los principales logros y deficiencias de los estudiantes que nos provee de información relevante para identificar cuáles son los conocimientos que necesitan ser reforzados y con ello orientar mejor nuestra acción pedagógica.
\end{abstract}

Palabras clave: Mapa de progreso, logro, Estadística

\begin{abstract}
:
In our country, the National Learning Standards are being developed using Learning Progress Maps as figure. Our descriptive research, used for the learning achievements on issues of Statistics and Probability in a targeted sample of students at the basic education level.. The identification of the main achievements and shortcomings of students provides us with relevant information to identify what knowledge they need to be reinforced and thus improve the pedagogical action.
\end{abstract}

Keywords: Progress Maps, achievements, Statistics

Modalidad: Ponencia.

IREM-PUCP

Pontificia Universidad Católica del Perú (PUCP)

arosorio@ pucp.edu.pe- eadvincula@pucp.edu.pe 
IV Encuentro sobre Didáctica de la Estadística, la Probabilidad y el Análisis de Datos

\section{Introducción}

En la actualidad, la Estadística es la herramienta más útil que se tiene para el trabajo de los datos y es requerida en el desarrollo de casi cualquier tarea que implica el análisis e interpretación de los datos. Esto es claro para muchos investigadores de la educación en Estadística.

La estadística es una de las disciplinas que más importancia han tenido desde los inicios mismos del hombre. En las últimas décadas, sus métodos y aplicaciones han permeado la mayoría de las áreas de la ciencia. La realidad es que se ha convertido en una disciplina que evolucionó para quedarse e incorporarse a la cultura de la sociedad moderna. Actualmente la estadística está mucho más relacionada con otras disciplinas que las matemáticas. Se ha usado como lenguaje y método de investigación científica en áreas tan diferentes como la lingüística, geografía, física, ingeniería, psicología y economía (ICMI/IASE, 2006).( Cueva \& Ibañez, 2008,p.34)

Las personas en general son expuestas a resultados estadísticos para respaldar un argumento de venta o la elección de una propuesta política. Tal es así que en época de elecciones los electores tienen a su disposición infinidad de resultados de estudios sobre las preferencias sobre algún partido político o algún proyecto o idea que sustente las propuestas de una agrupación en particular. Igualmente, las personas están expuestas a información que trabajan las diferentes instituciones públicas y que buscan que la población en general esté informada de diferentes aspectos relacionados con los temas para el mejor conocimiento de la realidad de un país.

Es necesario entonces que todas las personas dispongamos de un manejo adecuado de estos conocimientos estadísticos básicos y que podamos manejar las denominaciones o etiquetas con que se identifican estos conceptos dentro de la terminología de la estadística actual. Dónde es que podemos adquirir este aprendizaje, consideramos que la escuela es el mejor lugar para ello pues es el punto de paso obligado para la mayoría de ciudadanos de un país y haría que el conocimiento sea lo más universal posible. Lo que nos lleva a la clara necesidad de la enseñanza de la Estadística en la escuela.

A esta conclusión ya se llegó en diversos países y eso propició un proceso de inclusión de temas estadísticos en el currículo de Matemáticas de la escuela en general. El seguimiento de esta incorporación es parte de los trabajos de muchos investigadores en enseñanza de la Estadística, por ejemplo, Batanero, C. (2002) hace una recopilación de información sobre este punto y menciona la introducción de la enseñanza de la Estadística en Inglaterra y Argentina.

Según Holmes (2002), la enseñanza de la estadística y probabilidad fue ya introducida en 1961 en el currículo de Inglaterra en forma opcional para los estudiantes de 16 a 19 años que querían especializarse en matemáticas, con el fin de mostrar las aplicaciones de las matemáticas a una amplia variedad de materias .... Santaló (1980) ya presentaba un informe en una audiencia 1 Jornadas Interamericanas de Enseñanza de la Estadística. Buenos Aires, 2002. Conferencia inaugural internacional, indicando que la estadística se incluyó en la escuela secundaria en Argentina, para alumnos de 16 años 
en 1966 y para alumnos de 13 años en 1967. Más recientemente, Terán (2002) analiza los contenidos de la Ley Federal de Educación en Argentina, que la incluye desde la Educación General Básica al Polimodal.(Batanero, 2002, p.1)

La situación de la enseñanza de la estadística en la escuela en España también es presentada.

Aunque la enseñanza de la estadística ha estado presente en la escuela española en los últimos 20 años, encontramos una tendencia reciente a adelantar y renovar su enseñanza, haciéndola más experimental, en forma que se pueda proporcionar a los alumnos una experiencia estocástica desde su infancia (MEC 2006 a y b). En estas orientaciones curriculares observamos un fuerte incremento de los contenidos de estadística en la escuela primaria. (Batanero, Contreras \& Arteaga, 2011, p. 2)

Este proceso de inclusión también ha acontecido en nuestro país y lo podemos observar con los temas de Estadística y Probabilidad incluidos en el Diseño Curricular Nacional (2009). En nuestro caso los temas de Estadística se incluyen desde el primer grado de primaria. Sin embargo, a pesar de que se da la inclusión de los temas de Estadística y Probabilidad esto no asegura su enseñanza, es decir, es posible que la inclusión de los temas solo quede en el papel.

Esta problemática ya fue registrada por algunos investigadores.

En los programas de estudio de Matemática para segundo y tercer ciclo, del Ministerio de Educación Pública de Costa Rica (M.E.P.), se incluye entre los contenidos por estudiar, algunas nociones ligadas a los temas de Probabilidad y Estadística. Sin embargo es conocido que en repetidas ocasiones estos temas no se cubren, al menos en secundaria. Esto ocurre, entre otras razones, porque el tiempo lectivo que propone el M.E.P. para cubrir los programas a veces resulta insuficiente. Esto último, aunado al hecho de que dichos temas hasta el año 2003 no eran evaluados en las pruebas nacionales de conclusión de ciclo, ha provocado que muchos profesores los dejen de lado. Amén de esto, se puede agregar que muchos docentes no son conscientes de la importancia que puede tener para un estudiante poseer como parte de su cultura un buen manejo de la noción de incertidumbre.(Jimenez \& Jimenez, 2005, p.1).

Igualmente, Grima (2010) menciona que a pesar de la destacada presencia de la Estadística en las titulaciones universitarias y en muchas actividades profesionales, la estadística suele tener poco protagonismo en la enseñanza secundaria pues rara vez existe una asignatura específica de estadística, y más bien se presenta incluida en el libro de Matemáticas, mucha veces al final, de forma que si no da tiempo a verlo todo, esta es la parte que se queda sin impartir.

La consecuencia natural de esta problemática es el nivel con el que llegan los alumnos a un primer curso de Estadística a nivel universitario. El diseño de dichos cursos y podemos hacer referencia a los cursos en el ámbito de los Estudios Generales de nuestra universidad, se basan en que los estudiantes ya tienen un conocimiento previo de ciertos conceptos básicos y que solo es necesario refrescárselos muy rápidamente. La realidad 
lamentablemente es muy distinta, muchos de los estudiantes adolecen de los conocimientos esperados y por tanto el docente tiene que tomar entre dos posibles caminos: sigue la programación esperada sin tratar de reparar las deficiencias encontradas o hace un alto y trata de repararlas.

El primer camino provocará que estas sean subsanadas por cuenta de los alumnos y no necesariamente con el detalle o entendimiento necesario para poder trabajar temas más complejos o simplemente son arrastradas por los alumnos como conocimientos no adquiridos que provocaran dificultades al trabajar temas más complejos. El segundo camino provocará dificultades de tiempo y tal vez el sacrificio de algunos temas del curso que no se llegarán a ver o que no se revisarán con la profundidad requerida.

Luego la consecuencia de que existan diferencias entre el conocimiento esperado y el que se da en forma efectiva en los alumnos que llevan un primer curso de Estadística es altamente significativo para el avance del curso y por tanto, es de la mayor relevancia que el docente cuente con una referencia de cuáles son los posibles temas básicos en los que los estudiantes pueden presentar las mayores dificultades.

Los resultados de esta investigación serían relevantes para cualquier docente de un primer curso de Estadística a nivel universitario. Le permitirá contar con información sobre las posibles deficiencias que encontrará en sus futuros alumnos y comprobarlas mediante pruebas de entrada, con el fin de establecer las estrategias necesarias para la conducción de su curso.

Con los datos recogidos queremos confirmar que los temas básicos de Estadística y probabilidad que son enseñados durante la Educación Básica Regular permiten a los estudiantes de los ciclos 4 y 6 alcanzar las expectativas de aprendizaje previstas en el Mapa de progreso de Estadística y Probabilidad propuesto por el Instituto Peruano de Evaluación, Acreditación y Certificación de la Calidad de la Educación Básica (IPEBA).

\section{El Mapa de progreso de Estadística y Probabilidad}

Considerando que el aprendizaje es un proceso continuo, que se desarrolla a lo largo de la vida, los Mapas de Progreso posibilitan apreciar el avance progresivo de tal aprendizaje, facilitando la articulación de los niveles y etapas del sistema educativo. La elaboración de los Mapas de Progreso se realizó por un equipo integrado de especialistas de IPEBA y del Ministerio de Educación, asesorados por expertos nacionales e internacionales.

Los mapas de progreso están divididos en niveles. Los niveles indican lo que se espera que un estudiante haya aprendido al finalizar cada ciclo de la Educación Básica Regular. Los niveles muestran estos aprendizajes de manera sintética y empleando un lenguaje sencillo, con el fin de que todos puedan comprenderlos. 


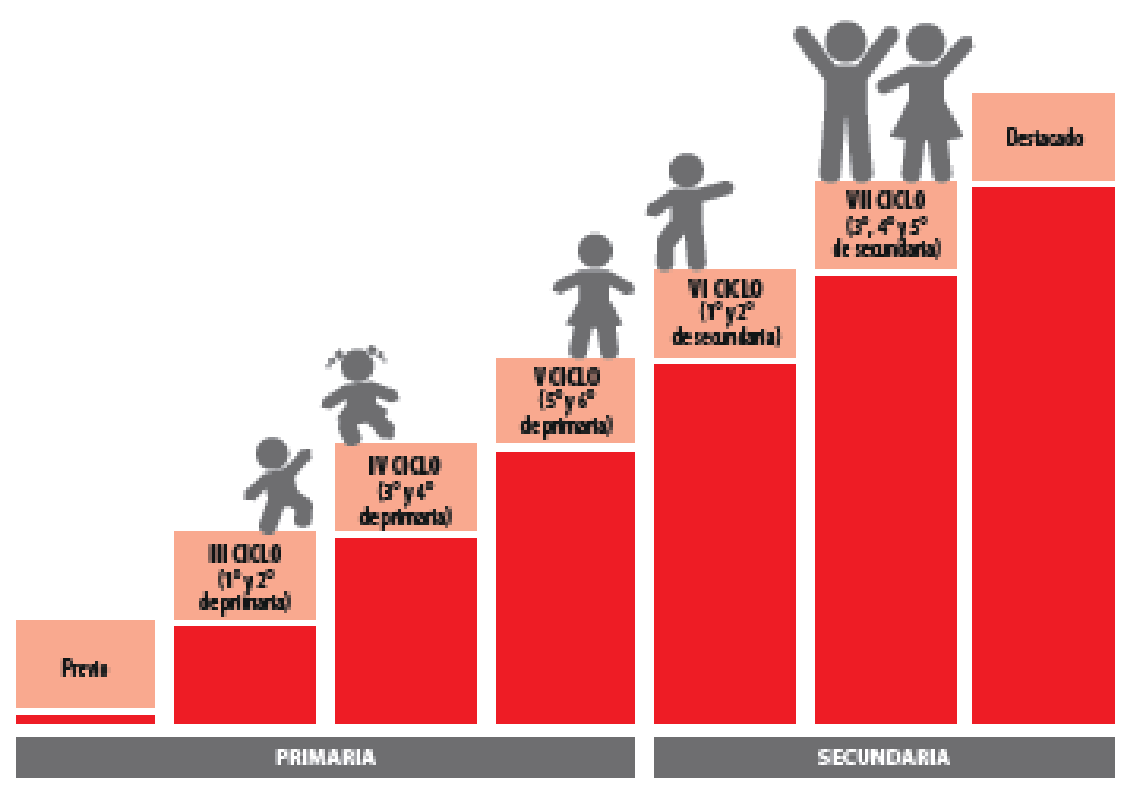

Cada nivel de un Mapa de progreso cuenta con un conjunto de indicadores de desempeño. Estos permitirán identificar claramente si los estudiantes lograron lo que indica el nivel correspondiente. Por tanto, los Mapas de Progreso son útiles porque le permiten a los docentes enfocarse en los aprendizajes centrales y observar cuán lejos o cerca están sus estudiantes del logro de estas metas de aprendizaje, para poder reorientar su acción pedagógica.

En particular, el Mapa de Progreso de Estadística y Probabilidad describe el desarrollo progresivo de la competencia para procesar e interpretar diversidad de datos transformándolos en información y analizar situaciones de incertidumbre para formular predicciones que permitan tomar decisiones adecuadas.

La descripción del progreso del aprendizaje en este mapa se realiza en base a tres aspectos:

a. Recopilación y procesamiento de los datos.

Implica el desarrollo de capacidades para trabajar con los datos, recopilarlos, clasificarlos, organizarlos, representarlos y determinar sus medidas descriptivas en función a un propósito, con la finalidad de brindar insumos para la interpretación de los mismos.

b. Interpretación y valoración de los datos.

Implica el desarrollo de capacidades para convertir en información los datos procesados mediante la lectura, interpretación, inferencia y valoración de la pertinencia y representatividad de los mismos con la finalidad de tomar decisiones.

c. Análisis de situaciones de incertidumbre.

Implica el desarrollo de capacidades para identificar, describir, modelar una situación aleatoria, determinar sus componentes (espacio muestral, el contexto y sus restricciones) y 
estimar la probabilidad de ocurrencia de los sucesos relacionados con ella, con la finalidad de predecirlos y tomar decisiones.

Dentro de cada nivel del Mapa de progreso de Estadística y Probabilidad se encontrarán indicadores de desempeño para cada uno de los aspectos descritos. Por tanto, nos basaremos en cada uno de estos indicadores para poder construir situaciones problemáticas que nos permitan verificar los conocimientos de los alumnos a analizar.

\section{Objetivos de la investigación}

El objetivo general de esta investigación es identificar el nivel de logro alcanzado por los estudiantes de la Educación Básica Regular, en relación a los contenidos de Estadística y Probabilidad esperados para su grado de estudios e identificar sus posibles deficiencias. Para lograr este objetivo, tenemos los siguientes objetivos específicos:

1. Determinar desde el mapa de progreso de Estadística y Probabilidad los objetos estadísticos que se establecerán como conocimientos básicos para cada aspecto y nivel.

2. Construir y validar los instrumentos que permitirán realizar las mediciones en los alumnos de los ciclos escogidos.

3. Determinar para cada alumno de la muestra el nivel en que se encuentra con respecto al mapa de progreso de Estadística y Probabilidad.

4. Establecer las diferencias que se presentan entre lo medido y lo esperado, con el fin de reportar adecuadamente a los interesados.

\section{Grupo de aplicación}

La muestra realizada fue del tipo no probabilística dirigida, y se escogieron a cinco colegios particulares de la ciudad de Lima. Tres de estos colegios integran el listado de colegios seleccionados por la PUCP para la admisión mediante la modalidad de Ingreso por Tercio Superior, esto era importante para nosotros puesto que el análisis de los resultados estaba dirigido a un primer curso de Estadística de la unidad de Estudios Generales Letras de la PUCP. Nuestra muestra estuvo compuesta por un aproximado de 380 alumnos del quinto grado de educación primaria y 396 alumnos del tercer año del nivel secundario. 


\section{Instrumentos de evaluación}

Los instrumentos utilizados en esta investigación se construyeron tomando como base el mapa de progreso de Estadística y Probabilidad. Este mapa como hemos explicado anteriormente, está dividido en siete niveles y tres aspectos. Dentro de la metodología de trabajo, no se midió a todos los alumnos en las tres dimensiones del mapa de progreso, esto por una cuestión de tiempo. La aplicación de un instrumento duraba en promedio 45 minutos y en general, todas las instituciones educativas solo nos permitían una hora de trabajo por aula. La elección de los alumnos para cada dimensión fue totalmente aleatoria.

Para la revisión de los enunciados de las preguntas y de los contenidos medidos, se aplicó una prueba piloto a un grupo de alumnos de dos entidades educativas. Se trabajó con 25 alumnos del centro Educativo José Carlos Mariátegui de Comas y 10 del Cristo Rey de Pueblo Libre. Se aplicaron los seis instrumentos diseñados y se identificaron las falencias de diseño, estas básicamente se concentraron en los contextos presentados. Los alumnos tuvieron problemas para identificar algunos términos, que no les resultaban familiares.

En nuestro trabajo los indicadores a considerar por cada aspecto y dentro de cada nivel fueron los siguientes:

\section{Aspecto: Recopilación y procesamiento de los datos.}

\begin{tabular}{|l|l|}
\hline \multicolumn{1}{|c|}{ Nivel } & \multicolumn{1}{|c|}{ Indicador } \\
\hline $\begin{array}{l}\text { Ciclo III (1er. y 2do. grado de } \\
\text { primaria) }\end{array}$ & Organiza datos en tablas simples \\
\cline { 2 - 2 } $\begin{array}{l}\text { Ciclo IV (3er. y 4to. grado de } \\
\text { primaria) }\end{array}$ & Oresenta datos mediante gráficos de barras \\
\hline \multirow{2}{*}{ Ciclo V (5to. y 6to. grado de primaria) } & $\begin{array}{l}\text { Presenta datos mediante gráficos para variables } \\
\text { cualitativas }\end{array}$ \\
\cline { 2 - 2 } & $\begin{array}{l}\text { Recopila datos mediante una encuesta en la que } \\
\text { formula preguntas y sus posibles respuestas }\end{array}$ \\
\hline Ciclo VI (1ro.y 2do. de secundaria) & $\begin{array}{l}\text { Determina la población usando criterios de } \\
\text { pertinencia }\end{array}$ \\
\hline
\end{tabular}


IV Encuentro sobre Didáctica de la Estadística, la Probabilidad y el Análisis de Datos

Aspecto: Interpretación y valoración de los datos.

\begin{tabular}{|l|l|}
\hline \multicolumn{1}{|c|}{ Nivel } & \multicolumn{1}{|c|}{ Indicador } \\
\hline $\begin{array}{l}\text { Ciclo III (1er. y 2do. grado de } \\
\text { primaria) }\end{array}$ & Lee información en tablas simples o gráficos. \\
\hline \multirow{4}{*}{$\begin{array}{l}\text { Ciclo IV (3er. y 4to. grado de } \\
\text { primaria) }\end{array}$} & $\begin{array}{l}\text { Interpreta información presentada en tablas } \\
\text { simples y de doble entrada }\end{array}$ \\
\cline { 2 - 3 } & $\begin{array}{l}\text { Interpreta información presentada en gráficos de } \\
\text { barras }\end{array}$ \\
\cline { 2 - 3 } Ciclo V (5to. y 6to. grado de primaria) & $\begin{array}{l}\text { Interpreta la moda de un grupo de datos } \\
\text { gráficos }\end{array}$ \\
\cline { 2 - 2 } & $\begin{array}{l}\text { Interpreta información no explícita presentada en } \\
\text { tablas. }\end{array}$ \\
\hline \multirow{4}{*}{ Ciclo VI (1ro. y 2do. de secundaria) } & $\begin{array}{l}\text { Interpreta y usa las medidas de tendencia central } \\
\text { reconociendo e interpretando la medida } \\
\text { representativa de un conjunto de datos }\end{array}$ \\
\cline { 2 - 2 } & $\begin{array}{l}\text { Infiere información de diversas fuentes } \\
\text { presentada en tablas y gráficos, la comunica } \\
\text { utilizando un lenguaje informal. }\end{array}$ \\
\hline
\end{tabular}

Aspecto: Análisis de situaciones de incertidumbre.

\begin{tabular}{|c|c|}
\hline Nivel & Indicador \\
\hline Ciclo II (Inicial 5años) & $\begin{array}{l}\text { Describe a partir de experiencia directa la } \\
\text { ocurrencia de sucesos cotidianos usando } \\
\text { expresiones coloquiales. }\end{array}$ \\
\hline \multirow{2}{*}{$\begin{array}{l}\text { Ciclo III (1er. y 2do. grado de } \\
\text { primaria) }\end{array}$} & $\begin{array}{l}\text { Identifica la imposibilidad de ocurrencia de } \\
\text { sucesos cotidianos }\end{array}$ \\
\hline & $\begin{array}{l}\text { Describe algunos posibles resultados de una } \\
\text { situación aleatoria por experiencia directa }\end{array}$ \\
\hline $\begin{array}{l}\text { Ciclo IV (3er. y 4to. grado de } \\
\text { primaria) }\end{array}$ & $\begin{array}{l}\text { Explica si la ocurrencia de un suceso es más } \\
\text { probable o menos probable que la de otros suceso } \\
\text { proveniente de la misma situación aleatoria }\end{array}$ \\
\hline \multirow{2}{*}{ Ciclo V (5to. y 6to. grado de primaria) } & $\begin{array}{l}\text { Determina todos los posibles resultados de una } \\
\text { situación aleatoria }\end{array}$ \\
\hline & $\begin{array}{l}\text { Interpreta la probabilidad de un evento mediante } \\
\text { el planteamiento clásico }\end{array}$ \\
\hline \multirow{2}{*}{ Ciclo VI (1ro. y 2do. de secundaria) } & $\begin{array}{l}\text { Identifica sucesos simples y compuestos } \\
\text { relacionados a una situación aleatoria propuesta }\end{array}$ \\
\hline & $\begin{array}{lll}\text { Interpreta las probabilidades } & \text { usando } \\
\text { planteamiento de frecuencias relativas. } & \end{array}$ \\
\hline
\end{tabular}




\section{Medición}

Nuestra expectativa de medición era determinar para cada alumno su nivel en el Mapa de progreso, teniendo en cuenta que se consideraría el nivel secuencial más alto alcanzado. Es decir, para que digamos que un alumno alcanzó el nivel del Ciclo $\mathrm{V}$ es que contesto acertadamente todos los indicadores de los conocimientos de los ciclos III, IV y V.

Para lograr la medición del logro de cada alumno se trabajó bajo el siguiente esquema:

I. Establecer desde cada respuesta esperada las características que debía presentar la respuesta correcta del alumno.

II. Determinar en base a las características establecidas si el alumno había desarrollado totalmente, parcialmente o no había desarrollado la respuesta correcta. Se utilizó una codificación para estos desarrollos y se incluyó en la codificación a las respuestas en blanco y las respuestas no relacionadas con lo solicitado.

III. Determinar el nivel de logro del alumno para cada pregunta respondida. Para ello se establecía desde el código establecido una nueva codificación.

IV. Determinar el logro de un nivel en base a los indicadores alcanzados del nivel indicado. Para que digamos que un alumno logro un determinado nivel, ha debido lograr todos los indicadores presentados de dicho nivel, si solo logro algunos indicadores diremos que el alumno se encuentra en proceso de logro de dicho nivel.

V. Determinar el nivel de logro dentro del mapa de progreso de un alumno, determinando el nivel más alto logrado. Para la determinación tuvimos en cuenta que el alumno debió lograr todos los niveles anteriores al adjudicado.

\section{Resultados}

Tenemos dos formas de analizar nuestros resultados, en términos de la medición del nivel alcanzado por cada alumno y en término de los logros de cada pregunta aplicada.

Para la primera forma, obtenemos el porcentaje de alumnos que ha alcanzado cada uno de los niveles del mapa de progreso y lo comparamos con los niveles esperados. Hay que tener en cuenta que los niveles esperados para cada grupo trabajado son los siguientes:

- Nivel primario se espera que cada alumno alcance los niveles del Ciclo III y IV, siendo el nivel del Ciclo V un nivel de logro destacado.

- Nivel secundario se espera que cada alumno alcance los niveles de los Ciclos III, IV, V y VI, siendo el nivel VII un nivel de logro destacado. 
Se ha encontrado que no todos los alumnos tienen un desarrollo secuencial, dado que por ejemplo, hemos identificado alumnos que tienen alcanzado el nivel del Ciclo III y el nivel del Ciclo V pero no el del Ciclo IV. Esos casos los hemos colocado como Otros, puesto que nuestro interés está en los alumnos que han desarrollado un conocimiento progresivo.

Para el aspecto Recopilación y procesamiento de los datos:

\begin{tabular}{|l|c|c|c|c|c|c|}
\cline { 2 - 7 } \multicolumn{1}{c|}{} & \multicolumn{3}{c|}{ 4TO DE PRIMARIA } & \multicolumn{3}{c|}{ 2DO DE SECUNDARIA } \\
\hline LOGRO & $\begin{array}{c}\text { Frecuencia } \\
\text { Absoluta }\end{array}$ & $\begin{array}{c}\text { Frecuencia } \\
\text { relativa }\end{array}$ & $\begin{array}{c}\text { Frecuencia } \\
\text { relativa } \\
\text { acumulada }\end{array}$ & $\begin{array}{c}\text { Frecuencia } \\
\text { Absoluta }\end{array}$ & $\begin{array}{c}\text { Frecuencia } \\
\text { relativa }\end{array}$ & $\begin{array}{c}\text { Frecuencia } \\
\text { relativa } \\
\text { acumulada }\end{array}$ \\
\hline SIN NIVEL & 35 & $28.00 \%$ & $28.00 \%$ & 39 & $29.55 \%$ & $29.55 \%$ \\
\hline CICLO III & 33 & $26.40 \%$ & $54.40 \%$ & 18 & $13.64 \%$ & $43.18 \%$ \\
\hline CICLOS III y IV & 38 & $30.40 \%$ & $84.80 \%$ & 21 & $15.91 \%$ & $59.09 \%$ \\
\hline CICLOS III , IV y V & 7 & $5.60 \%$ & $90.40 \%$ & 13 & $9.85 \%$ & $68.94 \%$ \\
\hline OTROS & 12 & $9.60 \%$ & $100.00 \%$ & 41 & $31.06 \%$ & $100.00 \%$ \\
\hline
\end{tabular}

Podemos concluir que solo el 36\% de los alumnos evaluados de primaria alcanzaron el nivel equivalente al Ciclo IV, que era el esperado dentro del mapa. En el caso de secundaria ningún alumno alcanzó el nivel equivalente al Ciclo VI y solo el 10\% de los alumnos evaluados alcanzó un nivel acorde con el final de la primaria (CICLOS III , IV y V).

Para el aspecto Interpretación y valoración de los datos:

\begin{tabular}{|l|c|c|c|c|c|c|}
\cline { 2 - 7 } \multicolumn{1}{c|}{} & \multicolumn{3}{c|}{ 4TO. DE PRIMARIA } & \multicolumn{2}{c|}{ 2DO. DE SECUNDARIA } \\
\cline { 5 - 7 } $\begin{array}{c}\text { NIVELES DE } \\
\text { LOGRO }\end{array}$ & $\begin{array}{c}\text { Frecuencia } \\
\text { Absoluta }\end{array}$ & $\begin{array}{c}\text { Frecuencia } \\
\text { Relativa }\end{array}$ & $\begin{array}{c}\text { Frecuencia } \\
\text { relativa } \\
\text { acumulada }\end{array}$ & $\begin{array}{c}\text { Frecuencia } \\
\text { Absoluta }\end{array}$ & $\begin{array}{c}\text { Frecuencia } \\
\text { Relativa }\end{array}$ & $\begin{array}{c}\text { Frecuencia } \\
\text { relativa } \\
\text { acumulada }\end{array}$ \\
\hline SIN NIVEL & 6 & $4.72 \%$ & $4.72 \%$ & 0 & $0.00 \%$ & $0.00 \%$ \\
\hline CICLO III & 83 & $65.36 \%$ & $70.08 \%$ & 65 & $51.59 \%$ & $51.59 \%$ \\
\hline CICLOS III y IV & 22 & $17.32 \%$ & $87.40 \%$ & 36 & $28.57 \%$ & $80.16 \%$ \\
\hline CICLOS III , IV y V & 15 & $11.81 \%$ & $99.21 \%$ & 25 & 19.84 & $100.00 \%$ \\
\hline OTROS & 1 & $0.79 \%$ & $100.00 \%$ & & & \\
\hline
\end{tabular}

Podemos concluir que solo el 39\% de los alumnos evaluados de primaria alcanzaron el nivel equivalente al Ciclo IV, que era el esperado dentro del mapa. En el caso de secundaria ningún alumno alcanzó el nivel equivalente al Ciclo VI y solo el $20 \%$ de los alumnos evaluados alcanzó un nivel acorde con el final de la primaria (CICLOS III, IV y V). 
Para el aspecto Análisis de situaciones de incertidumbre:

\begin{tabular}{|l|c|c|c|c|c|c|}
\cline { 2 - 7 } \multicolumn{1}{c|}{} & \multicolumn{3}{c|}{ 4TO DE PRIMARIA } & \multicolumn{3}{c|}{ 2DO DE SECUNDARIA } \\
\hline IND & $\begin{array}{c}\text { Frecuencia } \\
\text { Absoluta }\end{array}$ & $\begin{array}{c}\text { Frecuencia } \\
\text { relativa }\end{array}$ & $\begin{array}{c}\text { Frecuencia } \\
\text { relativa } \\
\text { acumulada }\end{array}$ & $\begin{array}{c}\text { Frecuencia } \\
\text { Absoluta }\end{array}$ & $\begin{array}{c}\text { Frecuencia } \\
\text { relativa }\end{array}$ & $\begin{array}{c}\text { Frecuencia } \\
\text { relativa } \\
\text { acumulada }\end{array}$ \\
\hline SIN NIVEL & 21 & $16.80 \%$ & $16.80 \%$ & 19 & $15.00 \%$ & $15.00 \%$ \\
\hline CICLO III & 50 & $40.00 \%$ & $56.80 \%$ & 38 & $29.90 \%$ & $44.90 \%$ \\
\hline CICLOS III y IV & 9 & $7.20 \%$ & $64.00 \%$ & 9 & $7.09 \%$ & $51.99 \%$ \\
\hline CICLOS III , IV y V & 7 & $5.60 \%$ & $69.60 \%$ & 7 & $5.51 \%$ & $57.50 \%$ \\
\hline OTROS & 38 & $30.40 \%$ & $100.00 \%$ & 54 & $42.52 \%$ & $100.02 \%$ \\
\hline
\end{tabular}

Podemos concluir que solo el 13\% de los alumnos evaluados de primaria alcanzaron el nivel equivalente al Ciclo IV, que era el esperado dentro del mapa. En el caso de secundaria ningún alumno alcanzó el nivel equivalente al Ciclo VI y solo el $6 \%$ de los alumnos evaluados alcanzó un nivel acorde con el final de la primaria (CICLOS III, IV y V).

Para la segunda forma, de acuerdo a los logros alcanzados por pregunta. Este análisis al detalle no es parte de los objetivos de esta investigación, pero se ha establecido algunas conclusiones desde cada pregunta para poder dar algunas recomendaciones generales a un profesor de un primer curso de Estadística a nivel universitario.

Sobre el aspecto de Recopilación y procesamiento de datos, los alumnos de la muestra de secundaria muestran:

- Dificultades para distinguir entre tabla de organización de datos y un gráfico de barras. La mayoría del 35\% que tienen como incorrecta la pregunta han hecho un gráfico en vez de la tabla solicitada o han hecho ambos. Hay que reforzar la diferencia entre organización de datos y presentación de datos.

- Dificultades en construir una tabla de doble entrada, aproximadamente el $40 \%$ de los alumnos de la muestra de secundaria, en vez de separar las frecuencias por género en la pregunta presentada, sumaban las frecuencias y construían una tabla simple. Establecer mecanismos para que los alumnos organicen datos clasificando por dos o más variables.

- Falta de decisión entre un gráfico de barras agrupadas que presentaba toda la información entregada y uno de sectores que presentaba información parcial, cuando se les pedía analizar cuál era el más adecuado para la presentación de los datos. Más del $50 \%$ de la muestra no pudo decidir. Habría que reforzar a los alumnos en el análisis de la presentación adecuada de la información, deben poder discriminar cuando la información está o no completa en un gráfico. 
- Dificultad para establecer, desde un grupo de preguntas, las que eran las más importantes para un estudio. Es necesario que los alumnos experimenten la creación de instrumentos de medición además de solo utilizarlos.

Sobre el aspecto de Interpretación y valoración de los datos, los alumnos de la muestra de secundaria muestran:

- Dificultades en interpretar información explicita y no explicita desde tablas y gráficos, aproximadamente un $40 \%$ de los alumnos lo han presentado. Sería adecuado que los alumnos tengan la oportunidad de extraer datos no directos desde tablas y gráficos para su uso, así se garantizaría que pueden hacer uso de la información allí mostrada.

- Dificultades para la utilización de la moda, no por no saber el mecanismo para hallarla sino porque no podían identificar el concepto. La mayor parte del $60 \%$ de alumnos que no pudieron contestar adecuadamente la pregunta sobre moda fue porque indicaban que no entendían a qué se refería el término. Sería bueno que los alumnos no solo repasen los procedimientos para poder hallar las medidas de tendencia central, sino que se garantice que pueden identificarlos por nombre.

- Dificultades en establecer qué medida de tendencia central debían aplicar cuando se les solicitaba verificar una afirmación sobre el promedio de un salón de clase. Al parecer darles un gráfico en vez de datos en un tabla, le dificultó el proceso. Es necesario que los alumnos conozcan dominen el uso de las medidas de tendencia central y que puedan utilizarlas indistintamente de donde vengan los datos.

- El $90 \%$ de la muestra de secundaria no pudo hacer ninguna inferencia desde un histograma de las notas de un salón de clase. Es necesario que los alumnos ejerciten el uso de los conceptos de la estadística descriptiva en información resumida en tablas y gráficos, y su significado dentro del contexto.

Sobre el aspecto de Análisis de situaciones de incertidumbre, los alumnos de la muestra de secundaria muestran:

- Dificultades para establecer si un evento es imposible o no para un experimento aleatorio. El 50\% de los alumnos de la muestra no pudieron establecer que todos los eventos simples propuestos eran posibles y el $90 \%$ no pudo establecer que un evento compuesto dado era imposible para la situación propuesta. Ejercitar los conceptos relacionados con experimento aleatorio.

- Dificultades para establecer algunos resultados desde un experimento aleatorio que presenta eventos simples formados por combinaciones. El $70 \%$ de los alumnos no pudieron establecer lo solicitado. Trabajar con experimentos aleatorios con espacios muestrales con eventos simples formados por combinaciones de resultados.

- Dificultades para establecer los espacios muestrales de experimentos aleatorios. En algunas preguntas llegaron a ser el $90 \%$ de los alumnos. Ejercitar los conceptos relacionados con experimento aleatorio.

- Dificultades para utilizar los planteamientos clásico y de frecuencias relativa para el cálculo de probabilidades. Más del $80 \%$ de los alumnos de la muestra no pudieron determinar las probabilidades solicitadas. Ejercitar el cálculo de probabilidades. 
IV Encuentro sobre Didáctica de la Estadística, la Probabilidad y el Análisis de Datos

\section{Conclusiones}

El Ministerio de Educación peruano, a través de la Unidad de Medición de la Calidad Educativa (UMC), viene aplicando desde el año 2007 la Evaluación Censal de Estudiantes (ECE). Esta evaluación consiste en la aplicación de pruebas estandarizadas a los estudiantes de segundo grado de primaria mediante una muestra nacional.

Los resultados de dichas pruebas en el área de matemática se tienen en el siguiente cuadro:

\begin{tabular}{|c|c|c|c|}
\hline \multirow{2}{*}{ MATEMÁTICA } & En inicio & En proceso & Satisfactorio \\
\cline { 2 - 4 } & $\boldsymbol{\%}$ & \% & $\%$ \\
\hline $\mathbf{2 0 0 7}$ & 56.5 & 36.3 & 7.2 \\
\hline $\mathbf{2 0 0 8}$ & 54.7 & 35.9 & 9.4 \\
\hline $\mathbf{2 0 0 9}$ & 49.2 & 37.3 & 13.5 \\
\hline $\mathbf{2 0 1 0}$ & 53.3 & 32.9 & 13.8 \\
\hline $\mathbf{2 0 1 1}$ & 51.0 & 35.8 & 13.2 \\
\hline $\mathbf{2 0 1 2}$ & 49.0 & 38.2 & 12.8 \\
\hline $\mathbf{2 0 1 3}$ & 50.8 & 32.3 & 16.8 \\
\hline
\end{tabular}

En base a los resultados presentados en http://sistemas02.minedu.gob.pe/consulta_ece/publico/index.php

Estas pruebas incluyen el contenido de Estadística y probabilidad según lo indicado en el Diseño Curricular Nacional 2009 para segundo grado, los temas se pueden ver en la siguiente imagen.

ESTADÍSTICA

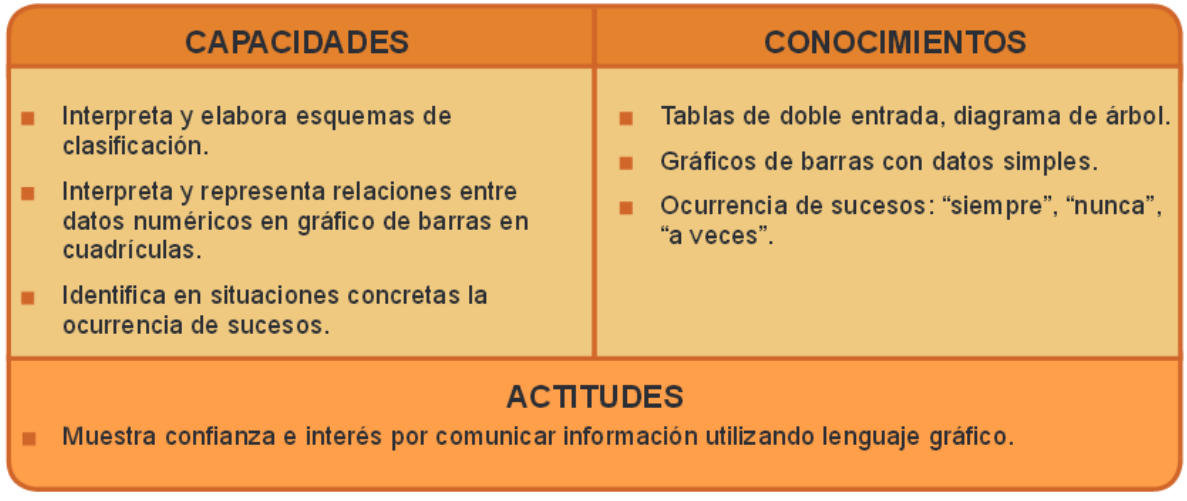

Que coinciden con los contenidos del nivel del Ciclo III del Mapa de progreso de Estadística y Probabilidad, 
A partir de los resultados de nuestra investigación acerca de los logros de los alumnos de cada grupo de medición para el Ciclo III obtenemos que los porcentajes de satisfactorios son:

\begin{tabular}{|c|c|c|c|}
\hline CICLO III & $\begin{array}{c}\text { Recopilación y } \\
\text { procesamiento de } \\
\text { los datos }\end{array}$ & $\begin{array}{c}\text { Interpretación y } \\
\text { valoración de los } \\
\text { datos }\end{array}$ & $\begin{array}{c}\text { Análisis de } \\
\text { situaciones de } \\
\text { incertidumbre }\end{array}$ \\
\hline $\begin{array}{c}\text { 4TO DE } \\
\text { PRIMARIA }\end{array}$ & $62.40 \%$ & $94.49 \%$ & $52.80 \%$ \\
\hline $\begin{array}{c}\text { 2DO DE } \\
\text { SECUNDARIA }\end{array}$ & $39.40 \%$ & $100.00 \%$ & $42.53 \%$ \\
\hline
\end{tabular}

Existe entonces en nuestra muestra un desarrollo de los contenidos estadísticos relacionados con el Ciclo III en los años posteriores al segundo grado de la primaria y este es más claro para los alumnos que actualmente se encuentran aún en el nivel primario. Pero este desarrollo se da más claramente en el aspecto de Interpretación de los datos, es decir, prácticamente todos los alumnos de nuestra muestra pueden leer e interpretar información desde tablas y gráficos a un nivel del Ciclo III. En los otros aspectos, de los alumnos que concluyeron el cuarto grado solo el $50 \%$ ha desarrollado satisfactoriamente los conocimientos en recopilación y procesamiento de datos y análisis de situaciones de incertidumbre. Mientras que en el caso de los alumnos que han concluido el segundo año de secundaria solo lo ha hecho un $40 \%$ en ambos aspectos.

Esto nos da una clara indicación que en la actualidad, la Estadística y la probabilidad, se están desarrollando más en la primaria que en años anteriores, pero a pesar del esfuerzo no se puede alcanzar aún los logros previstos en los momentos adecuados.

Como apoyar para que las metas sean alcanzadas en el momento justo, este por el momento es un esfuerzo que se encuentra en manos de los docentes. En este tema en particular el problema no solo radica en las estrategias de trabajo dentro del aula, sino todavía se está en una etapa de consolidación del dominio de estos temas por parte de los docentes. Hay varias investigaciones que nos hablan de este problema, en particular una de ellas lo indica como su resultado principal. "Estos resultados indica la necesidad de mejorar la formación de profesores en lo que respecta a conocimiento didáctico del contenido de estadística, en particular, con respecto al conocimiento de cómo los estudiantes aprenden y las dificultades que tienen con un determinado contenido matemático." (Arteaga, Batanero, Contreras, Cañadas, 2012,p. 141)

Nuestros pasos deben encaminarse ahora a apoyar la consolidación del conocimiento estadístico de los docentes de nuestro país y en ese sentido nuestros futuros trabajos se enfocarán, en conocer sus problemáticas más resaltantes y en buscar estrategias que permitan su superación. 
IV Encuentro sobre Didáctica de la Estadística, la Probabilidad y el Análisis de Datos

\section{Referencias}

[1] Arteaga, P., Batanero, C., Contreras, J., Cañadas, G. (2012) Evaluación del conocimiento de la estadística y los estudiantes en futuros profesores. Investigación en Educación Matemática XVI, 135 - 143

[2] Batanero,C. (2002). Los retos de la cultura estadística. Jornadas Interamericanas de Enseñanza de la Estadística, Buenos Aires. Conferencia inaugural. http://www.ugr.es/ batanero

[3] Batanero, C., Contreras, J. M. y Arteaga, P. (2011). El currículo de estadística en la enseñanza obligatoria. EM-TEIA. Revista de Educaçao Matematica e Tecnologica Iberoamericana. http://www.ugr.es/ batanero

[4] Cueva, J. e Ibañez, C. (2008, setiembre) Estándares en educación estadística: Necesidad de conocer la base teórica y empírica que los sustentan. Unión Revista Iberoamericana de Educacion matemática. Número 15, páginas 33 - 45. ISSN: 1815-0640

[5] Grima, P. (2010). Estadística: Enseñar y crear actitudes positivas a través de casos prácticos. Revista Iberoamericana de Educacion matemática. Número 24, páginas 11 - 26. ISSN: $1815-0640$

[6] Jimenez,L. y Jimenez, J. (2005, mayo). Enseñar probabilidad en primaria y secundaria? ¿Para qué y por qué?. Cidse-Revista virtual matemática- Educación e Internet, v6,n1. http://www.tec-digital.itcr.ac.cr/revistamatematica/contribuciones-v6-n1-may2005/artialeat/index.html

[7] IPEBA (2013). Mapas de Progreso del Aprendizaje. Matemáticas: Estadística y Probabilidad. InstitutoPeruano de Evaluación, Acreditación y Certificación de la Calidad de la Educación Básica. Hecho el Depósito Legal en la Biblioteca Nacional del Perú $N^{\circ}$ 2013-11912. ISBN 978-612-46406-4-3. en http://www.ipeba.gob.pe/estandares/MapasProgreso_Matematica_EstadisticaProbabilidad.p

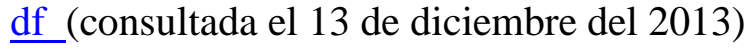




\title{
Desarrollo del Pensamiento Estadístico en Docentes de Educación Básica Regular mediante el Mapa de Progreso en Estadística y Probabilidad
}

\author{
Augusta Osorio Gonzales - Elizabeth Advíncula Clemente
}

\begin{abstract}
Resumen
Nuestra propuesta consiste en elaborar y aplicar talleres de capacitación para docentes de la Educación Básica Regular, que les permita desarrollar y ejercitar su pensamiento estadístico a la luz del Mapa de progreso en Estadística y Probabilidad elaborado dentro de los Estándares de Aprendizaje Nacionales. El mecanismo que estamos utilizando es el de bola de nieve, esto es, trabajamos con un grupo de docente cercanos o en relación con nuestra universidad por haber sido alumnos de algunos de nuestros diplomados y luego ellos nos van llevando con nuestra propuesta a sus propios planteles o ciudades. La invitación inicial se efectuó a 150 maestros de todo el país que han llevado en los últimos cinco años el Diplomado de Matemáticas para educación Primaria. Se les invitó a un taller gratuito en nuestra sede y a aquellos que estaban en provincias se les ofreció llevar el taller a sus ciudades. La propuesta incluye el acompañamiento y seguimiento de la implementación de las actividades propuestas por los docentes en sus instituciones educativas.
\end{abstract}

Palabras clave: Estadística, Mapa de progreso, talleres de capacitación

\begin{abstract}
In our country, the National Learning Standards are being developed using Learning Progress Maps as figure. Our proposal is to develop and implement training workshops for teachers of Basic Education, allowing them to develop and train their statistical thinking based on the Progress Maps in Statistics and Probability. The mechanism that we are using is the "snowball effect", It consists on working with a group of former students of our postgraduate school or teachers interested on our program, who after taking the course, promote the course proposal to their own cities. The invitation for the first course was extended to 150 teachers from all the country, who had taken part in previous courses of Mathematics teaching for elementary school on the last 5 years. The course was dictated in different headquarters, both Lima and provinces. The proposal includes the support and monitoring of the implementation of the activities proposed by the teachers in their educational institutions.
\end{abstract}

Keywords: Teaching, Progress Maps, Statistics, workshops

IREM-PUCP

Pontificia Universidad Católica del Perú

arosorio@ pucp.edu.pe - eadvincula@ pucp.edu.pe 


\title{
Uso de R para Mejorar el Aprendizaje de la Estadística en Alumnos Universitarios de Ciencias Sociales
}

\author{
MariCarmen González-Videgaray ${ }^{1}$, Rubén Romero-Ruiz ${ }^{2}$ y Nora del Consuelo Goris- \\ Mayans ${ }^{3}$
}

\section{Resumen:}

El aprendizaje de la estadística en el nivel universitario genera tanto problemas personales como institucionales. Por un lado, los estudiantes sufren con este tipo de asignaturas y llegan a desarrollar condiciones como la llamada "ansiedad estadística", que puede tener inclusive consecuencias fisiológicas en la salud de los individuos. Por otro lado, las altas tasas de reprobación en estas materias detienen el progreso de los alumnos y decrementan la eficiencia terminal. Esto suele ser aún más grave en carreras del área de Ciencias Sociales, ya que muchos estudiantes las eligieron por su bajo contenido de matemáticas y sienten temor ante la obligación de cursar este tipo de asignatura. Una forma de facilitar el acceso a la estadística es el trabajo en clase orientado a resolver problemas reales, de índole social. En este trabajo proponemos el uso de la metodología llamada "flip the classroom", junto con el tratamiento de situaciones con datos obtenidos directamente de la realidad, a través del entorno de computación y graficación estadística R, que es libre y gratuito. Los pasos principales son la formulación de un problema, la recolección de los datos, la selección del tratamiento estadístico, su aplicación en $\mathrm{R}$ y la interpretación de los resultados obtenidos. Se brindan las justificaciones para esta metodología y se discuten los resultados esperados.

Palabras clave: software libre, aprendizaje activo, entorno de programación, internet, web, aprendizaje basado en proyectos.

\begin{abstract}
:
Statistics learning at the higher education level creates problems both personal and institutional. For one side, students suffer with this kind of subjects and can develop conditions such as "statistics anxiety", which may even have physiological consequences on their health. On the other hand, high failure rates in these areas stop the progress of students and decrement terminal efficiency. This is usually more serious for students who major in Social Sciences, because many of them have chosen this area precisely for their low math, and are afraid of the obligation to take this kind of course. One way to facilitate statistics learning is by solving real problems at classroom, especially about social issues. We propose the use of the methodology called "flip the classroom" along with the treatment of data obtained directly from reality, through the $\mathrm{R}$ programming environment, which is free and open software. Key steps include problem formulation, data collection, statistical treatment selection, implementation in $\mathrm{R}$ and interpretation of the results. Justifications for this methodology are given and expected results are discussed.
\end{abstract}

Keywords: free software, active learning, programming environment, internet, web, project based learning.

Modalidad: Ponencia.

\footnotetext{
${ }^{1}$ Universidad Nacional Autónoma de México, Facultad de Estudios Superiores Acatlán, México, mcgv@unam.mx.

${ }^{2}$ Universidad Nacional Autónoma de México, Facultad de Estudios Superiores Acatlán, México, rubenr@unam.mx.

${ }^{3}$ Universidad Nacional Autónoma de México, Facultad de Estudios Superiores Acatlán, México, goris.nora@gmail.com.
} 


\section{Introducción}

\section{La enseñanza de la estadística}

En esta época en que se habla de la sociedad de la información y del conocimiento, la estadística forma parte de casi todos los planes de estudio de bachillerato, licenciatura y muchos del posgrado. Inclusive aparece en los niveles básicos. Se le reconoce como instrumento privilegiado del pensamiento científico y por lo tanto, se promueve su aprendizaje. Sin embargo, a pesar de múltiples y variadas iniciativas, su enseñanza continúa siendo poco exitosa e inclusive genera sufrimiento en algunos estudiantes.

La asignatura de estadística aparece en la mayoría de los planes de estudio de licenciaturas y posgrados. Esto se debe a su relevancia como instrumento para la investigación. La estadística guía la obtención y organización de los datos empíricos, así como su interpretación. Por ello, es indispensable su estudio en casi todas las áreas del conocimiento.

Muchos estudiantes han elegido ciertas áreas de estudio, en gran medida, porque tienen un bajo contenido de matemáticas. En México pocos alumnos eligen licenciaturas de ciencias y de ingeniería, porque han tenido malas experiencias con las matemáticas, entre otras razones. Esto ocasiona que para muchos estudiantes de áreas como las ciencias sociales, resulte difícil y atemorizador cursar la asignatura de estadística.

El problema es tan grave en algunos casos, que ha dado origen al término "ansiedad estadística", con síntomas tanto psicológicos como fisiológicos. La ansiedad estadística se ha definido como:

\footnotetext{
El desempeño caracterizado por preocupación extensa, pensamientos intrusivos, desorganización mental y excitación psicológica que se producen cuando una persona es expuesta a: contenidos, problemas, situaciones instruccionales o contextos evaluativos, relacionados con estadística, y comúnmente debilita la capacidad de logro en una gran variedad de situaciones psicológicas, ya que interfiere tanto en la manipulación de datos estadísticos como en la solución de problemas [1].
}

Onwuegbuzie y Wilson [2] realizaron una extensa revisión de la literatura sobre la condición de ansiedad estadística y detectaron que entre $66 \%$ y $80 \%$ de los estudiantes sufren de este problema. En particular, el $80 \%$ de los alumnos de posgrado presentan esta condición [3], que suele ser causa de procrastinación para graduarse. Es decir, la ansiedad estadística es un problema mayoritario entre los estudiantes, tanto de licenciatura como de posgrado.

La ansiedad estadística también parece ser la causa más importante por la cual muchos alumnos no concluyen sus investigaciones [4], aunque en esta actividad también intervienen problemas relacionados con la escritura académica y el uso de fuentes documentales. Esta situación es grave, puesto que la estadística es una asignatura que se estudia, de manera generalizada, en la educación media superior y superior, sobre todo por su estrecha relación con los métodos de investigación cuantitativos. Los alumnos de ciencias sociales requieren de un manejo adecuado de la estadística para obtener datos, organizarlos, presentarlos e interpretarlos. 
Pero no sólo eso, el pensamiento estadístico [5] y la cultura estadística son requisitos esenciales para la vida y la ciudadanía en el siglo 21. El pensamiento estadístico incluye aspectos como: comprender cabalmente los conceptos estadísticos clave; desarrollar la habilidad para explorar datos y obtener conclusiones a partir de ellos, así como elaborar argumentaciones basadas en la estadística [6]. Estas habilidades son necesarias para construir razonamientos válidos en muchas circunstancias que involucran el manejo de datos, gráficas o tablas. Prácticamente cualquier persona requiere tomar decisiones basadas en datos e información. Por lo tanto, el pensamiento estadístico es esencial para que estas decisiones sean correctas y permitan obtener beneficios, tanto personales como sociales.

Por su parte, la cultura estadística es un elemento que aparece hoy en día con gran frecuencia en el discurso mediático [7]. Gran parte de la información que se difunde en la televisión, radio, periódicos, revistas o internet, conlleva datos estadísticos. Se suelen presentar con frecuencia, por ejemplo, resultados de encuestas o de supuestas investigaciones científicas. Las personas deben determinar qué afirmaciones pueden considerarse válidas y cuáles no. La cultura estadística es indispensable para juzgar este tipo de discurso que, además, suele dar la apariencia de veracidad por el uso que hace de términos técnicos que no necesariamente son bien conocidos para las personas. Schmit [7] señala que la cultura estadística no sólo implica contar con conocimientos matemáticos, sino tener un pensamiento crítico, la capacidad de argumentar y persuadir con elementos válidos, así como interpretar estadísticas dentro de algún contexto particular. Como puede verse, la cultura estadística implica generar un pensamiento de orden superior. Por ello, algunos modelos de enseñanza como el modelo Melbourne [8] intentan, en primera instancia, convencer a los estudiantes de la relevancia que tiene adquirir una cultura estadística, tanto para sus proyectos académicos, como para su vida personal.

Adicionalmente, es notable cómo los organismos gubernamentales y no gubernamentales colocan cada vez más sus datos de manera accesible al público, en formatos que pueden ser procesados de manera estadística. Antiguamente se presentaban sólo como documentos de texto ya trabajados e interpretados. Esto es importante en las sociedades democráticas [9], puesto que permite a los ciudadanos ejercer cabalmente su derecho a la información, siempre y cuando posean realmente una cultura estadística. Los ciudadanos pueden usar los datos y construir sus propias interpretaciones. Por esta razón países con alto desarrollo como Australia han elaborado una "Declaración Estadística" [10] para lograr una sociedad informada en el siglo 21. El último párrafo de esta declaración indica que:

La habilidad para entender y evaluar estadísticas que permean nuestras vidas cotidianas es un bloque esencial dentro de las habilidades numéricas básicas. Mejorar las habilidades estadísticas debe ser parte del currículum de todos los niveles educativos, así como los programas de capacitación en el gobierno, los negocios y toda la comunidad [10].

Por todo lo anterior, resulta clara la importancia de promover que en todas las naciones se cuente con un pensamiento estadístico y una cultura estadística, entre toda la población. El principal enemigo de esta meta es la ansiedad estadística, que debe combatirse de manera frontal. Ante todo, debe tenerse conciencia de este problema y extender su difusión a todos los involucrados en la enseñanza de la estadística, tanto en ciencias sociales como en otras áreas. En segunda instancia, debe estudiarse y comprenderse este fenómeno, a través de la 
descripción y análisis de sus causas. Esto dará origen a mejores soluciones para mejorar la enseñanza de la estadística en las ciencias sociales.

\section{La ansiedad estadística}

La ansiedad estadística, de manera lógica, está relacionada con la "ansiedad matemática" [1] que es una condición aún más extensa y estudiada, que generalmente ocurre desde niveles básicos de educación, con sus propias características y causas. La ocurrencia de ansiedad matemática es un predictor de la ansiedad estadística. En gran medida, el miedo que tienen los estudiantes a las asignaturas de estadística está relacionado con una historia de confrontación poco exitosa con las matemáticas. Muchos de ellos han huido de carreras con contenido matemático y enfrentan, con diferentes niveles de angustia, la necesidad de cursar al menos una asignatura de estadística que aparece casi en todas las licenciaturas y posgrados.

Pero la ansiedad estadística no es igual a la ansiedad matemática ni es sólo causada por este tipo de problemas. Algunos autores han considerado que: el auto concepto que tiene un estudiante de sus habilidades matemáticas y computacionales [11], su autoestima y su nivel de perfeccionismo y el temor a fallar [2, 12], así como el miedo a los instructores [13], son también predictores importantes de la ansiedad estadística. Como hemos dicho, la estadística implica el pensamiento crítico, la elaboración de argumentos sustentados en hechos y razonamientos lógicos, así como el manejo de elementos gráficos como histogramas, diagramas de tallo y hoja, diagramas de dispersión, curvas normales, entre muchos otros.

Además, la estadística hoy en día requiere en gran medida del manejo, al menos rudimentario, de bases de datos y de programas computacionales. Si bien es posible hacer ejercicios sencillos con lápiz y papel o en el pizarrón, para efectuar análisis interesantes de datos reales casi siempre es indispensable contar con buenas bases de datos en algún formato digital, para luego explotarlas a través de un software de propósito específico. Algunas personas se sienten intimidadas ante bases de datos que parecen almacenadas en formatos poco intuitivos y amables. Más aún si los datos están en formato de texto plano, en el que son prácticamente ininteligibles, a menos que sean leídas, en forma correcta, por un software apropiado. Asimismo, el uso de paquetes estadísticos se suele asociar con procesos difíciles y complicados. La mera presentación de las interfaces de programas estadísticos puede causar a los alumnos una sensación de agobio por la cantidad de opciones que contienen.

En muchas ocasiones los cursos dan por hecho que los estudiantes son capaces de comprender y usar bases de datos. A veces se pide el uso de paquetes estadísticos sin dar suficiente explicación acerca de cómo se deben usar. Los alumnos suelen aprender los procedimientos de manera mecánica, tratando de dar gusto al profesor, sin tener una idea clara de qué debe hacerse y por qué. Uno de los primeros problemas para un alumno puede ser cómo encontrar una base de datos, cómo saber qué datos contiene y qué puede hacer con esos datos. Asimismo, al abrir la aplicación estadística, la multitud de opciones puede confundirlo y agobiarlo. 
También resulta difícil para los alumnos enlazar los datos, las medidas resumen como media o dispersión, las gráficas o las relaciones, con un discurso interesante y bien argumentado. Muchos cursos de estadística descontextualizan los problemas, convirtiéndolos en meros ejercicios matemáticos aislados, con lo cual se refuerza la tendencia a seguir pasos y procedimientos, sin saber por qué deben hacerse así y hacia dónde conducen. Así, el estudiante no aplica un verdadero pensamiento crítico, sólo memoriza secuencias de actividades.

Por otra parte, efectivamente existe un elemento de temor al docente o inclusive a los propios compañeros. El alumno no se siente en confianza para exponer sus dudas, porque teme ser atacado o ridiculizado, cosa que muchas veces sucede. Los profesores suelen sentirse presionados por concluir el temario y sienten molestia o estrés ante preguntas reiteradas de aspectos que ya se comentaron en clase. Muchos estudiantes sienten angustia de verse menos eficientes que sus compañeros y, si esto se generaliza, nadie hará preguntas en el grupo, lo cual dará la impresión de que todos entienden y siguen el ritmo de la clase.

También existe el miedo a la evaluación y la consiguiente calificación. En general, la calificación tiene consecuencias prácticas como aprobar o reprobar una asignatura, continuar o no un curso, graduarse o no. Además, la evaluación pone en tela de juicio el valor y el rendimiento del estudiante. Todo esto contribuye a rodear a la estadística de un ambiente poco propicio para el aprendizaje confortable.

\section{Cómo reducir la ansiedad estadística}

Como puede verse, la ansiedad estadística es un problema grave, generalizado y multifactorial. Por ende, el tratamiento no parece evidente y seguramente implica un conjunto de estrategias, más que una solución única. Por la importancia de este problema, muchos autores han propuesto diversos acercamientos interesantes. Entre ellos podemos citar los siguientes:

- Orientar el estudio de la estadística más a las aplicaciones prácticas [14] que a los desarrollos teóricos. Suele ser mucho más motivante aprender algo cuando se entiende cuál es el objetivo final de este aprendizaje. Los cálculos y las gráficas no son relevantes por sí mismos, sino por la información que producen y que puede ayudar a tomar buenas decisiones. Orientar la enseñanza a este tipo de aplicaciones puede incrementar la motivación de los alumnos.

- Promover que los docentes pongan cuidado especial en evitar la ansiedad estadística de sus alumnos [15]. El primer paso es, por supuesto, hacer conciencia entre los docentes de la existencia de la ansiedad estadística y de sus principales causas. Con sólo esta conciencia el profesor puede matizar y regular sus acciones, para evitar aquéllas que generan angustia en los alumnos. También puede ser conveniente hablar de este problema de manera abierta con los alumnos, hacerles saber que es un problema generalizado y que hay muchas formas de atacarlo. Esto suele darles un poco de tranquilidad y facilita el aprendizaje.

- Resolver sobre todo problemas y ejercicios relacionados con la vida cotidiana de los estudiantes [14]. Esto también contribuye a la motivación, hace más interesante la solución y facilita la comprensión tanto de los conceptos como de los procedimientos. 
- Enseñar con calma, a buen ritmo [14]. Esta idea es esencial. Gran parte del problema de los estudiantes es que perciben la carga de trabajo como excesiva y angustiante. El profesor pasa al siguiente tema cuando el primero no ha sido asimilado de manera correcta. Tal vez sea más conveniente revisar menos contenidos con más calidad, que apurar el paso para cubrir más temas, sin que el alumno pueda digerirlos de manera adecuada.

- Usar acercamientos novedosos y lúdicos. Por ejemplo, un grupo de autores ha planteado historias de detectives cortas para exponer temas de introducción a la estadística [16]. En este interesante trabajo, los autores idearon una serie de narraciones con personajes de ficción atractivos, que buscan explicar conceptos en forma divertida y sin elementos atemorizantes. Hacer este tipo de materiales es una excelente idea, pero requiere de un gran trabajo por parte de los docentes. Existen alternativas de gran calidad que pueden conseguirse en librerías, como "Conned Again, Watson”, de Colin Bruce, escrito en inglés, editado por Basic Books en 2001 y disponible en edición Kindle.

- Usar formas de presentación variadas y no matemáticas [8]. Esta sugerencia es consistente con la anterior. En algunos cursos de estadística se abusa de las demostraciones matemáticas y los procedimientos algebraicos, que no son estrictamente necesarios para que los estudiantes, sobre todo de ciencias sociales, puedan hacer bueno uso de la estadística y desarrollar una cultura al respecto. Además, esto ayuda a remontar el problema de la ansiedad matemática inherente.

En resumen, resulta de interés promover un acercamiento a la estadística que contemple, de manera integral y estratégica, todas las sugerencias anteriores.

\section{Objetivo del trabajo}

Por todo lo anterior, este trabajo tiene como objetivo presentar una alternativa para enseñar la estadística en las ciencias sociales a través de la metodología conocida como voltear la clase o "flip the classroom" [17]. Esta metodología, en resumen, pretende que en el salón de clase se realicen las actividades que tradicionalmente se dejan como tarea a los estudiantes, mientras que la explicación oral se deja como tarea. Para poder llevar a cabo esta metodología de manera cabal, es indispensable contar con el material apropiado, en algún soporte que permita que los alumnos dispongan de él sin problema, de preferencia en cualquier lugar y momento.

Para ello, se ha desarrollado una estrategia didáctica consistente en un libro de texto breve que relaciona pequeños textos literarios con temas estadísticos, y que promueve la solución de ejemplos reales cercanos, a través del manejo de Excel y del software libre R. A continuación se expone brevemente la propuesta en la sección de Material y Método. Actualmente se cuenta ya con el material de apoyo y las bases de datos. El software $\mathrm{R}$ y su interfaz RStudio, son de acceso libre y gratuito. El siguiente paso será realizar la investigación empírica que se describe en este documento. 


\section{Material y Método}

\section{Participantes}

Se tomará como muestra un grupo de estudiantes de la asignatura de estadística en el área de ciencias sociales, dentro de la Facultad de Estudios Superiores Acatlán, de la Universidad Nacional Autónoma de México. Se trata de una muestra de conveniencia, obtenida de la universidad pública más grande y más importante del país.

\section{Instrumentos}

Para medir la ansiedad estadística, se utilizará una traducción y adaptación del Statistical Anxiety Scale (SAS), desarrollado por Andreu Vigil-Colet, Urbano Lorenzo-Seva y Lorena Condon [18], con las consideraciones de Carmona Márquez [19] y las modificaciones pertinentes para el grupo de alumnos mexicanos, en el nivel de educación superior, en el área de ciencias sociales. Este inventario tiene un conjunto de 24 oraciones positivas que miden tres sub escalas: a) Ansiedad ocasionada por someterse a un examen; b) Ansiedad ocasionada por demandar ayuda; c) Ansiedad ocasionada por interpretar resultados.

Para la intervención pedagógica se utilizarán:

a) La metodología "flip the classroom", que requiere de un apoyo sustancial para que los estudiantes puedan tener acceso a los contenidos temáticos fuera del salón de clase, dejando el tiempo de clase para la resolución guiada y acompañada de problemas tipo. El apoyo didáctico se dará a través de un libro de texto que comprenderá los temas a revisar y los problemas tipo.

b) Como libro de texto se utilizará "Alicia en el País de las Estadísticas con $\mathrm{R}$ y Excel”, de MariCarmen González-Videgaray, ya concluido y en proceso de publicación. Este material ha sido elaborado con la visión de las estrategias ya mencionadas en la introducción y contempla las secciones mostradas en la figura 1.

c) Para el tratamiento de los ejemplos reales se utilizará el software Excel de Microsoft, así como el entorno de programación R [20], acompañado de su interfaz RStudio [21]. Los dos últimos son libres, gratuitos y pueden descargarse de internet, de los sitios señalados en las referencias.

d) Como fuentes de información se usarán bases de datos reales y confiables, disponibles vía internet, tales como INEGI [22], Banco de México [23], Banco Mundial [24], Organización Mundial de la Salud [25], entre otras.

\section{Procedimiento}

Se aplicará un procedimiento pre-test, post-test para medir los niveles de ansiedad estadística antes y después de la intervención pedagógica. Al inicio del semestre, se aplicará el cuestionario SAS, de manera anónima pero identificando a cada participante con un número que permitirá el seguimiento. El cuestionario se aplicará de manera electrónica, a través del complemento Questionnaire del ambiente virtual de aprendizaje Moodle. Este software permite la generación automática de bases de datos.

Se desarrollará el curso de estadística durante dos meses con apoyo del texto "Alicia en el País de las Estadísticas con R y Excel", poniendo énfasis en la solución de problemas con 
datos reales y con auxilio tanto del software libre R, como del entorno de programación, también libre, RStudio. Asimismo, se utilizará Excel para manejar bases de datos y también para resolver problemas estadísticos tipo.

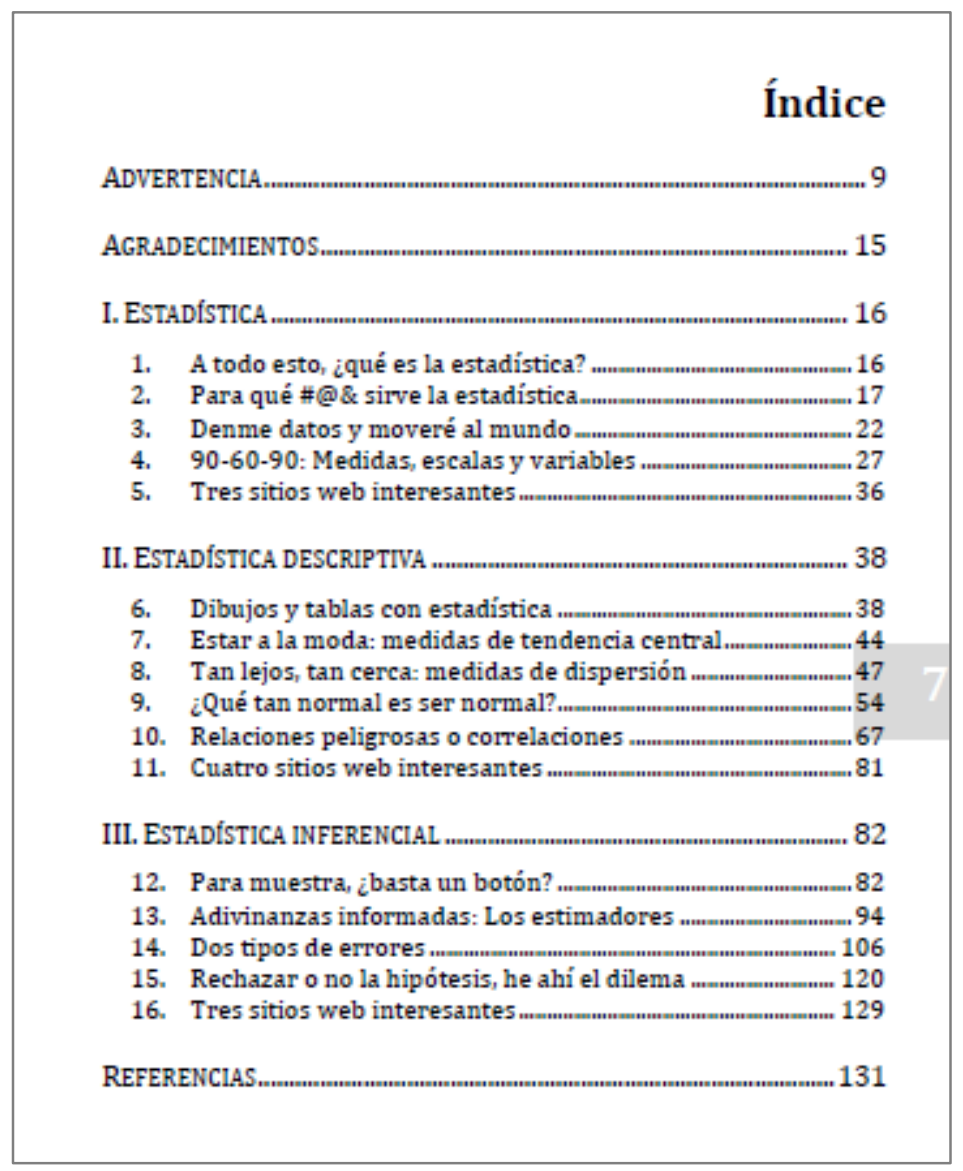

Figura 1: Índice del libro “Alicia en el país de las estadísticas con R y Excel”. Fuente: elaboración propia.

Al final de los dos meses, se aplicará nuevamente el cuestionario mencionado. Se compararán los resultados en los dos momentos utilizando pruebas de hipótesis para comparar dos proporciones y para comparar dos medias.

\section{Estadísticas}

Las bases de datos generadas serán almacenadas en hojas con formato CSV y los datos se procesarán con el software estadístico R. Se obtendrán estadísticas descriptivas y se efectuará una prueba t de comparación de medias pareadas.

\section{Resultados}

Se espera que los estudiantes modifiquen de manera favorable sus actitudes hacia la estadística después de promover el aprendizaje de esta asignatura a través de la intervención pedagógica ya descrita, que considera las estrategias que pretenden disminuir o evitar la ansiedad estadística. 
La idea fundamental detrás de la metodología "flip the classroom" es promover un aprendizaje activo, donde el estudiante construya su conocimiento y no únicamente repita procedimientos o conceptos. También se busca que el alumno esté convencido del valor de su aprendizaje, de modo que su objetivo, más allá de aprobar una asignatura, sea apropiarse de la estadística como instrumento para mejorar su pensamiento y su cultura.

\section{Discusión}

Consideramos que parte del problema que genera la ansiedad estadística es la falta de una estrategia didáctica integral, que incorpore, de manera crítica y reflexiva, el conjunto de alternativas que se han planteado para esta condición. Es posible que un acercamiento más completo ayude a los estudiantes a superar esta situación que es compleja y multifactorial.

Para ello, se plantea como metodología para la intervención didáctica la metodología "flip the classroom", que tiene la ventaja sustancial de promover un aprendizaje activo. En un importante y reciente artículo, Freeman et al. [26] insisten en que el aprendizaje activo es mucho más eficiente que las clases tipo conferencia, para el área de ciencias, matemáticas, ingeniería y tecnología. Básicamente se trata de que el estudiante construya su aprendizaje a través de hacer cosas, y no tanto de escuchar o ver exposiciones del profesor. De hecho, los autores sugieren que debe cambiarse la enseñanza basada en conferencias o exposiciones orales por parte del profesor, a una metodología donde el alumno tenga el papel principal. El profesor debe convertirse en una especie de acompañante o apoyo, lo más personalizado posible. Estas ideas son consistentes con el acercamiento a través de voltear la clase o "flipping the classroom".

Uno de los problemas principales para convertir estas ideas en realidad, es contar con los materiales didácticos apropiados y suficientes para sustituir la exposición oral del profesor. En ese sentido, consideramos que nuestra propuesta puede ser útil para la comunidad de habla hispana, una vez que se pruebe su eficacia en la investigación empírica.

Otro de los problemas reales en la enseñanza de la estadística, es el costo elevado de los paquetes estadísticos tradicionales como SPSS, SAS, Statgraphics o Statistica. Estos paquetes son sumamente poderosos y ofrecen interfaces atractivas e intuitivas. Sin embargo, resulta difícil para muchas instituciones y personas contar con esta posibilidad, debido a su alto costo. Nosotros proponemos un uso combinado de Excel con el entorno de programación R y la interfaz RStudio. Si bien Excel es un software comercial, su costo es accesible, su difusión es amplia y la mayoría de los estudiantes están familiarizados con él. Al complementarlo con $\mathrm{R}$, se puede contar con una herramienta estadística de gran potencia.

Probablemente la mayor dificultad al utilizar $\mathrm{R}$ es que su ambiente implica la escritura de instrucciones en texto plano. Parte de nuestra hipótesis es que, al redactar estas pequeñas frases, el estudiante tendrá una idea más clara de qué es lo que pretende hacer. Los paquetes estadísticos sofisticados muchas veces promueven que el estudiante oprima botones y seleccione de menús, de manera poco reflexiva, sin saber exactamente qué desea hacer y para qué. Creemos que puede ser interesante este acercamiento distinto, donde la interfaz prácticamente no ofrece nada, sino que el usuario debe especificar exactamente qué es lo que requiere. 
Por otro lado, R cuenta con una muy amplia documentación y un ecosistema con más de 5, 779 paquetes para resolver problemas y temas muy específicos [20]. Dentro del texto que se propone como guía para la intervención didáctica se explica cómo tener acceso a todos estos beneficios, cómo localizar información sobre $\mathrm{R}$ y cómo instalar paquetes. Además, $\mathrm{R}$ puede instalarse y utilizarse dentro de cualquier equipo, con cualquier sistema operativo. Inclusive puede funcionar en tabletas y en teléfonos inteligentes tipo iPhone.

Si los estudiantes aprenden a manejar el software estadístico libre R, podrán hacer uso de estos datos reales y obtener de ellos conclusiones interesantes, a través de procedimientos descriptivos o inferenciales como: tablas, gráficas, regresiones, estimaciones, intervalos de confianza o pruebas de hipótesis.

Consideramos que esta propuesta es factible y puede mejorar el resultado final de los estudiantes, ya que algunos autores han encontrado una relación curvilínea decreciente entre del desempeño en exámenes de estadística contra la ansiedad estadística [27]; es decir, si logramos disminuir la ansiedad estadística, el aprendizaje podrá ser placentero y las calificaciones serán mejores.

\section{Conclusiones}

Es indispensable que los educadores desarrollen formas de motivar a los estudiantes para el aprendizaje de la estadística y para utilizar software apropiado. Una de las formas de hacer esto es contar con una herramienta accesible para todos y sencilla de utilizar. Si bien el ambiente de programación R tiende a producir algún temor en los estudiantes, debido a que su funcionamiento es a través de instrucciones que deben escribirse de manera textual en la pantalla de la computadora, consideramos que es una herramienta atractiva para este propósito. Si se logra que los estudiantes adquieran una sensación de auto-eficacia en la resolución de problemas significativos, será posible avanzar en este ámbito [28]. También es fundamental lograr un ambiente donde todos, alumnos y profesor, puedan estar relajados [29] y disfruten de la aplicación de los procedimientos [30].

\section{Referencias}

[1] ZEIDNER, M. Statistics and mathematics anxiety in social science students: Some interesting parallels. British Journal of Educational Psychology. 1991; 61(3):319328.

[2] OnwuegbuzIE, A. J. \& Wilson, V. A. Statistics Anxiety: Nature, etiology, antecedents, effects, and treatments--a comprehensive review of the literature. Teaching in Higher Education. 2003; 8(2):195-209.

[3] OnwUEGbuZIE, A. J. Academic procrastination and statistics anxiety. Assessment \& Evaluation in Higher Education. 2004; 29(1):3-19.

[4] OnwUEGBUZIE, A. J. Writing a research proposal: The role of library anxiety, statistics anxiety, and composition anxiety. Library \& Information Science Research. 1997; 19(1):5-33.

[5] Meder, B. \& Gigerenzer, G. Statistical thinking: no one left behind. Probabilistic Thinking: Springer 2014:127-148. 
[6] Pfannkuch, M. \& Ben-Zvi, D. Developing teachers'statistical thinking. Teaching statistics in school mathematics-challenges for teaching and teacher education: Springer 2011:323-333.

[7] Schmit, J. Teaching Statistical Literacy as a Quantitative Rhetoric Course. American Statistical Association Joint Statistical Meetings, Vancouver, Canada. 2010

[8] Finch, S. \& Gordon, I. Statistical literacy for all: Teaching Critical thinking with data. Proceedings of The Australian Conference on Science and Mathematics Education (formerly UniServe Science Conference). 2011

[9] Arteaga, P., Batanero, C., Cañadas, G. \& Contreras, J. M. Las tablas y gráficos estadísticos como objetos culturales. Números. 2011; 76:55-67.

[10] NSS. Statistical Declaration:. 2008. [Fecha de consulta: 2014/29/07.] Disponible en:

http://www.nss.gov.au/nss/home.nsf/NSS/BF58B79DECE73EDFCA25750C00803 46F?opendocument

[11] BALOĞLU, M. Individual differences in statistics anxiety among college students. Personality and Individual Differences. 2003; 34(5):855-865.

[12] Onwuegbuzie, A. J. \& Daley, C. E. Perfectionism and statistics anxiety. Personality and Individual Differences. 1999; 26(6):1089-1102.

[13] OnwuegbuZIE, A. J. Statistics anxiety and the role of self-perceptions. The Journal of Educational Research. 2000; 93(5):323-330.

[14] Pan, W. \& TAng, M. Students' Perceptions on Factors of Statistics Anxiety and Instructional Strategies. Journal of Instructional Psychology. 2005; 32(3):205-214.

[15] PAN, W. \& TANG, M. Examining the Effectiveness of Innovative Instructional Methods on Reducing Statistics Anxiety for Graduate Students in the Social Sciences. Journal of Instructional Psychology. 2004; 31(2):149-159.

[16] D'ANDREA, L. \& WATERS, C. Teaching statistics using short stories: Reducing anxiety and changing attitudes. Sixth International Conference on Teaching Statistics, Cape Town, South Africa. 2002

[17] Bergmann, J. \& SAMS, A. Flip Your Classroom: Reach Every Student in Every Class Every Day: International Society for Technology in Education 2012.

[18] Vigil-Colet, A., Lorenzo-Seva, U. \& Condon, L. Development and validation of the Statistical Anxiety Scale. Psicothema. 2008; 20(1):174-180.

[19] CARMONA MÁrQueZ, J. Una revisión de las evidencias de fiabilidad y validez de los cuestionarios de actitudes y ansiedad hacia la estadística. Statistics Education Research Journal. 2004; 3(1):5-28.

[20] Gentleman, R. \& IhaKA, R. R Project. 2014. [Fecha de consulta: 2014/29/07.] Disponible en: http://www.r-project.org/

[21] Allaire, J. J. RStudio. 2014. [Fecha de consulta: 2014/29/07.] Disponible en: http://www.rstudio.com/

[22] INEGI. Instituto Nacional de Estadística y Geografía,. 2014. [Fecha de consulta: 2014/29/07.] Disponible en: http://www.inegi.org.mx/

[23] MéXico, B. D. Banco de México. 2014. [Fecha de consulta: 2014/29/07.] Disponible en: http://www.banxico.org.mx/

[24] The World BANK. Mexico | Data. 2014. [Fecha de consulta: 2014/04/26.] Disponible en: http://data.worldbank.org/country/mexico 
[25] Organization, W. H. Global Health Observatory (GHO). 2014. [Fecha de consulta: 2014/05/05.] Disponible en: http://www.who.int/gho/en/

[26] Freeman, S., Eddy, S. L., Mcdonough, M., Smith, M. K., Okoroafor, N., JORDT, H., et al. Active learning increases student performance in science, engineering, and mathematics. Proceedings of the National Academy of Sciences. 2014:201319030.

[27] KeEley, J., ZAYAC, R. \& CORREIA, C. Curvilinear relationships between statistics anxiety and performance among undergraduate students: Evidence for optimal anxiety. Statistics Education Research Journal. 2008; 7(1):4-15.

[28] Hsu, M. K., WANG, S. W. \& CHIU, K. K. Computer attitude, statistics anxiety and self-efficacy on statistical software adoption behavior: An empirical study of online MBA learners. Computers in Human Behavior. 2009; 25(2):412-420.

[29] Schacht, S. \& StewART, B. J. What's funny about statistics? A technique for reducing student anxiety. Teaching sociology. 1990:52-56.

[30] FORTE, J. A. Teaching statistics without sadistics. Journal of Social Work Education. 1995; 31(2):204-218. 


\title{
Argumentos utilizados por profesores de matemática para explicar conceptos asociados a la idea de aleatoriedad
}

\author{
Liliana Tauber ${ }^{1}$ \\ Resumen
}

En 2014, se ha aplicado una reforma en el nivel Secundario en Argentina, que exige modificaciones en la formación de los profesores de Matemática respecto de la Educación Estocástica. Con el objeto de contribuir a dicha formación, en este trabajo se analizan las respuestas de profesores de Matemática en ejercicio a una actividad utilizada en otras investigaciones sobre percepción subjetiva de la aleatoriedad. Los resultados muestran una mezcla de argumentos correctos e incorrectos, en los que se pueden percibir distintas creencias y sesgos. Este análisis podría servir de fundamento a la hora de elaborar actividades que puedan incluirse en los cursos de formación de profesores de Matemática que son los encargados de enseñar Estadística a nivel Secundario.

Palabras Clave: Educación Estocástica, Profesores de Matemática, aleatoriedad.

\begin{abstract}
In 2014, a reform has been implemented in the Secondary level in Argentina, which requires changes in the training of Mathematics teachers regarding the Stochastic Education. In order to contribute to such training, in this paper the responses of teachers of Mathematics, in an activity used in other research on subjective perception of randomness, are discussed. The results show a mixture of correct and incorrect arguments, which can be perceived different beliefs and biases. This analysis could serve as a basis when developing activities that may be included in the training of Mathematics teachers who are responsible for teaching statistics to Secondary level.
\end{abstract}

Key Words: Stochastic Education, Mathematics Teachers, Randomness.

\section{Modalidad: Ponencia}

\footnotetext{
${ }^{1}$ Facultad de Humanidades y Ciencias - Universidad Nacional del Litoral - Santa Fe - Argentina - mail: estadisticamatematicafhuc@gmail.com
} 


\title{
Combinatoria: ¿Por Qué Es Tan Difícil Para Algunos?
}

\author{
Víctor Hugo Vázquez Guevara ${ }^{1}$, Hugo Adán Cruz Suárez ${ }^{2}$, Francisco Solano Tajonar \\ Sanabria ${ }^{3}$, Fernando Velasco Luna ${ }^{4}$ y Hortensia Reyes Cervantes ${ }^{5}$
}

\begin{abstract}
Resumen
La combinatoria es una rama de la matemática muy útil cuando se trabaja con variables aleatorias discretas en el curso inicial de Probabilidad. En este trabajo se discutirá su campo de estudio, así como algunos problemas clásicos que pueden resolverse gracias a ella. Además, se presenta una discusión de algunas causas que pueden contribuir a que las técnicas y metodologías de la combinatoria no sean tan asimilables al primer contacto para algunos estudiantes tales como los son la falta de madurez matemática, la ausencia de una verdadera asimilación de los principios básicos de conteo, falta de imaginación y la insistencia de tratar de hallar reglas mecánicas para resolver problemas enumerativos; por citar algunas.
\end{abstract}

Palabras clave: Combinatoria, Probabilidad, Variable aleatoria discreta..

\begin{abstract}
.
Combinatorics is a very useful field of mathematics when one works with discrete random variable in the initial course of Probability. In this paper its range of action will be discussed as well as some classical problems which can be solved thanks to it. In addition, a discussion about some causes which may contribute to the hard assimilation of its techniques and methodologies such as lack of mathematical maturity, the absence of a true assimilation of the counting principles, lack of imagination and the insistence of finding mechanical rules for solving enumerative problems, is presented.
\end{abstract}

Keywords: Combinatorics, Probability, Discrete Random Variable

Modalidad: Ponencia.

\footnotetext{
${ }^{1}$ Benemérita Universidad Autónoma de Puebla. México, vvazquez@ @cfm.buap.mx

${ }^{2}$ Benemérita Universidad Autónoma de Puebla. México, hcs@fcfm.buap.mx

${ }^{3}$ Benemérita Universidad Autónoma de Puebla. México, ftajonar@fcfm.buap.mx

${ }^{4}$ Benemérita Universidad Autónoma de Puebla. México, fvelasco@fcfm.buap.mx

${ }^{5}$ Benemérita Universidad Autónoma de Puebla. México, hreyes@fcfm.buap.mx
} 


\section{Introducción.}

La combinatoria es una rama de las matemática cuyo nacimiento y desarrollo ha estado vinculado al de otras ramas tales como el Álgebra, Teoría de números y Probabilidad. Pertenece al área de las Matemáticas Discretas y estudia le enumeración, construcción y existencia de propiedades de configuraciones que satisfacen ciertas condiciones establecidas. En particular, la combinatoria enumerativa ó enumeración estudia los métodos para contar las distintas configuraciones de los elementos de un conjunto que cumplan ciertos criterios especificados. Ésta fue una de las primeras áreas de la combinatoria en ser desarrollada, quizás es por esto que cuando se habla de la combinatoria enumerativa se hace por medio del término combinatoria. Otras sub áreas de la combinatoria son: Teoría de particiones, Teoría de Gráficas, Teoría del diseño, Geometría finita, Teoría del orden por citar algunas.

\subsection{Un poco de Historia.}

Posiblemente el antecedente más antiguo de un problema de enumeración es el ofrecido por el libro I Ching que es el antiguo libro chino de las mutaciones (1200 a.C.) [1], se cree que describe la situación presente de quien lo consulta y predice el modo en que se reoslverá el futuro se adopta ante ella la posición correcta. Los símbolos que se consultan en este libro son llamados hexagramas y son formados por 6 líneas cada una de las cuales puede contener a algunos de dos símbolos; yin (línea interrumpida) y yang (línea sólida), el problema en este caso es el de saber el número de símbolos diferentes que pueden escribirse. En la siguiente figura se encuentran los 64 hexagramas posibles:

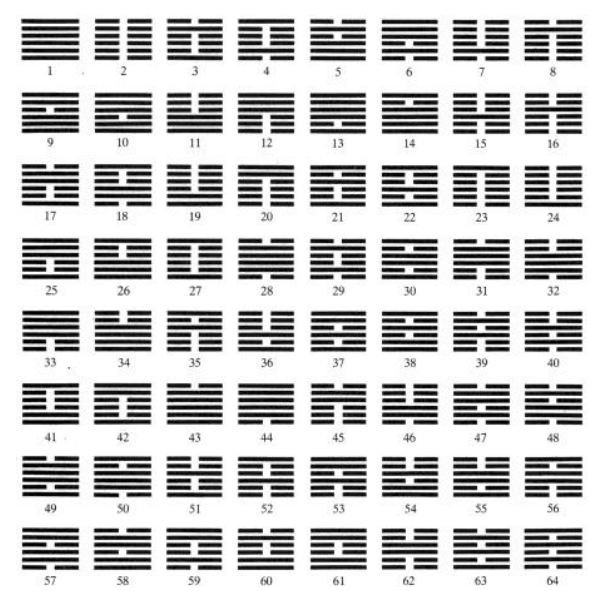

Figura 1. Hexagramas del I Ching

Otro antecedente procedente del mundo antiguo se encuentra en el problema 79 del papiro de Rhind (s. VII a.C.): Había un propiedad compuesta por 7 casa, cada casa tiene 7 gatos, cada gato se come 7 ratones, cada ratón se come 7 gramos de cebada, cada grano de cebada produce 7 medidas. ¿Cuánto suma todo?.

En el siglo VI a.C. el médico hindú Sushruta afirmaba que podía hacer 63 combinaciones con 6 ingredientes diferentes al tomar uno a la vez, dos a la vez, tres a la vez, etc. 
Existen otros problemas del mundo antiguo y del Medioevo sobre todo de índole religioso cuya solución está relacionada con conteo pueden hallarse en la literatura, en particular en el libro de Robin Wilson.

El filósofo y astrónomo Rabbi Abraham Ezra en 1140 estableció la simetría de los coeficientes binomiales pero fue Levu ben Gerson quien obtuvo una formula cerrada para éstos en 1321.

Ya en el siglo XVII, Frans van Schooten, Blaise Pascal y Marin Mersenne trabajaron en el llamado triángulo de Pascal de manera formal desde distintos puntos de vista; combinatorio, algebraico, musical. Sin embrago, se conocen versiones anteriores de este triángulo; por ejemplo, Al-Kraji en 1007, Zhu Shijie en 1303, Ibn Munim en 1303, Cardano en 1570, Tartaglia en 1556.

Es en este periodo que la palabra combinatoria hace su aparición con el trabajo Dissertatio de Arte Combinatoria de Wihem Leibniz en el que aborda entre otras cosas los problemas de permutaciones y combinaciones.

Jacob Bernoulli, al establecer las nociones básicas de Probabilidad en su Ars Conjectandi estableció y extendió el estudio muchas nociones combinatorias entre ellas; al igual que Leibniz, las combinaciones y las permutaciones.

En 1718 De Moivre en su libro Doctrine of Chances introduce el principio de inclusiónexclusión al analizar las probabilidades de ganar en juegos de azar, además expandió los coeficientes binomiales al caso multinomial.

Esta breve sección contiene así, un breve (y por supuesto incompleto) panorama del desarrollo de la combinatoria. Una discusión mas completa sobre la historia de la combinatoria puede encontrarse en [2].

\section{Principios de Conteo}

Gran parte de la Combinatoria Enumerativa se basa en dos principios de conteo: el aditivo y el multiplicativo, mismo que a continuación describiremos:

A grandes rasgos, el Principio Aditivo de Conteo establece que si se desea llevar a efecto una actividad, la cual tiene $n$ formas alternativas para ser realizada (obviamente diferentes), donde la primera de tales alternativas puede ser realizada de $N_{l}$ maneras, la segunda alternativa puede realizarse de $N_{2}$ formas, la tercera de ellas puede ser realizada de $N_{3}$ maneras, etc. Entonces esa actividad puede ser llevada a cabo de: $N_{l}+N_{2}+\ldots+N_{n}$ maneras.

Desde luego, este principio puede enunciarse sólo para dos actividades y extenderse por inducción a cualquier número finito de actividades. 
El principio multiplicativo es complemento del aditivo. Puede pensarse que este principio es utilizado cuando más de una actividad va a ser realizada una a continuación de la otra, un enunciado simple de este principio es el siguiente:

Si dos actividades se llevarán a cabo una después de la otra y si la primera de éllas puede realizarse de $N_{l}$ formas y para cada una de éllas la segunda actividad puede realizarse de $N_{2}$ maneras (hipótesis de homogeneidad) entonces, el número total de formas en que las dos actividades pueden realizarse es $N_{1} * N_{2}$.

Este principio puede ser generalizado a más de dos actividades por inducción pero es muy importante el cerciorarse que la hipótesis de homogeneidad se cumple.

Es usual que el abordaje del principio multiplicativo hallado en muchos libros de texto sea a través de diagramas de tipo de árbol ya que son una herramienta natural

Es con la correcta aplicación de estos principios que durante los cursos se llega a las fórmulas correspondientes al cálculo de permutaciones y combinaciones:

Problema 1. ¿De cuántas formas pueden acomodarse $n$ personas en una fila?

Observamos que es el principio multiplicativo la herramienta que debe ser utilizada pero en su versión generalizada ya que tenemos en total $n$ actividades (una por cada persona que será acomodada en la fila). La primer actividad (persona a ser acomodada) puede realizarse de $n$ formas distintas, para cada una de estas alternativas tenemos $n-1$ formas de realizar la segunda actividad, así que tenemos en total $n *(n-1)$ formas de realizar el acomodo de las dos primeras personas en la fila. Posteriormente, para cada una de estas $n *(n-1)$ formas de realizar las primeras dos actividades tenemos $n-2$ formas de realizar la tercera y por tanto tenemos $n *(n-1) *(n-2)$ formas de acomodar a las primeras tres personas en una fila. Continuando con este razonamiento tenemos que en total podemos acomodar a $n$ personas en una fila de $n$ ! formas y a cada una de estos acomodos se le suele llamar permutación.

Problema 2. ¿De cuántas formas podemos seleccionar un subconjunto de tamaño $k$ de un grupo de $n$ objetos (sin que el orden de selección importe, sólo la pertenencia o no de los objetos al subconjunto)?

La respuesta "inocente" a este problema usando el principio multiplicativo siguiendo los mismos argumentos de la solución del Problema 1 es $n *(n-1) * \ldots *(n-k+1)$, sin embargo esta respuesta cuenta más formas de las que en realidad existen ya que si consideramos un conjunto fijo de $k$ objetos la solución recientemente ofrecida considera como distintas a las $k$ ! permutaciones que pueden formarse con estos objetos; es decir, cada selección de $k$ elementos es considerada $k$ ! veces. Por tanto, la respuesta a este problema es

o en su forma más familiar

$$
n *(n-1) * \ldots *(n-k+1) / k \text { ! }
$$

$$
\mathrm{n} ! / \mathrm{k} !(\mathrm{n}-\mathrm{k}) !
$$

que denotaremos por $n C k$, y a cada uno de estos subconjuntos se le llamará una combinación de tamaño $k$. 
Nota 1. Con estas fórmulas a la mano, pueden resolverse muchos problemas típicos de la combinatoria enumerativa tales como: la fabricación de placas de automóviles, el sentado de personas en una línea y en una mesa circular, el acomodo de libros en un estante, la selección de comités, la enumeración de manos en el pókar, la distribución de pelotas en urnas, el cálculo del número de soluciones naturales (o enteras no negativas) de una ecuación lineal con coeficientes unitarios (por ejemplo $x+y+z=12$ ), la demostración de algunas propiedades de los coeficientes binomiales.

Una de las dificultades más comunes al resolver algunos problemas de combinatoria enumerativa es decidir si el orden de selección es o no relevante como puede evidenciarse en el siguiente problema de comités:

Problema 3. Supongamos que en un salón de clases tenemos 10 alumnos y un comité de tres personas debe ser formado. ¿Cuántos comités distintos pueden formarse si las responsabilidades de los tres miembros serán las mismas?

Este problema es resuelto con una combinación ya que en este caso el orden de selección no es importante; dicho de otra manera dado que las responsabilidades son las mismas para los tres miembros del comité, el comité Juan, Pedro y María es en esencia el mismo que el comité María, Juan y Pedro. Así, el número de comités bajo las condiciones establecidas es $10 \mathrm{C} 3=120$.

Problema 4. Bajo las mismas condiciones del Problema 3. ¿Cuántos comités pueden formarse si el comité debe tener un Presidente, un Secretario y un Tesorero?

En este caso, es claro que dos comités formados con las mismas personas pero arreglados en forma diferente son distintos; por ejemplo el comité Juan, Pedro y María afirma que Juan es el Presidente, Pedro el Secretario y María la Tesorera mientras que el comité María, Juan y Pedro afirma que María es la Presidente, Juan el Secretario y Pedro el Tesorero. Con esto, tenemos que el número de comités es $10 * 9 * 8=720=3$ !* $10 \mathrm{C} 3$.

\section{Conteo y Probabilidad}

Es posible introducir de manera natural los contextos en los cuáles algunas variables aleatorias discretas pueden ser empleadas y deducir así su función de masa de probabilidad a través de un conteo, por ejemplo la variable aleatoria Bernoulli, la Binomial, la Geométrica, la Hipergeomètrica y la Binomial Negativa.

A modo ilustrativo, exploremos los siguientes ejemplos:

Problema 5. Si lanzamos una moneda $n$ veces y nos preguntamos por el número de formas en que $k$ soles (en México las monedas tienen por un lado una figura a la que llamamos sol y por el otro lado un águila); y por tanto $n-k$ águilas, pueden ocurrir tenemos que la solución es $n C k$. En este caso, tenemos $k$ objetos iguales entre sí (los soles) y $n-k$ objetos iguales entre sí (las águilas) pero diferentes de los anteriores. Si dispusiéramos las formas en que estos $n$ objetos pueden acomodarse tendríamos como respuesta $n$ !, sin embargo los soles pueden permutarse entre sí de $k$ ! formas y las águilas de $(n-k)$ ! formas. Es por ésto que 
la respuesta es $n C k$. Lo cual al introducir la variable aleatoria binomial puede ayudarnos para deducir su función de masa de probabilidad.

Problema 6. Si lanzamos una moneda hasta que un número fijo de soles; digamos $r$, ocurran. ¿De cuántas formas pueden ocurrir los resultados de modo que sean necesarios $k$ lanzamientos?

Dada la naturaleza del problema, el $k$-ésimo lanzamiento necesariamente es un sol, es por esto que debemos contar el número de formas en que podemos arreglar $r-1$ soles y $k-r$ águilas (como en el caso del Problema 5, tenemos que tomar en cuenta que todos los soles son iguales entre sí al igual que las águilas). Así, tenemos que existen $(k-1) C(k-r)$ formas en que son necesarios $k$ lanzamientos para que ocurran $r$ soles. Este pequeño razonamiento puede llevarnos a la deducción de la función de masa de probabilidad de la variable aleatoria binomial negativa.

El caso de la variable aleatoria geométrica es un caso particular de la binomial negativa con $r=1$. Además, es fácil convencerse de que si lanzamos una moneda hasta que caiga sol sólo existe una forma en que esto ocurrirá en $k$ lanzamientos (para cada $k$ natural).

De manera similar se abordan problemas de probabilidad en los que el transfondo es un problema de combinatoria enumerativa así por ejemplo, los problemas señalados en la Nota 1 pueden trasladarse a problemas de probabilidad (muchas veces en problemas de resultados equiprobables) como lo muestran los siguientes ejemplos:

1. ¿Cuál es la probabilidad de que una placa de automóvil de 3 letras y 4 números tal que la primer letra siempre es " $T$ " y los números son mayores que 1000 fabricada al azar de modo que cada placa es igualmente probable sea múltiplo de dos (en la parte numérica) y tenga una " $\mathrm{X}$ " (en la parte literal)?

2. ¿Cuál es la probabilidad de que en un comité de 3 personas tomadas de un grupo de 10 tenga entre sus miembros a alguna persona en particular considerando que hay tres cargos distintos y que todas las selecciones son igualmente probables?

3. ¿Cuál es la probabilidad de que en una mano de pókar de 5 cartas se tenga alguna mano en particular: un par, dos pares, una tercia, un "full house", un pókar, etc.?

4. Si repartimos $k$ pelotas en $r$ urnas. ¿Cuál es la probabilidad de que todas las pelotas caigan en la primer urna?

Con lo expuesto anteriormente, se remarca el hecho de que la combinatoria enumerativa es una importante aliada de la probabilidad en el caso discreto y que su relación es totalmente natural. 


\section{Conclusiones Finales}

Dentro del primer curso de Probabilidad a nivel Licenciatura vale mucho la pena dedicar algún tiempo al tratado de algunos problemas básicos de combinatoria enumerativa así como a sus técnicas y sobre todo a esa forma de pensar; a nuestro juicio, tan particular que vincula tanto cierto grado de madurez matemática como de imaginación en situaciones cotidianas.

En la sección anterior, a través de ejemplos muy sencillos se mostró la intimidad que existe entre la combinatoria y la probabilidad. Pero, ¿Cuán bien asimilados están los principios básicos de conteo y sus alcances por parte de los estudiantes en el primer curso de Probabilidad?.

En la Benemérita Universidad Autónoma de Puebla, los estudiantes de las Licenciaturas de Matemáticas, Matemáticas Aplicadas y Acturía deben llevar su primer curso de Probabilidad después de aprobar por lo menos los cursos de: Matemáticas Básicas, Cálculo Diferencial, Cálculo Integral y Teoría de Ecuaciones. Es decir, son estudiantes que llevan al menos un año y medio en el nivel Licenciatura.

Sin embargo; en la experiencia de los autores de este trabajo, la proporción de estudiantes con vicios matemáticos que reflejan su inmadurez académica no es pequeña. Este hecho se ve reflejado en algunos casos por su búsqueda de una fórmula o estrategia que evite el tener que realizar una reflexión mas profunda del problema que se esté resolviendo, mientras que en otros ésto queda de manifiesto en cuestiones tan primordiales como el uso del sentido común para decidir cuál de los principios básicos de conteo ha de emplearse o bien, si ha de utilizarse una estrategia en la que el orden sea relevante.

Otra de las resistencias detectadas por parte de los estudiantes es el que, una vez "traducido" el enunciado al contexto de la combinatoria, pretenden resolverlo dentro de la teoría y no volver al contexto del problema hasta exponer su solución. Sin embrago, algunos problemas de combinatoria exigen que se acuda al enunciado del problema en cuestión en distintos puntos del desarrollo de su solución para efecto de ir incluyendo sus condiciones y restricciones.

Otro problema frecuente es el de no ser capaces de imaginar el contexto físico del problema para llevar a cabo la "traducción" señalada en el párrafo anterior. Lo cuál puede resultar particularmente grave dado que el estudio de la Probabilidad es importante en función de sus aplicaciones en situaciones prácticas.

Finalmente, debe tomarse en cuenta que es precisamente en el citado primer curso de Probabilidad que los estudiantes tienen su primer contacto con los problemas básicos de la combinatoria enumerativa, es por esto que quizá previo a este curso sea necesario uno de Matemática Discreta a fin de poder discutir con mayor detalle estos problemas y sobre todo adquirir de manera más pausada la sensibilidad requerida para encarar problemas de Probabilidad en el caso discreto. 
Bibliografía

[1] Molinero C., I Ching para todos, Longseller S.A., 2005.

[2] Wilson R. y Watkins J., Combinatorics: Ancient \& Modern Oxford University Press, 2013. 


\title{
Modelos Paramétricos en el Análisis de Supervivencia
}

Francisco Solano Tajonar Sanabria ${ }^{1}$, Yoanna Arenas Martínez ${ }^{2}$, Hugo Adán Cruz Suárez ${ }^{3}$, Víctor Hugo Vázquez Guevara ${ }^{4}$ y Hortensia Reyes Cervantes ${ }^{5}$

\section{Resumen}

El presente trabajo se encuentra ubicado en la línea del análisis de supervivencia, el cual está relacionado con el uso de modelos paramétricos, semi paramétricos para representar fenómenos relacionados con tiempos de vida, tiempos de supervivencia o tiempos de falla.

El objetivo principal del trabajo es presentar los conceptos y resultados básicos del análisis de supervivencia, entre los cuales destacan: la relación entre la función de supervivencia y la función de riesgo, que la función de densidad de probabilidad del tiempo de falla $T$ determina de manera única a la función de riesgo y recíprocamente. Los cuales permitirán abordar ejemplos y aplicaciones que se presentan en varios campos de investigación, como en el caso del cáncer. Además, se presentan los modelos paramétricos que son generalmente utilizados para el ajuste de tiempos de vida.

Palabras Clave: Análisis de supervivencia, Función de supervivencia, Función de densidad de probabilidad, Función de riesgo.

\begin{abstract}
This work is located on the line of the survival analysis, which is related to the use of parametric models, semi parametric models to represent times of life, survival times or times of failure-related phenomena.

The main objective of the work is to present the concepts and basic results of the survival analysis, including: the relationship between the survival function and the function of risk, that the time to failure $\mathrm{T}$ probability density function determines uniquely the function of risk and each other. Which will allow addressing examples and applications are presented in various fields of research, as in the case of cancer. In addition, present parametric models that are usually used for the adjustment of lifetimes.
\end{abstract}

Keywords: Survival analysis, Survival function, Function of density of probability, Function of risk,

\footnotetext{
${ }^{1}$ Benemérita Universidad Autónoma de Puebla. México, ftajonar@fcfm.buap.mx

${ }^{2}$ Benemérita Universidad Autónoma de Puebla. México, 212470815@fcfm.buap.mx

${ }^{3}$ Benemérita Universidad Autónoma de Puebla. México, hcs@fcfm.buap.mx

${ }^{4}$ Benemérita Universidad Autónoma de Puebla. México, vvazquez@fcfm.buap.mx

${ }^{5}$ Benemérita Universidad Autónoma de Puebla. México, hreyes@fcfm.buap.mx
} 


\section{Introducción.}

Comúnmente, al término estadística se le relaciona con el cálculo de promedios, porcentajes, etc., y con la presentación de los datos en forma tabular y en forma gráfica. Aunque las técnicas para resumir y presentar los datos son importantes, comprenden solo una parte de la estadística, la Estadística Descriptiva; la otra parte es la Inferencia Estadística, la cual se encarga de analizar datos colectados, y posteriormente inducir algún conocimiento sobre una población, donde los datos analizados son solo un subconjunto o muestra; esto es, el proceso va de lo particular a lo general.

La estadística es una herramienta ampliamente utilizada en la investigación científica. También se emplea en instituciones gubernamentales y educativas, en los negocios y en la industria.

El empleo juicioso de las técnicas estadísticas permite obtener conclusiones útiles a partir de datos numéricos.

En los últimos años el análisis de supervivencia ha desarrollado tópicos importantes en muchas áreas, especialmente en biología, medicina, ingeniería, teoría de confiabilidad, epidemiología, etc., con aplicaciones que van desde estudiar el desarrollo de alguna enfermedad, tiempos de vida en individuos con enfermedades terminales, falla en maquinaria, la durabilidad de electrodomésticos, hasta estudiar la resistencia de materiales al calor, etc., son solo algunas de las aplicaciones en donde el papel del análisis de supervivencia es importante.

Los fenómenos mencionados anteriormente no pueden predecirse o modelarse con algún modelo determinista, para más detalles ver ([5], [6]). Por ejemplo, supóngase que estamos interesados en determinar el tiempo de vida útil de un fusible puesto a funcionar en algún circuito, es claro que, este fallará de forma imprevista, en el sentido de que en un momento está funcionando bien y en el momento siguiente falla. Por el contrario, una barra de acero bajo una carga pesada se irá debilitando gradualmente en el transcurso de un periodo largo de tiempo. Se observa entonces que, la falla dependerá del fenómeno en estudio.

De forma que, según los comentarios anteriores, el uso de un modelo probabilístico parece ser un planteamiento realista para modelar cada uno de los fenómenos anteriores, ([2], [5], [10]).

Hay que señalar que existen varios modelos que se han propuesto para describir datos relacionados con tiempos de vida. Sin embargo, existen situaciones en donde los datos de tiempos de vida, no pueden ser ajustados por los modelos paramétricos clásicos. Entonces el problema básico dentro del análisis de supervivencia es dar y representar las distribuciones de tiempos de vida mediante modelos estadísticos y hacer inferencia sobre los parámetros de estos modelos. En este trabajo, se analizan modelos paramétricos para ajustar datos de tiempos de vida, haciendo énfasis en el modelo Weibull, por su amplia aplicación. En ([4], [9], [10]) se presenta el modelo Gama Generalizado, como una familia más general y que contiene como casos particulares a los modelos analizados. La dificultad de modelar mediante una gama generalizada es el trabajo numérico en el cálculo de los estimadores de esta distribución. 
2. ¿Qué es el Análisis de Supervivencia?

El Análisis de Supervivencia es una línea de la estadística inferencial que estudia y modela tiempos de falla o de vida de un individuo o un grupo de individuos sometido(s) a un tipo de "tensión" que puede ser un tratamiento, cirugía, etc., de esta forma se puede decir que el análisis de supervivencia se encarga de estudiar eventos-tiempo.

En la actualidad el uso de modelos paramétricos para representar fenómenos relacionados con tiempos de vida, tiempos de supervivencia o tiempos de falla, se ha desarrollado ampliamente con aplicaciones que van desde estudiar tiempos de vida en individuos con enfermedades terminales, la comparación de la efectividad de $k$ tratamientos buscando prolongar el tiempo restante de vida de estos individuos, así como en artículos de uso cotidiano bajo condiciones normales o de vida acelerada, garantía del funcionamiento de electrodomésticos durante $k$ unidades de tiempo, entrega de suplementos electrónicos confiables, dar confiabilidad o determinarla en el transporte público, describir el tiempo de vida de una amplia clase de productos como función de la temperatura, tales como aislantes eléctricos y dieléctricos, acumuladores, lubricantes y grasas, plásticos y filamentos de lámparas incandescentes, son solo algunos de los casos en donde el papel del Análisis de supervivencia o Teoría de confiabilidad juega un papel importante.

Vale la pena mencionar que existen varios modelos que se han propuesto en la literatura para describir datos de tiempos de vida, entre los modelos más usados se encuentran los modelos Exponencial, Gama, Weibull y Lognormal, sin embargo, no todos los conjuntos de datos se ajustan a dichos modelos, por lo que es importante proponer modelos más generales para explicar el comportamiento de este tipo de datos.

Un modelo que ha sido seguido por varios autores, para datos de tiempos de vida, es la distribución gama generalizada, ver ([7], [9], [10]), el cual es un modelo paramétrico general que incluye entre otros modelos a los ya mencionados anteriormente, desafortunadamente dicha distribución tiene limitantes significativas como lo es la estimación de sus parámetros por máxima verosimilitud ([1], [8]).

En el análisis estadístico, muchas veces los datos se refieren a observaciones de tiempos de vida o tiempos de falla, sin embargo, desde el enfoque del análisis de supervivencia, el análisis de estos datos es diferente a la de los datos comunes, ya que dentro de este se deben considerar conceptos como función de supervivencia, función de riesgo y otros conceptos básicos los cuales se mencionan a continuación

Antes de presentar los conceptos propios del análisis de supervivencia, es bueno considerar que para realizar un buen análisis de tiempos de vida se necesita lo siguiente:

1. Tener bien definido nuestro evento de interés.

2. Definir de forma apropiada el origen o inicio del estudio, es decir, indicar el comienzo de nuestro análisis. 
3. Definir la escala de tiempo.

Con lo anterior tenemos que el análisis de supervivencia es el conjunto de técnicas que nos permiten analizar, estudiar y modelar la variable (en este caso representa evento-tiempo) de interés. Esta variable, en general depende de otras variables que son explicables y que se denominan covariables del fenómeno, ver ([4]).

Sea $T$ una variable aleatoria que denota el tiempo hasta que ocurre algún evento específico. Este evento puede ser la muerte, la aparición de un tumor, el desarrollo de alguna enfermedad, la recurrencia de alguna enfermedad, fallo de equipo eléctrico, dejar de fumar, etc.

De forma más específica, en este trabajo, $T$ será una variable aleatoria continua, no negativa que representará a una población homogénea.

En seguida se presentan y analizan tres funciones que caracterizan a $T$ :

Función de Supervivencia: es la probabilidad de que un individuo sobreviva más del tiempo $t$.

Función de Riesgo: es la probabilidad de que un individuo de $t$ años experimente el evento en el siguiente instante de tiempo.

Función de densidad de probabilidad: es el modelo probabilístico que indica el comportamiento límite de la probabilidad en cada evento $\{t \leq T<t+\Delta t\}$ cuando $\Delta t \rightarrow 0$.

Si se conoce alguna de estas funciones, las otras dos pueden ser determinadas a partir de la función conocida. Estas funciones nos permitirán analizar diferentes aspectos de $T$, por ejemplo, que forma tiene su distribución de probabilidad, su función de riesgo, etc.

3. Conceptos y Resultados del Análisis de Supervivencia

Iniciamos presentando el concepto de función de supervivencia la cual es fundamental para definir y estudiar fenómenos aleatorios de tipo evento-tiempo, [6].

Definición 1. La función de supervivencia para la variable aleatoria (v.a) $T$ es la probabilidad de que un individuo viva más del tiempo t, esta función se denota por $S(t)$ y está dada por:

$$
S(T)=P(T \geq t) .
$$

En el caso de falla en artículos a $S(t)$ se le llama función de confiabilidad.

Esta función tiene las siguientes propiedades:

1. $S(t) S(0)=1$ es una función continua, monótona decreciente.

2. $S(0)=1$.

3. $S(\infty)=\lim _{n \rightarrow \infty} S(t)=0$ 
Note que

$$
S(t)=1-F(t-), \quad F(t-)=\lim _{x \rightarrow t-} F(x),
$$

donde, $F(t-)=\lim _{x \rightarrow t-} F(x)$.

Teorema 1. Sea $T$ una variable aleatoria continua, con densidad $f(t)$, entonces

$$
f(t)=-\frac{d S(t)}{d t}
$$

Demostración: Como $T$ es una variable aleatoria continua, se tiene que $\forall t \in \mathbf{R}, F(t-)=F(t)$, de donde,

$$
S(t)=1-F(t) \Rightarrow S^{\prime}(t)=-F^{\prime}(t) .
$$

Entonces

$$
f(t)=-\frac{d S(t)}{d t},
$$

que es lo que se afirmo en (2).

También, dado que,

$$
\int_{t}^{t+\Delta t} f(u) d u \approx f(t) \Delta t
$$

entonces, $f(t) \Delta t$ puede entenderse como la probabilidad aproximada de que el evento ocurra en el intervalo de tiempo $[t, t+\Delta t]$.

Otra función fundamental en el análisis de supervivencia es la función de riesgo. Esta función también es conocida como tasa de mortalidad en demografía, la función de intensidad en procesos estocásticos, [2].

Definición 2. La función de riesgo se define como sigue

$$
h(t)=\lim _{\Delta t \rightarrow 0} \frac{P(t \leq T<t+\Delta t \mid T \geq t)}{\Delta t} .
$$

Observación 1: $P[t \leq T<t+\Delta t \mid T \geq t]$ es la probabilidad de que un individuo de $t$ años experimente el evento en las próximas $\Delta t$ unidades de tiempo.

Entonces la función de riesgo es la razón o taza de falla instantánea de un individuo al tiempo $t$, dado que el individuo ha sobrevivido hasta ese momento $t$. Esto es, $h(t) \Delta t$ es aproximadamente igual a la probabilidad de que un individuo experimente el evento en el intervalo $[t, t+\Delta t]$ dado que ha sobrevivido hasta el tiempo $t$. 
Corolario 1.

$$
h(t)=\frac{f(t)}{S(t)}
$$

\section{Demostración:}

$$
\begin{aligned}
h(t) & =\lim _{\Delta t \rightarrow 0} \frac{P(t \leq T<t+\Delta t \mid T \geq t)}{\Delta t}=\lim _{\Delta t \rightarrow 0} \frac{F(t+\Delta t)-F(t)}{\Delta t S(t)} \\
& =\frac{1}{S(t)} \lim _{\Delta t \rightarrow 0} \frac{F(t+\Delta t)-F(t)}{\Delta t}=\frac{f(t)}{S(t)}
\end{aligned}
$$

puesto que $F^{\prime}(t)=f(t)$

Observación 2: como

$$
h(t)=\frac{f(t)}{S(t)}
$$

entonces,

$$
h(t)=-\frac{d}{d t} \ln (S(t))
$$

de aquí también se obtiene que:

$$
S(t)=\exp \left(-\int_{0}^{t} h(u) d u\right)
$$

Otra función básica que se emplea en el análisis de supervivencia es la función acumulativa de riesgo $H(t)$ la cual se define de la siguiente manera:

$$
H(t)=\int_{0}^{t} h(u) d u
$$

Aunque no profundizaremos en el uso de esta función, más adelante nos ayudará a expresar $f(t)$ en términos de $S(t)$ y $h(t)$.

\section{Observación 3:}

$$
H(t)=\int_{0}^{t} h(u) d u=-\int_{0}^{t} \frac{d}{d u} \ln (S(u)) d u=-\ln (S(t))
$$

Así que,

$$
S(t)=\exp (-H(t))
$$

Esto se puede verificar fácilmente usando la Observación 2 y el hecho de que $S(0)=1$ entonces, $\ln [S(0)]=\ln [1]=0$. 
La función de riesgo es particularmente útil para determinar apropiadamente la distribución de falla. Utilizando información cuantitativa acerca del mecanismo de fracaso, para describir la forma en la cual la probabilidad de experimentar el evento cambia con el tiempo. Esta tiene varias formas generales para porcentajes de riesgo distintos. La única condición para la función de riesgo es la no negatividad, es decir, $h(t) \geq 0$.

El comportamiento que sufre la función de riesgo nos dará información importante sobre las aplicaciones, por ejemplo:

1. Riesgo constante, los modelos que presentan este tipo de riesgo, son muy pocos, uno de ellos es el modelo exponencial.

2. Los modelos de riesgo creciente: se presentan con el proceso natural de envejecimiento.

3. Riesgo decreciente: son menos comunes, estos modelos se pueden ver en algún tipo de componentes electrónicos, $o$ en pacientes que experimentan algún tipo de trasplante.

4. Riesgo en forma de bañera: este tipo de riesgo es constante al principio y después es creciente, este riesgo es muy adecuado para estudiar el comportamiento de las poblaciones, que se siguen desde su nacimiento. Por ejemplo supongamos que se desea estudiar el tiempo de falla de artículos eléctricos, estos pueden experimentar fallas tempranas debido a partes defectuosas, después de algún tiempo el riesgo de fallar se estabiliza (falla constante) y finalmente, de riesgo creciente debido al desgaste del articulo.

5. Si el porcentaje de riesgo es creciente al principio, y eventualmente va decreciendo, entonces se dice que el riesgo tiene la forma de montículo o joroba.

El siguiente teorema nos permite relacionar a las tres funciones enunciadas anteriormente.

Teorema 2. Sea $T$ el tiempo para que ocurra una falla, $T$ es una variable aleatoria continua con densidad $f$ y f.d.a. $F$ con $F(0)=0$, entonces $f$ puede expresarse en términos de la función de riesgo de la siguiente manera:

$$
f(t)=h(t) \exp \left(-\int_{0}^{t} h(r) d r\right) .
$$

Demostración: Recuerde que,

$$
\begin{gathered}
S^{\prime}(t)=-f^{\prime}(t), \quad h(t)=\frac{f(t)}{S(t)}, \\
\ln [S(t)]=-\int_{0}^{t} h(r) d r,
\end{gathered}
$$

de aquí,

$$
S(t)=\exp \left(-\int_{0}^{t} h(r) d r\right),
$$


y de (4), tenemos:

$$
f(t)=h(t) S(t)
$$

Entonces la función de densidad de $T$ es,

como se afirmo en (6).

$$
f(t)=h(t) \exp \left(-\int_{0}^{t} h(r) d r\right),
$$

El resultado anterior nos dice que la función de riesgo proporciona mayor información acerca del mecanismo subyacente de falla que la función de supervivencia. Por esta razón, consideramos a la función de riesgo como el elemento dominante para el análisis de datos de supervivencia.

Hasta ahora lo que hemos visto, ha sido solo los conceptos básicos sobre análisis de supervivencia. En la siguiente sección se presenta los modelos más comunes para el análisis de supervivencia y se analizan las características que los distinguen.

\section{Modelos Paramétricos}

\section{MODELO EXPONENCIAL}

Existen muchas maneras de presentar a la función exponencial, pero sin duda la más interesante y la que presentamos en este trabajo, es la siguiente.

Estamos estudiando el tiempo transcurrido hasta que ocurra un evento, a la ocurrencia del mismo le estamos asociando un riesgo, entonces ¿puede ser que el riesgo sea constante?

La distribución exponencial desempeña un papel muy importante en la descripción de fenómenos, especialmente en el análisis de supervivencia. Inicialmente se uso en trabajos referentes al análisis de confiabilidad (componentes electrónicas) y con algunas limitaciones se extendió a estudios médicos, ([1], [2]).

Esta distribución tiene un solo parámetro ajustable (la media). Sin embargo, los métodos sobre los que se basa esta distribución son muy sensibles e incluso limitados.

Definición 3. Se dice que una variable aleatoria continua $T$, la cual toma valores positivos, tiene una distribución exponencial con parámetro $\alpha$ si su función de densidad de probabilidad está dada por:

$$
f(t)=\left\{\begin{array}{lc}
\frac{1}{\alpha} e^{-\left(\frac{t}{\alpha}\right)}, & \text { si } t>0 \\
0, & \text { cualquier otro caso. }
\end{array}\right.
$$

Lo anterior se denota por $T \sim \operatorname{Exp}(\alpha)$.

En la literatura existente, algunas veces a la f.d.p de $T$, también la definen como 


$$
f(t)=\left\{\begin{array}{lc}
\lambda e^{-\lambda t}, & \text { si } t>0, \\
0, & \text { cualquier otro caso. }
\end{array}\right.
$$

Se escoge en este estudio la parametrización dada por (7) debido a que, en este caso $E(\mathrm{~T})=\alpha$, ver [8].

Observación 4: La distribución exponencial generalmente se caracteriza como el tiempo de espera hasta la ocurrencia del evento.

En el análisis de supervivencia la caracterizamos como el tiempo transcurrido hasta el deceso.

Esta distribución tiene varias propiedades que son de interés, por ejemplo la función de riesgo es constante. De estas propiedades nos encargaremos enseguida, [3].

\section{PROPIEDADES}

\section{Función de Distribución Acumulativa}

La f.d.a. de la variable exponencial está dada por:

$$
F(t)=1-e^{-\left(\frac{t}{\alpha}\right)} \text {. }
$$

\section{Demostración:}

$$
\begin{aligned}
& F(t)=P(T \leq t)=\int_{0}^{t} f(r) d r=\int_{0}^{t} \frac{1}{\alpha} e^{-\left(\frac{r}{\alpha}\right)} d r=-\int_{0}^{\left(\frac{t}{\alpha}\right)} \exp (u) d u, \\
& \text { si } u=-\left(\frac{r}{\alpha}\right) \text {, entonces, } d u=-\left(\frac{d r}{\alpha}\right) \text {, y por lo tanto } F(t)=1-e^{-\left(\frac{t}{\alpha}\right)} \text {. o }
\end{aligned}
$$

\section{Función de Supervivencia}

$$
S(t)=e^{-\left(\frac{t}{\alpha}\right)} .
$$

\section{Demostración:}

$S(t)=P(T \leq t)=1-P(T<t)=1-F(t-)=1-F(t)$,

por ser $F$ continua; de manera que, $\forall t>0$, se tiene que

$$
S(t)=1-\left(1-e^{-\left(\frac{t}{\alpha}\right)}\right)=e^{-\left(\frac{t}{\alpha}\right)} .
$$

\section{Función de Riesgo}

A continuación, veremos que esta distribución tiene la propiedad de tener tasa de riesgo

$$
h(t)=\frac{1}{\alpha} .
$$




\section{Demostración:}

$$
h(t)=\frac{f(t)}{S(t)}=\frac{\frac{1}{\alpha} e^{-\left(\frac{t}{\alpha}\right)}}{e^{-\left(\frac{t}{\alpha}\right)}}=\frac{1}{\alpha}, \quad(\alpha \text { - Constante })
$$

El hecho de que el riesgo sea constante, nos indica que después de usar un artículo, la probabilidad de que falle, es la misma que cuando era nuevo, en otras palabras, el objeto no envejece. El siguiente resultado nos ayudará a comprender mejor esta situación.

TEOREMA 3: Si $T$ es el tiempo transcurrido para que ocurra una falla y $T$ es una variable aleatoria no negativa, entonces $T$ tiene una distribución exponencial, si y sólo si, tiene una tasa de riesgo constante.

\section{Demostración:}

$\Rightarrow$ Supongamos que $T$ tiene una distribución exponencial entonces por el resultado previo tenemos que $h(t)=\frac{1}{\alpha}$ donde $\alpha$ es constante.

$\Leftarrow \mathrm{Si} h(t)=\frac{1}{\alpha}$, entonces por el Teorema 2, podemos determinar de manera única a la función de densidad, de la siguiente manera

$$
f(t)=\frac{1}{\alpha} \exp \left(-\int_{0}^{t} \frac{1}{\alpha} d s\right)=\frac{1}{\alpha} \exp \left(-\left.\left(\frac{1}{\alpha} s\right)\right|_{0} ^{t}\right)=\frac{1}{\alpha} \exp \left(-\left(\frac{t}{\alpha}\right)\right)
$$

Por lo que concluimos que $f(t)$ tiene una distribución exponencial.

De esta manera queda demostrado el Teorema. 0

\section{MODELO WEIBULL}

En el punto anterior estudiamos un modelo en el que la tasa de falla es constante. Pero se sabe que en general, esto no siempre es cierto. En esta parte se trata un modelo que permite estudiar fenómenos en los que la tasa de falla no es constante.

Este modelo, es muy usado en el análisis de supervivencia, sobre todo en material eléctrico, aplicaciones industriales y en biomedicina, ([4], [5]).

Definición 4. Una variable aleatoria $T$ tiene una distribución Weibull, si existen parámetros $\lambda>0$ y $\beta>0$, tales que la variable $Z=\left(\frac{T}{\lambda}\right)^{\beta}$ tiene una distribución exponencial con media 1.

La función de densidad de $T$ esta dada por 


$$
f(t)=\left\{\begin{array}{lr}
\frac{\beta}{\lambda}\left(\frac{t}{\lambda}\right)^{\beta-1}\left(\exp \left(\frac{t}{\lambda}\right)^{\beta}\right), & \text { si } t>0 \\
0, & \text { cualquier otro caso. }
\end{array}\right.
$$

\section{PROPIEDADES}

\section{Función de Distribución Acumulativa}

La f.d.a. de la variable Weibull está dada por:

$$
F(t)=P(T \leq t)=1-\exp \left(\frac{t}{\lambda}\right)^{\beta} .
$$

\section{Función de Supervivencia}

$$
S(t)=\exp \left(\frac{t}{\lambda}\right)^{\beta}
$$

\section{Función de Riesgo}

$$
h(t)=\frac{\beta}{\lambda}\left(\frac{t}{\lambda}\right)^{\beta-1}
$$

\section{Algunos Comentarios}

Es importante mencionar que entre los modelos del análisis de supervivencia el exponencial y el Weibull son de los más utilizados para ajustar conjuntos de datos de tiempos de vida, sin embargo, existen otros modelos paramétricos que nos permiten estudiar tiempos de vida, una dificultad que generalmente presentan es que en muchos casos la estimación de sus parámetros es complicada y se tiene que recurrir a un método numérico.

Una aplicación del modelo Weibull, fue en 42 pacientes que sufren alguna enfermedad en el cerebro, estos datos siguen dicha distribución ver ([5]).

El modelo exponencial, es el que mejor se ajusta a pacientes con algún tipo de cáncer, ya que las características, de su función de riesgo y supervivencia, determinan cuando ocurren, cambios en dichos pacientes.

En problemas reales de supervivencia los modelos paramétricos no son suficientes y siempre se recurre a métodos no paramétricos y para tener una mejor información o realizar una mejor descripción del conjunto de datos se adicionan covariables. 


\section{Bibliografía}

[1] Alexander, M. Mood; Franklin, A. Graybill; Duane, C. Boes. (1974).Introduction to the Theory of Statistics. Third Edition. McGraw-Hill, Inc.

[2] Cox D. R. and Oakes, D. (1984), Analysis of Survival Data, Chapman and Hall.

[3] Harter, H. Leon and Moore, Albert H. (1965) Maximum Likelihood Estimation of the Parameters of Gamma and Weibull Population for Complete and Censored Samples. Technometrics, 7, 639-643.

[4] Kalbfleisch J.D. and Prentice R.L. (1980) The Statistical Analysis of Failure Time Data, New York John Wiley.

[5] Klein P. John and Moeschberger L. Melvin, Survival Analysis: Techniques for Censored and Truncated Data, New York: Springer-Verlag.

[6] Lawless J. F. Statistical Models and Methods for Lifetime Data. Second Edition Wiley Series.

[7] Parr, V.H. and J.T. Webster. (1965). A method for Discriminating Between Failure Density Functions Used in Rehability Predictions. Technometrics. 7:1-10

[8] Paun Garthwahwaite; Ian Jolliffe; Byron d Jones; (2002) Statical Inference Second Edition. Oxford Science Publications.

[9] Stancy, E. W. (1962). A Generalization of the Gamma Distribution. Ann. Math. Stat. 33:1187-1192.

[10] Stancy, E.W. and G.A. Mihram (1965). Parameter Estimation for a Generalized Gama Distribution. Technometrics. 7:349-358. 


\title{
Evaluación del ajuste de ítems en la teoría de respuesta al ítem
}

\author{
Kenner Ordóñez Lacayo ${ }^{1}$ \& Guaner Rojas Rojas ${ }^{2}$ \& Karol Jiménez Alfaro ${ }^{3}$
}

\begin{abstract}
Resumen
Las técnicas de análisis de las respuestas de las personas a un conjunto de ítems han sido ampliamente estudiadas en el contexto de la teoría de respuesta a los ítems. Desde la perspectiva del ajuste absoluto, la presentación mostrará la descripción del modelo logístico de dos parámetros sobre un conjunto de datos de respuestas de una muestra de aspirantes a ingreso a la Universidad de Costa Rica. La ponencia proporcionará los principales aspectos del modelo y los estadísticos empleados para el análisis del ajuste a los datos observados. Además, se mostrarán ejemplos que capturan la discrepancia entre el modelo y los datos.
\end{abstract}

Palabras clave: Teoría de Respuesta a los Ítems, Ajuste, Ajuste Absoluto.

\footnotetext{
${ }^{1}$ Prueba de Aptitud Académica de la Universidad de Costa Rica, Costa Rica. kenner.ordonez@ucr.ac.cr

${ }^{2}$ Prueba de Aptitud Académica de la Universidad de Costa Rica, Costa Rica. guaner.rojas@ucr.ac.cr

${ }^{3}$ Prueba de Aptitud Académica de la Universidad de Costa Rica, Costa Rica. karol.jimenez@ucr.ac.cr
} 


\title{
Una alternativa para la clasificación diagnóstica
}

\author{
Guaner Rojas Rojas ${ }^{1}$
}

\begin{abstract}
Resumen
Las herramientas de clasificación se emplean para definir categorías en función de atributos que reflejan las características de un ente. A partir de una matriz de atributos, se presentarán los elementos principales que contribuirían a un análisis de datos desde el enfoque de los modelos de diagnóstico cognitivo (MDC). Los MDC proporcionan información con un nivel adecuado de detalle y especificidad para apoyar a los profesores sobre la competencia de los estudiantes en un área determinada, por ejemplo en resolución de problemas. Los MDC que se presentarán varían en términos de la complejidad de la cantidad de parámetros. Adicionalmente, se mostrará cómo podrían utilizarse los MDC para reportar puntuaciones de las personas en ambientes tanto de pruebas estandarizadas como de aula.
\end{abstract}

Palabras clave: diagnóstico, clasificación, atributos.

${ }^{1}$ Universidad de Costa Rica, Costa Rica. guaner.rojas@ucr.ac.cr, guanerdavid@yahoo.com 
"IV Encuentro sobre Didáctica de la Estadística, la Probabilidad y el Análisis de Datos"

\title{
El Bootstrap como Herramienta para la Enseñanza de la Distribución Muestral
}

\author{
María Inés Rodríguez ${ }^{1}$ \& Héctor Agnelli ${ }^{2}$
}

\begin{abstract}
Resumen
La distribución muestral es un concepto básico para la adecuada comprensión de procedimientos inferenciales tales como test de hipótesis e intervalos de confianza. Existe un extenso conjunto de trabajos de investigación educativa en los que se reporta evidencia empírica acerca de que esta idea no es comprendida de manera adecuada. Creemos que la enseñanza de técnicas de remuestreo tales como bootstrap y test de permutación, además de brindar nuevas herramientas que puedan ser comprendidas y utilizadas por el futuro usuario de la estadística, contribuyen desde el punto de vista educativo a facilitar el aprendizaje de conceptos estadísticos claves tales como, distribución muestral, error estandar, intervalos de confianza y pruebas de significación. En este trabajo describimos la metodología bootstrap para el cálculo del error estandar de la mediana muestral.
\end{abstract}

Palabras clave: distribución muestral, remuestreo, error estandar, bootstrap.

\begin{abstract}
The sampling distribution is a basic concept for the proper understanding of inferential procedures such as hypothesis tests and confidence intervals. There is an extensive collection of works of educational research, reporting empirical evidence that this idea is not properly understood. We believe that teaching techniques such as bootstrap, resampling and permutation tests, in addition to providing new tools that can be understood and used by the future user of statistics, help from the educational point of view to facilitate the learning of key statistical concepts such as, sampling distribution, standard error, confidence intervals and significance tests. In this paper we describe the bootstrap methodology for calculating the standard error of the sample median.
\end{abstract}

Keywords: sampling distribution, resampling, standard error, bootstrap.

\footnotetext{
${ }^{1}$ Universidad Nacional de Río Cuarto, Argentina. mrodriguezbriguet@gmail.com

${ }^{2}$ Universidad Nacional de Rio Cuarto, Argentina. agnellih@ gmail.com
} 
IV Encuentro sobre Didáctica de la Estadística, la Probabilidad y el Análisis de Datos

\section{Introducción}

La prueba de hipótesis (y los valores p) es la metodología estadística más usada por los científicos experimentales aunque como muchas investigaciones lo ponen en evidencia es incorrecta su aplicación o inadecuada la interpretación del valor $\mathrm{p}$ o el nivel de significación (Vallecillos, 1996, Nickerson, 2000; Hubbard y Bayarri, 2003, Kline, 2004; Rodríguez y Albert, 2007). En un intento por reducir el impacto negativo de esta situación ha crecido una corriente que impulsa usar en su reemplazo los intervalos de confianza (IC). Esto ha llevado a trasladar la atención acerca del uso e interpretación que hacen los investigadores experimentales desde los tests a los IC, detectándose errores severos también en la interpretación de los mismos (Cumming, Willians, Fidler, 2004), (Belia, Fidler, Willians, Cumming, 2005). Además, en trabajos de investigación en los que se analizó la comprensión de IC por parte de los estudiantes se hallaron evidencias acerca de la incorrecta comprensión de su significado (Chance, del Mas, Garfield, 2004). Tratando de encontrar razones que expliquen estos comportamientos es conveniente analizar los conceptos que aparecen en la base constructiva de esos procedimientos inferenciales.

Esto conduce al análisis de una idea básica como es la de distribución muestral. Castro Soto y otros (2007) han recopilado un extenso conjunto de trabajos de investigación educativa en los que se reporta evidencia empírica acerca de que este concepto, no es comprendido de manera adecuada.

Por lo tanto, parece oportuno abordar el estudio de modos alternativos y a la vez complementarios de la enseñanza de la inferencia estadística y en particular, de la enseñanza de la distribución muestral. Desde esta posición creemos que las técnicas de remuestreo como el bootstrap y los tests de permutación, pueden contribuir a facilitar el aprendizaje de conceptos estadísticos claves como: distribución muestral, error estandar, intervalos de confianza y valores p. En este trabajo nos centraremos en el concepto de distribución muestral.

\section{Distribuciones Muestrales}

Sea $\mathrm{F}$ una distribución y $\theta$ un parámetro desconocido de la misma, pero fijo. Con el propósito de tener conocimiento acerca de $\theta$, se trabaja con una muestra aleatoria $X_{1}, \ldots, X_{n}$ de F. A partir de esta muestra se construye el estadístico $\hat{\theta}=\hat{\theta}\left(X_{1}, \ldots, X_{n}\right)$ que será utilizado como estimador de $\theta$. Pero para una muestra dada $X_{1}=x_{1}, \ldots, X_{n}=x_{n}$ ¿cuán bien aproxima $\hat{\theta}=\hat{\theta}\left(x_{1}, \ldots, x_{n}\right)$ a $\theta$ ? o en otros términos ¿cómo es la diferencia $\hat{\theta}-\theta$ ?. Dado que $\theta$ es desconocido la pregunta así formulada no tiene respuesta.

El análisis del comportamiento de los procedimientos inferenciales de la estadística clásica (a diferencia de la estadística Bayesiana) se basa en dar respuesta a esta otra pregunta ¿cómo será a la larga el comportamiento de este procedimiento? Es decir, basamos nuestra confianza en el funcionamiento de un procedimiento, no en una aplicación particular del mismo sino por su comportamiento en el muestreo repetido. 
IV Encuentro sobre Didáctica de la Estadística, la Probabilidad y el Análisis de Datos

Como $\theta=\hat{\theta}\left(X_{1}, \ldots, X_{n}\right)$ es una función de variables aleatorias esto significa que en el muestreo repetido $\theta$ presenta variabilidad entre muestras. Si establecido el tamaño n de la muestra pudiésemos tomar todas las posibles muestras de ese tamaño, podríamos calcular para cada una de ellas el valor del estimador $\theta=\theta\left(x_{1}, \ldots, x_{n}\right)$ y en consecuencia tener la distribución de $\hat{\theta}$ como variable aleatoria, es decir tendríamos la llamada distribución muestral de $\hat{\theta}$.

Conocida esta distribución podríamos preguntarnos como será en promedio el comportamiento de la diferencia $\hat{\theta}-\theta$, y esta cantidad es llamada el sesgo del estimador:

$$
\operatorname{Sesgo}=E_{\theta}(\hat{\theta}-\theta)
$$

También podemos analizar la variación de $\hat{\theta}$ alrededor de su esperanza y podríamos obtener el llamado error estandar de la estimación. De aquí la importancia de conocer la distribución muestral de los estimadores.

En general esperamos que la distribución muestral de $\hat{\theta}$ dependa del parámetro desconocido $\theta$, por lo tanto al estudiar las propiedades de la distribución muestral del estimador obtendremos información acerca del comportamiento de $\hat{\theta}$ como estimador en el muestreo repetido: ¿Cuál será su valor esperado?, ¿Cuál será su dispersión? ¿Cómo será la forma de su distribución? Pero ahora surge otra pregunta cómo hallamos $F_{\hat{\theta}}$, es decir, la distribución de $\hat{\theta}$. En general podemos se mencionan tres maneras distintas de arribar al conocimiento de la distribución de $\hat{\theta}$.

El primero de ellos, es el método analítico o teórico. A manera de ejemplo, en los cursos tradicionales de estadística se enseña cuál es la distribución muestral de la media cuando se tienen muestras que proviene de una distribución normal o se enuncia la distribución aproximada de la media acudiendo al Teorema Central de Límite. Pero esta metodología no se puede extender de una manera obvia a otros estimadores, como por ejemplo la mediana muestral. De hecho en los cursos básicos de estadística y en los textos destinados a estos cursos en general no se habla de la precisión de la mediana, aunque si se insiste en su uso como medida descriptiva valiosa para valores centrales de distribuciones asimétricas.

Un segundo método, lo constituyen las distribuciones obtenidas por simulación Monte Carlo. Volviendo al caso de la mediana se asume conocida la población a muestrear, se simulan muestras de esta población y se estudia entonces el comportamiento en el muestreo repetido del estadístico de interés, en este caso la mediana. La restricción que tiene este método es la suposición acerca del conocimiento de la población.

Una tercera opción para estudiar la distribución muestral, es utilizar el remuestreo, metodología que será bosquejada más adelante. 


\section{Problemas reportados Acerca de la Comprensión de la Distribución Muestral}

La comprensión del proceso de muestreo requiere distinguir entre dos propiedades básicas que tienen las muestras: la representatividad y la variabilidad. La primera de ellas referida a la similitud entre la muestra y la población queda asociada al diseño de la experiencia y la segunda es una característica vinculada con la variación entre muestras de una misma población extraídas en condiciones similares. En términos distribucionales y denominando $F_{\theta}$ a la distribución de la variable en la población, $\hat{F}_{\theta}$ a la distribución empírica de la variable en la muestra y $F_{\hat{\theta}}$ a la distribución muestral del estadístico algunos de los errores conceptuales vinculados con la comprensión de estas distribuciones son los siguientes:

a) Considerar a la distribución empírica de la variable en la muestra como una cabal representación de la distribución de la variable en la población sin importar el tamaño de la muestra

b) Confundir la distribución poblacional $F_{\theta}$ o a $\hat{F}_{\theta}$ con la distribución muestral del estadístico. Sobre esta última creencia cuando en las clases para desarrollar el concepto de distribución muestral se trabaja casi exclusivamente con muestras aleatorias de una distribución normal y se analiza el comportamiento de la media como estadístico, por cierto la media tiene también distribución normal- con la misma media aunque con varianza más pequeña- y por lo tanto involuntariamente se refuerza la idea del parecido necesario entre la forma de $F_{\theta}$ y la forma de $F_{\hat{\theta}}$, sin que quede claro que muestreando una población no normal, bajo las hipótesis del teorema central del límite, la distribución de la media es aproximadamente normal y por lo tanto distinta de la distribución original.

c) No distinguir claramente la distribución real del estadístico $F_{\hat{\theta}}$ con una eventual distribución asintótica utilizada como aproximación sin importar las condiciones de validez de esta aproximación -situación muy común para los estadísticos de los test de hipótesis-. Los errores señalados se convierten en obstáculos para la comprensión de conceptos fundamentales para la inferencia tales como errores estandar o valores $\mathrm{p}$.

Así a manera de síntesis podemos decir que para asimilar el concepto de distribución muestral los alumnos deben estar familiarizados con las ideas de variabilidad, distribución y muestreo aleatorio y ser capaces, al menos, de

- distinguir entre la distribución de las observaciones de una muestra y la distribución de un estadístico en muchas muestras seleccionadas aleatoriamente.

- describir como podría ser una distribución muestral para diferentes poblaciones y diferentes tamaños de muestra (basándose en el centro, la dispersión y donde se encontrarían la mayor parte de los valores)

- apreciar que cuando aumenta el tamaño de muestra menor es la variabilidad del estadístico muestral.

- Comprender que el error estandar es una medida de la variabilidad del estadístico. 
IV Encuentro sobre Didáctica de la Estadística, la Probabilidad y el Análisis de Datos

- Asumir que algunos valores del estadístico serán más o menos verosímiles que otros.

Estos conceptos son fundamentales para la adecuada comprensión e interpretación de IC, valores p, potencia y replicabilidad. Desde el punto de vista computacional están disponibles en la web, distintos simuladores que permiten al estudiante familiarizarse con estos conceptos seleccionando una entre varias distribuciones poblacionales, como así también para trabajar con diferentes tamaños de muestras, que contribuyen a comprender las ideas centrales de la inferencia estadística.

Presentada entonces la situación anterior, nos parece oportuno desde el punto de vista de la enseñanza, plantear en los cursos básicos la posibilidad de utilizar otras herramientas complementarias para la formación en inferencia estadística, como son los procedimientos de remuestreo. Se sabe que ellos constituyen herramientas útiles en situaciones complejas, que sin duda son enseñados a los futuros profesionales de la estadística. Estos métodos que no son presentados, salvo excepciones, en los cursos iniciales, prescinden del conocimiento las distribuciones muestrales teóricas y se basan en el muestreo repetido (remuestreo) de la misma muestra original. Nosotros creemos que por su esencia brindan la oportunidad de facilitar el aprendizaje de conceptos estadísticos claves.

\section{El Remuestreo}

Los test paramétricos tradicionales al igual que la construcción de intervalos de confianza están basados en el conocimiento de distribuciones muestrales ya sea del estadístico del test o de los estimadores puntuales en el caso de los intervalos de confianza. En cambio. Al generar este proceso de remuestreo varias veces se genera una distribución muestral que estima la distribución muestral teórica desconocida. (Yu, C.H.,2008).

Este proceso demanda un uso intensivo de la computadora, de allí que su actual vigencia práctica está dada por la disponibilidad de recursos computacionales veloces y baratos. En una etapa del proceso se utiliza la simulación Monte Carlo, pero en lugar de generar las simulaciones a partir de un modelo distribucional hipotético o postulado, tal cual es el uso habitual de las simulaciones Monte Carlo, en el remuestreo las simulaciones se hacen a partir de los datos reales que pertenecen a la muestra original disponible. Existen distintas técnicas de remuestreo, como ser: Validación cruzada, Jackknife, Test de permutaciones, Bootstrap, entre otras. En este trabajo nos centraremos en esta última metodología.

\section{1.- Bootstrap}

Efron (1979) introdujo el bootstrap como un método general para estimar la distribución muestral de un estadístico basándose en los datos observados. Un problema típico de la estadística involucra la estimación de un parámetro desconocido. Ante esta situación emergen dos preguntas básicas 1) ¿Qué estimador debe ser usado? 2) habiendo elegido el estimador ¿Cuán preciso es este estimador? 
El bootstrap es una metodología general destinada a responder a la segunda pregunta. Es una técnica basada en la potencia computacional como un sustituto del análisis teórico. El bootstrap permite determinar la distribución muestral de, virtualmente, cualquier estadístico computado a partir de una muestra. En los cursos tradicionales de estadística se enseña que si se quiere estimar la esperanza de una distribución se toma una muestra aleatoria y se calcula la media muestral como estimador del parámetro. Pero también los datos proporcionan para la precisión del estimador una estimación de su error estandar y a partir de éste se pueden construir intervalos de confianza. Como antes se ha señalado, en general no se habla de la precisión de la mediana y mucho menos de sus intervalos de confianza. El bootstrap permite, obviando ya sea deducciones matemáticas complejas, o los comportamientos asintóticos de los estimadores, superar estos obstáculos. Para esto el bootstrap aproxima la distribución muestral de la mediana sin apelar al conocimiento de la distribución poblacional, sino, considerando la distribución empírica de los datos. Es decir trata a los datos disponibles como una población y saca muestras aleatorias con reemplazo y de igual tamaño a la original, de dicha población. Por eso, este procedimiento se denomina remuestreo. Se puede notar que en estas muestras aparecerán valores repetidos de los datos originales como consecuencia del muestreo con reemplazo.

Para cada muestra se calcula la mediana y considerando una gran cantidad de estas muestras, por ejemplo 1000, se obtiene la distribución muestral aproximada de la mediana muestral y entonces se puede obtener una estimación de su error estandar. Esto que se ha hecho para la mediana se puede hacer para otros estimadores no importa cuan complicados sean. La técnica bootstrap permite también estimar el sesgo del estimador.

Por otra parte a partir de la obtención del error estandar se construyen intervalos de confianza. (DiCiccio, Efron,1996; Efron, 1993). El único requisito para alcanzar estos resultados es disponer de un software que permita calcular el estimador y generar el proceso del remuestreo (Boos, Stefanski, 2010).

Ejemplo: La siguiente tabla brinda los datos de una muestra aleatoria de tamaño $n=25$

\begin{tabular}{|l|l|l|l|l|l|l|l|l|l|l|l|l|}
\hline 1 & 4 & 6 & 12 & 13 & 14 & 18 & 19 & 20 & 22 & 23 & 24 & 26 \\
\hline 31 & 34 & 37 & 46 & 47 & 56 & 61 & 63 & 65 & 70 & 97 & 385 & - \\
\hline
\end{tabular}

El siguiente histograma revela la distribución asimétrica de esta muestra 
IV Encuentro sobre Didáctica de la Estadística, la Probabilidad y el Análisis de Datos

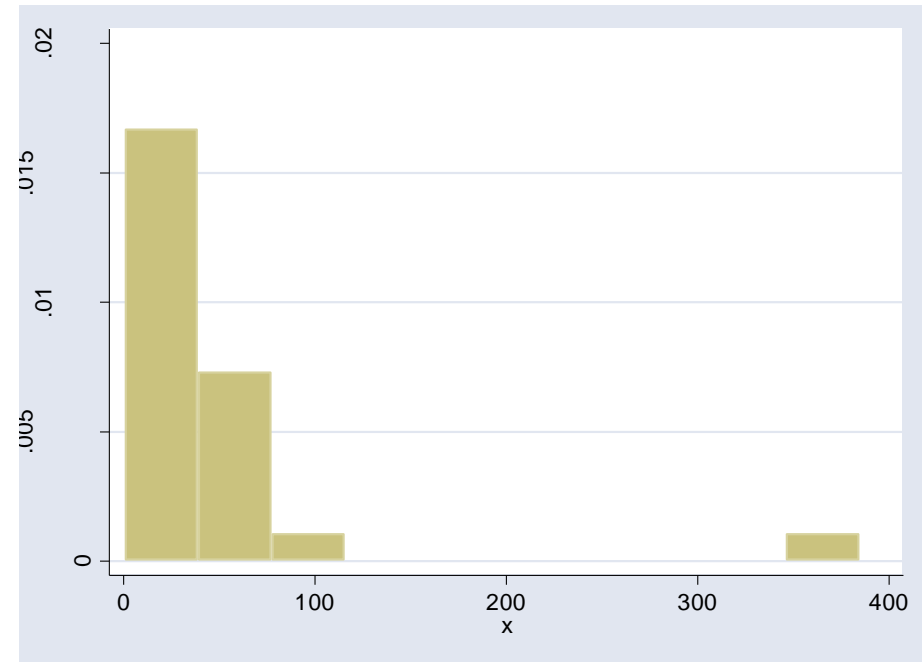

\section{Histograma de los valores muestrales}

La media es $\bar{x}=47,76$ y la mediana $m=26$. Por otra parte el error estandar de la media es $\mathrm{E} . \mathrm{E}($ media $)=14.8$, este error estandar es fácilmente calculado a partir del conocimiento del desvío estándar $\mathrm{S}$ de los datos como $S / \sqrt{n}$. Sin embargo, no existe una fórmula tan simple que entregue el error estandar de la mediana. Utilizando el software Stata se puede aplicar la metodología bootstrap aplicando el siguiente comando:

\section{- bootstrap "sum x, detail" r(p50), reps(1000) saving(rem)}

Esta instrucción indica que a partir de la muestra original se tomaran 1000 muestras con reemplazo todas de tamaño $n=25$ y para cada una de ellas se calculará la mediana. La salida que brinda el software mencionado, es la siguiente:

$\begin{array}{ccccccc}\text { Variable } \mid \text { Reps } & \text { Observed } & \text { sesgo } & \text { Std. Err. } & {[95 \% \text { I.C] }} \\ \text { m } & \text { | } 1000 & 26 & 2.67 & 7.57 & 19 & 46\end{array}$

Se observa que el error estandar de la mediana es E.E $(\mathrm{m})=7.57$. Este valor señala que para un conjunto de datos asimétricos como el que estamos analizando, la mediana es menos variable que la media.

El software también almacena en el archivo llamado rem los valores de las 1000 medianas y se puede solicitar tanto, un resumen de estos valores como un histograma de los mismos para tener una visión aproximada de cómo es la distribución muestral de la mediana. El resumen está presentado en la siguiente tabla

\begin{tabular}{cccccc} 
Variable I & Obs & Mean & Std. Dev. & Min & Max \\
\hline m | 1000 & 28.67 & 7.57 & 13 & 61
\end{tabular}


Aquí se observa que lo consignado como desvío estandar de la distribución muestral bootstrap es una estimación del error estandar de la mediana tal como había sido indicado en la salida exhibida anteriormente. Por otra parte la diferencia entre la media de estos 1000 valores (28.67) y el valor original de la mediana (26) es llamado el sesgo y es el valor 2.67, también antes consignado. Cabe señalar que menor sea el sesgo más centrada estará la distribución en el valor original del estadístico.

La metodología bootstrap también entrega un I.C para la mediana muestral. Ordenadas las medianas de menor a mayor se toman los valores que están en la posición 25 y 975 y estos valores determinan un I. C del 95\%, el I.C obtenido en nuestro ejemplo es [19-46] señalado en la salida. Cabe acotar que existen también otros procedimientos, aquí no mostrados, para calcular I.C utilizando bootstrap.

A continuación se presenta el histograma de la distribución empírica de las 1000 medianas obtenidas por bootstrap.

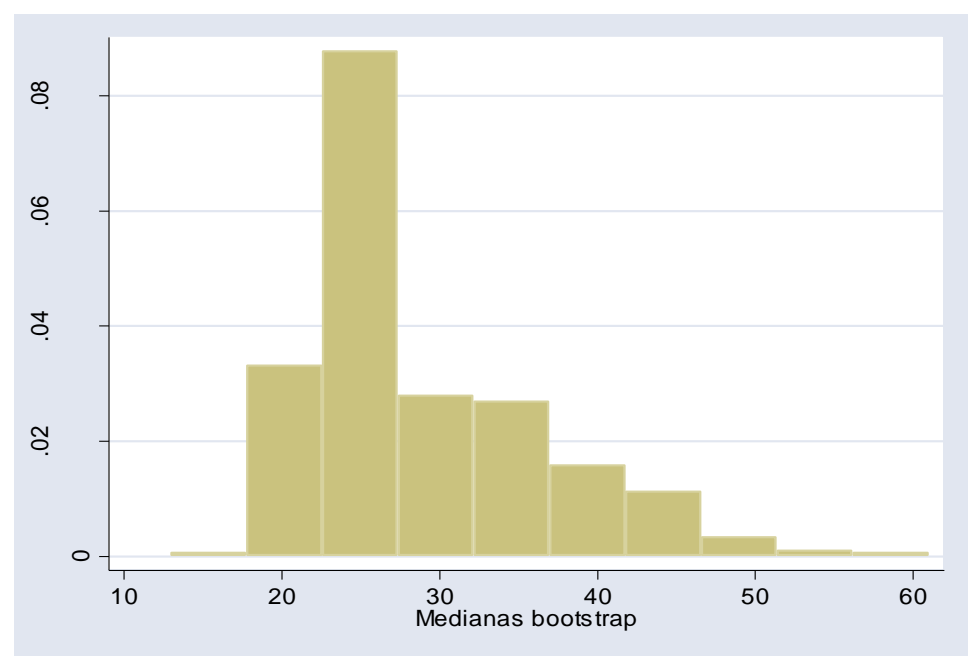

Histograma de los valores de las medianas

\section{Consideraciones}

La distribución muestral es un concepto básico y fundamental para la comprensión de la inferencia estadística, pero como se ha señalado su enseñanza y aprendizaje presenta dificultades. La construcción de la distribución de un estadístico a partir de un conjunto de datos es de difícil visualización, pues se la debe obtener ya sea mediante fórmulas o mediante simulaciones, pero en ambos casos haciendo supuestos distribucionales acerca de la población muestreada. El uso de la metodología boostrap, basada en el remuestreo, permite apreciar, prescindiendo de fórmulas, cómo varía un estadístico de muestra a muestra y por lo tanto, cómo se va construyendo la distribución muestral.

Por tal motivo, estimamos relevante continuar indagando acerca de cuáles son las maneras más adecuadas para introducir esta metodología basada en el uso intensivo del recurso computacional, como herramienta complementaria a la enseñanza tradicional de la estadística inferencial en los cursos básicos o introductorios de estadística. 
IV Encuentro sobre Didáctica de la Estadística, la Probabilidad y el Análisis de Datos

\section{Bibliografía}

[1] Belia, S., Fidler, F., Williams, J. and Cumming., G. Researchers misunderstand confidence intervals and standard error bars. Psychol. Methods, 2005,10:389-39

[2] Boos, D. Stefanski, L. Efron's bootstrap. Significance,2010,. Vol.7, 4, 186-188

[3] Castro Soto, A; Vanhoof, S; Van den Noorgate,W; Onghena, P. Students' misconceptions of statistical inference: A review of the empirical evidence from research on statistics education. Educational Research Review, 2007, 2.98-113

[4] Cumming, G.,Williams, J., \& Fidler, F. Replication and researchers' understanding of confidence intervals and standard error bars. Understanding Statistics, 2004, 3, 299311.

[5] Chance, B., delMas, R., \& Garfield, J. Reasoning About Sampling Distributions. In D. Ben-Zvi \& J. Garfield, The Challenge of Developing Statistical Literacy, Reasoning and Thinking, 2004, Dordrecht: Kluwer Academic Publishers.

[6] DiCiccio; T; Efron, B. Bootstrap Confidence Intervals. Statistical Science, 1996, Vol, 11 $\mathrm{N}^{\mathrm{o}} 3,189-228$.

[7] Efron, B. Bootstrap Methods:Another look at the Jacknife. The Annals os Statistics, 1979, 7, 1-26..

[8] Efron, B. An introduction to the bootstrap. New-York, Chapman \& Hall, 1993.

[9] Hubbard, R., and Bayarri, M.J. “Confusion Over Measures of Evidence ( $p$ 's) Versus Errors ( $\alpha$ 's) in Classical Statistical Testing," The American Statistician, 2003, 57, $171-182$.

[10] Kline, R.B. Beyond significance testing: Reforming data analysis methods in behavioral research. Washington DC, USA: APA, 2004.

[11] Nickerson, R. Null hypothesis significance testing: A review of an old and continuing controversy. Psychological Methods,2000, 5, 241-301.

[12] Rodríguez M.I., Albert A. Prueba de hipótesis estadística:estudio de dificultades conceptuales en estudiantes de grado y de postgrado. Memoria de la XI Escuela de Invierno de Educación Matemática. Red de Centros de Investigación en Matemática Educativa. 2007, 328-343. ISBN: 978-970-9971-14-9.

[13] Vallecillos, A. (1996).Inferencia estadística y enseñanza: un análisis didáctico del contraste de hipótesis estadísticas. Ed. COMARES. Granada. España

[14] Yu, C.H. Resampling: a conceptual and procedural Introduction. In J. W. Osborne (ed.). Best Practice in Quantitative Methods. Thousand Oaks, CA: Sage Publications, 2008. 


\title{
Nivel de razonamiento en la solución de problemas matemáticos y su relación con variables cognitivas, metacognitivas y la dificultad de la tarea
}

\author{
Alejandra Alfaro Barquero ${ }^{1}$ \& Greivin Ramírez Arce ${ }^{2}$
}

\section{Resumen}

Con el objetivo de identificar la relación existente entre el nivel de razonamiento matemático y las variables cognitivas, metacognitivas y de dificultad de la tarea, se aplicaron en el 2012 tres ejercicios algebraicos, tomados del Examen de Diagnóstico en Matemática, a una muestra de 52 estudiantes matriculados en cursos de matemática en el Instituto Tecnológico de Costa Rica (ITCR).

El nivel de razonamiento cognitivo se evaluó empleando el modelo taxonómico SOLO (Structure of Observed Learning Outcomes de Biggs y Collis, 1982). Asimismo, se estimó la dificultad de los ejercicios y los conocimientos matemáticos con el método de Rasch. Adicionalmente se utilizó como medida de habilidad el puntaje en la Prueba de Aptitud Académica del ITCR en el área matemática, el autoconcepto a partir del Cuestionario de Autoconcepto Matemático de Dermitzaki y Efklides (2000) y se midió la precisión metacognitiva como la concordancia entre los juicios de desempeño y el puntaje obtenido en el ítem.

Se encontraron diferencias significativas en el nivel de razonamiento cognitivo según la dificultad de los ítems, obteniendo mejores niveles de razonamiento en los ejercicios de mayor facilidad.

Asimismo, se evidenció asociación entre los conocimientos previos y el nivel de razonamiento cognitivo, no así en la habilidad y el autoconcepto matemático.

Palabras claves: experiencias metacognitivas, habilidad matemática, solución de problemas matemáticos, razonamiento cognitivo, conocimientos matemáticos, precisión metacognitiva y autoconcepto matemático.

\begin{abstract}
The purpose of this study was to identify the relation between the level of mathematical reasoning with cognitive and metacognitive variables, and also with the difficulty of the task. 52 students from Technological Institute of Costa Rica (ITCR) solved tree algebraic exercises taken from Diagnostic test of Mathematic.

The level of mathematical reasoning was defined utilizing SOLO Model (Structure of Observed Learning Outcomes of Biggs and Collis, 1982).The item's difficulties and the mathematical knowledge were determined by Rasch method. The scored obtained by mathematical area in ITCR Admission Test was utilized
\end{abstract}

${ }^{1}$ Instituto Tecnológico de Costa Rica, alealfaro@itcr.ac.cr

2Instituto Tecnológico de Costa Rica, gramirez@itcr.ac.cr 
by measure of ability and the Mathematical Self-concept Questionnaire (Dermitzaki. y Efklides, 2000) was applied. Finally, metacognitive precision was estimated with the concordance between judgments of self performance and the score in each item.

It was found higher means of mathematical reasoning in easier items. Also, association between prior knowledge and level of cognitive reasoning was evident, but not in the ability and mathematics self-concept.

Keywords: metacognitve experiences, mathematical skill, mathematical problem solving, cognitive development, mathematical knowledge, metacognitive accuracy, mathematical self-concept.

Modalidad: ponencia

El interés del presente estudio es identificar la relación existente entre la habilidad, los conocimientos, la dificultad de la tarea, el autoconcepto matemático y la precisión metacognitiva en relación con el nivel de razonamiento cognitivo alcanzado por los estudiantes del Instituto Tecnológico de Costa Rica (ITCR) en la resolución de ejercicios matemáticos.

Lo anterior es relevante considerando que los estudiantes universitarios ingresan con deficiencias en contenidos y habilidades de razonamiento matemático, lo que se evidencia en los resultados obtenidos en las pruebas de diagnóstico aplicadas en el ITCR en el 2010, a una muestra de 1315 alumnos matriculados en el curso de Matemática General, quienes obtuvieron, en promedio, una nota de 42,6 ( $\sigma=18.02)$ en escala de 0 a 100, con resultados aún más deficientes en el 2011 con una media de 35,34 ( $\sigma=16.12)$ (Ramírez y Barquero, 2010, 2011).

Estas deficiencias repercuten sobre el rendimiento académico obtenido en los cursos universitarios, explicando un $17.3 \%$ de la varianza en la nota del curso de Matemática General (Ramírez y Barquero, 2010). Ello se refleja igualmente en los porcentajes de aprobación de los cursos de Matemática General (47.64\%) y Calculo Diferencial e Integral (48.74\%) en el periodo 2006-2011 en el ITCR (Ramírez, Hernández, Alfaro, Blanco; 2012), lo que incide a su vez sobre la deserción en las universidades públicas (Barquero, 2009 y Guillén y Chinchilla, 2005 y 2007).

La situación es más preocupante si se considera que los estudiantes universitarios recibieron formación en temas de álgebra en secundaria, y a pesar de ello, experimentan dificultades en la resolución de nuevos problemas, presentando obstáculos epistemológicos (costumbres intelectuales que fueron útiles en situaciones previas, pero que después de un tiempo pueden obstaculizar la resolución exitosa de tareas afines, Bachelard, 1976), pues los conocimientos se transfieren de manera inadecuada a otros contextos, siendo difíciles de identificar y modificar para los individuos (Godino, en Ruiz, 2006).

Desde esta perspectiva, se propone como supuesto que el nivel de razonamiento cognitivo está asociado con el desempeño matemático, y constituye por lo tanto una herramienta fundamental para detectar obstáculos epistemológicos, mejorar la eficiencia de la transferencia de conocimientos adquiridos a nuevos problemas y la precisión metacognitiva; y consecuentemente, repercutirá en el rendimiento académico de los estudiantes. 
En este sentido el razonamiento cognitivo se definirá como el nivel de complejidad de los procesos mentales evidenciados por los individuos a través de las respuestas escritas en la solución de problemas algebraicos, utilizando el modelo taxonómico SOLO (Structure of Observed Learning Outcomes de Biggs y Collis, 1982), el cual jerarquiza el desarrollo cognitivo en categorías con orden progresivo según el nivel de madurez.

Para profundizar en el tema Ramírez, Chavarría y Mora (2010) realizaron un estudio en estudiantes del ITCR para analizar los tipos de errores cometidos por los estudiantes, los obstáculos evidenciados y el nivel de madurez de razonamiento cognitivo mostrado por los alumnos utilizando los cuatro niveles del modelo SOLO en expresiones algebraicas, concluyeron que la mayoría de los estudiantes se ubicaron entre los primeros dos niveles cognitivos (preestructural y uniestructural).

Resultados distintos encontraron Hernández y Cuevas (2014) en el curso de Cálculo Diferencial e Integral, en el ubicaron que los estudiantes se encontraban en el nivel multiestructural en el tema de álgebra. Por su parte Fuster (2014) utilizó el modelo SOLO para interpretar los resultados en pruebas de acceso a la universidad, concluyendo que las mismas se realizan desde las rutinas y no a partir de presupuestos teóricos de innovación.

Desde esta perspectiva se propone como hipótesis del estudio que el nivel de razonamiento cognitivo estará influido por otras variables cognitivas (la habilidad y los conocimientos matemáticos), variables de la tarea (dificultad), así como también por variables subjetivas como el autoconcepto matemático, pues éste último representa una valoración subjetiva de habilidad, y por lo tanto, dependerán en algún sentido de la competencia matemática del sujeto.

En lo pertinente al último concepto mencionado, Efklides, y Petkaki (2002), Efklides y Tsiora (2002) y Efklides (2006) encontraron una correlación moderada entre la autoconfianza matemática y la precisión metacognitiva; así como también entre las experiencias metacognitivas, la habilidad y autoconcepto matemático. En este sentido el autoconcepto repercute sobre las experiencias metacognitivas en la percepción del desempeño, retroalimentando el auto-concepto y generando una mayor eficacia regulatoria.

Así mismo, Alfaro (2014) encontró asociación negativa entre la precisión metacognitiva y la dificultad de la tarea, obteniéndose mejores niveles de precisión en ítems de menor dificultad.

Por otro lado, si bien el razonamiento cognitivo es importante para explicar el desempeño matemático de los estudiantes, existen también otras variables relevantes para los teóricos del aprendizaje que se han asociado con el desempeño, desde una perspectiva en la cual se concibe al individuo como un sujeto activo que ejerce una función de regulación sobre sus propios procesos cognitivos, modificando sus conductas y habilidades mentales a través del monitoreo y control de las variables motivacionales y cognitivas (Kitsantas, Huie y Winsler, 2008). Éstas últimas incluyen las capacidades cognitivas (o de procesamiento de información) así como también las habilidades metacognitivas (Arsal, 2010).

En este sentido Flavell (1979) definió la metacognición como cognición de la cognición, refiriéndose a los conocimientos que posee el individuo sobre sus propios procesos mentales, los cuales se vinculan con la metacognición a través de las funciones de control y monitoreo (Efklides, 2009). 
Al respecto algunos autores suponen la existencia de una estrecha relación entre habilidades cognitivas y metacognitivas. Así por ejemplo, Sastre-Riba (2011) señaló que las personas con altas capacidades intelectuales tienden a mostrar un mayor conocimiento metacognitivo, destacando la relación entre metacognición y funciones ejecutivas como procesos educativos de alto nivel. En este sentido, González (2010) subrayó también la vinculación bidireccional entre las estrategias de orden superior y la metacognición, ya que el desarrollo de estrategias impulsa el conocimiento metacognitivo y éstas a su vez, el desarrollo y generalización de estrategias cognitivas de orden superior.

Desde esta perspectiva en la cual se propone una asociación positiva entre cognición y metacognición, es de interés en este estudio evaluar la posible relación entre el nivel de razonamiento cognitivo y la precisión de las experiencias metacognitivas. Éstas últimas se definen como el grado de consciencia cognitiva o experiencia afectiva vinculada con tareas de aprendizaje (Flavell, 1979), percepciones subjetivas que resultan de los procesos mentales de control y monitoreo (Efklides, 2006).

Tales experiencias representan el grado de conciencia sobre los conocimientos y estrategias metacognitivas, las emociones asociadas con el afrontamiento de los desafíos cognitivos y la percepción de progreso o logro de las metas. A través de estas vivencias se generan una serie de sentimientos y estimaciones variadas, tales como las percepciones de dificultad, esfuerzo, tiempo requerido en la solución, la exactitud de la respuesta, la confianza y la satisfacción (Efklides, 2006).

En este caso en particular, las experiencias metacognitivas se evaluarán a través de los juicios de desempeño, los cuales pueden ser considerados como indicadores subjetivos del desempeño objetivo, lo que variará de individuo a individuo en el grado en que dichos juicios se acerquen o no a la realidad. Los juicios de desempeño son por tanto definidos como experiencias metacognitivas enfocadas a la evaluación de la calidad de la solución brindada en una tarea cognitiva y su respectiva valoración como correcta o incorrecta (Efklides, 2006).

A pesar de que estos juicios se basan en información externa e involucran procesos que tienden a ser más analíticos, ello no garantiza pleno grado de consciencia, transparencia, eficiencia y control sobre las propias conductas (González, 2010). Cabe suponer al respecto que los estudiantes que muestren mayores niveles de razonamiento cognitivo tenderán a mostrar mejor grado de precisión de los juicios del desempeño. Desde esta perspectiva la precisión se entenderá como el grado de concordancia entre las estimaciones de desempeño y la ejecución en la tarea, reflejo de la eficiencia de las funciones de control en situaciones de desempeño académico, a partir de la valoración del esfuerzo y tiempo invertido, así como de las estrategias empleadas (Efklides, 2006).

Este concepto de precisión de los juicios de desempeño ha sido estudiado también bajo la denominación de calibración, definido como los sucesos que resultan de la interacción entre el éxito subjetivo y el objetivo, frente a una tarea compuesta por un determinado número de reactivos en condiciones de incertidumbre. En este sentido las percepciones generadas pueden involucrar sesgos de sub o supra valoración, los cuales pueden ser modificados con la inclusión de variables ambientales críticas (Macbeth, Razumiejczk, 2012).

En esta línea algunos autores han encontrado evidencias de asociación entre las estimaciones de éxito y la ejecución en la tarea, tales son los casos de Beaudoin, y Desrichard (2011) quienes encontraron relación leve pero significativa entre los juicios de autoeficacia 
de memoria (MSE) y rendimiento en tareas de recuerdo, lo que tiende a incrementarse cuando los MSE son de dominio específico. A pesar de ello algunos autores tales como Everson (1997) y McCormick (2003) sugieren que la metacognición es una habilidad de dominio general, dadas las correlaciones evidenciadas entre las estimaciones de desempeño en ámbitos distintos entre sí, tales como en el área verbal y matemática.

En relación con la precisión metacognitiva, Tabor y Dunlosky (1997) y Rhodes y Tauber (2011) encontraron una mayor precisión en los juicios de aprendizaje (autopercepciones del grado de aprendizaje obtenido en tareas de memoria) cuando las estimaciones se emiten con retraso (unos minutos después de que se ha concluido la fase previa de estudio); comparado con quienes hacían sus estimaciones inmediatamente después de finalizar su periodo de aprendizaje, argumentando que los juicios que se expresan con dilación se vinculan con la memoria de corto y largo plazo (González, 2010), a diferencia de las estimaciones inmediatas, asociadas con la memoria de corto plazo.

La relación entre razonamiento cognitivo y precisión ha sido poco estudiada, es escaso lo que se conoce sobre el monitoreo y la regulación cognitiva en el campo matemático, pues los esfuerzos se han enfocado principalmente en el tema de la comprensión de textos (Winne y Nesbit, 2010), por lo que existen aún grandes vacíos en esta área.

Así mismo, al igual que se han evidenciado carencias en las habilidades matemáticas en población universitaria, también se ha encontrado que cerca de la mitad de los estudiantes de primer ingreso universitario no han alcanzado el pensamiento operacional formal, con pobres destrezas de monitoreo, tendencia al exceso de confianza (Winne y Nesbit, 2010) y limitaciones para percibir los errores cometidos (Efklides y Sideris, 2009, Moraitou y Efklides, 2009).

\section{Método}

\section{Participantes}

Se utilizó una muestra de 52 estudiantes, la cual fue tomada de Alfaro (2014), compuesta por un total de 495 estudiantes matriculados en los cursos de Matemática General (MG) y Cálculo Diferencial e Integral (CDI) en el segundo semestre de 2012 del Instituto Tecnológico de Costa Rica (ITCR).

La muestra de 52 estudiantes se seleccionó en forma aleatoria considerando una proporción similar de estudiantes de ambos cursos (46.2\% de MG y 53.8\% de CDI), 25\% mujeres y $75 \%$ hombres; con una media de edad de 18.76 años. La mayoría de ellos de primer ingreso universitario $(78.8 \%)$.

En el curso MG el 75\% presentó condición de repitencia, mientras que en el curso CDI el porcentaje fue de $32.1 \%$. La mayor proporción de reprobación en el curso MG es esperable considerando que el estudio se desarrolló en el segundo semestre, cuando sólo los estudiantes que aprobaron MG, matriculan por primera vez CDI, los demás repiten el curso. 


\section{Procedimientos}

Los instrumentos aplicados incluyeron una prueba de ejercicios matemáticos y una escala de autoconcepto matemático. Los mismos fueron aplicados durante las clases de los cursos de MG y CDI, previa autorización de la Escuela de Matemática, los docentes y los estudiantes (éstos últimos mediante consentimiento informado).

Los estudiantes completaron en primer lugar la prueba de conocimientos matemáticos constituida por 6 ítems provenientes de la Prueba de Diagnóstico en Matemática aplicada en 2010 y tres problemas tomados del Cuestionario de Experiencias Metacognitivas de Efklides (2006). Para cada uno de los ejercicios respondieron, luego de haber intentado dar solución al problema, qué tan correcta creían haber resuelto la tarea, utilizando una escala de 0 a 3 ( $0=$ nada, $1=$ poco, $2=$ bastante y $3=$ totalmente) para expresar sus juicios de desempeño.

Los conocimientos matemáticos se estimaron a partir de los resultados obtenidos en dicha prueba matemática con el método de Rasch, utilizando el paquete estadístico Winsteps. Se conceptualizaron como el nivel de dominio de conceptos evidenciado en las respuestas de solución a ejercicios matemáticos, que requieren para su acierto, el manejo de algunos contenidos matemáticos básicos (de nivel de primaria y secundaria). La prueba mostró una confiabilidad de .7, lo que se considera aceptable para fines de investigación, siempre y cuando los resultados obtenidos no tengan altas consecuencias para los individuos (Montero 2013 en Alfaro, 2014). Así mismo se realizó un análisis factorial confirmatorio para comprobar la adecuación de los indicadores empíricos utilizados en la medición de los constructos. Se obtuvieron al respecto niveles de ajuste adecuados (CFI .94, IFI .95, RMSEA $.05)$.

Por su parte, la dificultad de los ítems se definió como el grado de aptitud necesaria para resolver con éxito una tarea (Martínez, Hernández y Hernández, 2006); y se estimó también utilizando el método de Rasch. Los ítems obtuvieron puntajes aceptables de ajuste (entre .8 y 1.2). La dificultad se obtuvo con puntuaciones estandarizadas, siendo el ítem número tres el de mayor de mayor dificultad, seguido por el dos y el uno, como se muestra en la tabla 1 (Alfaro, 2014).

Tabla 1. Características psicométricas de los ítems matemáticos de acuerdo con el modelo de

\begin{tabular}{cccc}
\multicolumn{3}{c}{ Rasch } \\
\hline Ítem & Dificultad (Rasch) & Ajuste (IN.MSQ) & Tema \\
\hline 1 & -.96 & 1.04 & Factorización por agrupación \\
2 & -.46 & 1.11 & Simplificación algebraica \\
3 & .07 & 1.10 & Ecuación cuadrática \\
\hline
\end{tabular}

Posterior a la realización de la prueba matemática los estudiantes completaron una traducción al español del Cuestionario de Autoconcepto matemático de Dermitzaki y Efklides (2000), partiendo de la definición de dicho concepto como el nivel de competencia en el área matemática percibido por el propio individuo (Efklides y Vlachopoulos, 2012). Este instrumento estuvo constituido por 22 ítems y mostró una confiabilidad de .93 (Alfaro, 2014). Igualmente se realizó el análisis factorial confirmatorio alcanzándose indicadores de ajuste aceptables (CFI .89, IFI .9, RMSEA .08).

Adicionalmente, la habilidad matemática se obtuvo utilizando el puntaje obtenido en la Prueba de Aptitud Académica del ITCR en el área matemática de los estudiantes que habían resuelto previo a su ingreso a la universidad, con autorización del Comité de Examen 
de Admisión. Dicha prueba evalúa destrezas evidenciadas en la solución de tareas de índole numérica con ejercicios asociados con operaciones básicas, razonamiento deductivo/inductivo, interpretación de gráficos, resolución de problemas, razonamiento con figuras y razonamiento probabilístico (Moreira, 2010) y presentó valores de confiabilidad Alfa de Cronbach superiores a .9.

Por otra parte, el nivel de razonamiento cognitivo se definió utilizando la adaptación del modelo SOLO propuesto por Ramírez, Chavarría y Mora (2010), contemplando las categorías preestructural, uniestructural, multiestructural y relacional.

Según estos autores, en el nivel preestructural (el más básico), los estudiantes poseen información aislada de los conceptos y propiedades de los números reales que intervienen en la simplificación de expresiones algebraicas, no identifican correctamente el método que se debe emplear para la factorización o bien, confunden la tarea con resolver una ecuación. El estudiante conoce algunos conceptos pero no entiende como éstos se relacionan, ni qué significa resolver una ecuación.

En el siguiente nivel, uniestructural, los estudiantes realizan relaciones en forma correcta de algunos de los conceptos y propiedades de números reales en la simplificación y factorización de expresiones algebraicas. Identifican correctamente los conceptos y métodos de factorización y resolución de ecuaciones; sin embargo, en ocasiones realizan agrupaciones incorrectas, tiene problemas con priorizar las operaciones básicas y pierden una solución de la ecuación al realizar un despeje incorrecto; enfocándose en un solo aspecto relevante de la tarea.

En el nivel multiestructural, los estudiantes logran crear relaciones en forma correcta de los principales conceptos y propiedades que intervienen en la simplificación, factorización y solución de ecuaciones donde intervienen expresiones algebraicas simples o generalizadas. Realizan en forma correcta la agrupación de los términos semejantes y la extracción del factor común en cada una de estas agrupaciones; sin embargo, muestran algunas inconsistencias que no le permiten obtener la factorización correcta del polinomio. Reconocen qué es una ecuación y saben lo que tienen hacer para resolverla; sin embargo, no dan como respuesta el conjunto solución.

Finalmente en el nivel relacional, los estudiantes son capaces de simplificar, factorizar y resolver ecuaciones donde intervienen expresiones algebraicas. Factorizan polinomios como el producto de sus factores más simples, encuentran las soluciones y dan el conjunto solución de la ecuación propuesta.

Para la clasificación de las respuestas de los estudiantes según las categorías antes mencionadas se seleccionó una muestra de tres ejercicios (de los nueve resueltos por los estudiantes) vinculados con el tema de álgebra (factorización, simplificación algebraica y ecuación cuadrática). Cada ítem fue calificado otorgando una categoría de 1 a 4 , considerando los niveles contemplados en el modelo SOLO. Posteriormente se estimó un promedio de razonamiento por sujeto obtenido a partir de las categorías alcanzadas en los tres ítems.

Para ello previamente se instruyó a dos estudiantes de la Carrera de Enseñanza de la Matemática del ITCR por parte del docente de matemática: Msc. Greivin Ramírez. Este profesor formó parte del equipo que diseñó, aplicó y evaluó el Examen de Diagnóstico de 2010 (Ramírez y Barquero, 2010, 2011) y realizó varios estudios con la aplicación del 
modelo SOLO en estudiantes de secundaria y de grado universitario en temas de álgebra, estadística, probabilidad y geometría (Ramírez y Ballestero, 2007, Ramírez, Chavarría, Mora y Barahona, 2009, Ramírez, Chavarría y Mora, 2010).

Se siguió un proceso de entrenamiento en dos vías: la calificación de los ítems y la clasificación de los resultados obtenidos con la implementación del modelo SOLO. En el primer caso se realizaron varias sesiones de entrenamiento hasta garantizar un adecuado nivel de concordancia en los criterios de calificación entre los asistentes y el profesor experto, obteniéndose un grado de acuerdo casi perfecto según Mandeville (2005) en los coeficientes de correlación intra-clase: .941 y .946 (Alfaro, 2014).

Procedimiento similar se utilizó en el entrenamiento para la clasificación de las respuestas de los estudiantes según la adaptación del modelo SOLO hasta lograr porcentaje de concordancia superior al $90 \%$ entre los asistentes y el docente.

Finalmente la precisión metacognitiva se obtuvo como un puntaje promedio de precisión de los juicios de desempeño obtenidos en los nueve ítems de la prueba matemática. Para ello cada ítem se calificó en escala de 0 a 3, utilizando la misma métrica empleada para estimar los juicios de desempeño.

La escala de juicios del desempeño obtuvo un confiabilidad Alfa de Cronbach de .79 y obtuvo indicadores de ajuste observables en el análisis confirmatorio (CFI .98, IFI, .98, RMSEA .036).

Posteriormente para estimar el error en la precisión metacognitiva se restó al juicio de desempeño de cada ítem el puntaje obtenido en la solución de dicho ejercicio matemático, de la siguiente manera JD1-Puntaje.Ítem1= error de estimación prospectivo 1, y así sucesivamente.

Un valor de cero (0) en el error de estimación significa que hubo precisión en el juicio de desempeño, dado que el puntaje asignado en la escala de juicio de desempeño se correspondió en forma perfecta con el puntaje obtenido en el ejercicio matemático. Por otro lado un puntaje de 3 refleja la mayor distancia obtenida entre el juicio de desempeño y el rendimiento en el ítem, y por tanto el mayor grado de error.

Seguidamente, para obtener el puntaje de precisión se recodificaron en orden inverso los valores absolutos obtenidos en el error de estimación. De este modo, un valor de error de estimación de 0 se reconvirtió en un valor de 3; ya que representa el máximo puntaje de precisión obtenida para cada ítem.

Con las medidas obtenidas se implementó el procedimiento estadístico denominado análisis de senderos (path analysis) con el paquete estadístico Lisrel 8.54; el cual es utilizado para establecer relaciones de causalidad unidireccionales entre variables observadas (Cea, 2002), con el objetivo de evaluar un modelo explicativo sobre la relación entre la habilidad, los conocimientos y el autoconcepto en el área matemática con el nivel de razonamiento cognitivo y su posible vinculación con la precisión metacognitiva.

Así mismo, se realizó un análisis de varianza con medidas repetidas con el paquete estadístico SPSS para identificar la relación existente entre el nivel de razonamiento cognitivo y la dificultad de la tarea. Este método se utiliza para evaluar el efecto de la dificultad de los ítems considerando dicha variable como condición intra-sujeto, ya que todos los niveles de dificultad (contenidos en los tres ítems) se aplicaron un mismo grupo de 
estudiantes, contrario a lo que ocurre en un anova tradicional, en el cual se tendría una muestra de sujetos diferentes para resolver cada ítem (supuesto de medidas independientes).

\section{Resultados}

Las estadísticas descriptivas y correlacionales se resumen en las tablas 2 y 3. Cabe agregar además, que ninguna de las variables cumplió con el supuesto de normalidad de acuerdo con la prueba de Shapiro-Wilk.

Tabla 2. Medias y desviaciones estándar de las diferentes escalas y medidas

\begin{tabular}{cccccc}
\hline Medida & $\mathrm{M}$ & $\mathrm{DE}$ & Escala & Mínimo & Máximo \\
\hline Desempeño en la prueba matemática & 15.62 & 5.53 & $0-26$ & 3 & 26 \\
Estimación de conocimiento matemáticos & .2865 & .76 & Puntajes z & -1.89 & 2.23 \\
Autoconcepto matemático & 3.31 & .661 & $1-5$ & 2.09 & 4.76 \\
Precisión retrospectiva & 2.13 & .351 & $0-3$ & 1.40 & 2.89 \\
Habilidad matemática (admisión) & 57.38 & 13.60 & $0-100$ & 31.71 & 87.8 \\
\hline
\end{tabular}

Todas las variables mostraron asociación entre sí de manera significativa, a excepción de la precisión retrospectiva con el autoconcepto matemático. Así mismo, con respecto al razonamiento cognitivo las variables que evidenciaron mayor relación con dicho constructo fueron la precisión retrospectiva y los conocimientos matemáticos (mayores a 0.3 ).

Tabla 3. Correlaciones entre las variables

\begin{tabular}{lccccc}
\hline & 1 & 2 & 3 & 4 & 5 \\
\hline Examen admisión prueba matemática (1-100) & 1 & & & & \\
Puntaje autoconcepto matemático & $0,230^{* *}$ & 1,000 & & & \\
Puntaje precisión retrospectiva & $0,188^{*}$ & $-0,025$ & 1,000 & & \\
Conocimientos Matemáticos & $0,361^{* *}$ & $0,232^{* *}$ & $0,398^{* *}$ & 1,000 & \\
Nivel de razonamiento cognitivo & $0,299^{* *}$ & $0,235^{* *}$ & $0,313^{* *}$ & $0,666^{* *}$ & 1,000 \\
\hline
\end{tabular}

$* \mathrm{p}<0,05$

$* * \mathrm{p}<0,01$

Por otra parte, para evaluar la asociación entre nivel de razonamiento cognitivo y la dificultad de la tarea (ítem) se realizó un análisis de varianzas con medidas repetidas; sometiendo a prueba la hipótesis nula $\mathrm{H} 0$ : los promedios de nivel de razonamiento de los estudiantes son iguales en los tres niveles de dificultad de los ítems.

Para ello se evaluó el supuesto de esfericidad (las varianzas de las diferencias de los niveles de dificultad son iguales cuando se comparan dos a dos); comprobándose el cumplimiento del mismo $(\mathrm{p}=0.933)$. En concordancia con lo anterior se analizó la aproximación univariada (efectos intra-sujeto), pues este procedimiento es más potente y recomendado en muestras pequeñas.

Los resultados permiten rechazar la hipótesis nula y concluir que los promedios de nivel de razonamiento de los estudiantes son distintos en los tres niveles de dificultad de 
los ítems, como se muestra en la tabla 4. Se identificaron diferencias significativas $(\mathrm{p}=0.001)$ en las medias de los niveles de razonamiento según la dificultad de la tarea, en ítem $1(\mathrm{M}=3.32, \sigma=1.11)$, ítem $2(\mathrm{M}=2.83, \sigma=1.06)$ e ítem $3(\mathrm{M}=2.71, \sigma=0.8)$, evidenciando que a menor dificultad del ítem los estudiantes alcanzan mayor nivel de razonamiento; evidenciándose una tendencia de asociación lineal mediante la prueba de contrastes intra-sujetos $(\mathrm{F}(1)=12.83, \mathrm{p}=0.001)$.

Tabla 4. Análisis de varianzas para los niveles de razonamiento cognitivos de los estudiantes según la dificultad de la tarea con medidas repetidas

\begin{tabular}{lccccc}
\hline Variable dependiente & gl & $\begin{array}{c}\text { Suma de } \\
\text { cuadrados }\end{array}$ & $\begin{array}{c}\text { Media } \\
\text { cuadrática }\end{array}$ & F & Sig. \\
\hline Dificultad del ítem & 2 & 10.76 & 5.38 & 7.028 & .001 \\
\hline
\end{tabular}

Por otro lado, para medir la relación entre el razonamiento cognitivo con las variables precisión metacognitiva de los juicios de desempeño y la habilidad, el autoconcepto y los conocimientos en el área matemática se utilizó el análisis de senderos (path analysis), cuyos resultados se muestran en la figura 1.

Para facilitar la interpretación del modelo cabe aclarar que en la representación gráfica de un análisis de senderos las figuras cuadradas representan variables observables; y las líneas que se establecen entre estas indican relaciones de causalidad entre las variables independientes (aquellas utilizadas como punto de partida de las flechas) y las variables dependientes (a las cuales apunta cada flecha). El valor numérico que aparece al lado de cada flecha representa el grado de relación entre las variables; es decir, el cambio producido en la variable dependiente cuando se modifica en una unidad la variable independiente. Así mismo para cada variable dependiente se calcula un error, lo que representa el porcentaje de varianza de la variable dependiente no explicada por la o las variables independientes incluidas en el modelo.

Para aceptar el ajuste del modelo los indicadores CFI, IFI deben alcanzar valores iguales o superiores a .9, el RMSEA puntajes iguales o inferiores a .05 y la Chi cuadrada será mejor cuanto más pequeña y su probabilidad superior a 0.05 (Cea, 2002).

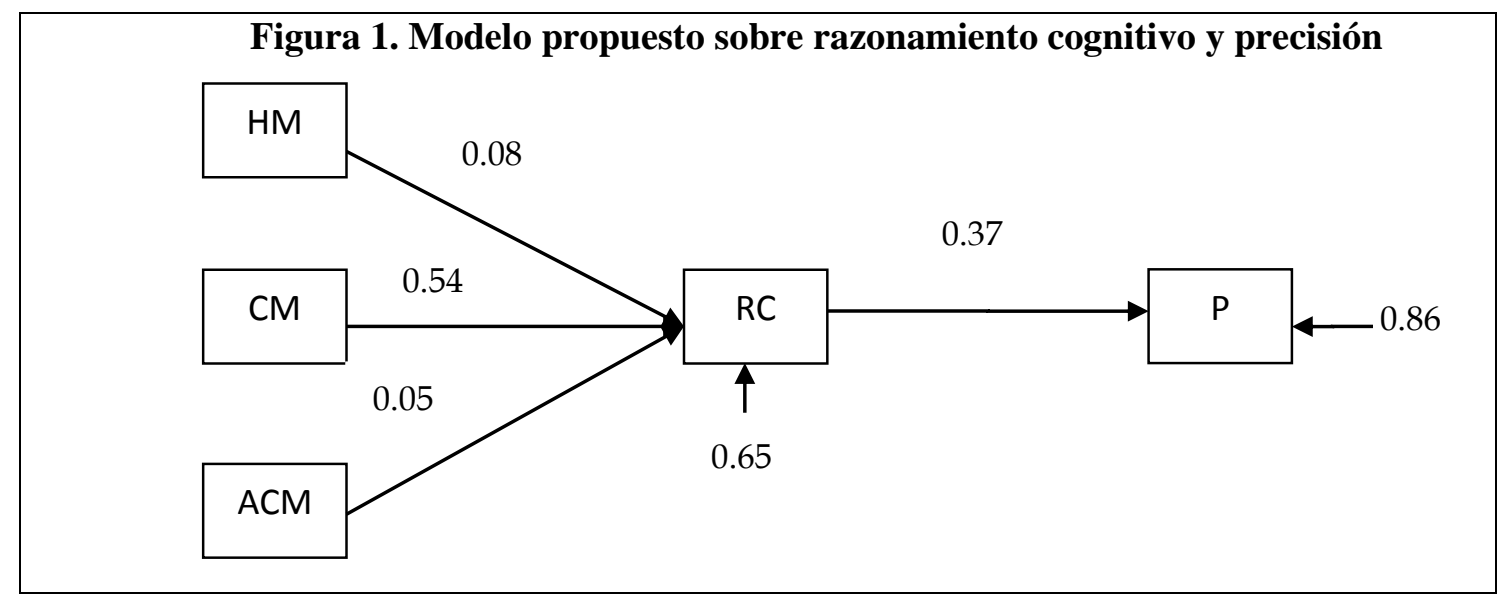




\section{Variables Independientes}

HM: Habilidad matemática

CM: Conocimientos matemáticos

ACM: Autoconcepto matemático
Variables dependientes

RC: Nivel de razonamiento cognitivo

P: Precisión de los juicios de desempeño

La figura 1 muestra los resultados obtenidos al evaluar el modelo propuesto, el cual mostró indicadores favorables (CFI de .97, el IFI de .97, el RMSEA de .053 y la Chi cuadrada $\left.\chi^{2}(9)=12.84 .22, \mathrm{p}=0.17\right)$. A pesar de ello, las variables habilidad y autoconcepto matemático mostraron una asociación cercana a cero, por lo que se consideró conveniente eliminarlos del modelo. Con esta modificación se obtuvieron los resultados mostrados en la figura 2.

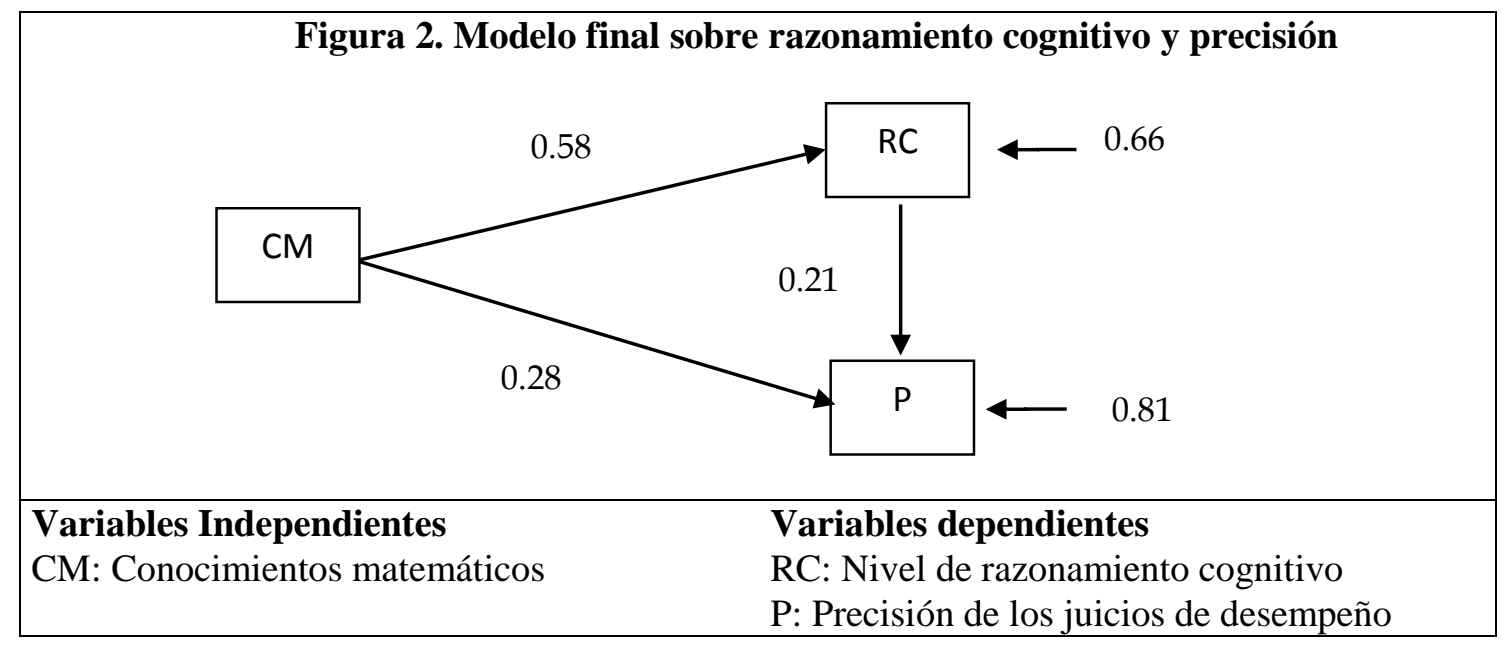

De acuerdo con el modelo final se obtuvo un ajuste perfecto (CFI e IFI: ambos con valor 1 , RMSEA de .000 y la Chi cuadrada $\left.\chi^{2}(1)=0, p=1\right)$. En este modelo los conocimientos matemáticos mostraron efecto sobre el razonamiento cognitivo y sobre la precisión. La pendiente de la recta evidencia que, en promedio, por cada incremento en la unidad de medida de los conocimientos matemáticos aumenta en 0.58 el razonamiento cognitivo alcanzado por los estudiantes y 0.28 la precisión de los juicios de desempeño. Así mismo, también se encontró asociación entre el razonamiento cognitivo y la precisión, en la cual por cada incremento en una unidad de medida del nivel de razonamiento cognitivo, la precisión de los juicios del desempeño aumenta 0.21 puntos. La varianza explicada por los conocimientos matemáticos sobre el razonamiento cognitivo fue del $34 \%$, mientras que para el modelo de precisión la varianza explicada por los conocimientos matemáticos y el razonamiento cognitivo fue del $19 \%$.

\section{Discusión}

Los resultados obtenidos aportan evidencias que sustentan la hipótesis en favor de la asociación entre los conocimientos matemáticos y la dificultad de la tarea sobre el razonamiento cognitivo, no así en el caso de la habilidad y el autoconcepto matemático.

No hay evidencia suficiente en este estudio para explicar el por qué estas dos últimas variables no mostraron vinculación con el nivel de razonamiento cognitivo; sin embargo, es posible conjeturar que la habilidad podría estar siendo medida también a través de la prueba de contenidos matemáticos, partiendo de la asociación evidenciada entre conocimientos y 
habilidad. En este sentido, si bien es cierto que dicha prueba evalúa conceptos previamente adquiridos, la transferencia adecuada de los mismos frente a problemas y contextos novedosos exigen para su acierto también algún grado de flexibilidad mental. Siendo así, la habilidad podría estar siendo implícitamente evaluada a través de los ejercicios de conocimiento, reduciendo el efecto directo de la habilidad sobre el razonamiento cognitivo.

Por otra parte, en relación con las variables que resultaron significativas en el estudio, dichos resultados destacan la interrelación existente entre las variables de contexto (la dificultad de las tareas) y las condiciones intra-sujeto (los conocimientos matemáticos) sobre el nivel de razonamiento cognitivo; el cual, si bien puede considerarse una característica de la capacidad intelectual del individuo que tiende a ser muy estable, su grado de éxito o madurez dependerá de la correspondencia entre las demandas cognitivas y el nivel de competencia de la persona. En este sentido es más probable que un mayor número de estudiantes evidencien altos niveles de madurez en sus razonamientos frente a exigencias menores que ante otras de mayor dificultad, en las que sólo quienes muestren mejor conocimiento y destreza podrán tener más criterio para razonar sus respuestas.

De este modo, se concluye que la dificultad de la tarea muestra una asociación inversa con el razonamiento cognitivo. Estos hallazgos son congruentes con los obtenidos por Alfaro (2014), en el cual se encontró una relación también inversa entre la dificultad de los ítems y la precisión metacognitiva; así como también asociación positiva entre esta última y los conocimientos previos; comportamiento muy similar al mostrado por dichas variables con respecto al razonamiento cognitivo.

En este sentido es válido retomar los planteamientos de González (2010), quien subrayó la existencia de una vinculación bidireccional entre las estrategias de orden superior y la metacognición. Desde esta perspectiva el nivel de razonamiento cognitivo y la precisión metacognitiva podrían también mostrar un desarrollo bidireccional, lo que explicaría por qué tanto la dificultad de la tarea como la competencia matemática mostraron un comportamiento muy similar frente a ambas variables.

Lo anterior también explicaría la asociación positiva encontrada entre el nivel de razonamiento cognitivo y la precisión metacognitiva, lo que a su vez respalda la tesis de Sastre-Riba (2011) de que las personas con mayores capacidades intelectuales tienen a presentar igualmente mejor desempeño metacognitivo.

\section{Bibliografía}

Alfaro, A. (2014). Experiencias metacognitivas y su relación con la autoeficacia, la habilidad matemática, la dificultad de la tarea y el rendimiento académico (Tesis maestría inédita). Instituto de Investigaciones Psicológicas, Costa Rica.

Arsal, Z. (2010). The effects of diaries self-regulation on strategies of preservice science teachers. International Journal of Environmental \& Science Education, 5 (1), 85 103.

Bachelard. (1976). La formación del espíritu científico. 5 ed. México: Siglo Veintiuno, editores, S.A. 
IV Encuentro sobre Didáctica de la Estadística, la Probabilidad y el Análisis de Datos

Barquero, J. (2009). Análisis de Rendimiento Académico en la carrera de Ingeniería en Electrónica. Manuscrito inédito. Departamento de Orientación y Psicología. Instituto Tecnológico de Costa Rica.

Beaudoin, M. y Desrichard, O. (2011). Are memory self-efficacy and memory performance related? A meta-analysis. Psychological Bulletin, 137 (2), 211-241.

Biggs, J. y Collis, F. (1982). Evaluating the quality of learning: The Solo Taxonomy. Academic Press, New York.

Cea, A. (2002). Análisis multivariable. Teoría y práctica en la Investigación Social. Madrid: Editorial Síntesis S.A.

Dermitzaki, I. y Efklides, A. (2000). Self-concept and its relations with cognitive and metacognitive factors regarding performance in specific domains of knowledge. Psychology: The Journal of the Hellenic Psychological Society, 7, 354-368.

Efklides, A. (2006). Metacognition and affect: What can metacognitive experiences tell us about the learning process? Educational Research Review 1, 3-14.

Efklides, A. (2009). Metacognition. Defining its facets and levels of functioning in relation to self-regulation and co-regulation. European Psychologist, 13 (4), 277-287. Doi. 10.1027/1015-5759.25.2.115.

Efklides, A. y Petkaki, Ch. (2005). Effects of mood on students' metacognitive experiences. Learning and instruction, 15, 215-431.

Efklides, A. y Tsiora, A. (2002). Metacognitive experiences, self-concept, and self regulation. Psychologia, 45, 222-236.

Efklides, A. y Sideris, G. (2009). Assessing cognitive failures. European Journal of Psychological Assessment, 25 (2), 67-72.

Efklides, A. y Vlachopoulos, S. (2012). Measurement of Metacognitive knowledge of self, task and strategies in Mathematics. European Journal of Psychological Assessment, 28 (3), 227-239. DOI:10.1027/1015-5759/a000145

Everson, H. (1997). Do metacognitive skills and learning strategies transfer across domains? Paper presented at the Annual Meeting of the American Educational Research Association.

Flavell, J. (1979). A new area of cognitive-developmental inquiry. American Psychologist, 34 (10), 906-911.

Fuster, C. (2013) Las pruebas de acceso a la Universidad y el control del aprendizaje de Historia. Historia e Identidades culturales. Actas V Simposio Internacional de Didáctica de las Ciencias Sociales en el ámbito Iberoamericano \& Congresso Internacional das XIII Jornadas de Educaçao Hsitórica, 1010-1021.

Guillén, E y Chinchilla, S. (2007).Variables asociadas a la repetición de cursos en el ITCR. Manuscrito inédito. Departamento de Orientación y Psicología. Instituto Tecnológico de Costa Rica.

Guillén, E. y Chinchilla, S. (2005). Detección de estudiantes en riesgo académico en el Instituto Tecnológico de Costa Rica. Revista Educación, 29 (2), 123-138.

González, J. (2010). Para una reconstrucción genealógica y epistemológica del concepto de metacognición. Revista de Psicología, 19 (1), 129-153. 
Hernández, A. y Cuevas, J. (2014). Análisis sobre el nivel de competencias en matemáticas básicas por parte de estudiantes de cálculo diferencial de nivel superior. En F. Santillán. Revista Iberoamericana para la Investigación y el Desarrollo Educativo. Jalisco, México.

Kitsantas, A., Huie, A. y Winsler, A. (2008). Self- regulation and ability predictors of academic success during college: a predictive Validity Study. Journal of Advanced Academics, 20 (1), 42-68.

Macbeth, G. y Razumiejczk, E. (2012). Modelado matemático del efecto del anclaje sobre el sesgo de subconfianza. Revista de Psicología (PUPC), 30 (2), 224-248.

Martínez, Hernández y Hernández (2006). Psicometría. Madrid: Alianza Editorial.

McCormick, C. (2003). Metacognition and learning. In W. M. Reynolds \& G. E. Miller (Eds.), Handbook of Psychology: Vol. 7. Educational psychology (pp. 79-102). NewYork: Wiley y Sons.

Mandeville, P. (2005). Tema 9. El coeficiente de correlación intraclase (ICC). Ciencia UANL, 8 (3), 414-416.

Moraitou, D. y Efklides, A. (2009). The Blank in the Mind Questionnaire (BIMQ). European Journal of Psychological Assessment, 25 (2), 115-122.

Moreira, T. (Julio, 2010). Tabla de especificaciones: Una experiencia de validación de la prueba de aptitud académica del Tecnológico de Costa Rica. Trabajo presentado en el VII Congreso Iberoamericano de Psicología. Federación Iberoamericana de Asociaciones de Psicología, Oviedo, España.

Ramírez, G., Hernández, M., Alfaro, A. y Blanco, R. (2012). Informe final RAMA TEC. Vicerrectoría de Vida Estudiantil y Servicios Académicos, Departamento de Orientación y Psicología y Escuela de Matemática de Instituto Tecnológico de Costa Rica.

Ramírez, G. y Barquero, A. (2011). Análisis de la prueba de diagnóstico de Matemática. Departamento de Orientación y Psicología y Escuela de Matemática del Instituto Tecnológico de Costa Rica.

Ramírez, G. y Barquero, A. (2010). Análisis de la prueba de diagnóstico de Matemática. Departamento de Orientación y Psicología y Escuela de Matemática del Instituto Tecnológico de Costa Rica.

Ramírez, G., Chavarría, J. y Mora, M. (2010). Análisis de las conceptualizaciones erróneas en conceptos de álgebra: un estudio con estudiantes universitarios de primer ingreso. Acta Latinoamericana de Matemática Educativa, 23. 95-103.

Ramírez, G., Chavarría, J., Mora, M. y Barahona, C. (2009). Análisis de las conceptualizaciones erróneas en conceptos de geometría y sistemas de ecuaciones: un estudio con estudiantes universitarios de primer ingreso. Revista Digital Matemática, Educación e Internet, 10 (1). Recuperado de http://www.tecdigital.itcr.ac.cr/revistamatematica/

Ramírez, G. y Ballestero, E. (2007). La centración en problemas de probabilidad basados en el razonamiento proporcional. Acta Latinoamericana de Matemática Educativa, 20. 46-68. 
IV Encuentro sobre Didáctica de la Estadística, la Probabilidad y el Análisis de Datos

Rhodes, M. \& Tauber, S. (2011). The influence of delaying Judgments of learning on metacognitive accuracy: A meta-analytic review. Psichological Bulletin, 137 (1), 131-148. Doi:10.1037/a0021705

Ruiz, A. (2006). Escuela francesa de didáctica de las Matemáticas y la construcción de una nueva disciplina científica, Costa Rica: San José. CIMM/UCR.

Sastre-Riba, S. (2011) Funcionamiento metacognitivo en niños con altas capacidades. Neurología, 52 (1), 11-18.

Tabor \& Dunlosky (1997). Age-Related Differences in Absolute but not relative metamemory accuracy. Psychology and Aging, 12 (1), 50-71.

Winne, P. y Nesbit, C. (2010). The psychology of academic achievement. Annual Review of Psychology, 61, 653-678. 


\title{
Mundial de Futbol Brasil 2014 y la probabilidad
}

\author{
Giovanni Sanabria Brenes ${ }^{1}$
}

\begin{abstract}
Resumen
A solicitud del periódico digital CRhoy de Costa Rica se elaboró un modelo probabilístico para predecir los resultados de algunos partidos de futbol del Mundial Brasil 2014. Las publicaciones generaron polémica y una serie de comentarios en redes sociales. El presente trabajo expone de forma general el modelo probabilístico realizado y analiza algunos comentarios para valorar como interpretan la probabilidad algunos docentes y ciudadanos en general.

Palabras clave: modelo probabilístico, concepto de probabilidad, cultura estadística.
\end{abstract}

${ }^{1}$ Instituto Tecnológico de Costa Rica - Universidad de Costa Rica, Costa Rica. gsanabriab@yahoo.com 


\title{
Uso Del Software Power Point a Través De La Metodología de Proyectos: Una Aplicación Para La Enseñanza De La Estadística En Secundaria.
}

\author{
Rocío Mora Fallas
}

Modalidad: Ponencia

\section{RESUMEN}

Dados los nuevos programas de estudio regidos por el Ministerio de Educación Pública, para el tercer ciclo de la educación general básica de Costa Rica, se pone en manifiesto cada vez más la necesidad de aplicar en las aulas metodologías para la enseñanza de corte constructivista. El presente trabajo plantea una breve exploración teoría de los beneficios que puede traer al docente de secundaria el utilizar recursos tecnológicos de fácil acceso; en este caso se hablara del software Power Point y las bases de datos reales como las que tenemos a nuestra disposición por medio del INEC, en contraste con el uso de metodologías clásicas constructivistas como lo son los proyectos estudiantiles; esto con el objetivo de motivar al estudiante a que pueda aprender estadística no solo como un contenido más en la asignatura de matemática si no de incentivar su importancia ya sea por cultura general de calidad o en un futuro profesional. Además se muestran ejemplos a manera de guía para el docente de cómo aplicar la conjunción de dichas metodologías en los salones de clase costarricenses.

. Palabras claves: Proyectos, Power Point, Estadística, Secundaria, Bases De Datos.

\begin{abstract}
According to the latest study programs given by the Ministry of Public Education for the third cycle of the basic general education in Costa Rica, it's clear the need to apply in the classrooms new constructivist methodological ways of teaching. This paperwork states a brief theoretical exploration of the benefits that could be given to the high school teachers by using or promoting the usage of easy technological resources such as Microsoft's Power Point along with the real data bases given by the National Institute of Statistics and Census or INEC, also, the usage of classic constructivist methodologies such as Student projects; with the objective of encouraging the alumni to learn about statistics not only as a common subject in the syllabus of the math courses but as a powerful knowledge in the everyday living as well as tool for the professional development in a significant amount of majors or career choices. Also this research shows examples of ways to guide the teachers of how to apply the this knowledge properly in the classrooms.
\end{abstract}

Keywords: Projects, Power Point, Statistics, High School. Data Bases. 


\section{Introducción}

Con la reforma de los planes de estudio para la educación en la asignatura de matemática en todos los niveles, pero en específico en secundaria, la estadística resalta con más importancia que en los planes de estudio anteriores, pueda deberse al gran valor que proporciona en diversas áreas profesionales dicha disciplina, por lo cual el Ministerio de Educación Pública intenta hacer frente a esta exigencias incluyendo, contenidos estadísticos en los programas de estudio para cada nivel educativo; sin embargo a pesar de que actualmente estos contenidos tienen más peso en el currículo de la matemática, el abismo existente entre lo que contienen los programas y lo que realmente se aplica y se enseña en la aulas es evidente, y una de las causas de este abismo es el hecho de el docente no sabe o no utiliza medios o estrategias educativas idóneas que hagan que el estudiante realmente aprecie y comprenda el valor de la estadística en su futuro profesional o en la vida en general.

En una investigación realizada en la Universidad de Minnesota, sobre la preparación de los educadores a la hora de enseñar estadística y el impacto en los estudiantes, enuncia entre sus principales conclusiones el hecho de que la mayor parte de los estudiantes reciben conocimientos estadísticos desde la primaria, y aun así llegan aún sistema universitario con conocimientos de probabilidad y estadística muy débiles o casi nulos (Ben-Zvi \& Garfield, 2008).

Lo que nos incita a pensar sobre el grado de responsabilidad del docente en este tipo de resultados. Una interesante tesis de graduación de la Universidad Nacional de Costa Rica (Arce, Alpizar Vargas, Arroyo Hernández, Gamboa Araya, \& Hidalgo Mora, 2004), realizó como parte de la misma un experimento donde entrevistaron en promedio a 100 educadores de la provincia de Heredia, y se les cuestiona sobre que contenido eliminarían o es prescindible del plan de estudios de matemática, a lo cual alrededor de un $15 \%$ responden que debería de eliminarse los contenidos referentes a probabilidad y estadística, por ser muy básicas y poco útiles y carecen de herramientas y bases suficientes y eficaces para enseñarlos de manera correcta.

Es en contexto donde radica la importancia de investigar y estudiar sobre medios didácticos y herramientas útiles en secundaria que doten a los docentes de la suficiente confianza para enseñar estadística de manera que esta se quede en los alumnos.

Este escrito explorará una propuesta metodológica ejemplificada para guiar al docente de secundaria e incentivarlo en indagar este tipo de metodologías y dar una motivación para enseñar estadística de la mejor manera posible. 


\section{Aprendizaje por medio de proyectos, con el uso particular del software Power Point.}

Sobre la metodología de los proyectos estudiantiles

William Heard Kilkpatrick, quien tiene el mérito de ser el introductor al método de proyectos, señalado como una figura fundamental de la educación progresista según la revista trimestral de educación: Perspectivas, en esta se habla extensamente de este método donde su esencia principal es explicar el por qué sirve el método y cuáles son sus pretensiones, que en contexto se sobreentiende que como objetivo principal de aplicación es la búsqueda de un propósito que mueva los gustos y deseos de los alumnos, en este revista nos habla acerca de la opinión de Kilkpatrick con respecto a la importancia de buscar este propósito antes del aplicar el método de proyectos:

El propósito es aquel impulso interior que hace perseverar al niño frente a obstáculos y dificultades. Esto genera una predisposición a los recursos interiores propios del conocimiento y el pensamiento. Los ojos y las manos se vuelven alertas. El propósito que actúa como meta orienta el pensamiento del niño, dirige su estudio del proyecto y los materiales, extrae lo necesario de sugerencias apropiadas y pone a prueba dichas sugerencias valorando su utilidad en relación al objetivo. El propósito, al contemplar un objetivo específico, define el éxito (UNESCO, 1997)

Nos queda claro como docentes, la importancia infundada desde la concepción del método de Kilkpatick de que el proyecto sea en cosas o temas que interesen de una u otra forma al discente, para que el proyecto educativo sea una vía de éxito en el proceso de enseñanza.

La idea de la aplicación de esta metodología constructivista está proyectada al trabajo en conjunto con un software eficiente donde el docente será solo una guía en el desempeño del trabajo de los alumnos, para esto el profesor deberá prepara guía de estudio con anterioridad donde el discente pueda explorar por sus propios medios todos los contenidos que se desean enseñar.

\section{Esquema 1: toma de decisión por parte del docente para el manejo de la metodología de proyectos ene aula educativa.}

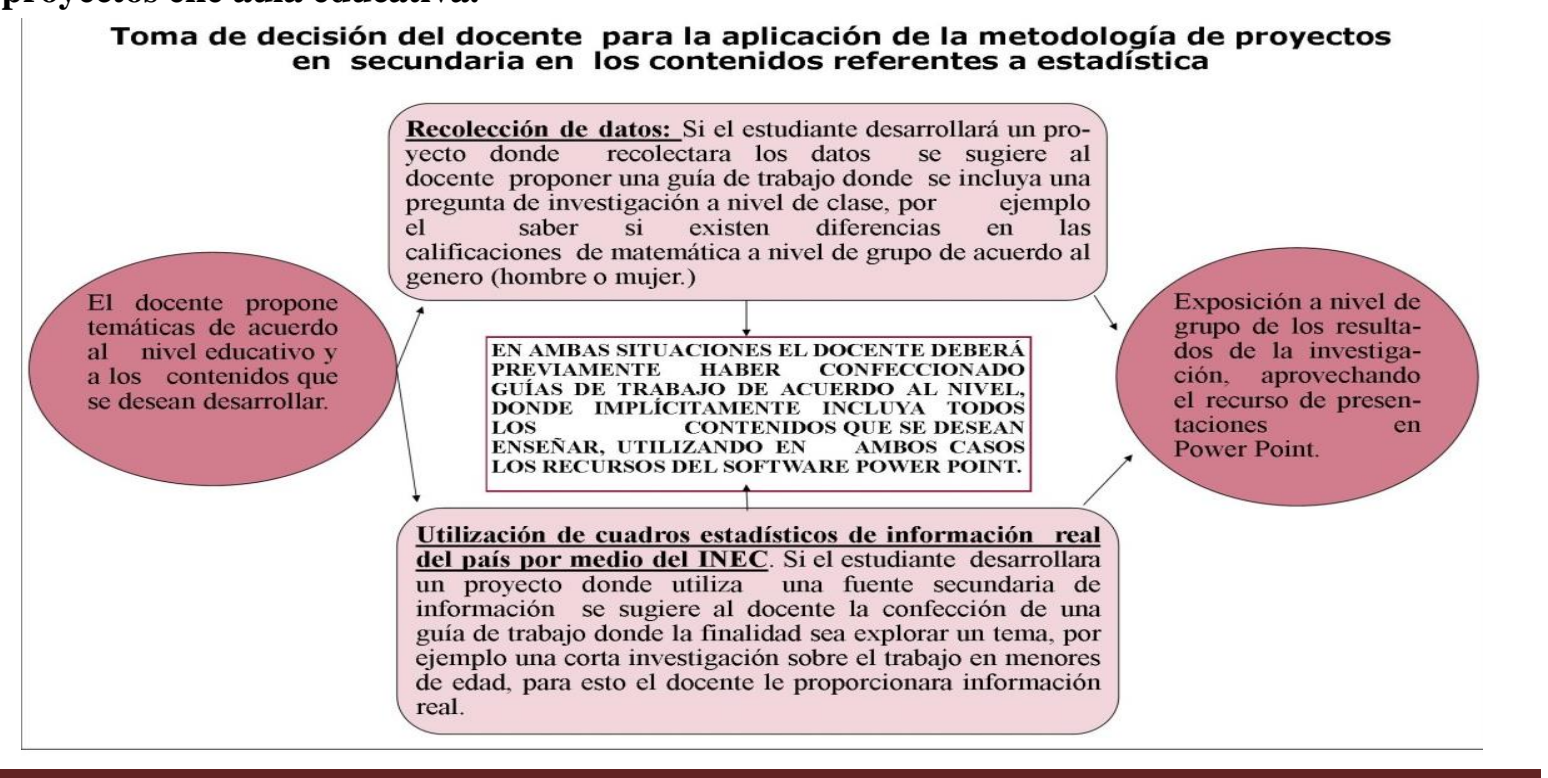




\section{Uso de un software eficiente}

Considerando la realidad tecnológica que vivimos, donde cada día y constantemente se inventa o se actualiza algo nuevo, donde todos quieren estar a la moda de los más reciente en el mercado. En este contexto el sector educativo no se queda atrás, Armenta, Larios \& Urrea (2010) nos dice:

Al maestro, en sus diversas funciones, se le exigen nuevas responsabilidades educativas en el trabajo con los estudiantes: que promueva competencias para la ciudadanía, que incorpore al aula las Tecnologías de la Información y la Comunicación con fines educativos, que conozca y aplique las nuevas teorías pedagógicas y didácticas, que desarrolle nuevos modelos de gestión del conocimiento escolar y de interacción con sus colegas y la comunidad educativa. (Armenta, Larios, \& Urrea, 2010)

Si bien este extracto es con respecto al sistema educativo mexicano, define a la perfección no solo la realidad de las exigencias educativas de ese País, sino de la gran mayoría de países latinos, que en competencia con sistemas educativos europeos o anglosajones se lleva una ferviente lucha por "mejorar" el sistema y estar al corriente de lo más novedoso, olvidando muchas veces lo más importante: el alumno; ya que si bien todas estas competencias en los sistemas educativos, suponen el ideal de lograr que nuestras jóvenes generaciones tengan potentes herramientas para lograr desempañarse en un mundo cada vez más competitivo, no hay que olvidar que cada estudiante que se encuentra en el aula de aprendizaje, tiene necesidades educativas distintas, contextos familiares diferentes, que los hacen vulnerables al cambio y al aprendizaje lo que repercute en que no todos puedan alcanzar la velocidad del cambio que se pretende.

Cabe rescatar la anécdota de la Ex Ministra de Educación de Chile

Estamos elevando drásticamente los estándares de tecnología en todas y cada una de las unidades escolares, llevándola hasta el aula y el escritorio de cada profesor y profesora. He visitado junto a la Presidenta de la República el sur de Chile, y tengo que decir que quedé preocupada por el exceso de tecnología instalada en los establecimientos escolares. En esta visita, al inaugurar tres liceos de una infraestructura espectacular en la nueva Región de los Ríos -en la zona de los lagos- no pude menos que preguntarme qué van a hacer los maestros con esta tecnología. Realmente es de última generación...Hay que tener en cuenta que nos encontrábamos en el sur de Chile, en un liceo rural. Yo pensaba en el desafío para los profesores, para los maestros y los directivos de estos liceos que tienen hoy el más alto nivel de tecnología disponible en el mundo. No es una cuestión de dinero, porque cuando se hace una inversión de esta naturaleza, no se puede comprar 
tecnología antigua, hay que comprar la más nueva. Es un gran desafío incorporar a estos liceos una tecnología tan moderna. (Jimenez, 2010)

Podemos canalizar que la situación expuesta se presenta en un liceo rural de Chile; sin embargo puedo decir que la realidad en Costa Rica no tiene gran diferencia, cada vez nos preocupamos más por estar actualizados, pero no nos preocupamos por utilizar lo que se tiene, ni reflexionamos si todo esto de verdad traerá consecuencias positivas, cabe resaltar que la situación anterior no solo representaba un reto para los alumnos: además de aprender los contenidos de su programa de estudios deben aprender a utilizar una tecnología de la que tal vez muchos no tengan el más mínimo conocimiento, mientras otros lo tienen totalmente, lo cual inclusive llega hasta generar discriminación, pero la situación no es solo esa, también como lo pudimos valorar es un gran reto para el docente.

La razón del porque toda la situación contextualizada, es apuntar al hecho de que como docentes y parte fundamental de un sistema educativo nos estamos olvidando de los esencial, de lo que si tenemos, de lo que podemos utilizar con facilidad y eficiencia, y con esto además de poder transmitir con eficacia a nuestros alumnos un conocimiento, transmitirles también el valor de apreciar la simpleza de las cosas y de que no se ocupa tener lo más novedoso, ni lo último del mercado para tener resultados importantes y de validez.

Con esto quiero referirme explícitamente a la enseñanza de la estadística en la educación secundaria. En la actualidad tenemos a disposición una buena base de datos de software estadísticos para facilitar los cálculos; sin embargo muchos apuntan a un desempeño profesional o para la educación superior, con lo cual si se hiciera su desempeño en secundaria podría dar más dificultades que soluciones.

Por mencionar algunos que se podrían adaptar a secundaria, se tienen los siguientes:

- Winstats: es un software libre con una interfaz gráfica bastante simple y poco llamativa y un tanto inflexible, sus herramientas son de poca utilidad en secundaria; sin embargo podría resultar útil en la elaboración de histogramas.

- StadiS: es de acceso libre, con características muy similares al Winstats, pero su implementación es un poco más complicada que es este último.

- Statgraphics: tiene una interfaz gráfica un poco más llamativa, su implementación no es tan complicada como los anteriores; sin embargo tiene la limitación de no ser de acceso libre.

Es este el punto idóneo para hacer mención a un software que por su característica "simpleza", es pasado por alto, hablo del programa del Microsoft Office: Power Point, su presentación es tan noble que es reducido a su implementación para presentaciones en 
diapositivas, que si bien es bastante útil en este ámbito, lo que podría ofrecer en el ámbito educativo es mucho más amplio de la implementación que se le da.

Sobre las características que debe de tener un software como medio didáctico, Moreno (2004) nos dice: "Una posible propuesta, referida a los medios en general, debería tener en cuenta al menos tres marcos de referencia como son: la funcionalidad de los medios, sus posibilidades didácticas, la fundamentación educativa; y los aspectos técnicos" (Moreno, 2004)

Si hablamos sobre la funcionalidad de un software en el aula nos referimos a que sea viable en costo y beneficios, que permita el control por parte de los usuarios, que sea de fácil ubicación y acceso, que permita la flexibilidad de uso. Con respecto a esto podemos agregar que nuestro software en estudio presenta sin ninguna dificultad todas estas características; aunque pertenece a la compañía Microsoft, podemos decir que es un software que se adquiere casi por "default" ya que su costo está incluido en la compra de los paquetes mínimos necesarios en un computador, si hablamos del sistema operativo Windows, que es el que normalmente se incorpora en los centros educativos. Por otra parte su interfaz gráfica es bastante atractiva y de fácil uso tanto para el docente como el discente. En cuanto a su fundamentación educativa y aspectos técnicos, nos referimos a que su adquisición sea fácil, sea económico, su mantenimiento sea sencillo, de fácil actualización, sea vigente, entre otras características, lo cual como lo discutimos anteriormente están presentes. En cuanto a lo referente sobre posibilidades didácticas, es precisamente el punto que se pretende abordar en esta sección de nuestro escrito, para ello lo haremos de manera ejemplificada en el aula educativa...

La idea esencial del recurso será no solo utilizarlo para la creación de gráficos a través de cuadros estadísticos, si no también instar a la investigación, hacer uso de la información del país, a través de la potente base de datos que se nos ofrece El INEC y valga decir que no solo en Costa Rica, también México, Colombia, España entre muchos otros países poseen bases de datos similares, de acceso público y presentadas en Excel que será en primera instancia la base para la manipulación de datos en Power Point.

\section{Propuesta metodológica}

Basándonos en el actual plan de estudios para la educación secundaria costarricense, se dará un recorrido sobre el uso del software Power Point en los distintos niveles educativos que podría emplearse, representado así una posible guía de usos que el docente podría darle al implementar el software como herramienta didáctica en el aula educativa.

Los contenidos de estadística en secundaria se limitan al tercer ciclo de la educación general básica, en el plan denominado: plan de transición se incluye al primer nivel del 
tercer ciclo: sin embargo los contenidos de estadística para el plan de estudios fijo se encuentran únicamente en el segundo y tercer nivel del mismo ciclo, aquí nos basaremos en el plan de estudios fijo. En primera instancia se mostrara una sugerencia de contenidos por niveles en los cuales el software podría ser de utilidad

Cuadro 1: Sugerencia de contenidos estadísticos en los cuales aplicar el uso de Power Point, incluidos en el plan de estudios para el tercer ciclo de la Educación General Básica de Costa Rica

\begin{tabular}{|c|c|c|c|}
\hline Nivel & Contenido & Utilidad & $\begin{array}{l}\text { Ventajas con respecto } \\
\text { a otros software }\end{array}$ \\
\hline \multirow[t]{2}{*}{ Octavo } & $\begin{array}{l}\text { Distribución de } \\
\text { frecuencia absoluta y } \\
\text { frecuencia relativa } \\
\text { (Recolección } \\
\text { de información) }\end{array}$ & $\begin{array}{l}\text { Confección de cuadros } \\
\text { estadísticos de frecuencia } \\
\text { absoluta y porcentual }\end{array}$ & $\begin{array}{l}\text { Interfaz gráfica } \\
\text { agradable, fácil uso e } \\
\text { implementación }\end{array}$ \\
\hline & $\begin{array}{l}\text { Gráfico de bastones, } \\
\text { gráfico de barras, } \\
\text { grafico circular. }\end{array}$ & $\begin{array}{l}\text { Confección de cada tipo de } \\
\text { grafico según la elección } \\
\text { adecuada del mismo }\end{array}$ & $\begin{array}{lr}\text { Fácil rimplantación, } \\
\text { interfaz } & \text { gráfica } \\
\text { agradable. } & \text { Fácil } \\
\text { manipulación de datos }\end{array}$ \\
\hline \multirow[b]{2}{*}{ Noveno } & $\begin{array}{l}\text { Tablas de frecuencia } \\
\text { absoluta y relativa con } \\
\text { variables continuas }\end{array}$ & $\begin{array}{l}\text { Confección de cuadros } \\
\text { estadísticos de frecuencia } \\
\text { absoluta y porcentual. }\end{array}$ & $\begin{array}{l}\text { Interfaz gráfica } \\
\text { agradable, fácil uso e } \\
\text { implementación }\end{array}$ \\
\hline & $\begin{array}{l}\text { Histograma y polígono } \\
\text { de frecuencia para } \\
\text { variables absolutas y } \\
\text { relativas en variables } \\
\text { continuas. }\end{array}$ & $\begin{array}{l}\text { Confección de histogramas y } \\
\text { polígonos de frecuencia }\end{array}$ & $\begin{array}{l}\text { Interfaz } \text { gráfica } \\
\text { agradable, fácil uso e } \\
\text { implementación. Fácil } \\
\text { manipulación de datos }\end{array}$ \\
\hline
\end{tabular}

(MEP, 2013)

Expondremos la siguiente situación a manera de ejemplo para desarrollar la implementación de un proyecto estudiantil utilizando el software Power Point.

Vamos a suponer que se estará trabajando con un grupo de Noveno, donde los objetivos académicos a cubrir son los siguientes:

- Construir tablas de frecuencia absoluta y frecuencia relativa.

- Representar gráficamente la información tabulada en forma de histograma y polígono de frecuencia.

- Determinar de la información que proporcionan las tablas estadísticas y los histogramas variables continuas (interpretación de datos) 
El docente propondrá una actividad inicial a manera de problema en el cual involucre a los estudiantes de la clase, con este problema desarrollará a manera de guía todos los conceptos que el estudiante debe de aprender, según los objetivos educativos a alcanzar por el discente; además se debe de tomar en cuenta que en algunos casos se trabaja solo con variables continuas o solo con variables discretas, por lo cual el docente debe de tener el cuidado tanto al proponer el ejemplo inicial como al proponer los temas. Por conveniencia educativa, se buscan temas que sean de la atención de los estudiantes, la propuesta radica en torno a alcanzar un objetivo el cual será el desarrollo de un proyecto que supone la investigación a nivel de clase o de institución de una pregunta de investigación, el docente podrá valorar dependiendo el nivel y el contenido a desarrollar si el estudiante recolectara los datos o si podrá utilizar información real (tomada del INEC) previamente proporcionada por el docente.

Siguiendo con nuestro ejemplo, una vez realizada la actividad inicial, supondremos que el docente dispondrá al grupo en grupo en sub grupos de acuerdo a temas para los cuales cuenta con los debidos datos del INEC.

Los temas que el docente puede exponer se sugieren que sean de relevancia social, como en este caso ya cuenta con el cuadro estadístico, los temas deberán ser ya delimitados, como en este caso expondremos la situación que un sub-grupo haya elegido estudiar sobre alguna temática relaciona a la cantidad de nacimientos de acuerdo a la edad de la madre, el docente deberá entregar una guía de estudio con su debido cuadro.

De acuerdo a los contenidos que se desean estudiar, una posible guía de desarrollo del proyecto pudiese ser:

\section{Figura 1: ejemplo de una guía de desarrollo de proyecto estudiantil.}

Guía de proyecto

Estudiantes: Fecha:

Analicen detalladamente el cuadro entregado antes de comenzar con la resolución de las preguntas propuestas.

Resuelva cada ejercicio propuesto, con ayuda de su equipo de trabajo.

1- Cuál es el objetivo de estudio que se pretende abarcar. (objetivos)

2- Cuál es la finalidad que desean lograr con el estudio del tema que desean abordar.

3- El estudio se hace sobre una muestra o sobre una población, justifique su respuesta. (puede consultar en páginas web según el estudio que esté realizando o con profesores del área de trabajo correspondiente.)

4- Qué tipo de variable utiliza el estudio proporcionado.

5- Realice una breve exploración teórica del tema con ayuda del docente.

6- Según la observación detallada de la información presentada en el cuadro, cual podrían inferir que es el objetivo de estudio del cuadro estadístico.

7- Con el cuadro mostrado, transcriba el cuadro a Power Point (debe abarcar todos los componentes de un cuadro estadístico) 
8- Menciones tres inferencias informativas que puedan hacer a través de la información mostrada en el cuadro. (puede apoyar su información con investigación de la web o ayuda del docente de la asignatura que corresponde la información presentada)

9- Construya en Power Point, con la información tabulada un histograma y polígono de frecuencias.

10- Infiera de manera grupal 4 conclusiones sobre el estudio realizado utilizando la información construida por el histograma.

11- Con base a toda la información recolectada, y con ayuda de su equipo de trabajo realice una presentación en Power Point a manera de informe sobre el estudio realizado.

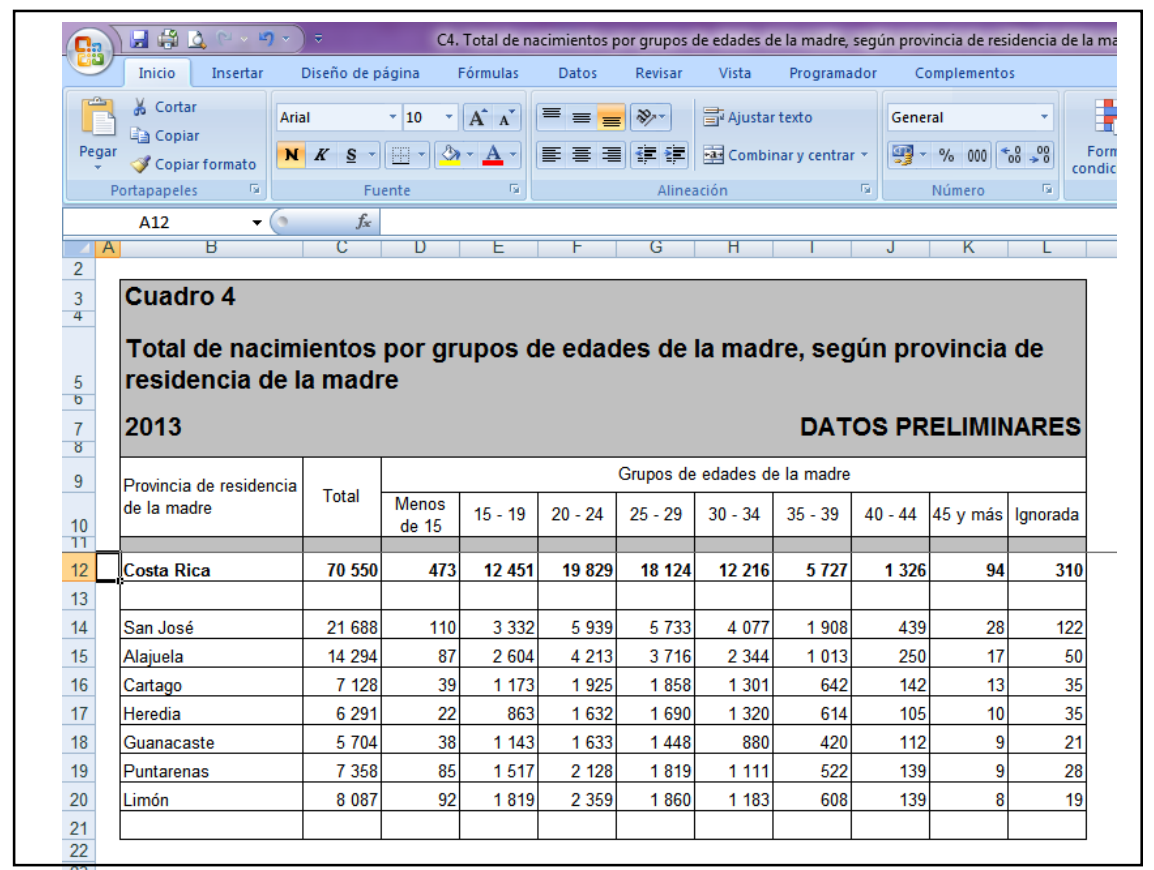

Cuadro 2: posible cuadro de información estadística que el docente podrá proponer a sus estudiantes, tomado de la página web del INEC.

Además deberá adjuntar la información del cuadro y como se trabajará en Power Point este puede ser entregado de manera digital, cabe destacar que la página del INEC proporciona la opción de solo obtener los datos sin el formato de cuadro estadístico, pero ya tabulados en Excel (también se puede descargar en formato pdf), esto tiene gran aporte pedagógico porque permite que el estudiante pueda poner en práctica conceptos sobre la elaboración de cuadros estadísticos, pero sin necesidad de tabularlos.

La guía de proyecto propuesta anteriormente es solo un ejemplo para el docente; sin embargo se recomienda en esta caso específico, mantener el punto final de la guía como conclusión del proyecto, inclusive podría darse que el grupo decida hacer un cartel con material concreto y no una presentación, pero para aprovechar el software se recomienda hacerlo con el mismo, siempre y cuando sea guiado por el docente. 
Una vez concluida la actividad inicial, entregado la guía de proyecto y la información requerida para hacerlo, el estudiante deberá empezar a trabajar con la guía constante de su docente.

Solo para reforzar el ejemplo propuesto, realizaremos el punto 9 de la guía de ejemplo. Como el punto 7 es solo de transcribir y confeccionarlo debidamente, se incita al lector a que lo realice a manera de práctica para explicar a sus alumnos.

\section{Realizando un histograma y polígono de frecuencia con Power Point.}

En este caso como tenemos la información tabulada en Excel, debemos tener ambos archivos abiertos

Pasos a seguir

1- Lo primero será abrir el software e ingresar a la sección de gráficos, donde cómo podemos ver a continuación, el software nos ofrece gran cantidad de tipos diferentes de cuadros, y otras opciones a elegir.

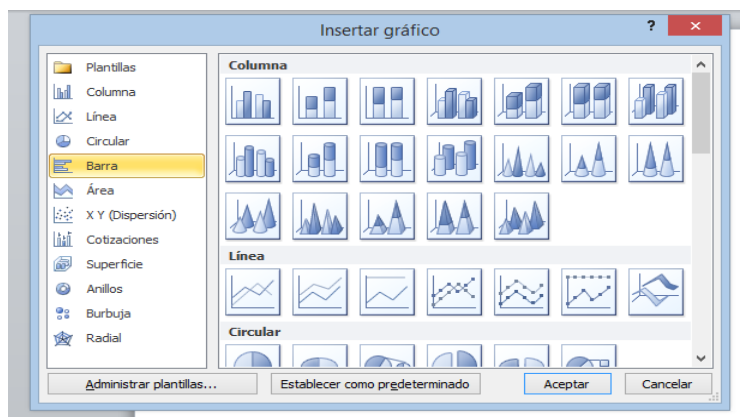

Figura 2: Opciones de gráficos que tiene el software PowerPoint.

2- Se seleccionara la primera opción de gráficos: Columna $\rightarrow$ columnas agrupadas, se obtendrá una vista como la siguiente:

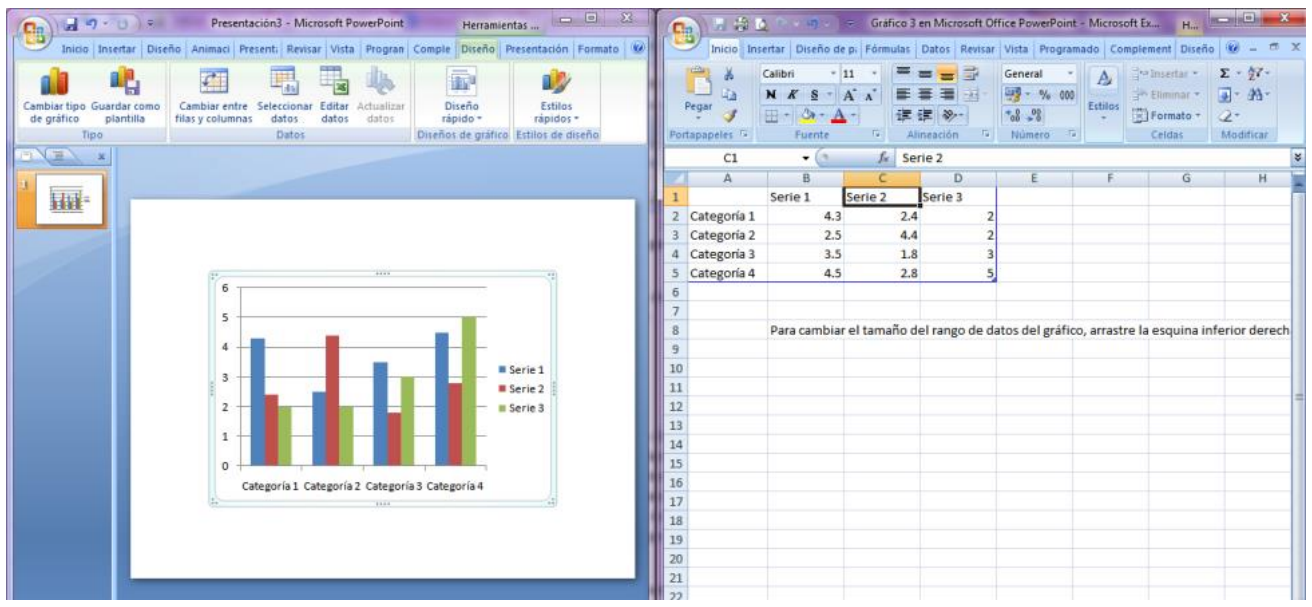

Figura 3: Vista inicial de al seleccionar la opción barras agrupadas de Power Point 
3- Supondremos que por el objetivo de indagación de los estudiantes, se ocupa graficar únicamente los datos correspondientes a la cantidad de nacimientos en la provincia de San José, para el año 2013, en valores absolutos, de esta forma en la vista anterior se borrara las columnas correspondientes a serie 2 y 3 , y se copiaran los datos requeridos insertando al principio y al final de las filas una nueva fila donde se digitara el valor 0 con respecto a la columna, se obtendrá una vista como la siguiente:

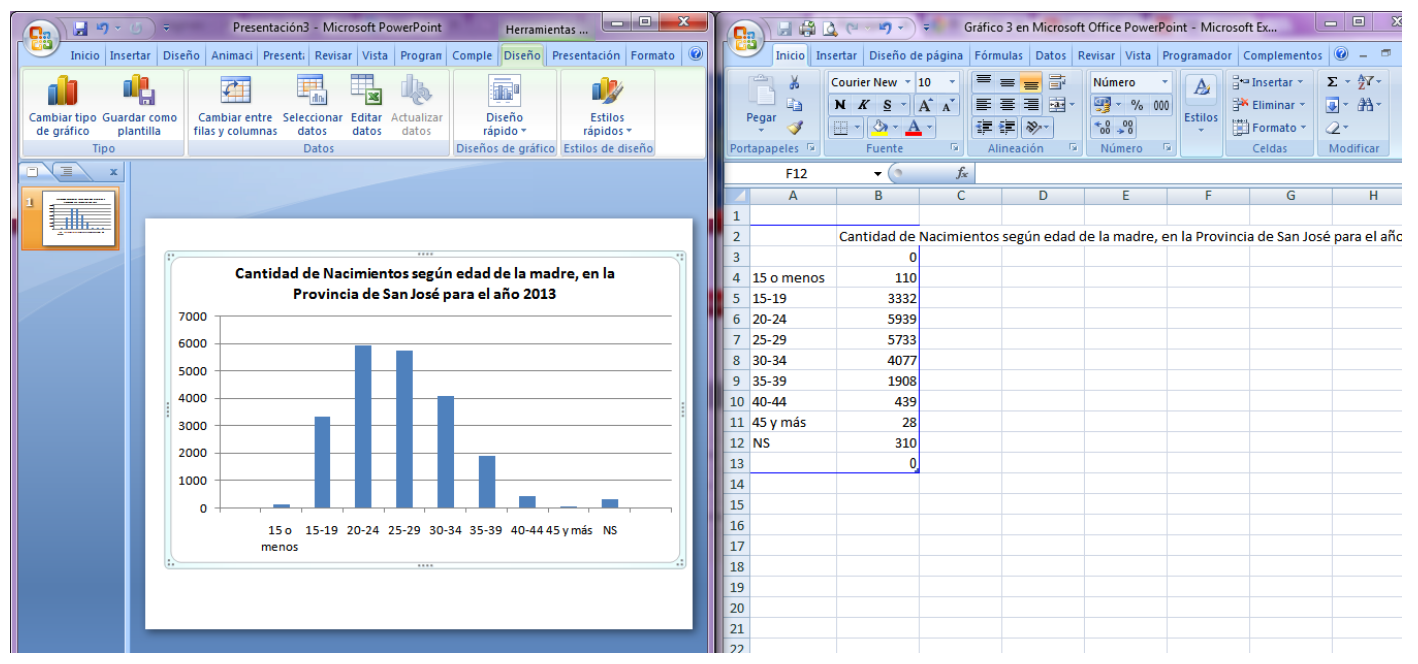

Figura 4: manipulación de un cuadro de barras agrupadas para la confección de un histograma en Power Point.

4- A continuación se deberá doblar la serie de datos, copiando la columna ya hecha.

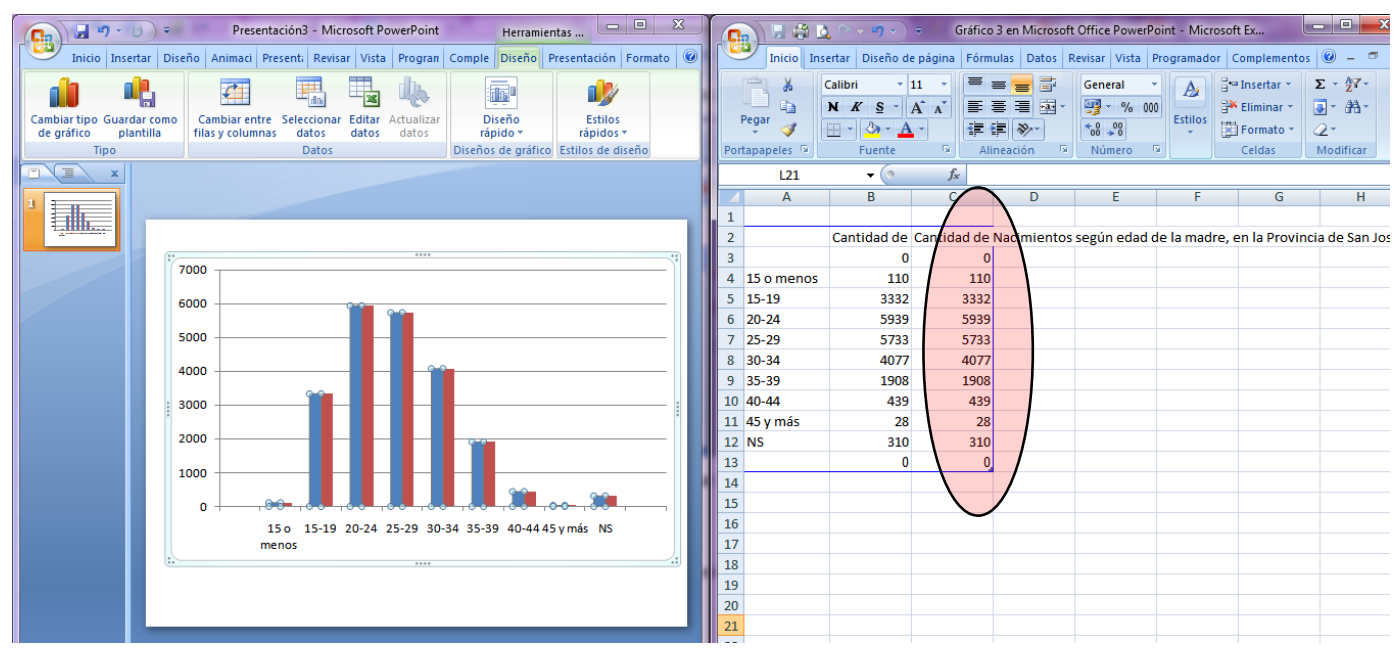

Figura 4: Manipulación de un cuadro de barras agrupadas 
5- Sobre la serie marcada en azul, en el botón derecho del mouse se cambia de grafico a lineal.

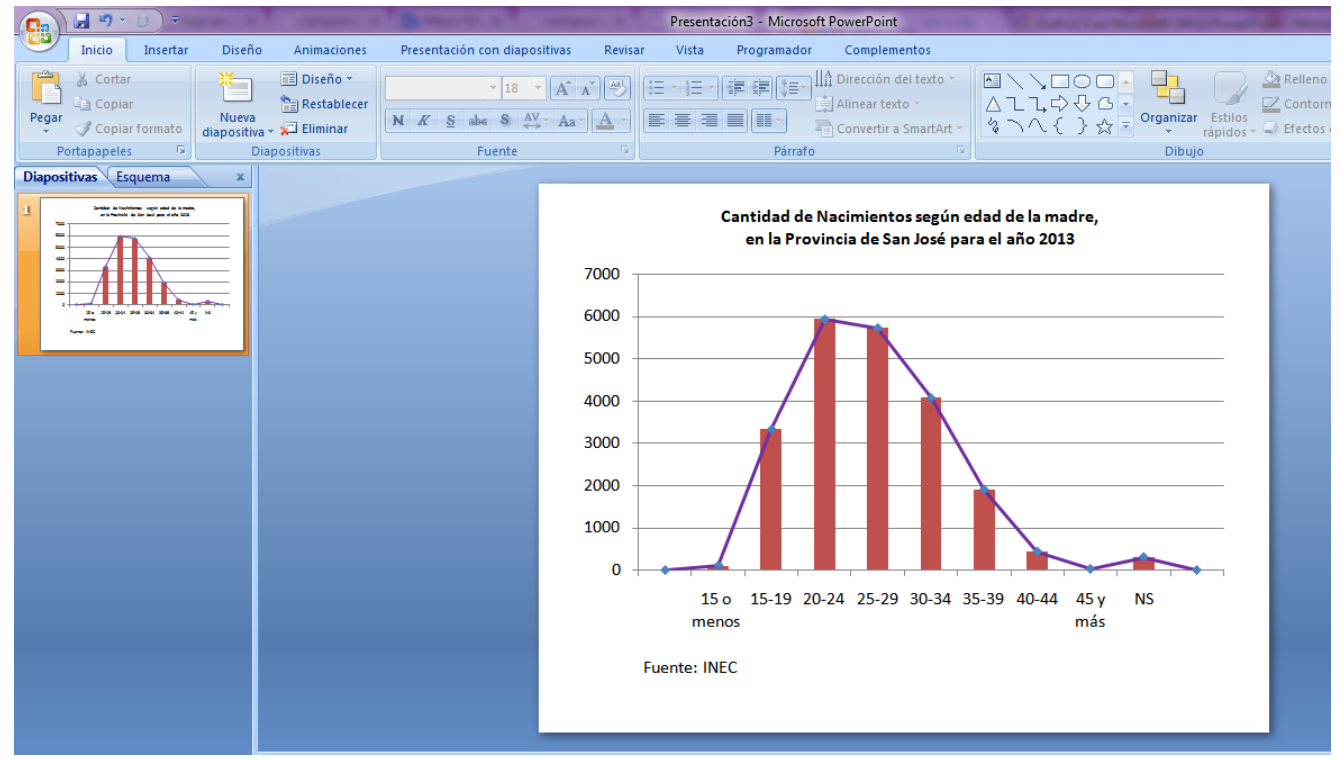

Figura 5: Creación del poligono de frecuencias

6- Producto final: como ya solo resta apilar las barras, con el botón derecho del mouse se deberá acceder a la opción: dar formato a serie de datos, y en la casilla intervalo, se corre a cero, de esta forma obtenemos:

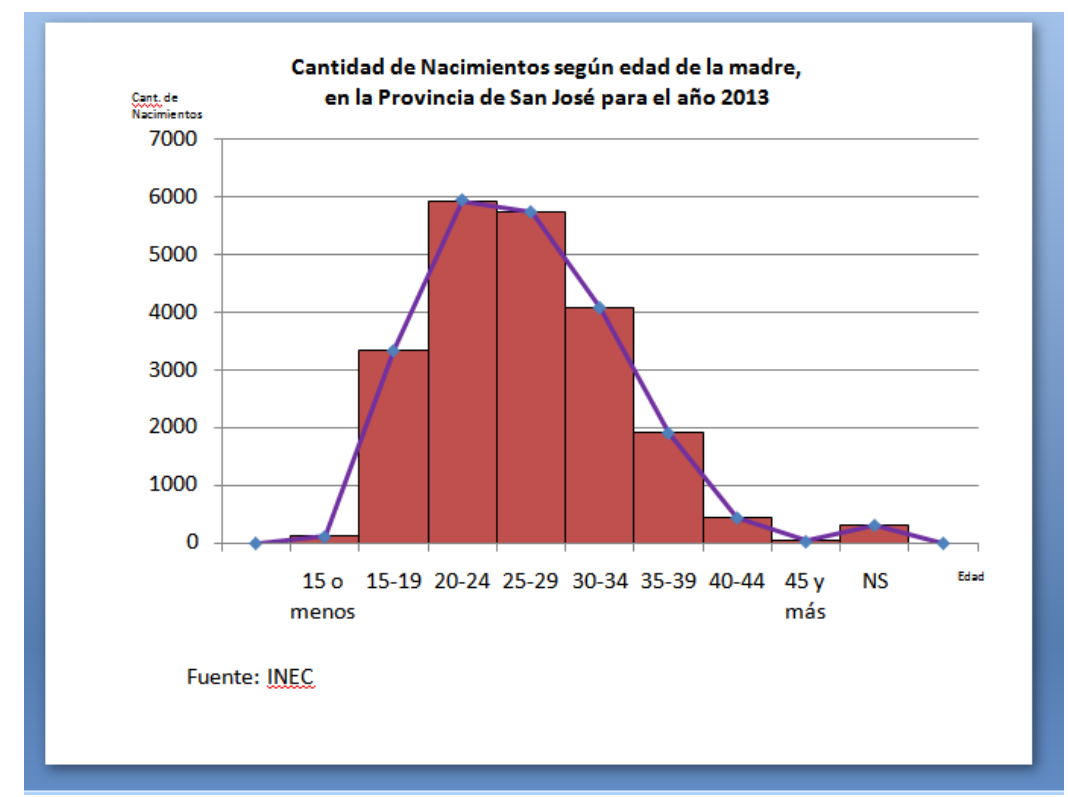

Figura 6: Grafico final, que se espera realice un estudiante. 


\section{Conclusiones}

Con esto hemos mostrado que la enseñanza de la estadística en secundaria no tiene por qué ser explícitamente destinada a las clases magistrales, podemos aplicar muchas metodologías propias de la corriente constructivista y enfocarnos en una educación más formativa, que inste al estudiante a interesarse de manera propia en el estudio de esta gran disciplina como lo es la estadística y que descubra en ella la gama de posibilidades que podrían encontrar, ya sea como medio que les permita realizar estudios de calidad e inclusive para aspiraciones profesionales.

Si bien en este escrito solo se abordó la enseñanza de la estadística instamos al docente a que explore más las metodologías propuestas y las aplique en contenidos de probabilidad.

Sabemos que para cualquier medio, recurso didáctico o propuesta metodológica que se desee aplicar existen limitaciones y beneficios en cuanto al proceso de enseñanza. Con lo referente a las limitaciones es importante conocerlas para saber cómo enfrentarlas en caso de que se presenten y bien, por obviedad la inminente importancia de saber qué beneficios traen para los estudiantes y el sistema educativo cada innovación que se realice en el mismo.

Con respecto a las propuestas comentada: aprendizaje por medio de proyectos e inclusión del software de Microsoft, se puede hablar de ciertas limitaciones que pueden encontrarse en el camino, entre ellas podemos citar las que provienen de carácter técnico:

- Falta de laboratorios de cómputo.

- Ausencia de herramientas que faciliten la labor del laboratorio como: proyector, programas computacionales, deficiencias en el hardware, entre otros.

- Dificultades por medio de la institución o alumnos para adquirir los mínimos materiales físicos a utilizar.

Ahora, si hablamos de limitaciones pedagógicas, de las más importantes que podríamos encontrar son:

- Carencia del conocimiento mínimo tecnológico por parte del docente en cuanto a utilizar las herramientas tecnológicas.

- Dificultad por parte del docente para trabajar con metodologías constructivistas.

- Discusiones de índole social o cultural entre los discentes.

- Predisposición negativa por parte del cuerpo estudiantil.

El hecho de que estas limitaciones existan, no quiere decir que la propuesta sea deficiente para el sistema, es mejor conocerlas para poder enfrentarlas con éxito, poniendo sobre una balanza si el bien educativo que estas puedan generar es mayor que la dificultad que se tenga que afrontar. 
Así como hablamos de dificultades técnicas, podemos hablar de benéficos de carácter técnico:

- Son propuestas adaptables casi que en cualquier centro educativo y a diversidad de contenidos educativos.

- La inversión económica es mínima y se puede realizar con materiales reciclables.

- En el caso del software, tanto Excel como PowerPoint son de fácil acceso y manipulación, tienen un costo relativamente bajo y son de fácil actualización.

Y claro lo más importante son los beneficios pedagógicos que estas puedan ofrecer, entre los que encontramos:

- La posibilidad de la interdisciplinaridad

- Posibilidad de aumentar el interés del estudiante hacia la estadista a fin de que reconozca su gran importancia y utilidad.

- Posibilidad por parte del docente de poder transmitir el conocimiento estadístico de manera más veraz y real al contexto social, cultural, familiar y ambiental del estudiante.

- Capacidad de poder inculcar valores humanos además de un conocimiento pedagógico.

- Inculcar el valor y la importancia del trabajo en equipo en los estudiantes.

Posibilidad de cambiar estereotipos negativos hacia la asignatura de matemática por parte de los estudiantes. 


\section{Bibliografía.}

Arce, A. A., Alpizar Vargas, M., Arroyo Hernández, J., Gamboa Araya, R., \& Hidalgo Mora, R. (2004). Enseñanza de la matemática: elementos para un diagnóstico. Heredia.

Armenta, M., Larios, N., \& Urrea, M. (2010). Secretaria de la Educación Pública: Secretaria de educación Pública: Programas de Formación Continua 2010-2011: Las matemáticas y su enseñanza en la escuela secundaria III. Recuperado el 12 de junio de 2014, de Edomex: http:/edu.edomex.gob.mx/opmatematicas/pdfs/des_curs_seciii.pdg

Ben-Zvi, D., \& Garfield, J. (2008). Preparing school teachers to develop students statistical reasoning. Minnesota: University of Minnesota.

Calderón, K. (2013). La Didáctica Hoy: Concepción y aplicaciones. San José, Costa Rica: EUNED.

Chavarría, S. (1998). La política Educativa hacia el siglo XXI: Propuesta y realizaciones. San José: Ministerio de Educación Pública.

Jiménez, M. (2010). Educación Secundaria. Derecho, inclusión y desarrollo: El movimiento de los pingüinos. Recuperado el 11 de junio de 2014, de UNICEF: http://www.unicef.org/argentina/spanish/Educacion_Secundaria(1).pdf

MEP. (2013). Transversalidad en los planes de estudio. Recuperado el 10 de junio de 2014, de Ministerio de educación Pública: http://www.mep.go.cr/sites/default/files/descargas/programasde-estudio

Moreno, M. (2004). Utilización de medios y recursos didácticos en el aula. Recuperado el 10 de mayo de 2014, de Universidad Complutense de Madrid: www.pendientemigracion.umc.es/info/doeprofe/isidro/merecur.pdf

UNESCO. (1997). William Herad Kilpatrick. Perspectivas: revista trimestral de educación, 8. 
IV Encuentro sobre Didáctica de la Estadística, la Probabilidad y el Análisis de Datos(IV EDEPA) - 2014

\title{
Análisis Estadístico sobre la aplicación de la Responsabilidad Social de la Empresa (RSE) en seis industrias textiles en El Salvador, 2014
}

\author{
Pedro Armando Ramos Alberto ${ }^{1}$. \\ Javier Eliseo Chávez Mancía ${ }^{2}$
}

\begin{abstract}
Resumen
Entiéndase Responsabilidad Social de la Empresa (RSE) como la acción voluntaria, humanista por parte de los empresarios en proporcionar beneficios que van más allá de las leyes y normas para la mejora de la sociedad, del medio ambiente y de la situación económica de sus empleados logrando de ésta forma ser competitivo en el área. Se seleccionaron en el presente trabajo de investigación seis industrias textiles para estudiar la Aplicación de la RSE: HILASAL, 4LEAGUE, F\&D, texhnos, IMPRESSION APPAREL y BCTC. Los objetivos del estudio se dirigieron a investigar el perfil de cada una de éstas, realizando un estudio exploratorio en las que se efectuaron análisis, se elaboraron indicadores, se hacen comparaciones y se usó la técnica estadística del análisis de correspondencia para identificar los niveles de aplicación con respecto a la RSE. Asimismo, se identifica si existen diferencias significativas en dicha aplicación en las diferentes industrias textiles y así establecer cuál es la empresa que lidera en la aplicación de la RSE.
\end{abstract}

Palabras clave: Industria textil, Indicadores, Responsabilidad Social de la empresa, diferencias significativas, técnicas estadísticas.

\begin{abstract}
There be understood Social Responsibility of the Company (SRC) as the voluntary action, humanist on the part of the businessmen in providing benefits that go beyond the laws and norms for the progress of the society, of the environment and of the economic situation of its personnel achieving of this one form to be competitive in the area. Six textile industries were selected in the present research work to study the Application of the SRC; HILASAL, 4LEAGUE, F\&D, TEXHNOS, IMPRESSION APPAREL and BCTC. The objectives of the study was conducted to investigate the profile of each of these, by performing an exploratory study in which analyzes were performed, developed indicators, comparisons are made and used the statistical technique of the analysis of correspondence so that they will enable us to identify the levels of implementation with respect to the CRS. Also, identify if there are significant differencesin the application in the different textile industries and thus establish which is the company that leads in the implementation of CSR.
\end{abstract}

Keywords: Textile industry, Indicators, Social Responsibility of the Company, significant differences, statistical techniques

\footnotetext{
${ }^{1}$ Universidad de El Salvador, El Salvador. pedro_ramalberto@yahoo.com

2 Universidad de El Salvador, El Salvador, cm05028@ues.com.sv
} 
IV Encuentro sobre Didáctica de la Estadística, la Probabilidad y el Análisis de Datos(IV EDEPA) $-2014$

Modalidad: Ponencia

\section{Referencias}

Peña, Daniel. Romo, Juan. (1997). Introducción a la Estadística para las Ciencias Sociales. McGraw-Hill. Madrid, España.

Martínez B., Ciro (2005).Estadística y Muestreo. ECOE EDICIONES. Bogotá, Colombia

Peña, Daniel ( ). Fundamentos de Estadística. Madrid, España

Visuata V. Bienvenido. (1989) Técnicas de Investigación Social. Limpergraf, S.A. Barcelona, España

Hernández S. Roberto. Fernández C. Carlos, Lucio, Baptista, Pilar.(2010). Metodología de la Investigación. México.

Pérez, Cesar(2005). Técnicas Estadísticas con SPSS 12. Person. Prentice Hall. Madrid. España.

Martin M. Quintin. Cabero M. (2008) Tratamiento Estadístico de datos con SPSS.Thomson.Editorial Paraninfo. Madrid, España 


\title{
Didáctica del Análisis de Datos para Estudiantes de Ingeniería
}

\author{
Andrea Ordóñez Delgado ${ }^{1}$
}

\begin{abstract}
Resumen
El presente trabajo brinda una propuesta para la enseñanza del análisis de datos en los estudiantes de primeros semestres de los programas de ingeniería. El análisis de datos es una herramienta muy útil para construir conceptos a partir de observaciones, recolecciones de datos y reconocimiento de patrones. Esta exploración le dará a los futuros ingenieros herramientas competentes.
\end{abstract}

Palabras clave: Análisis de datos, enseñanza, ingeniería.

${ }^{1}$ Escuela Colombiana de Ingeniería Julio Gravito, Colombia. paola.ordonez@mail.escuelaing.edu.co 


\title{
Evaluando Actitudes de los Estudiantes de la Universidad del Tolima Hacia la Estadística \\ Dicleny Castro Carvajal ${ }^{1}$ \& John Jairo Zabala Corrales ${ }^{2}$
}

\begin{abstract}
Resumen
Mediante la adaptación del test desarrollado por Schau (1995) SATS (Survey of Attitudes Toward Statistics, Test de actitudes hacia la Estadística), este trabajo muestra los resultados de la investigación y seguimiento realizado a los estudiantes de los programas de Historia, Sociología y Ciencias Políticas de la Facultad de Ciencias Humanas y Artes de la Universidad del Tolima, durante los últimos cuatro semestres, acerca de las actitudes hacia la Estadística luego de haber recibido un curso básico de Estadística o de Métodos Cuantitativos, para determinar qué aspectos del orden afectivo caracterizan a 121 estudiantes de dichos programas. El modelo validado por Schau (2004), describe cuatro dimensiones (Afectivo, Cognitivo, Valor y Dificultad). Una vez validado en la población objeto de estudio, se encontró un alfa de Cronbach de 0.76 mostrando consistencia entre las preguntas realizadas. Los resultados indican una alta correlación de las dimensiones Dificultad y de Valor, constituyéndose como lo afirma Batanero (2002) retos en la formación de una cultura Estadística.
\end{abstract}

Palabras clave: Test, Actitud, Enseñanza y Aprendizaje.

\begin{abstract}
By adapting the test developed by (Schau, 1995) SATS (Survey of Attitudes Toward Statistics (Attitude Test Statistics), this paper shows the results of research and monitoring carried out to students programs history, sociology and Political Science Faculty of Humanities and arts at the University of Tolima, over the last four semesters, about attitudes toward statistics after receiving a basic course in statistics or quantitative methods to determine which aspects of the affective 121 students characterize such programs, the model validated by Schau (2004) describes four dimensions (Affect, Cognitive Value and Difficulty.) once validated in the population under study, we found a Cronbach's alpha of 0.76 showing consistency between the questions asked. Results indicate a high correlation of Difficulty and Value dimensions, constituting as stated Batanero (2002) challenges in creating a culture Statistics.
\end{abstract}

Keywords: Test, Attitude, Teaching and Learning.

\footnotetext{
${ }^{1}$ Universidad del Tolima. Ibagué Colombia. diclenyc@ gmail.com

${ }^{2}$ Universidad del Tolima. Ibagué Colombia. johnjzabala@gmail.com
} 


\section{Introducción}

El objetivo final de la educación estadística, es formar ciudadanos que usen apropiadamente el pensamiento estadístico como la afirma Schau (2003), o en términos generales como lo propone Gal (2002), se pretende es proporcionar una cultura estadística, "que se refiere a dos componentes interrelacionados: a) capacidad para interpretar y evaluar críticamente la información estadística, los argumentos apoyados en datos o los fenómenos estocásticos que las personas pueden encontrar en diversos contextos, incluyendo los medios de comunicación, pero no limitándose a ellos, y b) capacidad para discutir o comunicar sus opiniones respecto a tales informaciones estadísticas cuando sea relevante",

la presencia de un curso de Estadística (o de métodos cuantitativos) en programas de ciencias sociales, proyecta establecer algunos elementos que incidan en la formación de profesionales junto a la mejora de una cultura estadística.

En Colombia se ha introducido la estadística en los planes de estudio desde 1974, y sus enfoques son tan diversos como las modalidades o especialidades a la que esté inscrito el curso. Es decir, la estadística como formación data de muchos años atrás, y los futuros profesionales, como lo relata Desrosières (2008) requieren utilizar la metodología estadística, a partir de la obtención de los datos, su tratamiento e interpretación.

Es preciso entender que gran parte de estos aprendizajes o la construcción de estos saberes, como lo plantea Moscoloni (2009), además de vencer las resistencias ofrecidas por los conceptos persistentes, transponer el rechazo o temor que produce la disciplina, se obtienen según, Estrada (2003) a partir de las actitudes propias o individuales hacia la estadística.

Frecuentemente se asocia la Estadística al quehacer cotidiano con el fin de presentar sus conceptos y como afirma Zabala (2001), esta práctica simplista se observa cuando ilustrando el análisis descriptivo de datos, damos énfasis a la operacionalidad insulsa, o en el caso extremo, la excesiva formalización matemática, hace perder el interés en el tema.

\section{CULTURA ESTADISTICA Y ACTITUD}

Para Batanero (2002),

"La cultura no es solamente conocimiento y capacidad. La parte emocional sentimientos, valores, actitudes son también componentes importantes de la educación. Una persona puede ser, por ejemplo, brillante en la resolución de problemas estadísticos y poseer un vasto conocimiento de conceptos y desconocer las aplicaciones de la estadística y el papel que juega en la sociedad. Podría conocer todo esto, y, sin embargo, odiar la materia, menospreciar su valor o estar convencido de que la mayor utilidad de la estadística es la posibilidad de usarla para manipular la verdad". 
Además Gal y colaboradores (1997), definen las actitudes como: "una suma de emociones y sentimientos que se experimentan durante el período de aprendizaje de la materia objeto de estudio". Son bastante estables, se expresan positiva o negativamente (agrado/desagrado, gusto/disgusto) y pueden referirse a elementos vinculados externamente a la materia (profesor, actividad, libro, método de enseñanza etc.). De acuerdo con Gal y Ginsburg (1994) las actitudes y creencias, especialmente las negativas, pueden tener un impacto directo en el clima de la clase y llegar a constituir un auténtico bloqueo del aprendizaje si no se controlan.

Según Gal, (citado por Batanero 2002), la cultura nos lleva a la imagen del subconjunto mínimo de habilidades básicas, que esperamos de todos los ciudadanos en contraposición a un conjunto más avanzado de conocimientos y capacidades, que solo algunos pueden adquirir.

\section{INSTRUMENTOS DE MEDICION DE ACTITUD HACIA LA ESTADISTICA}

Parafraseando a Estrada (2011), en la actualidad, de entre las múltiples técnicas de observación que se conocen (entrevistas, cuestionarios, etc.), el instrumento de medida de actitudes hacia la Estadística que todos los autores consultados utilizan, es la escala de actitud, porque sin olvidar el valor de complementariedad que tienen todas las técnicas para la observación, las escalas son procedimientos que se utilizan para determinar diferencias de grado o intensidad respecto a algún objeto actitudinal. Gal, Ginsburg y Schau (1997) afirman que los maestros de Estadística necesitan instrumentos para medir la actitud de sus estudiantes y así estar en condiciones de evaluar la efectividad de una experiencia de educación estadística. Para ello es necesario conocer las actitudes iniciales de los estudiantes hacia la Estadística y detectar cambios actitudinales durante y posteriormente a experiencias de educación estadística.

Se han desarrollado muchos instrumentos para medir la actitud, como lo reporta (Ferreyra, 2007), destacándose el Survey of Attitudes Toward Statistics (Test de actitudes hacia la Estadística, $\mathrm{SATS}^{3}$ ) desarrollado por Schau et al. (1995), en el se identifican cuatro componentes de las actitudes, según Schau (2004) ellas son: Afectivas, Cognitivas, Valor y de Dificultad. Las características de estas dimensiones se muestran a continuación:

$\checkmark$ Afectivo: sentimientos positivos o negativos hacia la Estadística;

$\checkmark$ Competencia cognitiva: percepción de la propia capacidad sobre conocimientos y habilidades intelectuales en Estadística;

$\checkmark$ Valor: utilidad, relevancia y valor percibido de la Estadística en la vida personal y profesional;

$\checkmark$ Dificultad: se refiere a la percibida de la Estadística como asignatura. Aunque un estudiante pueda reconocer el valor de una materia, sentir interés hacia la misma (componente afectivo) y pensar que tiene suficientes conocimientos y habilidades (componente cognitivo), puede llevarlo a considerar la materia como fácil o difícil.

\footnotetext{
${ }^{3}$ La encuesta está disponible en:

http://www.evaluationandstatistics.com/sitebuildercontent/sitebuilderfiles/sats28pre.pdf
} 
Por lo anterior, es fácil apreciar la implicación que poseen estas dimensiones en el aprendizaje de los estudiantes, y de allí, la relación importante que tienen estos componentes, con las preguntas del cuestionario S.A.T.S desarrollado por Schau et al. (1995).

Pérez, A. (citado por Gutiérrez y Enríquez, 2003), manifiesta: el objetivo que pretende el análisis de datos cualitativos, es la búsqueda de tendencias, tipologías, regularidades o patrones y la obtención de datos únicos de carácter ideográficos. Así pues, esta investigación se sustenta a partir del cuestionario de actitudes hacia la estadística SATS, que es el que utilizaremos en este trabajo con estudiantes de la Facultad de Ciencias y Artes de la Universidad del Tolima.

Para los objetivos que persigue este trabajo, es necesario encontrar una forma de identificar la importancia que están teniendo las cuestiones afectivas, cognitivas, de valor y de dificultad hacia la estadística, por parte de los estudiantes.

Sin embargo, no hay una forma universalmente establecida para medir las actitudes, por lo que Estrada, Batanero y Fortuny (2002) afirman:

Las actitudes aparecen como un fenómeno de difícil definición, debido a que no constituyen una entidad observable, sino que son construcciones teóricas que se infieren de ciertos comportamientos externos, frecuentemente verbales. (p.2)

Para Auzmendy (citado por Estrada R. 2002), las actitudes son aspectos no directamente observables sino inferidos, compuestos tanto por las creencias como por los sentimientos y las predisposiciones comportamentales, hacia el objeto al que se dirigen.

\section{METODOLOGIA}

Como ya se determinó, el propósito de esta investigación es la de caracterizar las actitudes hacia la Estadística de estudiantes de los programas de Historia, Sociología y Ciencias Políticas de la Facultad de Ciencias Humanas y Artes de la Universidad del Tolima, posteriormente de haber recibido un curso básico de Estadística o de Métodos Cuantitativos, para tal propósito se aplicó una adaptación en español del SATS (Survey of Attitudes Toward Statistics) el cual consta de 28 Items con cinco opciones de respuesta, para responder la encuesta, se le indicó al estudiante que tuviera en cuenta los enunciados, señalando, según su criterio en un solo cuadro, la siguiente escala: (1) muy en desacuerdo, (2) en desacuerdo, (3) indiferente, (4) de acuerdo y (5) muy de acuerdo.

Además, se clasifican los 28 ítems de acuerdo a Schau, C. (2004) scoring the SATS-28, en donde sugieren la clasificación de las componentes de las actitudes, la estructura en cuatro componentes definidos en Schau y cols. (1995),

El instrumento (test) en mención se aplicó a 121 estudiantes, de los semestres, segundo en adelante con seguridad de haber recibido un curso básico de Estadística o de Métodos 
Cuantitativos, constituyéndose este en la población objeto de estudio, se encontraron individuos que no contestaron todo el test, por tanto ellos no se consideraron en el análisis. Para el análisis estadístico nos apoyamos en el software libre PSPP ${ }^{4}$. El análisis se centrará en la operacionalidad de las variables descritas en el instrumento, referidas a las actitudes hacia la estadística y de las variables de clasificación: género, edad, semestre y programa que cursan los estudiantes.

\section{RESULTADOS}

El instrumento permitió analizar las actitudes hacia la Estadística de estudiantes de los programas de Historia, Sociología y Ciencias Políticas de la Facultad de Ciencias Humanas y artes de la Universidad del Tolima, posteriormente de haber recibido un curso básico de Estadística o de Métodos Cuantitativos, con un valor de fiabilidad de Cronbach de 0.76, nos permiten determinar la fiabilidad en la escala, como se muestra en la Tabla 1, en todos los ítems evaluados, se excluyen los casos en los cuales no se contestaron todos los ítems, es decir donde hubo celdas vacías.

Tabla 1. Determinación de la fiabilidad de Cronbach

\begin{tabular}{|l|c|c|}
\cline { 2 - 3 } \multicolumn{1}{c|}{} & $\mathrm{n}$ & $\%$ \\
\hline Casos validados & 100 & 82.64 \\
\hline Excluidos & 21 & 17.36 \\
\hline Total & 121 & 100 \\
\hline
\end{tabular}

\begin{tabular}{|l|c|}
\hline Alfa de Cronbach & 0.76 \\
\hline No. de items & 28 \\
\hline
\end{tabular}

Encontramos en las Tabla 2 y Tabla 3, la descripción de las variables edad y género, en ellas se muestra como las edades se distribuyen normalmente con un dato modal de 20 años y en lo que respecta al género decimos que hay una equidad en esa variable.

Tabla 2. Distribución de los estudiantes, respecto a la edad

\begin{tabular}{|c|c|c|}
\hline Edad & Frecuencia & Porcentaje \\
\hline 16 & 1 & $0.8 \%$ \\
17 & 2 & $1.7 \%$ \\
18 & 15 & $12.4 \%$ \\
19 & 25 & $20.7 \%$ \\
20 & 28 & $23.1 \%$ \\
21 & 15 & $12.4 \%$ \\
22 & 16 & $13.2 \%$ \\
23 & 9 & $7.4 \%$ \\
24 & 4 & $3.3 \%$ \\
25 & 3 & $2.5 \%$ \\
28 & 1 & $0.8 \%$ \\
Missing & 2 & $1.7 \%$ \\
\hline
\end{tabular}

${ }^{4}$ Disponible en: http://www.gnu.org/software/pspp/ 
Tabla 3. Distribución de los estudiantes, respecto al género

\begin{tabular}{|c|c|c|}
\hline Genero & Frecuencia & Porcentaje \\
\hline Masculino & 62 & $51.2 \%$ \\
Femenino & 58 & $47.9 \%$ \\
Missing & 1 & $0.8 \%$ \\
\cline { 2 - 3 } & 121 & $100 \%$ \\
\hline
\end{tabular}

La Tabla 4, indica la cantidad y proporción de estudiantes de acuerdo al programa académico que cursan, en un $43,8 \%$ corresponde al programa de ciencias políticas.

Tabla 4. Distribución de los estudiantes, según el programa

\begin{tabular}{|c|c|c|}
\hline Programa & Frecuencia & Porcentaje \\
\hline Historia & 38 & $31.4 \%$ \\
Sociología & 30 & $24.8 \%$ \\
Ciencias Política & 53 & $43.8 \%$ \\
\cline { 2 - 3 } & 121 & $100 \%$ \\
\hline
\end{tabular}

En la Tabla 5, se describe la cantidad de estudiantes según el semestre que cursan, como se observa la gran mayoría cursan semestre superior al tercero, y como se indicó, ellos ya han realizado un curso básico de estadística.

Tabla 5. Distribución de los estudiantes, según el semestre

\begin{tabular}{|c|c|c|}
\hline Semestre & Frecuencia & Porcentaje \\
\hline 2 & 7 & $5.8 \%$ \\
3 & 32 & $26.4 \%$ \\
4 & 29 & $24.0 \%$ \\
5 & 39 & $32.2 \%$ \\
6 & 14 & $11.6 \%$ \\
\hline
\end{tabular}

El test KMO (Kaiser, Meyer y Olkin) relaciona los coeficientes de correlación, observados entre las variables. Los coeficientes de correlación parcial entre las variables, cuanto más cerca de 1 tenga el valor obtenido del test KMO, implica que la relación entre las variables es alta. En este caso la prueba KMO arrojó un 0.604, permite concluir que las variables están asociadas y correlacionadas entre sí, y el procedimiento que se está realizando aplica para el tema y como el valor de probabilidad es mucho menor que 0.05 , es decir los valores de las correlaciones entre variables no son ceros, lo que significa que si hay correlación entre las variables.

Tabla 6. Prueba KMO y test de esfericidad de Barlett

\begin{tabular}{|rr|r|}
\hline \multicolumn{2}{|c|}{ KMO (Kaiser - Meyer - Olkin ) } & 0.604 \\
\hline Test de esfericidad de Barlett $\quad$ Chi-2 & 679.42 \\
Grados de libertad & 378 \\
Sig. & 0.000 \\
\hline
\end{tabular}


En la Tabla 7, muestra la composición de la escala de acuerdo a las componentes de las actitudes, sugeridas por (Schau2004) en Scoring the SATS-28, con los estadísticos resumen, en ella se observa que los promedios obtenidos en la prueba están un poco más altos de los teóricos y respecto a la desviación estándar, en general son pequeñas lo que se traduce en un buen grado de concordancia de las respuestas.

Tabla 7. Composición de la escala y resumen de estadísticos

\begin{tabular}{|l|c|c|c|c|c|c|c|c|}
\hline Componente & Ítems & $\mathrm{n}$ & Min & Max & Media & $\begin{array}{c}\text { Desv } \\
\text { típica }\end{array}$ & $\begin{array}{c}\text { Max } \\
\text { Posib }\end{array}$ & $\begin{array}{c}\text { Media } \\
\text { Teórica }\end{array}$ \\
\hline Afectivo & P01, P02, P11, P14, P15, P21 & 116 & 8 & 26 & 16.37 & 3.55 & 30 & 15 \\
\hline Cognitivo & P03, P09, P20, P23, P24, P27 & 115 & 6 & 29 & 17.13 & 3.74 & 30 & 15 \\
\hline Valor & P05, P07, P08, P10, P12, P13, P16, P19, P25 & 112 & 13 & 39 & 25.30 & 4.73 & 45 & 22.5 \\
\hline Dificultad & P04, P06, P17, P18, P22, P26, P28 & 111 & 9 & 28 & 20.88 & 4.08 & 35 & 17.5 \\
\hline Puntaje Tot & Todos & 100 & 42 & 111 & 79.34 & 12.40 & 140 & 70 \\
\hline
\end{tabular}

Tabla 8. Correlación de los ítems con los componentes rotados

\begin{tabular}{|l|c|c|c|c|c|}
\hline \multirow{2}{*}{ Item } & \multicolumn{5}{|c|}{ Componente } \\
\cline { 2 - 6 } & 1 & 2 & 3 & 4 & 5 \\
\hline Preg 21 & 0.653 & 0.182 & 0.164 & & \\
\hline Preg 11 & 0.598 & & & -0.337 & \\
\hline Preg 20 & 0.585 & 0.171 & & & \\
\hline Preg 03 & 0.553 & 0.389 & & & -0.201 \\
\hline Preg 10 & 0.537 & -0.137 & 0.151 & & 0.257 \\
\hline Preg 06 & 0.531 & & 0.301 & 0.143 & \\
\hline Preg 22 & 0.378 & 0.332 & -0.213 & & \\
\hline Preg 28 & 0.295 & 0.290 & & 0.184 & 0.273 \\
\hline Preg 02 & & 0.646 & & & 0.166 \\
\hline Preg 27 & 0.132 & 0.595 & 0.285 & & \\
\hline Preg 01 & & 0.561 & -0.48 & 0.175 & 0.196 \\
\hline Preg 18 & 0.238 & 0.544 & & & -0.294 \\
\hline Preg 26 & & 0.530 & & 0.263 & \\
\hline Preg 09 & 0.160 & -0.163 & 0.634 & & 0.254 \\
\hline Preg 24 & 0.226 & & -0.622 & 0.292 & 0.172 \\
\hline Preg 05 & 0.172 & 0.185 & 0.543 & 0.159 & \\
\hline Preg 19 & 0.304 & 0.198 & 0.540 & 0.172 & 0.236 \\
\hline Preg 23 & 0.153 & 0.367 & -0.496 & 0.150 & \\
\hline Preg 14 & 0.207 & 0.324 & 0.435 & -0.181 & -0.271 \\
\hline Preg 16 & & 0.176 & 0.342 & & 0.109 \\
\hline Preg 13 & 0.240 & -0.116 & & 0.697 & 0.123 \\
\hline Preg 17 & -0.247 & & 0.104 & 0.648 & \\
\hline Preg 15 & -0.216 & 0.272 & & 0.582 & \\
\hline Preg 08 & 0.373 & & -0.205 & 0.477 & \\
\hline Preg 07 & 0.249 & & & 0.240 & -0.644 \\
\hline Preg 12 & 0.343 & & 0.124 & 0.123 & 0.555 \\
\hline Preg 25 & & 0.401 & 0.153 & & 0.408 \\
\hline Preg 04 & & 0.141 & -0.217 & 0.289 & 0.385 \\
\hline
\end{tabular}

El resultado de la Tabla 8, reporta el análisis factorial, en el que se muestran los cinco factores que se presentan rotados, rotación varimax. El primer factor fue dominante explicando el $43.617 \%$ de la varianza total. Al analizar los componentes obtenidos en la 
Tabla 9, se concluye que para los estudiantes de Ciencias Sociales de la Universidad del Tolima, los factores no se destacan independencia, sino que aparecen mezclados lo que indica una relación de sentimientos.

Se registra en la Tabla 9, lo que corresponde a la Matriz de correlaciones, apoya la hipótesis de que las variables están significativamente correlacionadas, en especial tiene relevancia el coeficiente de correlación 0.811 respecto al puntaje total, lo que indica que en el componente Dificultad, los estudiantes ven la estadística como una asignatura difícil, y en el componente de Valor con un coeficiente de 0.777 nos indica que la educación estadística debe orientarse al valor como fundamento en la formación de los futuros profesionales en Ciencias Sociales.

Tabla 9. Matriz de correlaciones de Pearson.

\begin{tabular}{|c|c|c|c|c|c|}
\hline Componente & Afectivo & Cognitivo & Dificultad & Valor & Puntaje Tot \\
\hline Afectivo & 1 & $0.504^{* *}$ & $0.488^{* *}$ & $0.321^{* *}$ & $0.738^{* *}$ \\
\hline Cognitivo & & 1 & $0.386^{* *}$ & $0.455^{* *}$ & $0.746^{* *}$ \\
\hline Dificultad & & & 1 & $0.483^{* *}$ & $0.811^{* *}$ \\
\hline Valor & & & & 1 & $0.777^{* *}$ \\
\hline Puntaje Tot & & & & & 1 \\
\hline
\end{tabular}

** correlacionadas significativamente.

\section{CONCLUSIONES}

De acuerdo con los resultados anteriores, se puede afirmar que los estudiantes de Ciencias Sociales de la Universidad del Tolima, de los programas de Historia, Sociología y Ciencias Políticas, poseen una buena actitud hacia la estadística en los distintos componentes; se concluye que todas las dimensiones están íntimamente ligadas, contrario a la hipótesis de los autores del Test, de que los componentes son independientes entre sí.

El mayor puntaje promedio se obtiene del componente valor, sin embargo, todos los aspectos permanecen íntimamente ligados o relacionados, el componente dificultad se destaca, en los estudiantes de Ciencias Sociales, permaneciendo ligado entre los demás dimensiones.

Por último, el hecho de haber interrelación entre las componentes afectivas, se requiere de elaborar un estudio más minucioso de estos indicadores y así adoptar dentro del quehacer algunas estrategias que permitan asumir actitud de cambio.

Por lo demás, se debe concebir a la Estadística como una actividad que permite acceder al conocimiento de lo general y cuya enseñanza debe ser menos calculista, pero sí más emprendedora en la adquisición de habilidades para modelar y entender el mundo (Zabala, 2001). 


\section{Bibliografía}

Batanero, C. (2002) Los retos de la cultura estadística. Jornadas Interamericanas de Enseñanza de la Estadística. Buenos Aires, 2002. Conferencia inaugural. Disponible en: http://www.ugr.es/ batanero/ARTICULOS/CULTURA.pdf

Desrosiéres, A. (2008) Entre réalisme métrologique et conventions d'equivalence: les ambiguités de la sociologie quantitative: le renueve MODULAD, Paris, No 38, pp36-50. Disponible en: http://www.modulad.fr

Estrada, A., Batanero, C. y Fortuny J. (2002). Análisis de las actitudes y conocimientos estadísticos elementales en la formación del profesorado. Tesis doctoral. Universidad Autónoma de Barcelona. Recuperado de: http://www.uam.es/servicios/apoyodocencia/ice/tarbiya/pdf/articulos/Tarbiya038-079-090.pdf

Estrada, A. Roca A. (2003) Actitudes hacia la Estadística y su evaluación. Revista de Educación de Universidad de Granada (España), 2003, vol. 16, pp. 227-245. Disponible en: http://web.udl.es/usuaris/z4084849/docs/seiem2010.pdf

Estrada, A. (2011). Instrumentos de medición de actitudes hacia la estadística: la escala EAEE para profesores. En M.M. Moreno, N. Climent (Eds.), Investigación en Educación Matemática. Comunicaciones de los Grupos de Investigación de la SEIEM (pp. 233-251). Lleida. Disponible en: http://web.udl.es/usuaris/z4084849/docs/seiem2010.pdf

Ferreyra, M.F. Organista, Sandoval, J. (2007), La actitud hacia la estadística ¿influye en el aprendizaje de esta ciencia? Disponible en: http://promepca.sep.gob.mx/archivospdf/produccion/Producto1212141.PDF

Gal, Ido. (2002) Adults' Statistical Literacy: Meanings, Components, Responsibilities. International Statistica Review, 70(1), 1-25. Disponible en: http://www.stat.auckland.ac.nz/ iase/publications/isr/02.Gal.pdf

Gal, I., Ginsburg, L. y Schau, C. (1997). Monitoring attitudes and beliefs in statistics education. En I. Gal y J. Garfield (Eds.), The assessment chanllenge in statistics education (pp. 37-54). Amsterdam: IOS Press. Disponible en: http://www.stat.auckland.ac.nz/ iase/publications/assessbk/chapter04.pdf

Gal I. y Ginsburg, L. (1994). The role of beliefs and attitudes in learning statistics: towards an assesment framework. Journal of Statistics Education, 2(2). Disponible en: http://www.amstat.org/publications/jse/v2n2/gal.html

Gutiérrez, B. \& Enrique, O. (2003).Estrategias de evaluación que utilizan los docentes de la carrera de Educación Básica Integral de la Universidad de los Andes-Táchira. Capítulo 8: La Investigación. Recuperado de: http://www.tdx.cat/bitstream/handle/10803/8903/CorreOBCapitulo08Def.pdf?sequence=3

Moscoloni, N. (2009), Enseñanza de Estadística en Ciencias Sociales. U.N.R Journal, Año 2 Vol 01 Nov. 2009. Disponible en: 
http://rephip.unr.edu.ar/bitstream/handle/2133/1523/27-135-1-PB.pdf?sequence=1

Schau, C. (2003, August). Students' attitudes: the "other" important outcome in statistics education. Paper presented at the Joint Statistical Meetings, San Francisco, CA. Recuperado de: http://www.statlit.org/pdf/2003SchauASA.pdf

Schau, C. (2004). Scoring the SATS-28. Recuperado de:

http://www.evaluationandstatistics.com/sitebuildercontent/sitebuilderfiles/SATS28Scoring.pdf

Zabala, John J. (2001, Octubre). La Educación Estadística: Estamos en Mora. Notas Universitarias. CEDIP Centro de Estudios de Didáctica y Pedagogía. Coruniversitaria. Universidad de Ibagué. 


\title{
Un ejemplo metodológico de cómo aplicar la técnica ABP ( Aprendizaje Basado en Problemas) en Estadística
}

\author{
Grettel Gutiérrez Ruíz ${ }^{1}$ \& Lourdes Quesada Villalobos²
}

\begin{abstract}
Resumen
La ponencia presenta un ejemplo metodológico de cómo aplicar la técnica didáctica ABP (aprendizaje basado en problemas), para el estudio de temas básicos de estadística en secundaria o universidad. Se expondrá brevemente en qué consiste la técnica y los pasos que se requieren para implementarla. ABP es útil en cualquier área del conocimiento, en particular para Estadística se convierte en una herramienta importante para desarrollar habilidades de investigación y actitudes en los estudiantes relacionadas con este tema. El objetivo principal del $\mathrm{ABP}$ es que los estudiantes exploren e investiguen para adquirir su propio conocimiento. Esta técnica está siendo promovida por diferentes universidades, por ejemplo el Instituto Tecnológico de Monterrey (México).
\end{abstract}

Palabras claves: Técnica ABP, aprendizaje, resolución de problemas

\begin{abstract}
The presentation shows an example of how to apply the didactic technique PBL (problem based learning) to study basic statistics topics at high school or university level. The technique and the steps required to implement it are briefly explained. PBL is useful in any knowledge area, in particular in Statistics it is an important tool for developing student research skills and attitudes related to this topic. The main goal of PBL is that the students get the knowledge by their own exploration and research. This technique has been promoted by several universities, for example the Technological Institute of Monterrey (Mexico).
\end{abstract}

Keywords: PBL technique, learning, problem resolution.

Modalidad: Ponencia

\footnotetext{
${ }^{1}$ Instituto Tecnológico de Costa Rica y Liceo Otilio Ulate Blanco, ggutierrez@itcr.ac.cr

${ }^{2}$ Instituto Tecnológico de Costa Ricaloquesada@itcr.ac.cr
} 


\title{
Enseñanza de Sistemas Estocásticos de Control en las áreas de Inventarios y Finanzas
}

\author{
Hugo Cruz-Suárez ${ }^{1}$
}

\begin{abstract}
Resumen
En esta plática se presentarán los conceptos básicos de la teoría de control óptimo vía programación dinámica. Mediante ejemplos ilustrativos se mostrará el uso de la técnica de programación dinámica para la solución de problemas de optimización. Posteriormente, se introducirá de forma didáctica el uso de sistemas de control en las áreas de inventarios y finanzas.

Mediante simulación computacional se estudiará el comportamiento aleatorio de dichos sistemas, y en base a ello se propondrán estrategias de operación para optimizar su funcionamiento. En el caso de inventarios se determinará una estrategia que minimice el costo de operación, en función del stock del sistema. Para el caso de finanzas se presentará un modelo de consumo e inversión para el cual se determinará una posible estrategia de inversión en función del capital inicial del inversionista. Finalmente se comentarán las problemáticas a las que se enfrentan los estudiantes al iniciar en el área de control estocástico y una posible metodología que puede desarrollar el estudiante para la comprensión del tema.
\end{abstract}

Palabras clave: Procesos de Decisión de Markov, Programación Dinámica, Simulación.

${ }^{1}$ Facultad de Ciencias Físico-Matemáticas, Benemérita Universidad Autónoma de Puebla, México. hcs@fcfm.buap.mx 


\title{
Elementos de análisis factorial aplicados al estudio de cualidades psicométricas de la escala de "ansiedad matemática" de Fennema-Sherman
}

\author{
Luis Gerardo Meza Cascante ${ }^{1}$ \\ Evelyn Agüero Calvo ${ }^{2}$ \\ Zuleyka Suárez Valdés-Ayala ${ }^{3}$
}

\section{Resumen}

En esta ponencia se exponen los resultados de una investigación que indagó las características sicométricas de la escala de "ansiedad matemática" de FennemaSherman (1976) en 3725 estudiantes de la educación media costarricense matriculados en colegios diurnos oficiales en el año 2013, en la que se utilizaron técnicas del análisis factorial.

Palabras clave: ansiedad matemática, afecto, aprendizaje de la matemática, enseñanza de la matemática, escala de Fennema-Sherman, análisis factorial.

\section{Descripción del trabajo}

El estudio de la"ansiedad matemática" forma parte de los esfuerzos realizados en la Escuela de Matemática del Instituto Tecnológico de Costa Rica por avanzar en la investigación de las denominadas "respuestas afectivas", dado el creciente reconocimiento de que estas juegan un papel esencial en el proceso de enseñanza y aprendizaje de la matemática.

No existe unicidad en cuanto a la definición de ansiedad matemática. Pérez-Tyteca y Castro (2011) resaltan que las definiciones de ansiedad matemática suelen asumir implícitamente dos hechos. Por una parte, que la ansiedad matemática está relacionada con la ansiedad general (Hendel, 1980), con la ansiedad hacia los exámenes y con la producida por otras materias académicas, pero, por otra parte, también es específica (Hembree, 1990), es decir, la ansiedad matemática existe en personas que no tienen otros tipos de ansiedad (Morris, 1981)

En la investigación asumimos que la "ansiedad matemática" consiste en un sistema de respuestas de tipo afectivoque se caracteriza por la ausencia de comodidad que puede experimentar una personacuando enfrenta situaciones relacionadas con la matemática, tanto en su vida cotidiana como académica, que se manifiesta mediante una serie de síntomas como tensión, nervios, preocupación, inquietud, irritabilidad, impaciencia, confusión, miedo y bloqueo mental. (Pérez-Tyteca, Castro, Rico y Castro, 2011)

\footnotetext{
${ }^{1}$ Instituto Tecnológico de Costa Rica, Costa Rica. gemeza@itcr.ac.cr

${ }^{2}$ Instituto Tecnológico de Costa Rica, Costa Rica. evaguero@itcr.ac.cr

${ }^{3}$ Instituto Tecnológico de Costa Rica, Costa Rica. zsuarez@itcr.ac.cr
} 
La medición del constructo "ansiedad matemática" se puede realizar mediante escalas de tipoLikert, las que "miden de forma indirecta determinadas disposiciones mediante declaraciones verbales de opinión" (Visauta, 1989 citado por Burga, 2005). En otras palabras, al medir la "ansiedad matemática" mediante ese tipo de escalas se asume que es posiblelograrlo a través de manifestaciones verbales. (Pérez-Tyteca, 2012)

La escala de "ansiedad matemática" de Fennema-Sherman (1976)es una de las más reconocidasy ha sido validada a lo largo de más de 30 años (Pérez-Tyteca, 2012).

En esta ponencia se exponen los resultados de un estudio realizado con 3725 estudiantes de la educación media costarricense matriculados en colegios diurnos oficiales en el año 2013. La muestra estuvo integrada por un $48,1 \%$ de mujeres y 51,9 \% de hombres. La distribución por nivel educativo fue la siguiente: sétimo (20\%), octavo (19,9\%), noveno (20,8\%), décimo $(20 \%)$ y undécimo $(19,4 \%)$.

La investigación indagó sobre las características sicométricas de la escala de "ansiedad matemática" de Fennema-Sherman (1976), estudiando el índice de fiabilidad mediante la técnica del alfa de Cronbach y la unidimensionalidad aplicando técnicas de análisis factorial.

La unidimensionalidad implica que un sólo rasgo latente oconstructo se encuentra en la base de un conjunto de ítems (Hattie,1985).En la práctica, ningún instrumento resulta perfectamente unidimensional. Por tanto, la unidimensionalidad de un instrumento de medición se convierte en una cuestión de grado, razón por lo que se procura es tener instrumentos que en esencia muestren unidimensionalidad (Burga, 2005; Jiménez y Montero, 2013).

Aunque no existe una única línea metodológica para evaluarla unidimensionalidad (Burga, 2005), el análisis factorial sigue siendo una herramienta muy utilizada almomento de estudiar la dimensionalidad de un conjunto de ítemes(Jiménez y Montero, 2013).

Para evidenciar la razonabilidad de aplicar el análisis factorial se calculó el índice KaiserMeyer-Olkin (KMO) y se aplicó la prueba de esfericidad de Bartlett.

La medida de adecuación muestral deKMO expresa qué proporción de la varianza en las variables es varianza común (atribuible a factores subyacentes).Para la interpretación del coeficiente KMO se utilizaron las recomendaciones establecidas por Kaiser en 1974, citado por Frías-Navarro y Pascual (2012), a saber:

$$
\begin{aligned}
& 0,9<\mathrm{KMO} \leq 1,0: \text { Excelente adecuación muestral } \\
& 0,8<\mathrm{KMO} \leq 0,9: \text { Buena adecuación muestral } \\
& 0,7<\mathrm{KMO} \leq 0,8: \text { Aceptable adecuación muestral } \\
& 0,6<\mathrm{KMO} \leq 0,7: \text { Regular adecuación muestral } \\
& 0,5<\mathrm{KMO} \leq 0,6: \text { Mala adecuación muestral } \\
& 0,0<\mathrm{KMO} \leq 0,5: \text { Adecuación muestral inaceptable }
\end{aligned}
$$


La prueba de esfericidad de Bartlett se utiliza para contrastar la hipótesis nula de que la matriz de correlaciones es una matriz identidad, en cuyo caso no existirían correlaciones significativas entre las variables y el modelo factorial no sería pertinente (Bizquerra, 1989, citado por Dicovskyi, 2002).

La aplicación del análisis factorial, una vez evidenciada la conveniencia de utilizar la técnica mediante el calculo del coeficiente KMO (0.922) y el índice de esfericidad de Bartlet $(\mathrm{p}<0.05)$, se realizó indagando el cumplimiento de al menos uno de los siguientes criterios de unidimensionalidad:

a. La estructura de un factor dominante tras el análisis de los autovalores (Arias, 1996, citada por Rivas, Fierro, Jiménez y Berrocal, 1998)::

$$
\frac{\lambda_{1}}{\lambda_{2}}>5 \quad \text { o } \quad \frac{\lambda_{1}-\lambda_{2}}{\lambda_{2}-\lambda_{3}}>5
$$

b. El primer factor explica el $40 \%$ de la varianza (Carmines y Zeller, 1979, citados por Burga, 2005, p. 3).

c. Existencia de un codo en el gráfico de sedimentación después del primer autovalor (Céspedes, Cortés y Madrigal, 2011, p. 5).

Los resultados obtenidos muestran excelentes características psicométricas de la escala analizada: un valor de alfa de 0,876que supera el 0.8 mínimo como valor aceptable que recomienda Cea (1999) y el cumplimiento de los tres criterios de unidimensionalidad evaluados.

\section{Bibliografía}

Burga, A. (2005). La unidimensionalidad de un instrumento de medición: perspectiva factorial. Lima, Perú: Ministerio de Educación.

Cea, M.A. (1999) Metodología cuantitativa: estrategias y técnicas de investigación social. Madrid: Síntesis.

Céspedes, Y., Cortés, R. \& Madrigal, M. (2011). Validación de un instrumento para medir la percepción de la calidad de los servicios farmacéuticos del Sistema Público de Salud de Costa Rica. Revista Costarricense de Salud Pública, 20, 75-82. Recuperado de:

http://www.scielo.sa.cr/pdf/rcsp/v20n2/art2v20n2.pdf

Dicovskyi, L. (2002). Folletos del Curso "Estadística aplicada para análisis de encuestas en SPSS”. Estelí: ADESO. 
Fennema, E. \& Sherman, J. A. (1976). Fennema-Sherman mathematics attitude scales. Instruments designed to measure attitudes toward the learning of mathematics by males and females. JSAS Catalog of Selected Documents of Psychology, 6(31).

Frías-Navarro, D. \& Pascual, M. (2012). Prácticas del análisis factorial exploratorio (afe) en la investigación sobre Conducta del consumidor y marketing. En: Suma Psicológica, Vol. 19, Núm. 1, pp. 45-58

Hendel, D. (1980). Experiential and affective correlates of math anxiety in adult women. Psyclology of Women Quartely, 5(2), 219-230.

Hembree, R. (1990). The nature, effects, and relief of mathematics anxiety. Journal for Research in Mathematics Education, 21(1), 33-46.

Jiménez, K. \& Montero, E. (2013). Aplicación del modelo de Rasch, en el análisis psicométrico de una prueba de diagnóstico en matemática. Revista digital Matemática, Educación e Internet, 13(1), 1-23.

Morris, J. (1981). Mathematics Anxiety: Teaching to Avoid it. Mathematics Teacher. 74. 423-417.

Pérez Tyteca, P. (2012). La ansiedad matemática como centro de un modelo causal predictivo de la elección de carreras. Tesis para optar al grado de Doctora en Matemática con énfasis en Didáctica de la Matemática. Departamento de Didáctica de la Matemática, Universidad de Granada.

Pérez-Tyteca, P. ,Castro, E. , Rico, L. \& Castro, E. (2011). Ansiedad matemática, género y ramas de conocimiento en alumnos universitarios. Revista Enseñanza de las ciencias: revista de investigación y experiencias didácticas. Recuperado de:

http://fqm193.ugr.es/produccion-cientifica/revistas/ver_detalles/167625/

Pérez-Tyteca, P. \& Castro, E. (2011). La ansiedad Matemática y su red de influencias en la elección de carrera Universitaria. Recuperado de:

http://funes.uniandes.edu.co/1831/1/402_Perez2011Laansiedad_SEIEM13.pdf 


\title{
Aplicación del método de ecuaciones estructurales en el estudio del estilo de liderazgo de los directores de escuela o departamento académico en universidades costarricenses
}

\author{
Deyanira Meza Cascante ${ }^{1}$
}

\begin{abstract}
Resumen
En esta ponencia se exponen los resultados de una investigación que analizó si el estilo de liderazgo de los directores de escuela o departamento académico en universidades costarricenses está influido directamente por factores propios, tales como: servicio, proyección, autoconciencia y autoconocimiento y por rasgos del profesorado como: pensamiento crítico-participación activa, mediante la aplicación del modelo de ecuaciones estructurales.
\end{abstract}

Palabras clave: liderazgo, estilo de liderazgo, rasgos del director, rasgos del profesorado, gestión universitaria, ecuaciones estructurales.

\section{Descripción del trabajo}

El estudio de la influencia en el estilo de liderazgo de los directores de escuela o departamento académico en universidades costarricenses de factores propios del director y de rasgos del profesorado se enmarca dentro de los objetivos planteados en la tesis "Liderazgo universitario: Factores que determinan el estilo de liderazgo en directores de escuelas y departamentos académicos en universidades costarricenses", para optar por el grado de Doctor en Intervención Educativa.

En esta ponencia se exponen los resultados de un estudio realizado con 454 profesores y profesoras de escuelas y departamentos académicos de la Universidad de Costa Rica, Universidad Nacional y el Instituto Tecnológico de Costa Rica. La muestra de profesores está conformada en su mayoría por un $59,0 \%$ de hombres con respecto a las mujeres que representan el $41,0 \%$, siendo que un $74,4 \%$ de éstos labora jornada completa y el 25,66\% tiene jornada parcial, en su respectiva universidad.

El modelo de ecuaciones estructurales tiene como objetivo fundamental contrastar un modelo teórico sobre las relaciones entre variables independientes y variables dependientes. En la mayoría de estudios, se parte de supuestos teóricos ya establecidos que son de dominio público; sin que sea necesario un modelo teórico de alto nivel para someterlo a contraste, sino que puede ser un modelo de carácter personal, un simple conjunto de creencias acerca de cuáles pueden ser los factores explicativos, cuáles pueden ser las relaciones entre unas variables y otras.

\footnotetext{
${ }^{1}$ Instituto Tecnológico de Costa Rica. dmeza@itcr.ac.cr
} 
Un modelo de ecuaciones estructurales completo consta de dos componentes fundamentales: el modelo de medida que define las relaciones entre variables medidas (directamente observadas) y las variables latentes utilizadas. "Se modeliza como un factor común que subyace bajo una serie de variables observadas" (Uriel y Aldás, 2005, p. 492); y el modelo estructural -el que realmente se desea estimar- que contiene los efectos y relaciones entre los constructos y las ecuaciones estructurales que vinculan los constructos (Hair et al., 1999; Uriel y Aldás, 2005); este especifica la estructura causal hipotetizada entre variables latentes (construcciones teóricas no observadas directamente).

La evaluación del modelo de medida se efectuó a partir de la valoración de los índices de ajuste absoluto, incremental y de parsimonia, a saber:

\begin{tabular}{lc}
\hline \multicolumn{1}{c}{ Índices } & Significatividad \\
\hline Ajuste absoluto & \\
\hline CMIN/DF & Buen ajuste $<3$ \\
RMR & Buen ajuste $<, 05$ \\
RMSEA & Buen ajuste $<, 05$ \\
\hline Ajuste incremental & \\
\hline IFI & Buen ajuste $>, 9$ \\
TLI & Buen ajuste $>, 9$ \\
CFI & Buen ajuste $>, 9$ \\
\hline Parsimonia & \\
\hline PRATIO & Buen ajuste $>, 9$ \\
PNFI & Buen ajuste $>0,09$ \\
PCFI & Buen ajuste $>0,09$ \\
\hline
\end{tabular}

En el caso de la evaluación del modelo estructural se aplican los índices supra, siendo significativo si el estadístico supera $\pm 1,96$ (Tejedor, 2004).

Al no haber una definición unívoca sobre "liderazgo", ya que teóricos e investigadores formulan su propia conceptualización, la que es coincidente en definir a la influencia, el cambio y la ausencia de coerción como sus elementos constitutivos; la persuasión, la interacción, la construcción y el mantenimiento de un sentido de visión y cultura como rasgos distintivos del proceso de liderazgo, por lo que, para esta investigación se acuña el concepto de liderazgo como un proceso en el que se da una influencia recíproca entre el director y el profesorado, en la que estos formulan, orientan y mantienen la visión de la escuela o departamento, a fin de lograr metas consensuadas.

La revisión teórica ofreció un modelo de relación entre variables independientes factores constitutivos por rasgos de los directores, rasgos del profesorado y variables del contexto- y variables dependientes -estilos de liderazgo, concretados en cuatro estilos: transformacional, transaccional, no liderazgo e instruccional-. El análisis factorial exploratorio realizado de previo sobre las respuestas del profesorado permitió tamizar este listado de variables, o dimensiones (puesto que agrupa varias variables), siendo estas las que se incluyen en las hipótesis causales a comprobar con los diferentes modelos, a saber: 


\begin{tabular}{cl}
\hline \multicolumn{1}{c}{ Dimensión } & \multicolumn{1}{c}{ Constructo } \\
\hline Liderazgo transformacional & $\begin{array}{l}\text { Transformacional } \\
\text { Sentido del humor }\end{array}$ \\
\hline Liderazgo instruccional & Instruccional \\
\hline \multirow{2}{*}{ Rasgos de los directores } & $\begin{array}{l}\text { Servicio, proyección y } \\
\text { autoconciencia } \\
\text { Autoconocimiento }\end{array}$ \\
\hline \multirow{2}{*}{ Rasgos de los profesores } & $\begin{array}{l}\text { Pensamiento crítico- } \\
\text { Participación activa }\end{array}$ \\
\hline
\end{tabular}

De los análisis realizados a los modelos propuestos se concluye:

\section{a. Con respecto a la validación empírica del modelo de medida}

La formulación del modelo de medida con el fin de explicar y evaluar la contribución de cada ítem a la escala y el grado en que la escala mide los diferentes constructos, en todos los casos -tanto para la muestra total como para submuestrascumple con los criterios de evaluación; la valoración de los índices de ajuste absoluto, incremental y de parsimonia establece que el modelo hipotetizado es correcto y representa en forma sustantiva las relaciones entre las variables hipotetizadas y la población.

\section{b. Con respecto a la validación empírica del modelo estructural}

A partir de la representación de la estructura causal hipotetizada, en la que se muestran los efectos y las relaciones entre los constructos y las ecuaciones estructurales que les vinculan, mediante los criterios de evaluación: viabilidad de los parámetros estimados, adecuación de los errores estándar y la significación estadística de los parámetros y, de la valoración de los índices de ajuste absoluto, incremental y de parsimonia, a fin de establecer si el modelo hipotetizado ajusta a los datos, se tiene:

- Los constructos servicio, proyección y autoconciencia y, autoconocimiento que definen los rasgos de los directores influyen en la percepción que los profesores manifiestan acerca del estilo de liderazgo transformacional del director.

- El constructo servicio, proyección y autoconciencia influyen en el estilo de liderazgo instruccional del director percibido por el profesorado.

- El constructo pensamiento crítico-participación activa que describe los rasgos del profesorado, influyen en el estilo de liderazgo transformacional e instruccional del director, percibido por el profesorado." 


\section{Bibliografía}

Bass, B. (2000). El futuro del liderazgo en las organizaciones que aprenden. (I. Rentaría Trad). En Liderazgo y organizaciones que aprenden. III Congreso Internacional sobre Dirección de Centros Educativos (pp. 331-361). ICE, Universidad de Deusto. Bilbao, España: Ediciones Mensajero.

Batista, J.M. y Coenders, G. (2000). Modelos de ecuaciones estructurales. Cuadernos de Estadística. Madrid, España: Editorial La Muralla S.A.

Catena, A., Ramos, M., y Trujillo, H. (2003). Análisis Multivariado. Un manual para investigadores. Madrid, España: Editorial Biblioteca Nueva, S. L.

Cupani, M. (2008). Análisis de ecuaciones estructurales: conceptos, etapas de desarrollo y un ejemplo de aplicación. Revista Tesis Psicología, 1(1), 164-176. Recuperado de http://www.psychepersonality.com.ar/index.php/es/publicaciones/artículospublicados/6 2-articulo6

Díez, J. (1992). Métodos de análisis causal. Cuadernos Metodológicos, Num. 3. Centro de Investigaciones Sociológicas. Madrid, España.

Goleman, D. (1996). Inteligencia Emocional. Barcelona, España: Editorial Kairós, S.A.

Gorrochotegui, A. A. (1997). Manual de liderazgo para directivos escolares. Madrid: Editorial La Muralla, S. A.

Hair, J., Anderson, R., Tatham R. y Black, W. (1999). Análisis multivariante. Madrid, España: Prentice Hall.

Kelley, R. (1992). Líderes y seguidores: Cooperación mutua en beneficio de la empresa. España: McGraw Hill.

Kotter, J. (1990). El factor liderazgo. Madrid, España: Ediciones Díaz Santos S.A.

Lévy, J-P, Manera, J., García, J. M. y Cruz, I. (1999): Modelización con ecuaciones estructurales y variables latentes. Madrid, España. Editorial. Erica.

Lévy, J-P. (2003). Modelización y análisis con ecuaciones estructurales. En J-P. Lévy, y J. Varela, Análisis Multivariable para las Ciencias Sociales (pp.767-814). Madrid, España: Pearson Educación.

Manzano, A. y Zamora, S. (2009) Sistema de ecuaciones estructurales: Una herramienta de investigación. Cuaderno técnico 4., A.C. México, D.F.: Consejo Nacional de Evaluación para la Educación Superior.

McClelland, D. (1989). Estudio de la motivación humana. Madrid, España: Narcea. 
McGregor, D. (1975). El aspecto humano de las empresas. México: Editorial Diana.

Nieto, M. (2006). Prólogo del libro. En J-P Lévy, y J. Varela, Modelización con estructuras de covarianzas en Ciencias Sociales: temas esenciales, avanzados y aportaciones especiales. Madrid, España. Editorial Gesbiblo.

Ruiz, M. A.; Pardo, A. y San Martín, R. (2010). Modelos de ecuaciones estructurales. Papeles del Psicólogo, 31(1), 34-45. Recuperado de http://redalyc.uaemex.mx/src/ inicio/ArtPdfRed.jsp?iCve=77812441004

Tejedor, F. (2004) Análisis del modelo europeo de excelencia mediante la aplicación de modelos de ecuaciones estructurales (resumen del estudio), Sinergy Ingeniería de Gestión. Consultado el 28 de marzo del 2013 en http://www.sinergy.es/documentos/ 040701_Analisis_SEM_del_Modelo_EFQM_resumen.pdf

Tünnermann, C. (2009). El rol del docente en la educación superior del siglo XXI. Universidad Nicaragüense de Ciencia y Tecnología. Consultado el 9 de enero del 2013 en http://www.ucyt.edu.ni/Download/EL_ROL_DEL_DOCENTE_EN_LA_ E_S_DEL_SIGLO_XXI.pdf

Uriel, E. y Aldás, J. (2005). Análisis multivariante aplicado: Aplicaciones al marketing, investigación de mercados, economía, dirección de empresas y turismo. Madrid, España: Internacional Thompson Editores. 


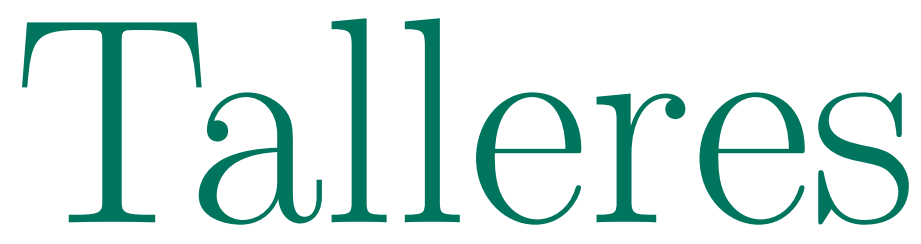


IV Encuentro sobre Didáctica de la Estadística, la Probabilidad y el Análisis de Datos

El uso de las Situaciones Aleatorias en la Enseñanza de la Probabilidad Augusta Osorio Gonzales

Resumen:

Se han realizado diversas propuestas que permiten enseñar el concepto de probabilidad. Estas se pueden observar en los distintos textos desde el nivel primario hasta el nivel universitario, pero también se puede evidenciar como casi siempre se deja un poco de lado lo que es el estudio de las situaciones aleatorias y su relación con el concepto de probabilidad. El taller que queremos presentarles mostrara actividades de un proceso de instrucción para la introducción de la probabilidad a nivel universitario y que se basa en el uso de las situaciones aleatorias como elemento básico en el desarrollo del tema de probabilidades. Este proceso ha sido analizado y su idoneidad validada en la tesis de maestría de la autora.

Palabras Claves: Situación aleatoria, probabilidad

Abstract:

There have been various proposals about teaching the concept of probability. These are presented in various texts from elementary school to university studies, but is also noticeable how the study of random situations and their relation to the concept of probability are being kept aside. The workshop will explain the activities, which involve the process of instruction of probability at the college level and it relation with the use of random situations as a basic element in the development of probabilities topic. This process has been analyzed and validated its suitability in the Master's thesis of the author.

Keywords: random situations, probability

Modalidad: Taller

IREM-PUCP

Pontificia Universidad Católica del Perú

arosorio@pucp.edu.pe 


\section{Antecedentes del proceso de instrucción}

Si un estudiante universitario no ha tenido la oportunidad de desarrollar su pensamiento aleatorio durante su preparación escolar, es previsible que se le presenten problemas durante su primer curso formal de Estadística en la universidad puesto que debe enfrentarse a una forma de pensar que no ha desarrollado previamente. ¿Cómo debe enfrentar un docente este problema?¿cómo se puede desarrollar en una persona una forma de concebir la realidad que no ha sido elaborada previamente durante un proceso continuo y progresivo? Estos son algunos de los desafíos que debe enfrentar cualquier profesor de un curso de Estadística básica dentro de casi todas las profesiones actuales.

La enseñanza del concepto de probabilidad esta intrínsecamente ligada con el concepto de situación aleatoria, experimento aleatorio y de posibilidad. Desgraciadamente son pocos los libros de Estadística general que desarrollan esta relación, y cuando lo hacen la muestran de una forma sumamente superficial. Entonces, si se desea hacer llegar a los alumnos ideas sobre las diferencias entre los conceptos de posibilidad y probabilidad, es necesario enfrentarlos a los conceptos y a las diferencias existentes mediante el uso de estos conceptos a ejemplos concretos. Dado que la bibliografía disponible no nos puede servir de apoyo en este tema puntual, el único medio en el que nos podemos apoyar es en la creación de actividades con ese fin. La reunión de las actividades creadas por la autora, forman un proceso de instrucción que dura alrededor de seis semanas y que cuenta con trabajos dentro y fuera de clase.

La propuesta está centrada en el establecimiento de la base necesaria para el entendimiento del concepto de probabilidad y en ese sentido, se trabajó un proceso de instrucción que permite a los estudiantes el manejo adecuado de las situaciones aleatorias. Las actividades de este proceso de instrucción favorecen el entendimiento de lo que es una situación aleatoria, cómo podemos definirla y de qué partes se compone. El proceso de instrucción se aplicó durante más de ocho semestres dentro del curso a cargo de la autora y fue sufriendo las modificaciones necesarias propias de la reflexión docente. Paralelamente fue necesario ir mejorando la visión teórica del proceso de introducción y se fue construyendo una visión de la realidad que permite colocar en ella todos los elementos que se desarrollaron. De esta manera podemos establecer cómo calcular la probabilidad de un suceso en particular de una situación aleatoria previamente definida.

\section{Descripción del proceso de instrucción}

Las características del proceso de instrucción se establecieron en base a una propuesta de solución que se procuró frente a cada una de las problemáticas detectadas en la enseñanza del concepto de probabilidad. Las problemáticas trabajadas están conformadas por las presentadas en Serradó, Cardeñoso y Azcárate (2006) y otras que la autora fue recogiendo durante los años en que aplicó el proceso de instrucción. A continuación alguna de las problemáticas consideradas: 


\begin{tabular}{|c|c|}
\hline PROBLEMATICA & PROPUESTA \\
\hline $\begin{array}{l}\text { Bennett (2000, citado } \\
\text { Serradó,Cardeñoso y Azcárate, 2006, } \\
\text { página 66) dice: "Las ideas intuitivas } \\
\text { sobre el azar pueden preceder a las ideas } \\
\text { formales y, si son correctas, pueden ser de } \\
\text { gran ayuda en el aprendizaje; pero en caso } \\
\text { contrario, pueden llegar a dificultar la } \\
\text { correcta comprensión de los conceptos." }\end{array}$ & $\begin{array}{l}\text { Trabajar los temas relacionados con las } \\
\text { situaciones aleatorias antes de formalizar } \\
\text { los conceptos mediante el uso de } \\
\text { actividades introductorias colaborativas. } \\
\text { Para evitar que el manejo de ideas } \\
\text { intuitivas sea errado, las actividades deben } \\
\text { ser monitoreadas con el suficiente número } \\
\text { de docentes. }\end{array}$ \\
\hline $\begin{array}{l}\text { Fischbein, Nello y Marino (1991, citado } \\
\text { en Serradó,Cardeñoso y Azcárate, 2006, } \\
\text { página 66), destacan las dificultades de los } \\
\text { sujetos en la comprensión de las nociones } \\
\text { de suceso simple y compuesto, que puede } \\
\text { ser un obstáculo para la posterior } \\
\text { comprensión de las nociones de sucesos } \\
\text { equiprobables, sucesos contrarios y } \\
\text { sucesos independientes. }\end{array}$ & $\begin{array}{l}\text { Presentar desde un inicio situaciones } \\
\text { problema donde los alumnos mismos } \\
\text { propongan los sucesos simples y } \\
\text { compuestos que analizarán para poder } \\
\text { evidenciar de forma temprana los } \\
\text { problemas que presenten en el } \\
\text { entendimiento de los conceptos. }\end{array}$ \\
\hline $\begin{array}{l}\text { El llamado sesgo de la equiprobabilidad, } \\
\text { de acuerdo con Lecoutre y Duran (1988, } \\
\text { citado en Serradó,Cardeñoso y Azcárate, } \\
2006 \text {, página 66), en el que los sujetos } \\
\text { consideran que los posibles resultados de } \\
\text { cualquier fenómeno son equiprobables } \\
\text { porque son materia del azar. }\end{array}$ & $\begin{array}{l}\text { Evitar utilizar las típicas situaciones } \\
\text { aleatorias de espacio muestral } \\
\text { equiprobable (lanzamiento de dados, } \\
\text { lanzamiento de monedas, juegos de cartas, } \\
\text { juegos con ruletas o rifas) como } \\
\text { situaciones problema para las actividades } \\
\text { del proceso de instrucción. }\end{array}$ \\
\hline $\begin{array}{l}\text { Batanero y Serrano (1999), nos proponen: } \\
\text { "Un tratamiento inadecuado de la forma } \\
\text { de contextualizar y referenciar los objetos } \\
\text { (acontecimientos, fenómenos, } \\
\text { experimentos aleatorios,...) puede } \\
\text { ocasionar un obstáculo didáctico en la } \\
\text { comprensión de la noción de aleatoriedad } \\
\text { y probabilidad." }\end{array}$ & $\begin{array}{l}\text { Evitar manejar diversos nombres para el } \\
\text { mismo concepto o en todo caso hacer } \\
\text { referencia siempre a las acepciones que } \\
\text { propongamos usar. Por ejemplo, decir } \\
\text { suceso o evento simple. Además, es } \\
\text { importante que el alumno tenga siempre la } \\
\text { oportunidad de analizar por sí mismo los } \\
\text { contextos antes que el docente se los } \\
\text { clarifique, de manera que pueda } \\
\text { interiorizar adecuadamente la situación } \\
\text { aleatoria problema y le sea más fácil } \\
\text { definir sus componentes. }\end{array}$ \\
\hline $\begin{array}{l}\text { Los alumnos proponen como posibles } \\
\text { situaciones aleatorias a situaciones que en } \\
\text { realidad no poseen la característica de } \\
\text { incertidumbre. }\end{array}$ & $\begin{array}{l}\text { Por la experiencia que tenemos con el } \\
\text { proceso de instrucción conocemos que } \\
\text { este problema se genera por dos } \\
\text { principales causas: el alumno no identifica } \\
\text { adecuadamente la acción a realizar dentro } \\
\text { de la situación propuesta y por tanto no } \\
\text { puede localizar la fuente de incertidumbre, } \\
\text { y el alumno no repara en el hecho de que } \\
\text { el ejecutante de la acción y la persona que } \\
\text { toma decisiones dentro de la misma es en } \\
\text { algunas ocasiones la misma persona. }\end{array}$ \\
\hline
\end{tabular}




\begin{tabular}{|l|l|}
\hline & $\begin{array}{l}\text { La solución radica, en el primer caso, en } \\
\text { enseñarles a los alumnos a realizar un } \\
\text { análisis previo de la situación propuesta } \\
\text { de manera que separen adecuadamente el } \\
\text { contexto propuesto de la acción que se } \\
\text { realiza durante la situación aleatoria. } \\
\text { Para el segundo caso, proponerles } \\
\text { ejemplos de situaciones problema donde } \\
\text { vean que a pesar de haber una acción a } \\
\text { realizar también hay una decisión que le } \\
\text { quita incertidumbre al resultado de su } \\
\text { ejecución. }\end{array}$ \\
\hline $\begin{array}{l}\text { Para el alumno es difícil establecer las } \\
\text { condiciones que se derivan de las } \\
\text { restricciones expresadas en el contexto de } \\
\text { una situación aleatoria y establecer que las } \\
\text { restricciones y condiciones afectan de } \\
\text { manera directa a la definición del espacio } \\
\text { muestral. }\end{array}$ & $\begin{array}{l}\text { Propiciar que el alumno coloque por } \\
\text { separado las restricciones provenientes del } \\
\text { contexto de la situación aleatoria } \\
\text { adicionales que tendrá en cuenta para el } \\
\text { análisis de los componentes de la } \\
\text { situación trabajada. } \\
\text { Considerar ejemplos concretos donde } \\
\text { variando las condiciones del contexto se } \\
\text { observa que cambia la forma de establecer } \\
\text { el espacio muestral. }\end{array}$ \\
\hline $\begin{array}{l}\text { Los alumnos se complican cuando tienen } \\
\text { que trabajar con espacios muestrales muy } \\
\text { grandes y no saben cómo representarlos. }\end{array}$ & $\begin{array}{l}\text { Hacer que tengan convenciones para } \\
\text { presentar los sucesos simples con el fin de } \\
\text { no tener dificultades para organizar los } \\
\text { espacios muestrales. }\end{array}$ \\
\hline $\begin{array}{l}\text { Los alumnos presentan problemas para la idea de que el espacio } \\
\text { situación aleatoria, no consideran todos } \\
\text { los casos posibles. }\end{array}$ & $\begin{array}{l}\text { muestral es todo lo posible y no todo lo } \\
\text { que nosotros creemos probable. }\end{array}$ \\
\hline $\begin{array}{l}\text { quar ejemplos extremos para poder hacer } \\
\text { andisualicen el problema. }\end{array}$ \\
\hline
\end{tabular}

Para el diseño de las actividades del proceso de instrucción se partió del hecho de que una vez que el alumno aprende a reconocer las situaciones aleatorias es cuándo el concepto de probabilidad toma sentido y va a procurarles información relevante. También se consideró que es importante que las situaciones aleatorias a trabajar durante todo el proceso de instrucción sean de interés para el alumno, lo mejor fue utilizar siempre situaciones de su entorno inmediato. Además se procuró que los mismos alumnos puedan llegar a plantear situaciones aleatorias a analizar y por eso el proceso de instrucción incluye una actividad de determinación de situaciones aleatorias. Y finalmente, es importante que el proceso apoye al alumno a reconocer los diferentes tipos de situaciones aleatorias que procurarán la existencia de los diversos planteamientos sobre el cálculo de probabilidades.

Las situaciones problema diseñadas para las diferentes actividades del proceso de instrucción tienen como eje central a la situación aleatoria y sus componentes. 
Específicamente, se busca que los alumnos puedan reconocer cuándo una situación propuesta es aleatoria y puedan determinar todos los componentes inherentes a ella. El conseguir este objetivo se evidenciará mediante el alcance de los siguientes objetivos parciales. Se espera que los alumnos:

1. Puedan mencionar algunos posibles resultados de una determinada situación, cuando tienen en consideración determinadas restricciones fijadas de antemano dentro del contexto de la situación que se está analizando.

2. Puedan diferenciar entre una situación de la vida cotidiana que incluye la característica de incertidumbre de una que no la tiene.

3. Puedan dar una posible situación aleatoria cuando se les da un determinado contexto de la realidad.

4. Puedan, dada una determinada situación aleatoria, determinar un suceso simple por extensión y por comprensión.

5. Puedan definir el espacio muestral de la situación aleatoria presentada.

6. Puedan, dada una determinada situación aleatoria, determinar un suceso compuesto por extensión y por comprensión.

7. Puedan determinar un suceso o evento compuesto por extensión, cuando les es presentada una determinada situación aleatoria y un suceso o evento compuesto por comprensión perteneciente a dicha situación aleatoria.

8. Puedan, dada una determinada situación aleatoria, determinar de un grupo de sucesos compuestos propuestos, cuál es el suceso compuesto que es componente de la situación aleatoria presentada.

9. Puedan definir apropiadamente un experimento aleatorio de su entorno. Brindado el contexto con sus condiciones o restricciones a tener en cuenta, el espacio muestral y un grupo de sucesos simples compuestos, ambos grupos por extensión y por comprensión.

La aplicación de todo el proceso de instrucción requiere seis semanas de programación de un curso de tres horas teóricas y dos prácticas a la semana. Además, los alumnos utilizaron tiempo fuera de clases: aproximadamente dos horas de práctica y un promedio de doce horas de trabajo para el desarrollo y revisión del trabajo de evaluación individual.

El proceso de instrucción consta de ocho actividades:

1. Actividad colaborativa para la introducción del concepto de situación aleatoria.

2. Actividad colaborativa para aplicar el concepto de situación aleatoria a un contexto dado.

3. Actividad colaborativa para describir los componentes de una situación aleatoria. 
4. Práctica en línea para repasar los conceptos vistos.

5. Prueba individual de conocimientos adquiridos, conceptos teóricos.

6. Práctica en parejas para poder aplicar el concepto de situación aleatoria y de sus componentes.

7. Desarrollo de trabajo individual de análisis de una situación aleatoria propuesta por el alumno.

8. Evaluación de los conceptos de situación aleatoria y de sus componentes, teórica y de aplicación.

Las actividades 1, 2, 3, 5 y 6 que se desarrollan dentro de las sesiones de clase y práctica del curso, tienen un diseño específico de tiempo para ser desarrolladas. En el caso de las tres primeras actividades, que van en la sesión de clases de la primera semana tomada para el proceso, estas se desarrollan en un lapso de 110 minutos. Se espera que la primera actividad se realice en 40 minutos, la segunda en 20 minutos y la última en 50 minutos. En el caso de las actividades 5 y 6 , que se realizan en la sesión de prácticas de la segunda semana, se espera que la parte individual se realice en 15 minutos y la de parejas en 60 minutos.

\section{Valoración de la idoneidad del proceso de instrucción}

El análisis de idoneidad tuvo como finalidad establecer si el proceso de instrucción diseñado presentaba los aspectos necesarios para que el alumno integre los significados relacionados al objeto en estudio, la situación aleatoria, a sus significados personales. La valoración de idoneidad se trabajó bajo las concepciones del Enfoque Ontosemiótico de la cognición e instrucción matemática (EOS) y se consideró solo dos facetas: la epistémica y la cognitiva.

Los descriptores o indicadores empíricos que ayudaron a la puesta en funcionamiento de la idoneidad didáctica como herramienta de análisis y valoración del proceso en estudio estuvieron basados en los propuestos por Godino, Bencomo, Font y Wilhelmi (2006). Desde los descriptores de idoneidad propuestos se determinó una serie de desviaciones que muestran los componentes del proceso de instrucción al tener como ideal la configuración epistémica de referencia considerada. Desde los resultados obtenidos nuestras interrogantes finales son ¿el proceso de instrucción cumple su objetivo? ¿Vale la pena invertir recursos en su mejora?

Con respecto al cumplimiento de los objetivos del proceso de instrucción, en Osorio(2012) se estableció que este proceso le permitió a los dos alumnos analizados, a pesar de iniciar el proceso sin ningún conocimiento previo sobre el tema, llegar a identificar, proponer y analizar adecuadamente una situación aleatoria. Esto nos da pie para indicar que el proceso cumple con su finalidad y por lo tanto, pensar que una mejora del proceso de instrucción es factible y valida. 
Debemos reconocer que a pesar de las desviaciones encontradas con referencia a los indicadores de idoneidad considerados, los alumnos pueden enfrentar los problemas propuestos, aunque en algunos casos requieren más tiempo para poder manejar los significados. Tenemos que uno de los alumnos analizados pudo manejar los significados casi desde un inicio del proceso de instrucción, mientras que el otro lo hizo recién al final del proceso.

Podemos también indicar que muchas de las desviaciones encontradas son consecuencia de la falta de presentación de los procedimientos y argumentos asociados a los significados pretendidos en el proceso de instrucción. Esta es la desviación más importante encontrada en el análisis, que fue más consecuencia de nuestro desconocimiento en materia de didáctica que en un desconocimiento del tema. ¿Cómo afecto esta falta de información a los alumnos? Por ejemplo, cuando un alumno tiene que decidir cuáles de las situaciones presentadas corresponden a un experimento aleatorio. En ese momento, el alumno tiene que concluir cuáles son los pasos del procedimiento que le llevan a establecer si la situación es o no un experimento aleatorio. Hemos observado durante el análisis de respuestas, que uno de los alumnos solo busca la característica de reproducibilidad dejando de lado el análisis de la incertidumbre. Esta deficiencia se puede subsanar si es que el alumno tiene a su disposición el procedimiento para establecer experimentos aleatorios.

Finalmente, no pensamos que este análisis es suficiente para poder terminar de elaborar un proceso de instrucción que nos lleve a lograr un cambio total en el pensamiento de los alumnos y los dirija a una nueva forma de ver la realidad. Este es solo un primer paso, que ayuda pero que por sí solo no hará todo el trabajo. Con este proceso hemos logrado que los alumnos se introduzcan a un entendimiento sobre de donde surgen la probabilidades, pero aún nos faltan hacer que trabajen con ellas y que además lleguen a construir los famosos modelos probabilísticos. Esto hace que tengamos que pensar en la elaboración de problemas que les permitan manejar la parte conceptual de los planteamientos de cálculo y procedimientos que los lleven a entender de donde surgen las variables aleatorias, tema fundamental para el trabajo en Inferencia estadística, y que está íntimamente ligado con el tema de experimentos aleatorios y modelos probabilísticos. Algunas de estas problemáticas ya las hemos ido trabajando y tenemos algunas ideas de cómo enfrentarlas, pero ahora viene el trabajo difícil, la elaboración de nuevos procesos de instrucción. Esta son algunas de las ideas que tenemos y que trabajaremos en el futuro. Nuestro propósito es llegar a establecer un mecanismo de enseñanza con el que un alumno pueda entender porque es necesario llegar a establecer modelos probabilísticos y como es que estos se determinan.

\section{Actividades a realizar durante el taller a presentar en el IV EDEPA}

Durante las tres horas que se trabajará este taller se desarrollarán las tres primeras actividades del proceso de instrucción, las que están vinculadas con el concepto de 
situación aleatoria y el reconocimiento de sus componentes. Se espera que los participantes desarrollen estas actividades y puedan probar su efectividad.

Los alumnos desarrollarán la primera actividad (Anexo 1) en los primeros 40 minutos del taller, durante ella se les presentará una posible situación real que analizarán para proponer algunos posibles resultados bajo las restricciones dadas. Posteriormente variarán dichas restricciones para que se analice el cambio que sufrirán los resultados de la nueva situación presentada. Estos análisis producirán el descubrimiento de los que caracteriza al tipo de situación presentada. Una vez ubicada esta caracterización se solicitará que brinden una situación que no posea dichas características.

Durante la segunda actividad (Anexo 2), que tomara aproximadamente 20 minutos, los participantes tendrán la oportunidad de definir otras situaciones de la realidad que cumpla con ser similares a la situación trabajada en el actividad 1. Este ejercicio busca que el participante establezca las diferencia entre las diferentes partes que compone la descripción de una situación aleatoria.

Durante la tercera actividad (Anexo 3), que tomara aproximadamente 50 minutos, los participantes tendrán la oportunidad de trabajar en la determinación de los componentes que conforman a una situación aleatoria. Tendrán la oportunidad de explicitar algunos de los sucesos de la situación y determinar su espacio muestral.

El tiempo restante del taller serán para poder presentar el resto de actividades del proceso de instrucción. Se revisarán ejemplos de dichas actividades y se revisará su importancia dentro del proceso. Finalmente, se podrá revisar el marco epistemológico que se tomó para la creación de todo el proceso de instrucción.

\section{Referencias bibliográficas}

- [1] Godino, J. D., Bencomo, D., Font, V. y Wilhelmi, M. R. (2006). Pauta de análisis y valoración de la idoneidad didáctica de procesos de enseñanza y aprendizaje de las matemáticas. Paradigma, Volumen XXVII, No 2, 221-252

- [2] Osorio, A. (2012). Análisis de la idoneidad de un proceso de instrucción para la introducción del concepto de probabilidad en la enseñanza superior. Tesis para optar el grado de Magister en Enseñanza de las Matemáticas. PUCP. http://tesis.pucp.edu.pe/repositorio/handle/123456789/4658 
IV Encuentro sobre Didáctica de la Estadística, la Probabilidad y el Análisis de Datos

- [3] Serradó,A. Cardeñoso,J. y Azcarate,P (2006). Los obstáculos en el aprendizaje del conocimiento probabilístico: su incidencia desde los libros de texto. TARBIYA, Revista de Investigación e Innovación Educativa del Instituto Universitario de Ciencias de la Educación Universidad Autónoma de Madrid №38.

\section{Anexo 1 - Ficha actividad 1 - Actividad colaborativa para la introducción del concepto de situación aleatoria.}

\section{Situación Propuesta}

Julia se encuentra conversando con su amiga Susana sobre la celebración de su primer año de casada. Julia le cuenta a Susana que su esposo Orlando le ha comentado que lo celebrarán el próximo jueves de una manera muy especial. Ella está tratando de imaginarse la sorpresa conociendo lo poco romántico que es Orlando y el poco tiempo que tienen para la celebración, con las justas contarán con unas tres o cuatro horas, puesto que Orlando está "full" con sus estudios para recibirse como médico.

Converse con su pareja de trabajo y juntos preparen una pequeña lista que considere las posibles formas de celebración que Julia podría imaginar, considerando que los recursos de la pareja no son muchos ya que Julia trabaja como secretaria en una pequeña oficina y Orlando recibe solo 1000 Nuevos soles por el trabajo que realiza.

A. Que nos faltaría conocer para poder poner un límite a las posibles formas de celebración?

B. Tomando en cuenta el punto A y pensando que solo tendrán tiempo para realizar una actividad de celebración, considere algunas posibles formas de celebración:

1.

2.

3

4.

C. Consideren que en el tiempo que disponen para celebrar Julia y Orlando, van a realizar dos actividades. 
IV Encuentro sobre Didáctica de la Estadística, la Probabilidad y el Análisis de Datos

D. Tomando en cuenta el punto A y C, considere algunas posibles formas de celebración:

Posibilidad 1.

Posibilidad 2.

Posibilidad 3.

Posibilidad 4.

E. ¿Cuál será el número total de posibilidades distintas que creen ustedes que puedan realizar Julia y Orlando para poder celebrar su aniversario?

F. ¿Qué cualidad posee la situación propuesta que impide que se conozca la forma de celebración?

Detalle una posible situación donde sí se pueda conocer el resultado de antemano.

Anexo 2 - Ficha actividad 2 - Actividad colaborativa para aplicar el concepto de situación aleatoria a un contexto dado.

Detalle una posible situación aleatoria que se dé, cuando se va a un paseo a la playa. 
Anexo 3 - Ficha actividad 3 - Actividad colaborativa para describir los componentes de una situación aleatoria.

Usted tiene que localizar a uno de sus compañeros de trabajo para coordinar una reunión y por tanto debe de llamar al número de celular que le ha proporcionado su jefe. Si usted cuenta con un celular recientemente cargado y con suficiente salgo, una vez que termina de digitar los números del teléfono que puede suceder con la llamada?

Trate de describir a continuación:

A. El contexto de la situación presentada

B. La acción implicada en la situación presentada

C. Las restricciones de la situación presentada

D. ¿Es esta una situación a la que se le puede denominar Experimento aleatorio?

Considerando que al conjunto de todos los resultados posibles se le denomina espacio muestral, determine el espacio muestral de la situación propuesta

Espacio muestral $=\{$ 
IV Encuentro sobre Didáctica de la Estadística, la Probabilidad y el Análisis de Datos

Considerando que a cada posible resultado se le denomina suceso o evento simple, determine 2 sucesos simples de esta situación.

\section{Evento 1: \{}

Evento 2: \{

Ahora, defina nuevamente cada suceso simple, pero hágalo por comprensión (es decir, utilice una oración para definirlo)

Evento 1:

Evento 2:

Considerando que a cada subconjunto del conjunto potencia del espacio muestral que no es un suceso o evento simple se le denomina suceso o evento compuesto, determine el suceso definido por

- Llegue a coordinar mi reunión. 


\title{
Propuesta didáctica para la enseñanza de la Estadística y la Probabilidad en Secundaria
}

\author{
Estibaliz Rojas Quesada ${ }^{1}$ \& Jeffry Barrantes Gutierrez ${ }^{2}$
}

\begin{abstract}
Resumen
En este taller se expondrán y crearán actividades lúdicas, en las cuales se detalla su construcción paso a paso, con el objetivo de brindar al participante diversas herramientas creativas para el desarrollo de los temas de Estadística y Probabilidad, tanto a nivel de primaria como de secundaria. Al final de cada construcción, el participante tendrá un material diferente y una guía de trabajo en caso que desee utilizarla en sus clases.
\end{abstract}

Palabras clave: Estadística, Probabilidad y Actividades Lúdicas.

\begin{abstract}
In this workshop you will be presented with fun activities, which will be explained to you step by step, and which look to provide to the participants creative tools for the development of subjects in the fields of Statistics and Probability, at both primary and secondary levels. At the end of each construction you will have different materials and a work guide which can be used in your lessons.
\end{abstract}

Keywords: Stadistics, probability, and fun activities.

${ }^{1}$ Universidad Estatal a Distancia, Costa Rica. erojasq@uned.ac.cr

${ }^{2}$ Universidad Estatal a Distancia, Costa Rica. jbarrantes@uned.ac.cr 


\title{
Utilización de Applets para favorecer la construcción de ideas fundamentales de la inferencia estadística informal
}

\author{
Liliana Tauber - María Jesús Bianchi y Mariela Cravero ${ }^{1}$
}

\begin{abstract}
Resumen
Desde la comunidad de educadores estadísticos, se ha expresado la preocupación por lograr una Alfabetización Estadística para todos. Existen diversas corrientes que coinciden en la definición de las ideas fundamentales que promueven la Alfabetización y el Razonamiento Estadísticos, lo cual a la larga formará el pensamiento estadístico. Tomando esto de referencia, realizamos una categorización de estas ideas, las cuales consideramos que son imprescindibles para lograr adquirir un Razonamiento Estadístico que permita formar ciudadanos estadísticamente cultos. En este taller presentaremos algunas actividades de simulación que permiten generar las ideas fundamentales relativas a: la variabilidad de las muestras aleatorias, a la de distribución muestral y al Teorema Central del Límite. Además, los asistentes tendrán oportunidad de experimentar utilizando simuladores en línea de distribución gratuita.
\end{abstract}

Palabras Clave: Ideas Estocásticas Fundamentales, Inferencia informal, Razonamiento Estadístico

\begin{abstract}
From the statistical community educators, has expressed concern about achieving Statistical Literacy for all. There are several trends that agree on the definition of the fundamental ideas that promote literacy and statistical reasoning, which eventually form the statistical thinking. Taking this baseline, we performed a categorization of these ideas, which we believe are essential to achieve Statistical Reasoning purchase order to form statistically literate citizens. This workshop will present some simulation activities for generating the fundamental ideas concerning: the random variability, the sampling distribution of and the Central Limit Theorem samples. In addition, attendees will have the opportunity to experience using simulators online free.
\end{abstract}

Key Words: Stochastics Fundamental Ideas, Informal Inference, Statistical Reasoning

\section{Modalidad: Taller de hora y media}

\footnotetext{
${ }^{1}$ Facultad de Humanidades y Ciencias - Universidad Nacional del Litoral - Santa Fe - Argentina - e-mail: estadisticamatematicafhuc@gmail.com, jesubianchi@hotmail.com, marielacravero@hotmail.com
} 
"IV Encuentro sobre Didáctica de la Estadística, la Probabilidad y el Análisis de Datos"

\section{Introducción}

Actualmente, el estudio de la alfabetización de los ciudadanos en distintas disciplinas se muestra como un tema central en la investigación didáctica (Gil y Vilches, 2006, Kemp, 2002, Gal, 2004; Ben-Zvi y Garfield, 2004, Schield, 2002, 2006; Batanero, 2009), así como también los estudios centrados en el análisis de la influencia de las actitudes sobre el rendimiento de los estudiantes o sobre el desempeño de los ciudadanos en diversas áreas del mundo del trabajo.

Además, desde la comunidad de los educadores estadísticos, en las últimas décadas, también se ha expresado la preocupación por lograr una Alfabetización Estadística básica para todos (Schield, 2006). Consideramos que para que un ciudadano logre estar científicamente alfabetizado, uno de los componentes principales es que haya logrado ser un ciudadano estadísticamente alfabetizado, ya que estamos convencidos que la estadística es la que provee de métodos de análisis a las ciencias, por lo cual si no se es culto estadísticamente, será más difícil poder comprender la metodología científica.

En consecuencia, a través del curso que presentamos en este trabajo, pretendemos realizar una breve delimitación de los conceptos estocásticos que son la base de la Alfabetización Estadística. Con este fin, partimos de la revisión de antecedentes en el tema y en función de ésta elaboramos una categorización inicial que nos permitirá luego tomar decisiones en relación con las actividades que se desarrollan en el curso. Por último, presentamos un análisis de las actividades propuestas en función de la categorización realizada.

\section{Revisión de literatura relacionada con la Alfabetización Estadística}

\subsection{Alfabetización Estadística y Ciudadanos estadísticamente alfabetizados}

Partimos de la postura de Wallman (1993), quien argumenta que la Alfabetización Estadística (que simbolizaremos como AE) es la habilidad para:

- entender y evaluar críticamente los resultados estadísticos que intervienen en la vida diaria $y$,

- apreciar las contribuciones que el pensamiento estadístico puede hacer en las decisiones personales, profesionales, públicas y privadas.

Dado que lo anterior puede resultar muy amplio a la hora de organizar una propuesta didáctica buscamos otros elementos teóricos que nos permitan clarificar la definición de Alfabetización Estadística. Es así que siguiendo las ideas planteadas por diversos investigadores en educación estocástica (Garfield, DelMas y Chance, 2003 y Ben-Zvi y Garfield, 2004) adherimos a la siguiente categorización que, actualmente es la más aceptada en la comunidad de educadores estadísticos, en la cual se diferencian los ejes fundamentales que diferencian a la Alfabetización Estadística, el Razonamiento Estadístico y el Pensamiento Estadístico:

- Alfabetización estadística (AE): es el conjunto de habilidades básicas que son usadas en la comprensión de información cotidiana y de resultados de investigaciones. Estas habilidades deberían permitir organizar datos, construir y presentar tablas y trabajar con distintos tipos de resúmenes de datos. También, implica una comprensión básica de conceptos, vocabulario y símbolos, y de la idea de probabilidad como medida de la 
"IV Encuentro sobre Didáctica de la Estadística, la Probabilidad y el Análisis de Datos"

incertidumbre. En otras palabras, la AE se define como la habilidad básica para pensar críticamente sobre argumentos basados en la evidencia.

- Razonamiento estadístico (RE): se puede considerar como la manera que las personas le dan sentido a las ideas e información estadística. Lo cual involucra hacer interpretaciones basadas en un conjunto de datos, representar o resumir datos. También involucra establecer relaciones adecuadas entre conceptos (p.e., centro y dispersión), o combinar ideas sobre los datos y el azar. Razonar, en este sentido, significa comprender y ser capaz de explicar procesos estadísticos y de interpretar, de manera global, los resultados estadísticos.

- Pensamiento estadístico (PE): involucra la comprensión de por qué y cómo se realizan las investigaciones en las que se utiliza la estadística como herramienta metodológica y las "grandes ideas o las ideas fundamentales" implícitas en ellas. Estas ideas incluyen la naturaleza de la variación y, cuándo y cómo usar los métodos más apropiados de análisis de datos, tales como resúmenes numéricos y gráficos. Desarrollar el PE implica resaltar la comprensión de la naturaleza del muestreo, cómo hacer inferencias a la población y cómo diseñar experimentos con el objetivo de establecer causas. Esto incluye, la comprensión de los modelos para simular fenómenos aleatorios y su aplicación en la estimación de probabilidades. Además de entender cómo, cuándo y por qué las herramientas inferenciales pueden usarse para fundamentar los procesos de investigación. El pensamiento estadístico también implica ser capaz de comprender y utilizar el contexto de un problema de investigación y dar conclusiones, reconocer y comprender los procesos completos (desde proponer preguntas para recolectar los datos hasta elegir el análisis y el test de hipótesis que corresponda). Finalmente, los pensadores estadísticos deben ser capaces de criticar y evaluar los resultados de un problema o de un estudio estadístico.

Por supuesto, los procesos de enseñanza y aprendizaje deberían fomentar diversos conceptos y competencias en cada proceso (AE, RE Y PE), de tal forma que luego de varios años de educación estadística se lograra formar un ciudadano estadísticamente alfabetizado que, aunque no sea un técnico estadístico, pueda ser un consumidor estadístico crítico. En consecuencia, consideramos que es fundamental pensar cuáles serían los conceptos y competencias estocásticos que pretendemos introducir a través de la enseñanza en los distintos niveles educativos. Con este fin adherimos al modelo teórico de Gal (2004), quien propone una interacción entre los conocimientos estocásticos básicos y otros procesos que deberían estar disponibles en las personas, y en consecuencia, en los estudiantes o profesores, para que ellos puedan comprender, interpretar, evaluar críticamente y reaccionar a los mensajes estadísticos encontrados en diferentes contextos. Este modelo asume que la Alfabetización Estadística involucra tanto un componente de conocimiento (compuesto de cinco elementos cognitivos: habilidades de alfabetización, conocimiento estadístico, conocimiento matemático, conocimiento del contexto y cuestiones críticas) como un componente disposicional (compuesto de dos elementos: postura crítica, creencias y actitudes). 
"IV Encuentro sobre Didáctica de la Estadística, la Probabilidad y el Análisis de Datos"

Los componentes y elementos en este modelo no deberían considerarse como entidades separadas sino como contextos dependientes, como un conjunto dinámico de conocimiento y aptitudes que juntos forman el comportamiento estadísticamente alfabetizado. La comprensión e interpretación de la información estadística requiere no sólo de conocimiento estadístico per-sé sino también la disponibilidad de otros conocimientos básicos tales como: habilidades de alfabetización, conocimiento matemático básico (fundamentalmente distinguir entre los distintos campos numéricos y tener conocimiento sobre proporcionalidad) y conocimiento del contexto. Sin embargo, la evaluación crítica de la información estadística (después de haber sido comprendida e interpretada) depende de elementos adicionales como la habilidad para realizar preguntas críticas y para tener una postura crítica, la cual se basa en ciertas creencias y actitudes hacia la Estadística y hacia las Ciencias en general.

Hemos basado esta parte del trabajo en el análisis de estos marcos teóricos, los cuales nos han permitido delimitar lo que es la Alfabetización Estadística y los elementos constitutivos de ésta. Este análisis nos permite además, identificar diversos conceptos que consideraremos fundamentales a la hora de diseñar actividades para la enseñanza de Estadística.

2.2 .

Ideas Fundamentales de la Alfabetización Estadística

La concepción de ideas fundamentales fue creada por Bruner (1960), quien indica que, en educación (de una determinada disciplina) se deberían seguir las líneas principales que ofrece la ciencia relacionada. Siguiendo a Goetz, (2008), una tesis básica de esta concepción radica en que es posible enseñar los principios básicos de un tema independientemente de la edad y el origen social de los destinatarios. Este enfoque se refiere al contenido de la educación estocástica (en nuestro caso particular) y también a la actitud que es característica para hacer estadística, por ejemplo, o cualquier otro tema. Así, en este sentido, la educación estocástica debe ser una copia no sesgada de la ciencia estadística. Por supuesto, el nivel de la educación debe ser diferente al nivel de la ciencia, pero esto no debería significar un obstáculo, sino un reto para que la didáctica de la estadística procure identificar los contenidos y los métodos típicos de la ciencia. Según Goetz (2008), además de las ideas fundamentales, cuando planificamos secuencias didácticas, deberíamos tener en cuenta las creencias básicas, tanto de los alumnos como de los docentes mismos. Goetz (2008), indica que se pueden distinguir dos tipos de creencias básicas: las normativas y las descriptivas. Las creencias normativas cumplirían una función similar a la de las ideas fundamentales, mientras que las creencias descriptivas, indican las creencias individuales relacionadas con los contenidos cognitivos. Estas últimas podrían considerarse como actitudes afectivas hacia la disciplina (en este caso, hacia la estadística). Según Goetz, una de las claves para descubrir las creencias es analizar los errores que los estudiantes cometen. Indicación que deberemos tener en cuenta a la hora de planificar nuestra enseñanza. En relación con las creencias de estudiantes y profesores de matemática, hemos encontrado a través de investigaciones previas (Tauber, 2010; Cravero, et. al., 2010) diversos tipos de creencias, por ejemplo: hemos encontrado que una gran proporción de profesores de matemática de nivel Medio, deciden no desarrollar conceptos estocásticos porque no se sienten seguros a la hora de resolver problemas, otros que plantean que la incertidumbre en los resultados estocásticos les provoca ansiedad por el hecho de no tener un único resultado. Como podemos concluir, estas creencias detectadas así como otras que hemos encontrado, influyen en los profesores a la hora de enseñar 
"IV Encuentro sobre Didáctica de la Estadística, la Probabilidad y el Análisis de Datos"

estadística. Por ejemplo, una de las consecuencias es que el profesor en muchas situaciones propone, un lote de datos, que generalmente no se sabe cómo se ha obtenido (o se ha tomado directamente de un libro en el que ni siquiera se plantea un contexto) y propone que el alumno calcule ciertas medidas como puede ser una media aritmética o una desviación pero sin relacionar con el tipo de variable, el tipo de distribución de frecuencias, etc. En otras palabras, es muy común que se propongan actividades puramente algorítmicas que de ninguna manera promueven la comprensión de las ideas estocásticas fundamentales. En consecuencia, consideramos que es necesario distinguir cuáles son las ideas fundamentales que deberían desarrollarse en la enseñanza formal para lograr que nuestros alumnos lleguen a ser ciudadanos estadísticamente alfabetizados.

Considerando que estas ideas fundamentales, en muchas ocasiones, pueden funcionar como obstáculos epistemológicos pero a la vez son el origen de muchos conceptos estadísticos, haremos una primera categorización basándonos en las recomendaciones realizadas por algunos educadores estadísticos (Schield, 2002; Batanero, 2001), quienes indican que es importante, a un nivel introductorio, incluir la enseñanza de:

- técnicas apropiadas que permitan describir proporciones y porcentajes expresados en lenguaje cotidiano que indiquen diversos significados en relación a los conceptos estocásticos que involucran. Por ejemplo: la sentencia "el porcentaje de hombres que son corredores", ¿tiene el mismo significado que: "el porcentaje de hombres entre todos los corredores"?

- lectura e interpretación de tablas y gráficos que involucren distintos tipos de proporciones, razones, tasas y porcentajes.

- Comparación entre proporciones y porcentajes. Por ejemplo: mostrar las diferencias, en relación con los significados y también en relación con el cálculo, entre las siguientes sentencias: Entre los fumadores, los hombres tienen el doble de probabilidades que las mujeres de contraer cáncer de pulmón. Los hombres tienen el doble de probabilidades que las mujeres de llegar a ser fumadores.

Como consecuencia de la revisión presentada y a partir de los trabajos que nuestro grupo ha desarrollado y de los de otros autores (Batanero, 2001; Meyer, 2006; Schield, 2002, 2006; Tauber, 2006), hemos realizado una categorización inicial de las ideas que consideramos fundamentales para la elaboración de secuencias didácticas para la enseñanza de Estadística. En los trabajos mencionados hacemos una extensa descripción de las ideas estocásticas que consideramos fundamentales para lograr formar a un ciudadano estadísticamente alfabetizado, en el presente trabajo, pretendemos mostrar actividades que permitan distinguir las siguientes ideas estocásticas fundamentales para iniciar la enseñanza de la inferencia estadística desde un punto de vista informal, a saber:

- Espacio muestral para eventos equiprobables.

- Probabilidad clásica y probabilidad frecuencial.

- Variable estadística y variable aleatoria.

- Frecuencias absolutas y porcentuales, marginales o condicionales. 
"IV Encuentro sobre Didáctica de la Estadística, la Probabilidad y el Análisis de Datos"

- Proporciones, probabilidades y porcentajes.

- Lectura e interpretación de gráficos estadísticos.

- Distribución de frecuencias, distribución de probabilidad y distribución muestral de un estadístico.

\section{Características del trabajo en el aula de estadística con proyectos}

En diversas investigaciones (Batanero, 2001; Batanero y Díaz, 2011) y planificaciones curriculares (N.C.T.M., 2000; Informe GAISE, 2010) se aconseja trabajar los conceptos estocásticos a partir del desarrollo de proyectos que favorezcan, no sólo la introducción de los conceptos sino también que permitan que el alumno aprecie el carácter metodológico de la Estadística. Otra de las recomendaciones que se realizan es, que la enseñanza basada en proyectos permita trabajar con datos reales y también con datos simulados.

En estos estudios, se plantean diversas razones que muestran las virtudes del trabajo con proyectos. Entre algunas de estas virtudes, una de las más importantes, como señalan Anderson y Loynes (1987), es que la estadística es inseparable de sus aplicaciones, y su justificación final es su utilidad en la resolución de problemas externos a la propia estadística. La historia de la estadística muestra también como ésta recibe ideas y aportes desde áreas muy diversas, donde, al tratar de resolver problemas diversos (por ejemplo: transmisión de caracteres hereditarios, medida de la inteligencia, etc.) se han creado conceptos y métodos estadísticos de uso general (correlación, análisis factorial).

Por otro lado, el trabajo con proyectos permite abordar un proceso de enseñanza y aprendizaje en la que se deben plantear preguntas, ideas, conjeturas que luego deberán comprobarse a través de la evidencia. Esta interacción no suele presentarse en las ejercitaciones planteadas en los libros de texto de uso habitual especialmente en el Nivel de educación Media. Por el contrario, en estas ejercitaciones generalmente se plantean actividades totalmente estructuradas en las que se dan directivas sobre las acciones a realizar, por ejemplo: "calcula la media aritmética". En estos casos, el alumno sólo acata la orden y utiliza un procedimiento puramente algorítmico, en el que se deja totalmente de lado todo el procedimiento estadístico que conlleva al cálculo de una media aritmética, por ejemplo, el análisis del tipo de variable, el tipo de distribución (si es muy asimétrica o si tiene valores alejados, etc.) entre otras cosas, lo cual lleva a un proceso que implica establecer relaciones entre los conceptos de variable estadística, distribución de frecuencias, medidas estadísticas que sean adecuadas al tipo de variable y de distribución, etc.

Como podemos apreciar, los dos procedimientos descriptos provocan dos tipos de aprendizajes muy diferentes: el primero, un aprendizaje acotado y totalmente descontextualizado y el segundo, que permite apreciar las relaciones que deben establecerse cuando una persona se enfrenta a un proceso de toma de decisiones.

En consecuencia, al trabajar con proyectos se coloca a los alumnos en la posición de tener que pensar en preguntas como las siguientes (Graham, 1987): ¿Cuál es mi problema? ¿Necesito datos? ¿Cuáles? ¿Cómo puedo obtenerlos? ¿Qué significa este resultado en la práctica? 
"IV Encuentro sobre Didáctica de la Estadística, la Probabilidad y el Análisis de Datos"

Como sugiere Holmes (1997), si los estudiantes trabajan la estadística por medio de proyectos, se pueden lograr aprendizajes más significativos, que pueden tener implícitas algunas de las siguientes características:

$\checkmark$ Los proyectos permiten contextualizar la estadística y hacerla más relevante. Si los datos surgen de un problema, son datos con significado y tienen que ser interpretados.

$\checkmark$ Se introducen ideas que no aparecen con los "datos inventados por el profesor o en el libro de texto", como por ejemplo: precisión, variabilidad, fiabilidad, posibilidad de medición, sesgo.

$\checkmark$ Se muestra que la estadística no se reduce a contenidos matemáticos.

\section{Metodología}

Ahora bien, dentro de este marco referencial, es que nos planteamos una necesidad inmediata, que es la transposición de estas ideas a la currícula de la enseñanza teniendo en cuenta distintos factores como los avances teóricos metodológicos de la Estadística en los últimos 50 años, especialmente dada por la irrupción del procesamiento automático de datos, la informatización de la sociedad misma y los avances metodológicos en otras disciplinas que "redescubren" en la estadística las implicaciones de su aplicación para la obtención de información del mundo real con distintos propósitos. Todas estas consideraciones confluyen para crear un escenario favorable, desde el punto de vista estadístico, para la enseñanza y aprendizaje de esta disciplina, y a su vez, dentro de este marco referencial de intervención en la toma de decisiones y de la relación entre la alfabetización estadística y la alfabetización científica.

Es así que el taller se centrará en la discusión de algunos conceptos fundamentales de la Alfabetización Estadística, las diversas formas de introducir dichos conceptos utilizando herramientas informáticas como simuladores y software de distribución libre. Toda esta discusión estará atravesada metodológicamente por el trabajo a través del planteo y resolución de proyectos en los que se utilice la Estadística como metodología de trabajo para obtener conclusiones.

Aunque en el taller prevemos desarrollar otras actividades, en este trabajo nos centraremos en el análisis de dos actividades basadas en la utilización de simuladores que nos ayudarán a obtener respuestas a las preguntas planteadas. Las actividades mencionadas pueden servir de apoyo en cursos introductorios de Estadística a Nivel Universitario y también pueden utilizarse en cursos de Nivel Medio, para este caso, especialmente la actividad 1. 
"IV Encuentro sobre Didáctica de la Estadística, la Probabilidad y el Análisis de Datos"

\subsection{Actividades que permiten introducir ideas fundamentales de la Alfabetización Estadística \\ 4.1.1. Actividad 1: "Distribuciones asociadas al lanzamiento de un dado"2}

A partir del experimento aleatorio asociado con el lanzamiento de un dado equilibrado de 6 caras, realiza las siguientes actividades:

a. Escribe todos los resultados que podrían aparecer en un lanzamiento de un dado equilibrado.

b. Si consideramos que el dado está equilibrado, ¿qué posibilidades hay de que aparezca cada uno de los resultados enumerados antes? ¿Qué razonamiento has seguido para obtener esas posibilidades?

c. Elabora una tabla en la que presentes los resultados posibles y sus probabilidades asociadas.

d. Si tuvieras que representar gráficamente la información presentada en la tabla, ¿qué gráfico elegirías? ¿A través de qué elementos podés fundamentar tu elección?

e. Confecciona el gráfico seleccionado y describe verbalmente las características de la distribución representada.

f. A continuación analizaremos si las características encontradas en la distribución de probabilidades del lanzamiento de un dado que has construido antes, se pueden observar cuando efectivamente lanzamos un dado. Para ello utilizaremos como herramienta de apoyo el simulador denominado "Modelo de Cajas" que se puede abrir desde la página web: http://nlvm.usu.edu/, el cual presenta la apariencia mostrada en la pantalla que presentamos en la Figura 1. Selecciona los números 1 al 6 para colocar en la "caja" de la derecha, de tal forma que te aparezcan como en la Figura 1 y también selecciona la opción que aparece al pie: "Mostrar Probabilidad Teórica". Una vez cumplidos estos pasos, pincha en el botón que dice "Iniciar" y espera hasta que en el indicador de la Cantidad de Selecciones aparezcan 10 selecciones y pulsa el botón "Pausar". Con este procedimiento el programa habrá simulado 10 lanzamientos de un dado y en el gráfico que aparece debajo te mostrará la distribución de los valores que han aparecido.

g. Teniendo en cuenta el gráfico obtenido y los valores que aparecen en la caja intermedia (entre los valores seleccionados y el gráfico), construye una tabla de

2 Para realizar esta actividad utilizaremos como herramienta de apoyo el simulador denominado "Modelo de Cajas" que se puede abrir desde la página web: http://nlvm.usu.edu/, una vez ubicados en la página, podemos elegir el idioma deseado (se encuentra al pie de la página) y luego seleccionaremos el módulo de Análisis de Datos y Probabilidad del nivel 9-12 (corresponde al periodo de 2ㅇ a $5^{\circ}$ a ño de la Educación Secundaria). 
"IV Encuentro sobre Didáctica de la Estadística, la Probabilidad y el Análisis de Datos"

frecuencias. Elabora un breve informe con las características de la distribución de frecuencias.

h. Compara las conclusiones extraídas en el punto e y en el punto g, a partir de la distribución de probabilidad y de la distribución de frecuencias respectivamente. ¿Puedes encontrar alguna similitud entre ambas distribuciones? ¿Encontraste diferencias? ¿Cuáles?

i. Ahora utiliza el botón de selección rápida y repite el proceso de los puntos f, g y h, para 50, 100 y 1000 lanzamientos de un dado. Describe verbalmente qué tendencias puedes observar a medida que se aumenta la cantidad de lanzamientos.

Figura 1. Simulador "Modelo de Cajas"

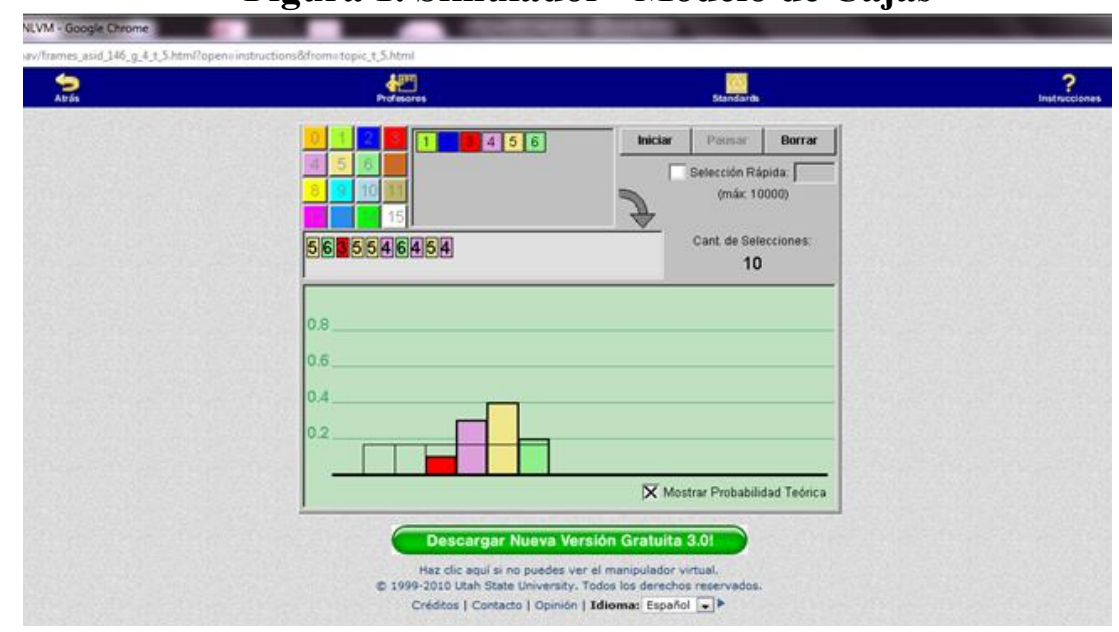

\subsubsection{Objetivos de la Actividad 1}

Algunos de los objetivos que se pretenden lograr a partir del desarrollo de la actividad 1 son:

$\checkmark$ Definir espacios muestrales para experimentos aleatorios con eventos equiprobables

$\checkmark$ Obtener probabilidades clásicas y empíricas a partir de la realización virtual del experimento.

$\checkmark$ Construir la distribución de probabilidad teórica del experimento

$\checkmark$ Construir la distribución de frecuencias empíricas a partir de la simulación del experimento aleatorio

$\checkmark$ Distinguir entre valores teóricos y empíricos

$\checkmark$ Introducir intuitivamente la Ley de los Grandes Números

\subsubsection{Contenidos de la Actividad 1}

A partir de esta actividad se pueden desarrollar los siguientes contenidos o conceptos estocásticos:

$\checkmark$ Distribución de probabilidad para eventos equiprobables.

$\checkmark$ Distribución de Frecuencias empíricas

$\checkmark$ Aproximación del modelo teórico a la distribución de frecuencias empírica 
"IV Encuentro sobre Didáctica de la Estadística, la Probabilidad y el Análisis de Datos"

$\checkmark$ Ley de los Grandes Números y estabilidad de las frecuencias

Los cuales estarán íntimamente relacionados con conceptos previos como por ejemplo:

$\checkmark$ Cálculo de probabilidades para espacios muestrales finitos

$\checkmark$ Definición de espacio muestral y de eventos simples

$\checkmark$ Concepto de frecuencias absolutas y relativas

\subsubsection{Extensión de la Actividad 1}

Esta actividad puede extenderse, considerando el espacio muestral que se obtiene al lanzar dos dados y definiendo la variable aleatoria: "Puntos obtenidos al lanzar dos veces un dado". En este caso, el simulador puede utilizarse de una manera similar a la planteada antes, sólo que se deberán seleccionar los valores correspondientes a la suma de puntos y considerar la distribución de probabilidad asociada ya que los eventos posibles no son equiprobables.

4.2.1. Actividad 2. "Generación de distribuciones muestrales"3

a. Ingresa en la página: http://onlinestatbook.com/stat_sim/sampling_dist/index.html, una vez ubicado en ella, pincha en el botón que indica: "Begin", te aparecerá una ventana como la que mostramos en la Figura 2. Por defecto aparecerá la distribución de una población sobre la cual trabajaremos a continuación. Analiza y describe las características estadísticas de la distribución presentada por defecto.

b. En el gráfico inmediatamente inferior, pincha en el botón que dice: "Animated", de esta manera se simulará una muestra de cinco elementos extraídos aleatoriamente de la población inicial. Construye la tabla correspondiente a los valores obtenidos en el gráfico (puedes hacerla en una hoja, o directamente cargarla en una hoja de cálculo de GeoGebra). La distribución presentada en este gráfico, ¿tiene alguna característica similar a la de la distribución poblacional?

c. Para cada muestra seleccionada, en el tercer gráfico, se presentará el valor de la media aritmética de la muestra correspondiente. Registra en otra tabla (o en otra columna de la hoja de cálculo) el valor obtenido.

d. Repite 10 veces el proceso indicado en los ítems b y c. Ahora considera la distribución del tercer gráfico, describe si encuentras alguna característica similar a la distribución poblacional o a las distribuciones de algunas de las muestras seleccionadas.

e. Sin borrar los valores que ya han sido seleccionados, ahora pincha en el botón que indica: "1000" ubicado en el segundo gráfico. En este caso, obtendrás los valores correspondientes a 1000 muestras de 5 elementos seleccionados aleatoriamente de la población (en otras palabras, estás repitiendo 1000 veces el proceso del punto b pero de una sola vez), y en el segundo gráfico no se representará nada porque allí sólo se representa cada una de las muestras por separado. Observa el tercer gráfico y describe

\footnotetext{
${ }^{3}$ Esta actividad utilizará como apoyo el applet "Sampling Distributions" que se puede abrir desde la página: http://onlinestatbook.com/stat sim/sampling dist/index.html
} 
"IV Encuentro sobre Didáctica de la Estadística, la Probabilidad y el Análisis de Datos"

nuevamente las características que presenta esta distribución. ¿Se puede determinar alguna característica similar a la población? ¿Cuál o cuáles? ¿qué diferencias observas entre ambas distribuciones?

f. ¿Cuál o cuáles piensas que son los motivos para encontrar esas similitudes y esas diferencias?

g. ¿Qué conclusiones podríamos sacar después de repetir el proceso de muestreo varias veces?

Figura 2. Simulador para generar distribuciones muestrales

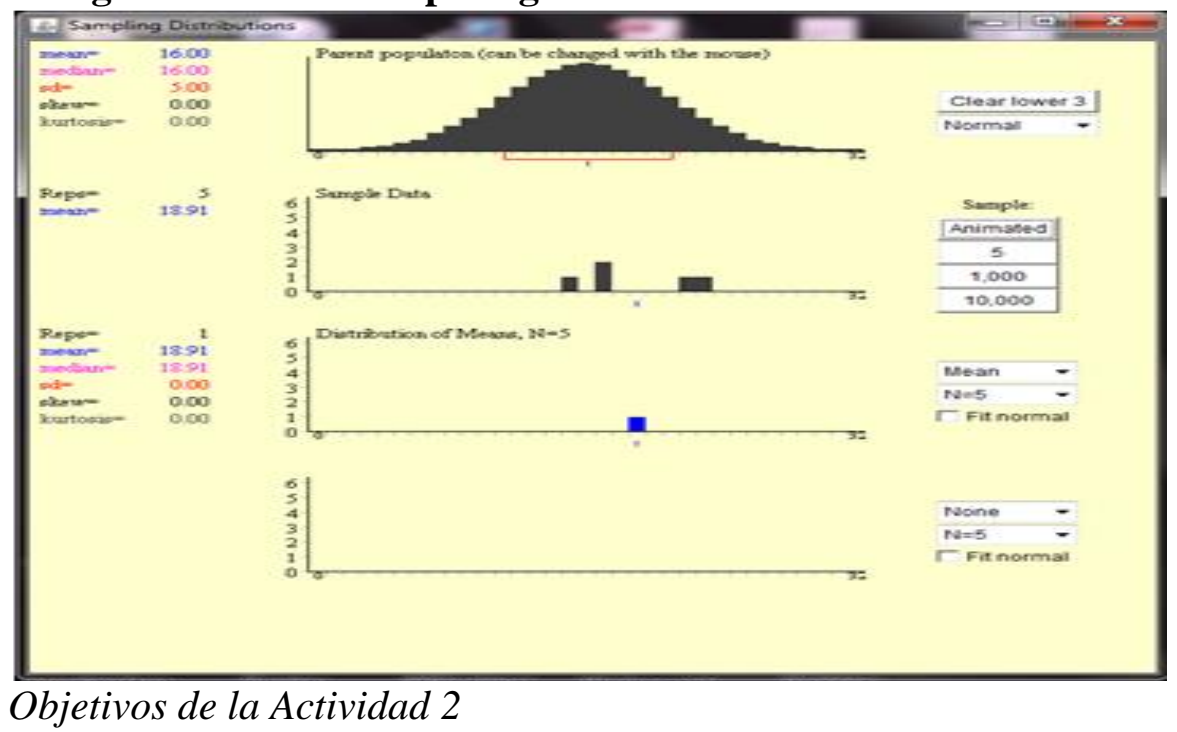

Algunos de los objetivos que se pretenden lograr a partir del desarrollo de la actividad 2 son:

$\checkmark$ Construir distribuciones de frecuencias asociadas a muestras de distintos tamaños.

$\checkmark$ Construir distribuciones muestrales de determinados estadísticos

$\checkmark$ Distinguir entre parámetros, estadísticos y estimadores

$\checkmark$ Introducir el concepto de error estándar de una distribución muestral

$\checkmark$ Valorar los fundamentos de la utilización de estadísticos para estimar parámetros

\subsubsection{Contenidos de la Actividad 2}

A partir de esta actividad se pueden desarrollar los siguientes contenidos o conceptos estocásticos:

$\checkmark$ Modelos de distribuciones de probabilidad.

$\checkmark$ Parámetros, estadísticos y estimadores.

$\checkmark$ Distribución de Frecuencias empíricas

$\checkmark$ Distribución Muestral de un estadístico

$\checkmark$ Aproximación del modelo teórico a la distribución de frecuencias empírica

$\checkmark$ Análisis de la bondad de ajuste del modelo teórico a partir de la aproximación de los gráficos 
"IV Encuentro sobre Didáctica de la Estadística, la Probabilidad y el Análisis de Datos"

$\checkmark$ Propiedades de la distribución muestral de un estadístico

$\checkmark$ Propiedades de los estimadores

$\checkmark$ Teorema Central del Límite

Los cuales estarán íntimamente relacionados con conceptos previos como por ejemplo:

$\checkmark$ Variable aleatoria

$\checkmark$ Variable estadística

$\checkmark$ Concepto de frecuencias absolutas y relativas

$\checkmark$ Medidas de tendencia central

$\checkmark$ Medidas de dispersión

$\checkmark$ Distribución de probabilidad Normal

\subsubsection{Extensión de la Actividad 2}

Aunque existen diversas maneras en las que podríamos extender esta actividad a partir del uso del simulador, en este caso proponemos una de entre todas las posibles. Como extensión de esta actividad puede resultar muy enriquecedor al realizar cada muestreo, utilizar el cuarto gráfico para generar la distribución muestral de otro estadístico, por ejemplo, de la Mediana. De esta forma se puede comparar la distribución muestral de la media y de la mediana, sus centros y dispersiones y de allí, obtener conclusiones sobre la bondad de cada estimador.

\section{Implicaciones para la enseñanza}

En esta oportunidad hemos presentado dos actividades que pueden trabajarse con el mismo carácter que un proyecto, en el sentido que los alumnos pueden interactuar entre sí y con el docente, estableciendo debates sobre el análisis que van realizando sobre las situaciones planteadas. Hemos desarrollado otros tipos de actividades (presentadas en otros trabajos, por ejemplo, Tauber, 2007) en los que se plantean proyectos basados en datos reales obtenidos a partir de bases de datos de organismos oficiales como la ONU, IPEC, etc., pero en esta ocasión pretendemos analizar las bondades del uso de applets educativos para introducir en la clase de estadística.

Como podemos observar, se intenta relacionar dos maneras de resolver las cuestiones planteadas: utilizando elementos clásicos como puede ser el papel y el lápiz y también utilizando herramientas informáticas que favorecerán el análisis solicitado en cada oportunidad.

A partir de estas actividades, podemos contribuir a la adquisición de competencias básicas, las cuales enumeramos a continuación:

Competencia en comunicación lingüística. Durante el desarrollo de cada actividad, los alumnos tienen oportunidad de ejercitarse en la construcción y comunicación del conocimiento y en la organización y autorregulación del pensamiento. Además, adquieren destrezas y actitudes (en el sentido usado por Gal, 2004) como puede ser formar un juicio crítico, generar ideas y disfrutar expresándose tanto de forma oral (exponiendo las conclusiones obtenidas a sus compañeros) como de forma escrita (redactando el informe solicitado). 
"IV Encuentro sobre Didáctica de la Estadística, la Probabilidad y el Análisis de Datos"

Competencia matemática. Puesto que en ambas actividades deben utilizar y relacionar números enteros, fraccionarios y decimales, los alumnos deberán aplicar operaciones básicas, símbolos, formas de expresión y razonamiento matemático. Utilizan proporciones y también ponen en práctica procesos de reflexión que llevan a la solución de los problemas o a la obtención de información, por medio del reconocimiento de las técnicas apropiadas. En las situaciones planteadas, los alumnos tendrán la oportunidad de integrar el conocimiento matemático con el conocimiento estadístico, por ejemplo, cuando obtienen la distribución de probabilidad del lanzamiento de un dado, están relacionando distintos campos numéricos con el concepto de probabilidad y de distribución de probabilidad. Por otro lado, cuando construyen gráficamente la distribución de probabilidad, tienen la oportunidad de apreciar que los ejes cartesianos se pueden utilizar en contextos diferentes, como puede ser justamente la construcción de una distribución de probabilidad si trabajamos con un concepto estocástico o de una función lineal, si trabajáramos específicamente en un modelo matemático o en la representación de la velocidad de un móvil si estamos representando una situación determinada en Física.

Competencia en el conocimiento y la interacción con el mundo físico. El trabajo propuesto a partir de estas actividades también permitirá identificar preguntas, elaborar conjeturas como por ejemplo en el Actividad 2, una conjetura que podría plantearse es que, si se toman muestras aleatorias independientes del mismo tamaño de una población con distribución normal y con determinadas media y varianza, entonces la distribución muestral de medias se aproximará a una distribución normal que tendrá la misma media de la población pero con una mayor concentración alrededor de la media que la distribución poblacional, lo cual corresponde al concepto de error estándar de la distribución de medias muestrales. Por supuesto, la conjetura que puede realizarse a partir del trabajo con la simulación, al comienzo puede ser de carácter intuitivo, justamente el trabajo en el aula y a través del tiempo llevará a formalizar estos resultados a través de los teoremas correspondientes. Por supuesto, este proceso podría llevar algunos años a través de la enseñanza hasta llegar a la definición de los teoremas. Otra cuestión que se genera a partir de estas actividades es que permitirán que el alumno obtenga conclusiones basadas en la evidencia que proporciona la simulación, lo cual le permitirá comprender el proceso de la inferencia y tomar decisiones. Asimismo, se procura una habilidad progresiva para poner en práctica los procesos y actitudes propios del análisis sistemático de una tarea y de la indagación científica, ya que podemos concebir estas actividades como pequeñas investigaciones cuyo objetivo en ambas actividades es encontrar los fundamentos de propiedades teóricas.

Tratamiento de la información y competencia digital. Cuando se propone a los alumnos que recojan la información correspondiente a través de las tablas de distribución o de los gráficos, estamos promoviendo que el alumno se enfrente a procesos de "recogida de datos" y "organización, análisis e interpretación de los datos". De esta manera permitimos que los alumnos se habitúen a buscar, obtener y procesar información para transformarla en conocimiento. En estos casos estamos contribuyendo al aprendizaje del uso de computadoras y de distintos tipos de software y a adquirir destrezas de razonamiento para organizar la información, relacionarla, analizarla, sintetizarla y hacer inferencias y deducciones de distinto nivel de complejidad.

Competencia social y ciudadana. A partir del trabajo propuesto se pueden adquirir conocimientos diversos y habilidades complejas que permitirán al alumno participar, tomar decisiones y responsabilizarse de las elecciones y conclusiones adoptadas. Además, permite 
"IV Encuentro sobre Didáctica de la Estadística, la Probabilidad y el Análisis de Datos"

concientizar de la importancia de la estadística en la sociedad actual, implicándose a través de procesos estadísticos. (Debemos aclarar que proponemos que estas actividades se realicen en grupos de 2 o 3 personas, dado que este tipo de trabajo fomenta la cooperación y la valoración del trabajo de los demás). Finalmente, ayuda a tener una actitud crítica y reflexiva en la valoración de la información disponible, contrastándola cuando es necesario, y respetando las normas de conducta acordadas socialmente.

Competencia para aprender a aprender. Se ejercita la curiosidad de plantearse preguntas, identificar y manejar las diversas técnicas y estrategias con las que afrontar una misma situación problemática y afrontar la toma de decisiones con la información de la que se dispone. Se ejercitan habilidades para obtener información y para transformar dicha información en conocimientos propios y relacionarla con conocimientos previos. Autonomía e iniciativa personal. Una de las virtudes (especialmente de la actividad 2) es que brinda una buena gama de posibilidades de apertura de la propia actividad, ya que podría plantearse de manera flexible $\mathrm{y}$, una vez descubiertas las primeras regularidades, permite analizar distribuciones muestrales de otros estadísticos y obtener conclusiones en función de ellos. Esto permite que los mismos alumnos puedan utilizar otros criterios de análisis, ejercitar su imaginación y llevar adelante las acciones necesarias para encontrar otros tipos de regularidades y, con el tiempo, lograr probar otras conjeturas. Otra virtud de las actividades planteadas es que permiten que el estudiante no dependa tanto del profesor, dado que tiene libertad para elegir las estrategias a seguir para llegar a sus conclusiones. Es por todo lo expuesto que consideramos un factor de gran relevancia en la enseñanza de la estadística, la utilización de software de distribución libre o de simulaciones que posibilitan la comprensión intuitiva de conceptos estocásticos. Esto se hace más relevante aún, considerando la disponibilidad que actualmente tienen los alumnos de secundaria a través del uso de las netbooks entregadas por los distintos organismos gubernamentales.

\section{Reflexiones Finales}

En este trabajo hemos descrito las características de dos actividades propuestas para el aula de estadística para el Nivel Medio y el Nivel Universitario (en un sentido introductorio en este último) basada en la utilización de software didáctico. También, hemos mostrado algunas de las virtudes de este tipo de trabajo, pero no debemos dejar de decir que el planteo de este tipo de propuestas debe ser siempre muy bien pensada y elaborada por el docente de acuerdo a sus propios de intereses de enseñanza y también a los intereses en relación con el aprendizaje que se pretende lograr en los alumnos.

En este sentido, es importante que el docente pueda comprometerse con las tareas planteadas, que es justamente uno de los defectos cuando se utilizan actividades extraídas de libros de texto, no sólo porque el docente no ha estado comprometido en la elaboración de esa tarea sino porque generalmente, los autores de libros de texto deben seguir ciertas normas y presentar cuestiones que no están pensadas para cada grupo en particular. Es por ello que fomentamos la idea de elaborar nuestras propias actividades en función del interés de los grupos con los que trabajamos y además, propiciamos la idea de utilizar las herramientas que están disponibles en internet y que pueden ayudarnos a proponer nuestros propios objetivos de enseñanza y aprendizaje. Lo único que puede limitarnos es nuestra propia imaginación. 
"IV Encuentro sobre Didáctica de la Estadística, la Probabilidad y el Análisis de Datos"

Debemos aprovechar además los resultados de estudios e investigaciones relacionados con la Educación Matemática y con la Educación Estadística, los cuales también nos aportarán ideas que pueden ayudarnos en la planificación de nuestras clases y de las actividades que propondremos a nuestros alumnos.

\section{Referencias Bibliográficas}

[1] Aliaga, M.; Cuff, C.; Garfield, J; Lock, R.; Utts, J. y Witmer, J. GAISE College Report. American Statistical Association. 2010. http://www.amstat.org/education/gaise/

[2] Anderson, C. W. y Loynes, R. M. The teaching of practical statistics. New York: Wiley. 1987.

[3] Batanero, C. Didáctica de la Estadística. Granada: Grupo de Investigación en Educación Estadística. 2001.

[4] Batanero, C. Retos para la formación estadística de los profesores. II Encontro de Probabilidade e Estatística na Scola. Universidade do Minho, 2009, Braga, Portugal.

[5] Batanero, C. y Díaz, C. Estadística con Proyectos. Facultad de Ciencias. Universidad de Granada. 2011.

[6] Ben-Zvi, D. y Garfield, J. Statistical Literacy, Reasoning and Thinking: goals, definitions and challenges. En: D. Ben-Zvi y J. Garfield (eds.), The challenge of developing statistical literacy, reasoning and thinking, pp. 3-15, 2004.

[7] Bruner, J. S. The process of education. Cambridge, MA: Harvard University Press. 1960.

[8] Cravero, M.; Redondo, Y.; Santellán, S. y Tauber, L. Relaciones entre Alfabetización Científica y Alfabetización Estadística. En: Actas de la III Reunión Pampeana de Educación Matemática. La Pampa. Argentina. 2010.

[9] Gal, I. Statistical Literacy: meanings, components, responsibilities. En: D. Ben-Zvi y J. Garfield (eds.), The challenge of developing statistical literacy, reasoning and thinking, pp. $47-78,2004$.

[10] Garfield, J.; Delmas, B. y Chance, B. The Web based ARTIST: Assesment Resource for improving Statistical Thinking. En: Assesmenton Statistical Reasoning to EnhanceEducational Quality of AERA Annual Meating, Chicago. 2003.

[11] Gil Pérez, D. y Vilches, A. Educación ciudadana y alfabetización científica: mitos y realidades. En: Revista Iberoamericana de Educación, № 42, pp. 31-54, 2006.

[12] Goetz, S. Fundamental ideas and basic beliefs in Stochastics. Theoretical Aspects and Empirical Impressions from the Education of Student Teachers. 2008. Disponible en: http://fplfachdidaktik.univie.ac.at/fileadmin/contributiongoetzrevised.pdf

[13] Graham, A. Statistical investigations in the secondary school. Cambridge: The Open University Centre for Mathematics Education. 1987.

[14] Holmes, P. Assessing project work by external examiners. En I. Gal y J. B: Garfield (Eds.), The assesment challenge in statistics education (pp. 153-164). Voorburg: IOS Press. 1997. 
"IV Encuentro sobre Didáctica de la Estadística, la Probabilidad y el Análisis de Datos"

[15] Kemp, A.C. Implications of diverse meanings for "scientific literacy". En: P.A. Rubba, J.A. Rye, W.J. Di Biase y B.A. Crawford (eds.): Proceedings of the 2002 Annual International Conference of the Association for the Education of Teachers in Science, pp. 1202- 1229, 2002, Pensacola, F.L.

[16] Meyer, R. El razonamiento inferencial estadístico como metodología y la formación de formadores en educación. Tesis Doctoral. Universidad Católica de Santa Fe. 2006.

[17] N.C.T.M. Principles and standards for school mathematics. Reston, VA; N.C.T.M. 2000.

[18] Schield, M. Three Kinds of Statistical Literacy: What should we teach? Proceedings of the Sixth International Conference on Teaching Statistics. 2002. Ed B. Phillips. Cape Town: International Statistical Institute and International Association for Statistical Education. Disponible en: http://www.stat.auckland.ac.nz/ iase.

[19] Schield, M. Statistical literacy survey analysis: reading graphs and tables of rates percentages. Proceedings of the Seventh International Conference on Teaching Statistics. 2006. Ed B. Phillips. Cape Town: International Statistical Institute and International Association for Statistical Education.

[20] Tauber, L. Meaning of stochastic concepts for mathematics students. Proceedings of the Seventh International Conference on Teaching Statistics. 2007. Ed A. Rossman y B. Chance. Salvador: International Statistical Institute and International Association for Statistical Education. Disponible en: http://www.stat.auckland.ac.nz/ iase.

[21] Tauber, L. Análisis de elementos básicos de alfabetización estadística en tareas de interpretación de gráficos y tablas descriptivas. Ciencias Económicas. Revista de la Facultad de Ciencias Económicas de la UNL. Año 8, 01, 53 - 67, 2010.

[22] Wallman, K. Enhancing Statistical Literacy: Enriching Our Society. En: Journal of the American Statistical Association, Vol 88, No 421, 1993.

\section{Referencias de applets:}

National Library of Virtual Manipulatives - Utah State University - Simulador "Modelo de Cajas": http://nlvm.usu.edu/

Simulador sobre Distribuciones Muestrales:

http://onlinestatbook.com/stat_sim/sampling_dist/index.html 


\title{
Un acercamiento a las pruebas de Hipótesis
}

\author{
José Juan Castro Alva ${ }^{1}$, Hortensia Reyes Cervantes ${ }^{2}$, Félix Almendra Arao ${ }^{3}$, Francisco \\ Tajonar Sanabria ${ }^{4}$, Hugo Cruz Juárez ${ }^{5}$
}

\begin{abstract}
Resumen
Tener una muestra representativa de la población de interés que sea aleatoria, independiente e idénticamente distribuida con una ley conocida, es importante en la estadística. La independencia es un factor clave para usar los conceptos teóricos de la probabilidad. Esto fortalece probabilísticamente a los datos para que el investigador pueda establecer aseveraciones acerca de uno o los parámetros de interés para tomar decisiones adecuadas que tengan validez estadística para toda la población. Y se supone en el taller que las observaciones tienen un comportamiento de tipo Bernoulli, Binomial o normal. Por medio de ejemplos se presentan las componentes que constituyen las pruebas de hipótesis.
\end{abstract}

Palabras clave: muestra, aleatoria, independiente, prueba de hipótesis.

\begin{abstract}
Having a representative sample of the population of interest is random, independent and identically distributed with a known law, is important in statistics. Independence is a key factor to use the theoretical concepts of probability. This strengthens the data probabilistically so that the researcher can establish claims about the parameters of interest to make suitable decisions that have statistical validity for the entire population. And it is assumed in the workshop, the observations have a behavior Bernoulli, Binomial or normal. Through examples, the components that make up the hypothesis tests are presented.
\end{abstract}

Keywords: sample, random, independence, hypothesis testing.

\footnotetext{
${ }^{1}$ Facultad de Ciencias Físico Matemáticas BUAP, México. jjcasatroa@gmail.com

${ }^{2}$ UPIITA del Instituto Politécnico Nacional, México. falmendra@ipn.mx

${ }^{3}$ Facultad de Ciencias Físico Matemáticas BUAP, México. hreyes@fcfm.buap.mx

${ }^{4}$ Facultad de Ciencias Físico Matemáticas BUAP, México. ftajonar@fcfm.buap.mx

${ }^{5}$ Facultad de Ciencias Físico Matemáticas BUAP, México. hcs@fcfm.buap.mx
} 


\section{Introducción}

El científico (médico, psicólogo, ingeniero, profesor, etc.) está interesado en resolver un problema, observa y analiza la naturaleza por un tiempo dado para encontrar relaciones que le parecen importantes usando sus teorías y experiencias. Plantea hipótesis y revisa los procedimientos en la literatura estadística que le permitan saber si es posible que ocurra o no con una cierta probabilidad. Esto muchas veces lo debe de repetir, bajo las mismas condiciones experimentales, para asegurarse de posibles errores y estar confiados de sus resultados. En la estadística siempre se necesitan muestras aleatorias independientes (m.a.i.), sin esta condición no se puede hacer inferencia.

Los científicos observan la naturaleza que los rodea plantean su conocimiento matemáticamente, eligen variables adecuadas para ser observadas, producen muestras y evalúan la información estadísticamente para tomar una decisión de rechazar o no rechazar la hipótesis con un error de equivocación para inferirla en toda una población.

Se presentan algunos conceptos básicos y sus componentes para comprender el concepto de pruebas de hipótesis.

\section{Objetivos}

- Comprender la importancia de tener una muestra aleatoria para tener resultados confiables.

- Identificar la variable aleatoria en el fenómeno de interés de tipo Bernoulli, Binomial y Normal.

- Identificar las componentes que se involucran en las pruebas de hipótesis.

\section{Desarrollo}

El uso de la probabilidad y estadística en forma adecuada nos permite tener un soporte matemático que apoya la modelación, predicción y explicación de muchos fenómenos de la vida real. Actualmente es la herramienta indispensable para tomar decisiones en la mayoría de las áreas del conocimiento que cuentan con datos.

Para introducirse en éste ámbito es importante describir en forma sencilla los siguientes términos útiles en la probabilidad y estadística. Ver Tabla 1.

Las hipótesis de investigación conducen al planteamiento de las hipótesis estadísticas y estas necesitan ser comprobadas mediante un método válido matemático (contraste de hipótesis).

Una prueba de hipótesis es una afirmación respecto a una característica de una población de interés. Cuando se contrasta una hipótesis, fijando un valor de equivocación, se comparan las predicciones que se deducen de ella con lo que se ha observado en la muestra: si coinciden dentro de un margen, la hipótesis es adecuada, sino la rechazamos. Cuando esto ocurre, nuevamente volvemos a plantear otra hipótesis que se acerque más a lo que observamos [3]. 


\begin{tabular}{|c|c|c|}
\hline Tipo & Descripción & Ejemplos \\
\hline Variable & $\begin{array}{l}\text { Es una característica que } \\
\text { toma valores distintos en } \\
\text { diferentes individuos }\end{array}$ & personas, animales, objetos \\
\hline Variable cuantitativa & $\begin{array}{l}\text { Es una característica que se } \\
\text { puede medir con un patrón } \\
\text { fijo }\end{array}$ & $\begin{array}{l}\text { Peso, } \\
\text { arterial, } \\
\text { viento. }\end{array}$ \\
\hline Variables cualitativas & $\begin{array}{l}\text { Estas variables no pueden } \\
\text { ser medidas con un } \\
\text { instrumento sólo se pueden } \\
\text { catalogar }\end{array}$ & $\begin{array}{l}\text { estado civil:, nivel } \\
\text { económico, belleza de una } \\
\text { persona. }\end{array}$ \\
\hline Variable aleatoria (V.a.) & $\begin{array}{l}\text { Los resultados son valores } \\
\text { fortuitos de un conjunto de } \\
\text { opciones, pero no se sabe } \\
\text { cuál ocurrirá cuando se lleve } \\
\text { a cabo el experimento }\end{array}$ & $\begin{array}{l}\text { Experimento: lanzar un } \\
\text { dado honesto } \\
\{1,2,3,4,5,6\} \text {. } \\
\text { Experimento: lanzar una } \\
\text { moneda, la cara puede ser de } \\
\{\text { águila, sol }\}=\{a, s\} .\end{array}$ \\
\hline V.a. continua & $\begin{array}{l}\text { Estas variables no dan saltos } \\
\text { o interrupciones entre ellas }\end{array}$ & $\begin{array}{l}\mathrm{X}_{1}=\text { peso de un hombre al } \\
\text { despertar, } 30<\mathrm{X}_{1}<100 \text {. } \\
\mathrm{X}=\text { tiempo que dura un foco } \\
\text { de una marca, } 0 \leq \mathrm{X}<\infty .\end{array}$ \\
\hline V.a. discreta & $\begin{array}{l}\text { Estas variables dan saltos o } \\
\text { interrupciones entre ellas }\end{array}$ & $\begin{array}{l}X_{1}=\{a, s\}, X_{2}=\{0,1\} . \\
X_{3}=\{1,2,3,4,5,6\} .\end{array}$ \\
\hline Muestra & $\begin{array}{l}\text { Es una parte de la población } \\
\text { elegida mediante un proceso } \\
\text { de selección aleatoria }\end{array}$ & $\mathrm{n}(n \leq N)$ \\
\hline Población & $\begin{array}{l}\text { Son todos los individuos que } \\
\text { cumplen una condición }\end{array}$ & $\mathrm{N}$ \\
\hline
\end{tabular}

Tabla 1. Algunos términos importantes de la estadística [5].

Una prueba de hipótesis consta de los siguientes puntos y se requieren en forma de aparición, [4]:

1. Los datos: El investigador debe de cuestionarse y revisar que los datos hayan sido realizados mediante un proceso aleatorio y comprender su naturaleza pues esto determina el tipo de procedimiento o análisis debe usarse.

Por ejemplo (Ejemplo 1) si son repeticiones independientes de un experimento Bernoulli $(p)$, con probabilidad de éxito $p$. Este tipo de experimentos se tienen cuando se lanza un dado o moneda honesta (que su estructura física no esté cargada hacia algún lado).

Otro ejemplo (Ejemplo 2) se tiene cuando un experimento Bernoulli ( $p$ ) se repite $n$ veces en forma independiente y después contamos el número de veces que ocurre una característica de interés: la aparición del número uno cuando se lanza un dado 20 veces o n puede interesar la cara del sol en la moneda, si la lanzamos 50 veces. 
Ejemplo 3. Cuando se tienen mediciones de pesos de personas o calificaciones de alumnos $(n>25)$, tenemos un experimento que tiene una variable de tipo continuo porque los valores que pueden tomar se encuentran en un intervalo que está contenido en los números reales. Los datos son normales

2. Las suposiciones: Las condiciones que se establecen en el experimento son importantes dentro de este procedimiento estadístico porque están relacionadas con las hipótesis que se establecen. Puede suceder que se especifique un valor concreto o un intervalo para un parámetro de la distribución de la variable.

Para el caso del Ejemplo 1, suponemos observaciones de tipo Bernoulli $\left(\mathrm{X}_{\mathrm{i}} \sim \operatorname{Ber}(p)\right.$ $i=1, \ldots, n)$ porque se realiza un procedimiento probabilístico que lo justifica.

3. Hipótesis estadística: En una prueba de hipótesis siempre se plantean dos hipótesis estadísticas que se proponen explícitamente: la hipótesis nula $\left(\mathrm{H}_{0}\right)$ y la hipótesis alternativa $\left(\mathrm{H}_{\mathrm{a}}\right)$. Estas hipótesis no deben tener elementos en común, por lo cual deben ser excluyentes sus conjuntos donde están definidos.

Una hipótesis alternativa es la hipótesis de prueba o también conocida como la hipótesis del investigador. La hipótesis nula es la negación de la hipótesis alternativa, es la hipótesis que se da por cierta.

Ejemplo 4. Supongamos que se quiere realizar un experimento de lanzar el dado 20 veces. Se establece el juego de hipótesis

$$
\mathrm{H}_{0} \text { : dado honesto vs } \mathrm{H}_{1} \text { : dado no honesto }
$$

Cuando se realiza el lanzamiento se cree que el dado es honesto a menos que el dado deshonesto pruebe lo contrario. Otra forma de escribirlo es

$$
\mathrm{H}_{0}: p=\frac{1}{6} \quad \text { vs } \quad \mathrm{H}_{1}: p \neq 1 / 6
$$

Ejemplo. Se va a reelegir el presidente de una compañía conocida.

$\mathrm{H}_{0}$ : el presidente no gana, gana el contrincante vs $\mathrm{H}_{1}$ : el presidente gana

$$
\mathrm{H}_{0}: p=\frac{1}{3} \quad \text { vs } \quad \mathrm{H}_{1}: p \neq 1 / 3
$$

4. Estadística de Prueba: Es una función de los valores muestrales que se utiliza para tomar una decisión [5]. Una muestra puede tomar diferentes valores y estos valores pueden tener diferentes valores en la estadística de prueba.

Para cualquier juego de hipótesis, se plantea un diagrama en donde se presentan las decisiones posibles que toma un investigador. Ver Cuadro 1. 


\begin{tabular}{|c|c|c|c|}
\hline & Situación real & y desconocida \\
\hline & & $\mathrm{H}_{0}$ es cierta & $\mathrm{H}_{0}$ no es cierta \\
\hline \multirow{2}{*}{$\begin{array}{l}\text { Decisión } \\
\text { tomada }\end{array}$} & Rechazar $\mathrm{H}_{0}$ & $\begin{array}{l}\text { Error tipo I } \\
\text { (Decisión mal } \\
\text { tomada) }\end{array}$ & $\begin{array}{l}\text { Decisión } \\
\text { correcta }\end{array}$ \\
\hline & $\begin{array}{l}\text { No } \\
\text { Rechazar } \mathrm{H}_{0}\end{array}$ & $\begin{array}{l}\text { Decisión } \\
\text { correcta }\end{array}$ & $\begin{array}{l}\text { Error tipo II } \\
\text { (Decisión mal } \\
\text { tomada) }\end{array}$ \\
\hline
\end{tabular}

Cuadro 1: Decisiones que se toman al plantear una prueba de hipótesis [1].

Las probabilidades de los errores que se cometen

$$
\begin{aligned}
& \alpha=P(\text { error tipo } I)=P\left(\text { rechazar } H_{0} \text { cuando es cierta }\right) \text { y } \\
& \beta=P(\text { error tipo } I I)=P\left(\text { no rechazar } H_{0} \text { cuando es falsa }\right)
\end{aligned}
$$

El investigador desea evaluar estadísticamente la evidencia que tiene (la muestra) para rechazar $\mathrm{H}_{0}$.

Denotemos por $\mathrm{R}=\{\mathrm{x} ; \mathrm{x}$ es una realización de $\mathbf{X}\}$, una prueba de hipótesis establece una partición de $\mathrm{R}$ en dos conjuntos donde $R=\mathbf{R}_{\mathrm{a}} \mathbf{U} \mathbf{R}_{\mathrm{r}}, \mathrm{R}_{\mathrm{a}}$ (región de aceptación) y $\mathrm{R}_{\mathrm{r}}$ (región de rechazo o región crítica).

- Para qué valores muestrales se toma la decisión de no rechazar $\mathrm{H}_{0}$.

- Para qué valores muestrales $\mathrm{H}_{0}$ es rechazada y $\mathrm{H}_{1}$ es no rechazada.

Toda prueba de hipótesis involucra un estadístico de prueba $\mathrm{T}(\mathbf{X})=\mathrm{T}\left(X_{1}, \ldots, X n\right)$ que depende de la muestra, también es una v.a. cuyo valor de la distribución de la muestra determinará la decisión de rechazar o no rechazar la hipótesis nula.

5.Distribución de la estadística de prueba. Usando resultados de probabilidad tenemos que:

Si las observaciones muestrales son de tipo Bernoulli (X $\sim \operatorname{Ber}(p)$ ), tendremos una distribución Binomial, para el caso de una prueba de hipótesis sobre el valor de $p$.

$$
X=\sum_{i=1}^{n} X_{i} \sim B \operatorname{in}(n, p)
$$

Si las observaciones muestrales son Normales, $\sigma^{2}$ conocido y $\mathrm{n}$ es grande, tendremos una distribución muestral Normal, para el caso de una prueba de hipótesis sobre el valor de la media $(\mu 0)$.

$$
\mathrm{Z}=\frac{(\bar{x}-\mu 0) \sqrt{n}}{\sigma} \sim N(0,1)
$$


Por ejemplo la distribución Bernoulli $(\mathrm{X} \sim \operatorname{Ber}(p))$ tiene un solo parámetro $(0<p<1)$ y es una distribución discreta, los experimentos que tienen dos resultados posibles. La variable aleatoria $\mathrm{X}$ puede tomar el valor de 0 o de 1 , donde $\mathrm{P}(\mathrm{X}=1)=p$ y $\mathrm{P}(\mathrm{X}=0)=1-p$. La probabilidad de éxito es $p$ y la probabilidad de fracaso es 1-p. La media y la varianza de una variable aleatoria Bernoulli son $E(X)=p$ y $\operatorname{Var}(X)=p(1-p)$, respectivamente. Cuando el experimento se repite $n$ veces (hay repeticiones independientes Bernoulli), la distribución de la v. a. se transforma y el número de éxitos es una Binomial $(\mathrm{X} \sim \operatorname{Bin}(\mathrm{n}, p)$ ), donde $\mathrm{X}$ tiene sus posibles valores de $0,1,2, . ., \mathrm{n}$. El número de éxitos en los $\mathrm{n}$ ensayos tiene una probabilidad de éxito de $p$, su medida central es $E(X)=n p$ y su varianza es $\operatorname{Var}(X)=n p(1$ p). En esta distribución $\theta=p$, sólo hay un parámetro.

Un método para estimar la proporción muestral de $\hat{p}$ (probabilidad de éxito), se realiza calculando a $\hat{p}=$ número de éxitos/número de ensayos $=\mathrm{X} / \mathrm{n}$.

Otra distribución muy conocida es la normal o la Gaussiana ( $X \sim N\left(\mu, \sigma^{2}\right)$ ) es conocida gracias al Teorema del límite central. La distribución tiene una v. a. que es continua, $-\infty<$ $\mathrm{X}<\infty$ con $-\infty<\mathrm{E}(\mathrm{X})=\mu<\infty \mathrm{y} \operatorname{Var}(\mathrm{X})=\sigma^{2} \geq 0$. La distribución es simétrica alrededor del cero, tiene colas que caen rápidamente y sus probabilidades están tabuladas en tablas de los libros de probabilidad y estadística. En esta distribución $\theta=\left(\mu, \sigma^{2}\right)$, hay dos parámetros, por lo cual tenemos un vector bivariado.

En los cursos de Probabilidad estos parámetros son fijos y conocidos, en los cursos de Estadística la suposición de independencia en una muestra es un término importante y determinante. También se ven algunas propiedades deseables de los estimadores, formas de compararlos, se exhiben métodos para estimar los parámetros y se presentan pruebas para analizar las muestras.

Supongamos que por algún motivo nos afirman que nuestras últimas observaciones aleatorias $\mathrm{X}_{1}, \ldots, \mathrm{Xn}$ (denotado por el vector $\mathbf{X}$ ) recolectadas tienen una distribución conocida, pero los parámetros que no son los adecuados. Por lo cual, debemos de realizar

6. Región de rechazo. Conjunto de valores que puede tomar la estadística de prueba y que dan evidencia de que la hipótesis de prueba es verdadera, son los valores de la estadística de prueba con los que se decide rechazar la hipótesis nula.

\section{Potencia de la Prueba [1]. Se define como}

$\delta(\mathrm{p})=1-\beta=\mathrm{P}\left(\right.$ rechazar $\mathrm{H}_{0}$ cuando el valor del parámetro es $\left.p\right)$

La función Potencia de una prueba de hipótesis con región de rechazo $\mathrm{R}_{\mathrm{r}}$ es una función que depende de $\theta$ y se define como $1-\beta(\theta)=P_{\theta}\left(X \in R_{r}\right)$. Una prueba de hipótesis adecuada

tiene una función Potencia cercana a uno para $\theta \in \Theta_{c}$ y cercana a cero cuando $\theta \in \Theta_{0}(\mathrm{el}$ subíndice significa que es un valor particular en la hipótesis nula ). En este contexto $\Theta_{0}$ representa un subconjunto no vacío del espacio de parámetros y $\Theta_{c}$ es un conjunto complementario a $\Theta_{0}, \Theta_{0} \cap \Theta_{c}=\varnothing$. 
Para $0 \leq \alpha \leq 1$, se define el tamaño de una muestra, como aquella prueba de hipótesis que cumple que $\sup _{\theta \mathbf{E} \theta_{\mathbf{e}}} \beta(\theta)=\alpha$. También una prueba tiene nivel $\alpha$ de significancia de la prueba, si cumple que $\sup _{\theta \mathbf{\epsilon} \theta_{\mathbf{0}}} \beta(\theta) \leq \alpha$.

A pesar que ambos errores son importantes, no es posible minimizarlos a la vez debido a que cuando el error tipo I se hace pequeño, el error Tipo II se hace grande y si se hace el error tipo II chico el otro aumenta (ver [1]), por lo cual se fijará el valor máximo que se está dispuesto a tolerar en el error tipo 1 y de las pruebas que cumplen la condición, se elegirá la prueba que tenga menor error tipo II.

Ejemplos de pruebas de hipótesis con una ley de probabilidad conocida.

Caso i) Pruebas de hipótesis para una distribución Binomial con n pequeña [2].

Esta distribución surge cuando hay $\mathrm{n}$ repeticiones independientes Bernoulli con probabilidad de éxito $p$. La v. a. $X=\sum_{i=1}^{n} X_{i} \sim B \operatorname{in}(n, p)$, si $p_{0} \in(0,1)$ aquí se pueden plantear los siguientes juegos de hipótesis.

a) $H_{0}: p \leq p_{0}$ en contra de (v.s.) $H_{1}: p>p_{0}$ donde su regla de decisión es Rechazar $H_{0}$ si $X \geq c$ donde $P\left(X \geq c\right.$ si $\left.p=p_{0}\right) \leq \alpha$, es una prueba de Cola derecha y es compuesta contra compuesta.

b) $H_{0}: p \geq p_{0}$ en contra de (v.s.) $H_{1}: p<p_{0}$ donde su regla de decisión es Rechazar $H_{0}$ si $X \leq c$ donde $P\left(X \leq c\right.$ si $\left.p=p_{0}\right) \leq \alpha$, es una prueba de Cola izquierda y es compuesta contra compuesta.

IV Encuentro sobre Didáctica de la Estadística, la probabilidad y el Análisis de Datos

c) $H_{0}: p=p_{0}$ en contra de (v.s.) $H_{1}: p \neq p_{0}$ donde su regla de decisión es Rechazar $H_{0}$ si $X \geq c_{1}$ si $p=p_{0}$ o Rechazar $H_{0}$ si $X \leq c_{\mathbf{2}}$ si $p=p_{0}$ donde $c_{\downarrow} 1$ y $c_{\downarrow} 2$ cumplen $P\left(X \geq c_{\downarrow} 1\right.$ si $\left.p=p_{1} 0\right)+P\left(X \leq c_{\downarrow} 2\right.$ si $\left.p=p_{\downarrow} 0\right) \leq \alpha$.

Caso ii). Pruebas de hipótesis para una distribución Binomial con n grande.

En este caso se usa la aproximación normal, el estadístico de prueba es, bajo $H_{\mathbf{a}}, Z_{\mathbf{0}}=\frac{\sum X_{i}-n p_{\mathbf{0}}}{\sqrt{n p_{\mathbf{0}}\left(1-p_{0}\right)}}$ 
Las reglas de decisión en a), b) y c)
a) Rechazar $H_{0}$ si $Z_{0} \geq z_{\alpha}$.
b) Rechazar $H_{0}$ si $Z_{0} \leq z_{\alpha}$.
c) Rechazar $H_{0}$ si $Z_{0} \geq z_{\frac{\alpha}{2}}^{o} \quad Z_{0} \leq-z_{\frac{\alpha}{2}}$ *

En el taller se exponen más ejemplos.

\section{Bibliografía}

[1] Infante Gil S., Pérez Zárate de Lara G., Métodos Estadísticos, Trillas, México, 1984.

[2] Navidi W., Estadística para ingenieros y científicos, Mc Graw Hill Interamericana, México, 2006. (ISBN 970-10-5629-9)

[3] Peña D. y Romo J., Introducción a la estadística para las ciencias sociales, Mc Graw Hill, 1999. (ISBN 844811617 8)

[4] Pérez Salvador B., Estadística para las ciencias sociales Vol. II, libros de texto y manuales de práctica, Universidad Autónoma Metropolitana, 1992. (ISBN 970-620-055-X)

[5] Wayne W. Daniel, Bioestadística, Limusa, 3ra. Edición, 1992. 


\title{
Aplicación de la Teoría de Modelos Multinivel Lineal y No-Lineal utilizando el software especializado HLM7.
}

\author{
Welman Rosa Alvarado ${ }^{1}$
}

\begin{abstract}
Resumen
Los modelos multinivel son básicamente un modelo de regresión de efectos mixtos, en donde se estudia una relación lineal entre dos o más variables en estudios realizados mediante un muestreo por agrupamiento, es decir, una técnica correlacional adecuada para analizar variaciones en las características de los sujetos que son miembros de un grupo que a su vez, hace parte de otra agrupación, o sea, mediciones que forman una estructura agrupada y jerárquica. El modelo permite la descomposición de la variación de una variable criterio (como por ejemplo, rendimiento) en sus componentes "dentro del grupo" (dentro-escuela, dentro-departamentos) y "entre grupo" (entre-escuela, entre-departamento) y el análisis de la asociación entre variables en esos niveles de agregación.
\end{abstract}

Palabras clave: multinivel, regresión jerárquica, niveles de agregación.

\begin{abstract}
Multilevel models are basically a regression model mixed effect, where a linear relationship between two or more variables in studies is studied by sampling for clustering, that is, an appropriate correlation technique to analyze variations in the characteristics of the subjects that are members of a group which, in turn, is part of another group, that is, measurements and forming a nested hierarchical structure. The model allows the decomposition of the variation of a criterion variable (eg, yield) components "in-group" (within-school, within-departments) and "between group" (between-school, between-department) and analysis of the association between variables at these levels of aggregation.
\end{abstract}

Keywords: multilevel, hierarchical regression, levels of aggregation.

${ }^{1}$ FEDECREDITO y UES, EL SALVADOR.welman_16@ hotmail.com 


\section{Introducción}

Los modelos multinivel (también conocidos como modelos jerárquicos) permiten establecer relaciones entre variables que se encuentran anidadas dentro de otras variables que les generan relaciones de dependencia y que requieren agruparse para estudiar tendencias y causalidades.

El ejemplo típico es el de analizar la relación entre nivel socioeconómico de los estudiantes y sus resultados en una prueba de selección para ingreso a una Universidad. Los estudiantes no son completamente independientes, ya que provienen de escuelas donde estaban formando parte de un grupo que les da ciertas características diferentes respecto a los estudiantes de otras escuelas. La correlación simple de nivel socioeconómico de los estudiantes vs calificaciones en la prueba, conduce a resultados diferentes si se agrupan y se hace el análisis de nivel socioeconómico de las escuelas vs calificaciones en la prueba.

El taller está estructurado de la siguiente manera:

En la primera parte se presentan los objetivos que se pretende alcanzar a partir de la aplicación de la teoría multinivel, describiendo brevemente la teoría de modelos multinivel lineal y no-lineal. Además, descripción de variables, técnica de análisis y la estrategia de análisis.

En la segunda parte, se analiza primero la descomposición de la varianza tanto del nivel individual como grupal a partir del modelo nulo o no condicional; segundo, se pretende responder a la pregunta ¿Cuál es el efecto que tienen las características pertenecientes a nivel individual y grupal respecto al constructo que se desea medir?

\section{Objetivos del Taller}

Abordar des un punto de vista teórico y aplicado el análisis de datos estructurados jerárquicamente mediante modelos de regresión jerárquica o multinivel. El taller consta de dos partes, la primera dedicada a la parte teórica de los modelos multinivel y la segunda, una presentación del software HLM7 en su versión educativa, el uso del programa con el caso de ejemplo e interpretación y análisis de resultados.

\section{Metodología de Aplicación Modelos Multinivel utilizando HLM.}

\section{Técnica de Análisis.}

Se utiliza la técnica de análisis estadístico por niveles múltiples. La cual es una técnica correlacional adecuada para analizar variaciones en las características de los individuos (Ej. Rendimiento académico) que son miembros de un grupo (Ej. Escuela), mediciones que forman parte de una estructura agrupada y anidada. La técnica permite la descomposición de la variación de una variable (Ej. Rendimiento) en sus componentes dentro de grupo y entre grupo, y el análisis de asociación entre variables en esos diferentes niveles de agregación. El modelo se compone de 
dos partes una aleatoria y otra fija, la primera muestra en cada nivel de agregación la estimación de la varianza de los parámetros determinados en la parte fija; la segunda con los parámetros que definen una línea promedio para todos los alumnos de todas las escuelas. Es importante resaltar que la inclusión de más variables en un modelo, permite estimar con mayor precisión el efecto de cada una de las variables, controlando por el efecto de las otras.

\section{Test Estadístico.}

Generalmente la técnica estadística que se utiliza para determinar si una variable es un factor asociado al resultado escolar de los estudiantes, es analizar el grado de asociación lineal, y para decidir si la asociación es estadísticamente significativa se usa el test de la razón de máxima verosimilitud. Trabajando con la hipótesis de nulidad de diferencia igual a cero, la diferencia entre los valores de máxima verosimilitud de dos modelos sigue la distribución de Chi-cuadrado, con grados de libertad igual al número de nuevos parámetros. Habitualmente para indicar el nivel de significación de cada estimación se usan como referencia el límite de probabilidad propuestos o utilizados por Fisher $(0.01,0.05,0.0025)$. En este trabajo se utilizará el límite de 0.05.

\section{Técnicas e instrumentos}

En esta etapa verificaremos la correspondencia entre la información contenida en el centro educativo y familia, con la información del estudiante perteneciente a ese centro educativo. El procedimiento a seguir se describe a continuación:

a) Realizar un análisis de regresión múltiple donde la variable dependiente será el factor o constructo a analizar sea rendimiento o repitencia del estudiante versus las variables contextuales, pertenecientes al entorno del centro escolar. El propósito de este análisis es conocer aquellas variables que afectan más al factor mencionado a través de un contraste estadístico de tal forma que aquellas variables que no sean estadísticamente significativas no serán consideradas en el modelo estudiantil.

b) Realizar análisis One-way: consiste en realizar un modelo multinivel donde se considera el promedio del rendimiento y repitencia del centro educativo más en error aleatorio para en n-estimó centro escolar. Construyendo de esta manera un modelo multinivel con una parte fija y otra aleatoria. El resultado será tres modelos multinivel one-way.

La estimación de los modelos nulos representa el punto de partida de todo análisis multinivel y presenta las siguientes formas funcionales y supuestos:

- Los modelos nulos no contienen ningún tipo de predictor (variable exógena), bien individual o grupal, excluyendo aquellas variables que pertenecen al nivel inferior o superior.

- El coeficiente intercepto equivale a la media global conformada por parte de la parte fija del modelo.

- Los términos de error del nivel escuela y del estudiante siguen una distribución normal con media igual a cero y varianzas iguales. 
- La varianza total es igual a la suma de las varianzas de $u_{0 j}$ y $e_{0 i j}$ y la importancia del grupo, la cual es la proporción de la varianza total atribuible a ese nivel $(\rho)$, aunque sin ningún control respecto del efecto de las variables de contexto.

\section{Temario a Impartir}

\section{PARTE 1:}

\section{Introducción a la Teoría de Modelos Multinivel.}

1. Antecedentes de los modelos multinivel

2. El modelo de ecuación estructural.

3. La naturaleza de los modelos multinivel.

4. Conjeturaciones previas al análisis multinivel.

5. Enfoques multinivel basado por análisis de regresión múltiple, ANOVA y ANCOVA.

6. El error de Medida.

7. El modelo con 2 niveles y notación básica.

8. Estimación de los parámetros para el modelo de componentes de varianza.

9. Estadística inferencial.

\section{PARTE 2:}

\section{Aplicación de Modelos Multinivel utilizando el software HLM7.0}

1. Objetivos de la aplicación.

2. Descripción de los datos.

3. Tipos de Variables.

4. Técnica de Análisis multinivel: Modelo Lineal y No-Lineal.

5. Test estadístico.

6. Estrategia de análisis: niveles de agregación.

7. Análisis e interpretación de Razones de Probabilidad en un Modelo No-Lineal.

8. Validación del modelo multinivel lineal y no-lineal. 


\section{Bibliografía}

[1] Browne, W.J. And J. Rasbash, Multilevel Modelling (1999). Institute of Education, University of London.

[2] Cervini, Rubén (2002). Desigualdades Socioculturales en el Aprendizaje de Matemática y Lengua de la Educación Secundaria en Argentina.

[3] Ferrão. Leite. Beltrão (2001). Introdução à modelagem multinível em Avaliação Educacional. Ministério do Planejamento, Orçamento e Gestão Instituto Brasileiro de Geografia e Estatística IBGE Escola Nacional de Ciências Estatísticas. Rio de Janeiro.

[4] Goldstein, Harvey. Rasbash. Yang. Geoffrey, Woodhouse (1993). Sally Thomas. A multilevel analysis of school examination results. Oxford review of education, vol 19, No. 4. Goldstein. Tutorial in biostatistics Multilevel modelling of medical data. Institute of Education; University of London; London; U.K. Goldstein, H. (1997). Methods in school effectiveness research. School effectiveness and school improvement.

[5] Gujarati, Damodar N. Econometría Básica. Tercera edición, United States Military Academy, West Point, 1997.

[6] Joop J. Hox, 2010. Multilevel Analysis. Techniques and Applications Quantitative Methodology Series. Second Edition.

[7] Montgomery. Peck. Vining (2002). Introducción al Análisis de Regresión Lineal. Primera edición Mexico.

[8] Naderiand J. Mace (2002). Education and Earnings; A Multilevel Analysis A Case Study of the Manufacturing Sector in Iran. Management and Planning Organisation, Tehran, Iran. University of London, London, U.K. 


\title{
Simulación de eventos probabilísticos con Excel
}

\author{
Kendall Eduardo Rodríguez Bustos ${ }^{1}$
}

\begin{abstract}
Resumen
En este taller se utilizará el software Excel para modelar experimentos estocásticos de la probabilidad desde un enfoque frecuencial. Los participantes trabajarán con actividades guiadas donde podrán apreciar la riqueza didáctica de la simulación en la resolución de problemas de variables aleatorias y así, comprender el concepto de la Ley de los Grandes Números en la teoría probabilística.
\end{abstract}

Palabras clave: Simulación, Probabilidad frecuencial, Aleatoriedad, Excel.

\begin{abstract}
In this workshop will be used the Excel software for modeling stochastic experiments of the probability from a frequential approach. Participants will work with guided activities where you will be able to appreciate the richness of the didactic simulation in the resolution of problems of random variables and, to understand the concept of the Law of Large Numbers in the probabilistic theory.
\end{abstract}

Keywords: Simulation, Frequential probability, Randomness, Excel.

${ }^{1}$ Tecnológico de Costa Rica, Costa Rica. kendall2412@gmail.com 


\title{
Introducción a la Construcción de Tablas y Gráficas
}

\author{
Gerardo Josué Cruz Márquez ${ }^{1}$
}

\begin{abstract}
Resumen
"Introducción a la Construcción de Tablas y Gráficas" es un taller educativo que tiene como objetivo ejemplificar a los docentes las ventajas de exponer a los estudiantes a experiencias de aprendizaje en las cuales manipulen objetos concretos y construyan en base a esa realidad tangible los conceptos y procedimientos matemáticos deseados, en este caso en el área de la estadística. Además es una exhortación a investigar y experimentar con metodologías innovadoras al momento de planificar actividades relacionadas a la introducción de nuevos conceptos y procedimientos matemáticos con el afán de desarrollar en los dicentes, más que memorización de unadefinición o un algoritmo, la construcción y comprensión de un concepto o un procedimiento matemático coherente, con significado y en relación con sus conocimientos previos.
\end{abstract}

Palabras clave: Didáctica, estadística, tablas y gráficos, primaria.

\begin{abstract}
"Introducción a la Construcción de Tablas y Gráficas" is an activity whose purpose is to exemplify for teachers the advantages of exposing students to learning experiences in which they manipulate concrete objects and on the basis of this tangible reality they can build mathematic concepts and procedures, in this case in the field of statistics. Moreover it constitutes a call for research and experimenting with innovative methodologies when planning activities related with the introduction of new mathematic concepts, with the ambition to develop the construction and understanding of a coherent, meaningful mathematical concept or procedure amongst the students which would go beyond mere memorization of a definition or algorithm.
\end{abstract}

Keywords: Didactic materials, statistics, charts and graphs, elementary education.

\section{¿Estadística?}

\section{Introducción}

Hoy en día, sin discusión alguna, la estadística es una de las partes de la matemática con mayor uso en la vida cotidiana y laboral. Gracias a ella el ciudadano común se informa del estado del tiempo para el resto de la semana y conoce las calles menos transitadas a distintas horas del día; el fanático del futbol puede seguir de cerca la cantidad de partidos jugados, perdidos, empatados, de goles a favor y de goles en contra de sus equipos favoritos de forma fácil y práctica; y el director de un colegio puede monitorear el índice de

\footnotetext{
${ }^{1}$ Universidad Pedagógica Nacional Francisco Morazán, Honduras.cruzmarquezg@gmail.com
} 
IV Encuentro sobre Didáctica de la Estadística, la Probabilidad y el Análisis de Datos

reprobación y de aprovechamiento de los estudiantes en los distintos espacios pedagógicos de la institución educativa.

El simple hecho de escuchar la palabra estadística trae a nuestra mente imágenes de tablas, gráficos y porcentajes de nacimientos, impuestos, poblaciones, deudas, ingresos, etcétera. Pero la estadística es mucho más que eso, estamos hablando de una ciencia antigua, casi tanto como la escritura misma, además es tan versátil que está inmersa desde simples eventos cotidianos como lanzar una moneda hasta en complejos procesos técnicos y científicos como el cálculo de la bolsa de valores.

Entonces, ¿qué es la estadística? El Diccionario de la Lengua Española en su duodécima segunda edición define el término estadística como la "rama de la matemática que utiliza grandes conjuntos de datos numéricos para obtener inferencias basadas en el cálculo de probabilidades". Esta definición resalta uno de los aspectos más importantes sobre la estadística: el carácter de ciencia matemática que esta posee.

Por su parte Ruiz (2004), haciendo énfasis en el fin que la estadística persigue y los procesosque conlleva, la define como la "ciencia que trata de la recopilación, organización, presentación, análisis e interpretación de datos numéricos con el fin de realizar una toma de decisión más efectiva”.

Mientras que Conde considera que la estadística "trata del recuento, ordenación y clasificación de los datos obtenidos por las observaciones, para poder hacer comparaciones y sacar conclusiones". En esta definición se especifica la manera en que la estadística se hace de los datos con los que trabaja: mediante la observación (medición o cálculo).

Considerando las aproximaciones anteriores podemos concluir que el término estadística hace referencia a la ciencia, rama de la matemática, que se encarga de la recolección, organización, análisis, representación e interpretación de datos con el fin de sintetizar una situación y hacer predicciones de eventos futuros.

\section{Educación Matemática}

Según la base de datos de la UNESCO, hasta el año 2010, la educación primaria en América Latina y El Caribe abarca el $94.24 \%$ de la población respectiva, sin embargo muestra que la tasa de repitencia en ese nivel es de $4.83 \%$ y la tasa de deserción estudiantil alcanza un $8.31 \%$. La educación secundaria nos presenta un panorama aún más alarmante al respecto, con solo un $72.16 \%$ de cobertura, una tasa de repitencia del $5.90 \%$ y una tasa de deserción estudiantil de $15.48 \%$.

Señalados por estos y otros factores como ser la revolución del conocimiento científico, la globalización, los gigantescos avances tecnológicos, el auge por la metodología y la didáctica en la enseñanza de las distintas áreas del conocimiento y principalmente el bajo rendimiento académico a todos los niveles de escolaridad, máxime en el área de matemáticas, es que como docentes nos preguntamos: ¿qué podemos hacer para mejorar la instrucción matemática y el rendimiento estudiantil en esta área? 
IV Encuentro sobre Didáctica de la Estadística, la Probabilidad y el Análisis de Datos

En su investigación respecto al rendimiento Benitez, Gimenez y Osicka (2000) ubican entre los principales factores del mismo "las metodologías de enseñanza utilizadas y la dificultad de emplear una enseñanza personalizada por parte de los docentes". En este punto la famosa frase del político, científico e inventor estadounidense Benjamin Franklin: "dime y lo olvido, enséñame y lo recuerdo, involúcrame y lo aprendo" ha cobrado vigencia.

Es así que hoy en día somos testigos de la inclusión de una gran variedad de recursos tecnológicosy de diversas técnicas didácticas dentro de los salones de clases. Además se ha vuelto común encontrar una gran variedad de novedosos materiales y recursos como tangrams, geoplanos, puzzles, varillas, troqueles, etcétera utilizados con fines didácticos todo esto con el objetivo de involucrar más a los estudiantes en el proceso de aprendizaje, desarrollar en ellos la compresión de los conceptos y procedimientos matemáticos y de esta manera poder llevarlos un paso adelante de la memorización y la aplicación de algoritmos. Bajo este nuevo enfoque se pretende que "el estudiante aprenda matemática haciendo matemáticas". (Torres, s.f.)

Puesto en evidencia la importancia que tiene el involucrar realmente a los estudiantes en el proceso de enseñanza-aprendizaje es que como docentes planteamos experiencias didácticas en las que los alumnos, principalmente los más pequeños, pueden manipular objetos concretos y que esto les ayude a identificar, conocer y apropiarse de los conceptos o procedimientos matemáticos que deseamos. Dentro de estas experiencias didácticas se destaca una en particular: los talleres educativos.

\section{Descripción de Actividades}

En el uso coloquial, la palabra taller hace referencia al lugar donde un grupo de personas construyen, reparan o crean algo. Así, por ejemplo, hablamos de talleres de mecánica, talleres de electrodomésticos, etcétera.

Considerando eso González (1987) define taller en un ámbito educativo como "un lugar donde varias personas trabajan cooperativamente para hacer o reparar algo, lugar donde se aprende haciendo junto con otros". Esa definición nos permite visualizar a groso modo las ventajas y la pertinencia de los talleres educativos en el aprendizaje significativo de los estudiantes.

Por su parte Gil (2010) al mencionar lo dicho por H. G. Wells considera que "el pensamiento estadístico será un día tan necesario para el ciudadano eficiente como la capacidad de leer o escribir". Actualmente eso no está lejos de nuestra realidad, ya que cuesta trabajo concebir a una persona que no esté expuesto al uso de tablas, gráficos o algún tipo de proceso estadístico en su labor profesional o en su vida cotidiana, debido a que estas están inmersas en todos los ámbitos posibles, desde los anuncios televisivos hasta las noticias en los medios de comunicación impresos.

Es por esto que el taller propuesto: Introducción a la Construcción de Tablas y Gráficas tiene como objetivo generalejemplificar las ventajas de exponer a los estudiantes a experiencias de construcción de tablas y gráficas mediante un proceso completo de recolección, clasificación, análisis, interpretación, representación de datos y exposición de resultados. 
IV Encuentro sobre Didáctica de la Estadística, la Probabilidad y el Análisis de Datos

Dicho taller está dividido en dos grandes secciones, la primera, es una simulación en la cual los participantes actuarán como estudiantes de secundaria. Esta se realiza con la intención de proporcionarles a los participantes una mejor perspectiva sobre el uso, las ventajas, desventajas, aspectos a mejorar y a considerar acerca de este tipo de actividades incluyentes en los salones de clase.

Esta simulación está dividida en dos partes la primera es una breve introducción teórica e histórica al concepto de estadística que tiene como objeto suplir los conocimientos previos que los estudiantes reales deberían tener y la segunda es la realizaciónde la actividad sugerida para estudiantes, esta se realizará con la ayuda de los facilitadores y siguiendo una gruía de trabajo pre-elaborada.

Durante la realización de esta actividad sugerida los participantes deberán recolectar, clasificar y representar datos mediante una tabla debidamente estructurada y generar gráficos básicos (de faja, de barras y circulares) en base a la misma. Todo esto haciendo énfasis en la comprensión de los conceptos y procedimientos matemáticos que involucra.

Al finalizar la simulación pasamos a la etapa de reflexión docente en la que los participantes podrán compartir acerca del trabajo realizado, las ventajas, desventajas, aspectos a mejorar y a considerar durante el desarrollo de este tipo de actividades con estudiantes reales, este es el paso nuclear en el cumplimiento de los objetivos del taller.

\section{Consideraciones finales}

La actividad sugerida para los estudiantes del taller educativo puede adecuarse al grado educativo y tema deseado, por ejemplo, podríamos aumentar la elaboración y el rigor científico de las construcciones realizadas o incluir elementos como el cálculo de medias, modas, etcétera procurando mantener el énfasis en la comprensión de los conceptos y los procedimientos matemáticos.

Como actividad docente este taller tiene como objetivo principal mostrar a los participantes las ventajas que tiene que los estudiantes conciban una estadística tangible, útil y cercana a ellos. Sustituir los problemas de situaciones desconocidas y vacías por situaciones concretas y relacionadas a su realidad cercana.

También pretende exhortar a los docentes a investigar y experimentar metodologías innovadoras al momento de desarrollar sus clases con la intención de despertar el interés y la motivación de los estudiantes en la clase, principalmente en el área de matemáticas en la cual estos son realmente escasos.

Finalmente, es importante tener presente el uso adecuado de recursos didácticos que nos permitan desempeñar nuestra labor docente de la mejor manera posible y cumplir a cabalidad nuestro objetivo profesional el cual es formar integralmente. 
IV Encuentro sobre Didáctica de la Estadística, la Probabilidad y el Análisis de Datos

\section{Bibliografía}

[1] Bellei, C. (UNESCO). "Situación Educativa de América Latina y el Caribe". Ediciones del Imbunche: Santiago, Chile. 2013.

[2] Benítez, M; Gimenez, M. y Osicka, R. [en línea]. "Las asignaturas pendientes y el rendimiento académico: ¿existe alguna relación?". (2000). En: http//fai.unne.edu.ar/links/LAS\%2...20EL\%20RENDIMIENTO\%20ACADEMICO.ht $\mathrm{m}$

(consultado el 1 de agosto, 2014)

[3] Conde, C. [en línea]. "Introducción a la estadística". CEPA. En:joseramoncj.files.wordpress.com/2009/03/estadistica.pdf (consultado el 1 de agosto, 2014)

[4] Diccionario de la lengua española. "Estadística". [En línea]. Madrid, España: Real Academia Española.

[5] Gil, A. "Proyectos de estadística en primaria". Revista Números, 2010, 75, p. 121-129, ISSN 1887-1984.

[6] González-Cuberes, M. "El Taller de los Talleres. Aportes al desarrollo de Talleres educativos". 1987.

[7] Ruiz, D. "Manual de estadística". Aumed: Sevilla, España. 2004.

[8] Torres, M. [en línea] "Papiroflexia y matemáticas". En: www.rinconmaestro.es/matematicas/geometria/geometria19.pdf (consultado el 28 de julio, 2014) 
IV Encuentro sobre Didáctica de la Estadística, la Probabilidad y el Análisis de Datos

\title{
Anexo 1: Información del taller
}

\author{
Facilitador: \\ Lic. Gerardo Josué Cruz Márquez \\ Participantes: \\ Profesores de séptimo grado
}

Tema:

Didáctica de la estadística / Propuestas de trabajo en la enseñanza de la estadística

\section{Propósitos:}

$>$ Ejemplificar las ventajas de exponer a los alumnos a experiencias de construcción de tablas y gráficas mediante un proceso completo de recolección, clasificación, análisis, interpretación, representación de datos y exposición de resultados.

$>$ Despertar en los docentes el interés sobre el uso de materiales y técnicas innovadoras en los salones de clases.

\section{Materiales (30 participantes):}

- 25 hojas de papel cuadrícula.

- 5 empaques de dulces M\&M’s (60 g)

- 35 platos desechables pequeños.

- 5 set de diez crayones.

- 5 marcadores negros.
- 10 hojas de papel blanco tamaño cartulina.

- 3 compases.

- 5 reglas plásticas.

- 5 tijeras.

- 1 cinta adhesiva

Cronograma de actividades:

\begin{tabular}{c|c|l}
$\begin{array}{c}\text { Tiempo } \\
(\mathrm{min})\end{array}$ & Actividad & \multicolumn{1}{c}{ Descripción } \\
\hline 5 & Inicio & $\begin{array}{l}\text { - Bienvenida } \\
\text { - Sugerencias generales para el desarrollo del taller }\end{array}$ \\
\hline
\end{tabular}

Etapa de Simulación

\begin{tabular}{|c|c|c|}
\hline 5 & Introducción & $\begin{array}{l}\text { - Presentación inicial } \\
\text { - Breve historia y definición de la estadística } \\
\text { - Formación de equipos de trabajo }\end{array}$ \\
\hline 15 & \multirow{4}{*}{$\begin{array}{l}\text { Actividad } \\
\text { sugerida }\end{array}$} & - Recolección de datos \\
\hline 10 & & - Clasificación de datos \\
\hline 15 & & - Análisis y representación de datos \\
\hline 15 & & - Exposición de resultados \\
\hline
\end{tabular}

Etapa de Reflexión Docente

\begin{tabular}{l|l|l}
\hline 25 & Reflexión
\end{tabular}

Valoración del trabajo realizado

Reflexión acerca del uso de esta técnica en los salones de clases, sus ventajas y desventajas. 


\section{Anexo 2: Guía de trabajo}

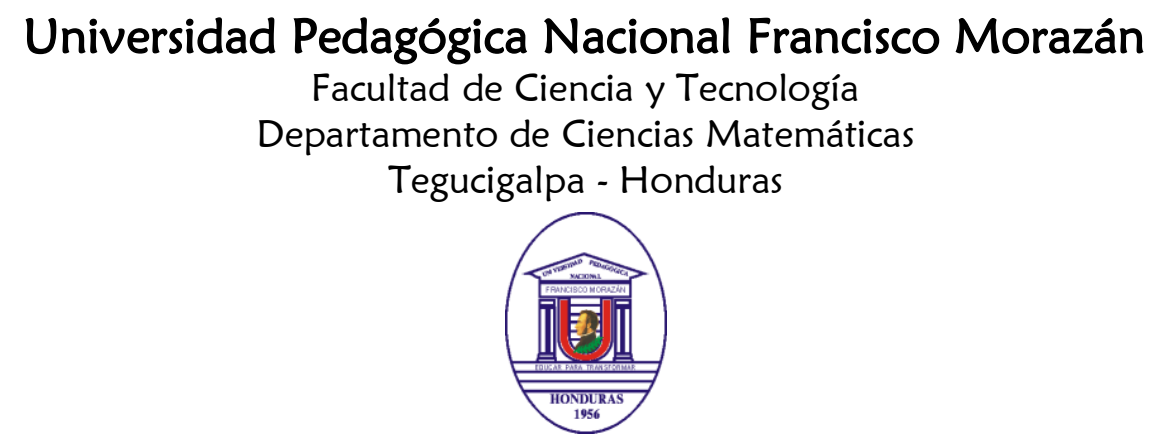

Taller: Introducción a la Construcción de Tablas y Gráficas Actividad Sugerida

Nombre del docente:

\section{Objetivos:}

1) Recolectar, clasificar y representar datos mediante una tabla estructurada.

2) Interpretar tablas de datos y generar gráficos básicos (de faja, de barras y circulares).

Instrucciones generales: Lea detenidamente cada una de las indicaciones y resuelva lo que a continuación se le pide, en caso de dudas consulte al facilitador.

Materiales por grupo:

- 3 hojas de papel cuadrícula.

- Empaques de dulces M\&M's (60 g)

- 2 hojas de papel blanco tamaño cartulina.

- 7 platos desechables pequeños.

- Set de diez crayones.

- Marcador color negro.

- Compás.

- Regla plástica.

- Tijera.

- Cinta adhesiva.

\section{Indicaciones:}

\section{Etapa 1: Recolección de datos}

1- Abrir el empaque de dulces y vaciarlo en uno de los platos plásticos.

2- Separar los dulces. Cada participante se encargará de separar un color de dulces del paquete y cada color de dulces debe quedar en un plato plástico distinto.

¿Cuántos colores de dulces distintos contiene el paquete?

¿Cuántos dulces contenía el paquete de dulces?

¿Cuántos dulces de cada color?

3- Comparar los resultados con los obtenidos por grupos cercanos. 


\section{Etapa 2: Clasificación de datos}

\section{Indicaciones:}

1- Representar los resultados obtenidos en la etapa anterior mediante una tabla que especifique los colores de los dulces, la cantidad de dulces de cada color y la cantidad total de dulces del empaque.

\section{¿De qué color de dulces hay más?}

\section{¿De qué color de dulces hay menos?}

\section{¿Hay colores de dulces con la misma cantidad?}

\section{Etapa 3: Análisis y representación de datos}

\section{Indicaciones:}

1- Cortar tiras de papel cuadrícula de diez cuadrados de alto y el mayor anchoque permita la página de papel cuadrícula.

2- Pegar las tiras recortadas por el altoa manera de generar un rectángulo de cuadrícula de diez cuadrados de alto y el mayor ancho posible.

3- Pintar dicho rectángulo tomando en cuenta la tabla creada anteriormente. Por cada dulce pintar trescolumnas de dicho color en la tira de cuadrícula. Cuando se culmine de representar todos los dulces de un color se comienza a pintar con el color siguiente hasta representar todos los colores encontrados. El resultado obtenido debe ser similar al mostrado en la figura 1.

Figura 1:

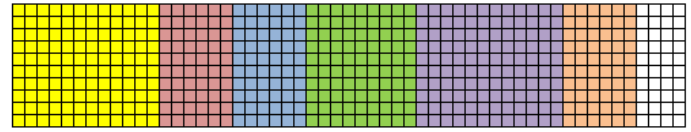

En este punto se nombrarán los gruposcomo A, B o C ya que cada uno realizará un trabajo específico:

\section{Indicaciones (grupos A):}

1- Pegar el rectángulo por el reverso a manera de crear una banda que muestre todos los colores de dulces encontrados en su exterior. Como muestra la figura 2.

2- Con ayuda de un compás dibujar en el papel blanco una circunferencia y marcar el centro de la misma.

3- Colocar la banda en el centro de la circunferencia trazada y marcar donde se encuentran los cambios de colores.

4- Trazar radios de la circunferencia que pasen por cada una de las marcas realizadas y colorear las secciones del círculo del color correspondiente en la banda de colores.

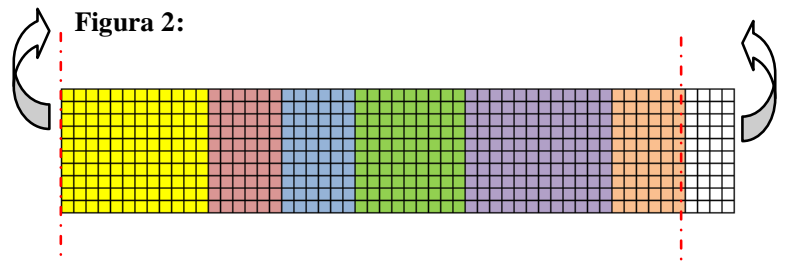


IV Encuentro sobre Didáctica de la Estadística, la Probabilidad y el Análisis de Datos

\section{Indicaciones (grupos B):}

1- Recortar el rectángulo de cuadrícula por cada uno de los colores pintados. Obteniendo seis rectángulos más pequeños pero de un solo color.

2- En la hoja de papel blanco marcar una línea horizontal. Pegar sobre ella de manera vertical y distribuidosuniformemente los rectángulos obtenidos. Como muestra la figura 3.

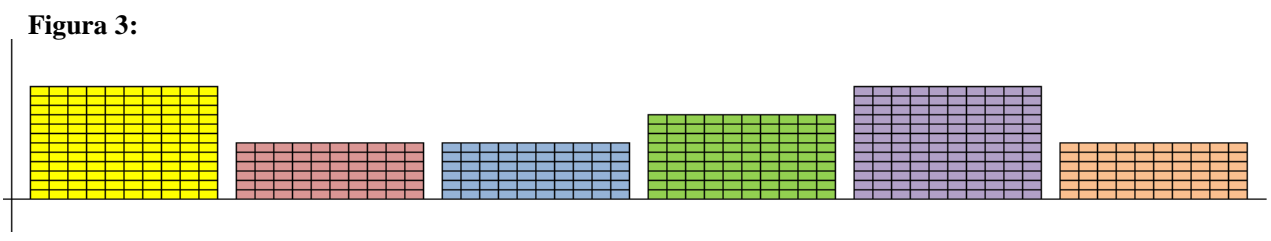

\section{Indicaciones (grupos C):}

1- Recortar el papel cuadrícula restante y dejar únicamente el papel coloreado.

2- Pegar la tira de papel coloreado sobre el papel blanco.

¿Qué representa la tabla construida?

¿Qué representa la gráfica construida?

\section{Indicaciones:}

\section{Etapa 4: Exposición de resultados}

1. Con ayuda de las respuestas a las preguntas anteriores asignar un nombre a la tabla y a la gráfica construida.

2. Etiquetar la cantidad de dulces que representa cada sección coloreada en el gráfico.

3. Presentar la tabla y gráfico construido al resto de los participantes, explicando la cantidad de dulces por color que encontraron, qué color de dulces hay más, que color de dulces hay menos, cual es el total de dulces, etc. 


\title{
Introducción al uso del software $\mathbf{R}$
}

\author{
Luis Rojas Torres ${ }^{1}$
}

\begin{abstract}
Resumen
En este taller se detalla en qué consiste el ambiente de programación para análisis estadístico $\mathrm{R}$ y se da una introducción de la sintaxis necesaria para realizar cálculos sencillos con números reales, aplicar funciones a un vector de datos, creación de un conjunto de datos, exportación de conjuntos de datos, instalación de paquetes y creación de gráficos básicos. Además, se muestra la flexibilidad que presenta R en la manipulación de la información de los objetos, lo cual ejemplifica la idoneidad de este software para la programación en el campo de la Estadística.
\end{abstract}

Palabras clave: R, software estadístico, análisis estadístico

${ }^{1}$ Universidad de Costa Rica. luismiguel.rojas@ ucr.ac.cr 


\title{
Geogebra como herramienta de simulación en eventos de probabilidad geométrica
}

\author{
Noé Alonso Navarro Martínez ${ }^{1} \&$ Kendall Eduardo Rodríguez Bustos ${ }^{2}$
}

\begin{abstract}
Resumen
Este taller tiene como objetivo presentar el concepto de la Generalización de Laplace que permite realizar cálculos de probabilidad geométrica en eventos probabilísticos relacionados con las medidas de longitudes, áreas y volúmenes de cuerpos y/o representaciones geométricas.
\end{abstract}

Se propone utilizar el software gratuito Geogebra para modelar experimentos estocásticos relacionados con la temática planteada. Por lo que los participantes trabajarán con actividades guiadas donde podrán apreciar la riqueza didáctica de la simulación en problemas de variables aleatorias.

Por último, es importante destacar el concepto de la Ley de los Grandes Números en la aproximación de cálculos de probabilidades en las simulaciones de eventos aleatorios.

Palabras clave: Ley de Laplace, Probabilidad Geométrica, Simulación, Geogebra.

\begin{abstract}
In this workshop is designed to introduce the concept of the Generalization of Laplace that allows you to perform calculations of probability in geometric probability events related to the measures of length, areas and volumes of bodies and/or geometric representations.

It is proposed to use the free software Geogebra for stochastic modeling experiments related to the issue raised. By what the participants will work with guides activities where will be able to appreciate the richness of the didactic problems in simulation of random variables.
\end{abstract}

Finally, it is important to emphasize the concept of the Law of Large Numbers in the approximation of calculations of probabilities in the simulations of random events.

Keywords: Law of Laplace, Geometric Probability, Simulation, Geogebra.

Modalidad: Taller

\footnotetext{
1 Tecnológico de Costa Rica, Costa Rica. zomnavarro@gmail.com

2 Tecnológico de Costa Rica, Costa Rica. kendall2412@gmail.com
} 


\title{
Exelearning como Recurso Didáctico para la Enseñanza de la Estadística en Secundaria
}

\author{
Carlos Monge Madriz ${ }^{1} \&$ Steven Gabriel Sánchez Ramírez ${ }^{2}$
}

\section{Resumen}

El presente taller se basará en la resolución de problemas de estadística y la elaboración de guías didácticas afines a la temática. Los asistentes al taller aprenderán a utilizar la plataforma digital educativa de "Exelearning", misma que les permite confeccionar unidades interactivas en las cuales se pueden incorporar multimedia, applets o actividades de retroalimentación. Con el fin de que el material producido pueda ser implementado para el trabajo en el aula o subido a un sitio web y accesado desde casa por el alumno.

Palabras clave: Exelearning, resolución de problemas, estadística, tecnologías, uso de la creatividad, material didáctico.

\begin{abstract}
Workshop attendees will learn to use digital educational eXeLearning platform, allowing them to make same teaching units which can incorporate multimedia, applets and feedback activities. In order that the material produced can be implemented to work in the classroom or uploaded to a website and accessed from home by the student.
\end{abstract}

Keywords: EXeLearning, problem solving, statistics, technology, use of creativity, teaching materials.

\section{Introducción}

La implementación de nuevos programas de estudio en matemáticas por parte del Ministerio de Educación Pública, permitió la introducción de 5 ejes disciplinares al currículo, de los cuales, el taller que se describirá a continuación, enfatizará principalmente en la proyección de:

- La resolución de problemas como estrategia metodológica principal.

- El uso inteligente y visionario de tecnologías digitales.

\footnotetext{
${ }^{1}$ Instituto Tecnológico de Costa Rica - cmongem27@gmail.com

${ }^{2}$ Instituto Tecnológico de Costa Rica - stevengabriel26@gmail.com
} 
Al estudio de la estadística y sus aplicaciones se le había dado menos importancia al punto de llegar a ser un tópico rezagado en los programas de matemáticas en años anteriores. En la reforma matemática implementada en el 2012, se da una potenciación a la enseñanza y el aprendizaje de la estadística para su aplicabilidad en la vida diaria.

Sin embargo, por la ausencia de estos temas, el docente no ha estado capacitado en la confección de materiales didácticos afines a esta temática. Consecuentemente, el objetivo de este taller es brindar herramientas tecnológicas para la confección de recursos didácticos en la enseñanza de la estadística mediante la resolución de problemas.

En el taller propuesto les brindará a los y las participantes herramientas que les permitan visualizar la plataforma exelearning como recurso didáctico, además de que la utilicen para la resolución de problemas estadísticos, conozcan su estructura y los elementos que lo componen para la elaboración de guías didácticas.

\section{La plataforma exelearning como recurso didáctico}

En concordancia con el Ministerio de Educación Pública y la incorporación de la tecnología en el aula se puede manifestar que:

"El sentido de la contextualización y la manipulación con los entornos reales se puede alterar con los medios tecnológicos. En la resolución de problemas donde puede intervenir la tecnología se requiere incluir otras habilidades y procesos que están asociados a la relación interactiva entre conocimiento, pedagogía y tecnología, condiciones que son parte cada vez más de las generaciones de estudiantes que asisten a la escuela (la manipulación de artefactos, relación especial con procesos visuales, multitarea, "conectividad social", etc.). Y esto no refiere solamente a artefactos, las posibilidades que ofrece Internet para la comunicación (donde la distancia se relativiza) permiten trabajar con problemas (y con proyectos) de una forma enteramente distinta a la que se realizaría sin esos medios." (MEP, 2012, p.32)

De acuerdo con el párrafo anterior, exelearning cumple con los parámetros propuestos por el MEP. Por ejemplo la integración de la tecnología, la didáctica y la incorporación de nuevas metodologías para la resolución de problemas.

\section{III. ¿Qué es exelearning?}

Según Blanes, M (2011, p.1) exelearning es una aplicación para la creación y edición de contenidos multimedia, orientada a la docencia. De este modo, la tarea de realizar contenidos más o menos complejos, se hace sencilla y está al alcance de cualquiera que se lo proponga.

Debido a la interactividad de esta plataforma, se convierte en un recurso de gran utilidad para el docente en la enseñanza de la estadística. Por ello, se sabe que para el estudio de 
esta rama de las matemáticas, es indispensable la visualización e interacción de gráficos, datos, muestras, tablas, registros, frecuencias, entre otros.

Consideramos que algunas de las ventajas de utilizar exlearning en el aula son:

- Permite la fácil y sencilla confección de unidades didácticas.

- Es abierto a la adaptabilidad con otros tipos de software externos a él.

- Se obtiene un resultado con gran atractivo visual.

- Es ágil en cuanto al estudio y enseñanza de las matemáticas.

- Es un software libre.

- El producto final puede ser incorporado a una página web.

\section{Exelearning, estadística y resolución de problemas}

En un mundo cargado de información y datos que son expuestos por distintos medios de comunicación, tales como los periódicos, la radio, la televisión y más aún el Internet, influenciados por masas políticas, sociales o económicas; nos vemos en la obligación de ser entes críticos, meticulosos, reflexivos e interrogativos al momento de analizar dicha información. Consecuentemente se requiere del fomento de habilidades de razonamiento estadístico que permitan el estudio de información y datos para una mejor toma de decisiones.

Todas estas habilidades de razonamiento se convierten en material para que el docente las desarrolle en las lecciones de matemáticas. Con la finalidad de que el estudiante las adquiera y las aplique a las situaciones que se le presenten en su diario vivir. Los escenarios en donde aparecerán estas distintas situaciones vienen enmarcados dentro de un contexto que involucra una problemática.

Es así que el docente en su planeamiento no puede obviar y dejar de lado una marcada metodología de resolución de problemas estadísticos. Es por ello que de acuerdo con Brousseau (1996) citado por Godino (1995) "las situaciones didácticas deben configurarse de tal modo que el alumno tenga que resolver problemas cuya apertura sea agradable en la que destaquen distintas fases de acción, formulación de conjeturas y validación, que el profesor deberá completar con la fase de institucionalización de los conocimientos puestos en juego" (p.10)

Ya que el profesor tiene dentro de su esquema metodológico una orientación a la resolución de problemas, le corresponde en segunda instancia la búsqueda de actividades con altos contenidos didácticos que involucren procesos de reflexión, crítica, razonamiento y discusión, siempre enfocados en el establecimiento de estrategias para la resolución de los cuestionamientos. Es aquí en donde Begg (1997) mencionado por Batanero (2000) señalan 
que la estadística es un buen camino para fomentar capacidades de comunicación, tratamiento de la información, uso de las tecnologías y del trabajo cooperativo.

Estas actividades didácticas orientadas a un aprendizaje significativo deben optar por una mayor interacción, que involucre visualización y una gran dosis de interactividad, por consecuencia la tecnonología se puede convertir en un excelente aliado.

"No sólo encontramos información estadística en la prensa y medios de comunicación o en los textos de otras asignaturas, sino que la propia Internet está empezando a modificar las relaciones docentes - con o sin participación voluntaria de los profesores. Es evidente que los profesores - en los diversos niveles educativos- hemos de aceptar que la rapidez del cambio tecnológico e implicarnos en él, si queremos guiar de algún modo la educación estadística, y crear una verdadera cultura estadística en la sociedad.” (Batanero, 2000, p. 9).

En concordancia con lo anterior, el docente tiene que tener una actitud de apertura hacia el uso de nuevas metodologías basadas en recursos digitales, y la plataforma de exelearning puede ser un primer acercamiento a ello, además de la integración del software con internet. Como se mencionó anteriormente, exelearning brinda un paquete para la construcción de unidades didácticas interactivas para el estudiante, que pueden ser alojadas en algún sitio web y accesadas por el alumnado desde casa.

La plataforma permite ambientar al estudiante en la problemática estadística a solucionar, poniendo a su disposición una serie de herramientas multimediales como gráficas, tablas imágenes, videos, o tutores animados. Todos estos elementos se convierten en medios valiosos para potenciar de manera atractiva el desarrollo del razonamiento estadístico, fundamentando la opinión de National Council of Teachers of Mathematics (NCTM), citado por Gamboa (2007), "Las representaciones deberían ser tratadas como un elemento esencial para apoyar la comprensión del estudiante sobre los conceptos y relaciones matemáticas, comunicar acercamientos matemáticos, argumentos y conocimientos de sí mimo y de otros; reconociendo las conexiones entre los conceptos matemáticos y aplicar las matemáticas a situaciones problema reales a través de la modelación.” (p.23)

Y es así como exelearning se convierte en un arma completamente útil para la modelización de problemas, dado que la incorporación de applets confeccionados en otros software como GeoGebra o JClick, llenan de dinamismo la actividad resolutiva, mediante el establecimiento de distintas vías de resolución, llevadas a cabo por la manipulación de animaciones o mediante el desarrollo de juegos.

"Cada uno de los ambientes computacionales que pueden emplear, proporcionan, condiciones para que los estudiantes identifiquen, examinen, y comuniquen distintas ideas matemáticas" (Gamboa, 2007, p.9) 
Exelearning es una recolección de dichos ambientes de aprendizaje, engloba un conjunto de acciones que permiten el trabajo y el estudio de aspectos de índole teórico como de índole práctico. Y ya que el aprendizaje estadístico requiere del estudio teórico de diversas definiciones o metodologías, exelearning no deja ser un complemento que añade dinamismo a esta actividad. Razonar, argumentar plantear y resolver problemas, comunicar, representar y conectar, son los procesos matemáticos que desea fomentar el MEP y que la plataforma a enseñar en este taller, permite que el docente, como manifiesta Sánchez (2014, p. 9), se actualice, tome el reto de cambiar la forma de dar clases e inyecte creatividad al momento de dar las lecciones de matemáticas.

\section{Elementos que componen el software exelearning}

En esta sección se abordaran los componentes principales con las que se trabajaran en el taller para la elaboración de las guías didácticas enfatizadas en tópicos de estadística en secundaria.

$\mathrm{Al}$ inicio los asistentes al taller recibirán un primer acercamiento al uso de la plataforma de exelearning, se describirá principalmente es uso de:

- Estructura

- Menú principal

- I Devices

- Área trabajo

Debido a que exelearning es una herramienta para la elaboración de unidades didácticas, la misma tendrá que subdividirse en diferentes apartados. Por ello es importante seguir una estructura adecuada, misma que se encuentra en la interfaz de exelearning de la siguiente manera: 


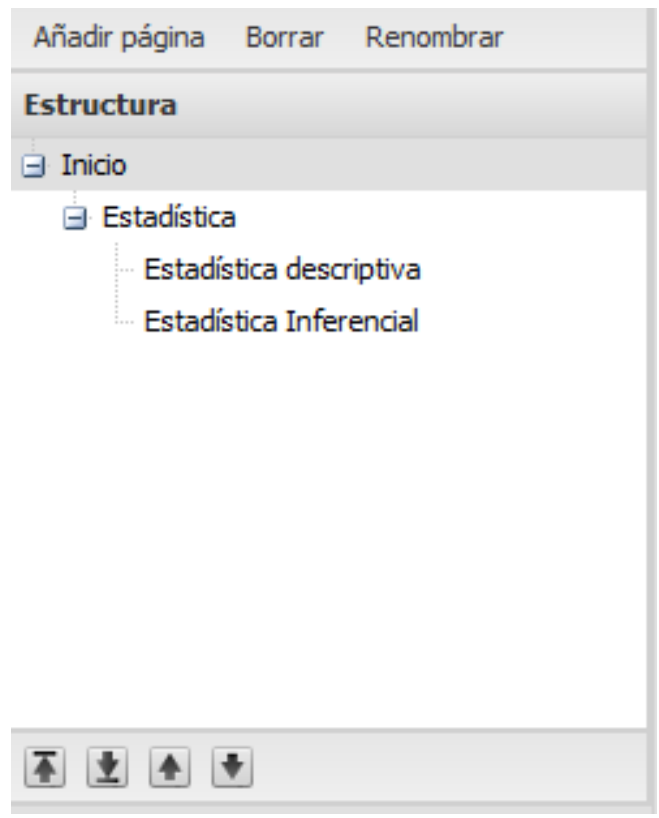

Al ser una plataforma interactiva es necesario que cuente con diferentes estilos de presentación, gestión de archivos, manuales de ayuda.

Archivo * Utilidades * Eștilos * Ayuda *

Por otro lado, exelearning incluye un área de trabajo, en la cual se visualiza toda la información o contenidos que se han venido elaborando.

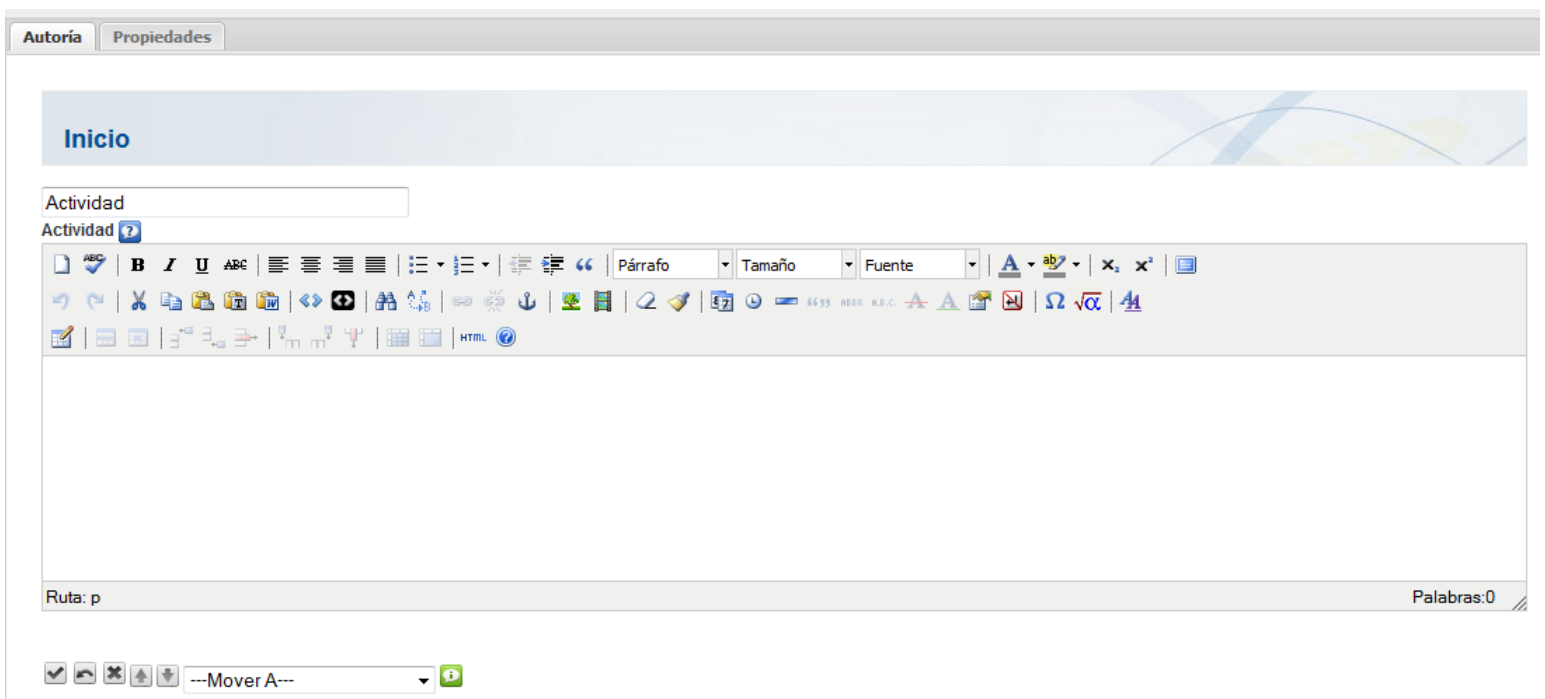

Por último para la confección de guías didácticas en el área de trabajo es necesario la inclusión de diversas actividades que fomenten distintas destrezas en el estudiante. Es por lo anterior que se muestran las idevices, en total, la plataforma contiene 18 distintas idevices, sin embargo las de mayor utilidad para la enseñanza de la estadística son: 
- Texto libre

Este elemento facilita la incorporación de texto, con la finalidad de presentar cualquier información. Además se cuenta con la ventaja de poder insertar imágenes o videos.

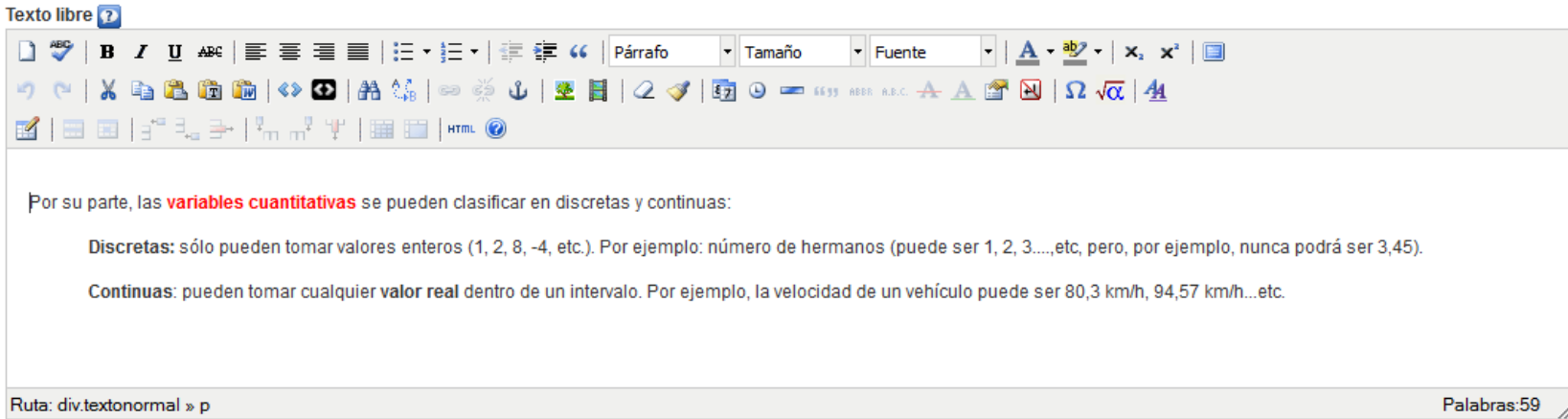

\section{- Reflexión}

Este idevice tiene la funcionalidad de agregar una opción a la unidad didáctica que le permita al estudiante crear una reflexión o plantearse un cuestionamiento. Por lo general existe la posibilidad de agregar una retroalimentación como guía del profesor.

\section{Reflexión}

¿Cuál considera usted que es la principal importancia que le agrega la estadística a su diario vivir?

\section{Pulse aquí}

\section{- Objetivos}

En esta sección se pueden incorporar los objetivos o habilidades a desarrollar en cada una de las unidades didácticas, además se puede incluir videos o imágenes. 
- Galería de imágenes

Incorporando esta actividad a la unidad didáctica, se cuenta con la posibilidad de crear un álbum con imágenes de una misma temática, que el estudiante puede seleccionar para posteriormente ampliar. Es de gran utilidad para la incorporación de gráficos o tablas.

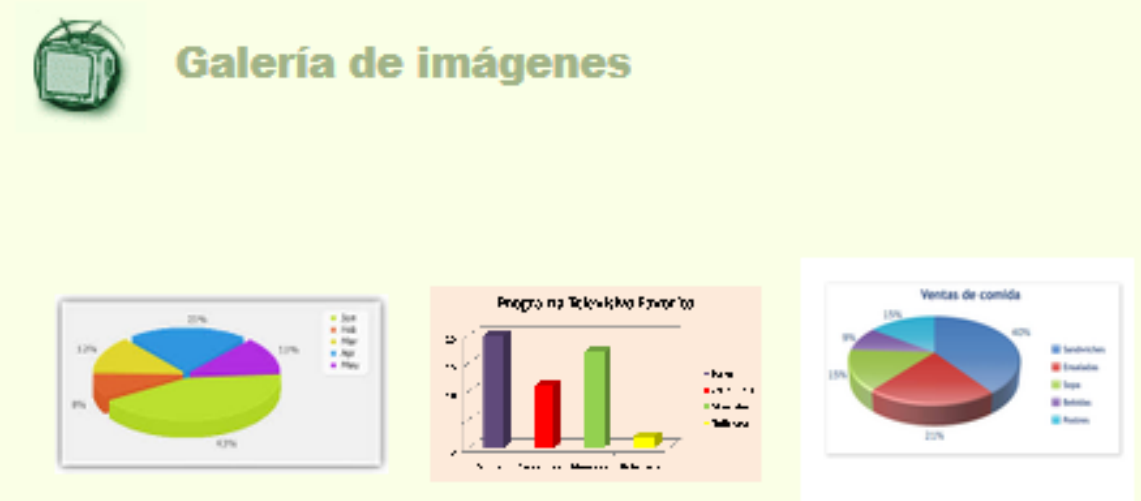

- Lupa

Esta actividad permite la visualización de una imagen al ampliarla utilizando una lupa. Es de gran importancia para el estudio de gráficos o tablas.

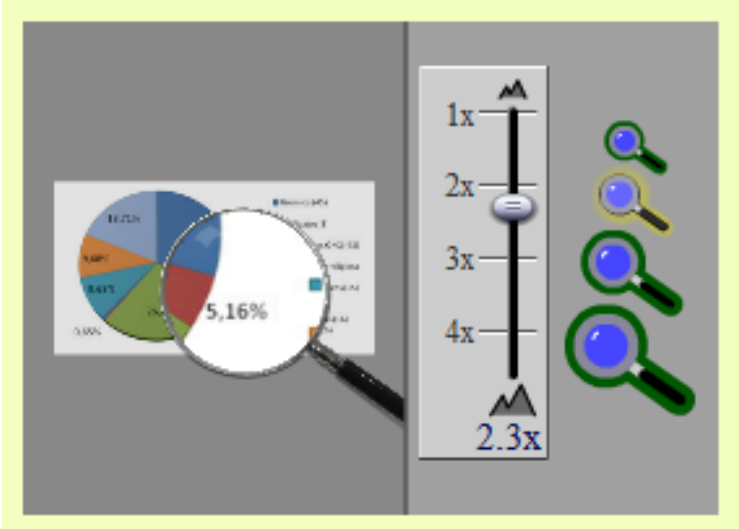


- Applet de Java

Con este elemento se pueden incorporar distintas actividades creadas en otras plataformas como Geoegebra, Jclic o Descartes. En el caso de estadística se podrían incluir applets en Geogebra que muestren animaciones, gráficos o tablas y puedan ser manipulables.

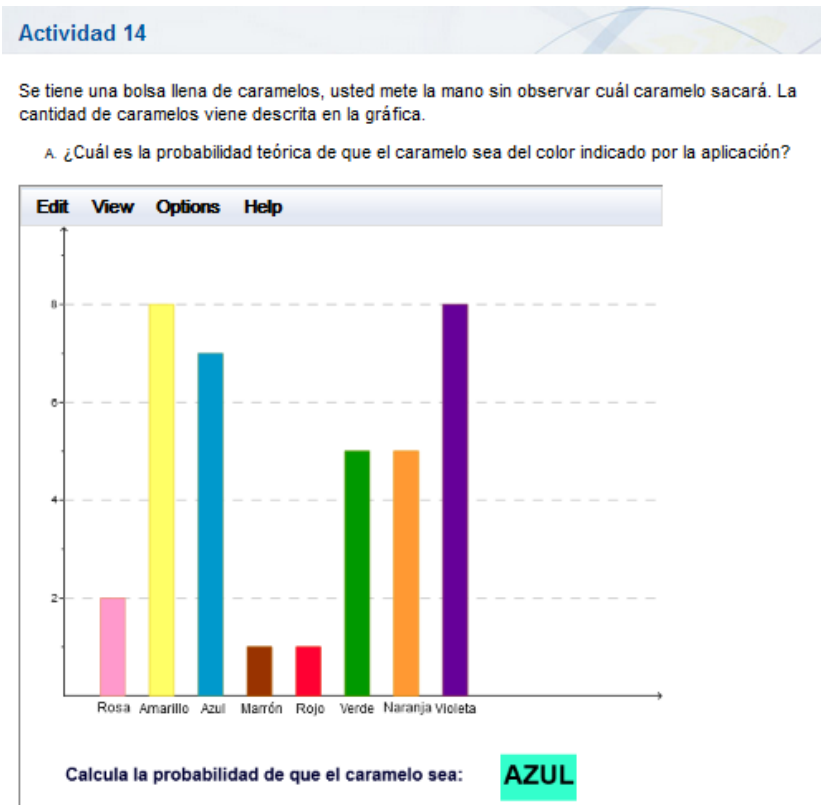

- Rellenar huecos

Con esta actividad, se le presenta al estudiante un texto en el que deberá completar las palabras faltantes en los espacios enmarcados.

Lea el párrafo que aparece abajo y complete las palabras que faltan. 
- Preguntas de selección múltiple

Con este idevice se le presentan al estudiante una serie de preguntas de las cuales puede elegir una o más respuestas. Permite agregar sugerencias para retroalimentar el trabajo del estudiante.

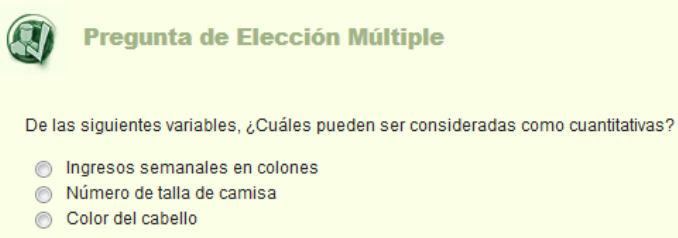

\section{Conclusión}

“...se sabe que para el profesor es un reto cambiar la forma de dar clases, pero la tecnología está cambiando todo en el entorno y los docentes también deben actualizarse por el bienestar de sus educandos" (Sánchez, 2014, p.9). Como lo dice Sánchez, en la cita anterior, los docentes deben de aprovechar los recursos tecnológicos para dar sus clases, pues se dará un mejor entendimiento de la materia, en este caso la estadística, utilizando la plataforma exelearnig, además de propiciar gran interés hacia el tema. Los y las estudiantes divagarán mas en el tópico propiciando el desarrollo de distintas destrezas con el fin de que las puedan utilizar como recurso para la toma de desiciones, ánalisis de información y datos estadísticos.

Por consiguiente, la finalidad de este taller es que los docentes puedan actualizarse utilizando tecnologías para dar clases interactivas, implementando nuevas metodologías, y confeccionando materiales didáticos orientados hacia la enseñanza de la estadística, pues este tópico ha tenido mas auge en los nuevos planes del Ministerio de Educación Pública. 


\section{Bibliografía}

[1] Batanero, C. (2000). “¿Hacia a dónde va la educación estadística?” En: http://www.ugr.es/ batanero/ARTICULOS/BLAIX.pdf (consultada el 27 de agosto, 2014)

[2] Gamboa, R. (2007). "Uso de la tecnología en la enseñanza de las matemáticas". En: http://cimm.ucr.ac.cr/cuadernos/cuaderno3/cuaderno3_c1.pdf (consultada el 25 de agosto, 2014)

[3] Godino, J. (1995)." ¿Qué aportan los ordenadores a la enseñanza y el aprendizaje de la estadística?". En: http://www.ugr.es/ batanero/ARTICULOS/ORDENA.pdf (consultada el 25 de agosto, 2014)

[4] Ministerio de Educación Pública. "Programas de estudio en matemáticas". San José, Costa Rica: MEP.2012.

[5] Monje, A. (2013). "Manual eXeLearning 7.2. Hacemos fácil lo sencillo". En: http://exelearning.net/html_manual/exe_es/index.html (consultado el 12 de agosto, 2014)

[6] Red Iberoamericana de TIC y Educación. (s.f.). "Creación de materiales didácticos con eXeLearning". En: http://www.riate.org/apls/moodle/web/course/view.php?id=4 (consultado el 12 de agosto, 2014)

[7] Sánchez, S. "Principios básicos para la creación de animaciones interactivas con el software Geogebra". II Encuentro Centroamericano de Matemática Educativa (2014). 


\title{
Resolviendo problemas de probabilidad de Distribución Binomial utilizando Geogebra
}

\author{
Franklin Hernández Clavera ${ }^{1}$ \& Rebeca Arce Núñez ${ }^{2}$
}

\begin{abstract}
Resumen
El presente taller aborda la resolución de problemas sobre Distribución Binomial utilizando el software libre Geogebra. Se utilizarán los programas Movie Maker y aTube Catcher para editar y grabar videos de las acciones en la pantalla de la computadora conforme se resuelven los problemas proporcionados. Se pretende que los participantes generen videos con sus propias explicaciones que permitan enseñar la distribución binomial de una forma dinámica. Este taller va dirigido a profesores de secundaria y universidad.
\end{abstract}

Palabras clave: Resolución de problemas, distribución binomial, software libre, videos educativos.

${ }^{1}$ IPEC Arabela Jiménez, Costa Rica. fhclaver@gmail.com

${ }^{2}$ Colegio Nocturno de Cartago, Costa Rica. rebear9034@gmail.com 


\title{
La integración de habilidades en la resolución de problemas de Probabilidad y Estadística
}

\author{
Edwin Chaves Esquivel ${ }^{1}$, Ricardo Poveda Vásquez ${ }^{2}$, Jonathan Espinoza González ${ }^{3}$, Claudia \\ Martínez Pacheco ${ }^{4}$ y Miguel González Ortega ${ }^{5}$
}

\begin{abstract}
Resumen
Se propone realizar un taller, en donde se plantean algunos problemas para la implementación de tópicos de probabilidad y estadística en secundaria, siguiendo lo propuesto en los programas de estudio de Matemáticas. El propósito básico de este taller consiste en ejemplificar el tipo de problemas que el docente puede implementar en el aula, para lograr un trabajo estudiantil exitoso, de modo que se involucran paralelamente diferentes conocimientos probabilísticos o estadísticos, mediante la integración de diferentes habilidades. Pero además, se analiza la puesta en práctica de los fundamentos teóricos estructurados en estos programas, por medio del método de los cuatro pasos para resolución de los problemas, la implementación de los diferentes procesos matemáticos y de las diferentes etapas metodológicas.

Los problemas planteados, por su estructura y nivel de dificultad, son susceptibles para que los profesores puedan_utilizarlos en sus aulas con las adecuaciones del caso.
\end{abstract}

Palabras clave: Resolución de problemas matemáticos, enseñanza de la estadística y probabilidad, programas oficiales de matemáticas en Costa Rica

\section{Introducción}

Los programas vigentes del Ministerio de Educación Pública (MEP, 2012), propone al docente aprovechar el potencial de las Matemáticas como herramienta fundamental para el desarrollo de las diferentes disciplinas científicas. En este currículo se plantea como objetivo principal la búsqueda del fortalecimiento de las capacidades cognoscitivas para enfrentar los retos de la sociedad moderna, donde el conocimiento, la información, y la demanda de habilidades y capacidades de razonamiento lógico y de toma de decisiones basadas en evidencia concreta tienen especial relevancia.

Para lograr este objetivo, se plantean dos propósitos básicos: primeramente que cada estudiante asuma la responsabilidad de participar activamente en la construcción del

\footnotetext{
${ }^{1}$ Escuela de Matemática-UNA, Escuela de Estadística-UCR. echavese@gmail.com

${ }^{2}$ Escuela de Matemática-UNA. ricardopovedav@gmail.com

${ }^{3}$ Escuela de Matemática-UNA. espinozaj25@gmail.com

${ }^{4}$ Escuela de Matemática-UNA. claudia.martinez.pacheco@una.cr

${ }^{5}$ Escuela de Matemática-UNA. Ministerio de Educación Pública. mago1310@gmail.com
} 
aprendizaje, y en segundo lugar que la acción docente se enfoque a la generación de suficientes situaciones de aprendizaje tanto en cantidad y calidad que faciliten el logro del primer propósito en forma motivadora.

Se promueve una acción de aula centrada en la resolución de problemas vinculados con contextos reales, físicos, sociales y culturales. Por medio de esta estrategia se promueve una identificación de cada estudiante con el entorno y el uso de las Matemáticas como herramienta para comprender y modelar las situaciones de la cotidianidad estudiantil. En este sentido, la mediación pedagógica adopta premisas fundamentales constructivistas, tal como lo establece la política educativa vigente para el país, la cual propicia construcción activa de los aprendizajes por parte del mismo estudiante.

Con este enfoque, se promueve el desarrollo de habilidades vinculadas directamente a las áreas matemáticas. El aprendizaje de los conceptos o conocimientos disciplinares por medio de la resolución de problemas debe ser concentrada en cuatro pasos básicos:

(1) propuesta de un problema,

(2) trabajo estudiantil independiente,

(3) discusión interactiva y comunicativa,

(4) clausura o cierre. (MEP, 2012; p.14)

El desarrollo de un problema por parte de los estudiantes, puede ocupar una o más lecciones. Esta propuesta se contrapone con la enseñanza tradicional que ha estado centrada en el desarrollo de los tópicos matemáticos en abstracto por parte del docente, con el planteo de ejemplos y prácticas rutinarias y mecánicas. El planteo y resolución de problemas constituye una constante durante todo el proceso educativo, lo que incluye el reforzamiento, movilización y aplicación de los conocimientos aprendidos. (MEP, 2012; p.15)

Aunque se promueve el empleo de problemas vinculados con contextos reales; no obstante, la implementación de problemas abstractos para situaciones particulares constituyen también una importante herramienta del proceso educativo. Con esto se pretende la construcción de capacidades para la manipulación de los objetos matemáticos cuya naturaleza es abstracta. La estrategia asumida se propone fundamentar pedagógicamente el paso desde lo concreto a lo abstracto. (MEP, 2012; p.15)

Una de las principales críticas que señalan los docentes sobre la implementación de esta estrategia didáctica, consiste en la cantidad de tiempo que se requiere para lograr cada una de las habilidades planteadas en el programa. Aseguran que la cantidad de problemas que deben ser propuestos, y el tiempo que se debe destinar para cada uno de ellos superan las posibilidades reales en cuanto al tiempo que se destina a las lecciones de Matemáticas actualmente.

En este sentido, el taller que se propone realizar con docentes de Matemáticas, viene a contrarrestar estas críticas. Para ello, se presentan actividades académicas que evidencian que por medio de un solo problema es posible integrar diferentes conocimientos matemáticos, que posibilitan en los estudiantes la adquisición de varias habilidades al mismo tiempo. 
Esta propuesta se desarrolla en el área de Estadística y Probabilidad, cuya implementación también ha causado zozobra en los docentes, debido a que en los nuevos programas de estudio, estas áreas tienen un mayor peso del que se les había venido dando en los programas anteriores.

\section{Proceso de mediación pedagógica para desarrollar capacidades cognitivas superiores}

Para las diferentes áreas matemáticas, en los programas de estudio, por un lado, se proponen habilidades vinculadas con las áreas matemáticas, y por otro lado, se plantean procesos que favorecen la reproducción de capacidades cognitivas transversales. Estas dimensiones están intimamente asociadas: los procesos matemáticos adoptados se introducen a partir de tareas para el aprendizaje en las que se persigue el desarrollo de habilidades específicas. (MEP, 2012; p. 26). Sin embargo, las el proceso que vincula el desarrollo de las habilidades específicas, las capacidades cognitivas y la competencia matemática es complejo, y de difícil identificación; pero aún más difícil es evaluar.

Producto de lo anterior, en el documento se aclara que ni los conocimientos matemáticos ni las habilidades específicas generan por sí solas, capacidades cognitivas más amplias que puedan encaminar hacia la competencia matemática. Para ello se requiere una acción de aula capaz de favorecer dicho propósito. Para ello se requiere diseñar lecciones con situaciones de aprendizaje que permitan la realización de los procesos matemáticos.

Sin embargo, no solamente el diseño de situaciones de aprendizaje es importante, sino también la labor docente durante la ejecución de estas situaciones. En este sentido, el docente deber ser un apoyo y el complemento ideal para la labor que realizan los estudiantes.

Por lo anterior, el diseño de las actividades didácticas y la labor docente en el aula son elementos claves para la realización de los procesos matemáticos. Esta labor requiere de un proceso de planificación de las lecciones, para poder generar una mediación pedagógica dirigida a la obtención de habilidades específicas y, por ende, se desarrollen capacidades básicas y la competencia matemática.

Para lograr este fin, tal como se ha citado previamente, la estrategia didáctica propuesta es la resolución de problemas, la cual se adapta plenamente a los propósitos previos. Al respecto se indica:

Colocada ya en contexto educativo, la resolución de problemas debe integrar al menos dos propósitos:

- aprendizaje de los métodos o estrategias para plantear y resolver problemas,

- aprendizaje de los contenidos matemáticos (conceptos y procedimientos) a través de la resolución de problemas. (MEP, 2012; p. 32)

Por medio del primer propósito se hace énfasis en los medios (heurísticas, estrategias y métodos) que requiere un problema para ser exitoso en la acción educativa. Mientras que 
con el segundo propósito se propone generar una acción de aula que posibilite generar un aprendizaje matemático vinculado con un contexto específico. Se incorporan, preferiblemente, problemas reales, en entornos físicos y socioculturales.

En el enfoque que se beneficia aquí, la escogencia de un problema para el desarrollo de una lección debe estar establecida por los propósitos de aprendizaje de un conocimiento matemático y el desarrollo educativo que se realiza, y no, por ejemplo, por las estrategias o técnicas que supone para su solución. Aunque, sin duda, existe relación entre un problema rico en posibilidades de solución y los fines de un buen aprendizaje.

Los contextos donde un problema puede emerger pueden ser diversos. Una situación de salud en el país, asuntos económicos, ambientales, culturales. Contextos escolares, familiares, comunitarios, profesionales, científicos. Pero también un problema puede diseñarse a partir de pasajes de la historia de las Matemáticas, de una representación artística donde es posible encontrar matemáticas, incluso un juego, un rompecabezas, un video, etc.

Un problema es un planteamiento o una tarea que busca generar la interrogación y la acción estudiantil utilizando conceptos o métodos matemáticos, implicando al menos tres cosas:

- que se piense sobre ideas matemáticas sin que ellas tengan que haber sido detalladamente explicadas con anterioridad,

- que se enfrenten a los problemas sin que se hayan mostrado soluciones similares,

- que los conceptos o procedimientos matemáticos a enseñar estén íntimamente asociados a ese contexto. (MEP, 2012; p. 34)

Además, se indica en el documento que la complejidad de un problema debe posibilitar una acción cognitiva, no pueden ser simples acciones rutinarias. Se puede poner en los siguientes términos: una tarea matemática constituye un problema si para resolverla el sujeto debe usar información de una manera novedosa. En el caso que el individuo pueda identificar inmediatamente las acciones necesarias se trata de una tarea rutinaria. (MEP, 2012; p. 34)

\section{Estadística y Probabilidad en los programas de estudio}

En los programas actuales, el área de Estadística y Probabilidad adquiere un mayor relieve del que se le había venido dando en los planes de estudio previos. Desde su inclusión en los programas de Matemáticas de 1995, estos temas se incluyeron parcialmente en Primaria; pero además no se le dio continuidad en la secundaria. 


\section{Programas oficiales de Matemáticas, peso relativo de las diferentes áreas por año y ciclo}

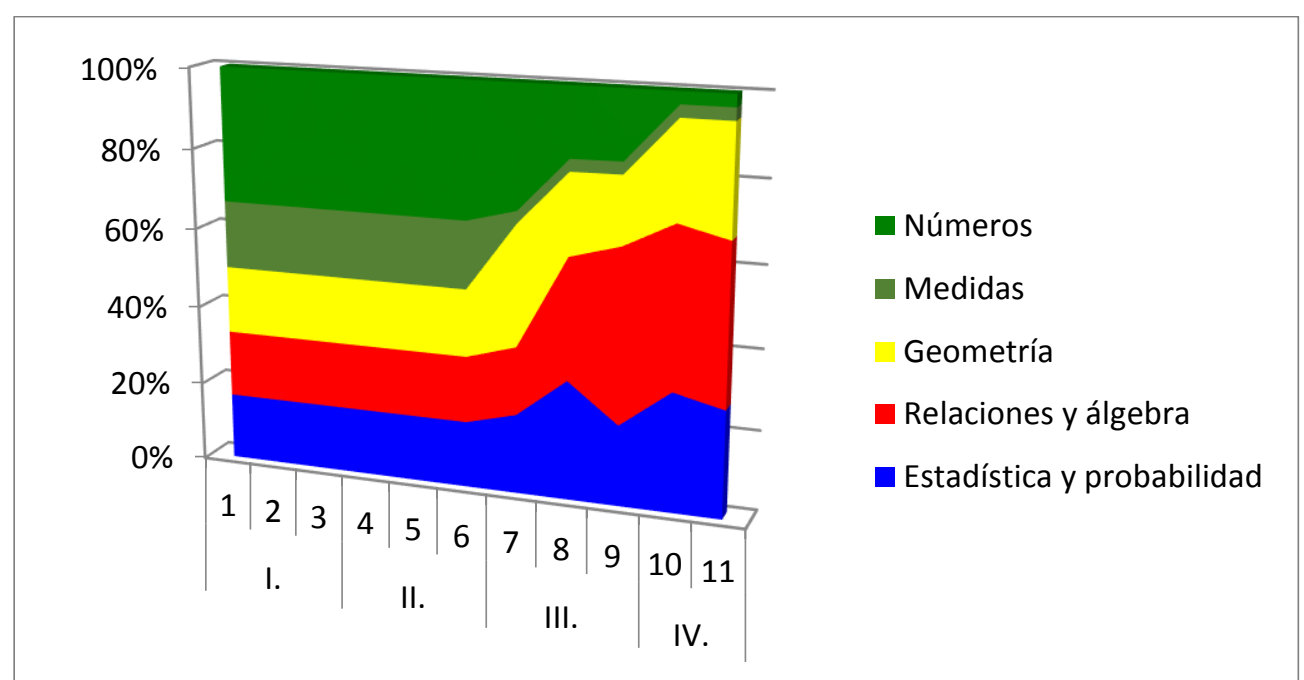

Fuente: Ruiz, A. Fundamentos Teóricos de los Programas de Estudio de Matemática. Conferencia impartida en II Encuentro Provincial de Educación Matemática, Filadelfia, Guanacaste, 2012.

Con la inclusión de una mayor cantidad de conceptos de Estadística y Probabilidad dentro de los programas vigentes, se ha pretendido favorecer el pensamiento aleatorio y el desarrollo de habilidades dirigidas a abordar situaciones de incertidumbre en la vida cotidiana. Pero también la generación de habilidades para utilizar evidencia estadística en la resolución de problemas contextuales. Con esto se propicia lograr una cultura estadística en los jóvenes, tal como ha sido resaltado por Batanero (2004), al fundamentar la necesidad de incluir el razonamiento estocástico en la educación primaria y secundaria.

La importancia de los análisis estadísticos se fundamenta en la resolución de problemas vinculados con datos, en donde el principio de variabilidad es el eje conductor que visualiza la importancia de empleo de diferentes técnicas para el manejo de información. Desde este punto de vista, se atribuye como principal función de la Estadística la de identificar, describir e interpretar el patrón de variación de grupos de datos, con el propósito de descubrir el mensaje que proporcionan en función del problema que les dio origen. Para ello, se discute la implementación de diferentes técnicas de recolección y resumen de información, así como la presentación por medio de cuadros, diagramas o gráficos, cálculo y uso de medidas estadísticas de posición y variabilidad. En esta etapa, se promueve la adquisición del razonamiento estadístico que trasciende lo procedimental, tal como lo señalaron Wild y Pfannkuch (1999), los cuales establecieron que para la promoción del razonamiento estadístico deben tomarse en cuenta cinco componentes básicos:

i. Reconocer la necesidad de los datos: una gran cantidad de problemas de la cotidianidad deben ser analizados considerando información pertinente y válida, que otorga evidencia concreta para decidir. 
ii. Transnumeración: básicamente consiste en cambiar las representaciones de los datos para favorecer una mejor comprensión en relación con el mensaje que transmiten. En esta etapa, se pasa de datos brutos a diferentes representaciones, en busca de aquella que pueda dar un mejor significado para el análisis que se realiza.

iii. Variación: resulta de vital importancia que los estudiantes puedan percibir la variabilidad que se presenta en los datos, la cual genera la incertidumbre sobre el mensaje que comunican. La función principal de la Estadística consiste establecer estrategias para explicar esta variabilidad y el impacto que provoca en cada caso.

iv. La Estadística como un conjunto de modelos: los diferentes objetos estadísticos, sea un cuadro, un gráfico, una medida u otros más elaborados, tienen como propósito utilizar los datos para modelar su patrón de variabilidad. Se requiere lograr la sensibilidad para diferenciar los modelos de acuerdo con el tipo de dato, pero guardando las diferencias entre cada modelo y los datos mismos.

v. Contexto, Estadística y Síntesis: se indica que el razonamiento estadístico se establece al momento en que se vincula el problema generado de un contexto particular con el modelaje estadístico para realizar la síntesis de los hallazgos. Al momento de hacer estadística se puede estudiar detalladamente el comportamiento de los datos para determinar los patrones, pero dichos patrones deben responder al contexto de los datos.

Aunque son conceptos globales, resulta de fundamental importancia tomarlos en cuenta al momento de formular problemas para la enseñanza de la disciplina.

Por su parte, para el estudio de la probabilidad, además de la valoración de los conceptos anteriores vinculados con el razonamiento estadístico, se propone iniciar con las ideas primarias que los estudiantes pueden tener sobre la disciplina y los diferentes conceptos que se relacionan con ella. En este sentido, la construcción del concepto de probabilidad se promueve a partir de las ideas intuitivas (denominadas intuiciones primarias) con que los niños ingresan a la escuela a partir de ciertos juegos. Con el análisis de estas intuiciones primarias, la acción educativa debe favorecer intuiciones secundarias mejor articuladas y más fundamentadas. Esta propuesta se fundamenta en los estudios de Fischbein (1975) en este campo. De esta manera, se procura que paulatinamente se vayan incorporando conceptos más elaborados tales como eventos más y menos probables, eventos equiprobables, resultados simples a favor de un evento, con los cuales sirven de base para construir el concepto clásico o laplaciano de probabilidad. Estos conocimientos básicos, deben permitir que hacia la secundaria se formalicen otros conocimientos como, la definición frecuencista de probabilidad, la ley de los grandes números, los axiomas de Kolmogorov y algunas propiedades que se desligan de ellos. En síntesis, por medio del estudio de las probabilidades se pretende modelar las situaciones aleatorias con el propósito de favorecer la toma de decisiones en condiciones de incertidumbre. 


\section{Propuesta de taller:}

Mediante el trabajo en grupos se plantean los siguientes problemas

\section{PROBLEMA 1}

A) Debido a los problemas de infraestructura vial que aquejan nuestro país, una organización internacional decidió brindar apoyo a uno de los cantones para que invierta en la construcción de carreteras, puentes peatonales, pasos a desnivel, entre otros. Para ello brindará una donación de \$500 millones. Pero para hacer más justa la escogencia, decidió seleccionar aleatoriamente al cantón que iba a ser beneficiado con dicha donación mediante el siguiente procedimiento.

En una tómbola se incluyen 81 bolitas idénticas en tamaño y peso. Cada una tiene el nombre de uno de los cantones de Costa Rica. Se selecciona una bolita al azar y el cantón seleccionado será aquel cuyo nombre está escrito en la bolita.

De acuerdo con la información anterior:

a) ¿Cuál cantón tiene más posibilidades de recibir la donación, el cantón de San José que actualmente cuenta con 287619 habitantes o el cantón de Dota en el cuál viven 6946 personas?

b) ¿Es posible que sea seleccionado el cantón de Flores ubicado en Heredia si éste es el cantón del país que posee la menor cantidad de $\mathrm{km}^{2}$ de territorio?

c) ¿Cuál cantón de Costa Rica tiene menos probabilidad de ser elegido para recibir la donación? ¿Cuál tiene más posibilidades?

d) ¿Cuál es la justificación que fundamenta las respuestas dadas a las preguntas a), b) $y c)$ ?

B) Se ha decidido donar el dinero a una de las provincias de Costa Rica, para ello se selecciona aleatoriamente una de las 81 bolitas (cada bolita contiene el nombre de un cantón), y la provincia beneficiada será aquella a la que pertenece el cantón seleccionado. ¿Es equitativa esta forma de seleccionar una de las provincias? Justifique su respuesta.

C) Tomando como referencia el análisis efectuado en los problemas anteriores, proceda a resolver las siguientes situaciones:

a) Suponga que las siguientes bolas se incluyen en la bolsa de papel:

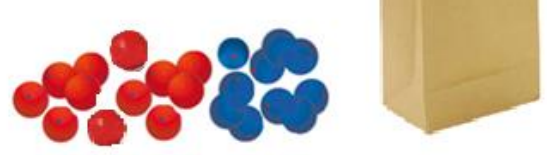

Si se extrae al azar una de las bolas, 
a1) ¿Cuál color es más probable? Justifique su respuesta.

a2) ¿Qué cambios realizarías para que los eventos: extraer una bola azul y extraer una bola roja sean igualmente probables?

b) Considere ahora cinco bolsas como las que se muestran a continuación (todas las bolas son del mismo tamaño).

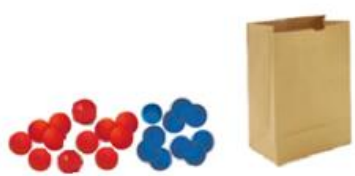

Caso 1

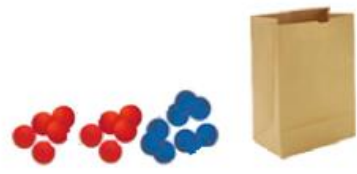

Caso 2

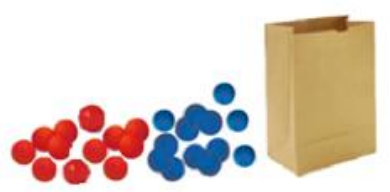

Caso 3

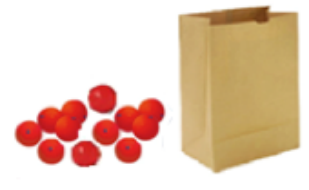

caso 4

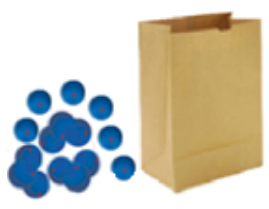

Caso 5

b1) ¿En cuál de los cinco casos es más probable seleccionar una bola roja? Justifique su respuesta.

b2) Si se consideran únicamente los casos 1, 2 y 3 ¿En Cuál de los tres es más probable obtener una bola roja?

\section{Análisis del problema 1}

Propósito de la actividad:Esta actividad se ideó para introducir la definición clásica o Laplaciana de Probabilidad. Mediante la solución de tres problemas, el lector estableció una estrategia para determinar la posibilidad de ocurrencia de un evento y su comparación con otros.

\section{Conocimientos y habilidades: octavo año}

\begin{tabular}{|l|l|}
\hline Conocimientos & Habilidades \\
\hline Eventos & Determinar eventos y sus resultados a favor dentro de una \\
Resultados favorables a un evento & situación aleatoria. \\
Eventos simples y compuestos & Clasificar eventos en simples o compuestos. \\
Evento seguro, evento probable, evento & Identificar eventos seguros, probables e imposibles en una \\
imposible. & situación aleatoria determinada. \\
\hline
\end{tabular}




\begin{tabular}{|l|l|}
\hline $\begin{array}{l}\text { Probabilidad } \\
\text { Eventos más probables, menos probables e } \\
\text { igualmente probables } \\
\text { Definición clásica (o laplaciana) }\end{array}$ & $\begin{array}{l}\text { Diferenciar entre eventos más probables, menos probables e } \\
\text { igualmente probables, de acuerdo con los puntos muestrales } \\
\text { a favor de cada evento. }\end{array}$ \\
Determinar la probabilidad de un evento como la razón \\
entre el número de resultados favorables entre el número \\
total de resulta- dos. \\
Valorar la importancia de la historia en el desarrollo de la \\
teoría de probabilidad.
\end{tabular}

En los problemas A) y B) el total de casos posibles es el mismo (81 cantones), por lo que el criterio empleado para resolverlos consiste en determinar el número de resultados a favor de cada evento. Éste es el principio básico que establece cuáles sucesos son más o menos probables en diferentes situaciones -juegos y situaciones aleatorios- de la vida real. Sin embargo, el problema C) propone situaciones en las que el total de casos no es el mismo. Para resolver este problema debe calcularse la razón entre la cantidad de resultados a favor de cada evento y el total de casos posibles. Éste es precisamente el principio que fundamenta el concepto clásico de probabilidad.

Ejes disciplinares incluidos en los programas de estudio: A través de este problema se integran los siguientes ejes disciplinares:

- Resolución de Problemas. Los estudiantes deben idear estrategias para resolverlo. No es un problema cuya solución es inmediata.

- Uso de la tecnología. Podría emplearse la calculadora como una herramienta para simplificar los cálculos.

- Contextualización activa. El problema está relacionado con situaciones de la vida cotidiana como los problemas de infraestructura que tiene el país o bien la selección de un cantón de Costa Rica, es decir, se utilizan elementos del contexto.

- Actitudes y creencias, particularmente:

- Perseverancia

- Confianza en la utilidad de las matemáticas

- Respeto, aprecio y disfrute de las Matemáticas

- Participación activa y colaborativa

- El uso de la historia de las matemáticas. En este caso se utiliza la historia para justificar la importancia del concepto de equiprobabilidad.

\section{Procesos}

En la resolución de los tres problemas pueden activarse los cinco procesos matemáticos que se proponen en los programas de estudio. Razonar y argumentar se manifiesta al momento de argumentar los resultados obtenidos. Plantear y resolver problemas pues su resolución no es trivial y se debe diseñar una estrategia y métodos adecuados para resolverlo. Por ejemplo hay que valorar si la estrategia de determinar los resultados simples favorables de cada evento les permitirá resolver el problema. Comunicar se activa cuando se expresan de forma oral las estrategias que les permitieron resolver el problema así como los resultados y argumentos matemáticos que justifican la solución.Conectar debido a que en los problemas se emplean habilidades de las áreas Números (comparar fracciones propias utilizando los símbolos $<,>0=0$ mediante su expansión decimal) y Relaciones y Álgebra (analizar la 
proporción entre dos cantidades numéricas). Por último, Representar se evidencia pues se debe idear una forma de representar la noción matemática "12 de las 22 bolas son rojas" la cual puede representarse mediante la fracción propia 12/22 o mediante el número decimal 0,54 .

\section{Elementos de la organización de la lección}

\section{Etapa 1: El aprendizaje de conocimientos}

Este problema se emplea para la generación de nuevo conocimiento, mediante su implementación se pretende que los estudiantes adquieran las habilidades vinculadas con la identificación de eventos más y menos probables, o eventos equiprobables; mediante la identificación del número de resultados a favor de cada evento. Del mismo modo, se posibilita la identificación de la probabilidad de un evento como la razón de resultados a favor entre el total de resultados, siempre que dichos resultados sean equiprobables, concepto que tradicionalmente se conoce como definición clásica o laplaciana de probabilidad.

\section{Propuesta de un problema}

Se recomienda presentar los tres problemas de forma independiente, es decir, hacer un cierre del problema A) antes de presentar el B). De la misma forma resolver el problema C) después de presentar una solución al B). Esto permitirá guiar al estudiante a la construcción de la definición clásica de probabilidad.

\section{Trabajo estudiantil independiente}

Se espera que los estudiantes identifiquen estrategias para resolver los problemas. Por ejemplo en el problema A) deben identificar que en la selección del cantón no importa su extensión o la cantidad de habitantes que poseen, sino que todos tienen la misma posibilidad de ser elegidos. Es decir los eventos son equiprobables.

En el caso del problema B), los eventos no son equiprobables pues el número de cantones por provincia no es el mismo. La siguiente tabla muestra la cantidad de cantones por provincia.

Costa Rica: Número de Cantones por provincia. 2014

\begin{tabular}{lc}
\hline Provincia & No. de cantones \\
\hline San José & 20 \\
Alajuela & 15 \\
Cartago & 8 \\
Heredia & 10 \\
Puntarenas & 11 \\
Guanacaste & 11 \\
Limón & 6 \\
Total & $\mathbf{8 1}$ \\
\hline
\end{tabular}


Es evidente que la provincia de San José tiene más posibilidades de ser elegida pues es la que tiene mayor cantidad de cantones (20) y la que menos tiene es Limón, ya que es la que tiene menor cantidad (6).

En el tercer problema, se espera que los estudiantes, después de reflexionar pueden deducir que para realizar un análisis comparativo entre los diferentes casos, se debe considerar no solamente la cantidad de bolas rojas, sino también el total de bolas rojas y azules en cada caso. Para ello se espera que puedan establecer la relación entre estos dos datos, es decir la razón de bolas rojas entre el total de bolas por bolsa, de modo que la mayor probabilidad de bola roja se obtiene en el caso en que dicha razón tome el mayor valor numérico. Así, aunque en los casos 1 y 3 las bolsas tienen la misma cantidad de bolas rojas (12), la posibilidad de seleccionar una bola roja en estos casos no es la misma, pues la cantidad total de bolas es diferente ( 22 y 25 respectivamente). La bolsa del segundo caso tiene menos bolas rojas (10), pero el total de bolas también es menor que en los casos 1 y 3 (18). En el cuarto caso hay la misma cantidad de bolas rojas que azules (es decir es igualmente probable seleccionar una bola roja que una azul) y en el quinto no hay bolas rojas, en este caso específico, se espera que el estudiante identifique que es imposible seleccionar una bola roja.

Para determinar en cuál de los casos es más probable seleccionar una bola roja los estudiantes deben buscar la proporción de bolas rojas en cada uno de ellos, la cual se muestra a continuación:

\begin{tabular}{cc}
\hline Caso & Proporción \\
\hline 1 & $12 / 22=0,55$ \\
2 & $12 / 25=0,48$ \\
3 & $10 / 18=0,56$ \\
4 & $12 / 12=1$ \\
5 & $0 / 15=0$ \\
\hline
\end{tabular}

De acuerdo a los datos de la tabla anterior

a1) Si se consideran los cinco casos es más posible seleccionar una bola roja en el cuarto caso

b2) Si se consideran los casos 1, 2 y 3 en el tercer caso es más posible seleccionar una bola roja.

Los casos 4 y 5 , permiten deducir también que la probabilidad del evento seguro es uno y la probabilidad del evento imposible es cero. Con ello, se deduce también que la probabilidad de cualquier evento es un valor numérico entre cero y uno.

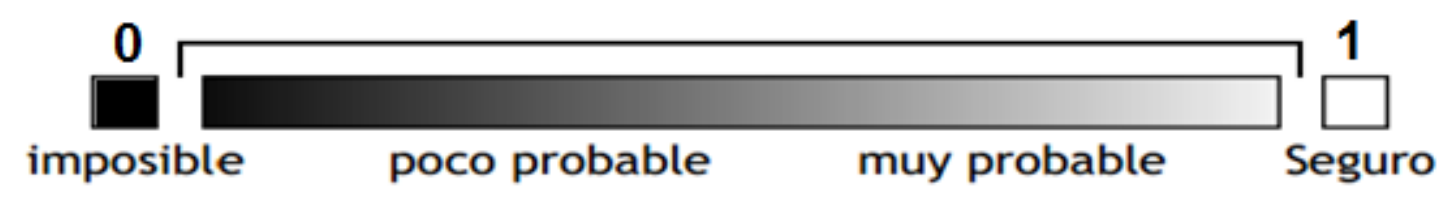

\section{Discusión interactiva y comunicativa}


Se espera que los estudiantes compartan con sus compañeros los hallazgos obtenidos. Se debe prestar atención a las estrategias utilizadas y a posibles errores que puedan surgir

\section{Clausura o cierre}

El docente enuncia el concepto de probabilidad clásica. Puede emplearse el problema para enunciar las reglas básicas de probabilidad.

\section{PROBLEMA 2}

Ante las sequías reiteradas que se han venido presentando en diferentes zonas del país, se hace necesario propiciar un proceso de ahorro de este líquido. Proceda a leer el siguiente artículo:

EL CONSUMO DE AGUA POR PERSONA EN LOS PAÍSES
DESARROLLADOS ALCANZA LOS 300 LITROS FRENTE LOS
8O QUE RECOMIENDA LA ORGANIZACIÓN MUNDIAL DE LA
SALUD (OMS)

El consumo de agua por persona en los países desarrollados puede alcanzar los 300 litros diarios, ante los 25 que se consumen en zonas subdesarrolladas y los 80 litros que recomienda la Organización Mundial de la Salud (OMS) para las necesidades vitales e higiene personal. No obstante, el consumo medio mundial de litros de agua por persona es de 1800 litros diarios si se suman las actividades en las que se utiliza el agua para la agricultura y ganadería un 75 por ciento, e industria un 8 por ciento.

Para concienciar a los ciudadanos de la necesidad de adoptar una serie de hábitos de ahorro de agua, el grupo Eroski y la organización mundial de conservación WWF/Adena han puesto en marcha la campaña "El agua, un recurso escaso, natural e imprescindible".

Eroski pretende sensibilizar a la población hacia una cultura respetuosa con el agua, posibilitando un ahorro efectivo de un recurso tan escaso a través de acciones de sensibilización para prevenir el derroche del agua y mostrando medidas que optimicen su aprovechamiento, con el propósito de que la población también se implique en el proyecto.

\section{AHORRO DEL AGUA}

Los estudios revelan que los seres humanos pueden llegar a desperdiciar una considerable cantidad de agua sin consumirla. Uno de los casos más alarmantes es el de los grifos deteriorados que permiten fugas de hastalo gotas por minuto provocando un desperdicio anual de 2000 litros de agua.

El uso diario de la ducha ${ }^{6}$, en vez del baño ${ }^{7}$, contribuye también de sobremanera a cimentar el ahorro de agua, pues pueden ahorrarse hasta 7300 litros de agua por persona al año. Además, si la ducha cuenta con economizadores de agua, la cifra de litros ahorrados asciende hasta los 14 600. Otro de los casos en los que se pueden ofrecer datos es en el capítulo de las cisternas, pues la instalación de
El agua es un recurso escaso, cada dos minutos muere un ser humano por falta de agua potable, algo difícil de comprender desde el mundo civilizado, que obtiene el líquido elemento sólo con abrir el grifo. Además, 20 por ciento de las especies de agua dulce corren peligro de extinción, víctimas de la contaminación o de la disminución de reservas.

La sequía que afecta a España no sólo se debe a una desigual distribución de precipitaciones entre la zona atlántica y la mediterránea, sino también al consumo desequilibrado que se realiza. España es el tercer país del mundo con mayor consumo por habitante, aunque en los últimos 75 años se ha producido una reducción del 30 por ciento del caudal circulante en los ríos, sólo 5 por ciento es atribuible a causas naturales.

\section{HÁBITOS DE CONSUMO RESPONSABLE}

La representante de WWF/Adena, Lucía De Stefano, señaló que "muchas veces no somos conscientes del impacto al medio ambiente que tienen nuestras acciones diarias". Por ello, se hace una serie de recomendaciones que según De Stefano "deben convertirse en hábitos diarios e interiorizarlos".

Entre ellos destacan evitar verter productos de limpieza por el desagüe, ya que dificultan la posterior depuración de las aguas; en el jardín regar al amanecer o al anochecer, ya que es cuando el agua tarda más en evaporarse, y escoger plantas autóctonas que consumen menos agua; cerrar el grifo al lavarse los dientes o los platos; tirar de la cadena del inodoro sólo cuando sea necesario y no utilizarlo como papelera; reparar los grifos que gotean con urgencia; ducharse en vez de bañarse; lavar la fruta y la verdura en un cuenco; o utilizar el lavavajillas y la lavadora sólo a plena carga.

Lucía De Stefano indicó que "el agua tenemos que utilizarla bien y sólo si la necesitamos", e incidió en la importancia de no abusar de jabones y detergentes, ya que estos "contaminan el agua", y aunque pase por la depuradora "nunca la devuelve a los rios en las mismas condiciones en que salieron de ellos".

De Stefano concluyó recordando que "el agua viene de ríos y vuelve a ellos, son arterias de vida en las que viven muchos organismos que participan en una cadena de vida que tenemos que conservar $y$ transmitir a generaciones futuras".

Tomado de la página Web

\footnotetext{
${ }^{6}$ Tomar un baño en la ducha.

${ }^{7}$ Tomar un baño en la tina.
} 
dispositivos de ahorro pueden lograr a que no se tiren 7600 litros de agua por persona.

En la actualidad, 26 países sufren la escasez de agua, pero la previsión es que para el 2025 sean 41 los países que presenten un déficit crónico de agua, afectando a 2800 millones de personas, 35 por ciento de los 8000 que para entonces habitarán el planeta.

En dos ciudades de Costa Rica, se seleccionaron muestras aleatorias de 26 personas cada una, de distintos estratos sociales y se midió la cantidad de agua que consumieron en un día cualquiera (en hectolitros). A continuación se presenta dicha información.

Ciudad 1: Consumo de agua por persona en hectolitros, para una muestra de 26 personas

\begin{tabular}{|l|c|c|c|c|c|c|c|c|c|c|c|c|c|}
\hline Persona & 1 & 2 & 3 & 4 & 5 & 6 & 7 & 8 & 9 & 10 & 11 & 12 & 13 \\
\hline Consumo & 0,69 & 0,71 & 0,76 & 0,76 & 0,81 & 1,00 & 1,03 & 1,17 & 1,17 & 1,31 & 1,35 & 1,35 & 1,57 \\
\hline \multicolumn{10}{|c|}{} \\
\hline Persona & 14 & 15 & 16 & 17 & 18 & 19 & 20 & 21 & 22 & 23 & 24 & 25 & 26 \\
\hline Consumo & 1,93 & 1,93 & 1,99 & 2,09 & 2,62 & 2,66 & 3,27 & 3,29 & 3,37 & 3,47 & 3,69 & 4,05 & 4,15 \\
\hline
\end{tabular}

Ciudad 2: Consumo de agua por persona en hectolitros, para una muestra de 26 personas

\begin{tabular}{|l|c|c|c|c|c|c|c|c|c|c|c|c|c|}
\hline Persona & 1 & 2 & 3 & 4 & 5 & 6 & 7 & 8 & 9 & 10 & 11 & 12 & 13 \\
\hline Consumo & 0,89 & 0,90 & 0,92 & 0,93 & 1,03 & 1,22 & 1,29 & 1,36 & 1,36 & 1,41 & 1,53 & 1,55 & 1,71 \\
\hline
\end{tabular}

\begin{tabular}{|l|c|c|c|c|c|c|c|c|c|c|c|c|c|}
\hline Persona & 14 & 15 & 16 & 17 & 18 & 19 & 20 & 21 & 22 & 23 & 24 & 25 & 26 \\
\hline Consumo & 1,80 & 2,00 & 2,12 & 2,22 & 2,31 & 2,39 & 2,45 & 2,78 & 3,12 & 3,14 & 3,22 & 4,99 & 6,20 \\
\hline
\end{tabular}

De acuerdo con dicha información, resuelva lo siguiente:

1) Construya un polígono de frecuencia porcentual, en cada caso, utilizando la siguiente distribución, que debe ser completada previamente

Consumo diario de agua por persona para una muestra aleatoria de las ciudades 1 y 2 (en hectolitros)

\begin{tabular}{lcccc}
\hline & \multicolumn{2}{c}{ Ciudad A } & \multicolumn{2}{c}{ Ciudad B } \\
\hline $\begin{array}{l}\text { Consumo } \\
\text { de agua }\end{array}$ & $\begin{array}{c}\text { Número de } \\
\text { personas }\end{array}$ & $\begin{array}{c}\text { Porcentaje } \\
\text { de personas }\end{array}$ & $\begin{array}{c}\text { Número de } \\
\text { personas }\end{array}$ & $\begin{array}{c}\text { Porcentaje } \\
\text { de personas }\end{array}$ \\
\hline De 0,50 a menos de 1,00 & 5 & & \\
De 1,00 a menos de 1,50 & 7 & & \\
De 1,50 a menos de 2,00 & 4 & & \\
De 2,00 a menos de 2,50 & 1 & & \\
De 2,50 a menos de 3,00 & 2 & & \\
De 3,00 a menos de 3,50 & 4 & & \\
De 3,50 a menos de 4,00 & 1 & & \\
De 4,00 a menos de 5,00 & 2 & & \\
Más de 5,00 & 0 & & \\
Total & $\mathbf{2 6}$ & & \\
\hline
\end{tabular}


2) Utilice diferentes medidas estadísticas y el gráfico anterior, para argumentar en cuál de las muestras se presentó un consumo de agua que se aleja en mayor medida de lo recomendado por la Organización Mundial de la Salud.

3) ¿Constituye la media aritmética o promedio una medida estadística de tendencia central adecuada para resumir estos datos? Razone su respuesta. En caso negativo, ¿cuál sería una mejor medida de tendencia central y por qué?

4) Construya un diagrama de cajas y con base en toda la información recolectada responda ¿En cuál de las muestras fue más homogéneo el consumo de agua? ¿Por qué? Analice nuevamente el resultado dado en la pregunta 2).

5) De acuerdo con la lectura realizada, ¿qué tan adecuado es el consumo de agua en estas dos ciudades, basados en las muestras observadas? Qué recomendaciones daría usted para mejorar esta condición.

\section{Análisis del problema 2}

Propósito de la actividad:Su propósito fue no sólo utilizar las medidas estadísticas de posición y variabilidad para caracterizar un conjunto de datos, si no destacar la importancia que tiene el combinar estas medidas y utilizar representaciones gráficas para analizar integralmente un conjunto de datos vinculados con un problema particular.

\section{Conocimientos y habilidades: décimo año}

\begin{tabular}{|l|l|}
\hline Conocimientos & \multicolumn{1}{|c|}{ Habilidades específicas } \\
\hline Medidas de posición & $\begin{array}{l}\text { 2. Resumir un grupo de datos mediante el uso de la moda, la } \\
\text { media aritmética, la mediana, los cuartiles, el máximo y el } \\
\text { mínimo, e interpretar la información que proporcionan dichas } \\
\text { - Moda }\end{array}$ \\
- Media aritmética & $\begin{array}{l}\text { 3. Identificar la ubicación aproximada de las medidas de } \\
\text { posición de acuerdo con el tipo de asimetría de la distribución } \\
\text { - Mediana }\end{array}$ \\
- Cuartiles & de los datos. \\
- Extremos & Medilizar la calculadora o la computadora para calcular las \\
- Máximo & Mínimo
\end{tabular}

\section{Conocimientos y habilidades: undécimo año}

\begin{tabular}{|l|l|}
\hline Conocimientos & Habilidades específicas \\
\hline $\begin{array}{l}\text { Medidas de variabilidad } \\
\text { - Recorrido }\end{array}$ & $\begin{array}{l}\text { 1. Identificar la importancia de la variabilidad para el análisis de datos. } \\
\text { - Recorrido intercuartílico }\end{array}$ \\
$\begin{array}{l}\text { - Variancia } \\
\text { - Desviación estándar }\end{array}$ & $\begin{array}{l}\text { análisis estadísticos y la necesidad de cuantificarla. } \\
\text { Resumir la variabilidad de un grupo de datos mediante el uso del } \\
\text { recorrido, el recorrido intercuartílico, la variancia o la desviación } \\
\text { estándar e interpretar la información que proporcionan. }\end{array}$ \\
$\begin{array}{l}\text { Representación gráfica } \\
\text { - Diagrama de cajas }\end{array}$ & $\begin{array}{l}\text { 5. Emplear la calculadora o la computadora para simplificar los cálculos } \\
\text { matemáticos en la determinación de las medidas de variabilidad. }\end{array}$ \\
& $\begin{array}{l}\text { 6. Utilizar diagramas de cajas para comparar la posición y la variabilidad } \\
\text { de dos grupos de datos. }\end{array}$ \\
& $\begin{array}{l}\text { 7. Resolver problemas del contexto estudiantil que involucren el análisis } \\
\text { de las medidas de variabilidad. }\end{array}$ \\
\hline
\end{tabular}


Ejes disciplinares incluidos en los programas de estudio: A través de este problema se integran los siguientes ejes disciplinares

- Resolución de Problemas. Los estudiantes deben aplicar sus conocimientos en medidas estadísticas para la resolución de este ejercicio y dado que no es trivial los argumentos que justifican las respuestas a cada una de las preguntas, puede ser ubicado en un nivel de Reflexión.

- Uso de la tecnología. Para el cálculo de las medidas podría emplearse la calculadora o una hoja de cálculo como una herramienta para simplificar los cálculos y hacer énfasis en el análisis.

- Contextualización activa. El ejercicio está relacionado con una problemática que no es ajeno al país: el consumo responsable del agua. El ejercicio en sí a parte de permitir la apropiación del conocimiento que se estipula, pretende crear conciencia sobre el uso racional de nuestros recursos naturales (el agua).

- Actitudes y creencias, particularmente:

- Perseverancia. Durante el análisis y la argumentación que cada estudiante propone para dar respuesta a las interrogantes planteadas.

- Confianza en la utilidad de las matemáticas. Viendo en ellas un medio que nos permite interpretar, caracterizar y modelar la realidad para la toma de decisiones a futuro.

- Participación activa y colaborativa. A la hora en que los estudiantes se organizan para la realización de cálculos, la confrontación de ideas, etc.

\section{Procesos}

En la resolución de este problema se activaron los cinco procesos matemáticos que se proponen en los programas de estudio.

- Razonar y argumentar. Se manifiesta en la resolución de las preguntas 2, 3,4, y 5, donde se solicita al estudiante una justificación de sus respuestas.

- Plantear y resolver problemas. Es inherente dada la naturaleza de este ejercicio.

- Comunicar: Se activa cuando los estudiantes comunican los resultados o respuestas, esto bajo el supuesto de que el docente habilite un espacio para dicha discusión.

- Conectar. Algunas áreas presentes son Números y Relaciones y Álgebra (uso de fórmulas).

- Representar. Se evidencia al utilizar representaciones gráficas y tabulares para argumentar y justificar los resultados o respuestas a las interrogantes planteadas.

\section{Elementos de la organización de la lección}

Movilización y aplicación de los conocimientos: Este problema-ejercicio corresponde a esta etapa pues se pide la aplicación directa de las medidas estadísticas para su resolución. No se pretende introducir un nuevo conocimiento.

Sin embargo, el Programa de Matemáticas es flexible en cuanto a que el docente puede organizar su lección de acuerdo a la etapa 1 (aunque se esté en la etapa 2). De ahí que a continuación se presenta una propuesta para desarrollar esta actividad siguiendo los cuatro momentos que se proponen en la etapa 1. 
Se recomienda al docente estar pendiente de que el estudiante realiza la lectura del artículo pues es conocido el poco hábito de lectura presente en ellos. Motivarlos a que se tomen su tiempo para entender la problemática que ahí se describe. Realizarle preguntas que permitan diagnosticar su comprensión sobre las ideas centrales presentes en ellas.

Con este problema se procura aplicar los conocimientos concernientes al uso de medidas de tendencia central y de variabilidad para caracterizar un conjunto de datos en un contexto cercano al estudiante. Se procura que los estudiantes se organicen en subgrupos para poder generar espacios de discusión en torno a la resolución del problema.

Se espera que los estudiantes puedan discriminar cuál o cuáles medidas estadísticas permiten responder de manera eficiente a cada uno de los planteamientos propuestos. A continuación se presentan los detalles que se esperan observar durante la etapa de resolución:

\section{Pregunta 1}

Cuando los estudiantes completan la distribución suministrada, se obtiene:

\section{Consumo diario de agua por persona para una muestra aleatoria de las ciudades A y B (en hectolitros)}

\begin{tabular}{lcccc}
\hline & \multicolumn{2}{c}{ Ciudad A } & \multicolumn{2}{c}{ Ciudad B } \\
\hline $\begin{array}{l}\text { Consumo } \\
\text { de agua }\end{array}$ & $\begin{array}{c}\text { Número de } \\
\text { personas }\end{array}$ & $\begin{array}{c}\text { Porcentaje } \\
\text { de personas }\end{array}$ & $\begin{array}{c}\text { Número de } \\
\text { personas }\end{array}$ & $\begin{array}{c}\text { Porcentaje } \\
\text { de personas }\end{array}$ \\
\hline De 0,50 a menos de 1,00 & 5 & 19,2 & 4 & 15,4 \\
De 1,00 a menos de 1,50 & 7 & 26,9 & 6 & 23,1 \\
De 1,50 a menos de 2,00 & 4 & 15,4 & 4 & 15,4 \\
De 2,00 a menos de 2,50 & 1 & 3,8 & 6 & 23,1 \\
De 2,50 a menos de 3,00 & 2 & 7,7 & 1 & 3,8 \\
De 3,00 a menos de 3,50 & 4 & 15,4 & 3 & 11,5 \\
De 3,50 a menos de 4,00 & 1 & 3,8 & 0 & 0,0 \\
Más de 4,00 & 2 & 7,7 & 1 & 3,8 \\
Total & 0 & 0,0 & 1 & 3,8 \\
\hline & $\mathbf{2 6}$ & $\mathbf{1 0 0}$ & $\mathbf{2 6}$ & $\mathbf{1 0 0}$ \\
\hline
\end{tabular}

Se puede confeccionar previamente plantillas que permitan a los estudiantes agilizar la elaboración de los polígonos de frecuencia y que ellos se enfoquen necesariamente en el trazo y la obtención de las marcas de clase (puntos medios). Cuando los estudiantes las elaboren, se obtendría una representación como la siguiente: 


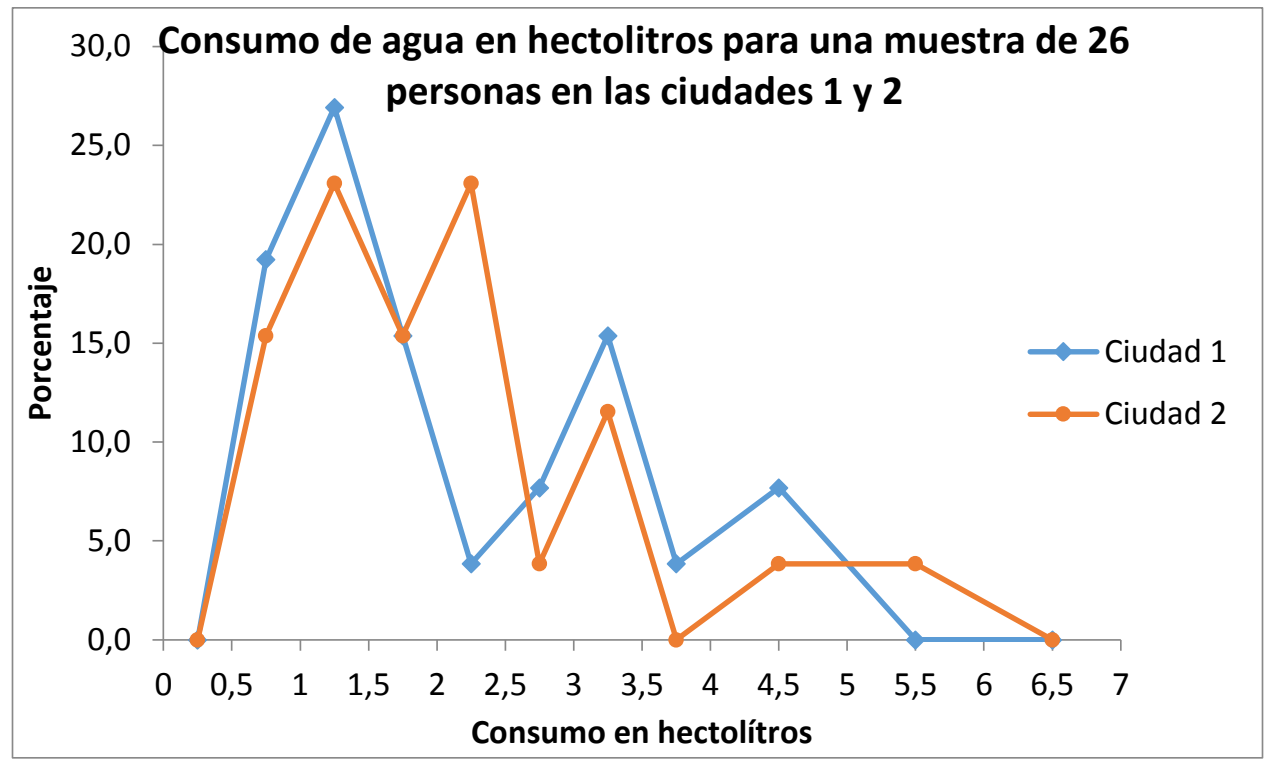

\section{Pregunta 2}

Para el cálculo de algunas medidas estadísticas, los estudiantes podrán hacer uso de la calculadora científica o una hoja de cálculo cuando sea posible. Lo importante es el análisis de dichas cantidades y la discusión que de ellas se pueda generar. No hay que valorar mayoritariamente la labor de cálculo. A continuación se detallan dichas medidas:

\begin{tabular}{|c|c|}
\hline \multicolumn{2}{|c|}{ CIUDAD 1 } \\
\hline MÍNIMO & 0,69 \\
\hline MÁXIMO & 4,15 \\
\hline RANGO & 3,46 \\
\hline PROMEDIO & 2,01 \\
\hline MEDIANA & 1,750 \\
\hline DESV & 1,14 \\
\hline Cuartil 1 & 1,02 \\
\hline Cualtil 3 & 2,96 \\
\hline
\end{tabular}

\begin{tabular}{|c|c|}
\hline \multicolumn{2}{|c|}{ CIUDAD 2 } \\
\hline MÍNIMO & 0,89 \\
\hline MÁXIMO & 6,20 \\
\hline RANGO & 5,31 \\
\hline PROMEDIO & 2,11 \\
\hline MEDIANA & 1,755 \\
\hline DESV & 1,26 \\
\hline Cuartil 1 & 1,26 \\
\hline Cualtil 3 & 2,42 \\
\hline
\end{tabular}

Si se considera el consumo medio o mejor denominado el consumo promedio, se tiene que en la ciudad 2 se produjo un mayor consumo. No obstante, al analizar el consumo mediano los resultados son prácticamente idénticos. Por esta razón se hace necesario realizar un análisis más integral, pues las representaciones gráficas generadas en el inciso anterior, tampoco evidenciaron un patrón claro. Pues tanto el mínimo consumo como el consumo máximo son mayores en la ciudad 2. Pero el cuartil 3 es mucho menor, por lo que la confusión se mantiene.Ante esta situación, los estudiantes pueden ofrecer diferentes respuestas muchas de ellas antagónicas entre sí, por lo que el docente debe estar atento para ofrecer la recomendaciones pertinentes.

\section{Pregunta 3}

En los casos en que existen importantes diferencias entre la mediana y el promedio, es una evidencia que existen valores extremos que provocan que el promedio se sesgue hacia esos valores extremos. En el problema planteado, existen valores de consumo de agua de unas 
pocas personas que sobrepasa el consumo de las demás personas, por ello, la media aritmética o promedio no es una medida adecuada de la tendencia central de los datos. En estos casos la mejor medida de tendencia central es la mediana, dado que indica el valor central, pues el $50 \%$ de las personas tiene un consumo inferior o igual y el otro $50 \%$ un consumo mayor o igual a dicho valor.

Por otro lado, la moda no es una medida que permita realizar comparaciones, en el sentido que en la ciudad 1 hay varias modas, mientras que en la ciudad 2 solamente una. Al agruparlos datos, pareciera evidenciarse que los datos presenta más de una moda.

\section{Pregunta 4}

Desde el punto de vista de la variabilidad, si se analizan los polígonos de frecuencias, el rango y la desviación estándar, todas muestran una mayor variabilidad en el consumo de la ciudad 2.

No obstante, para analizar la homogeneidad de los datos, resulta de relevancia analizar el diagrama de cajas, el cual se presenta a continuación:

\section{Consumo de agua en hectolitros para una muestra de 26 personas en las ciudades 1 y 2}

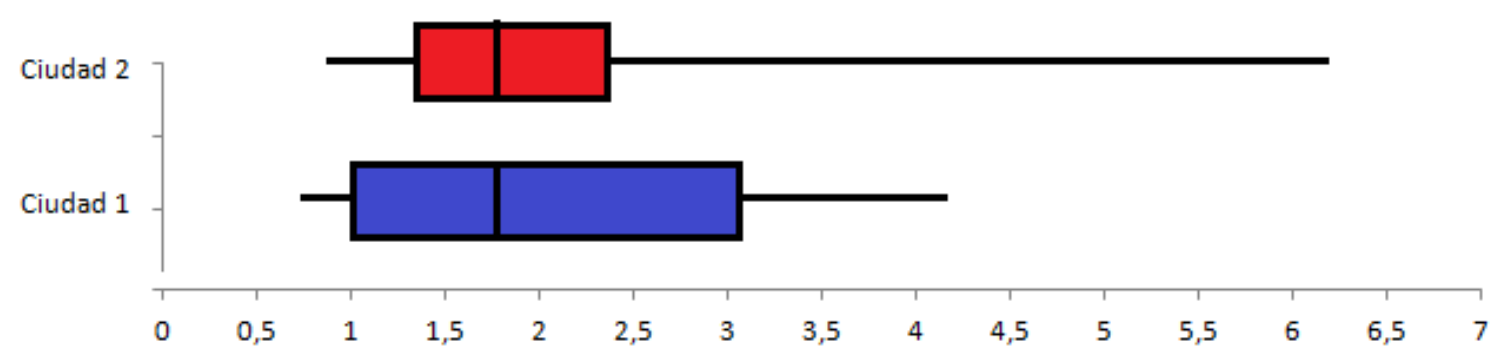

Puede notarse que a pesar de los resultados anteriores, el gráfico es contundente en indicar que la mayor variabilidad producida en la ciudad 2, obedece al $25 \%$ de la familias que más consumen agua, mientras que si se considera el $75 \%$ de las familias que menos consumen agua, los datos son más homogéneos en la ciudad 2.

Este resultado es relevante para efectos del análisis integral del problema, pues se evidencia que, aunque en ambas muestras el consumo de agua está muy por encima de lo que recomienda la Organización Mundial de la Salud, en la ciudad 2, para una gran mayoría de personas el consumo es inferior al de la ciudad 1, pero existen algunas personas que tienen un consumo mucho más alto, que afecta drásticamente las mediadas estadísticas, especialmente el promedio y la desviación estándar. 
Lo anterior refleja, que dentro de los análisis estadísticos, no es suficiente analizar una única medida, sino que se requiere llevar a cabo un análisis integral.

El análisis anterior, puede ser visualizado más simplemente con un diagrama de puntos, el cual refleja lo que se ha indicado previamente.

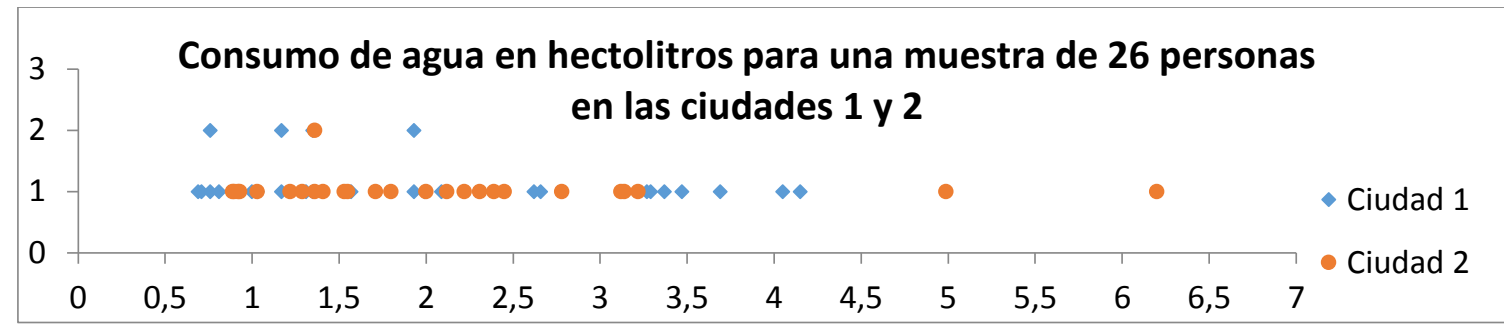

\section{Pregunta 5}

Si consideramos el consumo diario de agua propio de un país subdesarrollado como el nuestro y el consumo que recomienda la OMS, se puede observar que ambas muestras evidencian un uso inadecuado de este preciado líquido. El consumo mínimo de agua en la muestra de la ciudad B $(0,89 \mathrm{hl})$ supera a lo recomendado por esta organización $(0,80 \mathrm{hl})$ y que para el caso de la muestra de la ciudad A no está muy lejos. En otros términos, casi la totalidad de las personas entrevistadas en una u otra ciudad consumen más de lo que recomienda la OMS y de lo que corresponde a un país subdesarrollado.Al mismo tiempo, se espera que los estudiantes puedan ofrecer algunas recomendaciones tendientes a disminuir el consumo de agua.

Se espera que los estudiantes compartan con sus compañeros los hallazgos obtenidos. Se debe prestar atención a la forma en que los estudiantes justifican sus respuestas para garantizar que todas ellas están bien fundamentadas. Aquí, las representaciones gráficas les permitirán comprender más globalmente la situación.

Al final, el docente rescata las siguientes ideas:

- La utilidad de las medidas estadísticas en la caracterización de un conjunto de datos.

- Es importante considerar la distribución de los datos para garantizar que las medidas utilizadas son realmente representativas.

- Un análisis estadístico comparativo debe considerar las medidas de posición, de variabilidad y su relación con el patrón de variabilidad de los datos

- La mediaaritmética y la desviación estándar son muy sensibles a la presencia de valores extremos atípicos, pero puede compensarse observando la mediana, los cuartiles y un diagrama de cajas.

- La moda suela ser volátil y no representa bien al conjunto de datos

- Además de lo que se ha indicado previamente.

\section{Conclusión}

Con el taller planteado, se ha pretendido ejemplificar a los docentes de Matemáticas que en la implementación de los programas de estudio de esta área es posible integrar diversas 
habilidades mediante un solo problema. De este modo, con la aplicación de unos pocos problemas es factible abarcar varios conocimientos y habilidades específicas. Pero además, también se querido dejar en evidencia que si esta integración no se lleva a cabo, se estaría provocando una desarticulación del currículo, lo que implicaría un análisis aislado de conocimientos, y difícilmente se logren las habilidades generales propuestas en dicho programa.

En los dos problemas propuestos para el taller, se logró evidenciar la riqueza conceptual y didáctica que conlleva el planteamiento de situaciones didácticas que articulen diferentes conocimientos y habilidades. En el caso del problema vinculado con probabilidades, se ejemplificó la construcción de concepto clásico de probabilidad, desde la identificación de eventos más y menos probables, y de eventos equiprobables. La posibilidad de articular dichos conceptos, permite tener un conocimiento más integral de la disciplina, lo cual no se logra si los conocimientos se desarrollan en forma desarticulada. Esto también quedó en evidencia, con el segundo problema, en donde fue necesario realizar un análisis integral de representaciones gráficas con la identificación de medidas de posición y variabilidad para responder las interrogantes que generó el problema.

Aunque el primer problema estaba estructurado para generar conocimiento, mientras que el segundo estaba direccionado a la movilización y aplicación de conocimientos adquiridos, ambos dejan claramente evidenciado la puesta en práctica de los fundamentos teóricos establecidos en los programas de estudio. Además de la integración de habilidades que fue discutida previamente, entre otros componentes, se observa la presencia de los procesos matemáticos y de los ejes curriculares debidamente identificados en cada caso.

Finalmente, en relación con la enseñanza de la Estadística y la Probabilidad, los problemas han sido ilustrativos en relación con la flexibilidad que tiene el área para implementar los programas vigentes. Es importante rescatar la forma en que estos problemas, promueven el razonamiento estocástico, desde la perspectiva de los componentes básicos de sus componentes básicos, tal como se discutió en la sección 3. del presente documento.

\section{Referencias Bibliográficas}

Batanero, C. (2004). Los retos de la cultura estadística. Yupana. 1 (04), 27-37

Wild, C. and Pfannkuch, M. (1999). International Statistical Review, 67,3, 223-265.

Fischbein, E. (1975). The intuitive sources of probabilistic thinking in children. Reidel, Holanda.

MEP, (2012). Programas de Estudio en Matemáticas. Ministerio de Educación Pública. San José, Costa Rica 


\title{
Análisis descriptivos con EXCEL de ítems de selección única en pruebas de aula
}

\author{
Tania Elena Moreira Mora
}

\begin{abstract}
Resumen
El propósito de este taller es analizar los ítems de selección única con base en dos características: el nivel de dificultad y el índice de discriminación desde el enfoque de la teoría clásica de los test usando la herramienta de Excel.

El índice de dificultad y discriminación permitirá al docente identificar los ítems ambiguos, con un nivel de dificultad mayor a lo propuesto o errores de claves. Así como construir un banco de ítems por unidades temáticas.

El enfoque del taller será más práctico, centrado en los análisis descriptivos de los ítems, de tal manera que cada participante logre comprender y aplicarlos en sus pruebas de aula, con una herramienta estadística que es accesible para la mayoría. En concreto se analizará las frecuencias de las alternativas para valorar la calidad de los distractores, el índice de discriminación que contribuye a diferenciar entre los examinados con dominio y no dominio del conocimiento y el índice de dificultad que identifica la proporción de aciertos.

Por el enfoque práctico del taller, estos análisis descriptivos son básicos y pueden variar en muestras pequeñas como de las pruebas de aula. No obstante, proporcionan información más específica sobre aciertos y errores del estudiantado y, si se mantiene constante el tipo de muestra, se puede esperar resultados similares y, en general, contribuyen a mejorar la calidad de las pruebas.
\end{abstract}

Palabras clave: Dificultad, discriminación, pruebas de aula, ítems de selección única 


\section{Bibliografía}

De los Santos, G. Desarrollo, operación y evaluación de un módulo para capacitar a docentes en servicio para que mejoren sus exámenes de opción múltiple mediante el análisis gráfico de ítems.

En http://iide.ens.uabc.mx/blogs/mce/files/2011/05/Tesis-GUADALUPE-DE-LOS-SANTOSLAZARO.pdf. (Consultado el 12 de octubre del 2014). 2010

Gómez, I. Análisis evaluativo de calidad de la prueba objetiva tipo test (Preguntas de opción múltiple). En http://institucional.us.es/revistas/universitaria/13/art_8.pdf, (Consultado el 12 de octubre del 2014). 1998.

Hernández, R., Fernández, C. y Baptista, P. Metodología de la investigación (Quinta Edición). Perú: Mc. Graw Hill. 2010.

Morales, P. Análisis de ítems en las pruebas objetivas. En http://web.upcomillas.es/personal/peter/otrosdocumentos/AnalisisItemsPruebasObjetivas.pdf. (Consultado el 12 de octubre del 2014). 2012.

Abad, F.J., Garrido, J., Olea, J. y Ponsoda, V. Introducción a la Psicometría Teoría Clásica de los Tests y Teoría de la Respuesta al Ítem. En http://aprendeenlinea.udea.edu.co/lms/investigacion/file.php/39/ARCHIVOS_2010/PDF/IntPsico metria_aristidesvara_1_.pdf (Consultado el 17 octubre del 2014). 2006 


\title{
La enseñanza de la probabilidad geométrica en primaria y secundaria con ayuda de GEOGEBRA
}

\author{
Marylin Calderón Mora ${ }^{1}$
}

\section{Resumen}

El presente taller se enfoca en la resolución de una guía de ejercicios de probabilidad geométrica con ayuda del software de matemática GEOGEBRA. Dicha guía puede ser aplicada en los niveles de primaria y secundaria, cuando los conceptos geométricos y estadísticos ya han sido vistos en clase. Esta propuesta puede ser enriquecida con la iniciativa propia de cada docente, así mismo puede motivarlo a crear nuevos problemas.

Palabras clave: Probabilidad geométrica, área.

\begin{abstract}
This workshop focuses on the resolution of a guide of exercises of geometric probability with the help of a mathematics software called GEOGEBRA. Said guide may be applied to primary and secondary school, once the geometric and statistic concepts have been taught in class. This proposal may be enriched with the initiative of each teacher, it may also encourage the teachers to create new exercises.
\end{abstract}

Keywords: Geometric probability, area.

\section{Introducción}

Como parte de la reforma educativa aplicada en Costa Rica 2013 para el área de la matemática, toma relevante importancia en la educación integral de la matemática el estudio de la estadística y probabilidad. Estos temas desde hace varios años se había dejado de lado de los contenidos de la enseñanza secundaria. Estadística y Probabilidad no eran parte de los temas a evaluar en los exámenes de Bachillerato aunque si pertenecían a los contenidos de secundaria, por lo que muchos docentes e instituciones destinaban sus recursos a aquellos temas contenidos en dicha prueba.

Actualmente, a nivel internacional, la probabilidad y la estadística se consideran claves en la formación de los futuros ciudadanos, y se señala que su enseñanza inicie desde la pimaria, pues son una herramienta valiosa para la resolución de ciertos problemas. Además, con el uso de medios tecnológicos actuales se ha fortalecido la enseñanza.

${ }^{1}$ Colegio Saint Paul, Costa Rica, mary_cm@yahoo.com 
Los alumnos deben ampliar su visión de la probabilidad a más que monedas, naipes, dados o cualquier juego de azar, los problemas de probabilidad geométrica deben tener sentido, para que los jóvenes tome interés, y empezar con juegos es un muy buena estrategia de lograr captar dicho interés.

\section{Marco teórico}

Para desarrollar una estrategia de resolución de los problemas de probabilidad geométrica en importante que es estudiante domine un serie de conceptos y estrategias de resolución tanto de geometría como de probabilidad, además de la habilidad en cálculos sencillos de aritmética y muy importante, la habilidad del uso de un software adecuado de matemática, lo que se convertirá, si hay posibilidad del uso de este medio, en una forma ágil en la resolución y generación de los problemas de probabilidad geométrica.

Para definir el término Probabilidad Geométrica, primeros haremos una breve descripción de los términos que componen esta frase.

Epistemológicamente el término geometría deriva del griego geo (tierra) y metrón (medida), pero al pasar los siglos y gracias a matemáticos importantes como Euclides, Gauss, Lobatschwesky, John Bolyai, Sacchiary entre otros, se abrió el concepto de geo a todo el espacio y espacio conceptual-abstracto donde vivimos. Pensar en una geometría aplicada al algebra o a la probabilidad nos lleva a un espacio no tangible más conceptual, en donde el uso de la geometría permite relacionar conceptos teórico y abstractos, tomado así forma visual y creando una nuevas estrategias de razonamiento.

El concepto de probabilidad proviene del término en latín probabilitas, el cual se puede traducir como aquella posibilidad que hay entre diversas posibilidades de ocurrencia de un determinado evento. La Probabilidad es una rama de la matemática ya que está se encarga del estudio y medición cuantitativa de que un determinado hecho suceda u ocurra.

La medición de la ocurrencia de una evento $\mathrm{X}$ se denota como $\mathrm{P}(\mathrm{X})$ y está pertenece al intervalo $0 \leq \mathrm{P}(\mathrm{X}) \leq 1$.

Cuando $\mathrm{P}(\mathrm{X})=0$ se sabe con absoluta certeza que la ocurrencia del evento es nula, en otras palabras, es imposible que el evento ocurra, lo llamamos evento imposible.

Si $\mathrm{P}(\mathrm{X})=1$, se sabe con absoluta certeza que el evento va a ocurrir, lo llamamos evento seguro.

Las mediciones de las probabilidades se pueden expresar mediante:

- Notación decimal correspondiente a una fracción propia

- En notación de porcentaje \%, forma más utilizada comúnmente en el lenguaje. Esta es la forma más habitual de tener contacto con las probabilidades en periódicos, revistas, noticias, investigaciones... 
La probabilidad como medición cuantitativa se puede calcular de varios enfoques (Spiegel, 1970)

- Enfoque Clásico: $\quad P(X)=\frac{\text { Cantidad de eventos exitosos }}{\text { Cantidad total de posibles eventos }}$

- Enfoque frecuencias relativas

- Enfoque subjetivo

Otro concepto importante en el tema de las probabilidades es "el azar" o "fenómeno estocástico" definido como:

Se dice que un hecho o fenómeno es de naturaleza estocástica cuando su incidencia no está ligada ni vinculada absoluta o directamente a factores puramente causales propio de modelos deterministas, habiendo fenómenos o hechos cuya ocurrencia sucede en un contexto o margen de indeterminismo lógico o incertidumbre empírica, de forma aleatoria y al azar, motivo por el cual sus métodos de estudio son la estadística y la probabilidad, ya sea para el estudio del comportamiento estocástico de una o más variables (Pedraza, 2013)

Entonces, ahora podemos definir el concepto de Probabilidad como el conjunto de reglas que nos permiten determinar si un fenómeno tiene probabilidades de producirse fundamentado en el cálculo.

Sabiendo el significado de probabilidad y de geometría podemos tener una idea clara de lo que la probabilidad geometría estudia, tomamos problemas geométricos y los combinamos con la probabilidad de que un evento ocurra para calcular dicha probabilidad usamos concepto geométricos básicos como: áreas de polígonos, círculo y circunferencia, plano rectangulares, longitudes de segmentos, por ejemplo.

Con respecto al uso de la tecnología, es bien sabido el aporte de este medio a la enseñanza, para esta propuesta se usará el programa GEOGEBRA ya que por ser un software libre existe accesibilidad de bajarlo a cualquier medio interactivo de una forma sencilla, además el uso del programa en si, es muy fácil tanto para el profesor como para los alumnos. Pero es innegable el aporte, dinamismo e interés por parte de los alumnos del uso de un programa informático en el desarrollo de la resolución de los problemas.

Aun así, si no contáramos con los medios electrónicos para la resolución de los ejercicios, esto no sería un impedimento para llevar la probabilidad geométrica al aula, sus sencillos cálculos los pueden llevar a cabo los estudiantes de secundaria con o sin el uso de una calculadora. 


\section{Propuestas de taller}

\section{Problemas y estrategia de resolución del cálculo de probabilidad de áreas en polígonos.}

Guía de la propuesta del problema: Dibuje una región poligonal R y luego otra región poligonal $\mathrm{M}$ dentro de $\mathrm{R}$ ¿cuál es la probabilidad de que un punto $\mathrm{P}$ elegido al azar de la región $\mathrm{R}$ esté en la región $\mathrm{M}$ a la vez?

\section{Conocimientos a desarrollar:}

- Polígonos

- Cálculo de áreas de polígonos

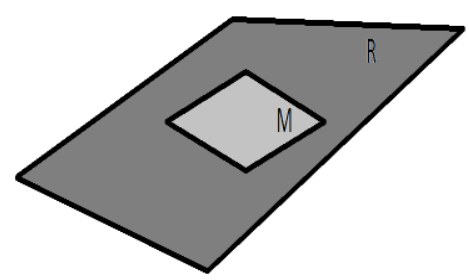

- Cálculo de probabilidad clásica

\section{Guía de estrategia de resolución.}

Se identifica el área posible donde se puede elegir el punto $\mathrm{P}$, la región $\mathrm{R}$, utilizando los datos de las longitudes los segmentos que forman la región $\mathrm{R}$, esta región puede ser cualquier polígono al cual los estudiantes puedan obtener el área.

Para calcular la probabilidad $\mathrm{P}(\mathrm{X})$ de que un punto $\mathrm{P}$ de la región $\mathrm{R}$ esté a la vez en la región $\mathrm{M}$ debemos calcular el área de ambas regiones y aplicamos el concepto de probabilidad clásica resolviendo:

$$
P(x)=\frac{\text { área de exito,región } M}{\text { área total, región } R}
$$

Con este sencillo cálculo podemos obtener la probabilidad de que se elija al azar un punto $\mathrm{P}$ dentro de la región $\mathrm{R}$ y que este esté contenido en de la región $\mathrm{M}$.

En este tipo de problema se pone en práctica conceptos de cálculo de áreas de polígonos, además de los conceptos de probabilidad, el estudiante debe ser capaz de intuir la aplicación de la fórmula de la probabilidad en la resolución del problema. 


\section{Uso de GEOGEBRA}

Si contamos con el software GEOGEBRA el problema puede iniciar con la construcción por parte de los alumnos de los polígonos.

Ejemplo: Usando GEOGEBRA, construya un polígono irregular de 5 lados cuyas medidas son $5,3,3,4$ y 7 , el que representa la región $\mathrm{R}$, otro polígono de tres lados regular de lado 2,5, y calcule la probabilidad de que un punto escogido al azar de la región del polígono de cinco lado esté dentro de la región del triángulo también.

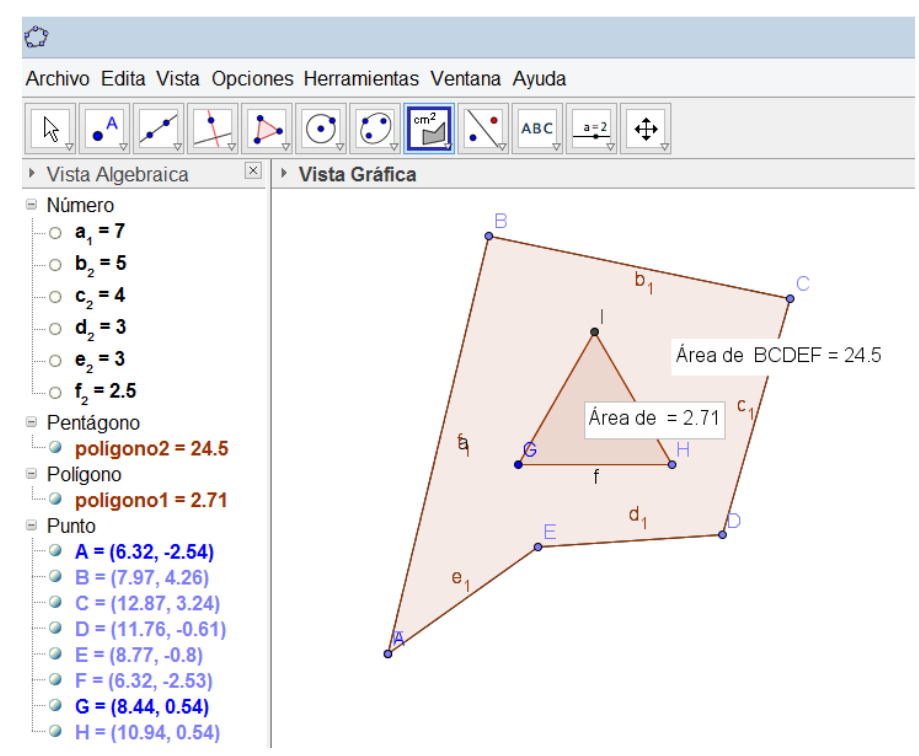

Sea $\mathrm{X}$ el evento que el punto $\mathrm{P}$ elegido al azar de la región del polígono de 5 lados, este en la región de triángulo también.

$P(X)=\frac{2,71}{24,5} \approx 0,1106$

\section{Respuesta del problema utilizando GEOGEBRA}

La respuesta dependerá de cómo se dibujen las regiones $\mathrm{R}$ y M. En el caso de las regiones hechas en GEOGEBRA, encontramos la probabilidad $11,06 \%$ de que, al elegir un punto al azar P de la región del pentágono irregular este esté en la región delimitada por el triángulo equilátero a la vez.

\section{Variaciones del problema}

Algunas modificaciones que se le pueden hacer al problema es trabajar con el área de polígonos irregulares, para los cuales el cálculo del área no es una simple aplicación de una fórmula, o variar el evento éxito; por ejemplo calcular la probabilidad de que el punto elegido esté dentro de la región $\mathrm{R}$ pero no en la región $\mathrm{M}$.

También podemos hacer variaciones en los cálculos de probabilidades más complejos como probabilidades conjuntas, unión e intersección de probabilidades. A nivel de $8^{\circ}$ y $9^{\circ}$ de secundaria del ciclo básico podemos aplicar estos problemas básicos, ya el cálculo de las probabilidades son parte del programa desarrollar. También podemos aplicar los conceptos de evento imposible y evento seguro. 


\section{Ejemplo de problema para desarrollar en el aula}

El problema lo podemos contextualizar con la realidad, por ejemplo:

"Se sabe que cayó una mancha pequeña de tinta sobre una bandera, la bandera tiene forma rectangular de dimensiones 30 $\mathrm{cm}$ y $18 \mathrm{~cm}$, en su interior tiene estampado el símbolo del colegio con forma de rombo de lado $5 \mathrm{~cm}$. ¿cuál es la probabilidad de que la mancha esté sobre el símbolo del colegio?"

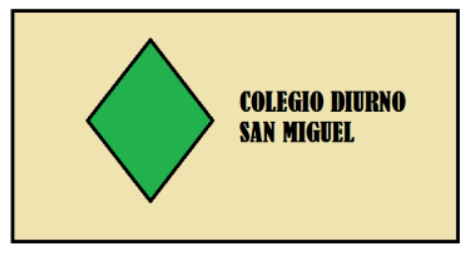

\section{Problemas y estrategia de resolución del cálculo de probabilidad de círculos.}

Guía de la propuesta del problema: Dibuje dos círculos concéntricos, identifique la región de la corona circular R. ¿Cuál es la probabilidad de que un punto $\mathrm{P}$ cualquiera dentro del círculo mayor esté en la corona circular R?

\section{Conocimientos a desarrollar:}

- Círculos

- Cálculo de áreas círculos

- Cálculo de probabilidad clásica

\section{Guía de estrategia de resolución.}

Se identifica el área posible donde se puede elegir el punto $\mathrm{P}$, la región $\mathrm{R}$, utilizando los datos de las longitudes los radios de los círculos y aplicando las fórmula de la corona circular, la región donde definimos el evento como éxito será la corona circular, mientras que la región o área del círculo mayor será la región total de elección del punto al azar.

Aplicamos el concepto de probabilidad clásica en donde

$$
P(x)=\frac{\text { área de exito, región de la corona circular }}{\text { área total del círculo mayor }}
$$

Con este sencillo cálculo podemos obtener la probabilidad de que se elija al azar un punto $\mathrm{P}$ que esté contenido exclusivamente dentro de la corona circular.

En este tipo de problema se pone en práctica conceptos de cálculo de áreas de círculos, además de los conceptos de probabilidad, el estudiante debe ser capaz de intuir la aplicación de la fórmula de la probabilidad en la resolución del problema. 


\section{Uso de GEOGEBRA}

Si contamos con el software GEOGEBRA el problema puede iniciar con la construcción por parte de los alumnos de dos círculos concéntricos dados los radios para formar la corona circular.

Ejemplo: Usando GEOGEBRA, construya una corona circular donde el radio del círculo mayor es 7 y el radio del círculo menos es 3 y calcule la probabilidad de que al elegir un punto $\mathrm{P}$ cualquiera que esté en la corona circular.

Sea $\mathrm{X}$ el evento que el punto $\mathrm{P}$ elegido al azar de la región de la corona circular.

$$
P(X)=\frac{153,94-28,27}{153,94}=\frac{125,67}{153.94} \approx 0,816
$$

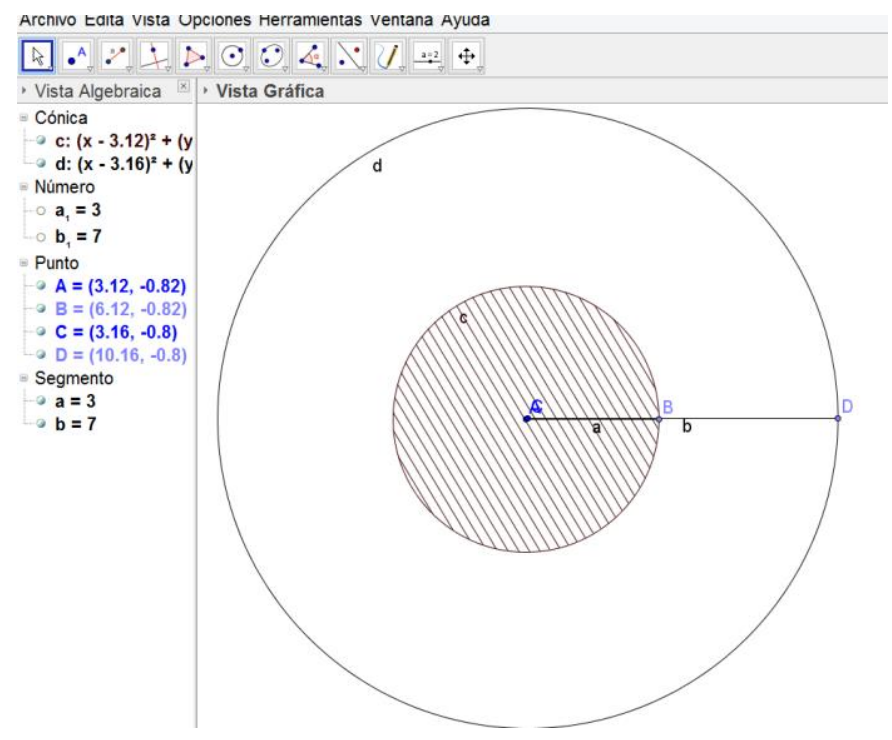

\section{Respuesta del problema utilizando GEOGEBRA}

La respuesta dependerá de cómo dibujemos los círculos, en el caso de los círculos dibujados con GEOGEBRA, la probabilidad de que un punto cualquiera dentro del círculo mayor esté en la corona circular $\mathrm{R}$ es de $81,6 \%$.

\section{Variaciones del problema}

Algunas modificaciones que se le pueden hacer al problema es comparar el cálculo de las probabilidades al variar el radio de uno de los círculos. También podemos hacer variaciones en los cálculos de probabilidades más complejos como probabilidades conjuntas, unión e intersección de probabilidades. A nivel de $8^{\circ}$ y $9^{\circ}$ de secundaria del ciclo básico podemos aplicar estos problemas básicos, ya el cálculo de las probabilidades son parte del programa desarrollar. También podemos aplicar los conceptos de evento imposible y evento seguro.

Otra variación que se puede aplicar es la combinación de áreas de polígonos con círculos, lo que se deja a la creatividad del profesor o maestro. 


\section{Ejemplo de problemas para desarrollar en el aula}

El problema lo podemos contextualizar con la realidad, por ejemplo:

"Se juega tiro al blanco, con el siguiente tablero, ¿Cuál es la probabilidad de obtener

a) 10 puntos?

b)5 puntos?

c)2 puntos?

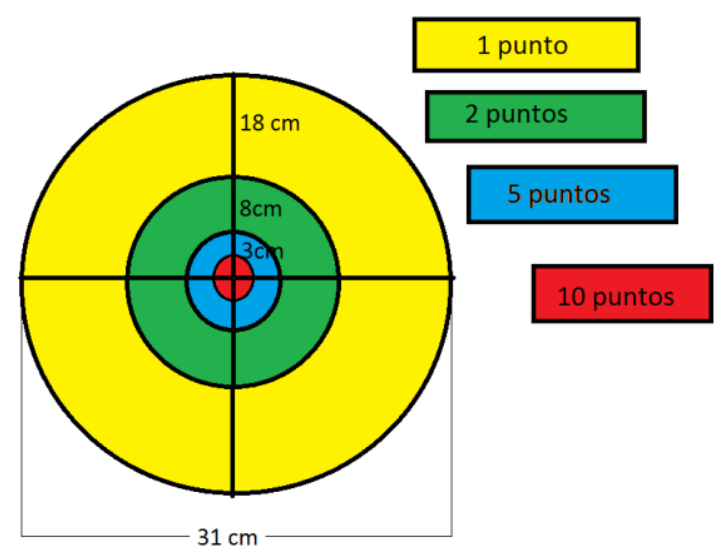

d)1 punto?

\section{Problemas y estrategia de resolución del cálculo de probabilidad de longitudes de segmentos.}

Guía de la propuesta del problema: Dibuje un segmento $\mathrm{AB}$, y los puntos $\mathrm{C}$ y $\mathrm{D}$ que estén contenidos en el segmento $\mathrm{AB}$. ¿Cuál es la probabilidad de que un punto $\mathrm{P}$ que pertenece a $\mathrm{AB}$ pertenezca al segmento $\mathrm{CD}$ ?

Conocimientos a desarrollar:

- Conceptos básicos de geometría.

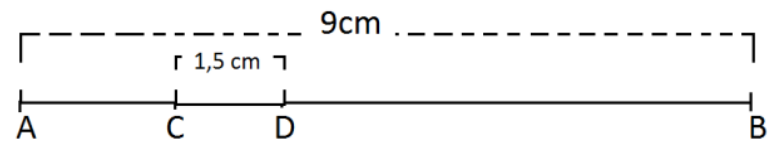

- Cálculo de probabilidad clásica

\section{Guía de estrategia de resolución.}

Teniendo la longitud del segmento $\mathrm{AB}$ y la del segmento $\mathrm{CD}$, se identifica que el éxito del evento será que el punto $\mathrm{P}$ al azar pertenezca al segmento $\mathrm{CD}$ por lo que el cálculo de la probabilidad es el cociente entre la longitud del segmento $\mathrm{CD}$ y la longitud del segmento AB.

Aplicamos el concepto de probabilidad clásica en donde

$$
P(x)=\frac{\text { longutid del segmeto } C D}{\text { longitud del segmento } A B}
$$


Con este sencillo cálculo podemos obtener la probabilidad de que se elija al azar un punto que pertenezca al segmento $\mathrm{AB}$ y que a la vez pertenezca al segmento $\mathrm{CD}$.

En este tipo de problema se pone en práctica conceptos de geometría, como punto, segmento, pertenencia a un segmento....además de los conceptos de probabilidad, el estudiante debe ser capaz de intuir la aplicación de la fórmula de la probabilidad en la resolución del problema.

\section{Uso de GEOGEBRA}

Si contamos con el software GEOGEBRA el problema puede iniciar con la construcción por parte de los alumnos de los segmentos, uno que esté contenido en el otro.

Ejemplo: Usando GEOGEBRA, construya dos segmentos AB con una longitud de 9 unidades lineales y el segmento CD con 1,5 unidades lineales. De forma que el segmento CD esté contenido en el segmento $\mathrm{AB}$ y calcule la probabilidad de elegir al azar un punto cualquiera del segmento $\mathrm{AB}$, este esté en el segmento CD a la vez, redondeé la cifra a dos decimales.

El evento éxito del ejercicio es que el punto esté en los $1,5 \mathrm{~cm}$ de

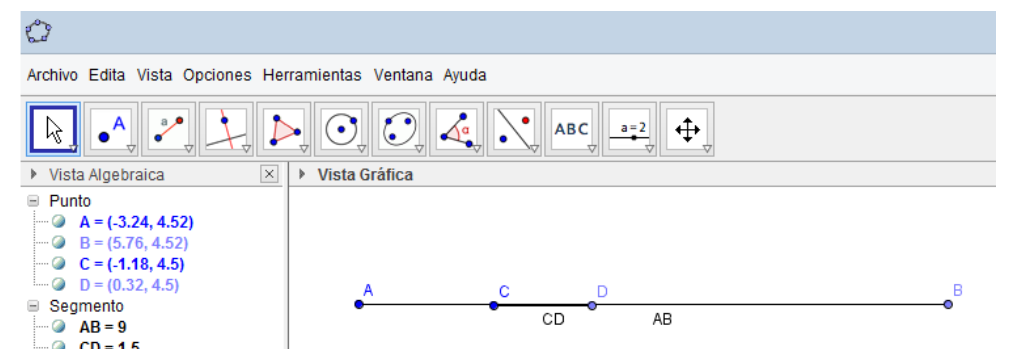
CD.

$P(X)=\frac{1,5}{9} \approx 0,17$

\section{Respuesta del problema utilizando GEOGEBRA}

La respuesta dependerá de la longitud del segmento $\mathrm{AB}$ construido con GEOGEBRA y la ubicación que se le dé a los puntos $\mathrm{C}$ y $\mathrm{D}$, por lo tanto, según la construcción, la probabilidad de que un punto que pertenece al segmento $\mathrm{AB}$ pertenezca a la ves al segmento $\mathrm{CD}$ es $17 \%$.

\section{Variaciones del problema}

Algunas modificaciones que se le pueden hacer al problema es trabajar con diferentes unidades, también se puede variar la pregunta del ejercicio y cambiar el evento éxito; calcular la probabilidad de que un punto al azar de $\mathrm{AB}$ no esté en el segmento $\mathrm{CD}$.

\section{Ejemplo de problema para desarrollar en el aula}

El problema lo podemos contextualizar con la realidad, por ejemplo: 
"Juan está de visita en la cuidad de Heredia, y toma en autobús para llegar al Universidad Nacional. Se supone que se encuentre con un amigo en la universidad a la 3 p.m. El autobús que le sirve pasa cada 10 minutos y el viaje a la universidad dura 8 minutos. Si Juan llega a la parada del bus a las 2: 48 p.m. ¿Cuál es la probabilidad de que Juan llegue puntual a ver a su amigo a 3 p.m.?"

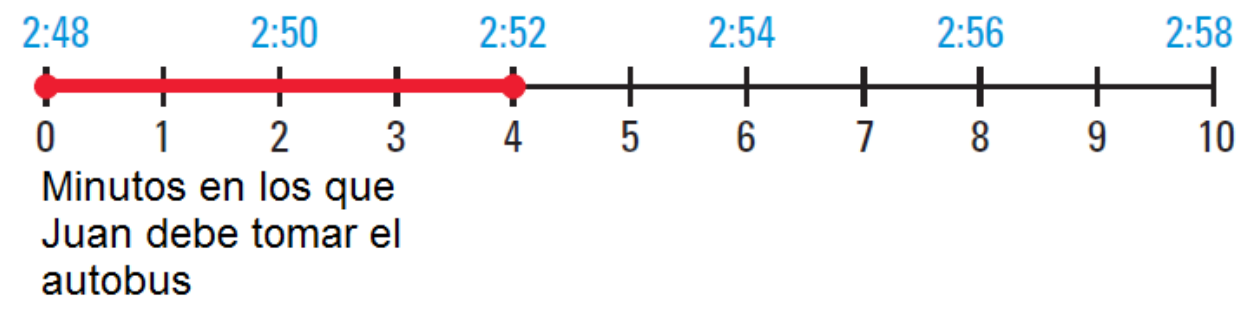

\section{Problemas y estrategias de resolución del cálculo de probabilidad de la ubicación de pares ordenados.}

Guía de la propuesta del problema: En un plano de coordenadas rectangulares grafique la recta $\mathbf{y}=\mathbf{- 2 x + 1 0}$ con $\mathbf{0}<\mathbf{x}<\mathbf{5}$ y $0<\mathbf{y}<\mathbf{1 0}$. Encuentre la probabilidad de que en un punto elegido al azar del plano la coordenada $\mathbf{x}$ sea menor que la coordenada $\mathbf{y}$

\section{Conocimientos a desarrollar:}

- Conceptos básicos de geometría.

- Concepto de ecuación de la recta.

- Puntos de intersección de rectas.

- Algebra.

- Grafica de rectas.

- Cálculo de áreas de polígonos.

- Cálculo de probabilidad clásica.

\section{Guía de estrategia de resolución.}

Primero se grafica la recta y la recta de los pares ordenados donde $\mathbf{x}=\mathbf{y}$, se identifican los polígonos

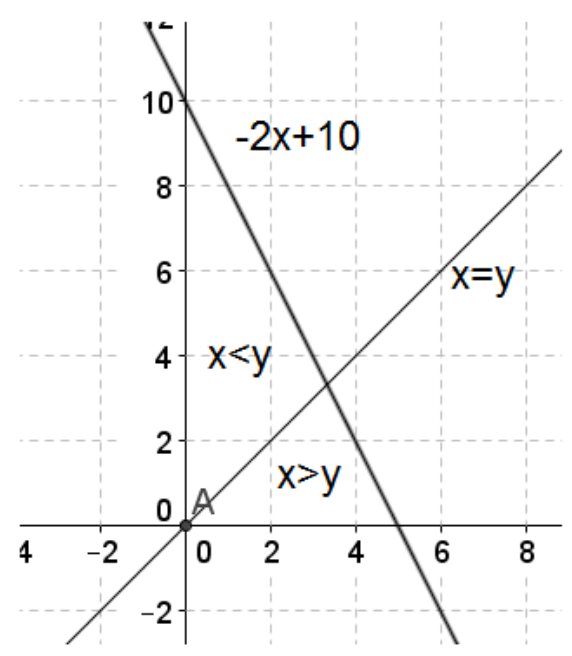
que se forman para obtener el área y calcular las probabilidades.

Sabemos que los pares ordenado que estén ubicados en la parte superior de la recta $\mathbf{y}=\mathbf{x}$ tendrán la forma $\mathbf{x}<\mathbf{y}$, y los pares ordenados que estén ubicados en la parte inferior de la recta $\mathbf{y}=\mathbf{x}$, tendrán la forma $\mathbf{x}>\mathbf{y}$. 
Aplicamos el concepto de probabilidad clásica en donde

$$
P(x)=\frac{\text { área del polígono donde } x>y}{\text { área total debajo de la recta }-2 x+10 \text { y los ejes }}
$$

Con este sencillo cálculo podemos obtener la probabilidad de que se elija al azar un punto que corresponda a un par ordenado en donde $\mathbf{x}>\mathbf{y}$ que pertenezca a la región delimitada por la recta y los ejes.

\section{Uso de GEOGEBRA}

Si contamos con el software GEOGEBRA el problema puede iniciar con la construcción por parte de los alumnos de la recta del problema y la recta $\mathbf{x}=\mathbf{y}$ para identificar las áreas de los polígonos en donde la condición $\mathbf{x}<\mathbf{y}$ se cumple.

Ejemplo: Usando GEOGEBRA: Calcule la probabilidad de que un punto elegido al azar pertenezca a la región delimitada por la recta $-2 x+10$ y los ejes, con la condición que $\mathbf{x}<\mathbf{y}$.

El evento éxito del ejercicio es que el punto elegido al azar esté ubicado dentro del área que cumple con la condición del problema.

$P(X)=\frac{8,21}{16,79+8,21} \approx 0,328$

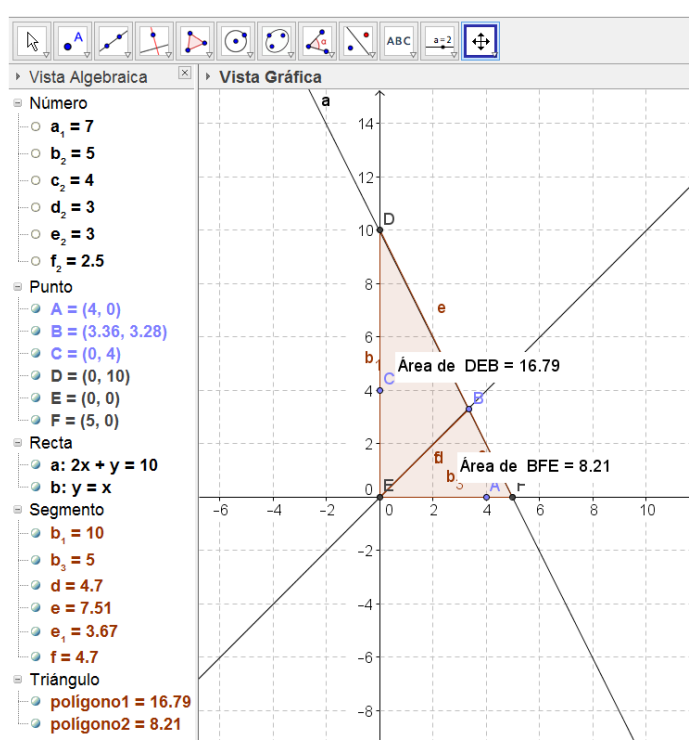

\section{Respuesta del problema utilizando GEOGEBRA}

Según las áreas de los polígonos obtenidos con GOEGEBRA; existe una probabilidad de 0,328 o $32,8 \%$ de elegir un punto al azar dentro del área delimitada por la recta $\mathbf{y}=\mathbf{- 2} \mathbf{x}+\mathbf{1 0}$ y los ejes de coordenadas que tenga la condición $\mathbf{x}<\mathbf{y}$.

\section{Variaciones del problema}

Algunas modificaciones que se le pueden hacer al problema es trabajar con diferentes ecuaciones de recta, intersección entre rectas y ejes, la estrategia de resolución del problema implica un alto dominio de los temas relacionados. 


\section{Ejemplo de problema para desarrollar en el aula}

El problema lo podemos contextualizar con la realidad, por ejemplo:

"Una empresa de productora de queso artesanal debe adquirir una combinación de dos tipos de e hierbas para la fabricación del queso con hierbas frescas, debe gastar un monto máximo de 10000 colones. El costo en hiervas de la compra se refleja en la ecuación $\mathbf{x}+\mathbf{y}=\mathbf{1 0 0 0 0}$, donde $\mathbf{x}$ y y son los dos tipos de hierbas frescas. ¿Cuál es la probabilidad de que la empresa compre más de la hierba $\mathbf{x}$ que de la $\mathbf{y}$ ? “

\section{Bibliografía}

Jose Barraqués F, J. G. (diciembre de 2009). Una propuesta para la enseñanza de la probabilidad en la universidad basada en la investigación didáctica. Obtenido de http://www.redalyc.org/pdf/405/40516671006.pdf

Marilú Rioseco G, R. R. (n.d.). Retrieved from www.oei.es/equidad/rioseco3.PDF

Pedraza, R. G. (2013). Estadística de la probabilidad o probabilidad estadística. Retrieved from http://probabilidadimposible.blogspot.com/2013/04/la-naturaleza-estocastica-de-la.html

Renato Allende O, H. T. (n.d.). Universidad Técnica Federico Santamaría . Retrieved from Departamento de Matemática:

http://hvillalobos.mat.utfsm.cl/Apuntes/A.04\%20Apunte\%20Cuarto\%20M\%F3dulo.pdf

Spiegel, M. (1970). Estadisticas. México: McGraw-Hill. 


\title{
Investigación Reproducible con Markdown-Knitr-R
}

\author{
Jesús Humberto Cuevas Acosta $1^{1}$
}

\section{Resumen}

En este taller se utilizará el lenguaje de marcado Markdown, el entorno de programación R, además del paquete Knitr como intermediario en la elaboración de un informe dinámico y reproducible producto de una investigación realizada en el campo de la estadística o el análisis inteligente de datos.

Palabras clave: investigación reproducible, educación estadística, Markdown, R, Knitr.

\section{Requerimientos}

a) Entorno de programación $\mathrm{R}$ versión 3.1.1 o superior

b) Entorno de Desarrollo Integrado: RStudio versión 0.98.1083 o superior

c) Paquetes de R: Knitr, markdown, xtable, rmarkdown, stargazer

d) Conexión a Internet

e) Videobeam

\footnotetext{
${ }^{1}$ Instituto Tecnológico de Chihuahua II, México. jesus.humberto.cuevas@ outlook.com
} 